
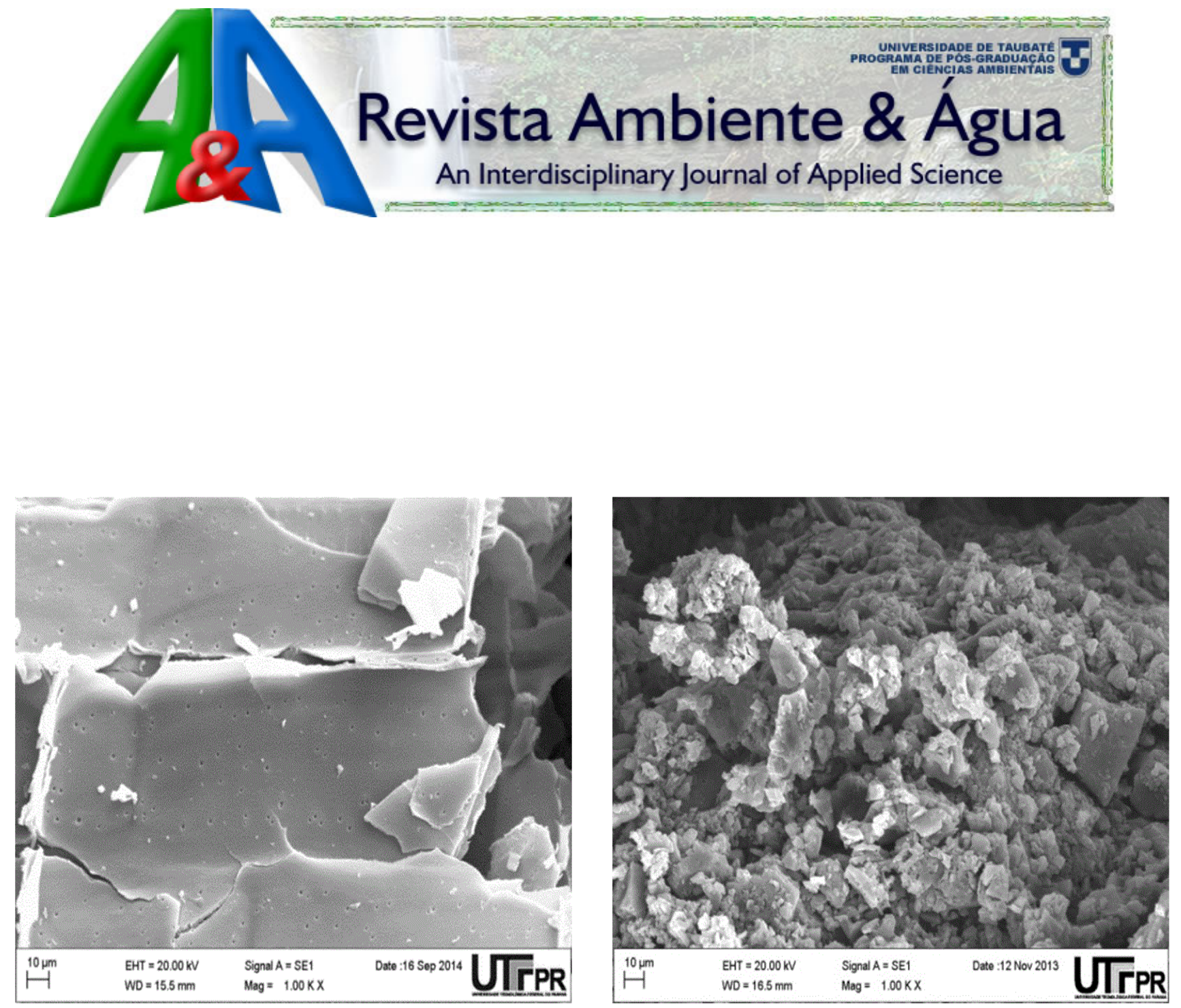

$\mathbf{N}=1980-993 X$ (Online) http://www.ambi-agua.net

$32^{\text {nd }}$ Edition of Revista Ambiente \& Água - An Interdisciplinary Journal of Applied Science, Taubaté, V. 11, N. 1, p. 4-238 Jan./Mar. 2016. (doi:10.4136/ambi-agua.v11.n1) 


\section{EDITORIAL BOARD}

\section{Editor}

\section{Getulio Teixeira Batista}

Instituto de Pesquisas Ambientais em Bacias Hidrográficas (IPABHi), SP, BR

Amaury Paulo de Souza

Ana Aparecida da Silva Almeida

Andrea Giuseppe Capodaglio

Antonio Evaldo Klar

Antonio Teixeira de Matos

Carlos Eduardo de M. Bicudo

Cláudia S. de C. M. dos S. Cordovil

Dar Roberts

Delly Oliveira Filho

Ehsan Noroozinejad Farsangi

Gabriel Constantino Blain

Giordano Urbini

Gustaf Olsson

Hélio Nobile Diniz

János Fehér

João Vianei Soares

José Carlos Mierzwa

Julio Cesar Pascale Palhares

Luis Antonio Merino

Marcelo dos Santos Targa

Massimo Raboni

Nelson Wellausen Dias

Petr Hlavínek

Richarde Marques da Silva

Sergio Francisco de Aquino

Silvio Jorge Coelho Simões

Stefan Stanko

Teresa Maria Reyna

Yosio Edemir Shimabukuro

Zhongliang Liu Beijing

\section{Associate Editors}

Universidade Federal de Viçosa (UFV), BR

Universidade de Taubaté (UNITAU), BR

University of Pavia, ITALY

Universidade Est. Paulista Júlio de Mesquita Filho (UNESP), BR

Universidade Federal de Viçosa (UFV), BR

Instituto de Botânica, IBT, BR

Centro de estudos de Engenharia Rural (CEER), Lisboa, Portugal

University of California, Santa Barbara, United States

Universidade Federal de Viçosa (UFV), BR

International Institute of Earthquake Engineering and Seismology(IIEES), Farmanieh, Tehran, Iran

Instituto Agronômico de Campinas, IAC, Brasil.

University of Insubria, Varese, Italy

Lund University, Lund, Sweden

Inst. Geológico, Sec. do Meio Amb. do Est. de SP (IG/SMA),BR

Debrecen University, Hungary

Instituto Nacional de Pesquisas Espaciais (INPE), BR

Universidade de São Paulo, USP, BR

Embrapa Pecuária Sudeste, CPPSE, São Carlos, SP, BR

Institute of Regional Medicine, National University of the Northeast, Corrientes, Argentina

Universidade de Taubaté, (UNITAU), BR

Polytechnic University of Milan, Milan, Italy

Inst. Bras. de Geogr. e Estatística (IBGE). Aracaju, SE, BR

Brno University of Technology República Tcheca

Universidade Federal da Paraíba, UFPB, BR

Universidade Federal de Ouro Preto, UFOP, BR

Univ. Est. Paulista Júlio de Mesquita Filho, UNESP, BR

Slovak Technical University in Bratislava Slovak, Eslováquia

Universidad Nacional de Córdoba, Argentina

Instituto Nacional de Pesquisas Espaciais (INPE), BR

University of Technology, China

\section{Nelson Wellausen Dias, PPGCA, UNITAU, IBGE, BR}

Theodore D`Alessio, FL, USA, Maria Cristina Bean, FL, USA

Getulio T. Batista, UNITAU, BR

Text Editors

Reference Editor

Layout Editors

Technical Support

Secretary and Communication
Liliane Castro, Bibliotecária - CRB/8-6748, Taubaté, BR

Vera L. F. Batista, IPABHi \& Getulio T. Batista, UNITAU, BR

Tiago dos Santos Agostinho, LAGEO, UNITAU, BR

Luciana Gomes de Oliveira, UNITAU, BR

Library catalog entry by Liliane Castro CRB/8-6748

Revista Ambiente \& Água - An Interdisciplinary Journal of Applied Science / Instituto de Pesquisas Ambientais em Bacias Hidrográficas. Taubaté. v. 11, n. 1 (2006)-

Taubaté: IPABHi, 2016.

Trimestral

ISSN 1980-993X

1. Ciências ambientais. 2. Recursos hídricos. I. Instituto de Pesquisas Ambientais em Bacias Hidrográficas. 


\section{TABLE OF CONTENTS}

\section{COVER}

Electronic photomicrographs of surface scan of: a) sugarcane bagasse and b) activated carbon; both magnified to $1.0 \mathrm{kX}$. Comparison between the retention of microcystin-LR by sugarcane bagasse and by activated carbon in natura applied as a bio-adsorbent in drinking waters.

Source: ALMEIDA, A. R. et al. Remoção de microcistina-LR da Microcystis aeruginosa utilizando bagaço de cana-de-açúcar in natura e carvão ativado. Rev. Ambient. Água, Taubaté, vol. 11 n. 1, p. 188-197, 2016. doi:10.4136/ambi-agua.1785

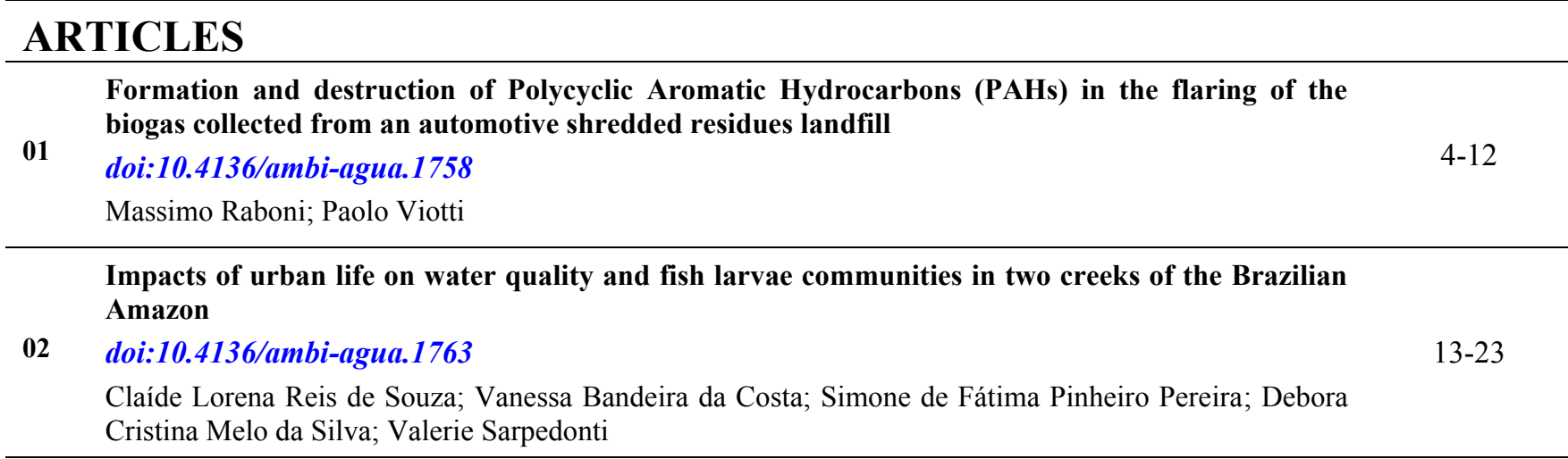

Histopathological assessment of $\mathrm{C57} \mathrm{BI} / \mathrm{J}$ mice organs exposed to tannery effluents doi:10.4136/ambi-agua.1694

03 Joyce Moreira de Souza; Abraão Tiago Batista Guimarães; Wellington Alves Mizael da Silva; Bruna de Oliveira Mendes; Dieferson da Costa Estrela; Aline Sueli de Lima Rodrigues; Adriana da Silva Santos; Guilherme Malafaia

Modelado espacial de incendios: una herramienta predictiva para el Bosque La Primavera, Jalisco México

04 doi:10.4136/ambi-agua.1536

Jose Luis Ibarra-Montoya; Francisco Martín Huerta-Martínez

Peroxidase de gramínea de Cerrado como alternativa no tratamento de efluentes agroindustriais

05 doi:10.4136/ambi-agua.1735

Raquel Pinheiro Reis Souza Ramalho; Paulo Sérgio Scalize; Samantha Salomão Caramori Modelagem do regime térmico de um reservatório tropical de abastecimento público, Juiz de Fora,
MG, Brasil
dob $10.4136 / a m b i$-agua.1544
Flaviane de Fátima Cândida de Souza; Celso Bandeira de Melo Ribeiro; Carlos Ruberto Fragoso Júnior;
Marcelo Henrique Otenio

Qualidade da água de três locais com potenciais fontes de contaminação no Rio Taquari, RS

07 doi:10.4136/ambi-agua.1789

Thais Muller; Camille Eichelberger Granada; Raul Antonio Sperotto

Aplicação e análise comparativa de três protocolos de avaliação rápida para caracterização da

08 paisagem fluvial

doi:10.4136/ambi-agua.1732

$85-97$

Jucimara Andreza Rigotti; Cesar Augusto Pompêo; Alessandra Larissa D’Oliveira Fonseca

Indicadores de produtividade aplicados à pesca artesanal do camarão sete-barbas, Penha, SC,

09 Brasil

doi:10.4136/ambi-agua.1659

Vicente Francisco Coelho; Joaquim Olinto Branco; Marco Antonio Harms Dias 
Estrutura comunitária de samambaias em mata ciliar: avaliação em gradiente de antropização

Ivanete Teresinha Mallmann; Vinícius Leão da Silva; Jairo Lizandro Schmitt

Mapa de aptidão do solo para a aplicação de lodo de esgoto: Bacia dos rios Piracicaba, Capivari e Jundiaí

11

doi:10.4136/ambi-agua.1714

Rodrigo Custódio Urban; Ricardo de Lima Isaac

Teores de Arsênio em solos de três regiões do estado de Santa Catarina

12 doi:10.4136/ambi-agua.1746

Leticia Cristina de Souza; Mari Lucia Campos; Gabriela Reichert; Carolina Natel de Moura

Potencial toxicológico de metais presentes em solos de cemitérios de Santa Maria - RS

doi:10.4136/ambi-agua.1723

13 Bruno Casanova Vilaverde Gomes; Gislaine Kummer; Márcia Maria da Silva Monteiro Pereira; Hécio José Izário Filho; Rodrigo Fernando dos Santos Salazar; Noeli Júlia Schüssler de Vasconcellos

Bioindicadores para avaliação do risco potencial de transmissão da esquistossomose no açude

14 Apipucos, Pernambuco

doi:10.4136/ambi-agua.1756

$156-161$

Rodrigo Moraes Loyo; Constança Simões Barbosa

Índices de qualidade da água e de estado trófico do rio Caiabi, MT

15 doi:10.4136/ambi-agua.1769

Grasiane Andrietti; Rosane Freire; Adriana Garcia do Amaral; Frederico Terra de Almeida; Milene

Carvalho Bongiovani; Roselene Maria Schneider

Análise multivariada para seleção de parâmetros de monitoramento em manancial de Juiz de Fora, Minas Gerais

16

doi:10.4136/ambi-agua.1590

176-187

Cézar Henrique Barra Rocha; Amanda Maia Pereira

Remoção de microcistina-LR da Microcystis aeruginosa utilizando bagaço de cana-de-açúcar in natura e carvão ativado

17 doi:10.4136/ambi-agua.1785

188-197

Aline Rafaela de Almeida; Fernando Hermes Passig; Thomaz Aurélio Pagioro, Priscila Tiemi Higuti do Nascimento; Karina Querne de Carvalho

Estudo da qualidade da água por meio de bioindicadores bentônicos em córregos da área rural e urbana

18

doi:10.4136/ambi-agua.1672

Sandro Morais Pimenta; Geraldo Resende Boaventura; Alfredo Palau Peña; Tiago Godoi Ribeiro

Pegada hídrica dos suínos abatidos na região do Corede Serra, RS, Brasil

19 doi:10.4136/ambi-agua.1688

Vania Elisabete Schneider; Sofia Helena Zanella Carra

Análise de procedimentos para seleção de áreas prioritárias em programas de pagamento por serviços ambientais hídricos

doi:10.4136/ambi-agua.1782

Ana Feital Gjorup; Elaine Cristina Cardoso Fidalgo; Rachel Bardy Prado; Azeneth Eufrasino Schuler 


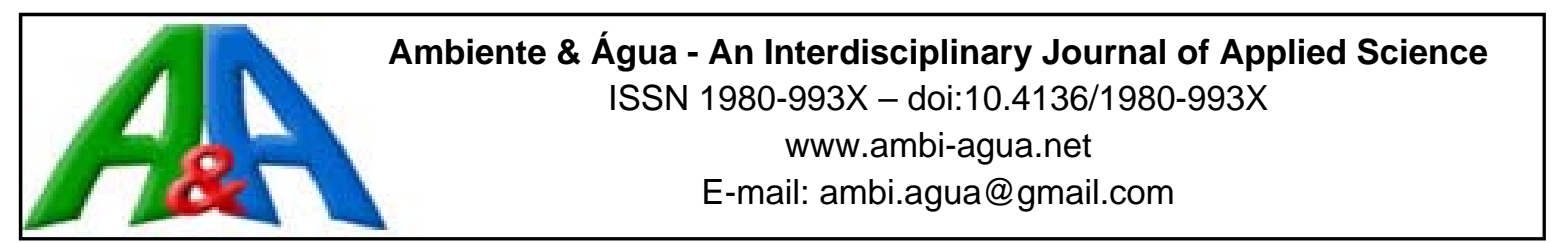

\title{
Formation and destruction of Polycyclic Aromatic Hydrocarbons (PAHs) in the flaring of the biogas collected from an automotive shredded residues landfill
}

\author{
doi:10.4136/ambi-agua.1758 \\ Received: 17 Sep. 2015; Accepted: 15 Dec. 2015 \\ Massimo Raboni ${ }^{1}$; Paolo Viotti ${ }^{2 *}$ \\ ${ }^{1}$ University LIUC - Cattaneo, 21053 Castellanza, VA, Italy \\ School of Industrial Engineering \\ ${ }^{2}$ University of Roma “La Sapienza”, Via Eudossiana 18, I-00184 Rome, Italy \\ Department of Civil and Environmental Engineering \\ *Corresponding author: e-mail: paolo.viotti@uniroma1.it, \\ mraboni@liuc.it
}

\begin{abstract}
The paper shows the results of the combustion in an enclosed flare of the biogas collected from an automotive shredded residues landfill. The results demonstrate that at $1,000^{\circ} \mathrm{C}$ and long combustion, several synthesis reactions lead to the formation of 4 to 6 rings of PAHs. This formation also involves the formation of compounds such as benzo(g,h,i)perylene, indeno(1,2,3-cd)pyrene and dibenzo(a,h)anthracene not present in raw biogas. However, the compounds most likely to form in combustion are benzo (a) anthracene and benzo (b) fluoranthene. The only exception is chrysene which is significantly destroyed. The experience has proved the total lack of formation of PAHs with only 2 and 3 aromatic rings.
\end{abstract}

Keywords: automotive shredded residues, biogas, combustion, PAHs.

\section{Formação e destruição de hidrocarbonetos aromáticos policíclicos (HAP) na combustão do biogás coletado de um aterro de resíduos de veículos desmantelados}

\section{RESUMO}

O artigo mostra os resultados da combustão em um flare enclausurado de biogás coletado de um aterro de resíduos de veículos desmantelados. Os resultados demonstram que a $1,000^{\circ} \mathrm{C}$ e combustão longa, várias reacções de síntese conduzem à formação de 4 a 6 anéis aromáticos policíclicos. Esta formação também envolve a formação de compostos, tais como o benzo(g,h,i)perileno, indeno(1,2,3-cd)pireno e dibenzo(a,h)antraceno não presentes no biogás bruto. No entanto, os compostos que mostraram a maior formação em combustão são o benzo(a)antraceno e benzo(b)fluoranteno. A única excepção é o criseno que sofre um efeito significativo de destruição. A experiência provou a total falta de formação de HAP com apenas 2 e 3 anéis aromáticos.

Palavras-chave: biogás, combustão, HAP, resíduos de veículos desmantelados. 


\section{INTRODUCTION}

Automotive Shredded Residues (ASRs) are generally disposed of in landfills, resulting in significant environmental and health issues (US-DHHS, 1995; Raboni et al., 2014). However, in some cases thermal treatment plants are used (Ciacci et al., 2010; Mancini et al., 2014). The ASR is characterized by the presence of hazardous inorganic compounds (particularly lead, cadmium, and copper), along with hazardous organic compounds derived from fuels, lubricating oils, paints, combustion residues, and solvents used in vehicle maintenance and cleaning (Torres et al., 2013). The composition of the ASR and the presence of hazardous inorganic compounds are well documented in the literature (Raboni et al., 2014; Santini et al., 2012; Mancini et al., 2014; Cossu et al., 2014). The leachate produced by these landfills has a significant amount of organic compounds and heavy metals and its treatment is carried out in a manner similar to the leachate from municipal solid waste landfills; in particular physicalchemical processes (Raboni et al., 2013) and biological activated sludge processes, and also MBR are applied (Torretta et al., 2013).

Biogas production from ASR landfills represents a very small share of the total production of biogas from different sources, which in Europe amounts approximately to 10 Mtoe $^{-1}$ (Eurobserv'Er, 2012; Raboni and Urbini, 2014; Raboni et al., 2015). However, it is characterized by the presence of a broad spectrum of hazardous organic compounds, in the categories of VOCs and PAHs, which are typical ubiquitous compounds as they are normally found in urban areas and along automotive routes (Mohamed et al., 2002). PAHs are typically generated by combustion processes, whereas the VOCs detected in the air are also connected to many to industrial activities; in some cases the VOCs are found in the exhaust air of water and wastewater stripping processes (Raboni et al., 2013).

With reference to hazardous organic compounds found in the biogas of ASR landfills, two recent studies measured the level of PAHs and VOCs in this biogas (Raboni et al., 2015; Urbini et al., 2014). Due to their high toxicity and carcinogenicity properties, it is essential to minimize the release of these compounds into the environment and, above all, into the atmosphere, in order to protect public health. While physical-chemical and thermal processes (Mancini et al., 2014; Innocenti et al., 2014) are regularly applied for the removal of these compounds from waste air streams, actually many studies and also real-scale applications are exploring biological treatment processes (Copelli et al., 2012; Mathur et al., 2007).

The main objective of the present research is to verify that PAHs may be destroyed by the combustion of biogas at high temperature in an enclosed ground flare. Attention was essentially focused on PAHs, as they represent the major risk to human health due to their toxicological and carcinogenic properties. In fact, dibenz(a,h)anthracene, benzo(a)pyrene, benzo(a)anthracene, benzo(b)fluoranthene, benzo(k)fluoranthene, chrysene and indeno(1,2,3-c,d)pyrene are considered probable human carcinogens (while acenaphthylene, anthracene, benzo(g,h,i)perylene, fluoranthene, fluorene, phenanthrene, and pyrene are not classified as carcinogenic) (US-DHHS, 1995). Dibenz(a,h)anthracene and benzo(a)pyrene especially are assigned the highest values of Toxicity Equivalent Factor (TEF), assessed in relation to carcinogenicity $(\mathrm{TEF}=5$ for $\operatorname{dibenz}(\mathrm{a}, \mathrm{h})$ anthracene; $\mathrm{TEF}=1$ for benzo(a)pyrene), while significantly smaller values are assigned to chrysene $(\mathrm{TEF}=0.01)$ and pyrene $(\mathrm{TEF}=0.001)$ (US-DHHS, 1995).

Indeed, several recent studies have shown that the formation and destruction of PAHs are concomitant phenomena, capable of leading to a net increase of these hazardous compounds in the output of combustion systems. This finding has also been verified in the combustion of methane and other light hydrocarbons.

The research was developed in an enclosed flare of a real-scale ASRs landfill. 


\section{EXPERIMENTAL SECTION}

\subsection{Combustion flare characteristics}

The landfill is equipped with 24 wells of biogas equally distributed over the entire landfill area, each discharging into two parallel, enclosed flares. The research was focused on one of the two flares fed by $700 \mathrm{Nm}^{3} \mathrm{~h}^{-1}$ of biogas.

The main features of the enclosed ground flare were as follows:

- diameter: $1.6 \mathrm{~m}$

- height of combustion area: $4 \mathrm{~m}$

- average temperature $1,000^{\circ} \mathrm{C}$

- average retention time: $3.6 \mathrm{~s}$

- combustion chamber lined with refractory (internally) and mineral wool (externally)

- multi-nozzle stainless steel burner

- combustion temperature measured with thermocouple (accuracy $\pm 1{ }^{\circ} \mathrm{C}$ )

- Measurement of Oxygen in the combustion chamber with a zirconinum oxide probe suitable for temperatures up to $1,400^{\circ} \mathrm{C}$ (accuracy: $\pm 1 \%$ ); Throughout the experiment, $\mathrm{O}_{2}$ was kept in the range $11-13 \%$ (average 12\%)

\subsection{Analyzed compounds}

The research was conducted in a three-month period during which 60 samples were collected on different days ( 30 of the raw biogas; 30 of the flare outlet).

The following compounds were detected:

a) sixteen PAHs in raw biogas and at the end of combustion flare: benzo(g,h,i)perylene, indenopyrene, dibenz(a,h)anthracene, benzo(a)pyrene, benzo(k)fluoranthene, benzo(b)fluoranthene, benz(a)anthracene, chrysene, pyrene, fluoranthene, anthracene, phenanthrene, fluorene, acenaphthene, acenaphthylene, naphthalene;

b) In raw biogas: $\mathrm{CH}_{4}, \mathrm{O}_{2}, \mathrm{~N}_{2}, \mathrm{CO}_{2}$ (with in addition, for a better characterization: $\mathrm{NH}_{3}$, $\mathrm{H}_{2} \mathrm{~S}$, Mercaptans, alkyl-sulfides: VOCs).

\subsection{Methods of sampling and analysis}

Measurement of $\mathrm{CH}_{4}, \mathrm{CO}_{2}, \mathrm{~N}_{2}$ and $\mathrm{O}_{2}$ in the raw biogas was done with an infrared analyzer LFG 20 (resolution $0.01 \%$ ). Pressure measurement was done with a micro-electronic manometer (accuracy $\pm 1 \%$ of full scale). Temperature and humidity measurement was done with a thermo-hygrometer (accuracy: $\mathrm{T} \pm 0.45^{\circ} \mathrm{C}: \mathrm{RU} \pm 1 \%$ of full scale).

The sampling and analysis of PAHs, in raw biogas and flare outlets, were performed with specific procedures for each class of compounds after the separation of particulates on filter membranes, and separation of condensate and adsorption of the organic compounds present in the vapor phase on a vial ORBO 43. The determination of PAHs was obtained by HPLC with fluorescence detection so as to combine high sensitivity with sufficient selectivity. The limit of analytical detection of single PAHs was $0.1 \mathrm{ng} \mathrm{Nm}^{-3}$.

\section{RESULTS AND DISCUSSION}

\subsection{Biogas composition}

Table 1 shows the macro-constituents (methane, carbon dioxide, oxygen and nitrogen) of the biogas composition, with additional minor components that may cause foul odors (ammonia, hydrogen sulphide, mercaptans and alkyl-sulfides). Biogas temperature is also indicated. 
Table 1. Biogas composition and temperature.

\begin{tabular}{lcccc}
\hline $\begin{array}{l}\text { Constituents } \\
\text { and temperature }\end{array}$ & $\begin{array}{c}\text { Unit of } \\
\text { measure }\end{array}$ & Mean & $\begin{array}{c}\text { Standard } \\
\text { Deviation }\end{array}$ & $\begin{array}{c}\text { Range } \\
\text { min-max }\end{array}$ \\
\hline $\mathrm{CH}_{4}$ & $\%$ & 48.0 & \pm 1.8 & $45.0-51.0$ \\
$\mathrm{CO}_{2}$ & $\%$ & 38.0 & \pm 2.2 & $35.0-42.0$ \\
$\mathrm{O}_{2}$ & $\%$ & 1.8 & \pm 0.4 & $1.30-2.30$ \\
$\mathrm{~N}_{2}$ & $\%$ & 12.0 & \pm 1.1 & $10.5-13.6$ \\
$\mathrm{NH}_{3}$ & $\mathrm{mg} \mathrm{Nm}^{-3}$ & 6.5 & \pm 2.2 & $3.60-11.20$ \\
$\mathrm{H}_{2} \mathrm{~S}$ & $\mathrm{mg} \mathrm{Nm}^{-3}$ & 3.6 & \pm 0.7 & $2.50-5.40$ \\
Mercaptans & $\mathrm{mg} \mathrm{Nm}^{-3}$ & 0.5 & \pm 0.2 & $0.25-0.80$ \\
Alkyl-sulfides & $\mathrm{mg} \mathrm{Nm}^{-3}$ & 3.4 & \pm 0.9 & $2.00-6.05$ \\
Temperature & ${ }^{\circ} \mathrm{C}$ & 54 & \pm 3.5 & $50.0-59.0$ \\
\hline
\end{tabular}

It can be observed that the main characteristics of the biogas are quite similar to that of MSW landfills with the sole exception of ammonia (much higher in MSW landfills due to the greater presence of organic nitrogen compounds in the waste).

For the objective of the present research, it is important to note that several VOCs were detected in the biogas, as potential precursors of PAH formation. In particular, toluene was found at the average concentration of $120.0 \mathrm{mg} \mathrm{Nm}^{-3}$, hexane at $75.0 \mathrm{mg} \mathrm{Nm}^{-3}$, trichloromethane at $55.5 \mathrm{mg} \mathrm{Nm}^{-3}$, acetone at $43.5 \mathrm{mg} \mathrm{Nm}^{-3}$ and minor presences were also found for xylene $\left(25.6 \mathrm{mg} \mathrm{Nm}\right.$ ), isopropanol $\left(20.2 \mathrm{mg} \mathrm{Nm}^{-3}\right)$, methyl-ethyl-ketone $\left(18.0 \mathrm{mg} \mathrm{Nm}^{-3}\right)$ and methyl-isobutyl-ketone $\left(14.5 \mathrm{mg} \mathrm{Nm}^{-3}\right)$.

\subsection{Effects of biogas combustion on PAHs}

Table 2 shows the main physical-chemical properties of the sixteen PAHs investigated. As known, PAHs are organic compounds with two or more aromatic rings in several structural configurations. Among them, those found in raw biogas are highlighted (grey background). Tables 3, 4, 5 and 6 show the results of flare combustion of the various PAHs. Each figure represents the PAHs with the same number of aromatic rings.

Tables 3 and 4 show that all PAHs with a relevant structural complexity (5 and 6 aromatic rings) are formed in combustion. This formation also affects three compounds such as benzo(g,h,i)perylene, indeno(1,2,3-cd)pyrene and dibenzo(a,h)anthracene not present in raw biogas.

However, the compound that shows the highest formation in combustion is benzo(a)anthracene, concentrations of which increase from the mean value of $6 \mathrm{ng} \mathrm{Nm}^{-3}$ to $71.5 \mathrm{ng} \mathrm{Nm}^{-3}$; also remarkable is the increase of benzo(b)fluoranthene. Turning to PAHs with four aromatic rings (Table 5), the behavior appears controversial, since chrysene predominates the destructive effect, while for pyrene and fluoranthene a relevant formation occurs, up to over $70 \mathrm{ng} \mathrm{m}^{-3}$ for both compounds. Finally, Table 6 shows that the six PAHs with only two or three aromatic rings, are not only not present in the raw biogas, but they do not form in combustion.

In order to discuss the result shown in the tables, it is first necessary to consider that many chemical organic compounds are not completely destroyed during the combustion processes, even at temperatures as high as $1,000^{\circ} \mathrm{C}$. Further, some organic compounds are synthesized in the combustion zone (and nearby) as a consequence of many secondary 
reactions. In a first decomposition step simple hydrocarbons and hydrocarbon radicals are formed in the gas phase (Figure. 1). These radicals are extremely reactive so that they give rise to several synthesis reactions, including cyclization. In this way, aliphatic hydrocarbons and aromatic hydrocarbons are formed which act as precursors to the formation of much more complex hydrocarbons, among which are the PAHs. Obviously the original presence of many VOCs in the raw biogas could be considered a more favorable condition for the synthesis of PAHs.

Table 2. Physico-chemical properties of PAHs. In grey background are PAHs detected in raw biogas (US-DHHS, 1995).

\begin{tabular}{llccccc}
\hline \multicolumn{1}{c}{ PAHs } & formula & n. rings & Mol.Wt & $\begin{array}{c}\text { Melting } \\
\text { Point }\left({ }^{\circ} \mathrm{C}\right)\end{array}$ & $\begin{array}{c}\text { Vap. Pressure } \\
\text { at } 25^{\circ} \mathrm{C}(\mathrm{mm} \mathrm{Hg})\end{array}$ & $\begin{array}{c}\text { Solubility } \\
\text { in } \begin{array}{c}\text { water at } 25^{\circ} \mathrm{C} \\
(\mu \mathrm{g} / \mathrm{L})\end{array}\end{array}$ \\
\hline Benzo(ghi)perylene & $\mathrm{C}_{20} \mathrm{H}_{12}$, & 6 & 276.4 & 273 & $1.03 \cdot 10^{-10}$ & 0.26 \\
indeno(1,2,3-cd)pyrene & $\mathrm{C}_{22} \mathrm{H}_{12}$ & 6 & 276.3 & 163.6 & $1 \cdot 10^{-11}-1 \cdot 10^{-6}$ & 6.2 \\
Dibenz(a,h)anthracene & $\mathrm{C}_{22} \mathrm{H}_{14}$ & 5 & 278.3 & 262 & $1 \cdot 10^{-10}\left(20^{\circ} \mathrm{C}\right)$ & 0.5 \\
Benzo(a)pyrene & $\mathrm{C}_{20} \mathrm{H}_{12}$ & 5 & 252.3 & 179 & $5.6 \cdot 10^{-9}$ & 2.3 \\
Benzo(k)fluoranthene & $\mathrm{C}_{20} \mathrm{H}_{12}$ & 5 & 252.3 & 215.7 & $9.59 \cdot 10^{-11}$ & 0.76 \\
Benzo(b)fluoranthene & $\mathrm{C}_{20} \mathrm{H}_{12}$ & 5 & 252.3 & 168.3 & $5.0 \cdot 10^{-7}\left(20^{\circ} \mathrm{C}\right)$ & 1.2 \\
Benz(a)antracene & $\mathrm{C}_{18} \mathrm{H}_{12}$ & 5 & 228.3 & $158-162$ & $2.2 \cdot 10^{-8}\left(20^{\circ} \mathrm{C}\right)$ & 10 \\
Crysene & $\mathrm{C}_{18} \mathrm{H}_{12}$ & 4 & 228.3 & $255-256$ & $6.3 \cdot 10^{-7}$ & 2.8 \\
Pyrene & $\mathrm{C}_{16} \mathrm{H}_{10}$ & 4 & 202.1 & 156 & $2.5 \cdot 10^{-6}$ & 77 \\
Fluoranthene & $\mathrm{C}_{16} \mathrm{H}_{10}$ & 4 & 202.3 & $108.8(*)$ & $5.0 \cdot 10^{-6}$ & $200-260$ \\
Anthracene & $\mathrm{C}_{14} \mathrm{H}_{10}$ & 3 & 178.2 & 218 & $1.7 \cdot 10^{-5}$ & $700\left(^{*}\right)$ \\
Phenanthrene & $\mathrm{C}_{14} \mathrm{H}_{10}$ & 3 & 178.2 & 100 & $6.8 \cdot 10^{-4}$ & $1,200\left(^{*}\right)$ \\
Fluorene & $\mathrm{C}_{13} \mathrm{H}_{10}$ & 3 & 166.2 & $116-117$ & $3.2 \cdot 10^{-4}\left(20^{\circ} \mathrm{C}\right)$ & 800 \\
Acenaphthene & $\mathrm{C}_{12} \mathrm{H}_{10}$ & 3 & 154.2 & 95 & $4.47 \cdot 10^{-3}$ & 1,930 \\
Acenaphthylene & $\mathrm{C}_{12} \mathrm{H}_{8}$ & 3 & 152.2 & $92-93$ & $29 \cdot 10^{-3}\left(20^{\circ} \mathrm{C}\right)$ & 3,930 \\
Naphthalene & $\mathrm{C}_{10} \mathrm{H}_{8}$ & 2 & 128.2 & $80.2(*)$ & $1.8 \cdot 10^{-2}(*)$ & $30,600\left(^{*}\right)$ \\
\hline
\end{tabular}

(*) Mrozik et al. (2003).

All PAHs have a high melting point, so they are normally in a solid state and in biogas are as fine particulate.

Table 3. Effect of flare combustion on PAHs with 6 aromatic rings (concentrations expressed as mean, standard deviation and range min-max).

\begin{tabular}{lcccc}
\hline \multirow{2}{*}{6 RINGS PAHs } & \multirow{2}{*}{ Flare position } & \multicolumn{3}{c}{ Concentration $\left(\mathrm{ng} \mathrm{Nm}^{-3}\right)$} \\
\cline { 3 - 5 } & & mean & sd & min-max \\
\hline \multirow{2}{*}{ Benzo(g,h,i)perylene } & in & $<0.1$ & $<0.1$ & $<0.1-<0.1$ \\
\multirow{2}{*}{ Indeno(1,2,3-cd)pyrene } & out & 4.3 & 0.8 & $2.6-5.5$ \\
& in & $<0.1$ & $<0.1$ & $<0.1-<0.1$ \\
& out & 1.8 & 1.8 & $1.1-2.6$ \\
\hline
\end{tabular}


Table 4. Effect of flare combustion on PAHs with 5 aromatic rings (concentrations expressed as mean, standard deviation and range min-max).

\begin{tabular}{llccc}
\hline \multirow{2}{*}{5 RINGS PAHs } & Flare & \multicolumn{3}{c}{ Concentration $\left(\mathrm{ng} \mathrm{Nm}^{-3}\right)$} \\
\cline { 3 - 5 } position & mean & sd & min-max \\
\hline \multirow{2}{*}{ Dibenz(a,h)anthracene } & in & $<0.1$ & $<0.1$ & $<0.1-<0.1$ \\
& out & 2.0 & 0.8 & $0.9-3.1$ \\
Benzo(a)pyrene & in & 1.8 & 0.6 & $1.1-3.6$ \\
& out & 3.5 & 1.8 & $1.2-5.9$ \\
Benzo(k)fluoranthene & in & 3.4 & 1.4 & $1.2-4.8$ \\
\multirow{2}{*}{ Benzo(b)fluoranthene } & out & 9.0 & 2.8 & $4.7-13.3$ \\
& in & 4.1 & 1.6 & $1.8-6.3$ \\
Benz(a)antracene & out & 19.6 & 7.2 & $9.9-32.6$ \\
& in & 6.0 & 1.9 & $3.0-8.6$ \\
& out & 71.5 & 13.5 & $54.0-98.0$ \\
\hline
\end{tabular}

Table 5. Effect of flare combustion on PAHs with 4 aromatic rings (concentrations expressed as mean, standard deviation and range min-max).

\begin{tabular}{|c|c|c|c|c|}
\hline \multirow{2}{*}{4 RINGS PAHs } & \multirow{2}{*}{ Flare position } & \multicolumn{3}{|c|}{ Concentration $\left(\mathrm{ng} \mathrm{Nm}^{-3}\right)$} \\
\hline & & mean & $\mathrm{sd}$ & $\min -\max$ \\
\hline \multirow[b]{2}{*}{ Chrysene } & in & 39.5 & 11.0 & $28.5-78.6$ \\
\hline & out & 21.1 & 6.6 & $11.5-33.6$ \\
\hline \multirow[b]{2}{*}{ Pyrene } & in & 2.3 & 0.6 & $1.2-4.5$ \\
\hline & out & 74.0 & 15.9 & $37.0-99.6$ \\
\hline \multirow{2}{*}{ Fluoranthene } & in & 2.2 & 0.7 & $1.1-3.8$ \\
\hline & out & 78.6 & 21.2 & $46.2-107.0$ \\
\hline
\end{tabular}

Table 6. Effect of flare combustion on PAHs with 3 and 2 aromatic rings (concentrations expressed as mean, standard deviation and range min-max).

\begin{tabular}{lcccc}
\hline $\begin{array}{l}\text { 2 \& } 3 \text { RINGS } \\
\text { PAHs }\end{array}$ & \multirow{2}{*}{ Flare position } & \multicolumn{3}{c}{ Concentration $\left(\mathrm{ng} \mathrm{Nm}^{-3}\right)$} \\
\cline { 3 - 5 } Anthracene & in & $<0.1$ & $<0.1$ & $<0.1-<0.1$ \\
& out & $<0.1$ & $<0.1$ & $<0.1-<0.1$ \\
Phenanthrene & in & $<0.1$ & $<0.1$ & $<0.1-<0.1$ \\
& out & $<0.1$ & $<0.1$ & $<0.1-<0.1$ \\
Fluorene & in & $<0.1$ & $<0.1$ & $<0.1-<0.1$ \\
& out & $<0.1$ & $<0.1$ & $<0.1-<0.1$ \\
Acenaphthene & in & $<0.1$ & $<0.1$ & $<0.1-<0.1$ \\
& out & $<0.1$ & $<0.1$ & $<0.1-<0.1$ \\
Acenaphthylene & in & $<0.1$ & $<0.1$ & $<0.1-<0.1$ \\
& out & $<0.1$ & $<0.1$ & $<0.1-<0.1$ \\
Naphthalene & in & $<0.1$ & $<0.1$ & $<0.1-<0.1$ \\
& out & $<0.1$ & $<0.1$ & $<0.1-<0.1$ \\
\hline
\end{tabular}




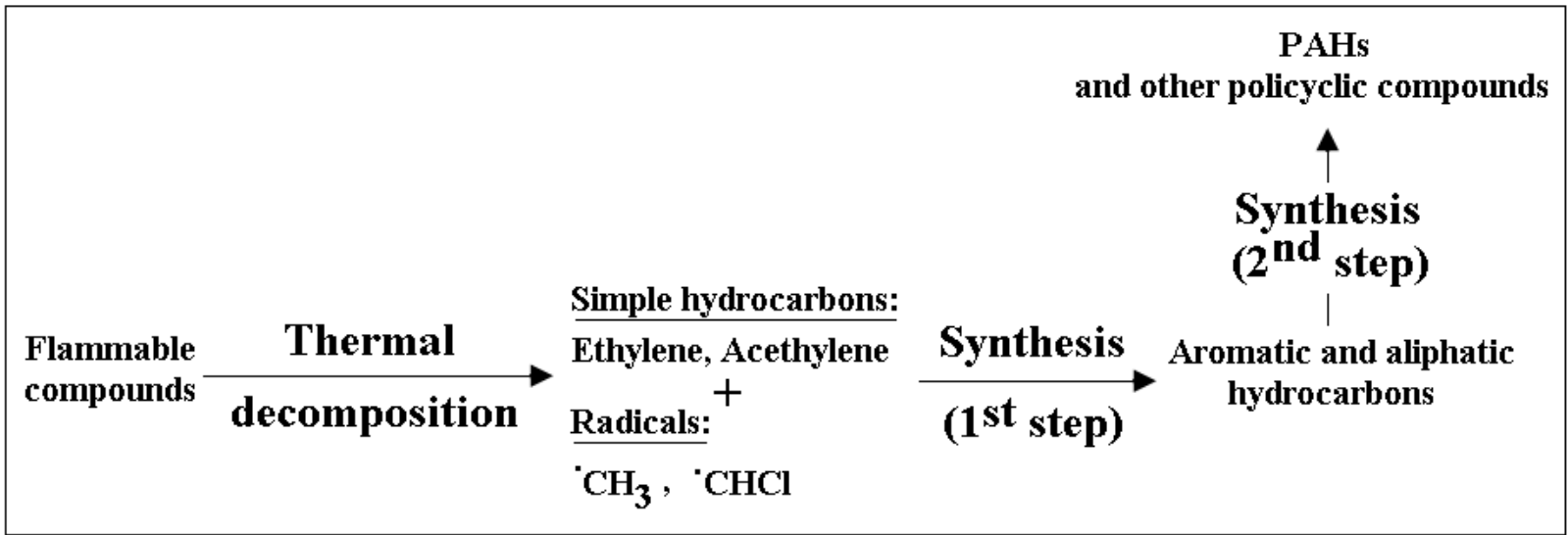

Figure 1. Pathway for PAH formation in a combustion chamber.

The results of this experience demonstrte that at $1,000^{\circ} \mathrm{C}$ and long residence time in combustion, all synthesis reactions lead to the formation of 4- to 6-ring PAHs. The only exception is chrysene, which undergoes significant destruction. This aspect requires further investigation to be fully understood. It is interesting to note that chrysene also differs from the other PAHs with high numbers of rings, for its better biodegradability (Urbini et al., 2014). The total lack of formation of PAHs with only 2 and 3 aromatic rings could be reasonably explained by the fact that the synthesis of high molecular weight PAHs is largely determined by condensation and dimerization reactions of the most simple PAHs (Siegmann and Sattler, 2000).

\section{CONCLUSION}

This study has proven that PAHs with a relevant structural complexity (5 and 6 aromatic rings) are formed in an enclosed flare combustion, operating at $1,000^{\circ} \mathrm{C}$ and $3.6 \mathrm{~s}$ residence time. This formation also produces compounds such as benzo(g,h,i)perylene, indeno(1,2,3-cd)pyrene and dibenzo(a,h)anthracene not present in raw biogas. However, the compound that has shown the highest formation in combustion is benzo(a)anthracene, the concentrations of which increase from the mean value of $6 \mathrm{ng} \mathrm{Nm}^{-3}$ to $71.5 \mathrm{ng} \mathrm{Nm}^{-3}$; the increase of benzo(b)fluoranthene is also remarkable. PAHs with four aromatic rings have shown controversial behavior, as chrysene has undergone a noticeable destruction (this aspect requires further investigation to be fully understood), while pyrene and fluoranthene have increased their concentrations to over $70 \mathrm{ng} \mathrm{m}^{-3}$ for both compounds. Instead, PAHs with only two or three aromatic rings, are not only not present in the raw biogas, but they do not form in combustion. The pathway for the synthesis of new PAHs can be explained as a consequence of many secondary reactions. In a first decomposition step, simple hydrocarbons and hydrocarbon radicals are formed in the gas phase. These radicals are extremely reactive, so that they give rise to several synthesis reactions, including cyclization. In this way, aliphatic hydrocarbons are formed, as well as aromatic hydrocarbons, which act as precursors of the formation of much more complex hydrocarbons, among which are the PAHs. The presence of many VOCs in the raw biogas could be considered a more favorable condition for the synthesis of PAHs. The total lack of formation of PAHs with only 2 and 3 aromatic rings could be reasonably explained by the fact that the synthesis of high molecular weight PAHs is largely determined by condensation and dimerization reactions of the simplest PAHs. 


\section{ACKNOWLEDGMENTS}

This research was supported by the ARF (Atheneum Research Funds) of Sapienza University.

\section{REFERENCES}

CIACCI, L.; MORSELLI, L.; PASSARINI, F.; SANTINI, A.; VASSURA, I. A comparison among different automotive shredder residue treatment processes. International Journal of Life Cycle Assessment, v. 15, n. 9, p. 896-906, 2010. http://dx.doi.org/10.1007/s11367-010-0222-1

COPELLI, S.; TORRETTA, V.; RABONI, M.; VIOTTI, P.; LUCIANO, A.; MANCINI, G. et al. Improving biotreatment efficiency of hot waste air streams: Experimental upgrade of a full plant. Chemical Engineering Transaction, v. 30, p. 49-54, 2012. http://dx.doi.org/10.3303/CET1230009

COSSU, R.; FIORE, S.; LAI, T.; MANCINI, G.; RUFFINO, B.; VIOTTI, P. et al. Review of Italian experience on automotive shredder residue characterization and management, Waste Management, v. 34, n. 10, p. 1752-1762, 2014.

http://dx.doi.org/10.1016/j.wasman.2013.11.014

EUROBSERV'ER. Biogas Barometer. Le Journal des Énergies Renouvelables, p. 67-79, 2012.

INNOCENTI, I.; VERGINELLI, I.; MASSETTI, F.; PISCITELLI，D.; GAVASCI, R.; BACIOCCHI, R. Pilot-scale ISCO treatment of a MtBE contaminated site using a Fenton-like process. Science of the Total Environment, v. 1, p. 485-486, 2014. http://dx.doi.org/10.1016/j.scitotenv.2014.01.062

MANCINI, G.; VIOTTI, P.; LUCIANO, A.; RABONI, M.; FINO, D. Full scale treatment of ASR wastes in a modified rotary kiln. Waste Management, v. 34, n. 11, p. 2347-2354, 2014. http://dx.doi.org/10.1016/j.wasman.2014.06.028

MATHUR, A. K.; MAJUMDER, C. B.; CHATTERJEE, S. Combined removal of BTEX in air stream by using mixture of sugar cane bagasse, compost and GAC as biofilter media. Journal of Hazardous Material, v. 148, n. 1-2, p. 64-74, 2007. http://dx.doi.org/10.1016/j.jhazmat.2007.02.030

MOHAMED, M. F.; KANG, D.; ANEJA, V. P. Volatile organic compounds in some urban locations in United States. Chemosphere, v. 47, n. 8, p. 863-882, 2002.

http://dx.doi.org/10.1016/S0045-6535(02)00107-8

MROZIK, A.; PIOTROWSKA, Z.; LABUZEK, S. Bacterial degradation and bioeremediation of polycyclic aromatic hydrocarbons. Polish Journal of Environmental Studies, v. 12, n. 1, p. 15-25, 2003.

RABONI, M.; TORRETTA, V.; URBINI, G.; VIOTTI, P. Automotive shredder residue: A survey of the hazardous organic micro-pollutants spectrum in landfill. Waste Management and Research, v. 33, n. 1, p. 48-54, 2014.

http://dx.doi.org/10.1177/0734242X14559300 
RABONI, M.; TORRETTA, V.; VIOTTI, P.; URBINI, G. Experimental plant for the physical-chemical treatment of groundwater polluted by municipal solid waste (MSW) leachate, with ammonia recovery. Revista Ambiente \& Agua, v. 8, n. 3, p. 22-32, 2013. http://dx.doi.org/10.4136/ambi-agua.1250

RABONI, M.; URBINI, G. Production and use of biogas in Europe: a survey of current status and perspectives. Revista Ambiente \& Agua, v. 9, n. 2, p. 191-202, 2014. http://dx.doi.org/10.4136/ambi-agua.1324

RABONI, M.; VIOTTI, P.; CAPODAGLIO, A. G. A comprehensive analysis of the current and future role of biofuels for transport in the European union (EU). Revista Ambiente \& Agua, v. 10, n. 1, p. 9-21, 2015. http://dx.doi.org/10.4136/ambi-agua.1492

SANTINI, A.; PASSARINI, F.; VASSURA, L.; SERRANO, D.; DUFOUR, J.; MORSELLI, L. Auto shredder residue recycling: mechanical separation and pyrolysis. Waste Management, v. 32, n. 5, p. 852-858, 2012. http://dx.doi.org/10.1016/j.wasman.2011.10.030

SIEGMANN, K.; SATTLER, K. Formation mechanism for polycyclic aromatic hydrocarbons in methane flames . Journal of Chemical Physics, v. 112, n. 2, p. 698-709, 2000. http://dx.doi.org/10.1063/1.480648

TORRES, E. A.; CERQUEIRA, G. S.; FERRER, T. M.; QUINTELLA, C. M.; RABONI, M.; TORRETTA, V. et al. Recovery of different waste vegetable oils for biodiesel production: A pilot experience in Bahia State, Brazil Waste Management, v. 33, n. 12, p. 2670-2674, 2013. http://dx.doi.org/10.1016/j.wasman.2013.07.030

TORRETTA, V.; URBINI, G.; RABONI, M.; COPELLI, S.; VIOTTI, P.; LUCIANO, A. et al. Effect of powdered activated carbon to reduce fouling in membrane bioreactors: a sustainable solution. Case study. Sustainability, v. 5, n. 4, p. 1501-1509, 2013. http://dx.doi.org/10.3390/su5041501

URBINI, G; VIOTTI; GAVASCI R. Attenuation of methane, PAHs and VOCs in the soil covers of an automotive shredded residues landfill: A case study. Journal of Chemical Pharmacy Research, v. 6, n. 11, p. 618-625, 2014.

UNITED STATES. Department of Health and Human Services - USDHHS. Toxicological profile for polycyclic aromatic hydrocarbons (PAHs). Atlanta: Agency for Toxic Substances and Disease Registry, 1995. 487. 


\begin{tabular}{|} 
Ambiente \& Água - An Interdisciplinary Journal of Applied Science \\
ISSN 1980-993X - doi:10.4136/1980-993X \\
www.ambi-agua.net \\
E-mail: ambi.agua@gmail.com
\end{tabular}

\title{
Impacts of urban life on water quality and fish larvae communities in two creeks of the Brazilian Amazon
}

\author{
doi:10.4136/ambi-agua.1763
}

Received: 26 Sep. 2015; Accepted: 25 Nov. 2015

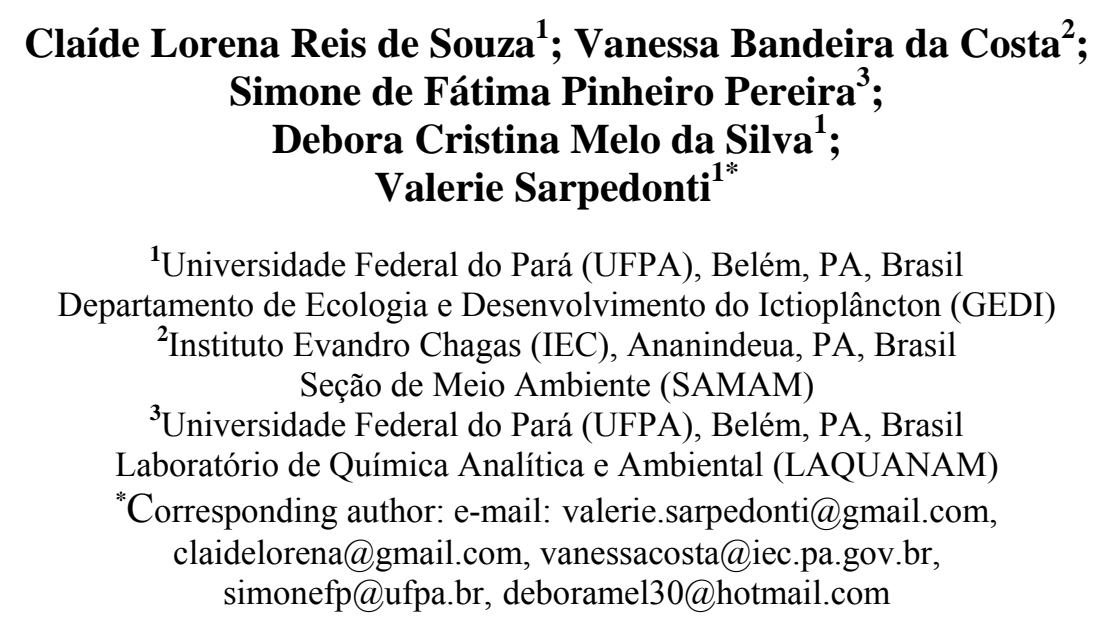

ABSTRACT

This study investigated the impact of anthropogenic activities in Belém City, Brazilian Amazon, by comparing water quality and fish larvae communities in two creeks that flow into the Guamá River. One creek crossed a poor and crowded suburb of Belém while the other was located in an island section that was declared an Environmental Protected Area in 1997. Two sampling points were set in each creek and monitored over eight hours once every three months over a one-year period. Strong variations of water quality were registered all year long and at all tides in Belém's mainland creek, along with, among other things, a very high number of thermotolerant coliforms. Few larvae were found. The water was considered unsuitable for human use and activities as well as for aquatic life. The island creek presented early signs of bacterial and nutrient contaminations during the rainy season, probably partly related to non-point source pollution. In both creeks, larvae communities were almost exclusively composed of clupeiforms. All larval development stages were encountered. Higher densities and proportion of newly hatched larvae were registered during the dry season and associated with the presence of nitrate. The results of the study show that adequate sewage and drainage systems must be developed in the city and suggest that it would be useful to conduct an integrated ambient monitoring study in Combú Creek.

Keywords: environmental protected area, pollution, urbanization.

\section{Impactos da vida urbana sobre a qualidade da água e comunidade de larvas de peixes em dois igarapés da Amazônia brasileira}

\section{RESUMO}

Este estudo teve como objetivo investigar o impacto das atividades antropogênicas da 
cidade de Belém pela comparação da qualidade da água e das comunidades de larvas de peixes em dois igarapés que desembocam no rio Guamá. Um dos igarapés atravessa um subúrbio pobre e populoso de Belém, enquanto o outro é localizado em uma ilha de Belém, declarada Área de Proteção Ambiental desde 1997. Dois pontos de coletas foram definidos em cada igarapé e monitorados durante oito horas, a cada três meses e durante um ano. $\mathrm{O}$ igarapé em região urbana apresentou fortes alterações na qualidade da água, durante o ano todo e em todas as marés, e isto deve se essencialmente a presença de um elevado número de coliformes termotolerantes. Poucas larvas foram encontradas. A água foi considerada imprópria para uso e atividades humanas, assim como para a vida aquática. O igarapé da ilha apresentou primeiros sinais de contaminação por nutrientes e bactéria durante o período chuvoso, parcialmente decorrente de fontes de poluição difusa. Em ambos os córregos, as comunidades larvais foram quase exclusivamente compostas de clupeiformes. Todos os estágios de desenvolvimento larval foram encontrados. Densidades e proporções mais elevadas de larvas recém eclodidas foram registradas durante a estação seca e associadas à presença de nitrato. Resultados apontam a necessidade de desenvolver um sistema de drenagem urbano para esgoto e água pluvial na maior brevidade, e recomenda um estudo de monitoramento integrado do igarapé na Área de Proteção Ambiental.

Palavras-chave: Área de proteção ambiental, poluição, urbanização.

\section{INTRODUCTION}

Water is one of the earth's most precious elements, upon which all life forms on the planet rely. Unfortunately, both the quality and quantity of water are currently threatened by human activities, lack of awareness and irresponsible behavior. While first-world countries may abuse water resources to support their lifestyles, developing countries struggle to provide basic needs to their citizenry (WWF, 2006). There are a variety of issues that make it difficult to bring the water crisis under control, such as the economic cost, politics, culture, education and lack of scientific knowledge. Over the past few decades megalopoli, such as Mexico City or Mumbai, have experienced a rapid and uncontrolled population increase that has led to severe water pollution in areas completely devoid of sanitation or drainage systems (SosaRodriguez, 2012).

Brazil, world's fifth-largest country both geographically and by population, also faces daily challenges to promote the 'right to the city' (Friendly, 2013). A study conducted in 2012 by the SNIS (Brazilian National Sanitation Information System ) of all 27 Brazilian state capitals indicated that the worst sanitation conditions are in Belém City, the capital of Pará State. Belém City is part of the Amazonian territory, and includes not only a continental urban center where approximately $98 \%$ of its population lives (1.4 million inhabitants in 2012), but also 39 poorly developed islands which represent 64\% of Belem's surface. In 1997, one of these islands, Combú Island, was declared an "Environmental Protected Area" (State law $6,083)$. The island is separated from Belém's mainland by the Guamá River, which also acts as a receptacle for contaminated waters deposited by the numerous creeks that flow through the city. Both the filthy living conditions in some suburbs, and their proximity to the protected island, suggest the need for quick implementation of water quality monitoring surveys in order to assess the actual levels of contamination in both impacted and supposedly nonimpacted areas, as a first and obligatory step to environmental risk management studies (Peptenatu et al., 2011).

This study compared water quality and fish larvae communities in two creeks, one that flows through a poor human settlement on Belém's mainland and another that passes through part of the Environmental Protected Area of Combú Island. Seasons, tides and larval 
development stages were considered. The results of the study will support follow-up studies of water pollution spreading from the city center towards the islands as well as of possible alterations of icthyoplankton communities. It is also hoped that, based on actual water quality data of the mainland creeks, the government may act to implement proper sanitation conditions and to protect aquatic resources that not only provide a source of food and livelihood for the local inhabitants, but also represent the diversity and richness of the Amazonian region.

\section{MATERIALS AND METHODS}

\subsection{Study area and Sampling}

The city of Belém, capital of Pará State, is located in the oligohaline zone of the Amazon region. It is surrounded by the Guamá River on its southern side, which carries large amount of suspended material from the Amazon and Tocantins river systems. Guamá River waters flow into Guajará Bay, on the eastern side of the city, considered the most exposed to human activities. Guajará Bay is then connected to Marajó Bay from which the waters flow into the ocean.

The two creeks selected for this study are located on opposite banks of the Guamá River: the Combú Creek, about $5 \mathrm{~km}$ long, crosses Combú Island, a preserved environment with few inhabitants that depend essentially on natural resources for their daily subsistence; and the Tucundubu Creek, about $4 \mathrm{~km}$ long, that passes through numerous human settlements which lack proper sanitation facilities. Two collection points were defined in each creek: downstream points $\mathrm{C} 1$ and $\mathrm{T} 1{ }^{\circ}\left(1^{\circ} 29^{\prime} 24.13^{\prime \prime S} / 48^{\circ} 27^{\prime} 41.93^{\prime \prime} \mathrm{W}\right.$ and $1^{\circ} 28^{\prime} 35.74^{\prime \prime} \mathrm{S} /$ $48^{\circ} 27^{\prime} 13.34^{\prime \prime} \mathrm{W}$, respectively) and $1 \mathrm{~km}$ inland points $\mathrm{C} 2$ and $\mathrm{T} 2$ (1 ${ }^{\circ} 29^{\prime} 53.16^{\prime \prime S}$ / $48^{\circ} 27^{\prime} 40.26^{\prime \prime} \mathrm{W}$ and $1^{\circ} 28^{\prime} 06.02^{\prime \prime} \mathrm{S} / 48^{\circ} 27^{\prime} 11.71^{\prime \prime} \mathrm{W}$, respectively) (Figure 1). Samplings were collected once per season in 2011 and 2012. Seasons in North Brazil are defined according to rainfall intensity: wet season (January to March), wet/dry transition season (April to June), dry season (July to September) and dry/wet transition season (October to December). Field trips occurred on two consecutive days (one day per creek) at the quarter moon; sampling was done at point 1 before sampling at point 2 and then returning to point 1 one hour later to conduct one more sampling set. Two pseudo-replicates were taken per sampling point. The overall survey lasted eight hours in order to include both ebb and flood tides, totaling about 32 samples day ${ }^{-1}$ creek $^{-1}$ (two pseudo-replicates per points, with two points per creek sampled eight times a day).

Fish larvae collection was done using a conical plankton net of $50 \mathrm{~cm}$ diameter, $330 \mu \mathrm{m}$ in mesh size, pulled by a small motorized vessel for 3 mins (license collection 18.152-1). Water volumes filtered at each trawl were assessed by a flowmeter placed at the net mouth. All samples were fixed in 4\% formaldehyde solution neutralized with sodium tetraborate.

In order to evaluate water parameters, sub-surface water was collected before each trawl using a five-liter 'Van Dorn' bottle. Chemical oxygen demand $\left(\mathrm{COD}_{5}-\mathrm{mg} \mathrm{L}^{-1}\right)$, biological oxygen demand (BOD - mg L $\mathrm{L}^{-1}$ ), dissolved oxygen $\left(\mathrm{DO}-\mathrm{mg} \mathrm{L}^{-1}\right)$, conductivity $(\mathrm{Cnd}-\mu \mathrm{S}$ $\mathrm{cm}^{-1}$ ) and total dissolved solids (TDS - $\mathrm{mg} \mathrm{L}^{-1}$ ) were assessed using a YSI 6600 multiparameters gauge; nitrate $\left(\mathrm{NO}_{3}^{-}-\mathrm{mg} \mathrm{L}^{-1}\right)$, ammonium $\left(\mathrm{NH}_{4}^{+}-\mathrm{mg} \mathrm{L}^{-1}\right)$ and phosphorus $\left(\mathrm{PO}_{4}{ }^{3-}-\mathrm{mg} \mathrm{L}^{-1}\right)$ concentrations were determined using an Ion Chomatography System (ICS DUAL 2000 DIONEX, USA); thermotolerant fecal coliforms (CTr most probable number MPN $100 \mathrm{ml}^{-1}$ ) were counted using a Collilert $-18^{\circledR} /$ Quanti-Tray ${ }^{\circledR} / 2000$ chromogenic substrate method from IDEXX Laboratories, Inc. and following the Standard Methods for the Examination of Water and Wastewater. 
a)

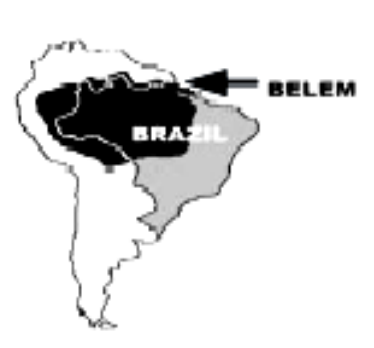

c)

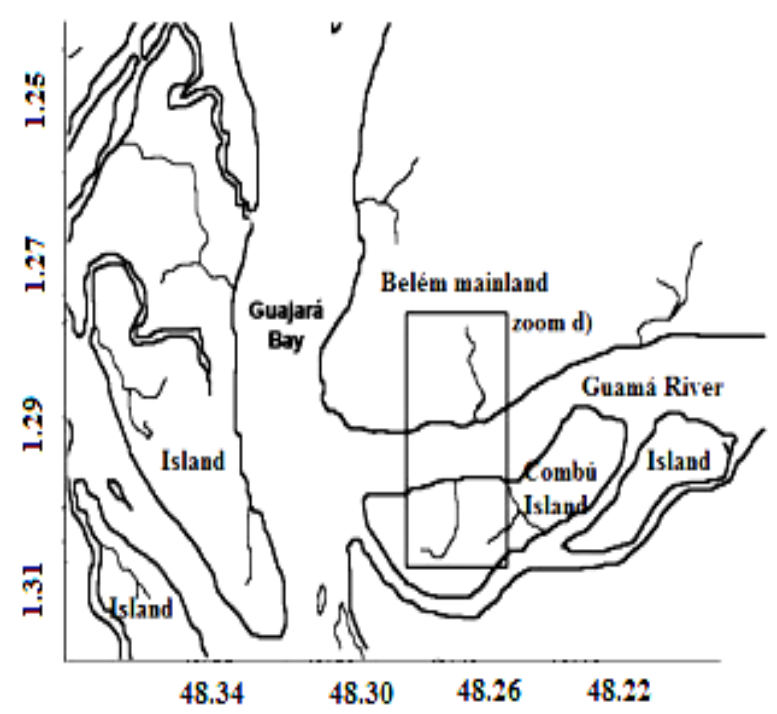

b)

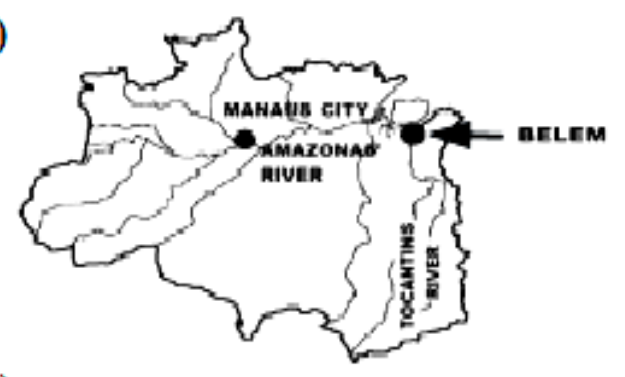

d)

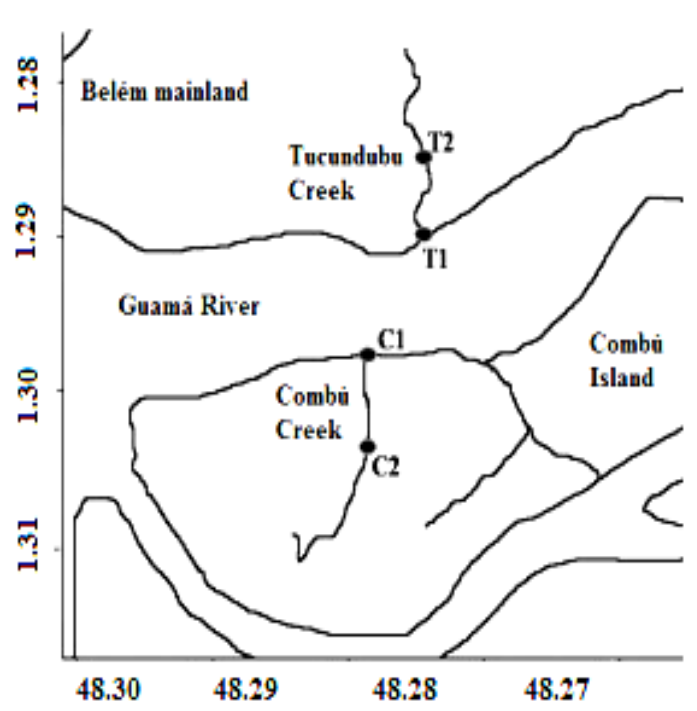

Figure 1. Study area a) South America, with the Amazonian territory (in black) and Brazilian borderline; b) Brazilian amazon and location of Belém City; c) Belém City including mainland area and some islands; and d) zoom on the sampling sites C1 and C2 in Combú Creek and T1 and T2 in Tucundubu Creek.

Larvae were separated from debris and other organisms using a stereomicroscope (Motic/Motion SMZ 134) and transferred to a 70\% alcohol solution. Subsequently, individuals were identified at the family level based, among other things, on fin characteristics, number of myomeres, shape, size of the digestive tract and pigment distribution. Fish larvae collections and literature were also consulted (Nakatani et al., 2001). Four larval developmental stages were separated based on the flexion of the notochord (pre-flexion, flexion and post-flexion stages) and presence / absence of the yolk-sac (yolk-sac stage).

\subsection{Data analysis}

Densities (number of larvae $100 \mathrm{~m}^{-3}$ ) were calculated, separated by seasons, sampling sites, tides, taxa and developmental stages. Data were $\log (x+1)$ transformed to increase the normality; similarity between densities was tested through analysis of variance (Hammer, 2001). Kolmogorov-Smirnov and Tukey tests were used to compare fish larvae mean densities and water parameters, respectively between ebb and flood tides, seasons and upstream/downstream sites. A Principal Component Analysis (PCA) was performed to relate water parameters with tides and seasons. The BIOENV routine based on Spearman correlation analysis was performed to assess which environmental variables were associated with fish larvae communities during the period of study. 


\section{RESULTS AND DISCUSSION}

\subsection{Water quality}

In developing countries, creeks or open drains act as the main collectors for urban pollution generated by industrial and domestic activities (Srivastava et al., 2011). Although Belém is not considered as a big industrial center, the lack of sanitation systems throughout the city renders the aquatic ecosystem vulnerable to heavy loads of organic material. This study showed that Tucundubu Creek is a good example of this. The creek presented both temporal and spatial changes $(\mathrm{p}<0.05)$ in water quality. Higher concentrations of thermotolerant coliforms, COD, TDS and conductivity were registered at ebbing, when the tide flushed city-polluted waters outward. Thermotolerant coliforms, $\mathrm{BOD}, \mathrm{PO}_{4}{ }^{3-}$ and N-products also presented statistically higher concentrations at the inner point, T2, closer to point sources of pollution (Table 1). Water quality improvement at $\mathrm{T} 1$ was possibly the result of water mixing between Tucundubu Creek and the Guamá River, suggesting that the latter may act as a natural barrier preventing or minimizing water crossing between the eastern margin of Belém and Combú Island. Further studies are recommended in order to develop a better understanding of how pollution spreads at the mixing zone before developing a new municipal sewage and waste water management strategy. Seasonal variations were observed with higher turbidity, DO, and N-products during the rainy season (Figure 2a). Rises in turbidity depending on the basin drainage area and water flow are commonly documented and related to sediment re-suspension in the water column (Costa et al., 2013). This process also causes water aeration and a consequent increase in DO. The high concentrations of nitrogen, and more especially nitrate, are within the range of those observed in other large Brazilian cities that suffer from contamination by domestic wastes, with occasional values above $10 \mathrm{mg}$ $\mathrm{L}^{-1}$ (e.g. Braga et al., 2000). Such was also the case for $\mathrm{PO}_{4}{ }^{3-}$ which, also presenting lower concentrations than in the study conducted by Braga et al. (2000), was considered too high for human use, fishing activities and aquatic life according to the norms established by the Brazilian Ministry of Environment (CONAMA 357/2005). Thermotolerant coliforms were very high and contributed to the classification of Tucundubu waters in class Group IV (CONAMA 357/2005), suitable only for navigation and harmony landscaping. Bacterial fecal pollution is one of the main causes of waterborne disease and mortality in developing countries (Sosa-Rodriguez, 2012). Such results should raise major concerns among governmental authorities as high bacterial concentrations were not only detected in the area of study but also in various locations scattered along the western margin of Belém City (Ribeiro, 2004) where children play in the water and fish with lines.

This study on Tucundubu Creek confirmed that water quality is the result of both the hydrological cycle and of anthropogenic activities in the watershed, with predictable seasonal variations related essentially to rain, and confined alterations of water properties through time and space due to human activities (Srivastava et al., 2011). On the contrary, results obtained in Combú Creek waters indicated no statistical differences between sampling sites or tides suggesting little contaminant input from riverines. Seasonal differences were observed, however, with higher concentrations of N-products, thermotolerant coliforms and suspended matter during the rainy season (Figure 2b). Nitrate, present in concentrations as high as those in Tucundubu Creek, suggested water deterioration within the preserved area. However, unlike Tucundubu Creek, which crosses city pavements and paved areas, Combú Creek is part of a wetland forest suggesting that its nutrients load was more likely a reflection of natural environmental conditions than a consequence of Belém's human activities. The contribution of non-point sources of pollution of nitrogen and phosphorus concentrations were largely debated by Howarth et al. (2000) as both parameters represent a major problem for the vital coastal waters of the United States. Thermotolerant coliforms, although much fewer than in 
Tucundubu Creek, did indicate bacterial pollution and a threat to human health. Even though their concentrations were usually not too high, they are responsible for water quality classification into Groups II or III (CONAMA 357/2005). Group II is suitable, among others, for human consumption after basic treatment, protection of aquatic communities and recreation while Group III can be used for human consumption after conventional or advanced treatment, recreational fisheries activities and irrigation. The deterioration of protected areas is observed worldwide and associated with poor environmental monitoring and understanding, which hampers the development of proper management policies (Leverington et al., 2010).

Table 1. Temporal mean and standard deviation followed by the median of water parameters at all sampling sites. $\mathrm{DO}=$ dissolved oxygen $\left(\mathrm{mg} \mathrm{L}^{-1}\right) ; \mathrm{BOD}=$ biological oxygen demand $\left(\mathrm{mg} \mathrm{L}^{-1}\right)$; $\mathrm{COD}=$ chemical oxygen demand $\left(\mathrm{mg} \mathrm{L}^{-1}\right)$; Cnd = conductivity $\left(\mu \mathrm{S} \mathrm{cm}{ }^{-1}\right)$; TDS = total dissolved solids $\left(\mathrm{mg} \mathrm{L}^{-1}\right) ; \mathrm{PO}_{4}{ }^{3-}=$ phosphorus $\left(\mathrm{mg} \mathrm{L}^{-1}\right) ; \mathrm{NH}_{4}{ }^{+}=$ammonium $\left(\mathrm{mg} \mathrm{L}^{-1}\right) ; \mathrm{NO}_{3}{ }^{-}\left(\mathrm{mg} \mathrm{L}^{-1}\right)=$ nitrate; $\mathrm{CTr}=$ thermotolerant fecal coliforms (most probable number MPN 100 $\mathrm{ml}^{-1}$ ).

\begin{tabular}{|c|c|c|c|c|c|c|c|c|}
\hline \multirow{2}{*}{ Parameters } & \multicolumn{8}{|c|}{ Sites } \\
\hline & $\mathrm{C} 1$ & Median & $\mathrm{C} 2$ & Median & $\mathrm{T} 1$ & Median & $\mathrm{T} 2$ & Median \\
\hline DO & $5.4 \pm 1.8$ & 6 & $5.4 \pm 1.8$ & 5.9 & $5.6 \pm 2.0$ & 5.8 & $5.2 \pm 1.8$ & 6.2 \\
\hline BOD & $6.2 \pm 2.7$ & 6.5 & $5.4 \pm 3.1$ & 4.5 & $5.6 \pm 4.0$ & 5.0 & $8.6 \pm 4.0$ & 7.0 \\
\hline COD & $22.3 \pm 11.3$ & 18.5 & $24.4 \pm 18.3$ & 20.0 & $23.8 \pm 16.9$ & 21.0 & $27.9 \pm 17.7$ & 38.0 \\
\hline Cnd & $28.4 \pm 9.9$ & 28.5 & $32.4 \pm 11.3$ & 30.5 & $96.9 \pm 112.7$ & 45.0 & $172.6 \pm 123.7$ & 226.0 \\
\hline TDS & $14.1 \pm 4.9$ & 14.5 & $16.2 \pm 5.6$ & 15.0 & $50.3 \pm 60.7$ & 22.0 & $87.1 \pm 64.4$ & 122.0 \\
\hline $\mathrm{PO}_{4}{ }^{3-}$ & $0.2 \pm 0.2$ & 0.1 & $0.24 \pm 0.2$ & 0.1 & $0.31 \pm 0.62$ & 0.1 & $0.4 \pm 0.7$ & 0.1 \\
\hline $\mathrm{NH}_{4}^{+}$ & $0.3 \pm 0.4$ & 0.1 & $0.1 \pm 0.1$ & 0.1 & $0.8 \pm 0.5$ & 0.1 & $3.0 \pm 3.1$ & 2.0 \\
\hline $\mathrm{NO}_{3}^{-}$ & $1.8 \pm 2.4$ & 0.8 & $4.5 \pm 9.9$ & 0.8 & $2.4 \pm 1.5$ & 1.6 & $3.8 \pm 5.6$ & 1.6 \\
\hline $\mathrm{CTr}$ & $3273 \pm 2646$ & 2734 & $2930 \pm 1914$ & 3255 & $641,021 \pm 1266$ & 14,800 & $1,249,507 \pm 1,773,882$ & 246,000 \\
\hline
\end{tabular}

\subsection{Fish larvae}

A total of 47 and 66 samples were collected in Tucundubu and Cumbú Creeks, respectively. Sampling effort difference was due to lower water levels in Tucundubu Creek at ebbing that made access to T2 difficult.

In Tucundubu Creek, sampling site $\mathrm{T} 1$ showed a total of 327 fish larvae, corresponding to a mean density of $25.100 \mathrm{~m}^{-3}$. No significant differences were registered between densities recorded during the $\mathrm{D} / \mathrm{W}\left(72.100 \mathrm{~m}^{-3}\right)$, wet $\left(5.100 \mathrm{~m}^{-3}\right)$ and dry $\left(23.100 \mathrm{~m}^{-3}\right)$ seasons. No larvae were encountered in W/D (Figure 3a). Site T2 accounted for a total of 51 larvae (7. 100 $\mathrm{m}^{-3}$ ), all sampled during the $\mathrm{D} / \mathrm{W}$ season $\left(21.100 \mathrm{~m}^{-3}\right)$. At both sampling sites, mean densities of larval fish were significantly greater during flood tides than during ebb tides.

A total of 1,803 fish larvae (mean of $89.100 \mathrm{~m}^{-3}$ ) was captured at $\mathrm{C} 1$; statistical differences in densities were observed only between the wet $\left(133.100 \mathrm{~m}^{-3}\right)$ and $\mathrm{D} / \mathrm{W}(54$. $100 \mathrm{~m}^{-3}$ ) seasons. At site C2, 2,344 larvae, corresponding to an overall density of $109.100 \mathrm{~m}^{-3}$, were captured. The dry season $\left(305.100 \mathrm{~m}^{-3}\right)$ presented densities statistically higher than during the other periods. The lower densities were registered in the W/D (mean of $34.100 \mathrm{~m}^{-3}$ ) (Figure 3a). There were no statistical differences between ebb and flood tide densities.

Larval fish communities were represented by the Orders Clupeiformes, Perciformes, Characiformes and Tetraodontiformes including six families: Clupeidae, Engraulidae, Pristigasteridae, Sciaenidae, Hemiodontidae and Tetraodontidae. In both creeks, Clupeiformes largely dominated, representing $98 \%$ of the catch. Clupeidae, Engraulidae, Pristigasteridae and Sciaenidae families were registered in Tucundubu Creek. Clupeids and engraulids 
represented $85 \%$ and $7 \%$, respectively, of the identified larvae. Clupeids prevailed at both points. In all seasons, more than half of captured larvae in Tucundubu Creek were in pre-flexion stage, including $4 \%$ of larvae with yolk-sacs that were found at both sites. The other stages of development showed a decrease of relative abundance from the pre-flexion to the post-flexion stages.

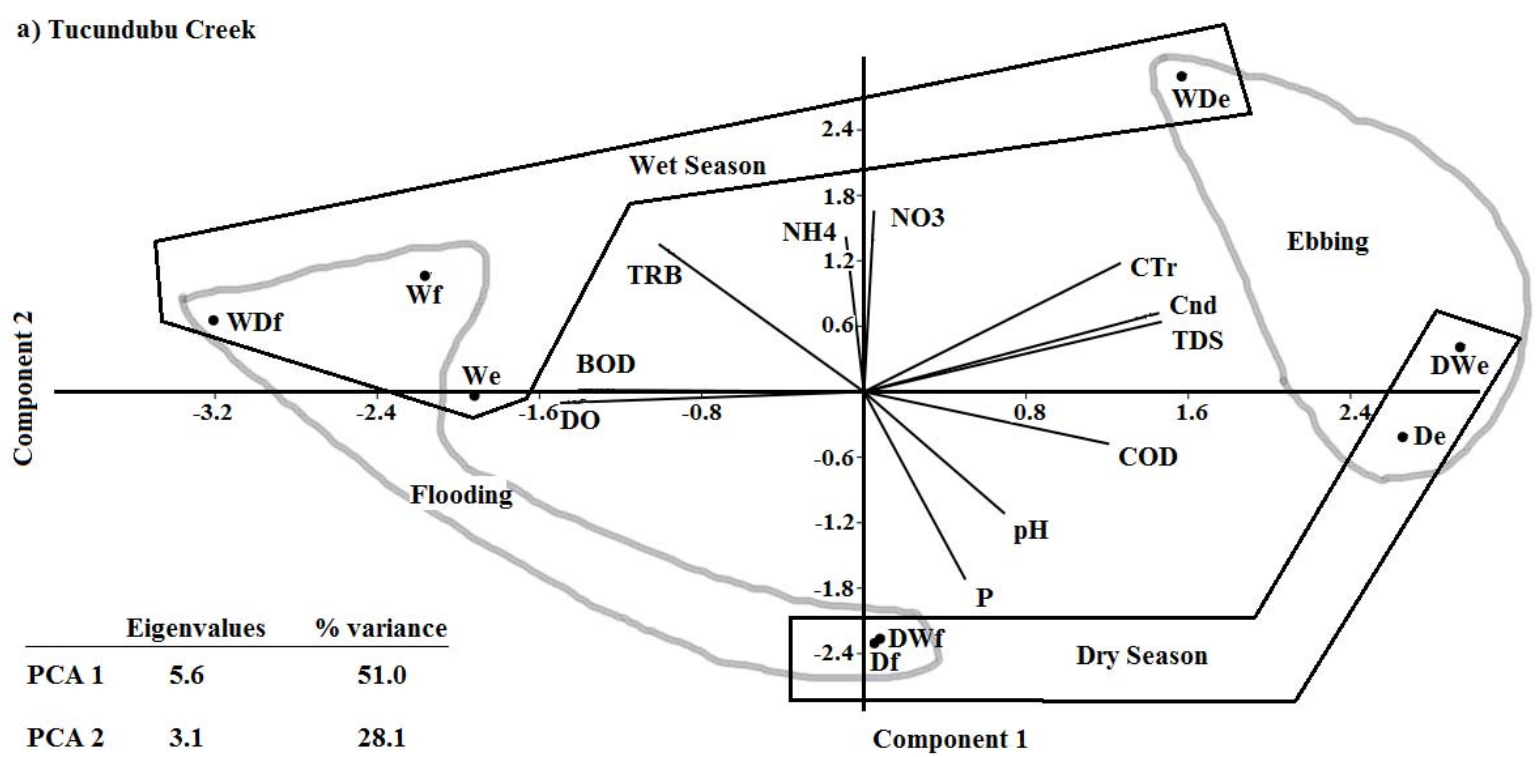

b) Combú Creek

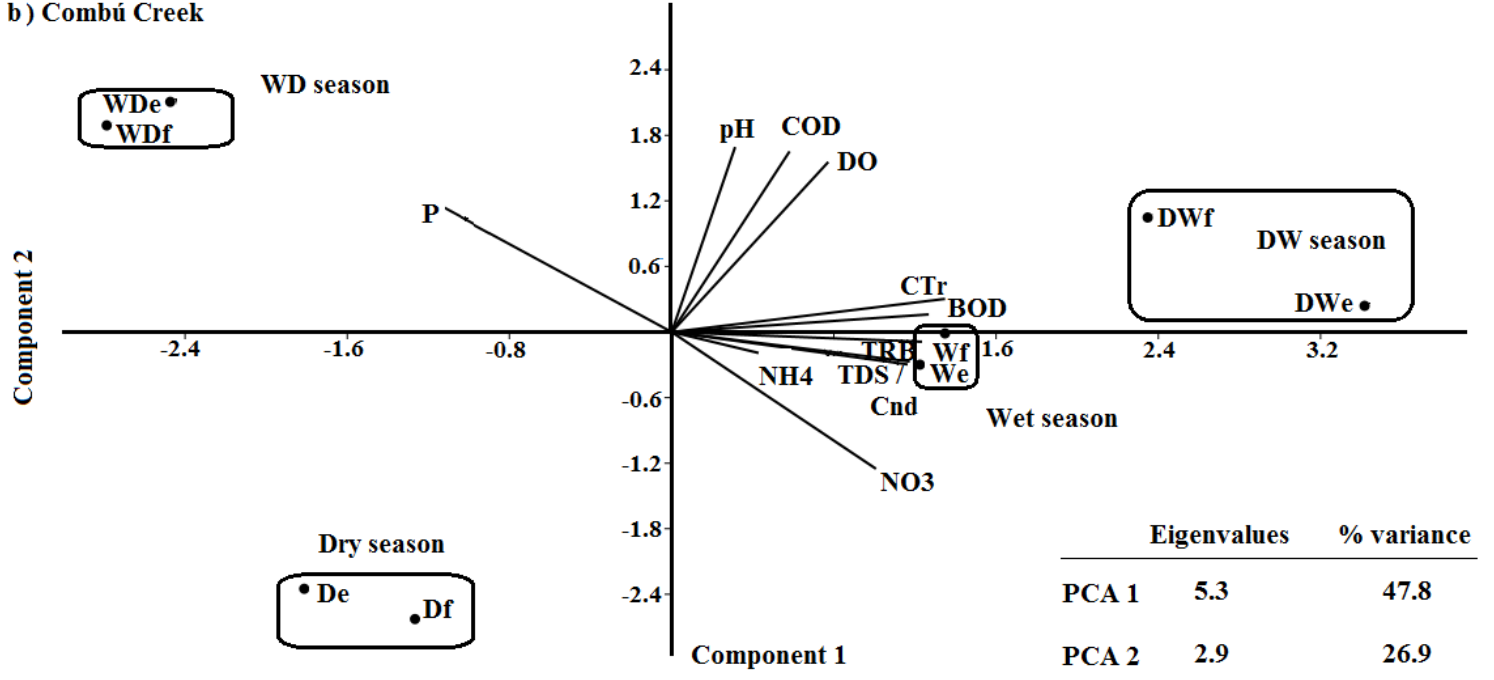

Figure 2. Principal Components Analysis applied on the environmental variables registered at Tucundubu (a) and Combú (b) creeks at all seasons and tides. Boxes represent possible case groupings. $\mathrm{DO}=$ dissolved oxygen $\left(\mathrm{mg} \mathrm{L}^{-1}\right)$; $\mathrm{BOD}=$ biological oxygen demand $\left(\mathrm{mg} \mathrm{L}^{-1}\right)$; $\mathrm{COD}=$ chemical oxygen demand $\left(\mathrm{mg} \mathrm{L}^{-1}\right) ; \mathrm{Cnd}=$ conductivity $\left(\mu \mathrm{Sm}^{-1}\right) ; \mathrm{TDS}=$ total dissolved solids $\left(\mathrm{mg} \mathrm{L}^{-1}\right) ; \mathrm{P}=$ phosphorus $\left(\mathrm{mg} \mathrm{L}^{-1}\right) ; \mathrm{NH}_{4}=\operatorname{ammonium}\left(\mathrm{mg} \mathrm{L}^{-1}\right) ; \mathrm{NO}_{3}\left(\mathrm{mg} \mathrm{L}^{-1}\right)$ $=$ nitrate; $\mathrm{CTr}=$ thermotolerant fecal coliforms (most probable number MPN $100 \mathrm{ml}^{-1}$ ); WD $=$ wet/dry season; $\mathrm{D}=$ Dry season; $\mathrm{D} / \mathrm{W}=$ dry $/$ wet; $\mathrm{W}=$ wet season, $\mathrm{f}=$ flood tide, $\mathrm{e}=\mathrm{ebb}$ tide. 
Combú Creek included all six families mentioned above. Hemiodontids and tetraodontids were captured only at $\mathrm{C} 2$ in the D/W transition period. Clupeiformes (Clupeidae, Engraulidae, Pristigasteridae families and non-identifies individuals) accounted for about $99 \%$ of the catch, with mainly clupeids. The other families were sporadic without relation to seasons or tides. All development stages were captured throughout the year with an overall prevalence of pre-flexion larvae (46\%), followed by larvae in flexion (38\%). The only exceptions were registered at $\mathrm{C} 1$ and $\mathrm{C} 2$ in the wet and dry season respectively, when flexion larvae were more abundant.

a)

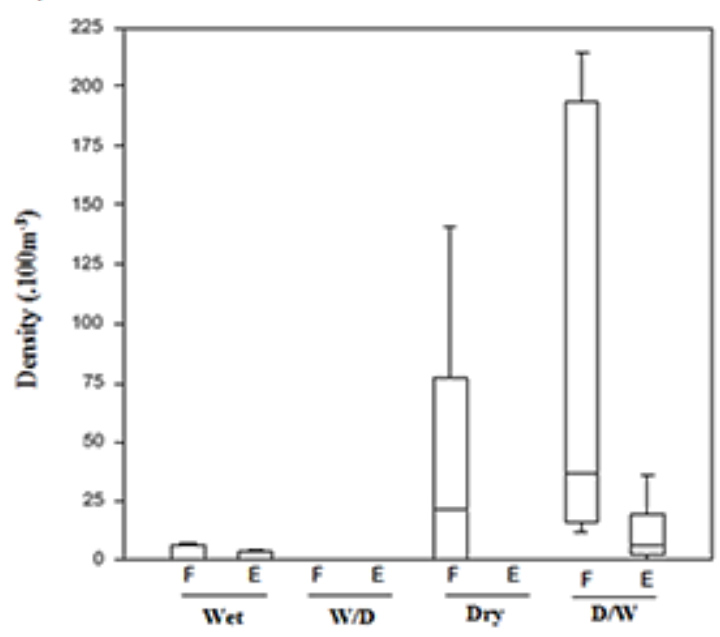

b)

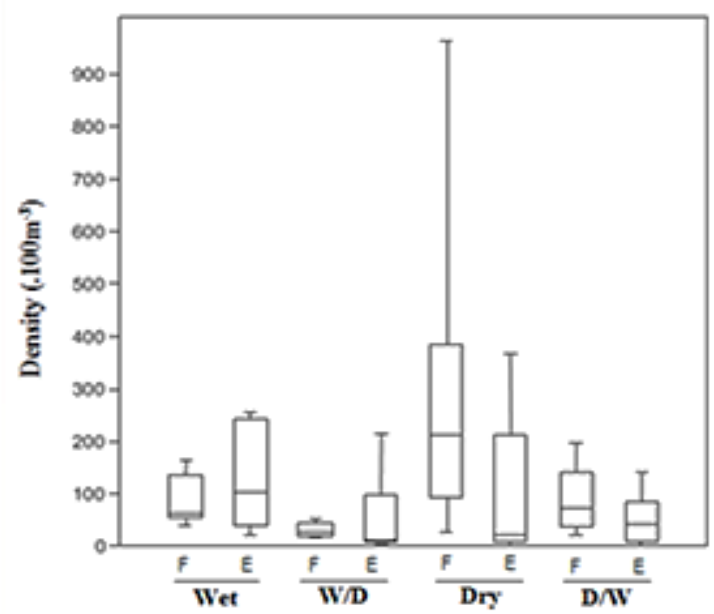

Figure 3. Box plots based upon larval densities at Tucundubu (a) and Combú bB) Creeks during the four seasonal periods, differentiating the ebb (E) and (F) flood tides. $\mathrm{W}=$ wet, $\mathrm{W} / \mathrm{D}=$ wet $/$ dry, $\mathrm{D}=$ Dry, $\mathrm{D} / \mathrm{W}=$ dry/wet seasons. Note that the scale differs between graphs.

BIOENV results showed that the environmental variables that best explained distribution patterns of fish larvae were thermotolerant coliforms $(-0.98)$ in Tucundubu Creek and $\mathrm{NO}_{3}{ }^{-}$in Combú Creek (0.48).

Differences in densities and, to a lower extent, diversity between both creeks pointed to unsuitable conditions for larval survival in Tucundubu Creek. The absence of open natural areas that could be used as nursery grounds as it is the case in Combú Creek could explain this result. However, water quality deterioration in Tucundubu Creek is most likely responsible for the changes in fish larvae densities, as reported in other impacted water courses (Konig et al., 2008) and sustained by BIOENV analysis result. The higher quantity of larvae registered at flooding and T1 indicated that individuals entered from the Guamá River into Tucundubu Creek, but that few larvae survived within the creek, unlike in Combú Creek where no differences were observed between tides and sites. It is noted that although Combú Creek presented higher density and diversity, both parameters remained low when compared to fish larvae communities encountered in the Amazonian Basin (Costa et al., 2011) or closer to the coast (Barletta-Bergan et al., 2002). This pattern is usually associated with all oligohaline areas that benefit few species but represent important nursery grounds (Sarpedonti et al., 2013). Clupeiformes' dominance has been documented in many Brazilian estuaries and worldwide, and associated with their extensive physiological plasticity.

The larval peak in density observed during the dry season agrees with the match-mismatch hypothesis that associates fish hatching with food availability (Cushing, 1990) that, in Belém, increases at the end of the dry season (Paiva et al., 2006). The importance of nutrients for the young's survival was sustained by BIOENV analysis result that indicated nitrates as the principal water parameter associated (positively) to larval 
communities in Combú Creek.

Fish larvae are considered good bio indicators for short-term quality changes in response to anthropogenic pressures (Ramos et al., 2015). In the study area, densities did reflect water quality with very few individuals in Tucundubu Creek. However, diversity was not considered appropriate for environmental impact assessments due to the naturally low number of fish species using the locale as a nursery ground.

\section{CONCLUSION}

This study found a drastic deterioration in the water quality of Tucundubu Creek associated essentially with extremely high levels of fecal coliforms and peaks of nutrients that rendered the environment unsuitable for human use and aquatic life. The results suggest an urgent need for authorities to develop new urban management plans in order to improve health and sanitation conditions. The protected area of Combú Island showed signs of contamination, although part of it was probably the result of non-point source pollution. A close follow-up study of the site is suggested, including hydrodynamic surveys, in order to determine the effect of Belem's mainland anthropogenic activities on the protected area. Fish larvae densities were considered suitable bio indicators for environmental impact assessments in the area, unlike the biodiversity index, which was determined to be unsuitable due to the naturally low number of taxa.

\section{ACKNOWLEDGMENTS}

The authors thank the Bureau of Environment (DEMA) staff and all laboratory colleagues for their assistance during field campaigns, the National Council for Scientific and Technologic Development (CNPq) for their financial support (Project N. 481157/2010-7) and the Institutional program for Graduates' Scholarship from Federal University of Pará (PIBIC / UFPA).

\section{REFERENCES}

BARLETTA-BERGAN, A.; BALETTA, M.; SAINT-PAUL, U. Structure and seasonal dynamics of larval fish in the Caete River Estuary in North Brazil. Estuarine, Coastal and Shelf Science, v. 54, p. 193-206, 2002. http://dx.doi.org/10.1006/ecss.2001.0842

BRAGA, E. S.; BONETTI, C. V. D. H.; BURONE, L.; BONETTI FILHO, J. Eutrophication and bacterial pollution caused by industrial and domestic wastes at the Baixada Santista Estuarine System, Brazil. Marine Pollution Bulletin, v. 40, n. 2, p. 165-173, 2000. http://dx.doi.org/10.1016/S0025-326X(99)00199-X

COSTA DA, A. J. G.; COSTA DA, K. G.; PEREIRA, L. C. C.; SAMPAIO, M. I.; COSTA DA, R. M. Dynamics of hydrological variables and the fish larva community in an Amazonian estuary of northern Brazil. Journal of Coastal Research, v. 64, p. 19601964, 2011.

COSTA DA, A. K. R.; PEREIRA, L. C. C.; da COSTA, R. M.; MONTEIRO, M. C.; de JESUS FLORES-MONTES, M. Oceanographic processes in an Amazon estuary during an atypical rainy season. Journal of Coastal Research, v. 65, p. 1104-1109, 2013.

CUSHING, D. H. Plankton production and year-class strength in fish populations: an update of the match/mismatch hypothesis. Advances in Marine Biology, v. 26, p. 249-293, 1990. 
FRIENDLY, A. The right to the city: theory and practice in Brazil. Planning Theory \& Practice, v. 14, n. 2, p. 158-179, 2013.

http://dx.doi.org/10.1080/14649357.2013.783098

HAMMER, Ø; HARPER, D. A. T.; RYAN, P. D. PAST: Paleontological statistics software package for education and data analysis. Paleontologia Electronica, v. 4, n. 1, p. 1-9, 2001 .

HOWARTH, R.; ANDERSON, D.; CLOERN, J.; ELFRING, C.; HOPKINSON, C.; LAPOINTE, B. et al. Nutrient pollution of coastal rivers, bays and seas. Issues in Ecology, v. 7, 2000.

KONIG, R.; SUZIN, C. H.; RESTELLO, R. M.; HEPP, L. U. Qualidade das águas de riachos da região norte do Rio Grande do Sul (Brasil) através de variáveis físicas, químicas e biológicas. Pan-American Journal of Aquatic Sciences, v. 3, n. 1, p. 84-89, 2008.

LEVERINGTON, F.; COSTA, K. L.; PAVESE, H.; LISLE, A.; HOCKINGS. A global analysis of protected area management effectiveness. Environmental Management, v. 46, n. 5, p. 685-698, 2010. http://dx.doi.org/10.1007/s00267-010-9564-5

NAKATANI, K.; AGOSTINHO, A. A.; BAUMGARTNER, G.; BIALETZKI, A.; SANCHES, P. V.; CAVICCHIOLI, M. et al. Ovos e larvas de peixes de água doce: desenvolvimento e manual de identificação. Maringá: UEM/Nupélia, 2001. 359 p.

PAIVA, R. S.; ESKINAZI-LEÇA, E.; PASSAVANTE, J. Z. O.; SILVA-CUNHA, M. G. G.; MELO, N. F. A. C. Considerações ecológicas sobre o fitoplâncton da Baía de Guajará e Foz do Rio Guamá (Pará, Brasil). Boletim do Museu Paraense Emílio Goeldi, v. 1, n. 2, p. 133-146, 2006.

PEPTENATU, D.; PINTILII, R. D.; DRAGHICI, C. Environmental risk management of urban growth poles regarding national importance. International Journal of Environmental Sciences and Technology, v. 8, n. 4, p. 737-746. 2011.

RAMOS, S.; CABRAL, H.; HELLIOTT, M. Do fish larvae have advantages over adults and other components for assessing estuarine ecological quality? Ecological Indicators, v. 55, p. 74-85, 2015. http://dx.doi.org/10.1016/j.ecolind.2015.03.005

RIBEIRO, K. T. S. A água e saúde humana em Belém. Belém: Cejup. 2004. 280 p. (Megan, 2)

SARPEDONTI, V.; ANUNCIAÇÃO, M. A.; BORDALO, A. Spatio-temporal distribution of fish larvae in relation to ontogeny and water quality in the oligohaline zone of a North Brazilian estuary. Bioto Neotropica, v. 13. n. 3, p. 55-63, 2013. http://dx.doi.org/10.1590/S1676-06032013000300007

SOSA-RODRIGUEZ, F. S. Assessing water quality in the developing world: an index for Mexico City. In: VOUDOURIS, K.; VOUTSA, D. (Eds.). Water quality monitoring and assessment. [S.1.]: InTech, 2012. p. 495-508. http://dx.doi.org/10.5772/32055

SRIVASTAVA, S.; SRIVASTAVA, A, S.; NEGI M. P. S.; TANDON, P. K. Evaluation of effect of drains on water quality of river Gomti in Lucknow city using multivariate statistical techniques. International Journal of Environmental Sciences, v. 2, n. 1, p. $1-7,2011$. 
WORLD WIDE FOUND FOR NATURE - WWF. World Water Assessment Programme. Water: a shared responsibility. Paris: UNESCO, 2006. p. 43. 


\begin{tabular}{|} 
Ambiente \& Água - An Interdisciplinary Journal of Applied Science \\
ISSN 1980-993X - doi:10.4136/1980-993X \\
www.ambi-agua.net \\
E-mail: ambi.agua@gmail.com
\end{tabular}

\title{
Histopathological assessment of $\mathrm{C} 57 \mathrm{Bl} / \mathrm{J}$ mice organs exposed to tannery effluents
}

\author{
doi:10.4136/ambi-agua.1694
}

Received: 01 Jul. 2015; Accepted: 05 Dec. 2015

\author{
Joyce Moreira de Souza ${ }^{1}$; Abraão Tiago Batista Guimarães ${ }^{1}$; \\ Wellington Alves Mizael da Silva ${ }^{1}$; Bruna de Oliveira Mendes ${ }^{1}$; \\ Dieferson da Costa Estrela²; Aline Sueli de Lima Rodrigues"; \\ Adriana da Silva Santos ${ }^{3}$; Guilherme Malafaia ${ }^{1,2^{*}}$ \\ ${ }^{1}$ Instituto Federal Goiano (IF Goiano), Câmpus Urutaí, Urutaí, GO, Brasil \\ Departamento de Ciências Biológicas \\ ${ }^{2}$ Universidade Federal de Goiás (UFG), Câmpus Samambaia, Goiânia, GO, Brasil \\ Programa de Pós-Graduação em Biodiversidade Animal \\ ${ }^{3}$ Instituto Federal Goiano (IF Goiano), Câmpus Urutaí, Urutaí, GO, Brasil \\ Departamento de Medicina Veterinária \\ *Corresponding author: e-mail: guilhermeifgoiano@gmail.com, \\ joycemsabio@gmail.com, abraaotbgbio@gmail.com, \\ wellington_8000@hotmail.com, brunaprofletras@outlook.com, \\ diefersonestrela@gmail.com, alineifgoiano@gmail.com, \\ adriana.santos@ifgoiano.edu.br
}

\begin{abstract}
The effluent produced in tanneries can cause environmental damage and public health problems when disposed of improperly. However, few toxicological studies have evaluated the effects of the intake of tannery wastewater by mammals. The objective of this study is the histological assessment of organs of $\mathrm{C} 57 \mathrm{Bl} / \mathrm{J}$ mice exposed to the intake of different concentrations of raw tannery effluents, beginning with the hypothesis that these effluents can cause damage to the histological structure of the organs. The animals were divided into the following groups: control ( $0 \%$ effluent) and exposed to $0.1 \%, 1 \%$, and $5 \%$ raw tannery effluent diluted in water, for a period of 120 days. For the histopathological evaluations, samples of the liver, kidney, spleen, heart, and lung were collected, fixed, and stained by the hematoxylin and eosin staining techniques. No anomalies were observed in kidney, spleen, heart, and lung fragments. Alterations were observed only in liver fragments. Moderate hydropic degeneration was detected in animals exposed to tannery effluents, mainly in the periportal space. A large number of necrotic hepatocytes $(\mathrm{p}<0.05)$ were detected, especially in animals exposed to higher tannery effluent concentrations. Further, the largest number of hepatocytes with karyomegaly $(\mathrm{p}<0.05)$ was observed in animals exposed to the highest effluent concentrations. Our study suggests that the observed alterations are related to the intake of tannery effluents and that these effluents cause changes that lead to an increase of free radical production and direct aggressions to hepatocyte membranes as well as to the appearance of hepatocellular karyomegaly.
\end{abstract}

Keywords: agro-industrial residues, animal model, histopathology, mice. 


\section{Avaliação histopatológica de órgãos camundongos C57Bl/6J expostos a efluentes de curtume}

\section{RESUMO}

Os efluentes produzidos nas indústrias curtumeiras podem causar danos ambientais e problemas de saúde pública quando descartados de forma inadequada. Porém, poucos estudos avaliaram os efeitos da ingestão de efluentes de curtumes em modelos experimentais mamíferos. O objetivo deste estudo foi realizar uma avaliação histológica de órgãos de camundongos C57BL/6J expostos à ingestão de diferentes concentrações de efluentes de curtumes, partindo da hipótese de que estes efluentes poderiam causar danos à estrutura histológica dos órgãos escolhidos para o estudo. Os animais foram divididos nos seguintes grupos: controle ( $0 \%$ de efluente) e expostos às porcentagens $0,1 \%, 1 \%$ e $5 \%$ de efluente bruto diluído em água, por um período de 120 dias. Para as avaliações histopatológicas, foram coletadas amostras de fígado, rim, baço, coração e pulmões, fixados e corados pela técnica de coloração com hematoxilina e eosina. Não foram observadas anomalias nos fragmentos de rim, baço, coração e pulmões. Foram observadas alterações apenas em fragmentos de fígado. Degeneração hidrópica moderada foi detectada em animais expostos à efluentes de curtumes, principalmente no espaço periportal. Foi detectado um grande número de hepatócitos necróticos $(p<0,05)$, em especial em animais expostos às concentrações mais elevadas de efluentes de curtume. Observou-se o maior número de hepatócitos com cariomegalia $(\mathrm{p}<0,05)$ em animais expostos às concentrações mais elevadas de efluentes. Nossos estudo evidência que as alterações observadas estão relacionadas com a ingestão de efluentes que podem levar ao aumento da produção de radicais livres e agressões diretas para membranas de hepatócitos, bem como o aparecimento de cariomegalia hepatocelular.

Palavras-chave: resíduos agroindustriais, modelo animal, histopatologia, camundongos.

\section{INTRODUCTION}

Industrial processes and human activities typically generate specific wastes that are composed of many different substances. Depending on the nature of these substances, such wastes can be harmful to the environment and to human health (Kraemer, 2006). In opposition to the amenities of modern society, the problems caused by such wastes are a serious threat to the current quality of life (Silva et al., 2012). The various types of waste generated include those that are produced by profitable industrial activities, such as bovine skin processing.

While this activity generates significant profits that contribute to the economic and social development of a country, it has been the subject of concern, primarily due to the production of a large amount of waste/effluent during bovine leather processing. As discussed by Gödecke et al. (2012), the leather tanning process requires several mechanical and chemical treatment processes that result in large amounts of waste as well as high concentrations of organic matter and various potentially toxic chemicals.

This problem is intensified by the fact that in many tanneries wastes/produced effluents are discharged into waterways incorrectly, causing a high risk of environmental contamination (Pacheco, 2005; Pinheiro, 2005; Ganem, 2007; Gödecke et al., 2012). Even after treatment in a treatment plant, tannery contains considerable organic and inorganic fillers such as acids, phenols, sulfates, and sulfides and toxic elements such as chromium, which is used during the tanning process (Batista e Alovisi, 2010).

To assess the impact of these effluents on organisms, toxicological studies have demonstrated teratogenicity in sea urchin species, the reduction of growth in microalgae, and a variety of toxic effects in micro-crustaceans (Oral et al., 2007). However, it is important to 
consider that while these organisms are suitable for determining lethality, toxicity must be evaluated in other ways because other signs and/or symptoms can be found in mammals. This limitation hinders the extrapolation of the obtained results to human beings. With respect to the effects of exposure to tannery effluents in experimental mammalian models, we highlight the work of Siqueira et al. (2011) and Moysés et al. (2014). In the first study, the authors demonstrated that Swiss mice that were exposed to different concentrations of tannery effluent diluted in water for 21 days exhibited a state of anxiety. However, Moysés et al. (2014) studied the neurotoxicity and hepatotoxicity induced by chronic exposure to tannery effluents and anxiety behaviors that are predictive of depression and memory changes. Those authors failed to observe changes in the evaluated parameters in rats.

Moreover, the analysis of the health of workers exposed to organic solvents and heavy metals associated with tannery effluents or wastes is widely discussed in the field of occupational toxicology (Shahzad et al., 2006; Salazar, 2008; Cuberos et al., 2009; Rumin et al., 2013); however, few basic studies investigated the effects of such wastes in an animal model.

Thus, the objective of this study was the histological assessment of different C57BL/6J mice organs exposed to water containing various raw tannery effluent concentrations, beginning with the hypothesis that these effluents can cause damage to the histological structure of the organs. We are not aware of any such study in the literature, and therefore this paper is a contribution to research on the effects of tannery effluents in the homeostasis of an organism.

\section{MATERIAL AND METHODS}

Male C57BL/6J mice obtained from the animal house of the Tropical Pathology and Public Health Institute of Goiás (Goiânia, GO, Brazil) were maintained in the Animal Laboratory of Biological Research of the Federal Institute of Goiás - Campus Urutaí (Urutaí, GO, Brazil). The animals were exposed to a normal light/dark cycle, and food and liquids were available ad libitum. A total of 32 animals, which were 21 days old, were divided into four groups: the control group, in which the animals received only drinking water that contained $0 \%$ tannery wastewater (see characterization in Table 1 ), and groups $0.1 \%, 1 \%$ and $5 \%$, which received raw tannery effluent diluted to the indicated concentrations in water. The animals were chronically exposed to the indicated concentrations of the raw tannery effluent, whose chemical characterization is presented in Table 1. The methodology of this study was consistent with the principles for ethical animal experimentation and was approved by the Ethics Committee for Animal Use of the Federal Institute of Goiás, GO, Brazil (protocol n. 18/2014).

The tannery effluent used in this study was obtained from a tannery located in Pires do Rio, GO, Brazil. The effluent provided by the tannery did not contain the element chromium because the effluent was removed from the steps of river operations (i.e., the steps prior to the tanning phase of bovine leather, which uses chromium salts). We chose to use this type of effluent because many tannery industries dispose of these wastes directly into the waterways that border the properties.

The animals in groups $0.1 \%, 1 \%$, and $5 \%$ were chronically exposed to raw tannery effluents diluted in water for 120 days. After this period, the animals were euthanized. They were anaesthetized with an intraperitoneal injection of $40 \mathrm{mg} / \mathrm{kg}$ pentobarbital, followed by cervical dislocation.

For the histopathological evaluations, the liver, kidney, spleen, heart, and lung were collected and fixed in $10 \%$ buffered formalin, embedded in paraffin blocks, and thin-sliced to $5 \mu \mathrm{m}$ thickness (Luna, 1968). The hematoxylin and eosin staining technique (HE) was used, 
following Behmer et al. (1976). The thin-session descriptions were carried out using a bright-field Carl Zeiss ${ }^{\circledR}$ optical microscope, model Jenaval, in order to compare the tissue structures of the organs removed from animals of the different experimental groups.

Table 1. Characterization of the raw tannery effluent, which was diluted in water at different concentrations and offered to C57BL/6J mice.

\begin{tabular}{|c|c|c|}
\hline Parameters ${ }^{1}$ & Tannery effluent & Potable water \\
\hline $\mathrm{pH}$ at $25^{\circ} \mathrm{C}(\mathrm{UpH})$ & 8.19 & 7.19 \\
\hline Turbidity (NTU) & 382.00 & $<1.00$ \\
\hline Turbidity ammonia nitrogen (mg. $\mathrm{L}^{-1}$ ) & 2.10 & 0.01 \\
\hline Total Nitrogen (mg. $\mathrm{L}^{-1}$ ) & 110.00 & 1.20 \\
\hline Nitrate (mg. $\mathrm{L}^{-1}$ ) & 23.00 & 0.30 \\
\hline Electrical conductivity at $25^{\circ} \mathrm{C}\left(\mu \mathrm{S} . \mathrm{cm}^{-1}\right)$ & 72.10 & 52.00 \\
\hline Total phosphorus (mg. $\mathrm{L}^{-1}$ ) & 33.61 & 0.11 \\
\hline Orthophosphate (mg. $\mathrm{L}^{-1}$ ) & 77.09 & 0.26 \\
\hline Biochemical oxygen demand (BOD) (mg. $\left.\mathrm{L}^{-1}\right)$ & $9,333.33$ & 0.50 \\
\hline Total solids (mg..$^{-1}$ ) & $82,190.00$ & 30.00 \\
\hline Dissolved Copper (mg. $\mathrm{L}^{-1}$ ) & $<0.01$ & 0.04 \\
\hline Dissolved Manganese (mg. $\mathrm{L}^{-1}$ ) & $<0.10$ & ND \\
\hline Dissolved iron (mg. $\mathrm{L}^{-1}$ ) & 1.91 & 0.09 \\
\hline Zinc (mg. $\left.L^{-1}\right)$ & $<0.01$ & 1.06 \\
\hline Sodium (mg. $L^{-1}$ ) & $5,680.00$ & 5.01 \\
\hline Magnesium (mg..$^{-1}$ ) & 243.20 & 1.21 \\
\hline Calcium (mg. $\left.\mathrm{L}^{-1}\right)$ & $2,805.00$ & 4.00 \\
\hline Sulfur $\left(m g . L^{-1}\right)$ & 833.33 & 1.00 \\
\hline Potassium (mg. $\mathrm{L}^{-1}$ ) & 122.00 & 1.60 \\
\hline Total organic carbon (TOC) (mg.L L $\left.^{-1}\right)$ & 93.32 & 8.20 \\
\hline
\end{tabular}

${ }^{1}$ The analysis of the raw tannery effluent and water followed the methodology recommended by the American Public Health Association (APHA et al., 2005). All analyses were performed in a commercial laboratory located in Goiania, GO, Brazil.

The alterations in affected organs were analyzed quantitatively. Specific counts for these alterations were carried out under the microscope using ten randomly chosen fields per thin section for each animal and 40x objective and 10x ocular lenses (magnitude 400x). The images were captured using the image analysis system TSView version 7.1.

Quantitative data were analyzed by analysis of variance (one-way ANOVA) with Tukey's test at $5 \%$ probability. The residual normality was verified using the Shapiro-Wilk test, and Bartlett's test was used to check the remaining homoscedasticity, using the $\mathrm{R}$ version 3.0.3 software (R Core Team, 2014). 


\section{RESULTS AND DISCUSSION}

No anomalies were observed in the kidney, spleen, heart, and lung fragments (images not shown). Alterations were observed only in liver fragments. Moderate hydropic degeneration was detected, mainly in the periportal space, in animals that drank water containing $5 \%$ of tannery effluent (Figure 1).

Studies focusing on histopathological alterations in mice exposed to the intake of tannery effluents are lacking in the literature, which makes the discussion of the data difficult. However, we believe that this alteration is related to varied factors but mainly to agents or elements contained in the effluents that damage the hepatocyte membranes and organelles by means, for example, of the generation of free radicals. The high sodium concentration in the effluent used (Table 1) may have contributed to the retention of the element in the organism, increasing hepatocyte intracellular osmotic pressure, allowing water to enter the cytoplasm, and causing cell isosmotic expansion.

Moysés et al. (2014) analyzed the parameters related to the hepatic oxidative stress, by means of the quantification of free radicals and analysis of lipid peroxidation in the livers of Wistar rats exposed to effluent intake. The authors did not observe any alteration in these parameters. However, it is worth mentioning that in the study of Moysés et al. (2014), the animals were exposed to effluent intake for only 30 and 45 days, which differs from our study, in which the animals were exposed to the effluents for 120 days. Also, the type of tannery effluent used by Moysés et al. (2014) as well as the rodent species and lineage was different from that used here. On the other hand, studies involving microalgae (Ajayan e Selvaraju, 2012) and fish (Prabakaran et al., 2007) corroborate the hypothesis that tannery effluents can significantly affect the production of species that react with oxygen and consequently the production of free radicals, damaging the structure and function of vital organs.

Another aspect observed here was the high number of necrotic hepatocytes, mainly in animals exposed to higher tannery effluent concentrations (Figures 2 and 3), which can also be related to the alterations that lead to the increase in the production of free radicals and direct aggressions to the hepatocyte membranes, creating hydrophilic channels through which the cell loses electrolytes and dies. It is known, for example, that Kupffer cells also generate free radicals in answer to liver damage (Friedman e Arthur, 1989) and, in this case, such cells can have contributed to necrotizing phenomena that occurred in these animals. Additionally, a high number of hepatocytes with karyomegaly were observed in animals exposed to the highest tannery effluent concentrations (Figures 2 and 3). It is emphasized that single hepatocytes or groups of hepatocytes with strikingly enlarged nuclei and frequently with prominent nucleoli were evaluated as karyomegaly.

Until now, we have not found any study in the literature that reports the presence of hepatocytes with karyomegaly in rodents exposed to tannery effluents. However, some studies have shown that the presence of karyomegaly in renal tubule cells has been frequently associated with chemical carcinogenicity in rodents, no matter the genotoxic potential of the administered compounds (Kitaura et al., 1999; Lock e Reed, 2006; Inoue et al., 2008; 2009). Kitaura et al. (1999), analyzing Long-Evans rats, observed that the karyomegaly is a typical histological characteristic observed in (kidney) proximal tubular epithelium before tumor development. More recently, Enzmann et al. (2013) showed that different carcinogens (diethylnitrosamine, DEN, 4-(N-methyl-N-nitrosamino)-1-(3-pyridyl)-1-butanone, NNK), evaluated in turkey eggs for in ovo carcinogenicity assay (IOCA), were responsible for the appearance of different hepatic alterations, including the hepatocellular karyomegaly. Therefore, these data signal the potential of tannery effluents to present carcinogenic characteristics (i.e., they are able to cause tumors in the liver) when animals are exposed to chronic and longer intake of such effluents. 


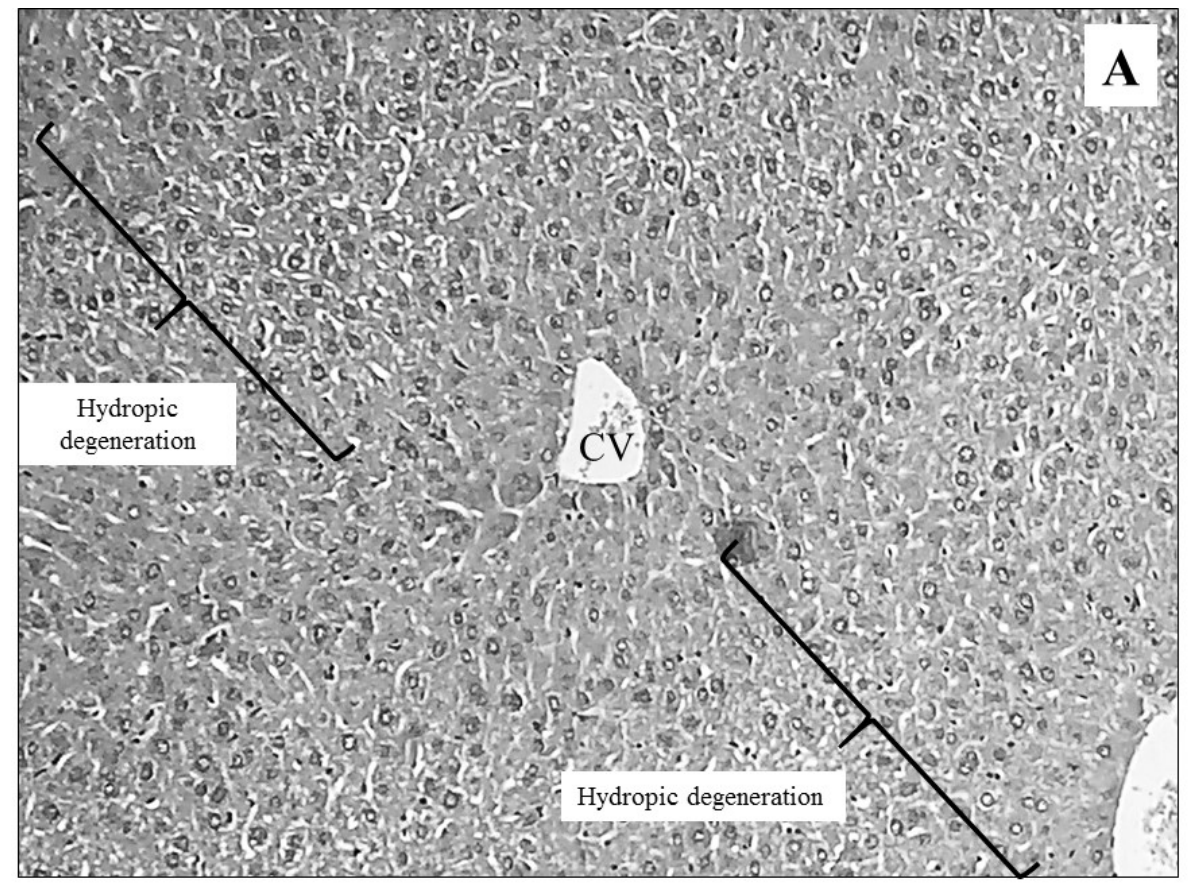

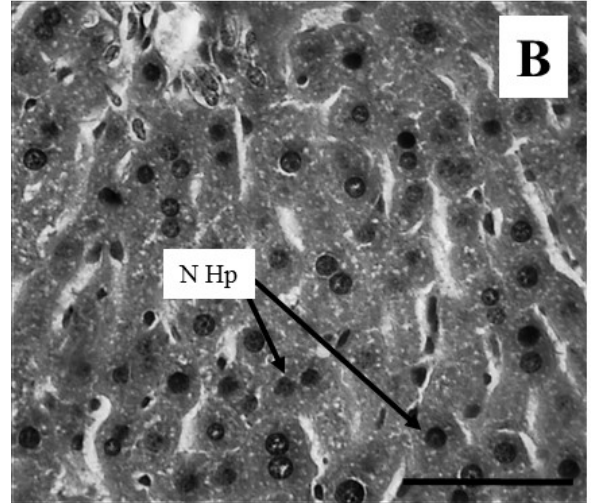

Control group

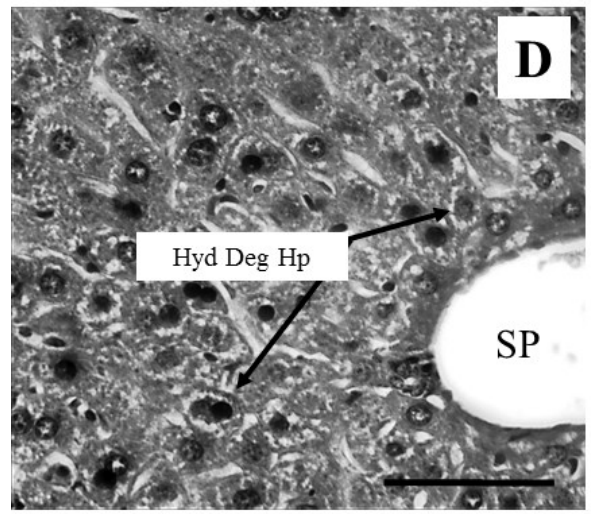

$1 \%$ group

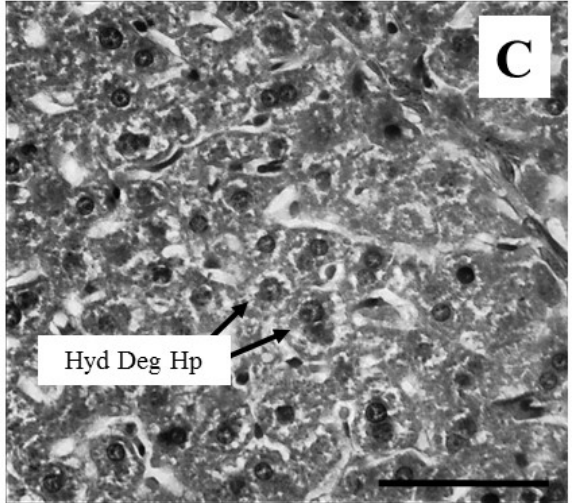

$0.1 \%$ group

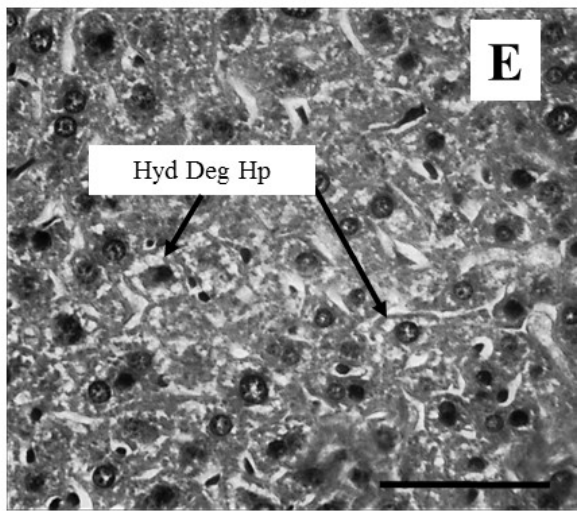

$5 \%$ group

Figure 1. Photomicrographs of distinct liver regions (A-E) of C57BL/6J mice exposed to intake of tannery effluent in different concentrations diluted in filtered water. In A, a marked hydropic degeneration in the mediozonal and periportal region is observed (animal from group 5\% of tannery effluent diluted in water). N Hp: Normal hepatocyte; Hyd Deg Hp: hepatocyte with hydropic degeneration; CV: centrilobular vein; SP: space port. Bar=100 $\mu \mathrm{m}$. HE stain. 


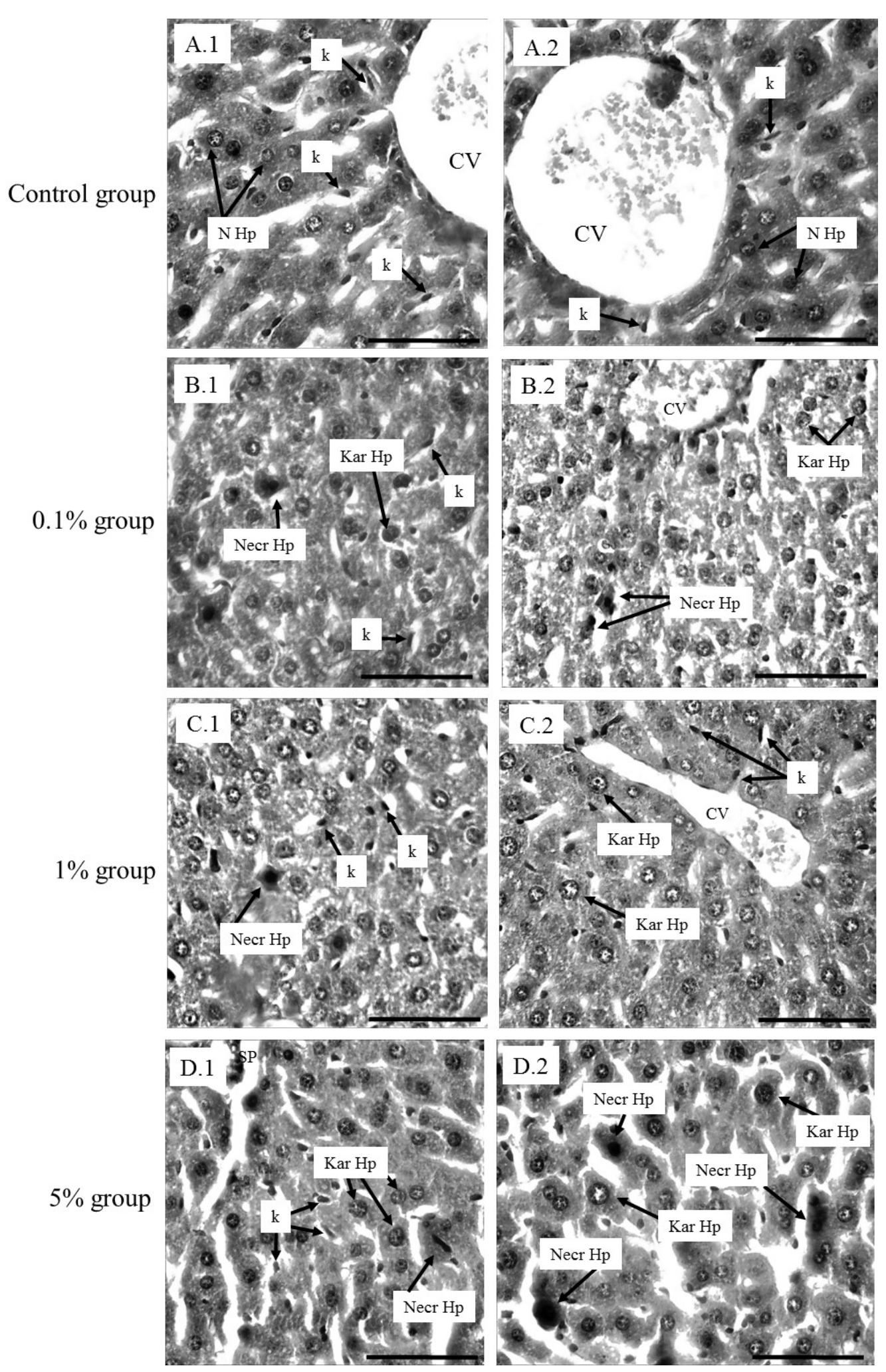

Figure 2. Photomicrographs representing distinct liver regions (A.1-D.2) of C57BL/6J mice exposed to intake of tannery effluents in different concentrations diluted in filtered water. A.1-A.2: control group; B.1-B.2: 0.1\% group; C.1-C.2: 1\% group; D.1-D.2: 5\% group. $\mathrm{N}$ Hp: Normal hepatocyte; Kar Hp: karyomegaly hepatocytes; k: Kupffer cell; Necr Hp: Necrosis in hepatocyte; CV: centrilobular vein; SP: space port. Bar=50 $\mu \mathrm{m}$. HE stain. 

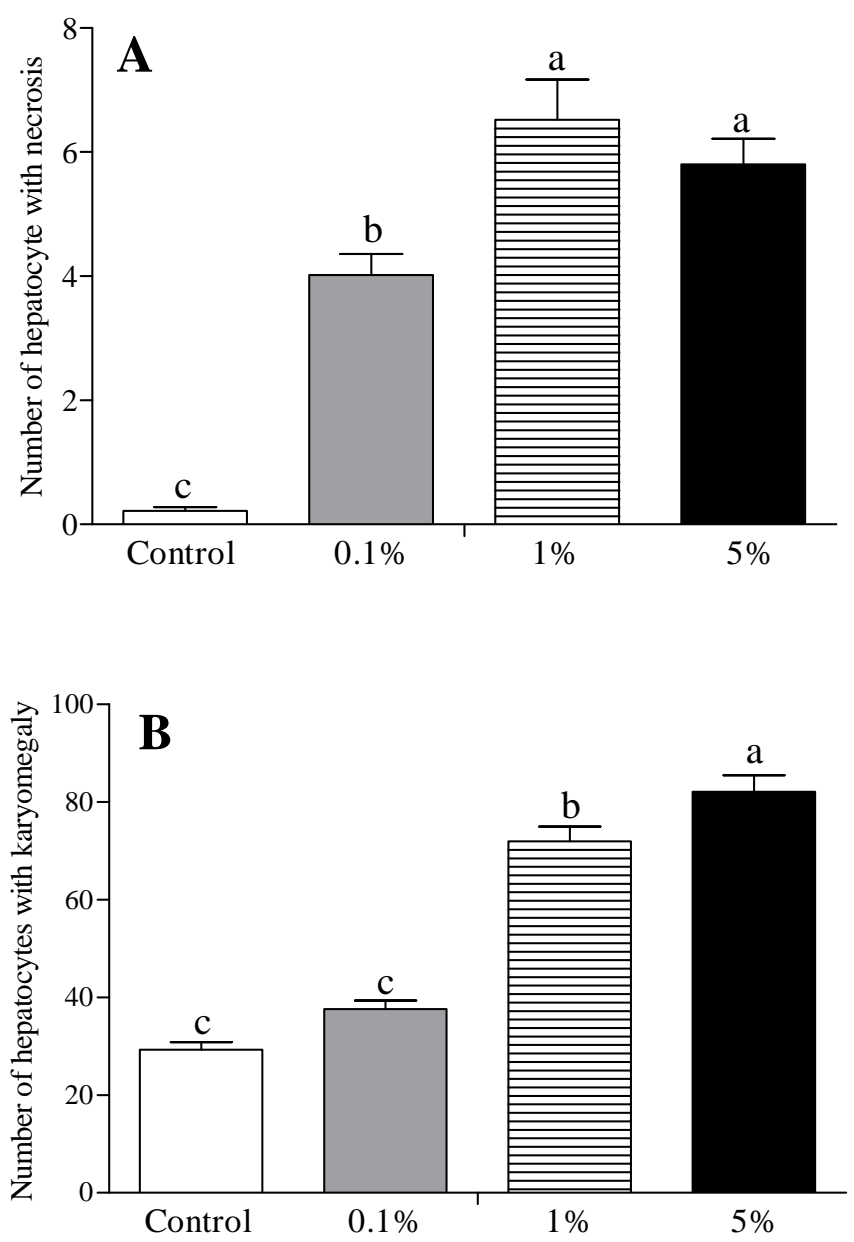

Figure 3. (A) Number of hepatocytes with karyomegaly and (B) with necrosis in liver of C57BL/6J mice exposed to intake of tannery effluents in different concentrations diluted in filtered water. Distinct letters indicate significant differences among the experimental groups by the analysis of variance (one-way ANOVA) at 5\% probability. Bars indicate mean \pm standard deviation $(n=5)$.

\section{FINAL CONSIDERATIONS}

Our study is a contribution to the knowledge on how the intake of tannery effluents can affect mice hepatocytes. Our results suggest that the alterations we observed are linked to changes that lead to the increase of free radical production and direct aggressions to hepatocyte membranes as well as the appearance of hepatocellular karyomegaly. On the other hand, studies that analyze the effects of effluent intake in mammal experimental models are rare; therefore, this opens the perspective of research to elucidate the biologic mechanisms related to the physical, physiological and anatomopathological effects in these animals.

Concerning histopathological alterations, a field of interest would be to better understand and influence the species that react with oxygen such as superoxide radicals, hydrogen peroxide, and hydroxyl radicals in the etiology of damage, especially if we consider that they are related to the toxicity of several xenobiotics and to the development of diseases. 


\section{ACKNOWLEDGMENTS}

The authors thank the Instituto Federal de Educação, Ciência e Tecnologia Goiano (Goiás, Brazil), for financing this study. They also thank the National Council for Scientific and Technological Development (CNPq - Conselho Nacional de Científico e Tecnológico, Brazil) for granting scholarships to the authors.

\section{REFERENCES}

AJAYAN, K. V.; SELVARAJU, M. Heavy metal induced antioxidant defense system of green microalgae and its effective role in phycoremediation of tannery effluent. Pakistan Journal of Biological Sciences, v. 15, n. 22, p. 1056-1062, 2012. http://dx.doi.org/10.3923/pjbs.2012.1056.1062

AMERICAN PUBLIC HEALTH ASSOCIATION - APHA; AMERICAN WATER WORKS ASSOCIATION - AWWA; WATER ENVIRONMENT FEDERATION - WEF. Standard methods for the examination of water and wastewater. $21^{\text {st }}$ ed. Washington, D.C., 2005.

BATISTA, M. M.; ALOVISI, A. M. T. Alterações de atributos químicos do solo e rendimento da cana soca pela utilização de lodo de curtume. Anuário da Produção de Iniciação Científica Discente, v. 13, n. 17, p. 387-396, 2010.

BEHMER, A. O.; TOLOSA, E. M.; FREITAS-NETO, A. G. Manual de técnicas para histologia normal e patológica. São Paulo: Art/Edusp, 1976.

CUBEROS, E.; RODRIGUEZ, A. I.; PIETRO, E. Niveles de cromo y alteraciones de salud en una población expuesta a las actividades de curtiembres en Bogotá, Colombia. Revista de Salud Pública, v. 11, n. 2, p. 278-289, 2009.

ENZMANN, H.; BRUNNEMANN, K.; IATROPOULOS, M.; SHPYLEVA, S.; LUKYANOVA, N.; TODOR, I. et al. Inter-laboratory comparison of turkey in ovo carcinogenicity assessment (IOCA) of hepatocarcinogens. Experimental and

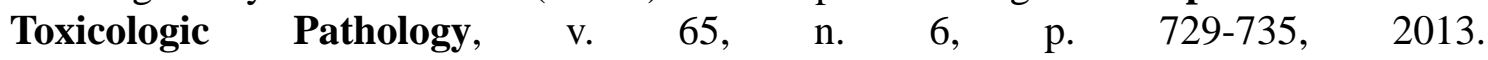
http://dx.doi.org/10.1016/j.etp.2012.09.007

FRIEDMAN, S. L.; ARTHUR, M. J. P. Activation of cultured rat hepatic lipocytes by Kupffer cell conditioned medium. Direct enhancement of matrix synthesis and stimulation of cell proliferation via induction of platelet derived growth factor receptors. Journal of Clinical Investigation, v. 84, n. 6, p. 1780-1785, 1989. http://dx.doi.org/10.1172\%2FJCI114362

GANEM, R. S. Curtumes: aspectos ambientais. 2007. Disponível em: http://bd.camara.gov.br/bd/bitstream/handle/bdcamara/ 1281/curtumes_aspectos_senna.pdf?sequence=1. Access: 20 May 2013.

GÖDECKE, M. V.; RODRIGUES, M. A. S.; NAIME, R. H. Resíduos de curtume: estudo das tendências de pesquisa. Revista Eletrônica em Gestão, Educação e Tecnologia Ambiental, v. 7, n. 7, p. 1357-1378, 2012. http://dx.doi.org/10.5902/223611705779

INOUE, K. M.; SHIBUTANI, M.; MASUTOMI, N.; TOYODA, K.; TAKAGI, H.; UNEYAMA, C. et al. A 13-week subchronic toxicity study of madder color in F344 rats. Food and Chemical Toxicology, v. 46, n. 1, p. 241-252, 2008. http://dx.doi.org/10.1016/j.fct.2007.08.002 
INOUE, K.; YOSHIDA, M.; TAKAHASHI, M.; SHIBUTANI, M.; TAKAGI, H.; HIROSE, $\mathrm{M}$. et al. Induction of kidney and liver cancers by the natural food additive madder color in a two-year rat carcinogenicity study. Food and Chemical Toxicology, v. 47, n. 6, p. 184-191, 2009. http://dx.doi.org/10.1016/j.fct.2008.10.031

KITAURA, K.; CHONE, Y.; SATAKE, N.; AKAGI, A.; OHNISHI, T.; SUZUKI, Y. et al. Role of copper accumulation in spontaneous renal carcinogenesis in Long-Evans Cinnamon rats. Japanese Journal of Cancer Research, v. 90, n. 4, p. 385-392, abr. 1999. http://dx.doi.org/10.1111/j.1349-7006.1999.tb00759.x

KRAEMER, M. E. P. A questão ambiental e os resíduos industriais. 2006. Disponível em: http://www.amda.org.br/imgs/up/Artigo_25.pdf Access: 14 Nov. 2014.

LOOCK, E. A.; REED, C. J. Trichloroethylene: mechanisms of renal toxicity and renal cancer and relevance to risk assessment. Toxicological sciences, v. 91, n. 2, p. 313-331, 2006.

LUNA, L. G. Manual of histologic staining methods of the Armed Forces Institute of Pathology. 13. ed. New York: McGraw-Hill, 1968. 258p.

MOYSÉS, F. S.; BERTOLDI, K.; SPINDLER, C.; SANCHES, E. F.; ELSNER, V. R.; RODRIGUES, M. A. S. et al. Exposition to tannery wastewater did not alter behavioral and biochemical parameters in Wistar rats. Physiology \& Behavior, v. 129, n. 1, p. 160166, 2014. http://dx.doi.org/10.1016/j.physbeh.2014.02.022

ORAL, R.; MERIÇA, S.; NICOLA, E.; PETRUZZELLI, D.; ROCCA, C. D.; PAGANO, G. Multi-species toxicity monitoring in a chromium-based leather tannery wastewater. Desalination, v. 211, n. 1, p. 48-57, 2007.

PACHECO, J. W. F. Curtumes: Série P+L. 2005. Disponível em: http://www.cetesb.sp.gov.br. Access: 5 Jan. 2013.

PINHEIRO, F. G. R. Avaliação do Potencial Poluidor de curtumes do Distrito Industrial de Icoaraci e influência sobre os recursos hídricos locais. 2005. 140f. Dissertação (Mestrado em Geologia) - Centro de Geociências, Universidade Federal do Pará, Belém, 2005.

PRABAKARAN, M.; BINURAMESH, C.; STEINHAGEN, D.; DINAKARAN MICHAEL, R. Immune response in the tilapia, Oreochromis mossambicus on exposure to tannery effluent. Ecotoxicology and environmental safety, v. 68, n. 3, p. 372-378, 2007. http://dx.doi.org/10.1016/j.ecoenv.2006.11.016

R CORE TEAM. R: a language and environment for statistical computing. Viena: $\mathrm{R}$ Foundation for Statistical Computing, 2014. Available in: http://www.R-project.org/. Access: 6 Jun. 2014.

RUMIN, C. R.; SILVA, D. B.; SOUZA, M. A. R. Intervenção em saúde do trabalhador em um curtume do Oeste Paulista. Revista Psicologia Organizações e Trabalho, v. 13, n. 2, p. 127-140, 2013.

SALAZAR, E. M. G. Economía ecológica frente a economía industrial. El caso de la industria de la curtiduría en México. Argumentos, v. 21, n. 56, p. 55-71, 2008.

SHAHZAD, K.; AKHTAR, S.; MAHMUD, S. Prevalence and determinants of asthma in adult male leather tannery workers in Karachi, Pakistan: a cross sectional study. BMC Public Health, v. 6, n. 1, p. 292-298, 2006. http://dx.doi.org/10.1186/1471-2458-6-292 
SILVA, A. S. S.; SOUZA, J. G.; LEAL, A. C. Qualidade de vida e meio ambiente: experiência de consolidação de indicadores de sustentabilidade em espaço urbano. Sustentabilidade em Debate, v. 3, n. 2, p. 177-196, 2012. http://dx.doi.org/10.18472 FSustDeb.v3n2.2012.8133

SIQUEIRA, I. R.; VANZELLA, C.; BIANCHETTI, P.; RODRIGUES, M. A. S.; STÜLP, S. Anxiety-like behaviour in mice exposed to tannery wastewater: the effect of photoelectrooxidation treatment. Neurotoxicology and Teratology, v. 33, n. 4, p. 481484, 2011. http://dx.doi.org/10.1016/j.ntt.2011.05.008 


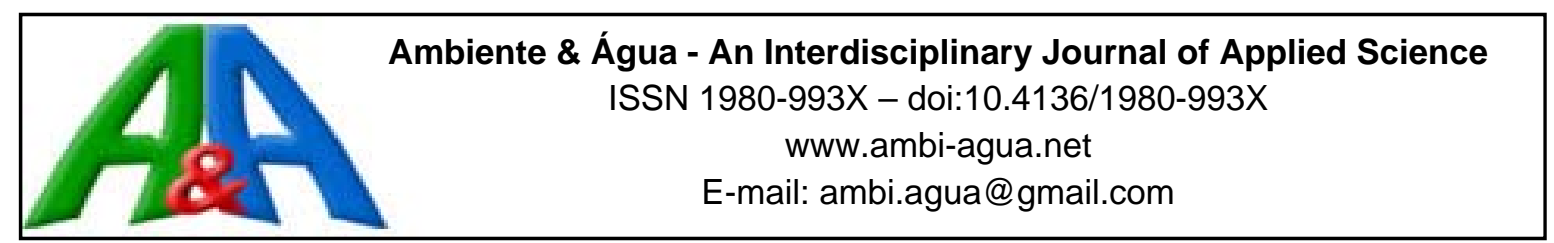

\title{
Modelado espacial de incendios: una herramienta predictiva para el Bosque La Primavera, Jalisco México
}

\author{
doi:10.4136/ambi-agua.1536
}

Received: 09 Oct. 2014; Accepted: 15 Dec. 2015

\author{
Jose Luis Ibarra-Montoya*; Francisco Martín Huerta-Martínez \\ Universidad de Guadalajara (UDG), Guadalajara, Jalisco, México \\ Centro Universitario de Ciencias Biológicas y Agropecuarias \\ Departamento de Ecología \\ *Autor correspondiente: e-mail: jibarra@ecologia.unam.mx, \\ fhuerta@cucba.udg.mx
}

\section{RESUMEN}

La interacción de diversos elementos de carácter socioeconómico, político y cultural, influidos por factores de paisaje y climáticos, son aspectos importantes en los regímenes de incendios. Sin duda la generación de modelos espaciales que integran esos elementos y factores, ayudan a predecir zonas potenciales de incendios con más exactitud. El Área de Protección de Flora y Fauna La Primavera (APFFLP) constituye el principal regulador climático de la zona metropolitana de Guadalajara, y presenta frecuentemente incendios forestales, por lo que representa un reto para la ciencia y la tecnología utilizarla como modelo para desarrollar metodologías que ayuden a predecir incendios forestales. El presente estudio se enfoca a la construcción de un modelo espacial que ayuda a determinar zonas potenciales de incendios en dicha área. El modelo integra variables meteorológicas, de paisaje, de combustibles, antropogénicas y/o de causalidad; y las ocurrencias históricas de los incendios comprendidos durante el periodo 1998-2012. Según el modelo, las variables que determinan las zonas con mayor potencial de incendios son: la pendiente (paisaje), la humedad relativa (meteorológica), el tipo de vegetación (causalidad) y el uso del suelo (antropogénicas). El modelo predice una zona extensa con alto potencial de incendiarse, ubicada en la parte central y noroeste del polígono del APFFLP; asimismo, existen zonas potenciales pequeñas y aisladas ubicadas en el oriente del polígono. La información generada en este estudio podría ayudar a la generación de mapas de riesgo locales y con ello, optimizar las acciones de manejo del fuego y restauración del Bosque La Primavera.

Palabras clave: área natural protegida, heterogeneidad ambiental, landscape, mapas de riesgo, MAXENT.

\section{Modelagem espacial de incêndios: uma ferramenta preditiva para o Bosque La Primavera, Jalisco México}

\section{RESUMO}

A interação de vários elementos de natureza socioeconômica, política e cultural, influenciados pela paisagem e fatores climáticos, são aspectos importantes do regime de fogo. Definitivamente, modelos espaciais que integram esses elementos ajudam a prever áreas 
potenciais de incêndio de forma mais precisa. A Área de Proteção da Vida Selvagem La Primavera (APFFLP) é o principal regulador do clima da região metropolitana de Guadalajara e, frequentemente, apresenta incêndios florestais, o que representa um desafio para a ciência e tecnologia o desenvolvimento de metodologias que possam ajudar prever estes incêndios. $\mathrm{O}$ presente estudo envolve a construção de um modelo espacial que ajuda a identificar áreas potenciais de incêndio na área. O modelo integra variáveis meteorológicas, paisagem, combustíveis, antropogênicas e/ou naturais; assim como, ocorrências históricas de incêndios durante o período 1998-2012. De acordo com o modelo, as variáveis que determinam as áreas de maior potencial de incêndios são: a declividade (paisagem), umidade relativa (tempo), tipo de vegetação (natural) e uso da terra (antropogênica). O modelo prediz uma grande área, com um elevado potencial de incêndio, localizado no polígono APFFLP central e noroeste; além disso, há pequenas zonas potenciais, isoladas ao leste do polígono. As informações geradas neste estudo podem dar suporte à geração de mapas de risco local e, assim, otimizar as ações de manejo do fogo e restauração do Bosque La Primavera.

Palavras-chave: área protegida, heterogeneidade ambiental, cenário, mapas de risco, MAXENT.

\title{
Spatial modeling of fires: a predictive tool for La Primavera Forest, Jalisco Mexico
}

\begin{abstract}
The interaction of various elements of socioeconomic, political and cultural nature, influenced by landscape and climatic factors, are important aspects of fire regimes. Space models that integrate these elements and factors help to more accurately predict potential fire areas. The Protected Area Wildlife La Primavera (APFFLP) is the main regulator of the climate of the Guadalajara metropolitan area, and forest fires frequently occur there. These represent a challenge for science and technology to develop methodologies that help predict forest fires. This study involves the construction of a spatial model that helps identify potential areas of fire in that area. The model integrates meteorological variables, landscape, fuels, anthropogenic and / or causality, and historical occurrences of fires during the period 1998-2012. According to the model, the variables that determine the areas of greatest fire potential are: slope (landscape), relative humidity (weather), vegetation type (causality) and land use (anthropogenic). The model predicts a large area with high potential for fire, located in the central and northwest APFFLP polygon; also, there are small, isolated potential zones in the eastern part of the polygon. The information developed by this study could support the generation of local risk maps, thereby optimizing the actions of fire management and restoration of the La Primavera forest.
\end{abstract}

Keywords: environmental heterogeneity, landscape, protected area, risk maps, MAXENT.

\section{INTRODUCCIÓN}

Los incendios forestales son el resultado de la interacción de diversos elementos de carácter socioeconómico, político y cultural que, influidos por factores de paisaje y climáticos, determinan la magnitud e intensidad de su comportamiento (Sánchez, 1989). Es por ello que predecir las ocurrencias de incendios forestales y entender sus factores asociados es un reto para la ciencia y es un proceso clave para diseñar estrategias de manejo del fuego (Gollberg et al., 2001). Diversos estudios se han enfocado a estudiar la interacción entre los factores físicos y climáticos del sitio (Flase y Ceccato, 1996; Sepúlveda et al., 2001; Giglio et 
al., 2003; Dryry y Veblen, 2008), en la mayoría de éstos se menciona que los incendios se correlacionan con las variaciones climáticas extremas y en algunos casos se ha explicado su ocurrencia desde una perspectiva geoespacial (Ávila-Flores y Pompa-Garcia, 2010). También se han desarrollado modelos que consideran las variables meteorológicas y de combustible para explicar el comportamiento de los incendios (Stolyarchuk, 1979; Mathur et al., 1984; Marcozzi et al., 1994; Mandallaz y Ye, 1997; Wybo et al., 1995; Gutiérrez et al., 1997; Gouma y Chronopoulou-Sereli, 1998; Leathwick y Briggs, 2001).

A nivel mundial existen sistemas que ayudan a identificar las áreas con peligro de incendio, destacan el Canadian Forest Fire Danger Index (Lee et al., 2002); Forest Fire Danger Index desarrollado en Australia (CSIRO, 2000) y el National Fire Danger Rating System, desarrollado por el Servicio Forestal de Estados Unidos de América (Deeming et al., 1978). En México no se cuenta con un sistema de identificación de áreas potenciales con peligro de incendio; es competencia del Centro Nacional de Control de Incendios Forestales (CENCIF) concentrar, procesar y distribuir la información de indicadores de riesgo y de los incendios forestales detectados en el territorio nacional a través de la detección satelital de puntos de calor (CONAFOR, 2014b; CONABIO, 2014).

Los modelos sobre incendios desarrollados para México han sido escasos y aislados y consideran solo la carga de combustibles forestales y algunas variables meteorológicas (Magaña, 1985; Zapata, 1991; Santillán, 1993). Por ejemplo, destacan los trabajos de Sepúlveda et al. (1999), quienes desarrollaron un modelo de combustibles en Baja California utilizando variables meteorológicas; Muñoz et al. (2005) generaron un modelo de comportamiento de incendios para la Sierra Madre Oriental y Pérez-Verdín et al. (2013) quienes desarrollaron un análisis espacio-temporal de la ocurrencia de incendios forestales en Durango, México; utilizando patrones de distribución espacial y análisis de estacionalidad y auto correlación de variables que afectan la magnitud de los mismos. Aún con estos esfuerzos, los modelos generados no utilizan todos los componentes y variables que interactúan para predecir la ocurrencia detallada de un incendio en una determinada zona; por lo que es necesario desarrollar modelos espaciales que integren variables meteorológicas, de combustibles y de paisaje con los factores de carácter socioeconómico, político y cultural y generar escenarios futuros de áreas potenciales de incendios, y puedan servir de apoyo a las autoridades correspondientes de combatir los incendios y en la toma de decisiones sobre la conservación, restauración y gestión ambiental.

El Área de Protección de Flora y Fauna Bosque La Primavera, (APFFLP), ubicada al oeste de la ciudad de Guadalajara, Jalisco, México, es el principal regulador del clima de la zona conurbada de Guadalajara, provee bienes y servicios a la población y alberga una gran diversidad de especies que mantienen una interacción con el bosque de encino y pino, y dependen casi exclusivamente de la existencia de este bosque y sus corredores ecológicos (Mexico, 2000). Desde el periodo comprendido entre los años 1910-1960, el Bosque La Primavera ha sido blanco del aprovechamiento intensivo de los recursos forestales maderables y no maderables, y se llevan a cabo actividades de agricultura, de uso público y especialmente la generación de incendios controlados durante la zafra en el cultivo de la caña de azúcar. Dadas estas actividades, el área presenta una alta frecuencia de incendios forestales que han llegado a afectar más de 11 mil hectáreas en un año (Mexico, 2012).

El objetivo del presente trabajo fue desarrollar un modelo espacial predictivo de áreas potenciales de incendios para el Área de Protección de Flora y Fauna Bosque La Primavera, México, tomando como base los datos históricos de la ocurrencia de incendios en el APFFLP durante el periodo 1998-2012 (Huerta-Martínez e Ibarra-Montoya, 2014) así como las variables de combustibles, meteorológicas, de paisaje y antropogénicas y/o de causalidad. 


\section{MATERIALES Y MÉTODOS}

\subsection{Sitio de estudio}

El APFFLP está ubicada en la región central del estado de Jalisco, entre las coordenadas extremas $103^{\circ} 28^{\prime}$ a $103^{\circ} 42^{\prime}$ de longitud oeste, y $20^{\circ} 32^{\prime}$ a $20^{\circ} 44^{\prime}$ de latitud norte. Está situada al centro de un conjunto de cinco valles (Tala, Tesistán, Toluquilla, Atemajac y San Isidro Mazatepec) pertenecientes a los municipios de Zapopan, Tala, y Tlajomulco de Zúñiga. La Primavera es el bosque más cercano a la ciudad de Guadalajara, localizándose al poniente de la misma, y está circundado por las siguientes carreteras: Guadalajara-Nogales al norte; Tala-Ahuisculco al oeste; Tlajomulco de Zúñiga-San Isidro Mazatepec al sur; y Guadalajara-Barra de Navidad y Periférico poniente de la Ciudad de Guadalajara desde la Avenida López Mateos hasta la Av. Vallarta, al este (Figura 1).

El APFFLP es un Área Natural Protegida (ANP) que tiene alrededor de 22 puntos de acceso mediante caminos de terracería y brechas, tres de los cuales se utilizan principalmente con fines recreativos: uno por la Av. Mariano Otero, en el lado oriente de esta zona, y dos en el lado norte, por el ejido La Primavera y Puente Las Tortugas.

Según la clasificación climática de Köppen, modificada por García (1973) para la República Mexicana, el clima predominante es templado subhúmedo $\mathrm{S}\left(\mathrm{w}_{1}\right)(\mathrm{w})$ y semicálido subhúmedo $(\mathrm{A}) \mathrm{C}\left(\mathrm{w}_{1}\right)(\mathrm{w})$. La temperatura media anual es de $20.6{ }^{\circ} \mathrm{C}$, siendo el mes más frío enero y el más cálido junio. El promedio anual de humedad es de 63\%. Los vientos dominantes provienen del suroeste, con una velocidad máxima $53 \mathrm{~km} \mathrm{~h}^{-1}$.

El APFFLP se asienta sobre la Sierra La Primavera, la cual se ubica en la provincia fisiográfica del Eje Neovolcánico, subprovincia Guadalajara, que se caracteriza por manifestaciones recientes de vulcanismo explosivo. Está considerada como la manifestación riolítica más reciente y compleja de toda la provincia del Eje Neovolcánico. Por su variación en cuanto a manifestaciones ígneas ácidas es uno de los relieves volcánicos con más diversidad de México, en el que se combinan domos anulares, mesetas, cerros y montañas que siguen las líneas de fractura de la caldera volcánica, y colinas irregulares modeladas por erosión. Si bien estas formas tienen un origen volcánico común, en la actualidad presentan influencia de las fuerzas denudatorias fluviales y tectónicas.

De acuerdo a la clasificación FAO/UNESCO, las unidades de suelo que constituyen el área comprendida en el APFFLP están representados por regosol y litosol. El regosol conforma el 92\% del ANP, el cual es derivado del intemperismo de la toba, pómez y riolita. En el restante $8 \%$ de la superficie en cuestión, se encuentran distribuidos suelos de tipo litosol, resultantes de procesos erosivos. Ambos suelos se caracterizan por presentar una profundidad efectiva máxima de acuerdo a lo siguiente: el $44 \%$ de la superficie con suelo presenta un espesor de 10 a $30 \mathrm{~cm}$; el $9 \%$ de 30 a $60 \mathrm{~cm}$ y el $47 \%$ restante es mayor de $60 \mathrm{~cm}$.

El $80 \%$ del suelo involucrado en el ANP presenta valores de contenido de materia orgánica menores al $2 \%$, lo que indica que no existe el suelo típico de bosque. La vegetación presente esta caracterizada principalmente por Bosque de encino, bosque de encino-pino, bosque de pino y bosque tropical caducifolio.

\subsection{Modelo conceptual}

Para la conformación del modelo espacial, fue necesario construir un modelo conceptual basado inicialmente en el publicado por Pompa (2012). Dicho modelo incluye cuatro componentes espaciales: 1) complejo de variables de combustibles, construido a partir de la existencia real de las especies dominantes (número); el $\mathrm{pH}$ del suelo; la profundidad de la hojarasca $(\mathrm{cm})$ y la concentración de carbono medida como la biomasa acumulada $\left(\mathrm{g} / \mathrm{cm}^{2}\right)$, con valores asignados en cada sitio de muestreo; 2) componente meteorológico, definido por la temperatura ambiental $\left({ }^{\circ} \mathrm{C}\right)$ y la humedad relativa $\left.(\%) ; 3\right)$ componente de paisaje, 
constituido por la pendiente (\%), la altitud (msnm), la exposición (azimut) y la fisiografía (raster); y 4) componente antropogénico y/o de causalidad, integrado por las variables de uso de suelo (raster), tipo de suelo (raster), tipo de vegetación (raster) y perturbación (\%). Su integración esquemática se muestra en la Figura 2.

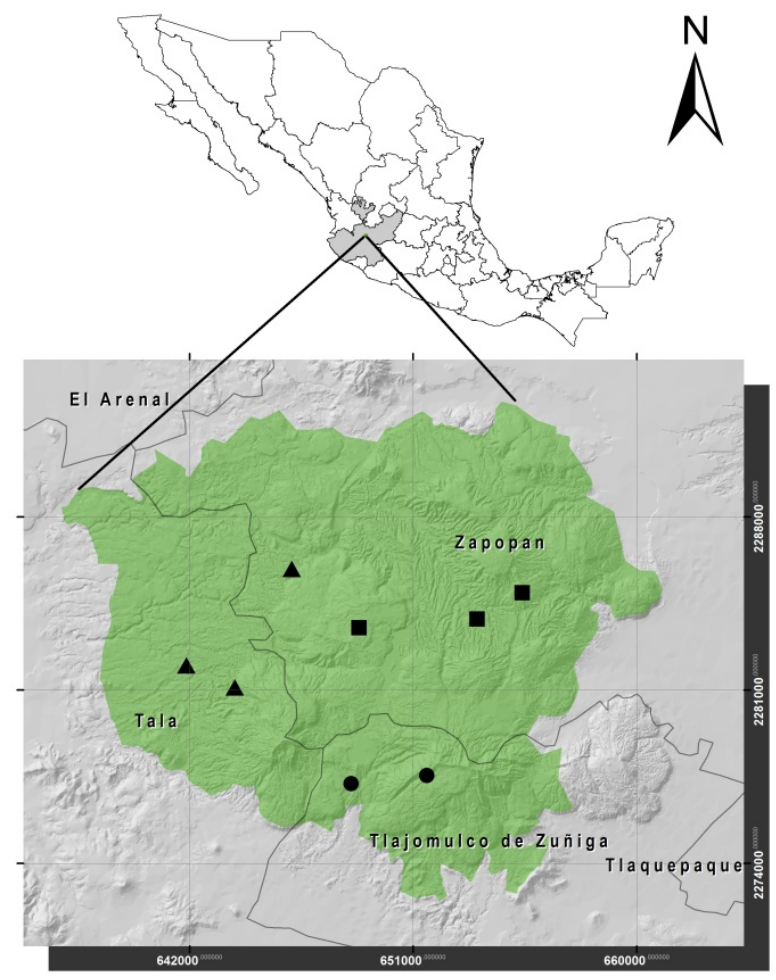

Figura 1. Mapa de localización geográfica del APFFLP, y ubicación de las estaciones de muestreo.

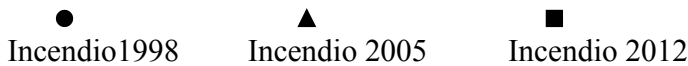

Modelo conceptual de la ocurrencia futura de incendios

- Especies dominantes

- $\mathrm{pH}$ del suelo

- Prof. de hojarasca

- Biomasa

Incendio1998

Incendio 2005

Incendio 2012

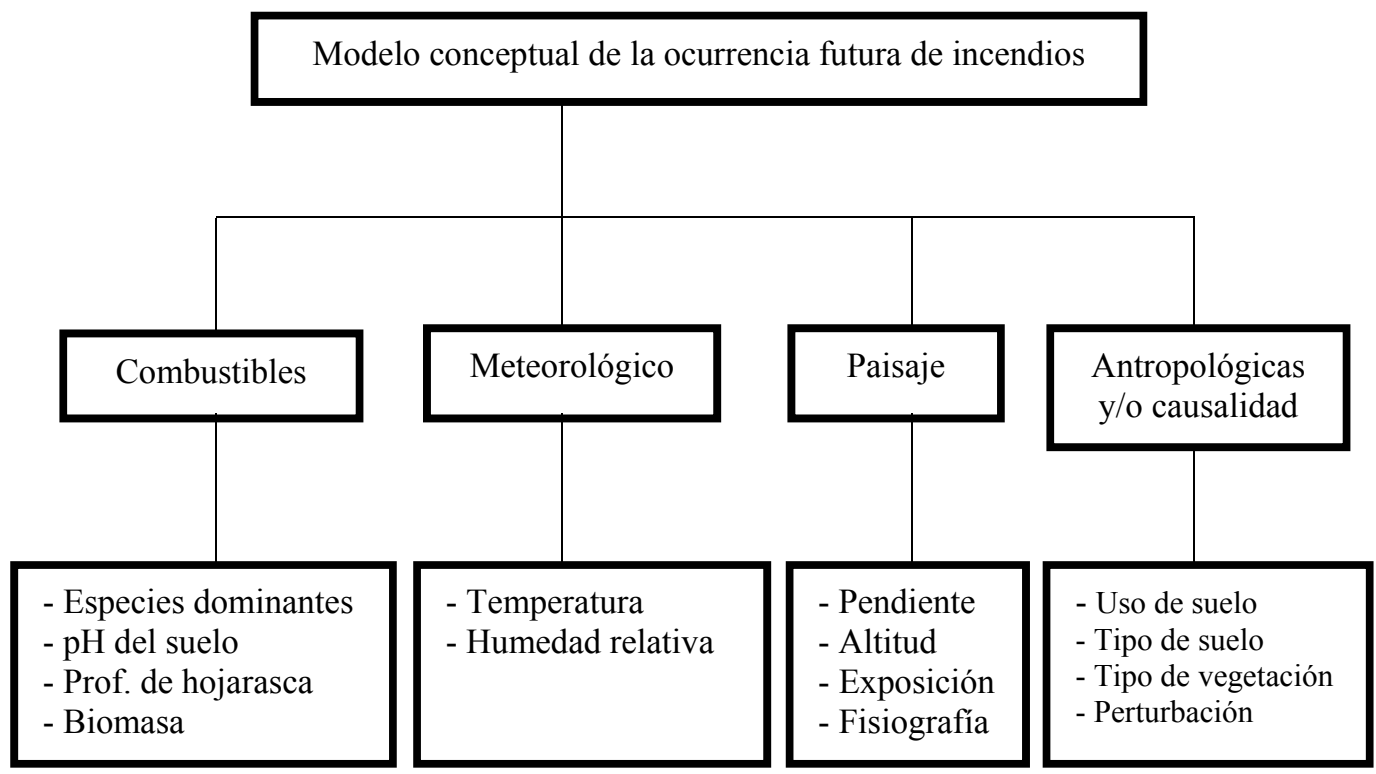

Figura 2. Esquema de la generación del modelo conceptual de ocurrencia futura de incendios forestales: Componentes y variables (Modificado de Pompa, 2012). 


\subsection{Selección de los puntos de muestreo}

Se eligieron ocho estaciones de muestreo ubicadas dentro del polígono del APFFLP; los sitios corresponden a las áreas quemadas por los incendios de los años: 1998, 2005 y

2012 (Figura 1, Tabla 1). Los sitios de muestreo fueron georreferenciados con un geoposicionador satelital (GPS map, $178 \mathrm{C}$ Sounder, marca GARMIN).

En cada una de las estaciones de muestreo se registraron catorce variables correspondientes al esquema del modelo conceptual de ocurrencia de incendios propuesto en este estudio (Figura 2).

Para el caso de la biomasa acumulada (combustibles forestales), la metodología consistió en la ubicación de una línea de $20 \mathrm{~m}$ de longitud cuya dirección fue definida aleatoriamente, para cada sitio de muestreo. Para el caso de la cama de combustibles forestales, se registró su profundidad a los 5, 10, 15, y $20 \mathrm{~m}$ de longitud de la línea. Además de colectó la hojarasca contenida en $1 \mathrm{~m}^{2}$ compuesto por cuatro cuadrantes de $0.25 \mathrm{~m}^{2}$ distribuidos a lo largo de la línea. Cada cuadrante fue pesado in situ y se tomó una muestra de $300 \mathrm{~g}$ para su posterior secado en el laboratorio. El peso seco de estas muestras fue relacionado con el peso del material colectado in situ y de esta forma se obtuvo la cantidad de este tipo de combustible por unidad de superficie.

Para determinar la altitud se empleó un modelo digital del terreno (MDT) con una resolución espacial de $30 \mathrm{~m}$, generado a partir de las curvas de nivel de INEGI; escala 1:50,000 (INEGI, 2014b).

Debido a la dificultad de representar espacialmente los agentes causales de incendios, y en virtud de la importancia de su integración en el modelo, se realizó el análisis de los rasgos geográficos asociados con actividades humanas. Chuvieco et al. (2000), García et al. (1999) y Almeida (1994), señalan que el desarrollo de un modelo de predicción de incendios siempre presenta dificultades debido a la incertidumbre asociada al comportamiento humano en relación con el fuego. En este caso los rasgos evaluados fueron el tipo de vegetación, uso de suelo, tipo de suelo y la perturbación.

La selección de las estaciones de muestreo, se basaron en encontrar los lugares adecuados para generar un mapa de interpolación por cada variable analizada. Por lo tanto las estaciones fueron seleccionadas según los criterios: i) ubicación dentro del área quemada para los años 1998, 2005 y 2012; ii) hacer una réplica en cada uno de los años; iii) incluir sitios con tipo de vegetación contrastante para análisis de la biomasa proveniente de diferentes especies de árboles.

\subsection{Identificación de las especies presentes en cada estación de muestreo}

Se identificaron las especies de árboles presentes en cada sitio, para ello se colectó material foliar de los individuos y se llevó a cabo la identificación utilizando las claves taxonómicas para encinos y pinos (Nixon y Muller, 1993; Vázquez et al., 2004; Manos et al., 1999); en aquellos organismos en donde se tuviera alguna duda se revisaron con el especialista. Se determinó que solo se analizaría la biomasa proveniente de los árboles (pinos y encinos) para dejar de lado la posible variación en la biomasa producida por las plantas anuales.

\subsection{Generación de coberturas de los parámetros}

Utilizando los datos obtenidos en campo de las diferentes variables, se construyeron las coberturas utilizando el polígono que delimita al APFFLP (Huerta-Martínez e IbarraMontoya, 2014). Estas coberturas, mapas tipo "raster" se generaron utilizando la técnica IDW de interpolación (Distancia Inversa Ponderada) (Delaney, 1999), en el Sistema de Información Geográfica ArcMap 9.2 ${ }^{\circledR}$ (GIS, ESRI, 1999-2001) enmascarando el análisis solo para el polígono del APFFLP. Para las variables de uso de suelo, tipo de vegetación, tipo de 
suelo y fisiografía, su utilizaron los shapes de INEGI (INEGI, 2014a), y el modelo digital de terreno (MDT). La resolución definitiva (tamaño de pixel) de todas las coberturas fue de $1 \mathrm{~m}^{2}$.

\subsection{Modelo espacial}

Se utilizó el enfoque de Máxima Entropía (MAXENT) para modelar las predicciones de incendios, para lo cual fue utilizada la versión de escritorio 3.3.0. de noviembre 2011 (Phillips et al., 2006). En general, este algoritmo detecta las relaciones no aleatorias entre dos conjuntos de datos; a) los registros georreferenciados de la presencia de los incendios, y b) un conjunto de coberturas tipo "raster", de datos digitales que representan a las variables de combustible, meteorológicas, de paisaje, antropológicas y de causalidad pertinentes para determinar las zonas potenciales de incendios (Phillips et al., 2006).

Una explicación detalla de los aspectos técnicos de MAXENT se puede revisar en Phillips et al. (2006), sin embargo el procedimiento general aplicado para construir los modelos espaciales en el APFFLP es el siguiente:

Utilizando las coberturas de los parámetros y los datos históricos (1998-2012) de ocurrencia de los incendios se llevó acabo el modelaje. El 75\% de los registros de presencia fueron utilizados como puntos de entrenamiento y el $25 \%$ como puntos de validación. Se utilizó un umbral de convergencia de $10^{-5}$ con 1000 iteraciones como límite superior para cada corrida. Para estimar la capacidad de predicción de cada modelo, se analizó el área bajo la curva (AUC), salida grafica donde se observa la capacidad de discriminación de una ausencia (especificidad) (Phillips y Dudik, 2008). El formato de salida analizado es el acumulativo (rango de 0 a 100, el cual indica una idoneidad de incendio potencial relativa de cada pixel mas no una probabilidad de ocurrencia del incendio (salida logística). Se uso la prueba de Jackknife (Sokal y Rohlf, 1995) para calcular la contribución relativa de cada variable al modelo, los resultados de esta evaluación se expresan con una medida conocida como "ganancia". Esta información es de suma importancia para evidenciar las características que deberían prescribir un incendio y que probablemente determinen el área potencial a incendiarse. Ambos análisis, el AUC y la prueba de Jacknife están implementados en MAXENT.

Debido a que MAXENT produce resultados diferentes de una corrida a la siguiente, utilizando la misma entrada de datos, se desarrollaron 100 modelos de predicción independientes para cada ocurrencia de incendio, y se eligieron un subconjunto de los mejores 10 modelos basado en dos criterios: i) fue seleccionado un primer conjunto de 20 modelos con menos del 10\% de error por omisión y, ii) de ellos, se seleccionaron los 10 modelos más cercanos a la media en las zonas donde han ocurrido los incendios. Estos 10 modelos fueron examinados en un Sistema de Información Geográfica (SIG), y se generó un mapa de consenso con los valores de pixel de 0 a 10, donde el 0 representa las áreas en las que todos los modelos predicen la ausencia de probabilidad de incendio y el 10 representa las zonas donde todos los modelos coinciden en la predicción de las zonas potenciales de incendios. Para disminuir la sobre predicción que pudiera presentar el modelo se decidió hacer un mapa utilizando las probabilidades entre $0.8,0.9$ y 1 .

\section{RESULTADOS Y DISCUSIÓN}

En el modelo espacial, la capacidad de predicción para los datos de prueba generó un AUC de 0.834 , lo que indica que la robustez del modelo para clasificar las presencias de los incendios fue buena (Parolo et al., 2008). Así mismo, las variables para todos los sitios que tuvieron el mayor poder de predicción cuando fueron analizadas con Jacknife de manera independiente fueron: la pendiente $(\mathrm{AUC}=0.91)$, la humedad relativa $(\mathrm{AUC}=0.82)$, el tipo de vegetación $(\mathrm{AUC}=0.83)$ y el uso de suelo $(\mathrm{AUC}=0.79)$, además, fisiografía resultó con poder 
de predicción en Rio Caliente, así como temperatura y pH en Cerritos Colorados (Tabla 2).

Tabla 1. Año de incendio y los sitios de muestreo. Se muestra también el uso de suelo y tipo de vegetación, así como las especies identificadas por cada localidad.

\begin{tabular}{|c|c|c|c|c|}
\hline Año & Localidad & U. suelo & Vegetación & Esp. Dominante \\
\hline \multirow{6}{*}{1998} & \multirow{4}{*}{1 Planillas } & \multirow{4}{*}{ Forestal } & \multirow{4}{*}{ Encino-Pino } & Quercus resinosa \\
\hline & & & & Quercus viminea \\
\hline & & & & Pinus oocarpa \\
\hline & & & & Q. coccolobifolia \\
\hline & \multirow{2}{*}{2 Poleo } & \multirow{2}{*}{ Forestal } & \multirow{2}{*}{ Pino-Encino } & Quercus resinosa \\
\hline & & & & Pinus oocarpa \\
\hline \multirow{7}{*}{2005} & \multirow{3}{*}{3 Cerro San Miguel } & \multirow{3}{*}{ Forestal } & \multirow{3}{*}{ Encino } & Quercus resinosa \\
\hline & & & & Quercus viminea \\
\hline & & & & Pinus oocarpa \\
\hline & \multirow{2}{*}{4 Cerro San Miguel 1} & \multirow{2}{*}{$\begin{array}{l}\text { Forestal/ } \\
\text { Pastoreo }\end{array}$} & \multirow{2}{*}{ Pino-Encino } & Quercus resinosa \\
\hline & & & & Pinus oocarpa \\
\hline & \multirow{2}{*}{5 Río Caliente } & \multirow{2}{*}{ Forestal } & \multirow{2}{*}{ Pino-Encino } & Quercus resinosa \\
\hline & & & & Pinus oocarpa \\
\hline \multirow{8}{*}{2012} & \multirow{2}{*}{6 Ejido López Mateos } & \multirow{2}{*}{ Forestal } & \multirow{2}{*}{ Pino-Encino } & Quercus resinosa \\
\hline & & & & Pinus oocarpa \\
\hline & \multirow{3}{*}{7 Cerritos Colorados } & \multirow{3}{*}{$\begin{array}{l}\text { Forestal/ } \\
\text { Zona } \\
\text { Geotermal }\end{array}$} & \multirow{3}{*}{ Pino-Encino } & Quercus resinosa \\
\hline & & & & Quercus viminea \\
\hline & & & & Pinus oocarpa \\
\hline & \multirow{3}{*}{8 Crucero Pedernal } & \multirow{3}{*}{ Forestal } & \multirow{3}{*}{ Pino-Encino } & Quercus castanea \\
\hline & & & & Quercus resinosa \\
\hline & & & & Pinus oocarpa \\
\hline
\end{tabular}

Tabla 2. Valores de AUC para catorce variables analizadas en los ocho sitios muestreados, según el modelo espacial. Las negritas significan un AUC $\geq 0.8$.

\begin{tabular}{lcccccccc}
\hline Variable & \multicolumn{10}{c}{ Localidad } \\
\hline & 1 & 2 & 3 & 4 & 5 & 6 & 7 & 8 \\
Tipo de Suelo & 0.64 & 0.68 & 0.34 & 0.48 & 0.49 & 0.42 & 0.36 & 0.45 \\
Humedad relativa & $\mathbf{0 . 8 4}$ & $\mathbf{0 . 8 0}$ & $\mathbf{0 . 8 5}$ & $\mathbf{0 . 8 3}$ & $\mathbf{0 . 8 9}$ & $\mathbf{0 . 8 6}$ & 0.69 & $\mathbf{0 . 8 9}$ \\
pH & 0.67 & 0.78 & 0.70 & 0.67 & 0.78 & 0.69 & $\mathbf{0 . 8 8}$ & 0.72 \\
Prof. Hojarasca & 0.38 & 0.47 & 0.49 & 0.40 & 0.67 & 0.74 & 0.47 & 0.47 \\
Biomasa & 0.45 & 0.56 & 0.67 & 0.58 & 0.49 & 0.55 & 0.62 & 0.61 \\
Temperatura & 0.63 & 0.76 & 0.65 & 0.37 & 0.71 & 0.79 & $\mathbf{0 . 8 3}$ & 0.73 \\
Fisiografía & 0.57 & 0.58 & $\mathbf{0 . 8 0}$ & 0.61 & $\mathbf{0 . 8 8}$ & 0.60 & 0.79 & 0.68 \\
Pendiente & $\mathbf{0 . 8 3}$ & $\mathbf{0 . 8 4}$ & $\mathbf{0 . 8 9}$ & $\mathbf{0 . 8 2}$ & $\mathbf{0 . 8 3}$ & $\mathbf{0 . 8 0}$ & $\mathbf{0 . 8 5}$ & $\mathbf{0 . 9 3}$ \\
Exposición & 0.67 & 0.57 & 0.68 & 0.69 & 0.51 & 0.55 & 0.75 & 0.78 \\
Perturbación & 0.70 & 0.79 & 0.62 & 0.69 & 0.79 & 0.61 & 0.61 & 0.68 \\
Uso de Suelo & $\mathbf{0 . 8 6}$ & $\mathbf{0 . 8 4}$ & $\mathbf{0 . 8 2}$ & $\mathbf{0 . 8 9}$ & $\mathbf{0 . 8 8}$ & $\mathbf{0 . 8 6}$ & $\mathbf{0 . 8 2}$ & $\mathbf{0 . 8 1}$ \\
Tipo de Vegetación & 0.78 & $\mathbf{0 . 8 9}$ & $\mathbf{0 . 8 4}$ & $\mathbf{0 . 8 9}$ & $\mathbf{0 . 8 7}$ & $\mathbf{0 . 8 8}$ & $\mathbf{0 . 8 3}$ & $\mathbf{0 . 8 9}$ \\
Altitud & 0.64 & 0.68 & 0.62 & 0.59 & 0.67 & 0.71 & 0.64 & 0.66 \\
Esp. Dominantes & 0.68 & 0.62 & 0.47 & 0.70 & 0.73 & 0.62 & 0.56 & 0.73 \\
\hline
\end{tabular}


Las predicciones del modelo espacial desarrollado en este trabajo muestran una zona continua con alto potencial de incendio ubicada en la parte central y noreste del polígono del APFFLP de aproximadamente 4,937 Ha (Figura 3), correspondiente al Ejido López Mateos. Cabe mencionar que parte de esta área ya fue incendiada en el año 2012 (Mexico 2012). Esta zona potencial de incendios predicha por el modelo espacial presenta pendientes pronunciadas y el tipo de vegetación es bosque de encino y de pino-encino.

Por otro lado, el modelo también predice pequeñas zonas aisladas potenciales de incendios de $28 \mathrm{Ha}$ en promedio y las cuales suman un total de $869 \mathrm{Ha}$, éstas se ubican en los Ejidos Los Chorros de Tala, la Lobera, la Hiedra, Agua Dulce, los Balnearios Las Tinajas y La Primavera. En estas zonas las variables más importantes son el uso de suelo y el tipo de vegetación.

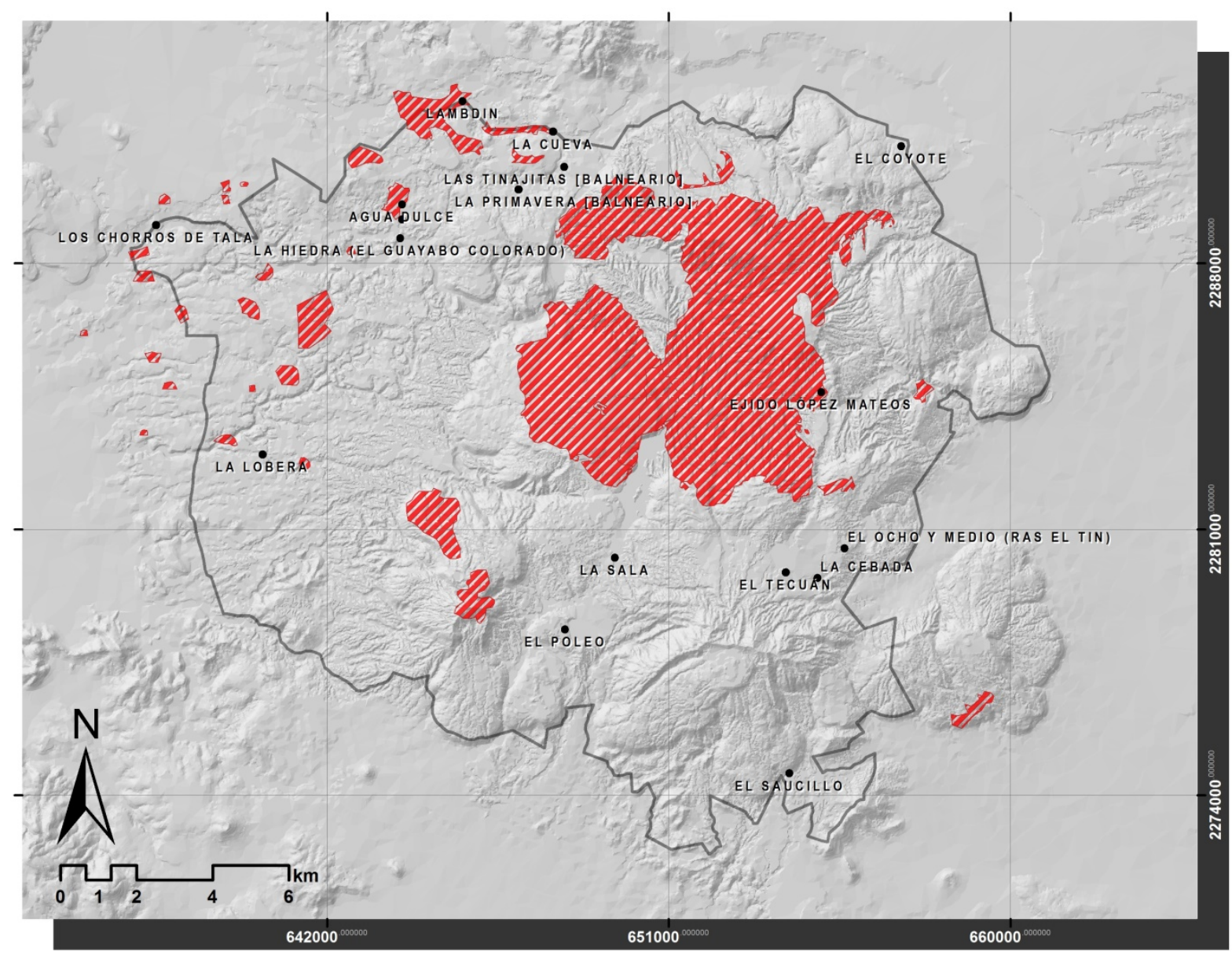

Figura 3. Zonas potenciales de incendio predichas por el modelo espacial.

Este estudio es de las pocas aplicaciones de modelado espacial para determinar zonas potenciales de incendio (Mandallaz y Ye, 1997; García et al., 1999; Muñoz et al., 2005; Pompa, 2012; Pérez-Verdín, 2013), y es el único hasta el momento que en el modelo integra variables de combustibles, meteorológicas, de paisaje y antropológicas y/o de causalidad, bajo un contexto geoespacial.

En este trabajo fue posible integrar las variables sugeridas en el modelo conceptual generado por Pompa (2012), para obtener un modelado espacial y un escenario de predicción, asimismo fue posible determinar las variables más importantes en la generación de incendios en cada sitio de muestreo. 
En estudios anteriores, (Mandallaz et al. 1997; García et al., 1999; Muñoz et al. 2005; Hernández-Leal et al., 2006), los predictores de incendios han sido en general a una escala regional, lo que hace difícil generar conclusiones a escala local. En este estudio, fue necesario generar microambientes dentro del APFFLP para representar la heterogeneidad real del sistema, tanto en los elementos socioeconómicos como en los factores ambientales, y las coberturas de las variables se realizaron a una alta resolución $\left(1 \mathrm{~m}^{2}\right)$ para utilizarlas como predictores, sobre las cuales se desarrolló el modelo de incendios. En este sentido, este estudio representa un esfuerzo novedoso para predecir la probabilidad de incendios a una escala espacial fina.

La heterogeneidad ambiental y complejidad espacial del APFFLP son altas, y están relacionadas con las variables meteorológicas y de paisaje, afectadas por los diferentes niveles de perturbación, usos de suelo, tipo de vegetación así como el tipo de suelo. Esta variabilidad ambiental en un área relativamente pequeña, se refleja en las ocurrencias históricas de los incendios. Por lo que las predicciones de incendios hechas en este trabajo indican a una zona local amplia y aislada, así como a un patrón de pequeñas zonas aisladas.

Los resultados sugieren que la pendiente (variable de paisaje), la humedad relativa (variable meteorológica), el tipo de suelo (variable antropogénica) y el uso de suelo (variable antropogénica); influyen de manera directa en la predicción de incendios en el APFFLP, lo que concuerda parcialmente con lo encontrado por Ruiz y Blanco (2004), quienes apuntan que el riesgo de incendios se incrementa proporcionalmente con la pendiente. El tipo y uso de suelo son variables importantes dentro de la predicción de incendios; ya que como se ha comentado en varias ocasiones, el hombre juega un papel importante en la ocurrencia de los incendios. De acuerdo con Olayo-González ${ }^{1}$, alrededor de $80 \%$ de la superficie afectada por estos eventos ha sido descuidos humanos.

En el caso particular del APFFLP se presentan actividades de agricultura, para lo cual se llevan a cabo incendios controlados durante la zafra en el cultivo de caña de azúcar, y actividades de recreación principalmente en dos áreas. Una de ellas se encuentra del lado norte por la entrada al ejido La Primavera, donde existen balnearios; esta zona es la de mayor carga de visitantes, registrándose cerca de 25,000 visitantes en temporada de Semana Santa. Por el lado oriente, se tiene una segunda vía de acceso en la prolongación de la Avenida Mariano Otero donde no obstante no haber ningún tipo de desarrollo, es la segunda área con mayor afluencia de paseantes, que durante Semana Santa suman 15,000 (Mexico, 2011).

Es posible que el origen potencial de los incendios en el APFFLP esté relacionado con las actividades antropogénicas: el uso de suelo, principalmente en el cultivo de caña de azúcar y las actividades de recreación. Sin embargo la magnitud y extensión de los incendios, así como la dirección de su alcance; podría estar dada por una combinación de variables meteorológicas y de paisaje; principalmente la humedad relativa y la pendiente respectivamente, que por un lado impulsarán el proceso del incendio y por otro lado dificultan el acceso a los brigadistas (Velasco-Herrera et al., 2013; Pompa García y Sensibaugh, 2014).

Estos resultados coinciden con el llamado Triángulo del Fuego, que establece que para que se produzca un incendio se necesitan tres elementos: calor, oxígeno y combustible; basta la ausencia de un elemento para que no se presente un incendio y la Gran Tríada la cual considera el factor topográfico, el tiempo atmosférico y los combustibles como los factores que intervienen en determinar el comportamiento del fuego (Mexico, 2011), De estos factores, el más importante es el tiempo atmosférico, que es el factor más variable y en ocasiones impredecible, lo que exige tener el mayor conocimiento y control para actuar con seguridad y eficiencia. Los elementos del tiempo atmosférico son: Temperatura, Humedad relativa y viento (velocidad y dirección), la interrelación de estos elementos dan como resultado

\footnotetext{
${ }^{1}$ Olayo-González, M. Gerente estatal Conafor Durango. Comunicación Personal. 6 de noviembre. 2012.
} 
condiciones particulares que cambian de manera temporal (a lo largo del día y de las estaciones) y espacial (cañones, laderas, valles), lo que también se refleja en el comportamiento del fuego, en su velocidad de propagación, en la altura de las llamas, en la intensidad de quema entre otros (CENAPRED, 2008).

La predicción del modelo es congruente con los obtenidos en diversos estudios, particularmente en lo referido a las causales de la ocurrencia del fuego (Ávila-Flores et al., 2010; Muñoz et al., 2005; Rodríguez et al., 2008; Meza y Sepúlveda, 2009; Pompa García y Sensibaugh, 2014). No obstante, ninguno incluye una plataforma que integre las variables en un modelo global de índole espacial.

Los hallazgos aquí documentados son muy importantes, sin embargo se requiere una mayor investigación, sobre todo en el diagnóstico del tiempo atmosférico, para lo cual se requiere de un registro sistemático a largo plazo sobre los elementos del clima, para así poder realizar a partir de datos reales, una simulación de diferentes escenarios probables, en función del peso específico de cada variable. Las aplicaciones del modelo pueden ser trascendentales en la dinámica del fuego sobre el ecosistema del APFFLP, en un contexto de cambio climático, y representa una información valiosa para los manejadores del bosque, quienes a partir de este modelo podrían definir sitios con alto potencial de ocurrir incendios y con ello optimizar los planes de prevención y supervisión y estrategias más efectivas de combate.

El modelo espacial generado presenta una solidez conceptual para determinar zonas potenciales de incendios, mediante su integración a partir de componentes de paisaje, meteorológicos, antropogénicos y/o causales y de combustibles. Su implementación a través de la plataforma desarrollada, constituye un aporte científico relevante y vanguardista en el modelaje de zonas potenciales de incendios en el APFFLP.

\section{CONCLUSIONES}

El modelo espacial desarrollado demostró ser una metodología confiable para predecir el potencial de ocurrencia de incendios en el APFFLP. En él, se destacan zonas potenciales, asiladas y pequeñas al oriente del polígono del APFFLP, las cuales históricamente se han quemado debido a las actividades propias para el cultivo de la caña de azúcar. Se destaca también una zona más grande ubicada en la parte noroeste del polígono que probablemente se incendie por las actividades de recreación y se magnifique por las condiciones topográficas y meteorológicas presentadas en esos sitios. La heterogeneidad ambiental del APFFLP, las actividades antropogénicas, las variables de uso de suelo, y la pendiente influyen en la ocurrencia del fuego.

Es esperanza para los autores, que este estudio pueda ayudar a identificar los sitios específicos en los que se recomienda realizar acciones preventivas específicas contra los incendios por parte de las autoridades correspondientes, y que tales esfuerzos puedan orientarse en una estrecha coordinación entre las comunidades locales, autoridades y la academia. De las variables que resultaron con alta importancia en la ocurrencia del fuego, la única que puede ser manipulada y controlada por el hombre es el uso de suelo, de tal forma que es en esta variable donde se debe incidir a fin de evitar la presencia de incendios forestales de la misma magnitud como las presentadas en los años 1998, 2005 y 2012.

\section{AGRADECIMIENTOS}

Agradecemos al Biol. Gerardo Cabrera Orozco, por su ayuda y cooperación en campo, así como a los miembros de SAP, Servicios Ambientales Profesionales. También agradecemos a la Biol. Karla Gutiérrez García por su apoyo en la construcción del SIG, y en 
la edición de los mapas de la APFFLP. Al CONACYT por su apoyo con la beca posdoctoral del Dr. José Luis Ibarra-Montoya.

\section{REFERENCIAS}

ALMEIDA, R. Forest fire risk areas and definition of the prevention priority planning actions using GIS. In: EUROPEAN CONFERENCE ON GEOGRAPHICAL INFORMATION SYSTEMS - EGIS, 5., 1994, Paris. Proceedings... Paris: EGIS Fundation, 1994.

ÁVILA-FLORES, D.; POMPA-GARCÍA, E. Spatial analysis of fire occurrence in the Durango State. Revista Chapingo. Serie Ciencias Forestales, v. 16, n. 2, p. 253-260, 2010 .

ÁVILA-FLORES, D.; POMPA-GARCÍA, M.; ANTONIO-NEMIGA, S.; RODRIGUEZTREJO, D.; VARGAS-PÉREZ, E.; SANTILLÁN-PEREZ, J. Driving factors for forest fire occurrence in Durango State of Mexico: A geospatial perspective. Chinese Geographical Science, v. 20, n. 6, p. 491-497, 2010. http://dx.doi.org/10.1007/s11769010-0437-x

CENTRO NACIONAL DE PREVENCIÓN DE DESASTRES - CENAPRED. Incendios Forestales. Zapopan: CONAFOR, 2008. P. 44.

CHUVIECO, E.; SALAS, F.; VEGA, C. Remote sensing and GIS for long-term fire risk mapping. Mega fires Project. In: CHUVIECO, E. (ed), A review of remote sensing methods for the study of large wildland fires. Madrid: Universidad de Alcalá, 2000. p. 91-107.

COMISIÓN NACIONAL PARA EL CONOCIMIENTO Y USO DE LA BIODIVERSIDAD - CONABIO. Incendio Forestales. 2014. Disponible en: http://www.conabio. gob.mx/informacion/geo_espanol/doctos/imagsatelite.html. Acceso en: 20 mayo 2014.

COMISIÓN NACIONAL FORESTAL - CONAFOR. Reporte nacional de incendios forestales. Guadalajara, Disponible en: http://www.mexicoforestal.gob.mx/files/ 120427reportenacionalincendis. Acceso en: 15 mayo 2014.

COMMONWEALTH SCIENTIFIC AND INDUSTRIAL RESEARCH ORGANIZATION CSIRO Forestry and Forest Products. Bushfire Behavior and Management. 2000. Disponible en: http://www.ffp.csiro.au/nfm/fbm/. Acceso en: 15 mayo 2014.

DEEMING, J.; BURGAN, R.; COHEN, J. D. The National Fire Danger Rating System. Washington, D.C.: USDA Forest Service, 1978.

DELANEY, J. Geographical information systems an introduction. Oxford: University press, 1999. $194 \mathrm{p}$.

DRYRY, S.; VEBLEN, T. Spatial and temporal variability in fire occurrence within the Las Bayas forestry reserve, Durango, México. Plant Ecology, v. 197, p. 299-316, 2008. http://dx.doi.org/10.1007/s11258-007-9379-5

FLASSE, P.; CECCATO, P. A contextual algorithm for AVHRR fire detection. International Journal of Remote Sensing, v. 17, p. 419-424, 1996. http://dx.doi.org/10.1080/01431169608949018

GARCÍA, E. Modificaciones al sistema de clasificación climática de Koppen. México: Instituto de Geografía, UNAM, 1973. 256 p. 
GARCÍA, V.; WOODARD, S.; ADAMOWICZ, P.; LEE, B. Dos modelos para la predicción de incendios forestales en Whitecourt Forest, Canada. Investigación Agraria, Sistemas y Recursos Forestales, v. 8, n. 1, p. 5-23, 1999.

GIGLIO, L.; DESCLOITRES, J.; JUSTICE, O.; KAUFMAN, J. An enhanced contextual fire detection algorithm for MODIS. Remote Sensing of Environment, v. 87, p. 273-282, 2003. http://dx.doi.org/10.1016/S0034-4257(03)00184-6

GOLLBERG, E.; NEUENSCHWANDER, F.; RYAN, C. Introduction: Integrating spatial technologies and ecological principles for a new age in fire management. International Journal of Wild land Fire, v. 10, p. 263-265, 2001.

http://dx.doi.org/10.1071/WF01047

GOUMA, V.; CHRONOPOULOU-SERELI, A. Wild land Fire danger zoning-A methodology. International Journal of Wildland Fire, v. 8, n. 1, p. 37-43, 1998. http://dx.doi.org/10.1071/WF9980037

GUTIERREZ, M.; MONEDERO, G.; SANDIA, A. Diseño de un mapa de incendios de vegetación en el Parque Nacional El Ávila apoyado en Modelos Digitales del Terreno. In: SIMPOSIO LATINOAMERICANO DE PERCEPCIÓN REMOTA 7., 1997, Mérida. Memorias..., Caracas: SELPER/Unidade Técnica de Sistemas. Instituto de Ingeneria, 1997.

HERNÁNDEZ-LEAL, P. A.; ARBELO, M.; GONZÁLEZ-CALVO, A. Fire risk assessment using satellite data. Advances in Space Research, v. 37, p. 741-746, 2006. http://dx.doi.org/10.1016/j.asr.2004.12.053

HUERTA-MARTÍNEZ, F. M.; IBARRA-MONTOYA, J. L. 15 Años de incendios en el Bosque La Primavera (Jalisco, México): Un acercamiento a sus posibles causas y consecuencias. CienciaUAT, v. 8, n. 2, p. 32-43, 2014.

INSTITUTO NACIONAL DE ESTADÍSTICA Y GEOGRAFIA - INEGI. Continuo Nacional del conjunto de datos vectoriales de uso de suelo y vegetación, 1:1 250 000 serie II. Jalisco, 2014a.

INSTITUTO NACIONAL DE ESTADÍSTICA Y GEOGRAFIA - INEGI. Continuo Nacional del conjunto de datos geográficos de la carta topográfica, 1:1 250000 serie II. Jalisco, 2014b.

LEATHWICK, J.; BRIGGS, C. M. Spatial prediction of wildfire hazard across. New Zealand: New Zealand Fire Service Commission, 2001.

LEE, B.; ALEXANDER, M.; HAWKES, B.; LYNHAM, T.; STOCK, B.; ENGLEFIELD, P. Information system in support of wild land fire management decision making in Canada. Computers and Electronics in Agriculture, v. 37, n. 1-3, p. 185-198, 2002. http://dx.doi.org/10.1016/S0168-1699(02)00120-5

MAGAÑA, O. Índices de peligro de incendios forestales. México: Instituto Nacional de Investigaciones Forestales, 1985. 
MANDALLAZ, D.; YE, R. Prediction of forest fires with Poisson models. Canadian Journal of Forest Research, v. 27, n. 10, p. 1685-1694, 1997. http://dx.doi.org/10.1139/x97-103MANOS, P.; DOYLE, J.; NIXON, K. Phylogeny, Biogeography, and Processes of Molecular Differentiation in Quercus Subgenus Quercus (Fagaceae). Molecular Phylogenetics and Evolution, v. 12, n. 3, p. 333-349, 1999. http://dx.doi.org/10.1006/mpev.1999.0614

MARCOZZI, M.; BOVIO, G.; MANDALLAZ, D.; BACHMANN, P. Influence of the weather on the forest fire danger index in Ticino Canton. Schweizerische Zeitschrift fur Forstwesen, v. 145, n. 3, p. 183-199, 1994.

MATHUR, R.; GOGATE, M.; MITTAL, R. Forest fire danger rating índices base don climate-a case study of West Dehra Dun Forest Division. Indian Forester, v. 110, n. 3, p. 223-241, 1984.

MEXICO. Secretaria de Medio Ambiente y Recursos Naturales - SEMARNAT. Programa de Manejo. Área de Protección de Flora y Fauna La Primavera. México, 2000. p.131.

MEXICO. Secretaria de Medio Ambiente y Recursos Naturales - SEMARNAT. Programas y Acciones en Reforestación, Conservación y Restauración de Suelos, Incendios Forestales y Sanidad Forestal de Ecosistemas forestales. Programa Nacional contra Incendios Forestales. México, 2011. p 65-106.

MEXICO. Secretaria de Medio Ambiente y Recursos Naturales - SEMARNAT. Programas y Acciones en Reforestación, Conservación y Restauración de Suelos, Incendios Forestales y Sanidad Forestal de Ecosistemas forestales. Programa Nacional contra Incendios Forestales. Mexico, 2012. p. 60-97.

MEZA, R.; SEPÚLVEDA, J. Caracterización del terreno y su influencia en los incendios forestales en Baja California, México. La Paz: INIFAP, 2009. www.mappinginteractivo.com/plantilla-egeo.asp?idarticulo=1603. Acceso en: 17 febrero de 2012.

MUÑOZ, R.; TREVIÑO, E.; VERÁSTEGUI, J.; JIMÉNEZ, J.; AGUIRRE, O. Desarrollo de un modelo espacial para la evaluación del peligro de incendios forestales en la Sierra Madre Oriental de México. Investigaciones Geográficas, v. 56, p. 101-117, 2005.

NIXON, K.; MULLER, C. The Quercus hypoxantha complex (Fagaceae) in Northeastern Mexico. Brittonia, v. 45, p.146-153, 1993. http://dx.do.org/10.2307/2807497

PAROLO, G.; ROSSI, G.; FERRARINI, A. Toward improved species niche modelling: Arnica montana in the Alps as a case study. Journal of Applied Ecology, n. 45, p. 1410-1418, 2008. http://d.doi.org/10.1111/j.1365-2664.2008.01516.x

PÉREZ-VERDÍN, G.; MÁRQUEZ-LINARES, M.; CORTÉS-ORTÍZ, A.; SALMERÓNMACÍAS, M. Análisis espacio-temporal de la ocurrencia de incendios forestales en Durango, México. Madera y Bosques, v. 19, n. 2, p. 37-58, 2013.

PHILLIPS, S. J.; ANDERSON, R. P.; SCHAPIRE, R. E. Maximum entropy modeling of species geographic distributions. Ecological Modeling, n. 190, p. 231-259, 2006. http://dx.doi.org/10.1016/j.ecolmodel.2005.03.026

PHILLIPS, S. J.; DUDIK, M. Modeling of species distributions with Maxent: new extensions and a comprehensive evaluation. Ecography, v. 31, p. 161-175, 2008. http://dx.doi.org/10.1111/j.0906-7590.2008.5203.x 
POMPA, G. Sistema de información geográfica de los ecosistemas de Durango. Mapa de climas. 2012. http://www.ujed.mx/sigeed/inicio.aspx. Acceso en: 01 febrero de 2012.

POMPA-GARCÍA, M.; SENSIBAUGH, M. Ocurrencia de incendios forestales y su teleconexión con fenómenos ENSO. CienciaUAT, v. 27, n. 2, p. 06-10, 2014.

RODRIGUEZ, T.; RAMÍREZ, H.; TCHIKOUE, H.; SANTILLÁN, J. Factores que inciden en la siniestralidad de los incendios forestales. Ciencia Forestal en México, v. 33, n. 104, p. 38-57, 2008.

RUIZ L., V.; BLANCO J., L. Comportamiento del fuego y evaluación del riesgo por incendios en las áreas forestales de México: un estudio en el Volcán la Malinche. In: Incendios forestales en Mexico. Metodos De Evaluación. Mexico: Universidad Nacional Autónoma de Mexico, 2004.

SÁNCHEZ, J. Los incendios forestales y las prioridades de investigación en México. México: Congreso Forestal Mexicano, 1989. p. 719-723.

SANTILLAN, P. Sistema para determinar indicadores de peligro de incendio forestal. Acuerdo de cooperación en materia forestal entre México y Finlandia. Helsinque: [s.n.], 1993. (Informe Técnico, n. 14)

SEPÚlVEDA, B.; ZUÑIGA, C.; OLGUÍN, E.; GOMERO, P. Implementación de un sistema de información geográfica para la prevención de incendios forestales en Baja California. Mexico: INIFAP; SAGAR, 1999.

SEPÚlVEDA, R.; MEZA, W.; ZUÑIGA, G.; SOLÍS, G.; OLGUÍN, E. SIG para determinar riesgo de incendios forestales en el noroeste de México. Mexico: Instituto Nacional de Investigaciones Forestales, Agrícolas y Pecuarias, 2001. p. 37.

SOKAL, R.; ROHLF, J. Biometry. The principles and practice of statistics in biological research. New York: State University of New York, 1995. 887 p.

STOLYARCHUK, L. Structure of the forest fire seasons in the regions west and east of lake Baikal. Lesnoe-Khozyaistvo, n. 7, p. 57-58, 1979.

VÁZQUEZ, M.; VALENCIA, S.; NIXON, K. Notes on red oaks (Quercus sect. Lobatae) in eastern Mexico, with description of a new species. Quercus hirtiifolia. Brittonia, v. 56, p. 136-142, 2004. http://dx.doi.org/10.1663/0007-196X(2004)056[0136:NOROQS] 2.0.CO;2

VELASCO-HERRERA, J.; FLORES-GARNICA, J.; MARQUEZ-AZÚA, B.; LÓPEZ, S. Áreas de respuesta homogénea para el muestreo de combustibles forestales. Revista Mexicana de Ciencias Forestales, v. 4, n. 15, p. 41-54, 2013.

WYBO, J.; GUARNIERI, F.; RICHARD, B. Forest-fire danger assessment methods and decision support. Safety Science, v. 20, n. 1, p. 61-70, 1995. http://dx.doi.org/10.1016/0925-7535(94)00067-D

ZAPATA, P. Determinación de indicadores de peligro potencial de incendios forestales con base en la cuantificación del material combustible. Chapingo: UACH, 1991. 


\begin{tabular}{|} 
Ambiente \& Água - An Interdisciplinary Journal of Applied Science \\
ISSN 1980-993X - doi:10.4136/1980-993X \\
www.ambi-agua.net \\
E-mail: ambi.agua@gmail.com
\end{tabular}

\title{
Peroxidase de gramínea de Cerrado como alternativa no tratamento de efluentes agroindustriais
}

\author{
doi:10.4136/ambi-agua.1735
}

Received: 20 Aug. 2015; Accepted: 26 Sep. 2015

\author{
Raquel Pinheiro Reis Souza Ramalho'; Paulo Sérgio Scalize"; \\ Samantha Salomão Caramori ${ }^{2 *}$ \\ ${ }^{1}$ Universidade Federal de Goiás (UFG), Goiânia, GO, Brasil \\ Escola de Engenharia Civil e Ambiental \\ ${ }^{2}$ Universidade Estadual de Goiás (UEG), Anápolis, GO, Brasil \\ Câmpus de Ciências Exatas e Tecnológicas (CCET) \\ *Autor correspondente: e-mail: sscaramori@gmail.com, \\ rprs.ramalho@hotmail.com, pscalize.ufg@gmail.com
}

\section{RESUMO}

A despoluição de águas residuais é ainda um desafio para a sociedade e comunidade acadêmica. Diante dos diversos materiais disponíveis para estudo destacam-se as enzimas, em função de sua especificidade para a despoluição e biodegradabilidade para descarte. Novas fontes de enzimas podem representar alternativas eficientes e de custo reduzido em relação às técnicas corriqueiramente utilizadas. Neste trabalho o perfil de peroxidase dos frutos de Echinolaena inflexa foi estudado para aplicação no tratamento de águas residuais. $\mathrm{O}$ teor de proteína encontrado foi 5,33 $\mathrm{mg} \mathrm{g}^{-1}$. As condições ótimas de reação foram: $50^{\circ} \mathrm{C}$, pH 7,5 a 0,1 mol L ${ }^{-1}$ de tampão fosfato e $15 \mathrm{~min}$. A enzima foi inativada somente após aquecimento a $94^{\circ} \mathrm{C}$ e teve sua atividade inibida quando incubada com ácido ascórbico a $10 \mathrm{mmol} \mathrm{L}^{-1}$, um inibidor clássico para peroxidases. Nos testes com fenóis e efluentes agroindustriais, a enzima foi capaz de degradar $87,5 \%$ do catecol, $67,8 \%$ do pirogalol e $39,1 \%$ do resorcinol e ainda apresentou $29,1 \%$ de capacidade de degradação de compostos fenólicos presentes no efluente bruto. Os resultados mostraram que a peroxidase de Echinolaena inflexa, uma nova fonte de enzimas, possui potencial para ser explorada como alternativa ao tratamento de águas residuais.

Palavras-chave: Echinolaena inflexa, fenóis, peroxidase, poaceae.

\section{Peroxidase of Brazilian Cerrado grass as an alternative for agro.industrial waste treatment}

\begin{abstract}
Decontamination of wastewater continues to be a challenge for society and the scientific community. Despite the availability of various materials for study, enzymes stand out due to their specificity for decomposition and biodegradability for disposal. New sources of enzymes may represent efficient and low-cost alternatives compared to routinely used techniques. In this survey, the peroxidase profile from Echinolaena inflexa fruits was studied for possible
\end{abstract}


applications in the treatment of wastewater. The protein content was found to be $5.33 \mathrm{mg} \mathrm{g}^{-1}$. The optimum reaction conditions were: $50^{\circ} \mathrm{C}, \mathrm{pH} 7.5$ at $0.1 \mathrm{~mol} \mathrm{~L}^{-1}$ of phosphate buffer for $15 \mathrm{~min}$. The enzyme was inactivated after $5 \mathrm{~min}$ at $94^{\circ} \mathrm{C}$ and was inhibited when incubated with ascorbic acid at $10 \mathrm{mmol} \mathrm{L}^{-1}$. In tests using phenols and agro industrial waste, the peroxidase was able to oxidase $87.5 \%$ of catechol, $67.8 \%$ of pyrogallol, $39.1 \%$ of resorcinol and still presented $29.1 \%$ of the degradation capacity of raw wastewater phenolic compounds. The results showed that the Echinolaena inflexa peroxidase, a new source of enzymes, is a potential alternative to wastewater treatment.

Keywords: Echinolaena inflexa, peroxidase, phenols, Poaceae.

\section{INTRODUÇÃO}

Um dos grandes impactos ambientais no mundo é a destinação inadequada de efluentes no meio ambiente. Efluentes não tratados promovem uma grave poluição hídrica, além de transportarem diferentes tipos de vírus, bactérias e protozoários nocivos à saúde pública (Mannarino et al., 2013). A partir dessa necessidade de tratamento, surgem diferentes processos, sendo alguns mais eficientes, baratos e ambientalmente corretos para a degradação de diversos compostos orgânicos.

As enzimas fazem parte desses processos e são reconhecidas como catalisadores biológicos, apresentando características importantes, tais como a atuação em condições amenas de reação, a alta especificidade, a biodegrabilidade e a alta eficiência catalítica. Dentre as classes de enzimas, as oxidorredutases possuem um dos principais papeis na degradação de compostos orgânicos, uma vez que catalisam reações de transferência de elétrons e as peroxidases, subclasse de oxidorredutases, oxidam os diferentes compostos na presença de peróxido de hidrogênio ou peróxidos orgânicos, gerando radicais livres (Chance e Maehly, 1955).

Dentro do contexto de tratamento de efluentes, cabe às peroxidases um papel de destaque, em função da sua capacidade em degradar substâncias tóxicas e persistentes. Segundo Neves e Silva (2007), essas enzimas são amplamente encontradas em vegetais, e estão ligadas à função fisiológica/bioquímica da planta. A maioria dos relatórios sobre a desintoxicação de águas residuais contaminadas com fenóis, cresóis e fenóis clorados usou peroxidase das raízes de Rábano Silvestre (Armoracia rusticana), na forma purificada com a denominação de HRP (Horseradish Peroxidase). Recentemente, peroxidases de outras fontes, como melão-de-são-caetano (Momordica charantia L.), nabo (Brassica rapa L.) e copaíba (Copaifera langsdorffii Desf.) foram sugeridas como alternativas a HRP (Akhtar e Husain, 2006; Maciel et al., 2006). Características deste processo, tais como o efeito do pH, concentração do substrato, tempo de reação e os efeitos de alguns aditivos tem sido estudados para otimizar as condições de reação (Hamid e Khalil-Ur-Rehman, 2009).

Dentre a diversidade de espécies existentes e com potencial para extração enzimática, temos a vegetação do Cerrado, o segundo maior bioma brasileiro, com mais de 6000 espécies distribuídas em sua região principal de ocorrência, o Planalto Central brasileiro (Ratter et al., 1997).

Estudos florísticos e fitossociológicos que analisaram o componente herbáceo do Cerrado encontraram um predomínio da família Poaceae, principalmente dos gêneros Andropogon, Axonopus, Paspalum, Echinolaena e Trachypogon. A espécie Echinolaena inflexa (Poir.) Chase, é uma gramínea nativa, amplamente distribuída e com alta produção de biomassa (Munhoz e Felfili, 2007). Embora essas espécies venham sendo utilizadas para vários fins, tais como medicinal e alimentar, ainda há necessidade de maiores estudos viabilizando outras formas de aproveitamento de seus espécimes. 
No presente trabalho foram produzidos extratos de Echinolaena inflexa a fim de encontrar atividade de peroxidase termoestável, uma vez que essas plantas ficam expostas a longos períodos sob o sol. Avaliou-se a aplicação das enzimas obtidas nesses extratos, na degradação de diferentes compostos fenólicos, como proposta no tratamento de efluentes agroindustriais.

\section{MATERIAL E MÉTODOS}

\subsection{Extração e avaliação da atividade de peroxidase de $E$. inflexa}

A gramínea E. inflexa foi coletada na trilha do Tatu, no Campus Dr. Henrique Santillo da Universidade Estadual de Goiás, localizado no município de Anápolis, estado de Goiás-Brasil, $16^{\circ} 22^{\prime} 53,6^{\prime \prime}$ de latitude Sul e 4856'41,9”'de longitude Oeste.

A parte selecionada para estudo foi composta dos frutos de Echinolaena inflexa, que foram coletados e triturados em moinho tipo martelo (TE-330 Tecnal), resultando em uma farinha que foi utilizada nos experimentos. A farinha foi embalada e estocada em recipientes de polipropileno a $-10^{\circ} \mathrm{C}$.

A obtenção dos extratos brutos foi avaliada de acordo com um planejamento fatorial $2^{3}$. Foram avaliados os parâmetros tempo de extração (30-90 min) e o pH de extração. Os extratos foram obtidos utilizando diferentes tampões: pH 6,0 - 8,0 fosfato de sódio 0,1 mol L ${ }^{-1}$ e pH 9,0 borato de sódio $0,1 \mathrm{~mol} \mathrm{~L}^{-1}$. Para o preparo dos extratos, $10 \mathrm{~mL}$ de tampão foram misturados a 0,5 g de farinha (Echinolaena inflexa), agitados (tempo de extração) e depois centrifugados (5000 rpm) por 15 min. Aquele tratamento, determinado pela combinação dos dois parâmetros, que demonstrou melhor desempenho na extração das enzimas estudadas foi selecionado para o preparo dos extratos utilizados ao longo deste trabalho.

Foram avaliados os parâmetros cinéticos $\mathrm{pH}$ e temperatura de reação, obedecendo a um planejamento fatorial $2^{4}$. O pH ótimo de reação foi avaliado pela incubação das amostras de extrato com soluções com pH diferentes. Em pH 5,0 foi utilizado o tampão acetato de sódio $0,1 \mathrm{~mol} \mathrm{~L}^{-1}$; entre o pH 6,0 e 8,0 o tampão fosfato de sódio $0,1 \mathrm{~mol} \mathrm{~L}^{-1}$. A temperatura ótima foi determinada pela variação da temperatura do ensaio de atividade enzimática, entre 30 e $60^{\circ} \mathrm{C}$, em intervalos de $10^{\circ} \mathrm{C}$, em banho-maria.

O tempo de reação com os substratos foi testado pelo acompanhamento da formação de produto por unidade de tempo, após a determinação dos parâmetros ótimos de extração e reação. A atividade de peroxidase das amostras foi medida mediante a variação dos seguintes intervalos de tempo: uma medida por minuto, entre 1 a $5 \mathrm{~min}$; uma medida a cada $5 \mathrm{~min}$, entre 5 e 20 min; uma medida a cada 10 min, entre 20 e 30 min e medidas a cada 30 min, entre 30 e $60 \mathrm{~min}$.

A análise do perfil enzimático foi realizada considerando-se a atividade de peroxidase. Esse ensaios foram efetuados de acordo com a metodologia de Halpin et al. (1989) utilizando pirogalol e peróxido de hidrogênio como substratos. Os resultados foram avaliados por medida espectrofotométrica a $420 \mathrm{~nm}$ e a leitura da atividade foi feita após 1 min de reação. A atividade enzimática foi medida em unidades de enzima $\mathrm{g}^{-1}$ de farinha (atividade inespecífica). Uma unidade de enzima foi considerada como aquela capaz de produzir um aumento equivalente a 0,100 na absorbância.

A concentração de proteínas totais (solúveis) foi determinada pela metodologia descrita por Bradford (1976), por meio de leitura espectrofotométrica, a $595 \mathrm{~nm}$. A quantidade de proteína, expressa em $\mathrm{mg} \mathrm{g}^{-1}$ de farinha, foi determinada por uma curva padrão de reagente Bradford com solução de albumina sérica bovina (Sigma-Aldrich, EUA), nas concentrações de 0,05 a $0,5 \mu \mathrm{g} \mu \mathrm{L}^{-1}$. A partir dos dados de concentração proteica calculou-se a atividade de específica de peroxidase, que foi expressa em unidade de enzima por mg de proteína. 
A estabilidade da peroxidase foi verificada pela incubação do extrato sob temperatura de ebulição $\left(94^{\circ} \mathrm{C}\right)$ durante 5, 10, 15, 30, 60 e 120 min, seguida de resfriamento em banho de gelo por 5 min, e leitura de atividade residual.

O efeito de substâncias inibidoras foi testado pela incubação a $37^{\circ} \mathrm{C}$ por $90 \mathrm{~min}$. de ácido cítrico e ácido ascórbico nas concentrações de $10 \mathrm{mmol} \mathrm{L}^{-1}$ e $50 \mathrm{mmol} \mathrm{L}^{-1}$, com extratos de $E$. inflexa.

\subsection{Uso potencial de peroxidase de $E$. inflexa para degradação de compostos fenólicos}

Foram realizados testes com compostos fenólicos e efluentes agroindustriais após estabelecimento dos parâmetros ótimos, baseados na metodologia de Akhtar e Husain (2006). As amostras de efluentes foram coletadas na Estação de Tratamento de Efluentes (ETE) do Distrito Agroindustrial de Anápolis (DAIA), localizada entre os paralelos $16^{\circ} 20^{\prime}$ e $16^{\circ} 30^{\prime} \mathrm{S}$ e os meridianos 48 $50^{\prime}$ e $49^{\circ} 00^{\prime} \mathrm{W}$. Na área do DAIA tem-se o Córrego Abraão, que está localizado em uma área de aproximadamente 29 ha. A carga orgânica atual contida no esgoto bruto, segundo o Departamento de Meio Ambiente do DAIA em 2015, após coleta de 24 h é de $3.750 \mathrm{~kg}$ DBO.dia ${ }^{-1}$. Em triplicata, $0,1 \mathrm{~mL}$ do extrato bruto foi incubado com 0,75 mL da solução de três compostos fenólicos: pirogalol, catecol e resorcinol $\left(1,0 \mathrm{mmol} \mathrm{L}^{-1}\right)$, bem como, com cada amostra de efluente: bruto, efluente tratado da Estação de Tratamento de Efluentes (ETE) do DAIA e a montante e a jusante do lançamento da ETE no Córrego Abraão, com adição de $0,15 \mathrm{~mL}\left(0,75 \mathrm{mmol} \mathrm{L}^{-1}\right)$ de peróxido de hidrogênio. Em seguida, a mistura foi agitada (vórtex) e incubada a $37^{\circ} \mathrm{C}$ por $90 \mathrm{~min}$. Posteriormente, foram retiradas alíquotas de 0,2 mL para a medida da concentração residual de fenóis pela metodologia de Lowry et al. (1951). Os tubos controle foram utilizados com as soluções de fenóis e peróxido de hidrogênio em substituição ao extrato enzimático e os tubos brancos foram constituídos de água destilada em substituição ao volume de extrato. $\mathrm{O}$ experimento foi realizado em triplicata, e a partir das médias e desvio padrão foram realizados gráficos de superfície usando o programa SigmaPlot versão 12.0.

\section{RESULTADOS E DISCUSSÃO}

Nos experimentos realizados com Echinolaena inflexa foi encontrada uma concentração de proteína solúvel de $5,33 \mathrm{mg} \mathrm{g}^{-1}$. A determinação da atividade enzimática dos extratos brutos de Echinolaena inflexa para peroxidase, em diferentes valores de $\mathrm{pH}$ e tempo de extração, permitiu encontrar as condições ótimas de extração destas enzimas. A relação entre $\mathrm{pH}$, tempo de extração e $\mathrm{U} \mathrm{g}^{-1}$ é demonstrada na Figura 1.

De acordo com os resultados obtidos, a região entre o $\mathrm{pH} 7,5$ e 8,5 foi a que apresentou atividade enzimática superior às demais, e por esta razão o $\mathrm{pH}$ 8,0 foi considerado como o ótimo para a extração das peroxidases da gramínea. A peroxidase de Echinolaena inflexa foi melhor extraída quando o $\mathrm{pH}$ de extração foi combinado à variação do tempo de extração. $\mathrm{Na}$ Figura 1 é também possível observar que a partir de 90 min obteve-se a maior quantidade de enzima ativa extraída.

As condições ótimas de reação mostraram que o efeito da temperatura numa reação enzimática pode ser observado inicialmente pelo aumento progressivo na atividade, devido ao aumento da velocidade de formação do produto e consequentemente consumo do substrato (Figura 2). Todavia, verifica-se que acima de $55^{\circ} \mathrm{C}$ começa a ocorrer perda gradativa da atividade, possivelmente devido à desnaturação proteica pelo calor, como resultado da perda da conformação nativa (Figura 2) 


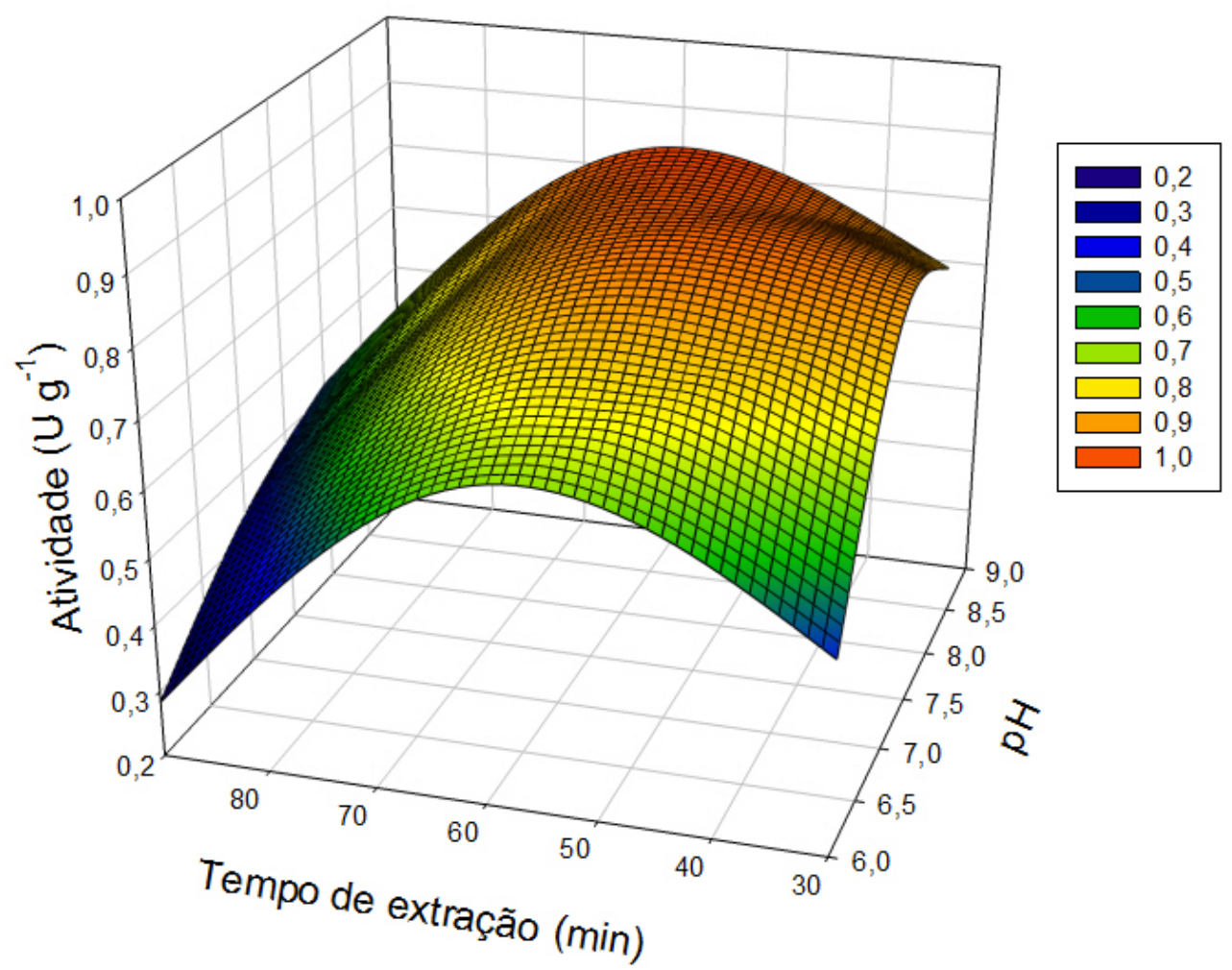

Figura 1. Efeito combinado entre o $\mathrm{pH}$ e o tempo de extração sobre a atividade de peroxidase de Echinolaena inflexa $\left(\mathrm{Ug}^{-1}\right)$.

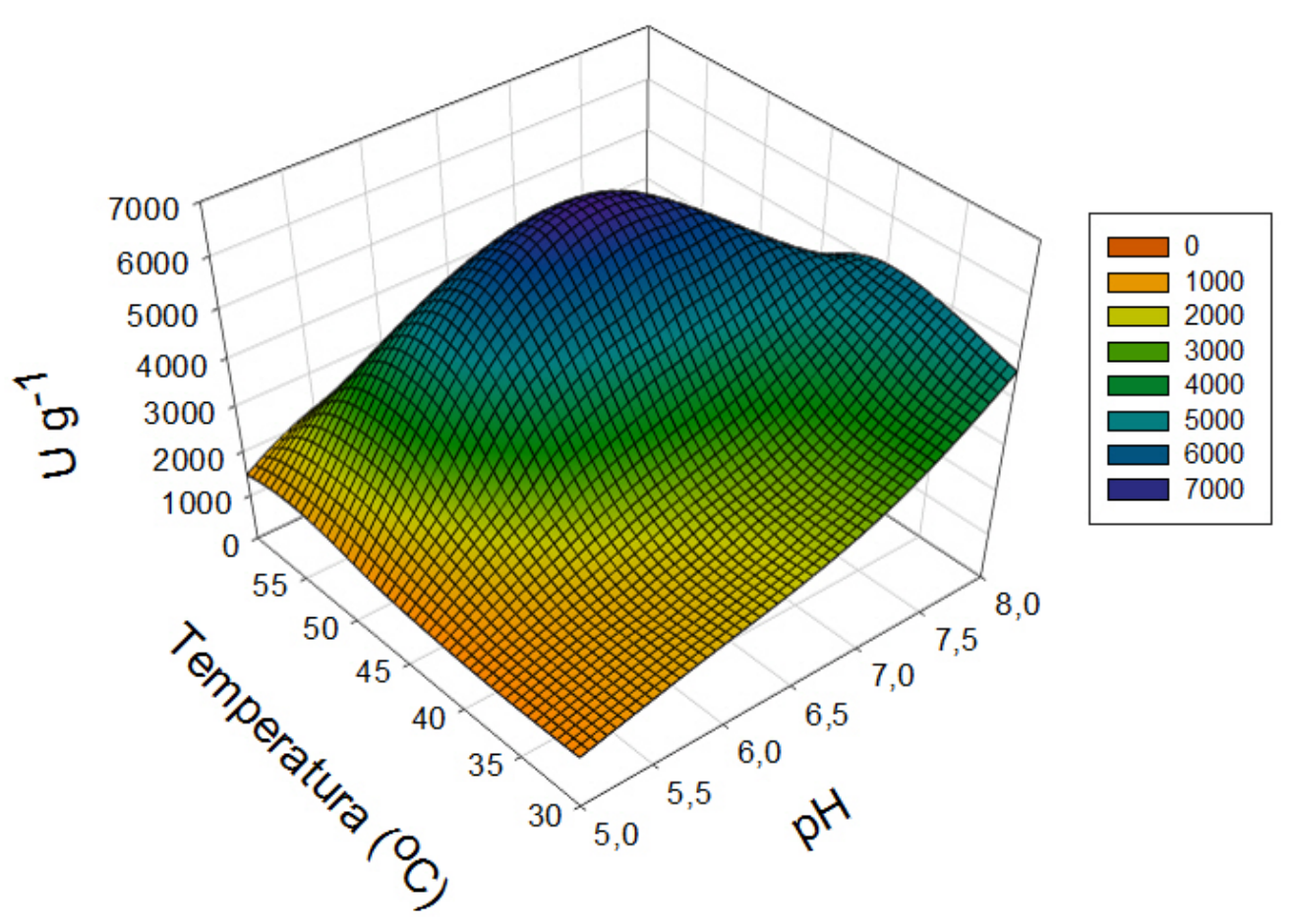

Figura 2. Efeito combinado entre o $\mathrm{pH}$ e a temperatura de reação sobre a atividade de peroxidase de Echinolaena inflexa $\left(\mathrm{U} \mathrm{g}^{-1}\right)$. 
A região que demonstrou condições ótimas de reação correspondeu aos ensaios realizados nas faixas de temperatura entre 45 a $55^{\circ} \mathrm{C}$ e de $\mathrm{pH}$ entre 7 e 8, faixa de $\mathrm{pH}$ semelhante ao utilizado no processo de extração para a espécie. Sob estas condições, a peroxidase de $E$. inflexa apresentou $6.250 \mathrm{U} \mathrm{g}^{-1}$ de farinha dos frutos.

Estes resultados são similares aos obtidos para as peroxidases de abacaxi e carambola, espécies também adaptadas a clima tropical, que apresentaram temperatura ótima para atividade na faixa de 50 a $55^{\circ} \mathrm{C}$ (Brito et al., 2005).

É possível ainda observar que, mesmo que a temperatura ótima tenha sido encontrada entre 45 a $55^{\circ} \mathrm{C}$, em todo o intervalo testado (entre 30 e $60^{\circ} \mathrm{C}$ ) há também atividade de peroxidase (Figura 2). Este comportamento pode ser atribuído à adaptação das gramíneas ao Cerrado. As plantas do bioma estão adaptadas a condições extremas, tais como alta insolação, solos rasos, secos e nutricionalmente pobres, além das temperaturas que variam grandemente ao longo do ano (Munhoz e Felfili, 2007).

Depois de estabelecidos esses parâmetros, o extrato da gramínea foi testado em função do tempo de reação, a $55^{\circ} \mathrm{C}$ (Figura 3). A influência do tempo de contato entre enzima e substrato foi significativa para os primeiros 10 min de reação, em que é possível observar aumentos bruscos na resposta enzimática. Após 15 min de tempo de reação o aumento na atividade passa a desacelerar, tendendo a estabilização.

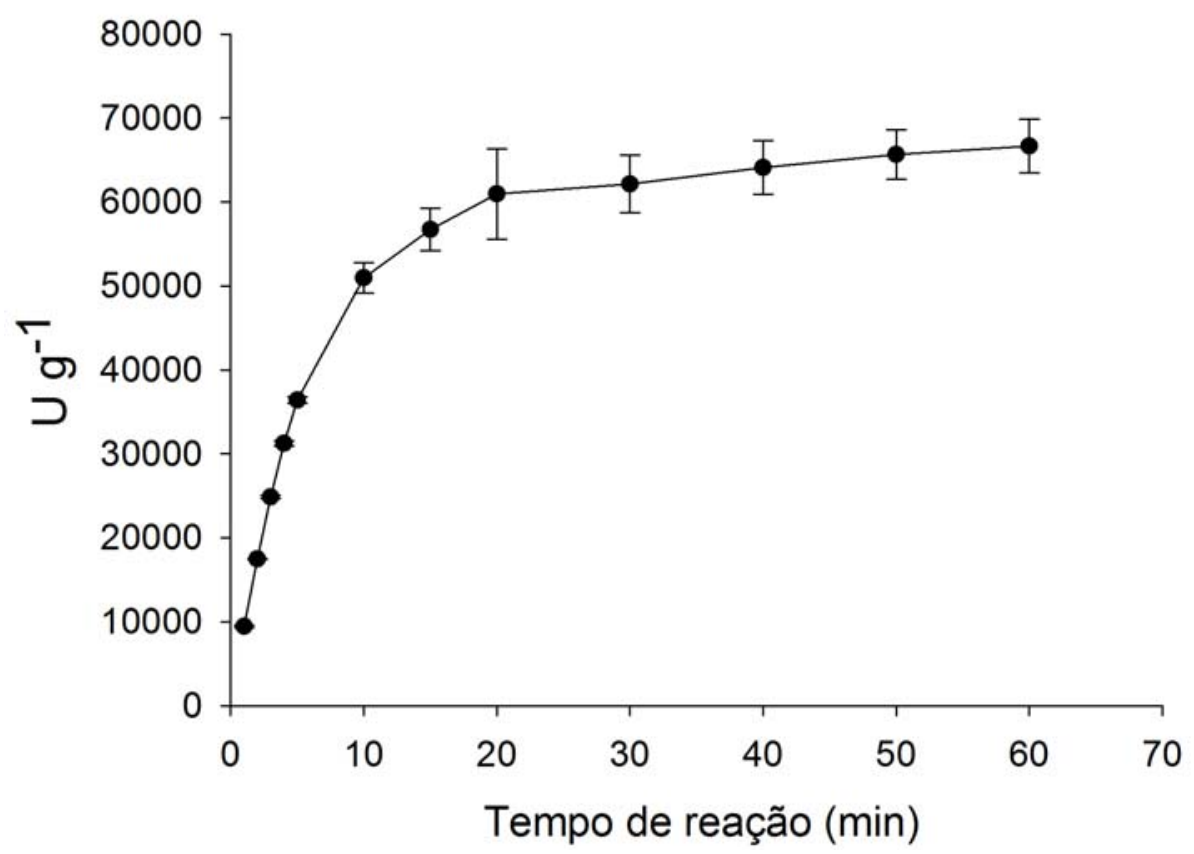

Figura 3. Efeito do tempo de incubação com os substratos pirogalol e peróxido de hidrogênio sobre a atividade de peroxidase em extrato aquoso de frutos de Echinolaena inflexa.

As peroxidases são uma das enzimas de maior estabilidade térmica presentes em frutas e vegetais (Berbicz e Clemente, 2001; Maciel et al., 2006). Este fato tem motivado grande interesse dos pesquisadores quanto aos estudos de inativação, tornando-a principal referência para se determinar quando o processamento térmico de frutas e vegetais foi ou não suficiente para manutenção de sua qualidade como alimentos (Neves e Silva, 2007).

A atividade de peroxidase diminuiu com o tempo de incubação na temperatura testada, no entanto manteve uma atividade de aproximadamente $11 \%$ após 5 min de tratamento. Com o aumento do tempo as atividades continuaram diminuindo, porém de forma mais lenta. Este resultado indica que a enzima é tolerante a temperaturas abaixo da ebulição. 
Berbicz e Clemente (2001) obtiveram resultados semelhantes a Echinolaena inflexa nos experimentos feitos com frutos de laranja. No tratamento térmico das enzimas de laranja a $80^{\circ} \mathrm{C}$, com 6 min a enzima manteve uma atividade entre 18 a 30\% em relação ao inicio do tratamento.

No tratamento térmico de peroxidases de carambola, goiaba e abacaxi aconteceu o mesmo padrão. A atividade da peroxidase diminuiu com o tempo, observando-se uma perda de aproximadamente $80 \%$ da atividade da enzima após tratamento térmico a uma temperatura de 75 a $85^{\circ} \mathrm{C}$ (Brito et al., 2005).

Nos testes com inibidores foi observada a boa capacidade de inibição da peroxidase pela ação do ácido ascórbico e menor inibição pelo ácido cítrico (Figura 4).

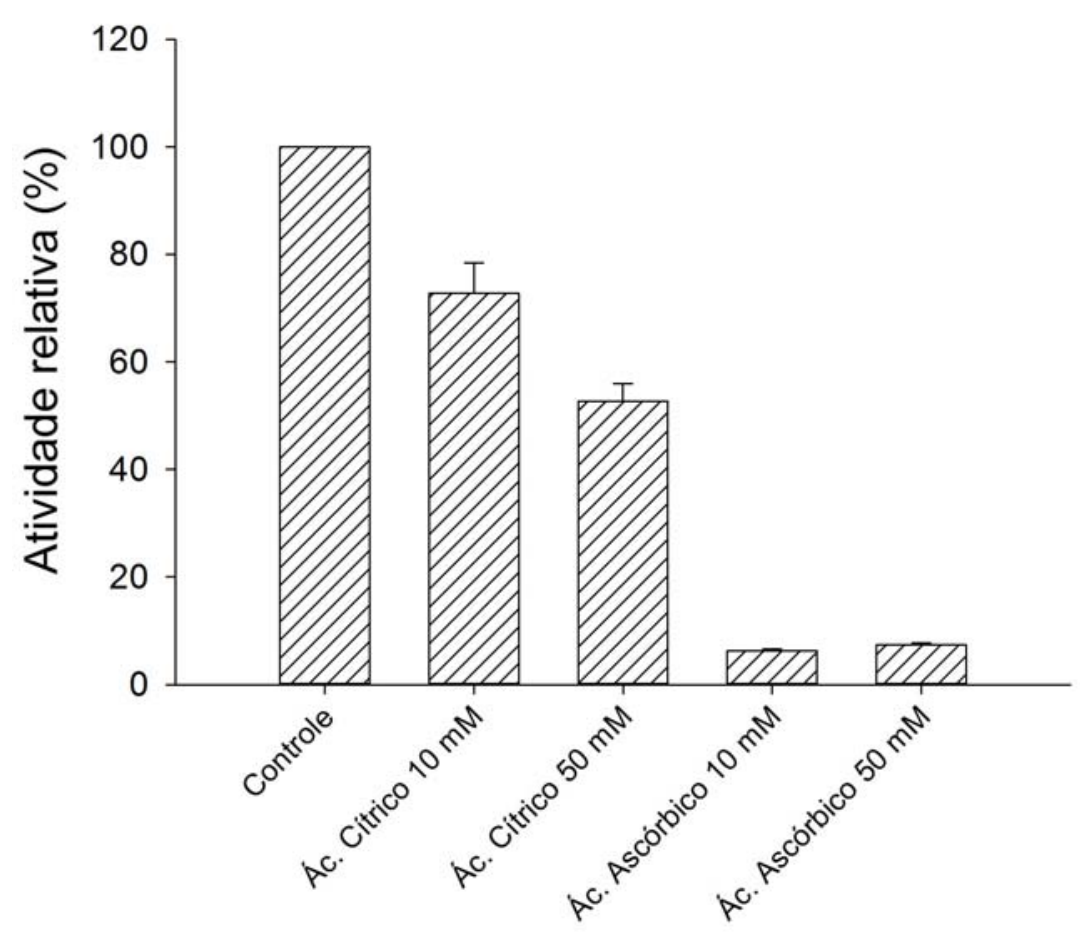

Figura 4. Efeito inibidor causado pelos ácidos na atividade de peroxidases de Echinolaena inflexa após 30 min.

O ácido ascórbico tem sido usado há mais de 50 anos como inibidor de escurecimento enzimático em alimentos de origem vegetal, muitas vezes combinado com ácidos orgânicos, tais como o ácido cítrico (Neves e Silva, 2007). O escurecimento resulta da degradação da antocianina pela ação de enzimas oxidativas, como polifenoloxidase (PPO), peroxidase (POD) e ascorbato oxidase. Os testes realizados corroboram o fato da enzima aqui estudada ser uma peroxidase clássica.

Neves e Silva (2007) também testaram o efeito do ácido ascórbico e do ácido cítrico no escurecimento enzimático. Os testes foram feitos com peroxidases de Yaco, e para elas o ácido ascórbico a $5 \mathrm{mmol} \mathrm{L}{ }^{-1}$ apresentou os melhores resultados nos seus experimentos, com uma redução de $27 \%$ de atividade em relação à amostra controle. O ácido cítrico se mostrou eficiente apenas em concentrações acima de $10 \mathrm{mmol} \mathrm{L}^{-1}$.

No caso deste trabalho o teste para avaliação de inibição de atividade nos extratos foi realizado para se confirmar se a atividade em questão era devido a ação de peroxidases. Assim como os testes com Yaco, os experimentos com Echinolaena inflexa tiveram grande inibição com ácido ascórbico mesmo em baixas concentrações. O papel do ácido ascórbico 
como inibidor se dá principalmente pela redução das quinonas em difenóis, evitando a formação de melaninas e na sua atuação inibidora enzimática como acidulante (Neves e Silva, 2007). O ácido cítrico também é amplamente empregado como acidulante e composto quelante.

A peroxidase de Echinolaena inflexa foi capaz de reagir com todos os compostos fenólicos testados e com amostras de efluente (Tabela 1). Entretanto, a maior capacidade de degradação de compostos fenólicos foi observada para o catecol, que foi de $87,5 \% \%$. Os resultados ainda demonstraram uma grande eficiência da enzima na degradação dos outros fenóis, sendo que foi capaz de degradar 67,83\% do pirogalol e 39,1\% do resorcinol.

Nos testes com efluentes agroindustriais (Tabela 1), a amostra de efluente bruto foi aquela em que a peroxidase de E. inflexa apresentou a maior capacidade de degradação de compostos fenólicos (29,1\%). Este resultado é interessante e promissor se levarmos em conta que uma amostra real de efluente dista em muito das condições laboratoriais otimizadas para os ensaios enzimáticos, em que há controle da concentração das espécies reagentes, da presença de contaminantes, do pH e da temperatura do meio, entre outros (Abd-Allah, 1999).

Tabela 1. Degradação de compostos fenólicos em amostras dos quatro pontos testados nos efluentes agroindustriais.

\begin{tabular}{lcc}
\hline \multicolumn{1}{c}{ Amostras } & $\begin{array}{c}\text { Capacidade de } \\
\text { degradação após 90 } \\
\text { min (\% média) }\end{array}$ & Desvio padrão \\
\hline $\begin{array}{l}\text { Córrego Abraão a montante do } \\
\text { lançamento do efluente }\end{array}$ & 7,3 & 12,7 \\
$\begin{array}{l}\text { Efluente bruto na entrada da ETE } \\
\text { Efluente tratado na saída da ETE }\end{array}$ & 29,1 & 5,2 \\
$\begin{array}{l}\text { Córrego Abraão a jusante do } \\
\text { lançamento do efluente }\end{array}$ & 11,0 & 0,5 \\
\hline
\end{tabular}

Klibanov (1983) realizou um dos primeiros trabalhos testando peroxidase em águas contaminadas. O autor usou HRP purificada e os experimentos mostraram o bom potencial, em alguns casos superior a 97\%, de descontaminação de fenóis, anilinas e outros compostos aromáticos de soluções aquosas.

Outros estudos recentes realizados com peroxidase livre e imobilizada tem-se mostrado igualmente eficientes no tratamento de fenóis e clorofenóis. Estudos mecanísticos com cloroperoxidases, peroxidase de rabanete, lignina peroxidase, manganês-peroxidase e lacase também têm sido realizados (Kamida et al., 2005).

A peroxidase de Rábano (HRP) é uma das mais estudadas, esta enzima catalisa a oxidação de vários substratos onde se incluem compostos aromáticos do tipo fenol, bifenol, anilinas, benzidinas e compostos heteroaromáticos relacionados. A HRP pode remover fenóis e aminas aromáticas diferentes da água, com eficiências de remoção para alguns poluentes superiores a 99\% (Akhtar e Husain, 2006). Outras fontes de peroxidase, como o melão-de-São-Caetano também já apresentaram boa capacidade para degradação de compostos fenólicos que ocorrem em efluentes de indústrias têxteis (Akhtar e Husain, 2006). O tratamento foi notavelmente eficaz em misturas de fenóis, mas a peroxidase solúvel deixou de funcionar depois de $3 \mathrm{~h}$, enquanto a peroxidase imobilizada continuou ativa. 


\section{CONCLUSÃO}

Este trabalho representa um primeiro esforço de se explorar novas alternativas ao tratamento de águas residuais. A capacidade de degradação de compostos fenólicos pela peroxidase de Echinolaena inflexa é comparável àquela estudada para outras fontes vegetais, mas com o diferencial de que a enzima continua ativa numa amostra real de efluente não-tratado.

O presente trabalho permitiu concluir que a gramínea Echinolaena inflexa apresentou um teor elevado de atividade da peroxidase, o que a torna fonte alternativa para aplicação em tecnologia enzimática. Os extratos de E. inflexa contendo peroxidase também foram capazes de degradar compostos fenólicos em condições controladas de laboratório e em amostras reais de efluente agroindustrial.

Entretanto, são importantes outros estudos para gerar reatores que possam tratar efluentes com compostos fenólicos de maneira alternativa e eficaz. Além disso, há a necessidade da realização de testes de caracterização de fenóis de cada efluente, para dessa forma entender a ação da enzima em águas de diferentes origens. A exploração de plantas do Cerrado, se feita de forma sustentável, pode resultar na descoberta e no desenvolvimento de ferramentas tecnológicas de baixo custo e de alta eficiência para a recuperação de águas contaminadas.

\section{AGRADECIMENTOS}

Ao Programa de Bolsas de Incentivo à Pesquisa PROBIP/UEG.

\section{REFERÊNCIAS}

ABD-ALLAH, A. M. A. Peroxidase Catalyzed Decontamination of Water Polluted with 2,4Dichlorophenols in Presence of Algae and Plant Materials. Journal of Abdulaziz University: Marine Sciences, v. 10, p. 41-45, 1999.

AKHTAR, S.; HUSAIN, Q. Potential applications of immobilized bitter gourd (Momordica charantia) peroxidase in the removal of phenols from polluted water. Chemosphere, v. 65, n. 7, p. 1228-1235, 2006. http://dx.doi.org/10.1016/j.chemosphere.2006.04.049

BERBICZ, F.; CLEMENTE, E. Avaliação da termoestabilidade e da regeneração da atividade da peroxidase extraída de laranja (Citrus spp.). Acta Scientiarum, v. 23, n. 5, p. 12391242, 2001.

BRADFORD, M. A rapid and sensitive method for the quantification of microgram quantities of protein-dye binding. Analytical Biochemistry, v. 72, p. 248-254, 1976. http://dx.doi.org/10.1016/0003-2697(76)90527-3

BRITO, C. A. K.; SATO, H. H.; SPIRONELLO, A.; SIQUEIRA, W. J. Características da atividade da peroxidase de abacaxis (Ananas comosus (L.) Merrill) da cultivar IAC Gomo-de-mel e do clone IAC-1. Ciência e Tecnologia de Alimentos, v. 25, n. 2, p. 244-249, 2005.

CHANCE, B.; MAEHLY, A. C. Assay of catalases and peroxidases. In: COLOWICK, S.P., KAPLAN, N. O. Methods in Enzymology. New York: Academic Press, 1955. V. 2, p. 764-775. 
HALPIN, B.; PRESSEY, R.; JEN, J.; MONDY, N. Purification and Characterization of Peroxidase Isoenzymes from Green Peas (Pisum sativum). Journal of Food Science, v. 54, n. 3, p. 644-649, 1989. http://dx.doi.org/10.1111/j.1365-2621.1989.tb04672.x

HAMID, M.; KHALIL-URREHMAN. Potential applications of peroxidases. Food Chemistry, v.115, n.4, p. 1177-1186, 2009. http://dx.doi.org/10.1016/j.foodchem.2009.02.035

KAMIDA, H. M.; DURRANT, L. R.; MONTEIRO, R. T. R.; ARMAS, E. D. Biodegradação de efluente têxtil por Pleurotus sajor-caju. Química Nova, v. 28, n. 4, p. 629-632, 2005. http://dx.doi.org/10.1590/S0100-40422005000400014

KLIBANOV, A. M. Peroxidase-Catalyzed Removal of Phenols from Coal-Conversion Waste Waters. Science, v. 221, n. 4607, p. 259-261, 1983.

LOWRY, O. H.; ROSBROUGH, N. J.; FARR, A. L.; RANDALL, R. J. Journal of Biological Chemistry, v. 193, p. 265-275, 1951.

MACIEL, H. P. F.; GOUVEA, C. M. C. P.; PASTORE, G. M. Obtenção de nova fonte de peroxidase de folha de Copaifera langsdorffii Desf. com alta atividade. Ciência e Tecnologia de Alimentos, v. 26, n. 4, p. 735-739, 2006.

MANNARINO, C. F.; MOREIRA, J. C.; FERREIRA, J. A.; ARIAS, A. R. L. Avaliação de impactos do efluente do tratamento combinado de lixiviado de aterro de resíduos sólidos urbanos e esgoto doméstico sobre a biota aquática. Ciência e saúde coletiva, v. 18, n. 11, p. 3235-3243, 2013. http://dx.doi.org/10.1590/S1413-81232013001100014

MUNHOZ, C. B. R.; FELFILI, J. M. Florística do estrato herbáceo-subarbustivo de um campo limpo úmido em Brasília, Brasil. Biota Neotropica, v. 7, n. 3, p. 205-215, 2007. http://dx.doi.org/10.1590/S1676-06032007000300022

NEVES, V. A.; SILVA, M. A. Polyphenol Oxidase from Yacon Roots (Smallanthus sonchifolius). Journal of Agricultural and Food Chemistry, v. 55, p. 2424-2430, 2007. http://dx.doi.org/10.1021/jf063148w

RATTER, J. A.; RIBEIRO, J. F.; BRIDGEWATER, S. The Brazilian Cerrado Vegetation and Threats to its Biodiversity. Annals of Botany, v. 80, p. 223-230, 1997. http://dx.doi.org/10.1006/anbo.1997.0469 


Ambiente \& Água - An Interdisciplinary Journal of Applied Science
ISSN 1980-993X - doi:10.4136/1980-993X
www.ambi-agua.net
E-mail: ambi.agua@gmail.com

\title{
Modelagem do regime térmico de um reservatório tropical de abastecimento público, Juiz de Fora, MG, Brasil
}

\author{
doi:10.4136/ambi-agua.1544
}

Received: 15 Oct. 2014; Accepted: 05 Nov. 2015

\author{
Flaviane de Fátima Cândida de Souza ${ }^{1 *}$; Celso Bandeira de Melo Ribeiro ${ }^{1}$; \\ Carlos Ruberto Fragoso Júnior ${ }^{2}$; Marcelo Henrique Otenio ${ }^{3}$ \\ ${ }^{1}$ Universidade Federal de Juiz de Fora (UFJF), Juiz de Fora, MG, Brasil \\ ${ }^{2}$ Universidade Federal de Alagoas (UFAL), Maceió, AL, Brasil \\ ${ }^{3}$ Empresa Brasileira de Pesquisa Agropecuária (EMBRAPA) Gado de Leite, Juiz de Fora, MG, Brasil \\ *Autor correspondente: e-mail: flavicandida@hotmail.com, \\ celso.bandeira@ufjf.edu.br, crubertofj@hotmail.com, \\ marcelo.otenio@embrapa.br
}

\section{RESUMO}

As alterações de temperatura da água e a dinâmica de estratificação térmica de um ecossistema aquático influenciam diretamente os processos físicos, químicos e biológicos. Devido às perturbações em que esses ambientes estão expostos, tais como mudanças climáticas e usos múltiplos da água, os estudos por meio de modelagem são importantes para suportar tomadas de decisão emergenciais e planejamentos futuros. Neste contexto, este trabalho teve como objetivo principal avaliar o comportamento térmico do reservatório de São Pedro utilizado para abastecimento de água, localizado no município de Juiz de Fora, MG, por meio da modelagem matemática com o modelo IPH-ECO para avaliar a temperatura superficial da água, com base em dados meteorológicos e hidrológicos, representando as forçantes e as condições de contorno, respectivamente. A modelagem do regime térmico mostrou correspondência significativa entre os valores simulados e observados, caracterizando o reservatório como um ambiente homogêneo. As simulações indicaram que a contribuição dos afluentes determina o padrão de distribuição espaço-temporal da temperatura da água no reservatório, limitando ou regularizando a sua variabilidade da temperatura.

Palavras-chave: hidrodinâmica, modelagem, temperatura da água.

\section{Thermal regime modeling of the public supply tropical reservoir at Juiz de Fora, MG, Brazil}

\begin{abstract}
Water temperature changes and the dynamics of thermal stratification of an aquatic ecosystem directly influence physical, chemical and biological processes. Due to disturbances in these environments, such as climate change and multiple water uses, studies which employ modeling are important to support emergency decision-making and future planning. In this context, this study evaluated the thermal behavior of the water supply reservoir of São Pedro, located in the city of Juiz de Fora, MG, using mathematical modeling with the IPH-ECO model to evaluate the surface temperature of water, based on meteorological and hydrological
\end{abstract}


data representing the forcing and boundary conditions, respectively. The modeling of the thermal system showed a significant correlation between predicted and observed values, characterizing the reservoir as a homogeneous environment. The simulations indicated that the tributary contribution determines the pattern of spatio-temporal distribution of water temperature in the reservoir, limiting or regulating the variability of its temperature.

Keywords: hydrodynamic, modeling, water temperature.

\section{INTRODUÇÃO}

A construção de reservatórios pode gerar impactos ambientais (i.e. eutrofização, sedimentação, toxicidade e veiculação de doenças), devido à transformação de um ambiente lótico em lêntico. Essas modificações nas condições de escoamento podem afetar, também, o regime térmico do sistema hídrico, além de introduzir novas condições químicas e biológicas (Ferreira e Cunha, 2013).

Os reservatórios são construídos em pontos estratégicos de uma bacia hidrográfica com o objetivo de atender diferentes demandas, tais como geração de energia, abastecimento, pesca, irrigação, recreação e aqüicultura (Christofolleti, 1980). Além destas também são muito utilizados para a regularização da vazão e amortecimento de cheias, apresentando-se como uma estrutura hidráulica de múltiplas finalidades para a sociedade.

No geral, a variação da temperatura de um corpo hídrico é ditada pelo regime climático natural e, associada à hidrodinâmica, afeta praticamente todos os fluxos ecológicos, como produção primária, nitrificação e respiração (Fragoso Jr. et al., 2009). Portanto, a avaliação da dinâmica do regime térmico auxilia na compreensão da variabilidade desses processos nos ambientes aquáticos.

Neste contexto, os modelos matemáticos se apresentam como ferramentas que ajudam na obtenção de uma visão dinâmica dos processos naturais, pois permitem a integração de dados dispersos espacialmente, com a possibilidade de prever diferentes situações ao simular cenários futuros (Rosman et al., 2001).

Conhecer a variação da temperatura de um corpo de água é particularmente importante por três motivos: (a) as descargas de efluentes em diferentes temperaturas podem causar efeitos negativos no ecossistema aquático; (b) a temperatura influencia as reações químicas e biológicas; e (c) a variação da temperatura afeta a densidade da água, e como consequência, altera os processos hidrodinâmicos e de transporte de massa (COPPE/UFRJ, 2007).

As condições de temperatura de um reservatório podem ser diferenciadas ao longo da coluna de água, por exemplo, promovendo um gradiente de densidade que forma uma barreira física que impede que compartimentos verticais se misturem (i.e. superfície e fundo). Essa barreira física cria zonas de circulação na coluna de água, amplificando o efeito da estratificação térmica. A ausência de variação térmica vertical, dentre outros fatores, facilita a homogeneização do corpo de água.

A compreensão do regime térmico em resposta ao clima e às condições hidrológicas é fundamental para o desenvolvimento de estratégias para gestão adaptativa da qualidade da água em reservatórios (Wang et al., 2012).

A modelagem do regime térmico de um reservatório depende do conhecimento das condições de contorno climáticas, que são as forçantes de temperatura do ar, radiação solar, evaporação, direção e intensidade do vento, precipitação e umidade, além dos dados hidrológicos que são as vazões de entrada e saída do reservatório.

Muitos e variados modelos de ecossistemas aquáticos foram desenvolvidos e publicados durante as últimas quatro décadas, com o objetivo de representar a variabilidade dos processos ecológicos. Dentre os modelos de qualidade da água utilizados em reservatórios 
podemos citar o IPH-ECO, desenvolvido no Instituto de Pesquisas Hidráulicas da Universidade Federal do Rio Grande do Sul, o SisBaHiA (Sistema Base de Hidrodinâmica Ambiental) desenvolvido na COPPE da Universidade Federal do Rio de Janeiro e o ELCOM (Estuary and Lake Computer Model) acoplado ao CAEDYM (Computacional Aquatic Ecosystem Dinamics Model) ambos desenvolvidos pelo CWR (Center of Water Research) da Universidade da Austrália.

No entanto, apesar de algumas tentativas terem sido feitas, trabalhos que tratam de abordagens integradas no espaço e no tempo em ecossistemas lacustres ainda são escassos (Mooij et al., 2010). Além disso, a complexidade de fatores envolvidos (i.e. poluição, usos múltiplos da água, espécies invasoras, alterações do uso e cobertura do solo na bacia) dificulta a análise e quantificação dos processos.

Assim, este trabalho tem como objetivo investigar o regime térmico do reservatório de São Pedro, localizado no município de Juiz de Fora (MG), por meio da modelagem hidrodinâmica, em duas dimensões na horizontal (2DH), com base nos dados meteorológicos e hidrológicos, utilizando o modelo ecológico desenvolvido no Instituto de Pesquisas Hidráulicas - IPH da Universidade Federal do Rio Grande do Sul, denominado IPH-ECO.

\section{MATERIAL E MÉTODOS}

\section{1. Área de estudo}

O Reservatório de São Pedro, também conhecido como Represa dos Ingleses ou Cruzeiro de Santo Antônio, localizada na Mesorregião da Zona da Mata Mineira/Microrregião de Juiz de Fora, está situada nas coordenadas $21^{\circ} 46^{\prime} 34,8^{\prime}$ ' $\mathrm{S} / 43^{\circ} 24^{\prime} 27,2$ '' $\mathrm{W}$, altitude média de $850 \mathrm{~m}$, possuindo $0,04 \mathrm{~km}^{2}$ de espelho de água próximo a mancha urbana do município de Juiz de Fora/MG, região oeste da cidade. Atualmente, consiste em um manancial urbano inserido em uma área apontada como vetor de crescimento pelo Plano Diretor do município (Juiz de Fora, 1999).

Criado em 1963, o reservatório teve início da sua operação somente em 1967, com o objetivo de abastecer parte da demanda do município. A produção de água da Estação de Tratamento de Água (ETA São Pedro), localizada no bairro São Pedro, é em média de $120{\mathrm{~L} . \mathrm{s}^{1}}^{1}$, variando de acordo com o nível do reservatório. Atualmente, este reservatório ainda constitui um importante manancial de abastecimento público, operado e mantido pela Companhia de Saneamento Municipal - CESAMA. Este manancial fica a $8 \mathrm{~km}$ da densa malha urbana e corresponde a 8\% do abastecimento da cidade (CESAMA, 2013).

Sob o clima Cwb (tropical de altitude) - clima mesotérmico com secas de inverno e chuvas concentradas de verão e verões brandos ou moderadamente quentes, na classificação de Koppen (Machado, 2010), a bacia hidrográfica do reservatório de São Pedro (BHRSP) ocupa uma área de 13,04 km² e seus principais afluentes são o Córrego São Pedro (margem esquerda) e o Córrego Grota do Pinto (margem direita). A bacia do córrego São Pedro tem seu exutório no rio Paraibuna que deságua na margem direita do rio Paraíba do Sul no município de Três Rios - RJ. A localização da bacia hidrográfica do reservatório de São Pedro na área urbana de Juiz de Fora é mostrada na Figura 1.

\subsection{O modelo IPH-ECO}

O modelo ecológico IPH-ECO é um sistema computacional desenvolvido no Instituto de Pesquisas Hidráulicas (IPH) da Universidade Federal do Rio Grande do Sul (UFRGS), voltado especialmente para o entendimento dos processos físicos, químicos e biológicos de corpos de água rasos e profundos, tais como lagos, reservatórios e estuários (Fragoso Jr., 2009). O modelo descreve as mais importantes interações hidrodinâmicas e abióticas, além dos principais processos bióticos, com a finalidade de auxiliar no entendimento de um 
determinado ecossistema aquático e está disponível para download em https://sites.google.com/site/iphecomodel/.

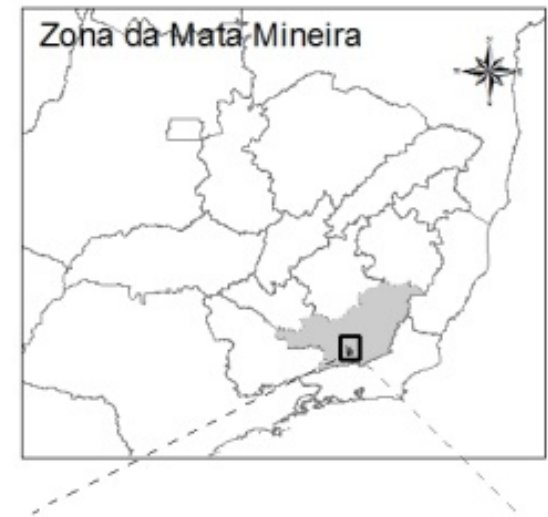

Área Urbana de Juiz de Fora
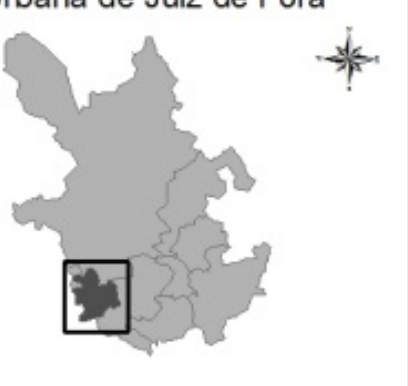

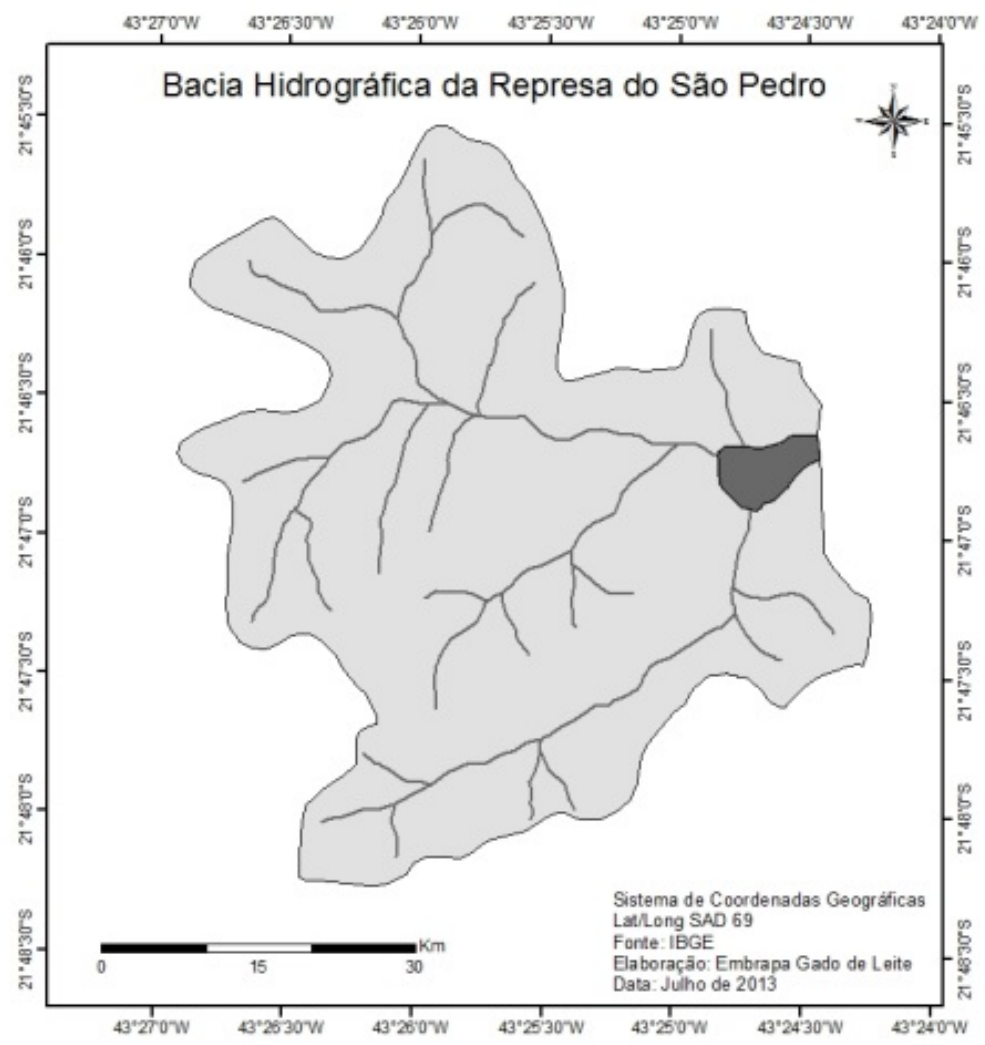

Figura 1: Localização da bacia afluente do reservatório de São Pedro.

Para este trabalho foi utilizado o módulo de balanço térmico associado ao módulo hidrodinâmico (bidimensional horizontal) do IPH-ECO para simulação da temperatura da água do reservatório de São Pedro. Uma vez que em corpos de água rasos, geralmente um modelo bidimensional na horizontal (2DH) é aconselhado, já que a dimensão horizontal predomina sobre vertical e nem sempre ocorre estratificação nestes sistemas (Rosman, 1999).

A escolha do IPH-ECO se baseou na possibilidade de representação do regime térmico para um reservatório raso e acesso à informações fundamentais para utilização do modelo por meio de curso específico, já que existem diversos modelos com variadas adequações e possibilidades de representação. Além disso, é preciso considerar o acesso a informações de forma documental ou até mesmo informal como base para utilização do modelo.

\subsection{Módulo hidrodinâmico}

As equações governantes para o módulo hidrodinâmico no IPH-ECO são derivadas das equações de Navier-Stokes para um escoamento turbulento sob a suposição de distribuição hidrostática de pressões. Estas equações são baseadas na conservação da massa e quantidade de movimento e podem ser escritas na forma diferencial (Equações 1, 2 e 3).

Equação da Continuidade:

$$
\frac{\partial \eta}{\partial t}+\frac{\partial[(h+\eta) u]}{\partial x}+\frac{\partial[(h+\eta) v]}{\partial y}=0
$$


Equação da Quantidade de Movimento:

$$
\begin{aligned}
& \frac{\partial u}{\partial t}+u \frac{\partial u}{\partial x}+v \frac{\partial u}{\partial y}=-g \frac{\partial \eta}{\partial x}-\gamma u+\tau_{x}+A_{h} \nabla^{2} u+f v \\
& \frac{\partial v}{\partial t}+u \frac{\partial v}{\partial x}+v \frac{\partial v}{\partial y}=-g \frac{\partial \eta}{\partial y}-\gamma+\tau_{y}+A_{h} \nabla^{2} v-f u
\end{aligned}
$$

em que:

t é o tempo;

u e v são as componentes da velocidade nas direções $\mathrm{x}$ e y no plano horizontal, respectivamente;

$\eta$ é elevação da superfície da água medida a partir de um nível de referência (Figura 2);

h é a profundidade medida a partir de um nível de referência;

g é a aceleração da gravidade;

f é o parâmetro de Coriolis; $\tau_{\mathrm{x}} \mathrm{e}$

$\tau_{\mathrm{y}}$ são as tensões do vento nas direções x e y, respectivamente;

$\nabla=\partial / \partial x \cdot \vec{i}+\partial / \partial x \cdot \vec{j}$ é um operador vetorial no plano $x-y ; e$

$\mathrm{A}_{\mathrm{h}}$ é o coeficiente de viscosidade turbulenta horizontal; e

$\gamma$ é coeficiente de atrito junto ao fundo.

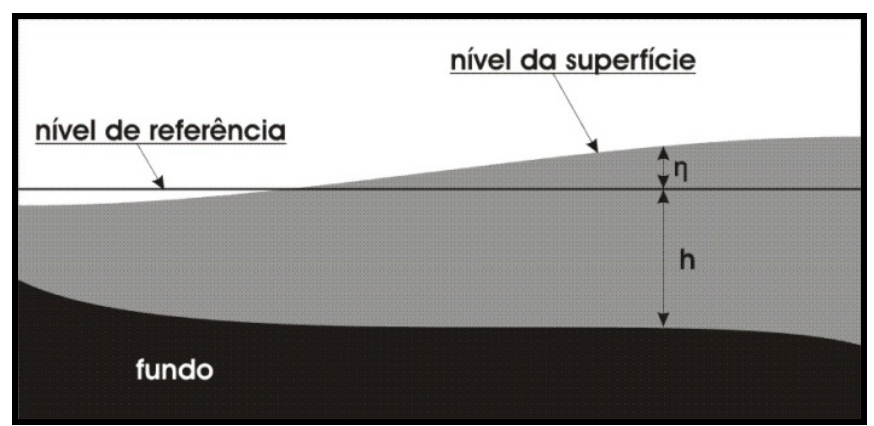

Figura 2. Elementos integrantes das equações de movimento.

O coeficiente de atrito junto ao fundo $(\gamma$ ) é estimado empiricamente (Equação 4).

$$
\gamma=\frac{g \sqrt{u^{2}+v^{2}}}{C_{z}^{2} H}
$$

em que:

$\mathrm{H}=\mathrm{h}+\eta$ é a profundidade total que corresponde à soma do nível de referência com $\mathrm{o}$ nível da superfície; e 
Cz é o coeficiente de atrito de Chèzy.

Como é comum em modelos de escoamentos, a tensão de atrito na superfície livre pelo vento é escrita em termos da velocidade do vento (Rosman, 1999; Equações 5 e 6).

$$
\begin{aligned}
& \tau_{x}=C_{D} \cdot W_{x} \cdot\|W\| \\
& \tau_{y}=C_{D} \cdot W_{y} \cdot\|W\|
\end{aligned}
$$

em que:

$C_{D}$ é o coeficiente de arraste do vento (valores utilizados 1 e -6);

Wx e Wy são as componentes do vetor velocidade do vento nas direções x e y em m.s ${ }^{-1}$, respectivamente, medidas a 10 metros da superfície livre e

$\|\mathrm{W}\|$ é o módulo do vetor velocidade do vento.

As equações de águas rasas (Equações de 1 a 3) não têm solução analítica genérica. Um esquema Euleriano-Lagragiano semi-implícito de diferenças finitas resolve numericamente as equações governantes para um número finito de pontos no espaço e no tempo (Casulli e Cheng, 1992). Este método necessita subdividir o domínio de aplicação em uma malha, comum número finito de células. As equações são discretizadas espacialmente em uma grade retangular que consiste em células computacionais quadradas com comprimento $\Delta \mathrm{x} e$ largura $\Delta y$.

\subsection{Balanço de calor}

No modelo IPH-ECO a temperatura da água é estimada a partir do balanço de calor entre a superfície da água e a atmosfera. Esse balanço é aplicação direta da primeira lei da termodinâmica para um fluido incompressível, a qual define que a variação da energia interna é igual à resultante dos fluxos de calor nos contornos do sistema. O balanço do fluxo de calor pode ser representado pela Equação 7.

$$
\Delta \mathrm{Q}=\mathrm{Q}_{\mathrm{RAOC}}^{\text {agua }}+\mathrm{Q}_{\mathrm{RAOL}}-\mathrm{Q}_{\mathrm{RWOL}}-\mathrm{Q}_{\mathrm{COND}}-\mathrm{Q}_{\mathrm{EVAP}} \pm \mathrm{Q}_{\mathrm{IN} / \mathrm{OUT}}
$$

em que:

$\mathrm{Q}_{\mathrm{RAOC}}^{\text {agua }}$ é o fluxo de calor referente à radiação atmosférica de onda curta,

$\mathrm{Q}_{\mathrm{RAOL}}$ é o fluxo de calor referente à radiação atmosférica de onda longa,

$\mathrm{Q}_{\mathrm{RwOL}}$ é o fluxo de calor referente à radiação da água de onda longa,

$\mathrm{Q}_{\mathrm{COND}}$ é o fluxo de calor referente à condução para a atmosfera,

$\mathrm{Q}_{\mathrm{EVAP}}$ é o fluxo de calor referente ao processo de evaporação e

$\mathrm{Q}_{\text {IN/OUT }}$ é o fluxo de calor resultante das entradas e saídas por advecção tais como escoamento superficial e subterrâneo, fluxo de afluentes, precipitação e fluxos de saída. $\mathrm{O}$ calor armazenado durante um intervalo de tempo pode ser convertido em temperatura (Equação 8). 


$$
S_{t}=\frac{\Delta Q}{\rho \cdot V \cdot c}
$$

em que:

V é o volume $\mathrm{em}^{3}$,

$\rho$ é a densidade da água em $\mathrm{kg} \mathrm{m}^{-3} \mathrm{e}$

$c$ é o calor específico da água em $\mathrm{J} \mathrm{kg}^{-1}{ }^{\circ} \mathrm{C}^{-1}$. A variabilidade temporal e espacial da temperatura da água pode ser avaliada para a equação de transporte de massa (Equação 9).

$$
\frac{\partial(H T)}{\partial t}+\frac{\partial(u H T)}{\partial x}+\frac{\partial(v H T)}{\partial y}=\frac{\partial}{\partial x}\left(K_{h} \frac{\partial(H T)}{\partial x}\right)+\frac{\partial}{\partial y}\left(K_{h} \frac{\partial(H T)}{\partial y}\right)+H \cdot S_{t}
$$

em que:

$K_{h}$ é o coeficiente de difusão horizontal $\mathrm{em} \mathrm{m}^{2} \cdot \mathrm{s}^{-1}$,

T é a temperatura da água em graus Celsious $\left({ }^{\circ} \mathrm{C}\right) \mathrm{e}$

$\mathrm{S}_{\mathrm{t}}$ é o termo de perdas e ganhos (dado pela Equação 8). Esta equação é resolvida numericamente por meio de um esquema explícito centrado de diferenças finitas.

\subsection{Amostragem e preparação dos dados para modelagem}

\subsubsection{Monitoramento da temperatura água}

A amostragem neste trabalho foi realizada a partir da seleção de três pontos representativos ao longo do espelho de água do reservatório, denominados Ponto 1, Ponto 2 e Ponto 3 (Figura 3).

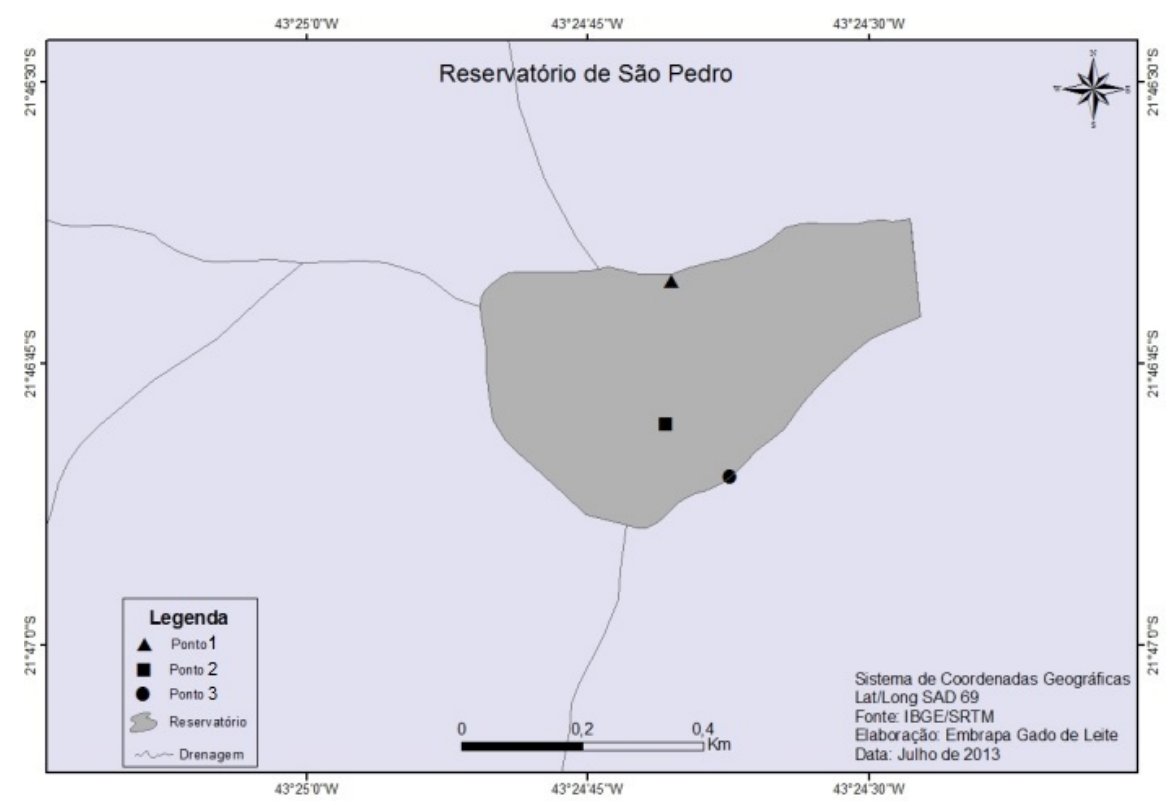

Figura 3: Distribuição dos pontos amostrais no reservatório de São Pedro.

A seleção de pontos para o monitoramento deste manancial foi uma etapa importante para se obter um referencial de qualidade do mesmo. Foram selecionados 3 pontos, dois pontos próximos às margens e um ponto na área central do reservatório. Foram realizadas 10 campanhas de amostragem, entre os meses de julho de 2010 a junho de 2011, 
intencionalmente com frequência mensal, porém nos meses de novembro de 2010 e março de 2011 não foi possível realizar campanhas devido às chuvas e dificuldades de deslocamento para acesso às áreas de coleta. As amostragens in situ foram realizadas com a utilização de uma sonda modelo HQ30d da Hach, com a finalidade de registrar a temperatura da água nos diferentes pontos selecionados no reservatório (Tabela 1).

Tabela 1. Valores de temperatura da água observados in situ.

\begin{tabular}{lccc}
\hline \multirow{2}{*}{ Meses } & \multicolumn{3}{c}{ Temperatura da água $\left({ }^{\circ} \mathrm{C}\right)$} \\
\cline { 2 - 4 } & Ponto 1 & Ponto 2 & Ponto 3 \\
\hline Julho/2010 & 19,08 & 19,23 & 19,16 \\
Agosto/2010 & 19,70 & 19,60 & 20,70 \\
Setembro/2010 & 22,70 & 23,00 & 23,40 \\
Outubro/2010 & 23,90 & 24,10 & 24,10 \\
Dezembro/2010 & 26,50 & 28,10 & 27,20 \\
Janeiro/2011 & 23,40 & 23,00 & 23,50 \\
Fevereiro/2011 & 27,00 & 27,20 & 27,20 \\
Abril/2011 & 22,20 & 21,90 & 22,10 \\
Maio/2011 & 18,80 & 19,10 & 18,70 \\
Junho/2011 & 18,90 & 18,70 & 18,90 \\
\hline
\end{tabular}

A temperatura da água foi monitorada próxima a superfície com cerca de 30 a $40 \mathrm{~cm}$ de profundidade controlado pela imersão do leitor da sonda utilizada na água. Estes dados foram comparados com os valores de temperatura estimados pelo IPH-ECO a partir dos dados climatológicos e dados das vazões de entrada e de saída do reservatório, os quais serão discutidos mais adiante.

Para a modelagem do regime térmico foi utilizado um intervalo de tempo de 10 segundos durante o período de simulação de 355 dias. Os principais dados de entrada do modelo são descritos a seguir.

\subsubsection{Dados meteorológicos}

Os dados meteorológicos foram fornecidos pela Estação Climatológica Principal situada na Universidade Federal de Juiz de Fora, operada e mantida pelo Instituto Nacional de Meteorologia na sede de Belo Horizonte/MG (INMET), são eles: temperatura do ar $\left({ }^{\circ} \mathrm{C}\right)$, evaporação $(\mathrm{mm})$, direção e intensidade do vento $\left(\mathrm{m}^{\mathrm{s}} \mathrm{s}^{-1}\right)$, precipitação $(\mathrm{mm})$ e umidade $(\%)$ com freqüência 00:00h/12:00h/18:00h. Os dados de radiação solar $\left(\mathrm{w} / \mathrm{m}^{2}\right)$ foram obtidos diretamente da Estação Climatológica Principal do INMET/MG com frequência de 1 hora, em sua página de internet. Esta estação dista cerca de $4,5 \mathrm{~km}$ do reservatório, o que garante pouca diferenciação das condições climáticas locais que poderiam afetar na comparação dos dados, como nebulosidade e precipitação.

\subsubsection{Levantamento batimétrico}

A batimetria do reservatório foi efetuada com a utilização de um Ecobatímetro (Garmin Fishfinder 140), aparelho que descreve a topografia do fundo do lago e os pontos foram marcados com um GPS (Garmin GPSMAP 76CSx) para garantir as amostragens nos mesmos pontos ao longo do monitoramento. Este levantamento foi realizado no dia 11 de julho de 2010, data referente ao primeiro monitoramento.

A batimetria revelou que a profundidade média do reservatório é de 2 metros (Figura 4), caracterizando-o como um reservatório raso, com predomínio da dimensão horizontal sobre a 
vertical, o que não justifica a aplicação de uma aproximação tridimensional. Desta forma, foi utilizada uma representação bidimensional na horizontal $(2 \mathrm{DH})$ do domínio. A discretização espacial consistiu em uma malha de $50 \times 50 \mathrm{~m}$ resultando em 450 células computacionais.

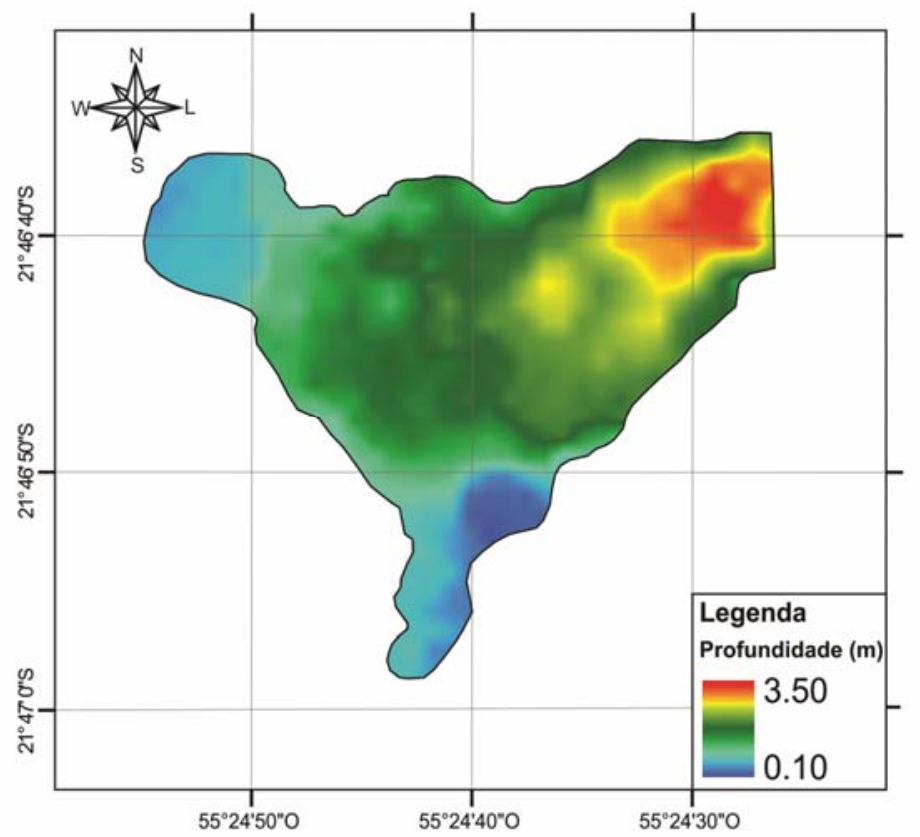

Figura 4: Batimetria do reservatório de São Pedro.

\subsubsection{Estimativa das vazões afluentes}

Uma estimativa das vazões de entrada do modelo foi obtida por meio do Método Racional Modificado (Euclydes, 1987) que resume todos os processos hidrológicos da bacia em um único coeficiente, o qual determina a parcela da chuva que se transforma em escoamento superficial (Equação 10).

$$
\mathrm{Q}=0,278 \cdot \mathrm{C} \cdot \mathrm{i} \cdot \mathrm{A} \cdot \varphi
$$

em que:

Q é a vazão na seção de interesse $\left(\mathrm{m}^{3} \mathrm{~s}^{-1}\right)$,

i a intensidade de chuva $\left(\mathrm{mm} \mathrm{h}^{-1}\right)$,

A é a área de drenagem na seção de interesse $\left(\mathrm{km}^{2}\right)$,

$\varphi$ o coeficiente de retardamento (adimensional), e

C é o coeficiente de escoamento superficial ou coeficiente de run off. Este valor é normalmente estimado em função do mapa de uso e ocupação do solo. Para bacia em estudo utilizou-se para os tipos de cobertura do solo os valores 0,20 para Mata, 0,80 para Área Urbana, 0,30 para Pasto/Solo Exposto e 0,0 para Água, conforme Tucci (2001).

O cálculo do coeficiente de retardamento é realizado com a Equação 11.

$$
\varphi=0,278-0,00034 . \mathrm{A}
$$

Para tanto é necessário o cálculo das áreas das bacias dos afluentes São Pedro e Grota do Pinto e seus respectivos coeficientes de retardamento.

O coeficiente de retardamento foi estimado com base em dados de vazão levantados 
in situ fazendo uso de um molinete hidrométrico nos afluentes São Pedro e Grota do Pinto. Os coeficientes de retardamento ajustados para os principais afluentes do reservatório foram: 0,2749944 para o São Pedro e 0,2768576 para o Grota do Pinto, cujas respectivas áreas de drenagem são $8,84 \mathrm{~km}^{2}$ e $3,36 \mathrm{~km}^{2}$.

A vazão de saída foi disponibilizada pela Estação de Tratamento de Água de São Pedro da Companhia de Saneamento Municipal - CESAMA que trata a água do reservatório de São Pedro e faz o controle horário dessa vazão para direcionar as operações dentro da estação.

\subsubsection{Saída do modelo}

Para a simulação da temperatura da massa de água do reservatório de São Pedro foram selecionados três pontos de saída do modelo (Pontos 1, 2 e 3), como mostra a Figura 5, com a finalidade de comparar os valores simulados com os valores observados em campo.

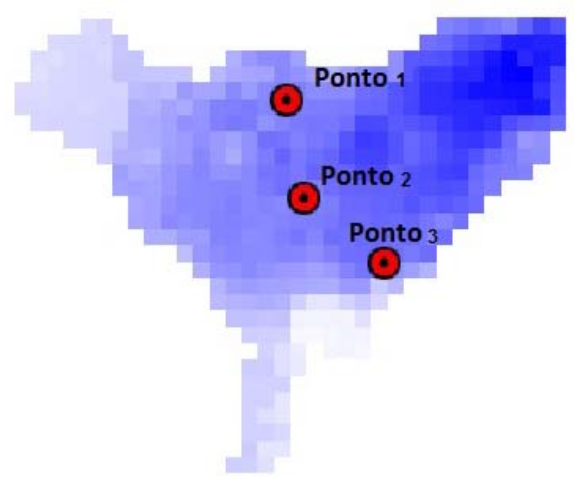

Figura 5: Pontos de saída selecionados no modelo IPH-ECO.

\subsubsection{Avaliação do desempenho do modelo}

O grau de correspondência entre a saída do modelo e os dados observados foi medido com a utilização de dois índices estatísticos: (a) a raiz do erro médio quadrático (REMQ), e (b) o coeficiente de Nash-Sutcliffe $\left(\mathrm{R}^{2}\right)$. $\mathrm{O}$ cálculo da raiz do erro quadrático médio e do coeficiente de determinação de Nash-Sutcliffe são apresentados nas Equações 12 e 13.

$$
\begin{aligned}
& \text { REMQ }=\sqrt{\frac{1}{N} \sum_{\mathrm{i}=1}^{\mathrm{N}}[\mathrm{Yobs}-\mathrm{Ycal}]^{2}} \\
& \mathrm{R}^{2}=1-\frac{\sum\left(\mathrm{Y}_{\mathrm{obs}}(\mathrm{t})-\mathrm{Y}_{\mathrm{cal}}(\mathrm{t})\right)^{2}}{\sum\left(\mathrm{Y}_{\mathrm{obs}}(\mathrm{t})-\overline{\mathrm{Y}}_{\mathrm{obs}}(\mathrm{t})\right)^{2}}
\end{aligned}
$$

em que:

REMQ é raiz do erro médio quadrático,

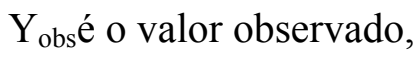

$\mathrm{Y}_{\text {cal }}$ é o valor estimado ou calculado pelo modelo,

$\mathrm{N}$ é o número total de valores,

$\mathrm{R}^{2}$ é o coeficiente de Nash-Sutcliffe e

$\bar{Y}_{\text {obs }}$ é a média dos valores observados. 


\section{RESULTADOS E DISCUSSÃO}

\subsection{Simulações da temperatura da água}

Ao final da simulação, o modelo IPH-ECO estimou a temperatura da água para os pontos de interesse em um período total de 355 dias, iniciando às 00:00 horas do dia 01/07/2010 e finalizando às 23:00 horas do dia 01/06/2011. Esse período de simulação corresponde ao período de monitoramento realizado no reservatório permitindo observar o comportamento térmico nos períodos seco e chuvoso ao longo do ano em questão. A comparação entre as temperaturas da água simuladas e observadas entre os meses de julho de 2010 a junho de 2011 nos pontos 1, 2 e 3 é mostrada, respectivamente, na Figura 6.
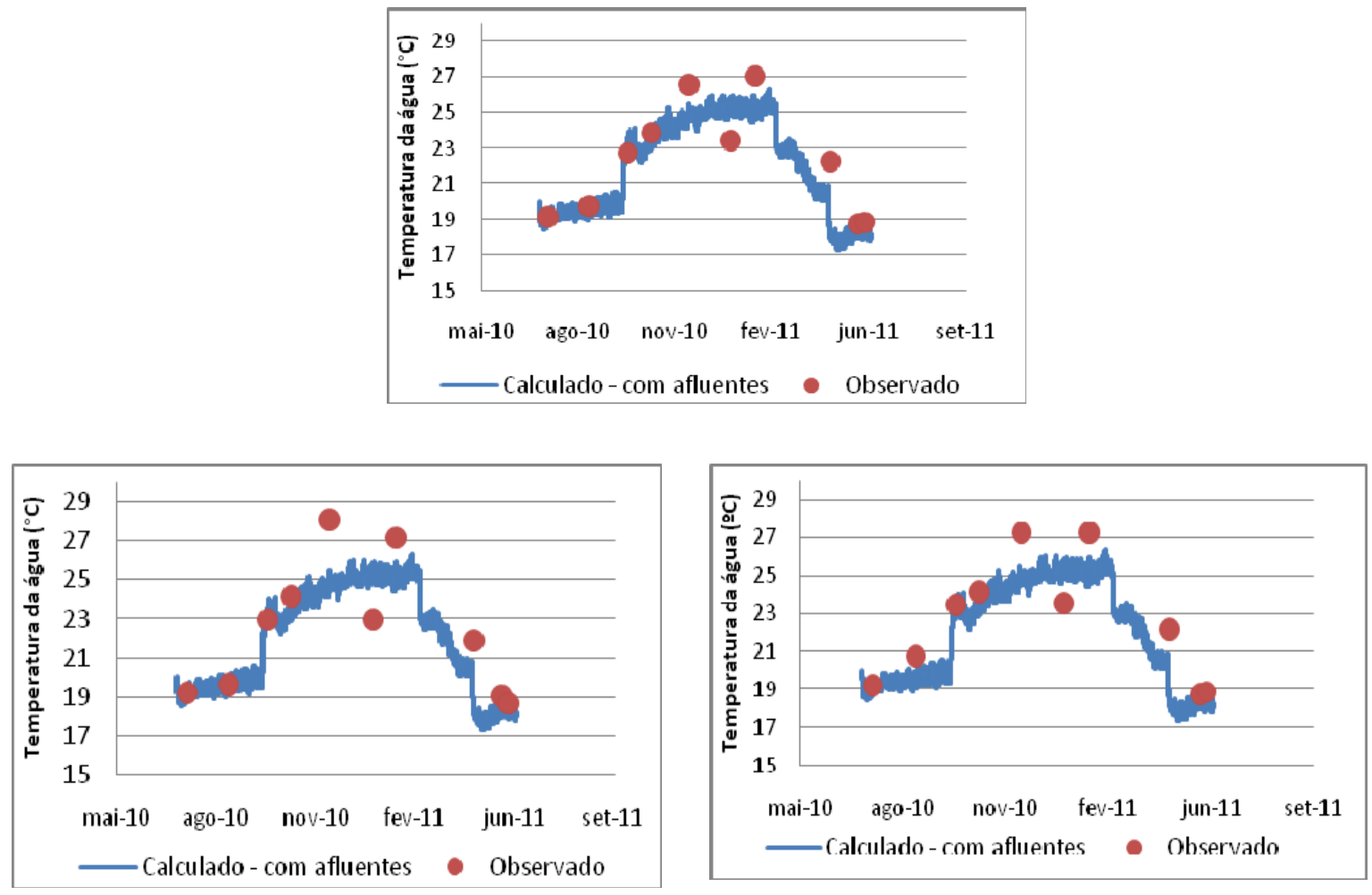

Figura 6. Variação da temperatura da água de Julho de 2010 a Junho de 2011. a) Comparação entre os valores de temperatura da água simulados (linha contínua) e observados (pontos) no Ponto 1 (área central do reservatório). b) Comparação entre os valores de temperatura da água simulados (linha contínua) e observados (pontos) no Ponto 2 (margem esquerda do reservatório). c) Comparação entre os valores de temperatura da água simulados (linha contínua) e observados (pontos) no Ponto 3 (margem direita do reservatório).

Ao se analisar a Figura 6 pode-se perceber que os valores simulados e observados apresentam boa correspondência ao longo do tempo. Os indicadores da eficiência do ajuste apresentaram bons valores ( $\mathrm{REMQ}<2 \%$ e $\left.\mathrm{R}^{2}>0,75\right)$ no período de simulação, caracterizando um grau de correspondência significativo para a modelagem da temperatura da água nos pontos de saída selecionados no IPH-ECO. Os dados observados e calculados apresentam correspondência direta com o clima tropical de altitude com temperaturas mais elevadas no período chuvoso (novembro a março) e mais brandas no período seco (abril a outubro).

Praticamente toda energia que controla o metabolismo de um reservatório advém da energia solar. Ferreira e Cunha (2013), ao desenvolver e aplicar um modelo unidimensional para simular o comportamento térmico do reservatório do Rio Verde, localizado na Região Metropolitana de Curitiba - PR, observaram que a formação estratigráfica no reservatório 
depende principalmente da incidência de radiação solar e que os ventos não seriam eficientes em misturar a coluna de água. Porém, para o reservatório de São Pedro, os dados de campo e simulações do comportamento térmico revelam proximidade dos valores de temperatura verificados nos três pontos amostrais caracterizando o reservatório como um ambiente homogêneo (Tabela 2).

Tabela 2. Cálculo da Raiz do Erro Médio Quadrático (REMQ) e coeficiente de determinação $\mathrm{R}^{2}$.

\begin{tabular}{|c|c|c|c|c|c|c|c|c|c|}
\hline & \multicolumn{6}{|c|}{ Temperatura $\left({ }^{\circ} \mathrm{C}\right)$} & \multirow{2}{*}{\multicolumn{3}{|c|}{ Cálculo dos índices por ponto }} \\
\hline & \multicolumn{3}{|c|}{ Valores Observados } & \multicolumn{3}{|c|}{ Valores Estimados } & & & \\
\hline & Ponto 1 & Ponto 2 & Ponto 3 & Ponto 1 & Ponto 2 & Ponto 3 & Ponto 1 & Ponto 2 & Ponto 3 \\
\hline Julho/2010 & 19,23 & 19,08 & 19,16 & 20,00 & 20,00 & 20,00 & 0,5928 & 0,8462 & 0,7055 \\
\hline Agosto/2010 & 19,6 & 19,7 & 20,7 & 19,47 & 19,50 & 19,44 & 0,0166 & 0,0387 & 1,5817 \\
\hline Setembro/2010 & 23,0 & 22,7 & 23,4 & 19,72 & 19,76 & 19,67 & 10,7662 & 8,6201 & 13,8823 \\
\hline Outubro/2010 & 24,1 & 23,9 & 24,1 & 23,44 & 23,45 & 23,44 & 0,4354 & 0,2049 & 0,4309 \\
\hline Dezembro/2010 & 28,1 & 26,5 & 27,2 & 24,67 & 24,65 & 24,70 & 11,7346 & 3,4109 & 6,2275 \\
\hline Janeiro/2011 & 23,0 & 23,4 & 23,5 & 25,06 & 25,07 & 25,06 & 4,2556 & 2,7805 & 2,4358 \\
\hline Fevereiro/2011 & 27,2 & 27,0 & 27,2 & 25,33 & 25,32 & 25,35 & 3,4848 & 2,8099 & 3,4048 \\
\hline Abril/2011 & 21,9 & 22,2 & 22,1 & 21,48 & 21,46 & 21,50 & 0,1791 & 0,5420 & 0,3616 \\
\hline Maio/2011 & 19,1 & 18,8 & 18,7 & 17,84 & 17,82 & 17,88 & 1,5806 & 0,9533 & 0,6792 \\
\hline Junho/2011 & 18,7 & 18,9 & 18,9 & 18,13 & 18,13 & 18,15 & 0,3203 & 0,5968 & 0,5677 \\
\hline REMQ & & & & & & & 1,83 & 1,44 & 1,74 \\
\hline $\mathbf{R}^{2}$ & & & & & & & 0,75 & 0,81 & 0,77 \\
\hline
\end{tabular}

\subsection{Representatividade do efeito dos afluentes no regime térmico do reservatório}

Com a finalidade de avaliar a influência das vazões afluentes no regime térmico do reservatório de São Pedro, foi realizada uma nova simulação retirando as vazões de entrada e saída. Os resultados desta simulação foram comparados com os dados observados e com a simulação original, considerando as vazões de entrada e saída (Figura 7).

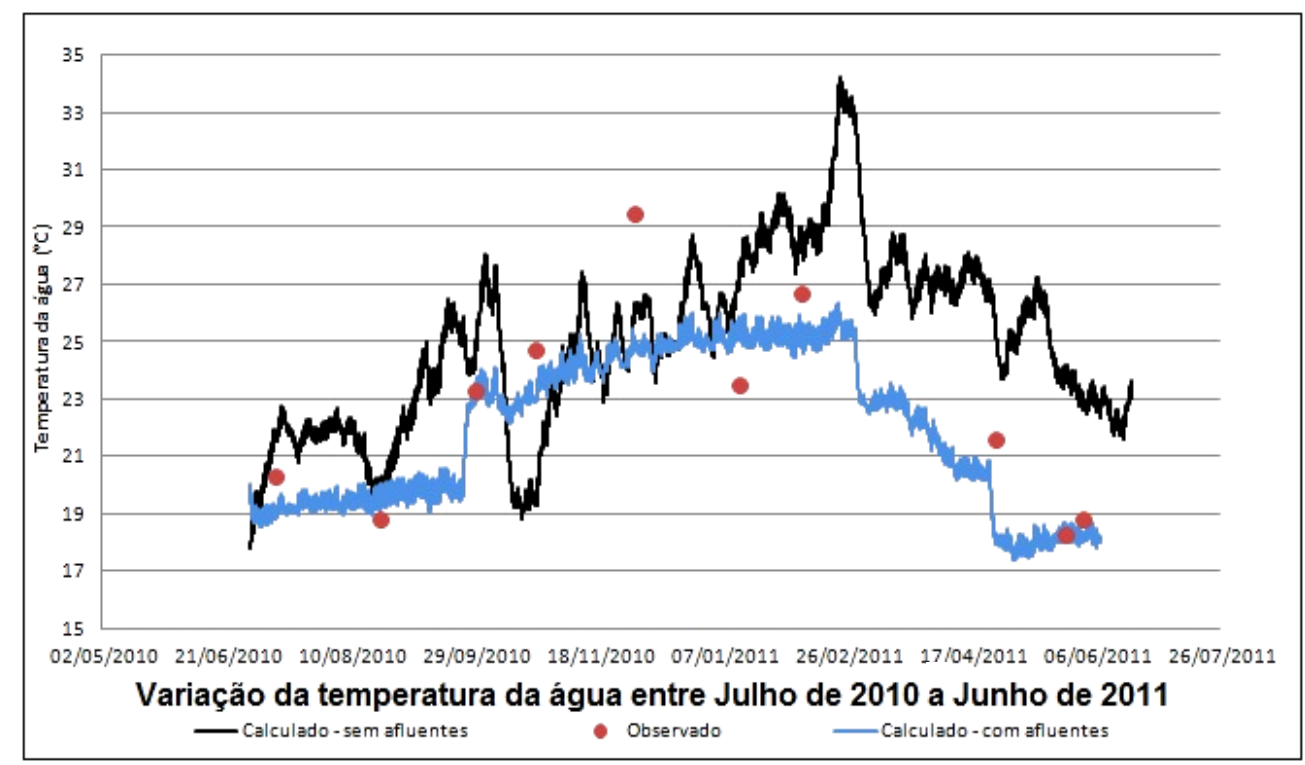

Figura 7. Variação da temperatura do reservatório com e sem os afluentes (Ponto 2). 
Nesta circunstância, mostra-se a influência dos afluentes no regime térmico do reservatório, pois a proximidade dos valores observados com os valores calculados mostrou-se relacionada ao fato de se considerar as vazões dos afluentes, ou seja, os afluentes Grota do Pinto e São Pedro controlam a temperatura do reservatório de São Pedro. Fato este também pode ser justificado por conta do tamanho do reservatório e as vazões dos afluentes, onde a mistura e distribuição das temperaturas das águas ocorrem de forma rápida, respeitando a sazonalidade imposta pelo clima tropical de altitude. As alterações climáticas influenciam sobremaneira a dinâmica de temperatura dos afluentes e consequentemente o reservatório.

Destarte, Fantin-Cruz et al. (2010) apontam que o regime térmico dos rios pode ser afetado por outras perturbações provocadas por ações humanas, como efluentes térmicos, redução no fluxo dos rios (irrigação, hidrelétricas) e lançamentos de águas a partir de barragens a montante dos rios. Os lançamentos que atingem os afluentes e o reservatório de São Pedro são basicamente representados por esgotos domésticos e não há barragens a montante. Eles apontam ainda que se devem considerar inclusive alterações na temperatura da água à jusante do reservatório, que são influenciadas por condições ligadas a variáveis operacionais (volume, descarga), limnológicas (tempo de retenção, padrão de estratificação e gradientes térmicos), além da posição da barragem ao longo do perfil longitudinal do rio.

O fluxo dos rios é capaz de influenciar na produtividade biológica de um corpo hídrico, interferindo em processos como eutrofização, ciclagem de nutrientes e atividades fotossintéticas. Para Collischonn e Motta Marques (2003), de um modo geral os rios trazem maior parte dos nutrientes e sedimentos para o interior dos lagos e reservatórios, por isso, a circulação originada pelas entradas desses afluentes é particularmente importante.

Os resultados apresentados por Pacheco et al. (2014), ao investigar o Reservatório do Funil, localizado no município de Resende - RJ, mostram que a variabilidade espacial da concentração de clorofila $(\mathrm{Chl})$ e $\mathrm{CO}_{2}$ está mais relacionada a mudanças no fluxo do rio ao longo do ano do que fatores ambientais, tais como temperatura do ar e radiação solar. Nesse sentido, quando o fluxo do rio atuar como regulador da temperatura de um reservatório, também será capaz de condicionar processos físicos, químicos e biológicos, visto que a temperatura é um fator físico que interfere diretamente na estrutura das comunidades biológicas e processos ecossistêmicos.

A variabilidade das vazões se apresentou conforme esperado para o contexto climatológico, com volumes maiores no período chuvoso e menores no período seco. A maior vazão ocorreu no mês de janeiro de $2011\left(0,2294 \mathrm{~m}^{3} / \mathrm{s}\right)$ no córrego São Pedro, cuja bacia possui a maior área de contribuição.

\section{CONCLUSÃO}

Foi possível representar o regime térmico do reservatório de São Pedro por meio da modelagem hidrodinâmica $2 \mathrm{DH}$, com base em dados meteorológicos e hidrológicos, utilizando o modelo IPH-ECO. O contexto climatológico como principal fator influenciador das condições térmicas da água a partir das trocas de calor da atmosfera com a superfície de água foi fundamental para realização deste trabalho, visto que os resultados das simulações mostraram valores de temperatura em consonância direta com as condições do clima tropical de altitude.

Nossos resultados mostraram temperaturas simuladas, em média $22^{\circ} \mathrm{C}$, próximas às temperaturas observadas, em média $22,21^{\circ} \mathrm{C}$. A simulação satisfatória do regime térmico é essencial para investigar os processos ecossistêmicos, uma vez que a temperatura é um fator que influencia diretamente nesses processos. Essa representatividade significativa no modelo suportaria testar cenários com mudanças de temperatura e garantir ações previamente necessárias para prevenir eventuais efeitos negativos à diversidade biológica, além da 
operação e manutenção do sistema de abastecimento.

A contribuição dos afluentes se mostrou muito importante para as simulações hidrodinâmicas, pois a variação das vazões limita ou regulariza as temperaturas do reservatório de São Pedro. Por se tratar de um reservatório pequeno e raso, as temperaturas são mais limitadas às vazões dos afluentes caracterizando, assim, uma baixa capacidade deste reservatório de regularização de suas temperaturas.

\section{AGRADECIMENTOS}

Os autores agradecem ao Programa de Pós-Graduação em Ecologia da Universidade Federal de Juiz de Fora (PGECOL), ao Departamento de Engenharia Sanitária e Ambiental da Universidade Federal de Juiz de Fora, à Embrapa Gado de Leite (CNPq), à Coordenação de Aperfeiçoamento de Pessoal de Nível Superior (CAPES) e ao Corpo de Bombeiros de Juiz de Fora pelo apoio ao trabalho. Os autores agradecem ao CNPq, Processo número 473854/20082 e à Embrapa código Infoseg 06.08.06.002.00.00 pelo apoio financeiro ao trabalho.

\section{REFERÊNCIAS}

CASULLI, V.; CHENG, R. Semi-implicit finite difference methods for three-dimensional shallow water flow. International Journal for Numerical Methods in Fluids, v. 15, p. 629-648, 1992. http://dx.doi.org/10.1002/fld.1650150602

COMPANHIA DE SANEAMENTO MUNICIPAL DE JUIZ DE FORA - CESAMA. Website. Disponível em: www.cesama.br. Acesso: em abr. 2013.

CHRISTOFOLLETI, A. Geomorfologia. São Paulo: Blucher, 1980.

COLISCHONN, W.; MOTTA MARQUES, D. L. Desenvolvimento de modelo bidimensional de transporte de sedimentos e nutrientes para lagos. In: CONGRESSO BRASILEIRO DE LIMNOLOGIA, 9., 2003, Juiz de Fora. Anais... Juiz de Fora: ICB; UFJF - MG, (2003).

EUCLYDES, H. P. Saneamento agrícola: atenuação de cheias: metodologia e projeto. Belo Horizonte: Rural Minas, 1987.

FANTIN-CRUZ, I.; TONDATO, K. K.; MARQUES, D. M.; PEDROLlO, O. Regime térmico em águas correntes e sua importância na estrutura do habitat e na biologia de organismos aquáticos. Revista Caminhos de Geografia, v. 11, n. 36, p. 295-307, 2010.

FERREIRA, D. M.; CUNHA, C. Simulação numérica do comportamento térmico do reservatório do Rio Verde. Revista Engenharia Sanitária e Ambiental, v. 18, n. 1, jan./mar. 2013.

FRAGOSO JR., C. R.; FERREIRA, T. F.; MOTTA MARQUES, D. M. L. Modelagem ecológica em ecossistemas aquáticos. 1. ed. São Paulo: Oficina de Textos, 2009. v. 1. $304 \mathrm{p}$.

FRAGOSO JR., C. R. Modelagem tridimensional da estrutura trófica em ecossistemas aquáticos continentais. 2009. Tese (Doutorado em Recursos Hídricos e Saneamento Ambiental) - Instituto de Pesquisas Hidráulicas, Universidade Federal do Rio Grande do Sul, Porto Alegre, 2009. 
INSTITUTO ALBERTO LUIZ COIMBRA DE PÓS-GRADUAÇÃO E PESQUISA DE ENGENHARIA - COPPE/UFRJ. Manual do Usuário do SisBaHia. 2007. Disponível em: http://www.sisbahia.coppe.ufrj.br/Arquivos_Gerais. Acesso em: jul. 2013.

JUIZ DE FORA. Prefeitura Municipal. Plano diretor de desenvolvimento urbano de Juiz de Fora. Juiz de Fora: FUNALFA, 1999.

MACHADO, P. J. O. (Org.) Diagnóstico físico-ambiental da bacia hidrográfica do Córrego São Pedro: um exercício acadêmico de gestão dos recursos hídricos. Ubá: Geographica, 2010.

MOOIJ, W. M.; TROLLE, D.; JEPPESEN, E.; ARHONDITSIS, G.; BELOLIPETSKY, P. V.; CHITAMWEBWA, D. B. R. et al. Challenges and opportunities for integrating lake ecosystem modeling approaches. Aquatic Ecology, v. 44, 2010. http://dx.doi.org/10.1007/s10452-010-9339-3

PACHECO, F. S.; SOARES, M. C. S.; ASSIREU, A. T.; CURTARELLI, M. P.; ROLAND, F.; ABRIL, G. et al. River inflow and retention time affecting spacial heterogeneity of chlorophyll and water-air $\mathrm{CO}_{2}$ fluxes in a tropical hydropower reservoir. Biogeosciences Discuss, v. 11, p. 8531-8568, 2014. http://dx.doi.org/10.5194/bgd-118531-2014

ROSMAN, P. C. C.; MASCARENHAS, F. C. B.; MIGUEZ, M. G.; CAMPOS, R. O.; EIGER, S. Métodos numéricos em recursos hídricos. 5. ed. Rio de Janeiro: ABRH, 2001.

ROSMAM, P. C. C. Subsídios para modelagem de sistemas estuarinos. In: ROSMAN, P. C. C.; MASCARENHAS, F. C. B.; MIGUEZ, M. G.; CAMPOS, R. O.; EIGER, S. Métodos numéricos em recursos hídricos. Rio de Janeiro: ABRH, 1999.

TUCCI, C. E. M. Hidrologia: ciência e aplicação. Porto Alegre: Ed. UFRGS, 2001.

WANG, S.; QIAN, X.; HAN, B.; LUO, L.; HAMILTON, D. P. Effects of local climate and hydrological conditions on the thermal regime of a reservoir at Tropic of Cancer, in southern China. Water Research, v. 46, n. 8, p. 2591-2604, 2012. http://dx.doi.org/10.1016/j.watres.2012.02.014 


\begin{tabular}{|} 
Ambiente \& Água - An Interdisciplinary Journal of Applied Science \\
ISSN 1980-993X - doi:10.4136/1980-993X \\
www.ambi-agua.net \\
E-mail: ambi.agua@gmail.com
\end{tabular}

\title{
Qualidade da água de três locais com potenciais fontes de contaminação no Rio Taquari, RS
}

\author{
doi:10.4136/ambi-agua.1789 \\ Received: 21 Oct. 2015; Accepted: 16 Nov. 2015 \\ Thais Muller; Camille Eichelberger Granada; Raul Antonio Sperotto* \\ Centro Universitário UNIVATES, Lajeado, RS, Brasil \\ Programa de Pós-Graduação em Biotecnologia (PPGBiotec) \\ *Autor correspondente: e-mail: rasperotto@univates.br, \\ thais_muller84@hotmail.com, cegranada@univates.br
}

\section{RESUMO}

O Vale do Taquari apresenta uma grande quantidade de indústrias alimentícias e curtumes, que lançam seus efluentes no Rio Taquari, além de um cemitério em Área de Preservação Permanente, localizado no município de Muçum. Este trabalho teve como objetivo realizar análises físico-químicas e microbiológicas da água do Rio Taquari e comparar pontos à montante e à jusante de três locais considerados potencialmente poluidores. Os locais escolhidos foram um cemitério e um curtume no município de Muçum, e um curtume seguido de um frigorífico de frangos no município de Roca Sales. Foram realizadas análises de $\mathrm{pH}$, turbidez, oxigênio dissolvido $\left(\mathrm{O}_{2}\right)$, nitrogênio total $(\mathrm{N})$ e coliformes termotolerantes. Realizou-se também o isolamento de Escherichia coli seguido de testes de resistência aos antibióticos neomicina, rifampicina, clindamicina e penicilina. As análises físico-químicas realizadas neste estudo mostraram que somente a turbidez, em quatro dos seis pontos analisados, apresentou valores acima do permitido pela legislação para águas de classe I, sendo que estes valores elevados provavelmente têm origem inorgânica. As bactérias isoladas no ponto à jusante do cemitério e à montante e à jusante das indústrias de Roca Sales foram sensíveis à neomicina, rifampicina e clindamicina, e resistentes à penicilina. Pode-se concluir, portanto, que mesmo com a liberação dos poluentes, não existe uma interferência significativa na qualidade da água nos três locais analisados do Rio Taquari.

Palavras-chave: análises físico-químicas, atividades antrópicas, efluentes.

\section{Water quality near three sources of potential contamination of the Taquari River - RS}

\begin{abstract}
The Taquari Valley has several food industries and tanneries which release their effluents into the Taquari River, as well as a cemetery in a Permanent Preservation Area in Muçum town. This study performed physicochemical and microbiological analyses of water from before and after three Taquari River sites considered potentially polluting and compared the results of those samples. The chosen sites were a cemetery and a tannery in Muçum and a tannery next to a poultry processing plant located in Roca Sales. We analyzed $\mathrm{pH}$, turbidity,
\end{abstract}


dissolved oxygen $\left(\mathrm{O}_{2}\right)$, total nitrogen $(\mathrm{N})$ and thermotolerant coliforms. We also isolated Escherichia coli and performed resistance tests to neomycin, rifampicin, clindamycin and penicillin antibiotics. The physicochemical analyses showed that only turbidity, in four out of the six analyzed spots, had values above the established threshold of class I water. These elevated values are probably of inorganic origin. Bacteria isolated from the samples collected after the cemetery and both before and after the Roca Sales industries were sensitive to neomycin, rifampicin and clindamycin, and resistant to penicillin. It can be concluded, therefore, that even the release of the pollutant effluents does not substantially interfere with the water quality of the three analyzed sites.

Keywords: anthropic activities, effluents, physicochemical analysis.

\section{INTRODUÇÃO}

A bacia hidrográfica do sistema Taquari-Antas situa-se na região nordeste do estado do Rio Grande do Sul, abrangendo uma área de $26.428 \mathrm{~km}^{2}$, com aproximadamente $530 \mathrm{~km}$ de extensão (Pimenta et al., 2012). Essa bacia nasce no extremo leste do Planalto dos Campos Gerais e tem suas nascentes nos municípios de Cambará do Sul, Bom Jesus e São José dos Ausentes, sendo em sua parte superior denominado de Rio das Antas; e em sua parte inferior, Rio Taquari. Seus principais afluentes pela margem esquerda são os rios Camisas, Tainhas, Lajeado Grande e São Marcos; e pela margem direita, Quebra-Dentes, Prata, Carreiro, Guaporé, Forqueta e Taquari-Mirim (Becker et al., 2013).

De modo geral, as bacias hidrográficas do Brasil, bem como a bacia Taquari-Antas, vêm sofrendo severas modificações que são geradas, na sua maioria, por atividades antrópicas (Becker et al., 2013), sendo que os escoamentos de esgotos são as vias de contaminação mais significativas (Marques et al., 2012). Guedes et al. (2012) relataram que a utilização desses recursos naturais para despejo das indústrias altera significativamente suas características, e o monitoramento da qualidade da água é um dos principais instrumentos para a gestão de recursos, pois possibilita verificar a dimensão do impacto, com o intuito de determinar estratégias para minimizá-lo.

As indústrias alimentícias ou que processam produtos animais como laticínios, frigoríficos e curtumes, são as principais fontes poluidoras. Essas indústrias, além de liberarem diversos poluentes, lançam também vários micro-organismos no ambiente (Iafigliola et al., 2000). Segundo Krewer et al. (2012), esses micro-organismos podem ser resistentes a diversos tipos de antibióticos, sendo que essa resistência pode ser atribuída ao uso de antimicrobianos, desinfetantes e suplementos alimentares utilizados nos processos de produção.

Os curtumes processam as peles de animais para que estas possam ser utilizadas na fabricação de diversos artigos de consumo. Nesse processamento, são utilizados produtos altamente tóxicos como sulfato de cromo, álcalis à base de mercúrio, sulfeto de sódio, cloreto de sódio, fungicida e óleo catiônico (Cunha e Shiraiwa, 2011). O processo com cromo, utilizado por cerca de $90 \%$ dessas indústrias, gera resíduos que necessitam de tratamento adequado, pois podem interferir significativamente no ambiente onde são liberados (Oliveira et al., 2008).

Os cemitérios, muitas vezes localizados em locais inadequados, também causam impacto devido à geração e percolação pelo solo de um líquido escuro chamado necrochorume. Esse líquido é resultado da decomposição dos corpos e contem substâncias orgânicas, inorgânicas e micro-organismos, podendo contaminar o solo e os recursos hídricos localizados nas proximidades (Silva e Malagutti Filho, 2010). A contaminação do meio ocorre principalmente no primeiro ano do sepultamento. O necrochorume é mais denso que a água, rico em sais 
minerais e pode conter ainda resíduos de tratamentos químicos hospitalares, como quimioterápicos, formaldeído e metanol, além de patógenos associados a mortes por doenças infectocontagiosas e óxidos metálicos como $\mathrm{Ti}, \mathrm{Cr}, \mathrm{Cd}, \mathrm{Pb}, \mathrm{Fe}, \mathrm{Mn}, \mathrm{Ni}$, que podem ser lixiviados dos adereços das urnas (Silva et al., 2009). A Resolução no 335 do CONAMA, de 3 de abril de 2003 (CONAMA, 2003), dispõe sobre o licenciamento ambiental de cemitérios e estabelece que os mesmos são considerados importantes fontes de contaminação, sendo que sua implantação necessita estar dentro dos critérios legais de proteção ambiental para proteger o solo e as águas subterrâneas.

Para obter uma estimativa da contaminação existente nas bacias hidrográficas, destino do despejo de indústrias como as citadas anteriormente, e também por contaminantes em suas margens, como é o caso do cemitério, é necessária a realização de análises que avaliem aspectos físicos, químicos e microbiológicos (Castro, 2006). Com base nos valores obtidos nessas análises é possível se verificar se os corpos hídricos encontram-se de acordo com o uso ao qual eles se destinam. A Resolução 357/05 do CONAMA (2005) estabelece os parâmetros físicos, químicos e microbiológicos para a classificação dessas águas. Essa resolução admite cinco classes: especial, I, II, III e IV, sendo que a classe especial pode ser utilizada para consumo humano apenas com desinfecção, enquanto que as classes I, II e III necessitam de tratamento simplificado, convencional e avançado, respectivamente. Os rios classificados nas quatro classes também são destinados à preservação dos ambientes aquáticos, à manutenção do equilíbrio natural das comunidades aquáticas, à irrigação, à recreação, à aquicultura, à pesca e à dessedentação de animais. Os rios de classe IV servem apenas para a navegação e harmonia paisagística.

O Rio Taquari serve como despejo final de efluentes das indústrias de couros, suínos e frangos da região do Vale do Taquari. Este mesmo rio é muito utilizado pela população para atividades de lazer. Devido a isso, é necessário um rigoroso controle de sua qualidade, assegurando boas condições sanitárias em sua utilização, bem como garantindo a sobrevivência da biota local. Assim, este trabalho teve como objetivo realizar análises físico-químicas e microbiológicas da água do Rio Taquari e comparar pontos à montante e à jusante de três locais considerados potencialmente poluidores. Os locais escolhidos foram um cemitério e um curtume no município de Muçum, e uma indústria de frangos seguida de um curtume no município de Roca Sales. Foram feitas análises de coliformes termotolerantes, $\mathrm{pH}$, nitrogênio total $(\mathrm{N})$, oxigênio dissolvido $\left(\mathrm{O}_{2}\right)$ e turbidez. Realizou-se também o isolamento de Escherichia coli seguido de teste de resistência aos antibióticos neomicina, rifampicina, clindamicina e penicilina.

\section{MATERIAL E MÉTODOS}

\subsection{Pontos amostrados e coleta de material}

Os pontos de coleta estão localizados na Bacia Taquari-Antas, mais precisamente na sua parte inferior, onde este se denomina Rio Taquari. A ocupação e o uso do solo, ao longo da referida bacia, são intensamente diversificados, apresentando municípios densamente povoados ou ainda aqueles onde a agricultura e a indústria são a principal atividade econômica. Além disso, alguns municípios concentram ambas as características. Conforme visto na Figura 1, os pontos avaliados estão compreendidos entre os municípios de Muçum e Roca Sales, sendo um cemitério e um curtume em Muçum e um curtume seguido de uma indústria de frangos no município de Roca Sales. Os pontos ficam em torno de cinquenta metros antes e após o local de despejo dos efluentes e foram denominados da seguinte forma: Local 1, abrangendo os Pontos 1 e $2(\mathrm{P} 1$ e $\mathrm{P} 2)$, respectivamente à montante e à jusante do cemitério em Muçum; Local 2, abrangendo os Pontos 3 e 4 (P3 e P4), respectivamente à 
montante e à jusante do curtume existente no município de Muçum; e Local 3, abrangendo os Pontos 5 e 6 (P5 e P6), respectivamente à montante e à jusante do curtume seguido de uma indústria de frangos existentes no município de Roca Sales. As coordenadas geográficas dos

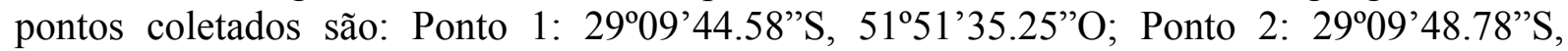

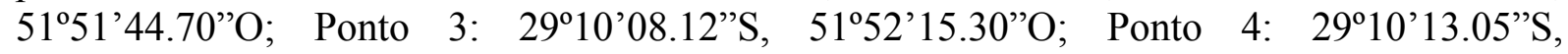

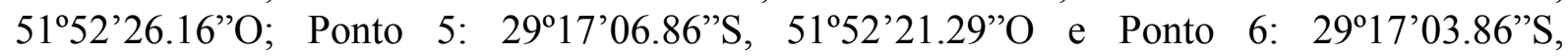
51 ${ }^{\circ} 52^{\prime} 34.65$ "O. As coletas (1 L para cada amostra) ocorreram nos meses de julho à novembro de 2013 e foram realizadas em triplicatas biológicas (totalizando 18 amostras por dia de coleta) e em triplicata experimental (coleta em três dias diferentes).

\subsection{Análises de coliformes termotolerantes}

As análises de coliformes termotolerantes procederam-se com base na metodologia da Instrução Normativa $n^{\circ} 62$ do Ministério da Agricultura, Pecuária e Abastecimento (MAPA), pela técnica do Número Mais Provável (NMP). Foram dispensados $10 \mathrm{~mL}$ da amostra em uma série de três tubos contendo meio Lauryl Sulfato de Sódio $\left(\right.$ OXOID $\left.^{\circledR}\right)$ com tubos de Durham, em concentração dupla, e $1 \mathrm{~mL}$ da amostra em uma série de três tubos contendo meio Lauryl Sulfato de Sódio $\left(\right.$ OXOID $\left.^{\circledR}\right)$ com Durham, em concentração simples. Efetuaram-se ainda duas diluições da amostra. Os tubos foram incubados a $36^{\circ} \mathrm{C}$ por no máximo 48 hs. Transcorrido este período, os tubos positivos (que apresentaram formação de gás dentro do Durham ou leve efervescência sob fraca agitação) foram inoculados para os tubos contendo meio Verde Brilhante bile $2 \%\left(\right.$ OXOID $\left.^{\circledR}\right)$ com Durham, e incubados a $36^{\circ} \mathrm{C}$ por 24 hs. Após esse período, os tubos positivos foram inoculados para tubos de caldo EC Medium (Acumedia ${ }^{\circledR}$ ) com Durham e incubados por no máximo 48 hs. Para cada caldo, anotou-se o número de tubos positivos. A partir da combinação de números correspondentes aos tubos que apresentaram resultado positivo, é verificado o número mais provável (NMP) de acordo com a tabela correspondente (MAPA), onde o resultado é expresso em NMP 100 $\mathrm{mL}^{-1}$.

\subsection{Análises físico-químicas}

Os aspectos físico-químicos como $\mathrm{pH}$, nitrogênio total $(\mathrm{N})$, oxigênio dissolvido $\left(\mathrm{O}_{2}\right)$ e turbidez foram analisados, respectivamente, utilizando-se os equipamentos: pHmetro (Digimed DM-20), TNM1 Schimadzu Total Nitrogen Measuring unit, medidor de Oxigênio Dissolvido portátil Digimed DM-4P e Turbidímetro Digimed DM-TU.

\subsection{Isolamento de Escherichia coli e teste de resistência aos antibióticos}

$\mathrm{O}$ isolamento de E. coli foi realizado segundo a metodologia descrita por Borges et al. (2003). A amostra foi diluída e inoculada em placas contendo ágar Mac Conkey (MC) $\left(\mathrm{OXOID}^{\circledR}\right.$ ) e incubadas a $37^{\circ} \mathrm{C}$ por 18 a 24 hs. As colônias lactose positivas (coloração rosa forte) foram inoculadas em ágar Eosin Methylene Blue (EMB) (OXOID ${ }^{\circledR}$ ). As colônias típicas de E. coli (escuras com brilho verde-metálico) foram preservadas em ágar Trypticase Soy Agar (TSA) $\left(\right.$ OXOID $\left.^{\circledR}\right)$ inclinado. No teste de resistência a antibióticos, cada isolado bacteriano preservado em ágar TSA foi inoculado em caldo Brain Heart Infusion (BHI) $\left(\mathrm{OXOID}^{\circledR}\right)$ por $24 \mathrm{hs}$ a $36^{\circ} \mathrm{C}$, e transferidos para placas de ágar Mueller Hinton $\left(\mathrm{BD}^{\mathbb{R}}\right)$, de acordo com os métodos descritos por Correa et al. (2005). Os antibióticos testados foram Neomicina (30 $\mu \mathrm{g})$, Rifampicina (5 $\mu \mathrm{g})$, Clindamicina (2 $\mu \mathrm{g})$ e Penicilina (G100).

\subsection{Análises estatísticas}

Os dados obtidos nas análises das amostras nos pontos à montante e à jusante foram comparados pelo teste $t$ de Student $(\mathrm{p} \leq 0,01)$ para determinar se os pontos de descarte de efluentes analisados modificam a qualidade das águas do Rio Taquari. Os três locais à 
motante foram comparados entre si usando-se One-Way ANOVA seguido de teste de Tukey $(\mathrm{p} \leq 0,05)$. O mesmo foi feito com os três locais à jusante do descarte. Os resultados também foram comparados com os parâmetros da Resolução 357/05 do CONAMA, de 17 de março de 2005, que dispõe sobre a classificação dos corpos de água e diretrizes ambientais para o seu enquadramento, bem como estabelece as condições e padrões de lançamento de efluentes.

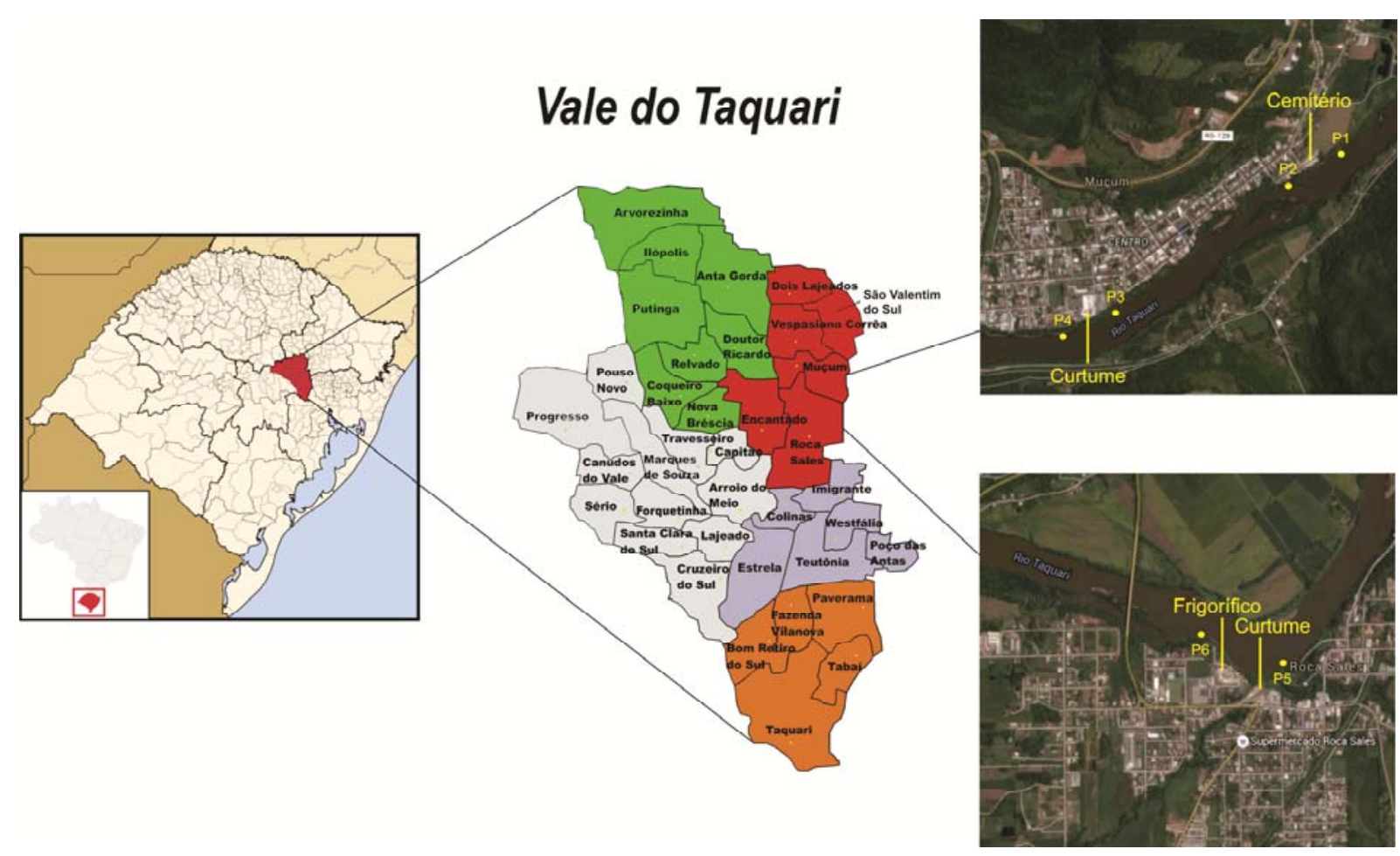

Figura 1. Locais de coleta na Bacia Taquari-Antas. Local 1, abrangendo os Pontos 1 e 2 (P1 e P2), respectivamente à montante e à jusante do cemitério em Muçum; Local 2, abrangendo os Pontos 3 e 4 (P3 e P4), respectivamente à montante e à jusante do curtume existente no município de Muçum; e Local 3, abrangendo os Pontos 5 e 6 ( $\mathrm{P} 5$ e P6), respectivamente à montante e à jusante do curtume seguido de uma indústria de frangos existentes no município de Roca Sales.

\section{RESULTADOS E DISCUSSÃO}

A Figura 2 mostra as análises físico-químicas e microbiológicas realizadas neste trabalho. A temperatura da água durante a análise de $\mathrm{pH}$ ficou entre 8 e $12^{\circ} \mathrm{C}$ em todas as amostras. De acordo com a Figura $2 \mathrm{a}$, as análises de $\mathrm{pH}$ não apresentaram diferenças entre os pontos à montante e à jusante de nenhum dos três locais analisados, sendo que os valores de $\mathrm{pH}$ ficaram próximos da neutralidade em todas as amostras coletadas. Quando comparadas entre si, as amostras dos pontos à montante não apresentaram diferenças. Nas amostras dos pontos à jusante, o ponto 2, localizado após o cemitério do município de Muçum, teve um valor maior que o ponto 6, localizado após as indústrias de Roca Sales. O fato de não haver diferenças entre os pontos à montante e à jusante pode ser explicado pela correção de $\mathrm{pH}$ feita nos efluentes antes de serem despejados nos corpos d'água (Ferreira Filho e Hang Chui, 2006). Cabe salientar que não ocorreu diferença estatística nem mesmo no local 1 (cemitério), onde o resíduo não sofre nenhum tratamento. A Resolução CONAMA 357/05 estabelece que, para as águas de Classe Especial, I e II, que são destinadas à preservação da vida aquática, a faixa de $\mathrm{pH}$ deve ser de 6,0 à 9,0 , sendo que o $\mathrm{pH}$ encontrado em todos os locais foi 
considerado propício à biota aquática.

A Figura $2 \mathrm{~b}$ mostra os resultados das análises de turbidez. Neste parâmetro foram observadas diferenças significativas entre os pontos à montante e à jusante em dois dos três locais avaliados. No cemitério (Local 1) e no curtume do município de Muçum (Local 2), a turbidez foi maior nos pontos à montante do que nos pontos à jusante. Quando comparadas as médias dos pontos à montante e à jusante, podemos constatar que a turbidez do ponto 1 , à montante do cemitério, é menor que dos demais pontos à montante, e a média da turbidez do ponto à jusante das indústrias de Roca Sales (Local 3) é maior que os demais pontos à jusante. A turbidez mais elevada pode estar associada a níveis mais altos de matéria orgânica ou inorgânica, como por exemplo, sólidos suspensos, devido a diversos fatores como o tipo de solo, uso do solo nas margens, temperatura e níveis de pluviosidade (Silva et al., 2008). A alteração na turbidez causa transtornos à biota aquática do rio, podendo interferir na fotossíntese e gerar um problema na cadeia alimentar, além de ser prejudicial a algumas espécies de peixes (Wilson, 2010). Segundo a Resolução 357/05, os valores para a turbidez considerados aceitos são de até 40 UNT para os rios de classe I, e 100 UNT para os rios de classe II. Somente os pontos 2 e 4 (à jusante do cemitério e à jusante do curtume de Muçum) apresentaram resultados inferiores a $40 \mathrm{UNT}$, ficando dentro do limite estabelecido para água de classe I. O ponto 1 (à montante do cemitério) apresentou 45,23 UNT, sendo enquadrada como água de classe II. Entretanto, os demais pontos (3, 5 e 6) ficaram acima de 100 UNT, e dessa forma poderiam ser utilizados somente para os usos aos quais as águas de classes III e IV se prestam, ressaltando que essas duas classes não possuem limites estabelecidos para a turbidez.

A Figura 2c mostra que as análises do $\mathrm{O}_{2}$ dissolvido não apresentaram alterações significativas entre os pontos à montante e à jusante dos três locais de coleta. No ponto à montante do cemitério (Local 1), foi verificado um valor menor que nos outros pontos à montante analisados (o curtume de Muçum e as indústrias de Roca Sales). O mesmo ocorre com o ponto à jusante do cemitério (P2), que apresenta menores valores que os outros dois pontos à jusante (P4 e P6). A Resolução 357/05 do CONAMA estabelece que para a preservação da vida aquática, o nível mínimo de $\mathrm{O}_{2}$ dissolvido para águas de classe I é de 6,0 $\mathrm{mg} \mathrm{L}^{-1}$, e de classe II é 5,0 $\mathrm{mg} \mathrm{L}^{-1}$. Os níveis encontrados nas nossas análises variaram de 8,21 $\mathrm{mg} \mathrm{L}^{-1}$ a $9,03 \mathrm{mg} \mathrm{L}^{-1}$, ficando dentro do limite estabelecido pela legislação para águas de classes I e II. A quantidade de $\mathrm{O}_{2}$ dissolvido nos corpos d'água é um dos indicadores mais importantes da qualidade dessa água. Levando em conta que um bom nível de $\mathrm{O}_{2}$ é de extrema importância para a respiração aeróbica dos organismos do rio em questão (Janzen et al., 2008), pode-se dizer que o Rio Taquari possui um ambiente propício aos organismos que o habitam em todos os seis pontos analisados.

As análises de $\mathrm{N}$ total mostraram diferenças entre os pontos à montante e à jusante dos três locais analisados (Figura 2d). Ocorreu um aumento significativo nos valores de $\mathrm{N}$ total em todos os pontos posteriores ao descarte dos efluentes (P2, P4 e P6). O Local 3 (curtume e indústria de frangos de Roca Sales), à montante e à jusante do despejo, apresentou os maiores níveis de $\mathrm{N}$ total. $\mathrm{O}$ Local 1 (cemitério) apresentou o menor nível de $\mathrm{N}$ em seu ponto posterior, quando comparado com os pontos posteriores dos locais 2 e 3 . $\mathrm{O} \mathrm{N}$ é um composto que promove o crescimento dos organismos e a eutrofização, que resulta na diminuição do $\mathrm{O}_{2}$ dissolvido e causa problemas à biota aquática (Timofiecsyk et al., 2012). O aumento do $\mathrm{N}$ total nos pontos à jusante pode estar relacionado aos efluentes despejados nesses locais. Estes efluentes são ricos em matéria orgânica e, quando não tratados de forma adequada, apresentam grande quantidade de $\mathrm{N}$ (Nunes et al., 2011). Cabe ressaltar que, apesar de ter ocorrido um aumento significativo nos pontos posteriores, todos os pontos possuem níveis 
de $\mathrm{N}$ total que variam de $0,52 \mathrm{mg} \mathrm{L}^{-1}$ a $1,12 \mathrm{mg} \mathrm{L}^{-1}$, ou seja, dentro do estabelecido pela legislação vigente (até $2,18 \mathrm{mg} \mathrm{L}^{-1}$ para águas de classes I e II em ambientes lóticos). Exceto para o local 3, o valor mais elevado nas análises de $\mathrm{N}$ dos pontos à jusante está em desacordo com o que foi observado para a turbidez, que foi maior nos pontos à montante dos locais $1 \mathrm{e} 2$ (cemitério e curtume de Muçum). A turbidez maior nesses pontos provavelmente seja devido à presença de compostos inorgânicos, por exemplo, sólidos suspensos (Wilson, 2010), que podem ser ressuspendidos do fundo por algum processo físico decorrente da geomorfologia do rio. Entretanto, mais estudos seriam necessários, em cada um dos locais analisados, para se chegar a uma conclusão.

Nas análises microbiológicas (Figura 2e), não houve diferença significativa entre os pontos à montante e à jusante de nenhum dos três locais avaliados. Quando os pontos à jusante foram comparados entre si, foi visto que o ponto 1 (cemitério) possui uma média maior que os pontos à jusante dos locais 2 e 3 (curtume e indústrias de Roca Sales). No cemitério (Local 1), os valores médios da contagem de coliformes das amostras à montante $\mathrm{e}$ à jusante ficaram em torno de $20 \mathrm{NMP} 100 \mathrm{~mL}^{-1}$. Tendo em vista que esse parâmetro pode estar relacionado à quantidade de matéria orgânica de origem fecal e é um indicativo da qualidade da água (Skorczewski et al., 2012), pode-se concluir que há uma quantidade maior de coliformes no ponto à jusante do cemitério, quando comparado aos demais pontos à jusante, e isso pode estar associado ao escoamento do necrochorume. Silva et al. (2009) ressaltou que o necrochorume liberado na decomposição dos cadáveres possui elevada quantidade de material orgânico, assim como uma alta concentração de micro-organismos, oriundos principalmente dos corpos que ainda não estão completamente decompostos. Cabe ressaltar que nas análises de $\mathrm{O}_{2}$ dissolvido, o ponto à jusante do cemitério apresentou um nível menor que os pontos à jusante do curtume e das indústrias de Roca Sales, reforçando a hipótese que esse ponto possui maior contaminação.

As amostras foram submetidas também ao isolamento de Escherichia coli seguido de testes de resistência a antibióticos. Não foram obtidas cepas no ponto à montante do cemitério (P1) e nos pontos à montante e à jusante do curtume de Muçum (P3 e $\mathrm{P} 4$, respectivamente). No ponto à jusante do cemitério (P2), obteve-se uma maior quantidade de cepas, indicando que existe uma grande quantidade desses micro-organismos no local. Este resultado é reforçado pelas análises dos coliformes termotolerantes e de $\mathrm{O}_{2}$ dissolvido. $\mathrm{O}$ fato de não isolarmos cepas nos pontos à montante do cemitério e do curtume de Muçum (P1 e P3, respectivamente), reforça a ideia de que a turbidez alterada nesses pontos seja de origem inorgânica. Nos pontos à montante e à jusante das indústrias de Roca Sales (P5 e P6, respectivamente), a quantidade de cepas isoladas foi a mesma. Nos testes com os antibiogramas, os três pontos ( $\mathrm{P} 2, \mathrm{P} 5$ e P6) mostraram sensibilidade à Neomicina e à Rifampicina. O crescimento de uma das cepas isoladas do ponto 2 foi retardado pela Clindamicina. Todas as cepas isoladas demonstraram resistência à penicilina. Segundo Dias et al. (2010), a E. coli é um micro-organismo resistente aos principais antibióticos, e é capaz de produzir $\beta$-lactamase, uma enzima que rompe os anéis $\beta$-lactâmicos e inativa as penicilinas.

Quando os valores obtidos nas análises são comparados com os parâmetros da legislação vigente, percebe-se que o Rio Taquari não pode ser enquadrado como um rio de classe I em todos os trechos analisados. Os valores obtidos nas análises de $\mathrm{pH}, \mathrm{O}_{2}$ dissolvido, $\mathrm{N}$ total e coliformes termotolerantes ficaram dentro dos padrões estabelecidos para a classe I em todas as amostras analisadas. Entretanto, as análises de turbidez de quatro dos seis pontos analisados (P1, P3, P5 e P6) apresentaram valores acima do máximo permitido para águas de classe I (40 UNT), sendo que em três desses pontos (P3, P5 e P6) os valores ficaram acima do máximo permitido para águas de classe II (100 UNT). 
A.

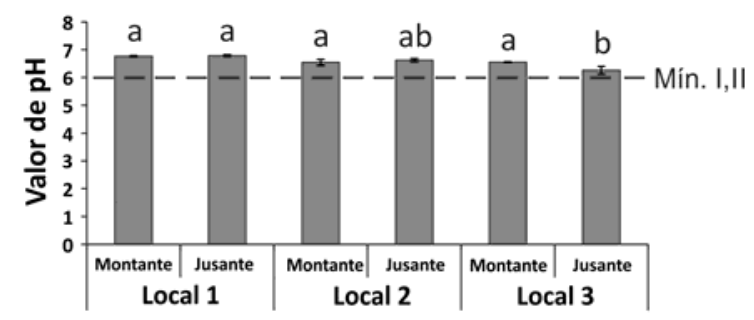

C.

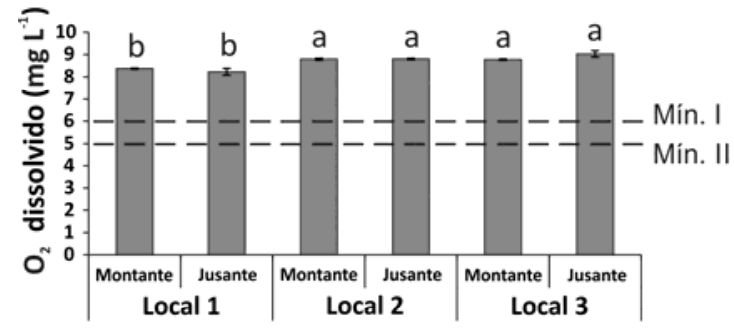

B.

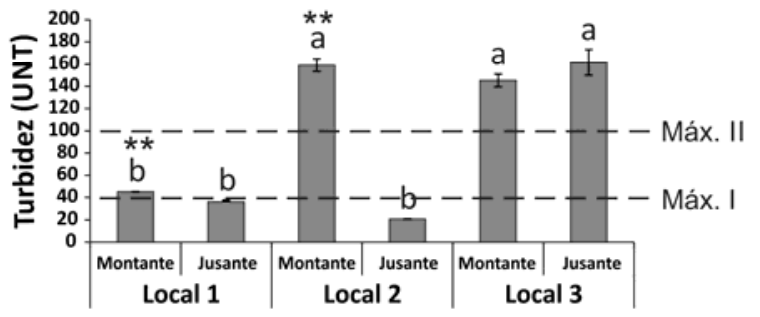

D.

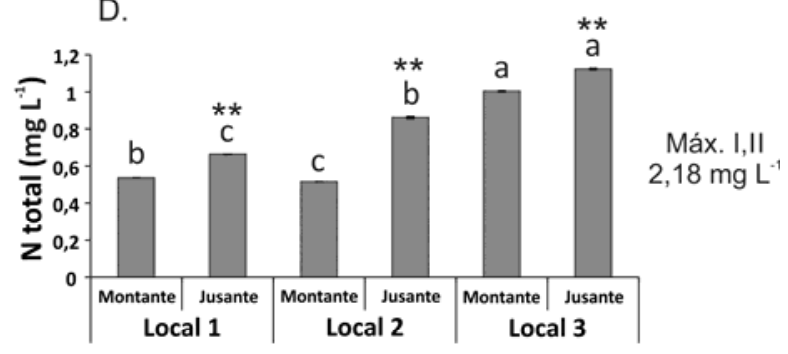

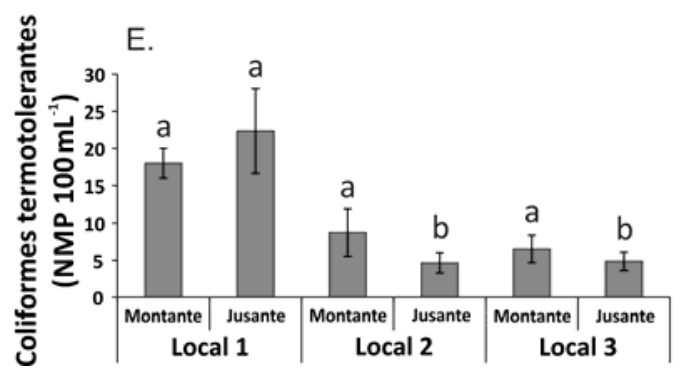

Figura 2. Análises físico-químicas e microbiológicas. (a) $\mathrm{pH}$; (b) Turbidez; (c) $\mathrm{O}_{2}$ dissolvido; (d) $\mathrm{N}$ total; (e) Coliformes Termotolerantes. Os locais 1, 2 e 3 correspondem, respectivamente, ao cemitério do município de Muçum, ao curtume do município de Muçum e às indústrias de frangos e de couros no município de Roca Sales. Os dados são mostrados como média \pm erro padrão. As análises estatísticas referem-se à comparação "à montante" X "à jusante" em cada um dos locais (teste $t$ de Student, $\mathrm{p} \leq 0,01$, mostrado por dois asteriscos), e à comparação, separadamente, dos três locais "à montante" e dos três locais "à jusante" (One-Way ANOVA, seguido de teste de Tukey, $p \leq 0,05$, mostrado por letras nas colunas). Máx. = Valor máximo permitido pela legislação vigente; Mín. = Valor mínimo permitido pela legislação vigente. UNT = Unidades Nefelométricas de Turbidez.

\section{CONCLUSÃO}

Com este estudo podemos concluir que, mesmo tendo uma intensa atividade antropogênica em suas margens, o Rio Taquari não apresenta índices de poluição alarmantes, podendo ser classificado como água de classe I de acordo com a maioria dos parâmetros analisados, exceto turbidez, cuja origem é provavelmente inorgânica. No momento não temos como afirmar o verdadeiro motivo da alta turbidez em alguns pontos, somente podemos especular que diferentes níveis de precipitação ou diferentes usos do solo nas margens poderiam afetar a turbidez desses pontos. Mesmo tendo um cemitério em atividade em sua margem e sendo o local de despejo dos efluentes de diversas indústrias do Vale do Taquari, os trechos analisados deste rio possuem águas com baixo nível de contaminação e, ao que tudo indica, o tratamento de efluentes utilizados pelas indústrias de couros e frangos entre os municípios de Muçum e Roca Sales é eficaz. 


\section{REFERÊNCIAS}

BECKER, F. G.; DE FRIES, L. C. C.; FERRER, J.; BERTACO, V. A.; LUZ-AGOSTINHO, K. D. G.; SILVA, J. F. P. et al. Fishes of the Taquari-Antas river basin (Patos Lagoon basin), southern Brazil. Brazilian Journal of Biology, v. 73, p. 79-90, 2013. http://dx.doi.org/10.1590/S1519-69842013000100010

BORGES, L. G. A.; DALLA VECHIA, V.; CORÇÃO, G. Characterization and genetic diversity via REP-PCR of Escherichia coli isolates from polluted waters in southern Brazil. FEMS Microbiology Ecology, v. 45, p. 173-180, 2003. http://dx.doi.org/10.1016/S0168-6496(03)00147-8

CASTRO, V. G. Utilização da água na indústria de alimentos. 2006. 45f. Monografia (Especialização em Higiene e Inspeção de Produtos de Origem Animal e Vigilância Sanitária) - Universidade Castelo Branco, São Paulo, 2006.

CONSELHO NACIONAL DO MEIO AMBIENTE - CONAMA. Resolução n 335 , de 3 de abril de 2003. Dispõe sobre o licenciamento ambiental de cemitérios. Diário Oficial [da] União, Brasília, n. 101, 28 maio 2003, p. 98-99.

CONSELHO NACIONAL DO MEIO AMBIENTE - CONAMA. Resolução nº 357, de 17 de março de 2005. Dispõe sobre a classificação dos corpos de água e diretrizes ambientais para o seu enquadramento, bem como estabelece as condições e padrões de lançamento de efluentes, e dá outras providências. Diário Oficial [da] União, Brasília, n. 53, 18 mar. 2005, p. 58-63.

CORREA, A. A.; FUENTEFRIA, D. B.; CORÇÃO, G. Antimicrobial resistance analysis among enterococci isolated from pig feces. Acta Scientiae Veterinarie, v. 33, p.155$159,2005$.

CUNHA, L. J.; SHIRAIWA, S. Aplicação do método eletromagnético indutivo na investigação da pluma de contaminação da água subterrânea por resíduos de cromo de curtume. Revista Brasileira de Geofísica, v. 29, p. 127-134, 2011. http://dx.doi.org/10.1590/S0102-261X2011000100009

DIAS, M. T.; SANTOS, P. C. R. F.; OLIVEIRA, L. A. T.; MARIN, V. A. Evaluation of antimicrobial sensitivity of Escherichia coli strains isolated from mussels (Perna perna L.). Ciência \& Tecnologia de Alimentos, v. 30, p. 319-324, 2010.

FERREIRA FILHO, E.; HANG CHUI, Q. S. Quality of measurements and neutralization of alkaline effluents with carbon dioxide. Engenharia Sanitária e Ambiental, v. 11, p. 169-174, 2006. http://dx.doi.org/10.1590/S1413-41522006000200010

GUEDES, H. A. S.; DEMETRIUS D. S.; ABRAHÃO A. A. E.; CELSO B. M. R.; ANTONIO T. M.; JOSÉ H. P. S. Application of multivariate statistical analysis in the study of water quality in the Pomba River (MG). Revista Brasileira de Engenharia Agrícola e Ambiental, v. 16, p. 558-563, 2012. http://dx.doi.org/10.1590/S141543662012000500012

IAFIGLIOLA, M. C.; MENTEN, J. F. M.; RACANICCI, A. M. C.; GAIOTTO, J. B. Copper and antibiotic as growth promoters in rations for broiler chickens. Revista Brasileira de Ciência Avícola, v. 2, p. 201-208, 2000. http://dx.doi.org/10.1590/S1516635X2000000300002 
JANZEN, J. G.; SCHULZ, H. E.; LAMON, A. W. Measurements of dissolved oxygen concentration at water surface. Engenharia Sanitária e Ambiental, v. 13, p. 278-283, 2008. http://dx.doi.org/10.1590/S1413-41522008000300006

KREWER, C. C.; GRESSLER, L. T.; COSTA, M. M.; KREWER, C. C.; VARGAS, A. C. Susceptibility to disinfectants and antimicrobial resistance profile in Escherichia coli isolates. Pesquisa Veterinária Brasileira, v. 32, p. 1116-1120, 2012. http://dx.doi.org/10.1590/S0100-736X2012001100007

MARQUES, R. F. P. V.; SILVA, A. M.; RODRIGUES, L. S.; COELHO, G. Impacts of urban solid waste disposal on the quality of surface water in three cities of Minas Gerais Brazil. Ciência e Agrotecnologia, v. 36, p. 684-692, 2012. http://dx.doi.org/10.1590/S1413-70542012000600010

NUNES, M. A. G.; KUNZ, A.; STEINMETZ, R. L. R.; PANIZ, J. N. G. Application of swine culture treated effluent for swine manure dilution and nitrogen removal by denitrification. Engenharia Agrícola, v. 31, p. 388-398, 2011. http://dx.doi.org/10.1590/S0100-69162011000200019

OLIVEIRA, D. Q. L.; CARVALHO, K. T. G.; BASTOS, A. R. R.; OLIVEIRA, L. C. A.; MARQUES, J. J. G. S. M.; NASCIMENTO, R. S. M. P. Use of leather industry residues as nitrogen sources for elephantgrass. Revista Brasileira de Ciências do Solo, v. 32, p. 417-424, 2008. http://dx.doi.org/10.1590/S0100-06832008000100039

PIMENTA, A. M; FURLANETTO, L. M.; ALBERTONI, E. F.; PALMA-SILVA, C. Water quality in the lotic area of the Antas river before and after the construction of the Monte Claro hydroelectric plant, south Brazil. Acta Limnologica Brasileira, v. 24, p. 314325, 2012. http://dx.doi.org/10.1590/S2179-975X2013005000001

SILVA, A. E. P.; ANGELIS, C. F.; MACHADO, L. A. T.; WAICHAMAN, A. V. Impacts of precipitation on the water quality of the Purus River. Acta Amazonica, v. 38, p. 733742, 2008. http://dx.doi.org/10.1590/S0044-59672008000400017

SILVA, R. W. C.; MALAGUTTI FILHO, W. Application of electrical imaging in the study of contamination from cemeteries. Geociências, v. 29, p. 343-354, 2010.

SILVA, R. W. C.; MALAGUTTI FILHO, W.; MOREIRA, C. A. Emprego do método da eletrorresistividade no estudo da contaminação subterrânea do cemitério municipal de Vila Rezende, Piracicaba - SP. Revista Brasileira de Geofísica, v. 27, p. 389-399, 2009. http://dx.doi.org/10.1590/S0102-261X2009000300007

SKORCZEWSKI, P. Abundance and distribution of fecal indicator bacteria in recreational beach sand in the southern Baltic Sea. Revista de Biología Marina y Oceanografia, v. 47, p. 503-512, 2012.

TIMOFIECSYK, A.; FAVARETTO, N.; PAULETTI, V.; DIECKOW, J. Carbon and nitrogen losses with liquid catlle manure from a clayey oxissol under no-till and natural rainfall. Revista Brasileira de Ciência do Solo, v. 36, p. 1924-1930, 2012. http://dx.doi.org/10.1590/S0100-06832012000600026

WILSONB, P. C. Water quality notes: water clarity (turbidity, suspended solids, and color). University of Florida, 2010. 


\begin{tabular}{|} 
Ambiente \& Água - An Interdisciplinary Journal of Applied Science \\
ISSN 1980-993X - doi:10.4136/1980-993X \\
www.ambi-agua.net \\
E-mail: ambi.agua@gmail.com
\end{tabular}

\title{
Aplicação e análise comparativa de três protocolos de avaliação rápida para caracterização da paisagem fluvial
}

\author{
doi:10.4136/ambi-agua.1732
}

Received: 19 Aug. 2015; Accepted: 03 Nov. 2015

\author{
Jucimara Andreza Rigotti ${ }^{1 *}$; Cesar Augusto Pompêo ${ }^{2}$; \\ Alessandra Larissa D'Oliveira Fonseca ${ }^{3}$ \\ ${ }^{1}$ Universidade Federal do Rio Grande do Sul (UFRGS), Porto Alegre, RS, Brasil \\ Programa de Pós-Graduação em Recursos Hídricos e Saneamento Ambiental \\ ${ }^{2}$ Universidade Federal de Santa Catarina (UFSC), Florianópolis, SC, Brasil \\ Departamento de Engenharia Sanitária e Ambiental \\ ${ }^{3}$ Universidade Federal de Santa Catarina (UFSC), Florianópolis, SC, Brasil \\ Departamento de Geociências \\ *Autor correspondente: e-mail: anrigotti@gmail.com, \\ cesar.pompeo@ufsc.br, alarissa.fonseca@gmail.com
}

\section{RESUMO}

Os sistemas fluviais são dotados de características, como a heterogeneidade e a variabilidade, que permitem a qualificação de uma paisagem fluvial. Os protocolos de avaliação rápida vêm se mostrando ferramentas úteis para a caracterização da integridade, principalmente na escala de trecho de rio. O objetivo deste trabalho foi aplicar três protocolos e avaliar o desempenho deles entre si e em comparação com os resultados obtidos por métodos analíticos de avaliação da qualidade da água e da comunidade de macroinvertebrados aquáticos. O escalonamento multidimensional não-métrico (nMDS) foi aplicado com os parâmetros de qualidade da água e o Índice do Estado Trófico (IET), e com os dados de abundância de macroinvertebrados bentônicos. Também foram calculadas correlações entre índices e os resultados finais dos protocolos. A viabilidade de utilização dos protocolos para caracterização do habitat se confirmou, pois houve compatibilidade entre os resultados nos três procedimentos. Apesar das particularidades, todos abordam de alguma forma os aspectos gerais que caracterizam a integridade dos rios, indicando a condição do habitat que melhor suporta a biodiversidade aquática em rios urbanos.

Palavras-chave: integridade do habitat, qualidade da água, rios urbanos.

\section{Application and comparative analysis of three rapid assessment protocols to characterize fluvial landscape}

\section{ABSTRACT}

Fluvial systems are endowed with features such as heterogeneity and variability which allow the classification of a fluvial landscape. Rapid assessment protocols have been useful tools for the characterization of integrity, especially in river segment scale. The objective of this paper was to apply three protocols in order to evaluate their performance in comparison with each other and with the results obtained by analytical methods of water quality 
assessment and an evaluation of the aquatic macro-invertebrate community. Non-metric multidimensional scaling (nMDS) was applied using water quality parameters, trophic state index (IET), and abundance data of benthic macro-invertebrates. In addition, the study determined correlations between the index and the protocols' final scores. The protocols' feasibility for habitat characterization was confirmed, since the results were compatible for all procedures applied. Despite some particularities, each protocol offered a particular description of general aspects that affect river integrity, indicating which habitat conditions best support aquatic biodiversity in urban rivers.

Keywords: habitat integrity, water quality, urban streams.

\section{INTRODUÇÃO}

$\mathrm{Na}$ ecologia da paisagem o conceito de corredor, que pode ser aplicado aos cursos d'água, é considerado um dos padrões essenciais da organização da paisagem, através do qual se processam fluxos importantes de energia, de materiais e de espécies (Saraiva, 1999). O termo paisagem ribeirinha ou fluvial foi usado por Ward (1998) para indicar uma perspectiva global e de processos associados ao sistema fluvial. Os padrões de biodiversidade são direta e indiretamente influenciados pela geomorfologia das paisagens fluviais, as quais podem ser compreendidas como uma organização hierárquica aninhada (Ward, 1998). Tal hierarquia se inicia com a bacia hidrográfica e se particulariza pelas divisões em segmentos, trechos de rio e micro-habitat.

Sob a denominação de protocolos de bioavaliação rápida - Rapid Bioassessment Protocols - Barbour et al. (1999) agruparam uma série de métodos empregados pelas agências de recursos hídricos dos Estados Unidos. Segundo estes autores, os protocolos foram delineados inicialmente para ser ferramentas de baixo custo capazes de determinar a capacidade do rio para dar suporte à vida aquática. Essas ferramentas podem incluir a avaliação de aspectos físicos do habitat, regime de fluxo, qualidade da água e bioindicadores. Nos últimos anos um número bastante elevado de protocolos foi desenvolvido, em vários locais e com diferentes objetivos de avaliação. Para caracterizar o habitat, por exemplo, o trabalho de Fernández et al. (2011) analisaram cinquenta deles.

Em um sentido geral, os protocolos de avaliação rápida são formados por conjuntos de procedimentos, baseados em critérios estabelecidos previamente conforme a finalidade do estudo. Tal abordagem é constituída pela observação em campo, registrada na forma de descrição, sistema de pontuação ou de classificação. Os protocolos têm a possibilidade de ser rápidos em comparação com outros métodos de avaliação, como da fauna bentônica; contudo, não necessariamente utilizam apenas a avaliação visual. O procedimento em campo pode ser acompanhado de medições diretas, por exemplo, a estimativa de velocidade e profundidade presente no Índice do Habitat Fluvial - IHF (Pardo et al., 2002) ou incluir de antemão o uso de outras ferramentas, como mapas e imagens (Ollero et al., 2011). Além do mais, mesmo os protocolos idealizados para que sejam preenchidos completamente com observações em campo exigem um estudo detalhado, com desenvolvimento e adequação ao local de aplicação, como demonstraram Guimarães et al. (2012).

O resultado do uso dos protocolos pode fazer parte dos estudos ecológicos dos cursos d'água (Barbour et al., 1999) e de estratégias de monitoramento (Rodrigues e Castro, 2008), bem como estar presente no contexto de recuperação de rios (Habberfield et al., 2014), pois a aplicação dos mesmos é bastante efetiva no conhecimento do sistema fluvial de modo amplo. Nesse sentido, os protocolos se tornam instrumentos úteis, de baixo custo e relativa facilidade de aplicação, sendo tais aspectos também amplamente reconhecidos (Barbour et al., 1999; Callisto et al., 2001; Rodrigues e Castro, 2008). 
Este artigo trata da aplicação de três protocolos de avaliação rápida na escala de trecho de rio, com objetivo de avaliá-los quanto à caracterização da integridade da paisagem fluvial, sendo a paisagem fluvial compreendida como o habitat formado pelo curso d'água e os processos a ele associados. Considerou-se que esta integridade abrange aspectos hidrológicos, geomorfológicos e ecológicos representados em diferentes níveis de detalhamento com emprego de indicadores apropriados. Alterações da estrutura física, conservação da vegetação ripária, regime de escoamento e qualidade da água são exemplos de parâmetros encontrados nestes protocolos. O desempenho de cada procedimento foi analisado por intermédio da comparação dos resultados dos protocolos entre si e com os resultados de qualidade da água e da diversidade da fauna bentônica.

Essa abordagem preenche uma lacuna na caracterização da geomorfologia das paisagens, que se refere à escala de trecho de rio, porém a avaliação e monitoramento das características físicas do habitat fluvial nessa escala carecem de padronização (Fernández et al., 2011). Assim, apesar da adaptação ser necessária em muitas circunstâncias, também há a necessidade de adotar certo grau de uniformidade nas estratégias de avaliação, para facilitar posteriormente o monitoramento. Destaca-se que o trabalho não visa a estabelecer qual é o melhor protocolo, mas mostrar e discutir a aplicação, os aspectos mais bem abordados e os pontos que podem ser melhorados. O trabalho pode contribuir na escolha de técnicas para novos estudos, além de colaborar com a discussão e aprimoramento deste tipo de abordagem.

\section{MATERIAL E MÉTODOS}

\section{1. Área de estudo e desenho amostral}

A bacia hidrográfica do Itacorubi se localiza em Florianópolis na porção central da ilha, entre as coordenadas $\mathrm{x}=743.137 \mathrm{~m} ; \mathrm{y}=6.941 .075 \mathrm{~m} \mathrm{e} \mathrm{x}=749.568 \mathrm{~m} ; \mathrm{y}=6.948 .682 \mathrm{~m}$ (Fuso 22S, Datum SAD-69). Ocupa a área de $27,7 \mathrm{~km}^{2}$, considerando como parte da bacia toda a drenagem que desemboca no manguezal do Itacorubi (Figura 1).

Os cursos d'água Córrego Grande, rio do Meio, Ana d'Ávila e Itacorubi são os principais formadores da drenagem natural desta bacia. Nestes rios foram escolhidos os trechos de amostragem, utilizando-se os critérios de semelhança na ordem de rio $\left(3^{\mathrm{a}}\right.$ e $4^{\mathrm{a}}$ ordem) e na área da bacia $(1,0$ a 3,5 km²). Estes critérios são relevantes para possibilitar a comparação dos dados e a aplicabilidade dos métodos. Além disso, considerou-se a localização dos trechos relativamente à ocupação urbana em áreas próximas, o que permitiu variabilidade de condições ambientais a serem avaliadas. O estudo procurou representar dentro do possível as condições da bacia hidrográfica em questão, considerando as limitações financeiras, de esforço amostral e de processamento dos dados, principalmente de fauna bentônica.

Na Figura 1 são identificados os seis trechos de amostragem: um no rio Itacorubi (IT); um no rio Ana d'Ávila (AD); dois no rio do Meio (M1) e (M2); e dois no rio Córrego Grande (C1) e (C2), todos com comprimento de $100 \mathrm{~m}$. Os trechos se localizam no que se pode denominar de curso superior e médio dos rios, caracterizados por altas declividades e velocidades. Não foi realizada aplicação no ambiente estuarino, para isso seria necessária adaptação ou escolha de procedimentos específicos para essas áreas. No início dos trechos foram medidos parâmetros de qualidade da água e coletadas amostras da fauna bentônica, para efetuar a comparação com os resultados finais dos protocolos.

\subsection{Protocolos de Avaliação Rápida}

Os procedimentos escolhidos para caracterizar a integridade da paisagem fluvial foram: o IHF - índice do Habitat Fluvial; o PAH - Protocolo Simplificado de Avaliação de Habitats; e o procedimento de avaliação da condição dos cursos d'água urbanos, que ao longo do seu 
processo de aperfeiçoamento foi denominado Índice de Integridade (InI). Os parâmetros avaliados por cada protocolo são apresentados na Tabela 1, informações específicas sobre a forma de aplicação podem ser obtidas diretamente nas referências.
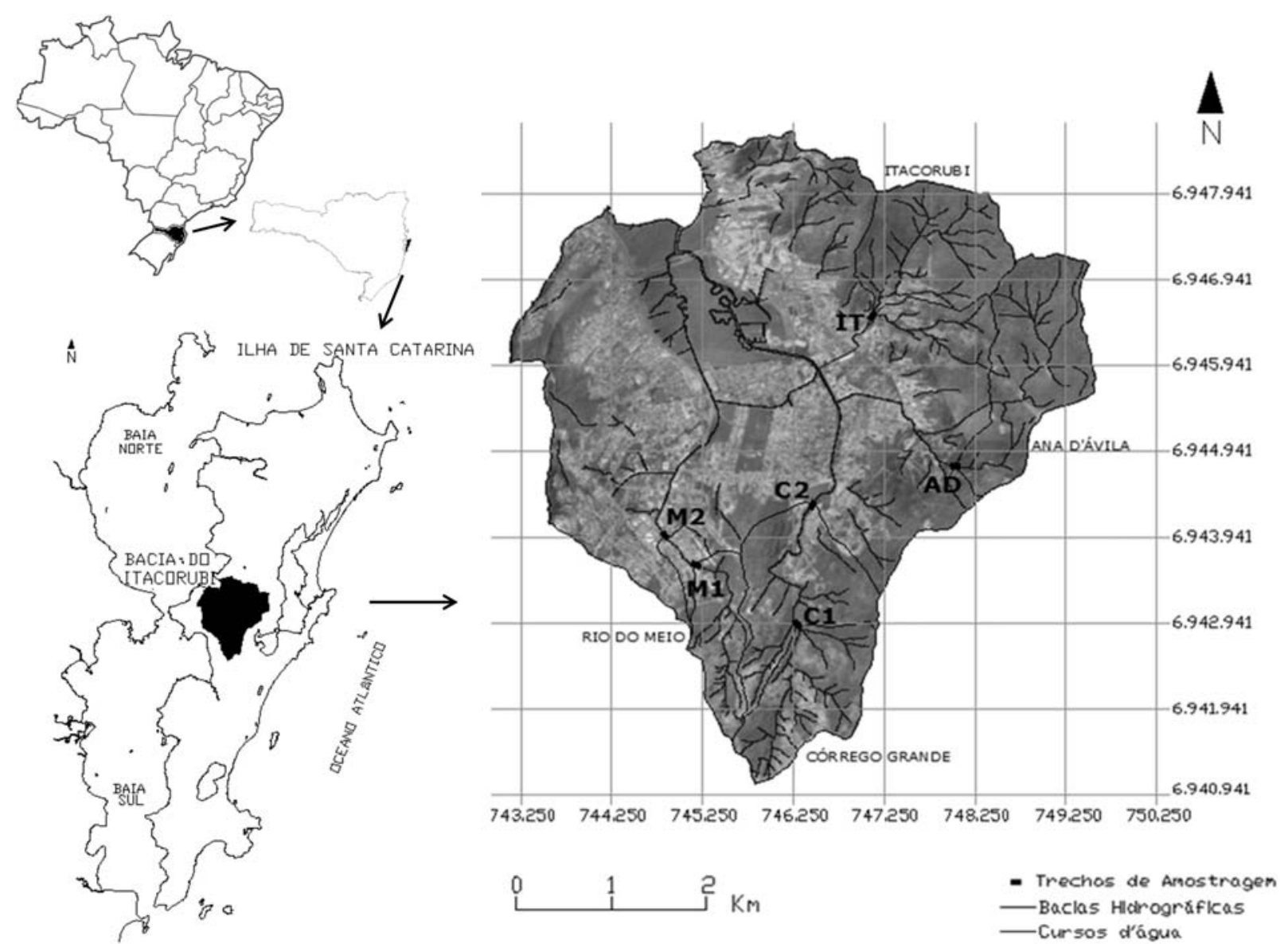

Figura 1. Localização da área de estudo - bacia hidrográfica do Itacorubi em Florianópolis-SC-Brasil - e dos trechos de amostragem em quatro cursos d'água pertencentes à bacia: nos rios Córrego Grande (C1 e C2), do Meio (M1 e M2), Ana d'Ávila (AD) e Itacorubi (IT).

O IHF caracteriza e pontua aspectos físicos do leito relacionados com a heterogeneidade e complexidade de habitats e que dependem em grande medida da hidrologia e do substrato existente (Pardo et al., 2002). Este procedimento tem sido frequentemente utilizado na Espanha e, para sua aplicação neste trabalho, realizou-se a tradução e adaptação dos termos utilizados, principalmente os relativos à vegetação aquática, tarefa que contou com o apoio de especialistas na área.

O PAH é uma modificação do protocolo elaborado por Hannaford et al. (1997), realizada por Callisto et al. (2001), para que ele seja utilizado em ecossistemas lóticos com diferentes níveis de preservação das características naturais, ou sob a influência antropogênica. Seu uso fornece uma avaliação geral e qualitativa de vários atributos dos habitats (Callisto et al., 2001).

O InI é resultado de pesquisas sobre a revitalização de cursos d'água em área urbana (Dalla Costa e Pompêo, 2008; Pompêo et al., 2011) com objetivo de quantificar e qualificar a integridade dos rios. O procedimento completo envolve a descrição qualitativa de cada trecho e a aplicação em todo o percurso do rio, visando obter comparações para indicação de ações prioritárias de revitalização, porém no escopo deste trabalho o protocolo foi aplicado apenas ao trecho de interesse. 
A fim de tornar possível a análise comparativa entre os protocolos, realizou-se uma parametrização da escala de seus resultados finais de forma que fossem representadas com valores entre 0 e 100 . Portanto, não se efetuou a comparação específica entre cada parâmetro que compõe os procedimentos e, sim, o resultado final de cada um dos protocolos.

Todos os protocolos foram aplicados nos dias 10 e 11 de novembro de 2014, uma vez que a avaliação das modificações sazonais não fez parte do estudo. A análise da variabilidade entre os resultados, caso os procedimentos fossem utilizados por diferentes pessoas com ou sem treinamento prévio não foram abordadas neste trabalho. Essa análise já foi discutida, entre outros, por Callisto et al. (2002) e Guimarães et al. (2012), os quais mostraram que não houve diferença significativa entre as aplicações.

\subsection{Qualidade da água}

Em cada local de amostragem foram medidos parâmetros in situ: temperatura e condutividade (Condutivímetro YSI-EC 300); oxigênio dissolvido (Oxímetro YSI - 55); pH (pHmetro YSI- 100 AC). Em seguida, coletaram-se amostras de água que foram acondicionadas, mantidas no escuro e sob refrigeração até os procedimentos em laboratório. As amostras de água foram homogeneizadas e, com uma alíquota, medida a turbidez (Turbidímetro Alfa-Kit ${ }^{\circledR}$ ). O restante de cada amostra foi filtrado e congelado para a análise de fósforo inorgânico dissolvido e pigmentos fitoplanctônicos, seguindo a metodologia de Grasshoff et al. (1999). Os pigmentos (clorofila-a e feoftina-a) foram extraídos com etanol $80 \%$. Os parâmetros foram escolhidos por serem efetivos na identificação de alterações da qualidade da água geradas pela urbanização, principalmente a eutrofização, e por permitirem o cálculo do Índice de Estado Trófico (IET).

\subsection{Macroinvertebrados bentônicos}

Os macroinvertebrados foram coletados com rede do tipo Surber, malha de $500 \mathrm{~m}$ de abertura. As amostras, fixadas em campo e conservadas com álcool $70 \%$, foram triadas no laboratório em bandeja e sob microscópio estereoscópico (total de 6 amostras por local). Os organismos foram contados e identificados até o menor nível taxonômico possível. Os principais guias de identificação seguidos foram: Mugnai et al. (2010); Merritt e Cummins (1984). Adicionalmente, após o processo de triagem da fauna, efetuou-se a análise de biomassa e teor de matéria orgânica com o substrato que ficou retido na rede de coleta.

\subsection{Análise dos dados}

A comparação dos resultados foi efetuada através da relação entre os protocolos e a avaliação das condições básicas da cadeia alimentar aquática (estado trófico) e da qualidade do habitat para os demais níveis, com ênfase na macrofauna bentônica. Para o tratamento dos dados foram utilizadas ferramentas da estatística multivariada, que permitem a visualização integrada dos resultados tanto dos índices como dos parâmetros de qualidade da água.

Para avaliar a condição de eutrofização dos cursos d'água, estimou-se o Índice de Estado Trófico (IET), adaptado por Lamparelli (2004). Os valores do IET são distribuídos em seis classes: ultraoligotrófico (IET $\leq 47)$; oligotrófico $(47<$ IET $\leq 52)$; mesotrófico $(52<$ IET $\leq$ 59); eutrófico (59 < IET $\leq 63)$; supereutrófico $(63<$ IET $\leq 67)$; hipereutrófico (IET > 67). Este índice fornece informações sobre a comunidade fitoplanctônica associada à concentração de nutrientes (Lamparelli, 2004). Assim, em conjunto com os outros parâmetros medidos, o IET indica as alterações na cadeia trófica, resultantes da urbanização da bacia hidrográfica a montante dos trechos de rio estudados. Além disso, constitui-se de uma alternativa proposta por Fasola et al. (2011) para quantificar o parâmetro que avalia a poluição no curso d'água, no protocolo de avaliação da integridade (InI). 
Tabela 1. Parâmetros que compõe cada protocolo: Índice do Habitat Fluvial (IHF), de acordo com Pardo et al. (2002); Protocolo Simplificado de Avaliação do Habitat (PAH), conforme Callisto et al. (2001); Índice de Integridade (InI), segundo Pompêo et al. (2011).

Índice do Habitat Fluvial (IHF)

Inclusão de corredeiras - sedimentação em poços

Frequência de corredeiras

Composição do substrato

Regimes de velocidade/profundidade

Porcentagem de sombra no leito

Elementos de heterogeneidade

Cobertura de vegetação aquática

Protocolo Simplificado de Avaliação do Habitat (PAH)
Tipos de fundos (peixes)
Frequência de remansos (ou curvas)
Tipos de substrato
Deposição de lama
Depósitos sedimentares
Alteração no canal do rio
Características do fluxo das águas
Presença de vegetação ripária
Estabilidade das margens
Extensão da vegetação ripária

Índice de Integridade (InI)

Corte ou remoção da vegetação

Substituição parcial ou total da vegetação nativa por espécies exóticas

Processos erosivos visíveis nas margens

Presença de sedimentos nas margens

Obstruções do leito por acúmulo de sedimentos

Retificação da seção transversal

Mudança de revestimento da seção transversal

Canalização ou tamponamento do trecho

Corte de meandros

Retirada da água sem modificação estrutural do curso d'água

Retirada da água com modificação estrutural do curso d'água

Obstruções por pontes e travessias inadequadas

Presença de resíduos sólidos depositados nas margens ou no leito menor do curso d'água

Lançamento de efluentes, conexões de esgoto

Lançamento de águas pluviais urbanas

Presença de edificações residenciais, comerciais, industriais ou da administração pública

Presença de áreas de esporte, lazer ou infraestrutura pública

A expressão da diversidade de organismos que indica a qualidade do habitat é complexa, portanto para a avaliação da comunidade de macroinvertebrados foram calculados vários índices: número de espécies $(\mathrm{S})$; número de indivíduos $(\mathrm{N})$; índice de riqueza de espécies de Margalef (d); índice de Pielou (J'); índice de Shannon-Wiener (H'); e índice de Simpson

Rev. Ambient. Água vol. 11 n. 1 Taubaté - Jan. / Mar. 2016 
(1- $\lambda$ ), no programa PRIMER 6. Adicionalmente, calculou-se o índice EPT composto da porcentagem dos taxa Ephemeroptera, Plecoptera e Trichoptera, considerados grupos sensíveis à poluição; e, em contraposição, a porcentagem do grupo oligochaeta, que é considerado um grupo resistente de organismos (Ferreira et al., 2012).

A relação entre todos os resultados foi avaliada pelo escalonamento multidimensional não-métrico (n-MDS), um método de ordenação que permite a visualização das relações de similaridade entre os locais. Desta forma, os resultados do estudo (parâmetros de qualidade da água, IET e abundância da fauna) são utilizados como dados de entrada para gerar a análise. Os resultados dos protocolos não foram utilizados para gerar os diagramas por uma questão de balanceamento de dados, pois para os parâmetros e dados de abundância há um número maior de amostras do que o número de aplicação dos protocolos.

Dois diagramas foram gerados, nos quais se sobrepôs o coeficiente de correlação de Spearman $(\rho)$. O tamanho dos vetores representa o valor do coeficiente, e o raio do círculo é o valor máximo $(\rho=1)$, foram representados os dados com $\rho>0,70$. O primeiro nMDS foi efetuado com os resultados dos parâmetros qualidade da água e do IET. Esses dados foram submetidos à normalização e com eles foi gerada a matriz de dissimilaridade (a partir da distância Euclidiana). Neste primeiro diagrama, o coeficiente de Spearman relaciona os resultados dos protocolos aos dados que foram utilizados para gerar o nMDS, ou seja, ele mostra a correlação entre os resultados dos protocolos com os parâmetros qualidade da água e o IET. O segundo nMDS foi realizado utilizando-se os dados de abundância da fauna, para os quais se aplicou a transformação logarítmica, a matriz gerada foi baseada na distância BrayCurtis. Neste diagrama o coeficiente de Spearman correlaciona os resultados dos protocolos e dos índices de diversidade aos dados de abundância da fauna. Deste modo, aqui a correlação foi calculada entre uma matriz de resultados (índices bióticos e protocolos), que é diferente dos dados utilizados para gerar o nMDS (dados de abundância).

Por fim, os resultados finais padronizados para todos os protocolos foram representados graficamente. Também foram apresentados os resultados numéricos dos coeficientes de correlação entre cada protocolo e entre os protocolos e os índices, para os índices com valores mais representativos. Os gráficos e as análises de correlação foram feitas no programa $\mathrm{R}$ (R Core Team, 2015).

\section{RESULTADOS E DISCUSSÃO}

Os protocolos se mostraram representativos na caracterização da integridade da paisagem fluvial nos trechos de amostragem, identificando diferenças entre esses locais conforme sua integridade. De acordo com os parâmetros de qualidade da água e o IET, o primeiro nMDS apresentou uma distribuição indicando um gradiente dos trechos menos poluídos para os mais poluídos, (Figura 2-a). Pela sobreposição do coeficiente, percebe-se que houve correlação positiva entre os resultados dos protocolos e dos parâmetros: oxigênio dissolvido e matéria orgânica particulada; e correlação inversa com o Índice de Estado Trófico - IET. Já no segundo nMDS, para os dados de fauna, houve uma diferenciação maior em dois grupos (Figura 2-b), um formado pelos trechos de rio com maior influência da área urbana (IT, M2 e C2) e outro dos trechos sob menor influência (AD, M1, C1). Neste nMDS o índice que melhor se correlacionou diretamente foi o índice Ephemeroptera, Plecoptera e Trichoptera EPT e inversamente o número de indivíduos $(\mathrm{N})$.

Apesar do segundo nMDS, relativo aos dados de abundância, ter mostrado o agrupamento entre os trechos mais urbanizados, mesmo entre eles observou-se diferenças nos valores dos índices de diversidade da fauna (Tabela 2). Isso mostra a importância de se utilizar várias métricas na estimativa desta diversidade. $\mathrm{O}$ trecho $\mathrm{C} 1$ no rio Córrego Grande se 
destaca nos valores dos índices EPT e riqueza de espécies (d). Já para a porcentagem do grupo oligochaeta, o rio Itacorubi (IT) se sobressai, uma vez que este valor represente, na média das amostras, $46 \%$ dos organismos encontrados.
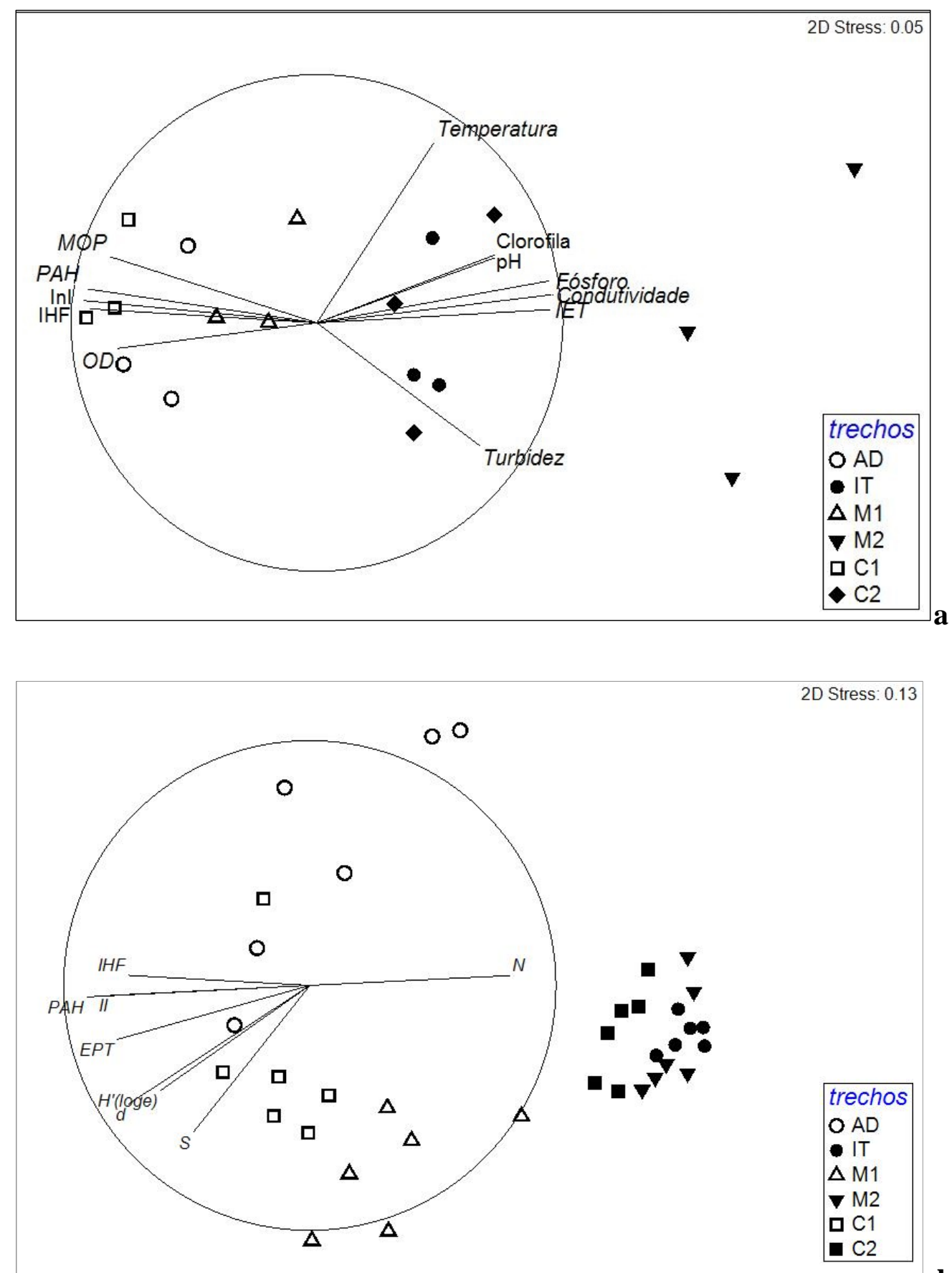

Figura 2. a) nMDS para os resultados de qualidade da água $(\mathrm{n}=3)$, sendo IHF (índice do Habitat Fluvial), PAH (Protocolo Simplificado de Avaliação de Habitats) e InI (Índice de Integridade) os protocolos e os parâmetros abreviados são: OD: oxigênio dissolvido; MOP: matéria orgânica particulada. Os parâmetros $\mathrm{pH}$ e clorofila ficaram sobrepostos na análise. b) nMDS para os dados de abundância $(n=3)$, sendo IHF, PAH e InI os protocolos e as métricas são: S: número total de espécies; N: número total de indivíduos; d: índice de riqueza de espécies (Margalef); J': índice de homogeneidade de Pielou; H': índice de diversidade de Shannon-Wiener; 1- $\lambda$ ': índice de Simpson. 
Tabela 2. Resultados gerais dos índices calculados para os trechos de amostragem, sendo os trechos: AD- no rio Ana d'Ávila; IT- no rio Itacorubi; C1 e C2- no rio Córrego Grande; M1 e M2- no rio do Meio. Índice de Estado Trófico (IET) para a qualidade da água e para a fauna: número total de espécies $(\mathrm{S})$; número total de indivíduos $(\mathrm{N})$; índice de riqueza de espécies (Margalef) (d); índice de homogeneidade de Pielou (J'); índice de diversidade de Shannon-Wiener (H'); índice de Simpson (1- $\lambda$ ); Porcentagem de Ephemeroptera, Plecoptera e Trichoptera (EPT); Porcentagem de Oligochaeta (oligo).

\begin{tabular}{cccccccccc}
\hline Trechos & IET & $\mathrm{S}$ & $\mathrm{N}$ & $\mathrm{d}$ & $\mathrm{J}$ & $\mathrm{H}^{\prime}$ & $1-\lambda$ & $\mathrm{EPT}$ & oligo. \\
\hline $\mathrm{C} 1$ & 46 & 14 & 102 & 2,87 & 0,74 & 1,95 & 0,78 & 27,8 & 1 \\
$\mathrm{AD}$ & 47 & 7 & 247 & 1,36 & 0,53 & 1,03 & 0,52 & 4,5 & 0 \\
$\mathrm{M} 1$ & 50 & 13 & 200 & 2,42 & 0,64 & 1,62 & 0,69 & 9,1 & 4 \\
$\mathrm{IT}$ & 56 & 9 & 2148 & 1,00 & 0,48 & 1,01 & 0,55 & 0,5 & 46 \\
$\mathrm{C} 2$ & 59 & 10 & 1360 & 1,30 & 0,37 & 0,83 & 0,39 & 0,2 & 11 \\
M2 & 67 & 6 & 1322 & 0,66 & 0,49 & 0,82 & 0,45 & 0,0 & 14 \\
\hline
\end{tabular}

A correlação mostrou valores acima de 0,80 para os três protocolos em relação ao Índice de Estado Trófico (IHF x IET $-\rho=0,92$; PAH x IET $-\rho=0,90$; InI x IET $-\rho=0,95$ ) e ao índice Ephemeroptera, Plecoptera e Trichoptera (IHF x EPT $-\rho=0,82$; PAH x EPT $-\rho=0,81$; InI x EPT $-\rho=0,86$ ). Esses resultados apontam para a efetividade dos protocolos na avaliação de trechos de rios distintos em termos de grau trófico e composição da fauna.

Os valores padronizados de cada protocolo, para os locais correspondentes, mostraram que houve uma mesma tendência de resultados (Figura 3). Isso se comprova pelo valor da correlação dos protocolos entre si: IHF x PAH: $\rho=0,899$; IHF x InI: $\rho=0,986$; PAH x InI: $\rho=0,943$. Contudo, percebe-se pelo gráfico que houve variação entre os resultados dos protocolos para um mesmo trecho, principalmente no trecho M2 do rio do Meio.

Colocando-se os locais com os valores dos índices em ordem decrescente, da maior para a menor integridade, verificou-se que todos os protocolos reconheceram os mesmos trechos de maior integridade: C1 (no rio Córrego Grande) e AD (no rio Ana d'Ávila), localizados a montante das áreas mais urbanizadas. Também o trecho de menor integridade foi o M2 no rio do Meio, o qual se encontra canalizado com revestimento em concreto no fundo e pedra argamassada nas margens. Já os trechos C2 e IT, no rio Córrego Grande e no rio Itacorubi, respectivamente, apresentaram muita similaridade entre si. Apesar de não ter sido feito teste estatístico, a similaridade foi observada pelo nMDS. Nesta análise não houve diferenciação entre as amostras desses trechos e, do mesmo modo, no IET os trechos C2 e IT apresentaram grau mesotrófico. Quanto à posição dos trechos na ordenação, é compreensível que não houvesse unanimidade entre os protocolos, pois eles mostraram condições de integridade muito semelhantes.

Embora se tenha percebido a consistência entre os resultados das análises e coeficientes de correlação quanto aos resultados dos protocolos, é relevante discutir algumas particularidades que refletem a aplicabilidade desses protocolos na avaliação da qualidade do habitat em trechos de rios urbanos. No IHF, que prioriza as características físicas do habitat, os trechos: M1 no rio do Meio e IT no rio Itacorubi obtiveram a mesma pontuação. Esse resultado pode ser atribuído ao fato de que a diferença entre esses locais está na qualidade da água e não propriamente na estrutura física. Esta diferença foi indicada no IET, em que o trecho M1 se caracterizou como oligotrófico e o trecho IT como mesotrófico. 


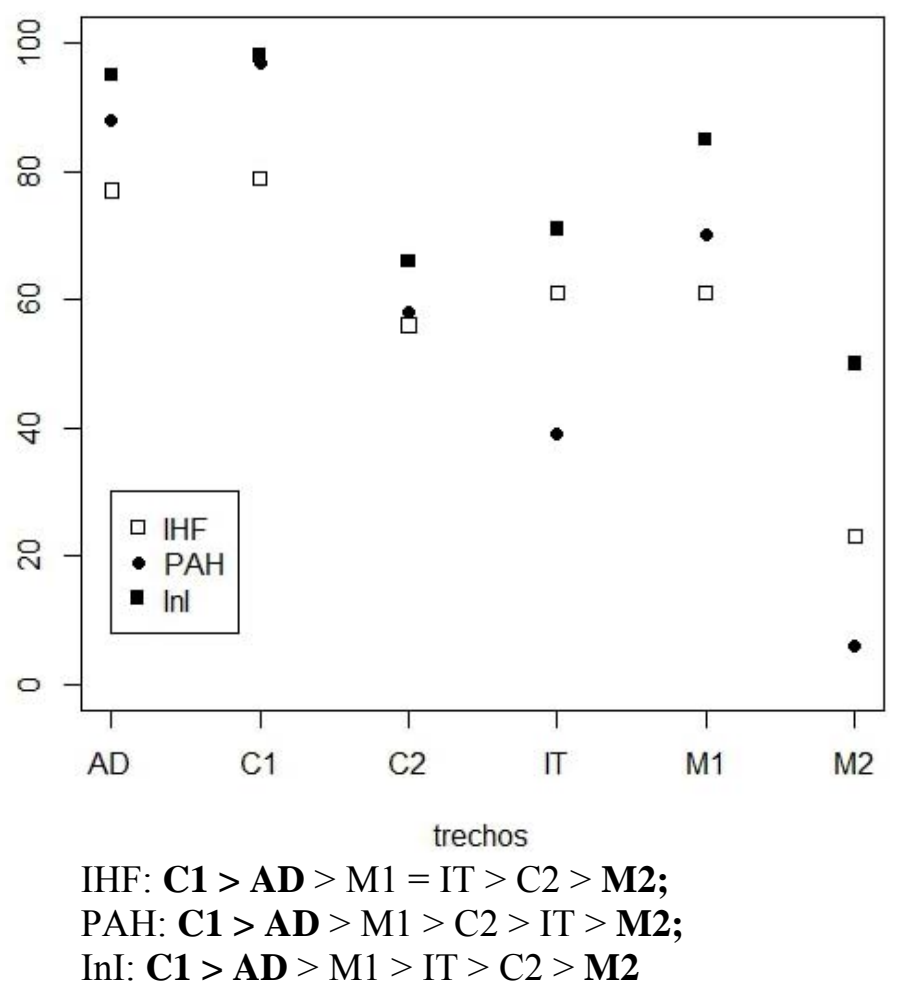

Figura 3. Gráfico dos valores finais dos protocolos, sendo os trechos: AD- no rio Ana d'Ávila; IT- no rio Itacorubi; $\mathrm{C} 1$ e $\mathrm{C} 2-$ no rio Córrego Grande; $\mathrm{M} 1$ e M2- no rio do Meio. Valores dos índices em ordem decrescente, da maior para a menor integridade.

Nota-se que o InI possui valores mais otimistas em relação aos outros dois protocolos. Isso se deve, em parte, pela sua formulação que foi baseada nos impactos sofridos nos cursos d'água, no contexto de degradação gerada pela urbanização. No meio urbano, um curso d'água considerado íntegro não necessariamente é isento de toda e qualquer alteração, como seria um ambiente de referência em conservação. Para o IHF e o PAH, por seu turno, a referência é um local sem perturbações; sua aplicação em área urbana tende então a gerar valores menores, pois a urbanização geralmente é acompanhada de fortes modificações na estrutura dos rios.

Em relação à fauna, os valores das correlações com os protocolos são menores, apontando para o fato de que os organismos não possuem respostas diretas como os parâmetros de qualidade da água, pois há inúmeros fatores interferentes e grande complexidade em quantificá-los. Por tal motivo, a avaliação da variedade de atributos do habitat, juntamente com outros índices, é crítica para a interpretação dos dados bióticos (Barbour et al., 1999). Além disso, é de fundamental importância a análise conjunta com o uso de várias métricas, bem como a relevância da qualificação dos grupos de organismos. Apenas a riqueza de taxa presentes e as características puramente físicas do habitat não fornecem tudo sobre a diversidade do local, pois há fatores relacionados ao ciclo de vida, às preferências e às tolerâncias biológicas dos organismos (Diaz et al., 2004).

A perspectiva de habitat proposta por Diaz et al. (2004), na qual o habitat é mais do que o substrato ou estrutura, é fundamental para a compreensão dos resultados. Embora a estrutura física seja elemento-chave, há as outras qualidades de apoio, como o suporte trófico e a biologia dos organismos em si. Desta forma, os protocolos que contemplam aspectos da 
qualidade da água são mais completos. No caso, o protocolo InI contempla esse aspecto no parâmetro lançamento de efluentes, inclusive com possibilidade de quantificação (Fasola et al., 2011). Contudo, este protocolo não aborda algumas características físicas essenciais para o habitat da fauna, como o regime de velocidade, a composição do substrato e a heterogeneidade, quesitos estes que são melhor abordados no IHF e PAH.

A maneira que os autores Callisto et al. (2002) encontraram de suprir a necessidade da parte de qualidade da água foi a aplicação conjunta do $\mathrm{PAH}$ com outro protocolo rápido, desenvolvido pela EPA (US Environmental Protection Agency). A adaptação e a aplicação de protocolos associados para complementar a avaliação, nesse sentido, revelam-se bastante viáveis. Pode-se dizer o mesmo da melhoria de protocolos existentes. Eles têm potencial para aumentar o nível de detalhamento, com inclusão de outros aspectos, sem que necessariamente passem a envolver os mesmos custos dos procedimentos analíticos convencionais. Afinal, os protocolos possuem também o objetivo de aproximar a linguagem científica, muitas vezes complicada, das necessidades enfrentadas pelos gestores e das expectativas das pessoas (Gregory et al., 2008; Guimarães et al., 2012), tornando mais fácil a apropriação social do saber científico.

O uso dos protocolos oferece uma visão geral da qualidade ambiental, que pode ser utilizada pelos gestores de recursos hídricos como instrumentos complementares (Rodrigues e Castro, 2008). Além disso, também são empregados: como instrumentos de educação ambiental (Guimarães et al., 2012); na formação profissional (Callisto et al., 2002); na caracterização hidromorfológica na escala de trecho de rio (Fernández et al., 2011); no monitoramento ambiental (Pardo et al., 2002) e na perspectiva de recuperação de rios (Habberfield et al., 2014). Isso ilustra a gama de aplicação e a utilidade da ferramenta. Os protocolos estão em constante aperfeiçoamento; testar sua aplicabilidade faz parte desse processo de melhoria.

\section{CONCLUSÃO}

Os três protocolos aplicados - Índice do Habitat Fluvial (IHF), Protocolo Simplificado de Avaliação de Habitat (PAH) e Índice de Integridade (InI) - mostraram bom desempenho para o reconhecimento da integridade do habitat fluvial. A escolha do método mais adequado depende dos objetivos do estudo, pois cada um possui suas particularidades e limitações. $\mathrm{O}$ IHF caracteriza melhor a estrutura física do rio, porém a integridade do habitat também depende da qualidade da água, item que é melhor avaliado pelos outros protocolos (PAH e InI). O emprego de técnicas associadas ou em conjunto com procedimentos analíticos torna o estudo mais completo. No entanto, a similaridade dos resultados, dos protocolos entre si e com as demais análises, mostrou a viabilidade de sua utilização na área de estudo. Para dar continuidade a essa perspectiva de trabalho poderão ser realizadas outras aplicações, que permitam a validação por teste estatístico, incluindo um maior número de trechos. Utilizar estes e outros procedimentos se mostra necessário para testar e avaliar a validade dos métodos e, desta forma, contribuir com a melhoria e padronização das técnicas de avaliação na escala de trecho de rio.

\section{AGRADECIMENTOS}

Agradecemos ao INCT-TMCOcean (projeto de pesquisa CNPq n ${ }^{\circ} 573.601 / 2008-9$ ) pelo apoio financeiro para realização dos campos, à Coordenação de Aperfeiçoamento Pessoal de Nível Superior (CAPES) pela bolsa de estudos e ao Programa de Pós-Graduação em Geografia da Universidade Federal de Santa Catarina (UFSC). 


\section{REFERÊNCIAS}

BARBOUR, M. T.; GERRITSEN, J.; SNYDER, B. D.; STRIBLING, J. B. Rapid bioassessment protocols for use in streams and wadeable rivers: periphyton, benthic macroinvertebrates and fish. 2. ed. EPA 841-B-99-002. Washington, D.C.: USEPA, 1999.

CALLISTO, M.; MORETTI, M.; GOULART, M. Macroinvertebrados bentônicos como ferramenta para avaliar a saúde de riachos. Revista Brasileira de Recursos Hídricos, v. 6, n. 1, p. $71-82,2001$.

CALlistO, M.; FERREIRA, W. R.; MORENO, P.; GOULART, M.; PETRÚCIO, M. Aplicação de um protocolo de avaliação rápida da diversidade de habitats em atividades de ensino e pesquisa (MG-RJ). Acta Limnologica Brasiliensia, v. 14, n. 1, p. 91-98, 2002.

DALLA COSTA, S.; POMPÊO, C. A. Revitalização de cursos d'água em áreas urbanas: caracterização e avaliação da degradação ambiental na zona de proteção legal do Rio Córrego Grande. In: SIMPÓSIO DE RECURSOS HÍDRICOS SUL-SUDESTE, 2., 2008, Rio de Janeiro. Anais... Rio de Janeiro: ABRH, 2008.

DIAZ, R. J.; SOLAN M.; VALENTE, R. M. A review of approaches for classifying benthic habitats and evaluating habitat quality. Journal of Environmental Management, v. 73, p. 165-181, 2004. http://dx.doi.org/10.1016/j.jenvman.2004.06.004

FASOLA, G. B.; SIMONASSI, J. C.; POMPÊO, C. A. Análise de variáveis físico-químicas e biológicas da água para um Protocolo de Avaliação Rápida da integridade de cursos d'água em áreas urbanas In: DIAS, V. L. N.; PET GEOGRAFIA UDESC (Orgs.). Cadernos do Observatório Geográfico da Grande Florianópolis do PET Geografia UDESC.1. ed. Florianópolis: Insular, 2011. p. 80-104.

FERNÁNDEZ, D.; BARQUÍN, J.; RAVEN, P. J. A review of river habitat characterisation methods: indices vs. characterisation protocols. Limnetica, v. 30, n. 2, p. 217-234, 2011.

FERREIRA, W. R.; RODRIGUES, D. N.; ALVES, C. B. M.; CALlisto, M. Biomonitoramento de longo prazo da Bacia do Rio das Velhas através de um índice multimétrico bentônico. Revista Brasileira de Recursos Hídricos, v. 17, n. 3, p. 253-259, 2012.

HANNAFORD, M. J.; BARBOUR, M. T.; RESH, V. H. Training reduces observer variability in visual-based assessments of stream habitat. Journal of the North American $\begin{array}{llllllll}\text { Benthological Society, v. 16, n. 4, p. } & \text { 853-860, }\end{array}$ http://www.jstor.org/stable/1468176

GUIMARÃES, A.; RODRIGUES, A. S. L.; MALAFAIA, G. Adequação de um protocolo de avaliação rápida de rios para ser usado por estudantes do ensino fundamental. Revista Ambiente \& Água, v. 7, n. 3, p. 241-260, 2012. http://dx.doi.org/10.4136/ambiagua.996

GRASSHOFF, K.; EHRHARDT, M.; KREMLING, K. Methods of seawater analysis. 3. ed. Weinheim: Verlag Chemie, 1999. 634 p. 
GREGORY, K. J.; BENITO, G.; DOWNS, P. W. Applying fluvial geomorphology to river channel management: Background for progress towards a palaeohydrology protocol. Geomorphology, v. 98, p. 153-172, 2008.

http://dx.doi.org/10.1016/j.geomorph.2007.02.031

HABBERFIELD, M. W.; BLERSCH, S. S.; BENNETT, S. J.; ATKINSON, J. F. Rapid geomorphic and habitat stream assessment techniques inform restoration differently based on levels of stream disturbance. Journal of the American Water Resources Association, v. 50, n. 4, p. 1051-1062, 2014. http://dx.doi.org/10.1111/jawr.12156

LAMPARELli, M. C. Grau de trofia em corpos d'água no estado de São Paulo avaliação dos métodos de monitoramento. 2004. 119 f. Tese (Doutorado em Ciências na Área de Ecossistemas Terrestres e Aquáticos) - Instituto de Biociências, Universidade de São Paulo, São Paulo, 2004.

MERRITT, R. W.; CUMMINS, K. W. (Orgs.) An introduction to the aquatic insects of North America. 2. ed. Iowa: Kendall/Hunt, 1984. 722 p.

MUGNAI, R.; NESSIMIAN, J. L.; BAPTISTA, D. F. Manual de identificação de macroinvertebrados aquáticos do estado do Rio de Janeiro. 1. ed. Rio de Janeiro: Technical Books, 2010. 176p.

OLlero, A.; ASKOA, I.; GONZALO, L. E.; ACÍN, V.; BALlARÍN, D.; DÍAZ, E. et al.The IHG index for hydromorphological quality assessment of rivers: updated version. Limnetica, v. 30, n. 2, p. 255-262, 2011.

PARDO, I.; ÁlVAREZ, M.; CASAS, J.; MORENO, J. L.; VIVAS, S.; BONADA, N. et al. El hábitat de los ríos mediterráneos. Diseño de un índice de diversidad de hábitat. Limnetica, v. 21, n. 3/4, p. 115-133, 2002.

POMPÊO, C. A.; RIGOTTI, J. A.; FREITAS FILHO, M. D. Urban stream condition assessment. In: INTERNATIONAL CONFERENCE ON URBAN DRAINAGE, 12. 2011, Porto Alegre. Anais... Porto Alegre: ABRH, 2011. p. 1-7.

R CORE TEAM. R: a language and environment for statistical computing. Vienna: $R$ Foundation for Statistical Computing, 2015.

RODRIGUES, A. S. L.; CASTRO, P. T. A. Protocolos de avaliação rápida: instrumentos complementares no monitoramento dos recursos hídricos. Revista Brasileira de Recursos Hídricos, v. 13, n. 1, p. 161-170, 2008.

SARAIVA, M. G. A. N.. O rio como paisagem: gestão de corredores fluviais no quadro do ordenamento do território. Lisboa: Fundação Calouste Gulbenkian, 1999. 512p.

WARD, J. V. Riverine landscapes: biodiversity patterns, disturbance regimes, and aquatic conservation. Biological Conservation, v. 83, n. 3, p. 269-278, 1998. http://dx.doi.org/10.1016/S0006-3207(97)00083-9 


\begin{tabular}{|} 
Ambiente \& Água - An Interdisciplinary Journal of Applied Science \\
ISSN 1980-993X - doi:10.4136/1980-993X \\
www.ambi-agua.net \\
E-mail: ambi.agua@gmail.com
\end{tabular}

\title{
Indicadores de produtividade aplicados à pesca artesanal do camarão sete-barbas, Penha, SC, Brasil
}

\author{
doi:10.4136/ambi-agua.1659
}

Received: 08 May 2015; Accepted: 21 Nov. 2015

\section{Vicente Francisco Coelho $^{1 *}$; Joaquim Olinto Branco ${ }^{2}$; Marco Antonio Harms Dias ${ }^{2}$}

\author{
Universidade do Vale do Itajaí (UNIVALI), Itajaí, SC, Brasil \\ ${ }^{1}$ Programa de Pós-Graduação em Ciência e Tecnologia Ambiental \\ ${ }^{2}$ Centro de Ciências Tecnológicas da Terra e do Mar (CCTMar) \\ *Autor correspondente: e-mail: vicacoelho@hotmail.com, \\ branco@univali.br,mdias@univali.br
}

\section{RESUMO}

Utilizando ferramentas da contabilidade ambiental para coleta e organização de informações, referentes ao mercado e organização do trabalho da comunidade de pescadores artesanais de Penha, SC, realizou-se a pesquisa de forma descritiva e exploratória das práticas produtivas. Esta pesquisa baseou-se em duas fontes de dados e referências bibliográficas para padronização de informações. Calcularam-se indicadores de produtividade, objetivando avaliar a possibilidade do uso deles na gestão ambiental do recurso. Foram levantados os insumos consumidos, custos, quantidades produzidas e comercializadas, destinos e valores de venda, sazonalidade de esforço e capturas. Com base neles, foi possível se calcular indicadores de produtividade ambiental e econômica, em ciclos mensais e anuais, que permitiram evidenciar o período de defeso. Tais métodos exigem muito esforço em coleta e controle de dados, sobretudo em uma comunidade tradicional de pesca artesanal desassociada de rígidos controles necessários essenciais ao estudo sistêmico da produtividade e da organização econômica.

Palavras-chave: gestão da produção, contabilidade ambiental, Xiphopenaeus kroyeri.

\section{Productivity indicators applied to seabob shrimp fishing, Penha SC, Brazil}

\section{ABSTRACT}

This study used environmental accounting tools to collect and organize information related to the market and work organization of the artisan fishing community of Penha, SC. This research was based on two sources of data and references to standardize information. We calculated productivity indicators and evaluated the possibility of using them for environmental management of this resource. We calculated the consumed inputs, costs, produced and marketed quantities, destinations and sales values, and the seasonality of effort and catches. Based upon these, it was possible to calculate indicators of environmental and economic productivity, in monthly and annual cycles that allowed us to identify the best periods in which to close the shrimp harvesting season. Such methods, however, require great 
effort for data collection and tracking, especially in a traditional community of artisanal fisheries lacking the strict control needed for systemic study of productivity and economic organization.

Keywords: environmental accounting, production management, Xiphopenaeus kroyeri.

\section{INTRODUÇÃO}

A pesca artesanal é uma das principais atividades, tradicionalmente realizada no município de Penha SC (26 46'10' S 48 $38^{\circ}$ '45'), que envolve um elevado contingente de famílias, especialmente dependentes da pesca de arrasto do camarão sete-barbas (Xiphopenaeus kroyeri) (Branco, 2005). Com uma área de $60,3 \mathrm{~km}^{2}$, esse município teve seu desenvolvimento social e econômico inicial, centrado na pesca. Atualmente, conta com uma população de 28 mil habitantes e IDHM de 0,743 e PIB per capta de R\$ 14.784,68 (IBGE, 2015).

Modelos artesanais de pesca e aquicultura são relevantes, ao empregar $90 \%$ da força de trabalho e contribuir com $50 \%$ da produção mundial do setor pesqueiro (Carneiro et al., 2014). A pesca do camarão sete-barbas (Xiphopenaeus kroyeri), por sua importância na geração de renda e alimento (Bail e Branco, 2007), pode ser considerada um patrimônio natural, ativo ambiental, e juntamente com a cultura de trabalho dos pescadores artesanais de Penha, um bem comum no litoral catarinense, que ainda demanda planos de monitoramento e políticas públicas adequadas de gestão. Fundamentado em seu valor intrínseco como patrimônio biológico, o camarão deve ser conservado pela importância ecológica e valor antropogênico, ao sustentar todo um modo de vida (Alho, 2008).

As políticas públicas voltadas à pesca ainda não conseguem promover uma gestão deste patrimônio, ao excluir aspectos intrínsecos à atividade da pesca artesanal, como mercado e organização do trabalho (Carneiro et al., 2014). O presente trabalho visa preencher essa lacuna informacional, com contribuição de agentes da comunidade de pescadores de Penha, SC. Os participantes, voluntários, ao responder os questionamentos acerca de seus métodos de produção, possibilitaram o início de um entendimento dessas questões.

A administração da produção, além de contribuir para o entendimento e a organização do trabalho, procura atender a relação custo-benefício, na perspectiva econômica (Pfitscher, 2004; Martins e Laugeni, 2006). Entretanto a crescente conscientização ambiental da sociedade tem provocado cobrança das ações e responsabilidade de conduta das organizações, dos três setores, que intervém no meio ambiente (Silva Gomes et al., 2012). Nesse contexto surge a contabilidade ambiental, com ferramentas de gestão, buscando suprir informações sobre ativos e passivos ambientais intrínsecos ao sistema produtivo, respectivamente, elementos de origem natural ou biológica capazes de gerar benefícios econômicos e custos relacionados ao consumo desses, bem como os relacionados à produção indesejada de insumos, emissões e descartes (Cruz et al., 2009).

A aplicação desta ferramenta ainda não atingiu seu estado da arte no contexto ambiental. Publicações relacionadas ainda não conseguem responder adequadamente toda a complexidade do tema (Reis et al., 2011), principalmente as centradas em sustentabilidade, que procuram evidenciar macro processos administrativos e econômicos em gestão de grandes grupos empresariais (Araujo e Ramos, 2015). Entretanto, associada a dados de produtividade, pode demonstrar a redução de custos ambientais e uso eficiente dos recursos naturais (Pfitscher, 2004; Silva e Cruz, 2014).

A produtividade é uma medida de desempenho muito comum na administração da produção, seu cálculo é simples e o resultado expressa um indicador de eficiência, entretanto sua complexidade está associada à delimitação sistêmica dos processos produtivos e a 
contabilização de suas entradas: recursos, máquinas, energia, sistemas, equipamentos e pessoas, bem como as saídas: produtos e subprodutos na forma de bens ou serviços, descartes e emissões (Martins e Laugeni, 2006). Desta forma, não estão diretamente associados aos conceitos de ativo e passivo ambientais, por exemplo, o camarão que "entra" no processo de pesca como um ativo, a gerar valor posteriormente durante a comercialização. Ou um efluente que "sai” de uma atividade qualquer, com custo para seu tratamento, portanto um passivo.

No contexto ambiental, o uso do indicador de produtividade material, não se trata necessariamente de uma forma de medir impacto, entendendo esse como taxas de transformação do ambiente inerentes à realização da atividade antropogênica (Kristensen, 2004). Dado às relações de causa e efeito dos processos produtivos (Campos, 2004), pode ser tratada como uma medida de pressão ambiental (Kristensen, 2004), onde processos mais eficientes podem resultar, por exemplo, em menor consumo de madeira por quilograma de camarão pescado, o que por definição é positivo, contudo não impede o corte de arvores.

Esse trabalho ao utilizar pressupostos da contabilidade ambiental, para organizar informações intrínsecas à atividade produtiva da pesca artesanal, com a finalidade de possibilitar o cálculo de indicadores de produtividade, a partir de um estudo de caso, objetiva avaliar a possibilidade de uso desses indicadores na gestão ambiental de um recurso visto como um bem comum, e, dessa forma, evidenciar críticas aos métodos de controle de dados no contexto da pesca artesanal.

\section{MATERIAL E MÉTODOS}

Com o uso de técnicas de levantamento de dados, foram realizadas entrevistas estruturadas, sobre fundamentos metodológicos clássicos da administração da produção, para delimitação sistêmica dos processos produtivos (Martins e Laugeni, 2006). A qual é iniciada com a definição do processo de pesca, que inicia quando o pescador sai com a embarcação, finalizando com o desembarque e acondicionamento do produto pescado, com potencial de comercialização - saída de ativo ambiental da atividade. Tem como entrada: a biomassa total extraída e uma embarcação adequada ao uso (óleo diesel, gelo e trabalho do pescador).

Para que a embarcação esteja em condições adequadas ao uso, são necessários processos de apoio ao funcionamento da pesca como reparos nos motores, pintura e manutenção de cascos. Como entradas desses, embarcações com deficiência em seus sistemas de flutuação, propulsão ou com necessidade de pintura, bem como: madeira, tinta, óleo lubrificante, horas de serviço de profissionais especializados ou dos próprios pescadores.

A intervenção em campo ocorreu no município de Penha, SC, em local previamente identificado (Bail e Branco, 2007), como praia, rancho de pesca, trapiches, peixaria e, em alguns casos, nas próprias residências dos pescadores. Contando com uma amostra não probabilística, sobre 30 pescadores artesanais, de um total de 60 atuantes na cooperativa local, em 2012. A partir dessa, foi possível levantar custos, quantidades e a frequência média demandada dos serviços e insumos, sobre os quais foi calculada a média mensal de consumo de cada item. Entretanto os dados materiais foram indicados em uma grande variedade de unidades de medida de volume, a exemplo, embalagens de dois litros de tinta, de um litro de óleo e metros lineares de madeira.

A transformação dos dados volumétricos para quilogramas ocorreu com auxílio de bibliografia especializada. Para óleos lubrificantes e tintas, especificações de densidade obtidas em fichas técnicas de produtos similares (Petrobras, 2011; International Paint, 2009a; 2009b). Já a madeira, tanto a usada em manutenção, quanto o total da frota, foram estimadas com auxilio de um manual de construção de embarcações de madeira (FAO, 2008).

Também, foram utilizados dados coletados em 2010 de 81 pescadores e 71 embarcações, procedentes de cinco mil controles de diário de bordo, no município de Penha, SC, cedidos 
pela Empresa de Pesquisa Agropecuária e Extensão Rural de Santa Catarina (Epagri), que forneceram informações de quantidades e valores, produzidos e comercializados ao longo dos meses, bem como o destino da comercialização desses (atravessadores, peixarias e etc.). Além da produção, forneceu dados da sazonalidade de esforço, como consumo de óleo diesel e serviços de pesca.

A produtividade é calcula através da divisão de saídas por entradas, de acordo com o escopo da análise (Martins e Laugeni, 2006). No presente estudo foi calculada sobre o somatório total das entradas e saídas, ao longo dos meses, não considerando a fauna acompanhante, em duas unidades de medidas: quilogramas e reais, respectivamente, produtividade material (pressão ambiental) e produtividade econômica (rentabilidade).

\section{RESULTADOS E DISCUSSÃO}

O principal desafio em utilizar indicadores de produtividade numa comunidade tradicional de pescadores, não está na forma de cálculo, mas na sistemática de controle dos dados. A contabilidade, aplicada ao contexto ambiental, tem caráter interdisciplinar, procurando analisar informações, que auxiliem os gestores na definição de metas ambientais (Silva Gomes et al., 2012). Demandas que, além de exigir um gestor capacitado a lidar com questões técnicas econômicas e ambientais, exige dos pescadores participação do processo de monitoramento, transparência com as informações e equidade de atuação.

\subsection{Organização econômica dos pescadores artesanais}

As embarcações foram reparadas, a cada $36 \pm 23$ meses, pintadas anti-incrustações com $6 \pm 4$, tinta comum em $12 \pm 5$ meses, e os motores receberam manutenção em $45 \pm 17$ meses.

As atividades de apoio combinadas consomem 16\% do volume material, nas quais o item madeira representa $11 \%\left(10 \mathrm{t} \mathrm{ano}^{-1}\right)$; enquanto que, no processo da pesca estima-se um consumo de $77 \mathrm{t}_{\text {ano }}{ }^{-1}$ de óleo diesel ou $79 \%$ da massa de insumos, que representa em torno de 189 mil reais e $49 \%$ do valor total (Tabela 1). Todas as atividades de manutenção consomem aproximadamente $51 \%$ do montante de recursos financeiros aplicados na operação. Os valores foram levantados a partir do valor médio de mercado para cada item e estão expressos em Reais ( $\mathrm{R} \$)$.

A Tabela 1 apresenta-se sobre uma lógica de processo produtivo, considerando seus principais insumos, esforço de trabalho e suas relações econômicas, denominados de “entradas” para cada embarcação, em unidades, quilogramas, semanas de trabalho, horas de pesca e valores. Para uma real avaliação do impacto na comunidade os dados são explicitados por embarcação e frota. Considerou-se como frota pesqueira dessa comunidade a quantidade de 52 embarcações, pois foi o maior registro de operação no período de maior intensidade, nos meses de junho e julho.

Do total de produto pescado, apenas o montante comercializado, aproximadamente 205 toneladas de camarão sete-barbas (valor médio 3,22 $\mathrm{R} \$ \mathrm{~kg}^{-1}$ ) e $40 \mathrm{t}$ de outras espécies $\left(2,85 \mathrm{R} \$ \mathrm{~kg}^{-1}\right.$ ) (Tabela 2) perfazem um faturamento aproximado de 782.083,00 R\$ ano-1 . Os principais compradores são os atravessadores, mercados de peixes locais e peixarias.

Dentre os produtos comercializados, existem diferenças no valor e quantidade destinada a compradores (113 t a 2,91 $\mathrm{R} \$ \mathrm{~kg}^{-1}$ para atravessadores e $1,7 \mathrm{t}$ a $4,90 \mathrm{R} \$ \mathrm{~kg}^{-1}$ para consumidor final), evidenciando possíveis estratégias de ganho de produtividade, apenas pelo controle e redirecionamento de oferta (Ritzman e Krajewski, 2004; Martins e Laugeni, 2006). Contudo, os pescadores atuam de forma individual, diminuindo suas capacidades de barganhas nas negociações mercadológicas. 
Tabela 1. Entradas de materiais e serviços e sua relação econômica, em um ano de captura do camarão sete-barbas, na Armação do Itapocoroy, Penha.

\begin{tabular}{|c|c|c|}
\hline Entradas & Consumo embarcação & Consumo frota \\
\hline \multicolumn{3}{|c|}{ Materiais (kg ano ${ }^{-1}$ ) } \\
\hline Madeira & 206,25 & $10.725,00$ \\
\hline Tinta Comum & 31,64 & $1.645,28$ \\
\hline Tinta Anti-incrustante & 11,80 & 613,60 \\
\hline Consumo de Óleo Lubrificante & 29,26 & $1.521,52$ \\
\hline Diesel & $1.488,75$ & $77.415,00$ \\
\hline Gelo & 124,20 & $6.458,40$ \\
\hline Camarão sete-barbas & $4.151,35$ & $215.870,30$ \\
\hline Outras Espécies & 785,66 & $40.854,20$ \\
\hline Total & $6.828,91$ & $355.103,30$ \\
\hline \multicolumn{3}{|c|}{ Trabalho (Semanas ano ${ }^{-1}$ ) } \\
\hline Serviço de marceneiro & 1,19 & 61,88 \\
\hline Serviço de Pintura & 1,50 & 78,00 \\
\hline Serviço mecânico & 0,37 & 19,24 \\
\hline Total & 3,06 & 159,12 \\
\hline Serviço de pesca (Horas ano-1) & $1.147,10$ & $59.649,20$ \\
\hline \multicolumn{3}{|c|}{ Econômico (R \$ ano-1 $)$} \\
\hline Madeira & 832,76 & 43.303,52 \\
\hline Serviço de marceneiro & 714,81 & $37.170,12$ \\
\hline Tinta Comum & 508,24 & $26.428,48$ \\
\hline Tinta Anti-incrustante & 310,00 & $16.120,00$ \\
\hline Peças de Reposição & 713,33 & $37.093,16$ \\
\hline Serviço mecânico & 193,33 & $10.053,16$ \\
\hline Consumo de Óleo Lubrificante & 450,08 & $23.404,16$ \\
\hline Diesel & $3.631,09$ & $188.816,68$ \\
\hline Gelo & 61,40 & $3.192,80$ \\
\hline Total & $7.415,04$ & $385.582,08$ \\
\hline
\end{tabular}

A individualidade na tomada de decisão, dos pescadores, pode acarretar em prejuízos à gestão ambiental, ao controle das informações e ao próprio recurso biológico, desarmônico aos pressupostos de uma adequada gestão de bens comum (Lipietz, 2012). A competição e a necessidade de renda podem conduzir pescadores a negligenciar aspectos ligados ao bem comum a sua volta, que por não pertencer a ninguém, pode ser superexplorado e destruído (Lipietz, 2012). Já uma proposta de monitoramento de dados, para ganhos de produtividade pode estar em risco, antes mesmo de utilizada, caso não haja uma uniformidade da coleta de dados, pois a contabilidade ambiental apenas será eficaz à gestão, caso o controle das informações atenda a critérios de geração e transmissão de informações padronizadas, fidedignas e com agilidade (Kalikoski et al., 2009; Pereira et al., 2011). 
Tabela 2. Destino dos produtos comercializados (saídas) na pesca em um ano de captura, na Armação do Itapocoroy, Penha.

\begin{tabular}{lrrrrrr}
\hline Destino do Produto & \multicolumn{2}{c}{ Camarão sete-barbas } & & \multicolumn{2}{c}{ Outras Espécies } \\
\cline { 2 - 3 } \cline { 6 - 7 } Comercializado & \multicolumn{1}{c}{$\mathrm{kg}$} & \multicolumn{1}{c}{$\mathrm{R} \$$} & & $\mathrm{~kg}$ & $\mathrm{R} \$$ \\
\hline Atravessador & $112.859,50$ & $328.546,00$ & & $3.939,80$ & $8.497,20$ \\
Peixaria & $64.408,50$ & $219.900,80$ & & $27.867,90$ & $81.563,12$ \\
Consumo Próprio & $1.420,50$ & $1.728,00$ & & $2.054,00$ & $5.519,60$ \\
Beneficio Próprio & $15.136,50$ & $69.997,00$ & & $1.940,00$ & $5.100,10$ \\
Benefício de Terceiros & $7.205,00$ & $22.814,50$ & & 325,00 & 328,00 \\
Direto ao Consumidor Final & $1.703,30$ & $8.343,50$ & & $3.737,00$ & 17971,75 \\
Restaurantes & 168,00 & 672,00 & & - & - \\
Sem Informação & $2.922,00$ & $10.118,00$ & & 233,20 & 711,60 \\
Doação & 95,00 & 246,00 & & 190,20 & 26,40 \\
\hline Total de saídas & $\mathbf{2 0 5 . 9 1 8 , 3 0}$ & $\mathbf{6 6 2 . 3 6 5 , 8 0}$ & & $\mathbf{4 0 . 2 8 7 , 1 0}$ & $\mathbf{1 1 9 . 7 1 7 , 8 0}$ \\
\hline
\end{tabular}

Comunidades tradicionais de pescadores têm sua organização social e hierárquica definidas, assim um novo modo de trabalho deve surgir de uma realidade existente (Lipietz, 2012). Conhecer as necessidades individuais deste grupo, forças motrizes do processo, (Kristensen, 2004), é fundamental em uma estratégia de monitoramento de produtividade.

\subsection{Principais fatores que influenciam a produtividade}

O indicador de produtividade não tem uma unidade de medida, pois se trata de uma medida relativa de eficiência. Com o valor de saída de produtos de 246.205,40 kg e entrada de insumos de 355.103,30 kg, resulta em um indicador de produtividade material anual de 0,69, assim, para cada 1,0 kg de insumos materiais que entram no processo, 0,69 kg efetivamente transformam-se em produtos; já com uma produção de 782.083,60 R\$ e custo de 385.582,08 $\mathrm{R}$ \$ obteve-se o indicador de produtividade econômica anual de 2,03, para cada real aplicado no processo retornam 2,03 $\mathrm{R} \$$.

Quanto à produtividade material mensal, apenas em junho $(0,80)$ e julho $(0,71)$ ficaram acima da média anual, enquanto que, maio apresentou a menor eficiência material com um indicador de 0,46. Já a produtividade econômica mensal, confirmou junho como mais produtivo, com um indicador calculado de 5,11. Os meses de fevereiro, julho, agosto e outubro, também se mantiveram acima da média anual (Figura 1).

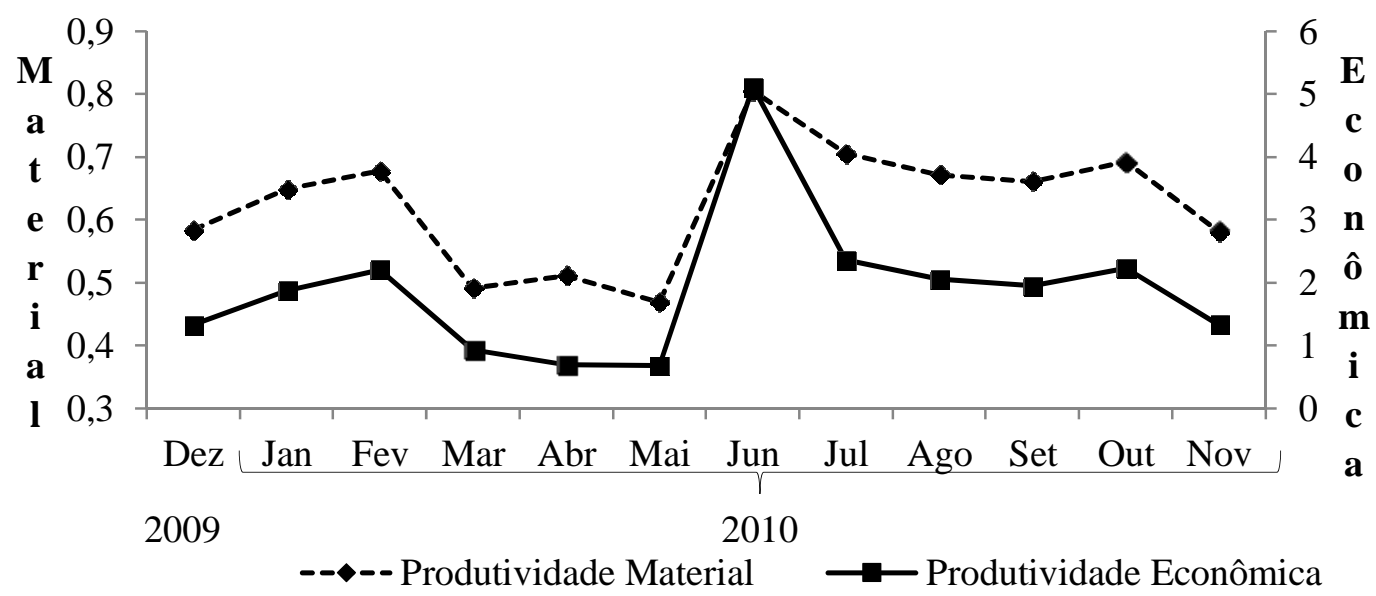

Figura 1. Indicadores de produtividade material e econômica mensais, na pesca de camarão sete-barbas da Armação do Itapocoroy, Penha. 
Na ausência de planos de gestão e estratégias de atuação bem definidas, durante o espaço de tempo analisado, evidenciar o efeito do período de defeso é uma contribuição do método, podendo auxiliar gestores em pesquisas futuras, com uso de indicadores de produtividade na formação de metas estratégicas numéricas e formas de controle direcionando as ações de gestão de forma eficaz (Ritzman e Krajewski, 2004). Mesmo com o grande incremento do esforço, o ganho de eficiência material e econômica após o defeso é significativa, dado um incremento considerável na quantidade produzida.

Existem duas linhas para o ganho de produtividade, aumentar o valor ou a quantidade das saídas ou reduzir os das entradas. Já o uso do indicador exige um controle de dados exato (Kalikoski et al., 2009; Pereira et al., 2011), no caso da pesca o mais rígido possível, sobre os quais deve ser construído um gráfico de produtividade linear ao longo do tempo (Ritzman e Krajewski, 2004). A atividade da gestão passa a ser estratégica, planejando ações para ganhos de produtividade, para que seu sucesso possa ser avaliado graficamente (Campos, 2004; Martins e Laugeni, 2006). Desta forma, por exemplo, a existência de mais de um período de defeso ao ano, poderia resultar em significativa redução de consumo de óleo diesel, para produzir a mesma quantidade total no período. Entretanto a concentração de oferta pode reduzir o preço do produto, jogando contra o incremento de produtividade econômica.

Indicadores de produtividade como ferramenta de gestão ambiental, pode avaliar a viabilidade e o sucesso de diferentes estratégias de pesca, como formas e frequências de atuação sobre pesqueiros, rotas percorridas, tipos de redes, uso de equipamentos, metas de produção e múltiplos períodos de defeso ao longo do ano. Sendo capaz de expressar rentabilidade e pressão ambiental, seu uso pode trazer benefícios ao longo do tempo, através de planejamento, execução, controle e avaliação corretiva, PDCA, de diferentes estratégias para melhoria contínua do processo (Campos, 2004) de forma sustentável, uma vez que permite avaliar tais melhorias em um escopo econômico ecológico (Pfitscher, 2004).

Formular um plano de controle, para cálculo de indicador de produtividade, com base em uma ou mais entradas, depende do escopo de estudo e objetivo estratégico (Martins e Laugeni, 2006). O insumo óleo diesel, com consumo anual aproximado de 77 t (79\%) e 189 mil reais (49\%), é significativo para produtividade material e econômica. Avaliá-lo individualmente, sobretudo sobre o ponto de vista material, ou seja, quantidade de massa produzida por de óleo diesel consumido, pode ser um indicador de pressão ambiental (Kristensen, 2004) relevante ao processo de pesca, facilitando as atividades de controle.

Excluir a fauna acompanhante é fundamental no cálculo do indicador de produtividade ambiental proposto no presente trabalho. Seu volume produzido é tão grande e a eficiência geral do processo é tão baixa que, considerá-la no cálculo, faz com que a linha do gráfico fique horizontal, perdendo seu potencial de indicar a produtividade, uma vez que as varrições dessa passam a ser mínimas (Coelho, 2014). Entretanto a utilização da fauna acompanhante, para gerar renda auxiliar a pesca artesanal do camarão sete-barbas (Branco, 2005; Baile Branco, 2007), é uma alternativa de modernização ecológica de produção e oportunidade bioeconômica, voltadas ao desenvolvimento de tecnologia e processos produtivos, transformando descarte em subproduto (Pereira e González, 2014), que tem como resultado ganhos de produtividade por aumento de produção, saídas de processos (Ritzman e Krajewski, 2004; Martins e Laugeni, 2006).

Aproveitar a fauna acompanhante para aumentar a renda dos pescadores pode ser viável (Branco, 2005; Bail e Branco, 2007), mas não é a única forma de ampliar as saídas do processo produtivo. Estratégias comerciais voltadas à valorização dos produtos podem incrementar a produtividade, sem aumento efetivo da quantidade produzida (Martins e Laugeni, 2006), por meio do aperfeiçoamento dos métodos e valorização das atitudes ambientais que, conforme Pfitscher (2004) são ferramentas eficientes na manutenção do meio ambiente. Nesse sentido, o valor de mercado da biodiversidade, deve considerar não apenas 
os custos operacionais para disponibilização do produto, mas também o valor do sistema de conservação e dos ciclos biológicos, bem como o valor do serviço social e o modo de vida tradicional (Alho, 2008).

\subsection{Sazonalidade de produção}

Os maiores esforços médios mensais de pesca foram praticados em junho (18 dias), logo após o período de defeso do camarão sete-barbas (Figura 2). Juntamente com agosto, meses de inverno, tem a pressão mais intensa da frota artesanal sobre o estoque de camarões, com até 52 embarcações atuando diariamente, reduzindo no verão para 36 a 29 embarcações (dezembro/09, novembro/10), e a poucas no defeso do camarão.

Com a aplicação dos recursos produtivos da pesca, existe a entrada dos recursos naturais através da captura, sendo registradas aproximadamente 216 t de camarão sete-barbas e $41 \mathrm{t}$ de outras espécies, distribuídas entre peixes, moluscos e crustáceos. Quanto à entrada mensal de recursos, junho se destaca como de maior aporte médio diário por embarcação, com aproximadamente $91 \mathrm{~kg}$ de pesca (Figura 3).

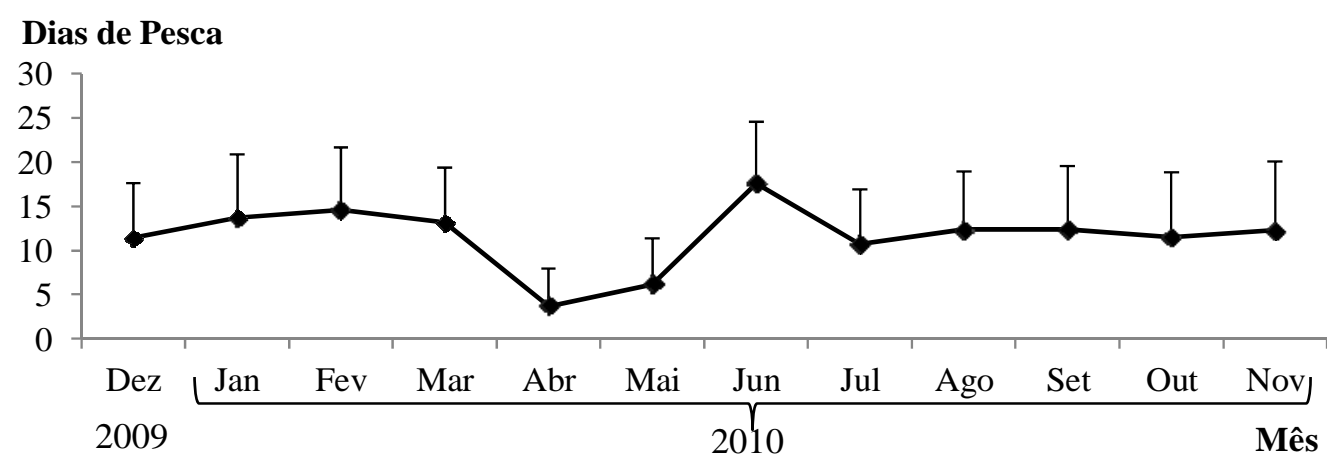

Figura 2. Média de esforço mensal em dias de pesca por embarcação na Armação do Itapocoroy, Penha, SC.

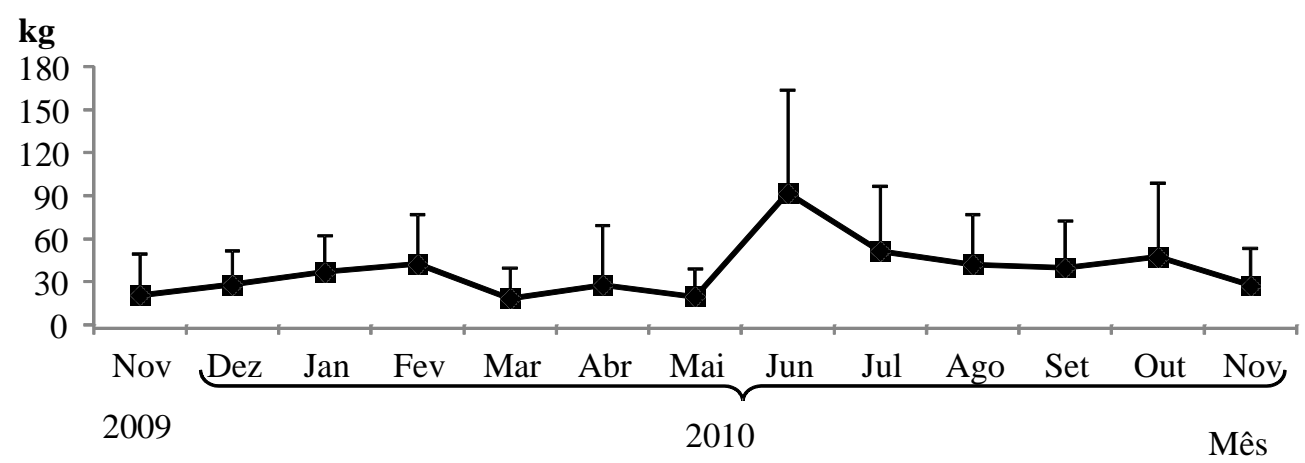

Figura 3. Média diária de produção de pescado por embarcação, na Armação do Itapocoroy, Penha.

Apesar do aumento de entradas com a elevação do esforço em junho, após o defeso do camarão (março, abril e maio), ocorre maior desempenho, eficiência e menor custo por unidade produzida. O efeito dessa interrupção na população é fundamental, pois incrementa a biomassa capturada (Branco, 2005), influenciando a produtividade em junho. Para manutenção do bem comum, controle do esforço de pesca deve passar, portanto, por outras 
medidas: repousos biológicos, fechamentos temporários, medidas técnicas, que já vêm sendo propostas pelos pescadores há muito tempo (Sann, 2012).

Nesse sentido, uso de indicadores mensais, pode se tornar um critério modulador do exercício da atividade, possibilitando comparar a pressão ambiental resultante de diferentes esforços ao longo dos anos (Kristensen, 2004), alterando as metas de atuação operacional e, até mesmo, as sazonalidades de produção. Também o dimensionamento e modernização de frota devem adaptar-se às novas realidades e metas, a fins de gerenciar o bem comum (Sann, 2012).

Um plano de monitoramento de produtividade, com objetivo de gestão do bem comum para a pesca artesanal do camarão em Penha SC, pode embasar-se em alguns pressupostos como metas de produção e um sistema de cotas individuais transferíveis, tal qual proposta pela Comunidade Europeia (Sann, 2012); e um Estado atuando como o guardião do bem comum, obtendo previamente o consentimento esclarecido da comunidade local, antes de conceder-lhe acesso em um processo de redistribuição de resultados, o qual não se trata de uma mera relação mercantil, mas uma recompensa pelo esforço aplicado (Lipietz, 2012).

Pesquisas futuras devem avaliar as forças motrizes do sistema, necessidades individuais e setoriais da pesca artesanal do camarão sete-barbas, dados de estado do meio ambiente (Kristensen, 2004) e suas relações com a produtividade, bem como atuação em sistemas cooperados e individuais.

\section{CONCLUSÃO}

Ao ser capaz de demonstrar os efeitos do defeso, um evento extremamente importante para a pesca artesanal do camarão sete-barbas, os indicadores de produtividade demonstram eficácia para ser aplicado neste contexto. Como indicador de pressão ambiental, pode ser usado exclusivamente para demonstrar o desempenho da atividade antropogênica.

Com melhor desempenho após o defeso, a produção é mais eficiente neste período ecológica e economicamente. O indicador de produtividade material (pressão ambiental) mais elevado, após a parada obrigatória, mostra menor consumo recursos por unidade pescada nesta época, que no resto do ano. Também é mais rentável (produtividade econômica) mesmo com grande incremento de esforço, porém no conjunto, com o mesmo aproveitamento do bem comum no decorrer de um período anual.

Frente à dificuldade para obter informações nesse contexto, evidencia-se que uma forma de simplificar a coleta de dados, para cálculo de indicadores de produtividade material, é com o controle exclusivo das quantidades consumidas de óleo diesel e produzidas de pescado, dado a grande representatividade deste insumo materialmente. Já para o cálculo da rentabilidade é necessário ainda, ao mínimo, os valores de negociação destes insumos e produtos, bem como os custos totais de manutenção de equipamentos e embarcações. No decorrer de médias históricas, com necessidade de replicação desse estudo tanto no mesmo local, quanto em outros similares, poder-se-á avaliar a gestão e preservação desse patrimônio biológico de forma mais abrangente.

A visão tradicional da pesca artesanal está desassociada das rígidas exigências de exatidão da coleta de dados, necessários aos estudos sistêmicos da produtividade e da organização econômica, bem como mensuração de impactos e tendências. Incluir estes processos gerenciais no dia-a-dia desta população tradicional demandará inclusão social, em um processo de educação socioeconômica e ambiental, para proporcionar clareza das metas produtivas, argumentos de vendas e leitura dos resultados. Nesse contexto, entender o patrimônio biológico como bem comum, que pertence a todos e a ninguém, é fundamental para um modelo de pesca mais produtivo, embasado em controle, socialmente justo e sustentável. 


\section{AGRADECIMENTOS}

Os autores gostariam de agradecer ao Programa de Pós-Graduação em Ciência e Tecnologia Ambiental da Universidade do Vale do Itajaí. Ao CNPq (Conselho Nacional de Pesquisa) pela bolsa de produtividade do pesquisador JOB. Ao apoio e disponibilidade de acesso dos pescadores artesanais da localidade de Penha, SC, que permitiram realizar a coleta de dados desse artigo. Bem como as contribuições dos revisores anônimos que contribuíram consideravelmente no trabalho.

\section{REFERÊNCIAS}

ALHO, C. J. R. The value of biodiversity. Brazilian Journal of Biology, v. 68 n. 4. 2008. http://dx.doi.org/10.1590/S1519-69842008000500018

ARAUJO, A. O.; RAMOS, M. C. P. Limitações dos relatórios de sustentabilidade para análises custo-benefício de ações sociais e ambientais. Contextus - Revista Contemporânea de Economia e Gestão, v. 13, n. 1, p. 132-155, 2015.

BAIL, G. C.; BRANCO, J. O. Pesca artesanal do camarão sete-barbas: uma caracterização sócio-econômica na Penha, SC. Brazilian Journal of Aquatic Science And Technology, v. 11, n. 2, p. 25-32, 2007.

BATISTA, M. Avaliação do impacto do plano de ordenamento da área marinha do parque natural da Arrábida na pesca comercial local. 2007. 97f. Dissertação (Mestrado) - Universidade de Lisboa, 2007.

BRANCO, J. O. Biologia e pesca do camarão sete-barbas Xiphopenaeus kroyeri (Heller) (Crustacea, Penaeidae), na Armação do Itapocoroy, Penha, SC. Revista Brasileira de Zoologia, v. 22, n. 4, p.1050-1062, 2005. http://dx.doi.org/10.1590/S010181752005000400034

CAMPOS, V. F. TQC - Controle da qualidade total: no estilo japonês. 8. ed. Belo Horizonte: INDG, 2004. 224p.

CARNEIRO, A. M. M.; DIEGUES, A. C. S.; VIEIRA, L. F. S. Extensão participativa para a sustentabilidade da pesca artesanal. Desenvolvimento e Meio Ambiente, v. 32, p. 8199, 2014. http://dx.doi.org/10.5380/dma.v32i0.35949

COELHO, V. F. Análise do processo produtivo da pesca artesanal do camarão setebarbas em penha, SC: indicadores e gestão ambiental. 2014. 73f. Dissertação (Mestrado em Ciência e Tecnologia Ambiental) - Universidade do Vale do Itajaí, Santa Catarina. 2014. 73p.

CRUZ, C. F.; MARQUES, A. L.; FERREIRA, A. C. S. Informações ambientais na contabilidade pública: reconhecimento de sua importância para a sustentabilidade. Sociedade, contabilidade e gestão, v. 4, n. 2, p. 47-60, 2009.

FOOD AND AGRICULTURE ORGANIZATION OF THE UNITED NATIONS - FAO. Projetos para barcos de pesca: 2 barcos com fundo em $\mathrm{V}$ construídos com tábuas ou compensado. Documento técnico sobre as pescas 134 Rev. 2. Roma, 2008. Disponível em: http://es.scribd.com/doc/20553752/ projetos-para-construcao-de-barcos. Acesso em: 01 maio 2015. 
INSTITUTO BRASILEIRO DE GEOGRAFIA E ESTATÍSTICA - IBGE. Dados do município de Penha, SC. $2015 . \quad$ Disponível em: http://www.cidades.ibge.gov.br/xtras/perfil. php?lang=ecodmun=421250. Acesso em: 01 maio 2015.

INTERNATIONAL PAINT. Ficha de informação de segurança para produtos químicos: YKA923, Tinta Toplac Squall Blue, vol. 1, 14 Ago. 2009a.

INTERNATIONAL PAINT. Ficha de informação de segurança para produtos químicos: YBA993, Tinta Micron Optima Activator Black, Part B, vol. 8, 6 Ago 2009b.

KALIKOSKI, D. C.; SEIXAS, C. S.; ALMUDI, T. Gestão compartilhada e comunitária da pesca no Brasil: avanços e desafios. Ambiente \& Sociedade, v. 12, n. 1, p. 151-172, 2009.

KRISTENSEN, P. The DPSIR Framework. Aarhus: National Environmental Research Institute of Denmark, 2004. 10p.

LIPIETZ, A. Questões sobre os “bens comuns”. Revista Passerelle, n. 6, p. 20-26, 2012.

MARTINS, P. G.; LAUGENI, F. P. Administração da produção. 2. ed. São Paulo: Saraiva, 2006. 562p.

PEREIRA, D. L.; PILATTI, L. A.; FASCINA. M. M.; ZAHAIKEVITCH, E. V.; CRUZ, J. C.; FRANCISCO, A. C. A controladoria e sua relação com o planejamento estratégico: o caso de uma multinacional no interior do Paraná. Revista Eletrônica Fafit/Facic, v. 2, n. 2, p. 11-21, 2011.

PEREIRA, V. C.; GONZÁLEZ, S. R. O debate acerca das insuficiências da modernização ecológica para pensar a sustentabilidade ambiental na agricultura em tempos de mudanças climáticas. Observatorio de la Economía Latinoamericana, v. 199, p. 115, 2014.

PETROBRAS. Fichas de informação de segurança de produto químico: BR0109 Óleo Diesel S500, vol.1, 03 de Janeiro; BR0006_P Lubrax Náutica Diesel, vol. 0.4P, 28 de Janeiro 2011.

PFITSCHER, E. D. Gestão e sustentabilidade através da contabilidade e controladoria ambiental: estudo de caso na cadeia produtiva de arroz ecológico. 2004. 252f. Tese (Doutorado em Engenharia de Produção) - Universidade Federal de Santa Catarina, Florianópolis, 2004.

REIS, L. G.; NOGUEIRA, D. R.; TARIFA, M. R. Uma análise histórica das publicações existentes sobre o tema contabilidade ambiental. Revista de Estudos Contábeis, v. 2, n. 3, p. 90-97, 2011.

RITZMAN, L. P.; KRAJEWSKI, L. J. Administração da produção e operações. São Paulo: Prentice Hall, 2004. 419p.

SANN, A. L. A questão da sobrecapacidade e dos direitos de pesca. Resposta ao livro verde das pescas da Comissão Europeia. Revista Passerelle, n. 6, p. 51-54, 2012.

SILVA, A. R.; CRUZ, C. V. O. A. A importância da contabilidade ambiental no mundo globalizado. UNOPAR Científica Ciências Jurídicas e Empresariais, v. 8, p. 81-87, 2014. 
SILVA GOMES, S. M. D.; SAMPAIO, M. S.; AZEVEDO, T. C.; SLOMSKI, V. G. Proposta para o ensino da controladoria ambiental nos cursos de graduação de ciências contábeis nas IESS brasileiras. Revista de Gestão Social e Ambiental, v. 6, n. 1, p. 177-189, 2012. 


\begin{tabular}{|} 
Ambiente \& Água - An Interdisciplinary Journal of Applied Science \\
ISSN 1980-993X - doi:10.4136/1980-993X \\
www.ambi-agua.net \\
E-mail: ambi.agua@gmail.com
\end{tabular}

\title{
Estrutura comunitária de samambaias em mata ciliar: avaliação em gradiente de antropização
}

\author{
doi:10.4136/ambi-agua.1717 \\ Received: 28 Jul. 2015; Accepted: 26 Nov. 2015

\section{Ivanete Teresinha Mallmann*; Vinícius Leão da Silva; Jairo Lizandro Schmitt} \\ Universidade Feevale, Novo Hamburgo, RS, Brasil \\ Centro de Ciências da Saúde \\ *Autor correspondente: e-mail: ivamallmann@terra.com.br, \\ suicinivleao@hotmail.com,jairols@feevale.br
}

\section{RESUMO}

As matas ciliares são essenciais para a manutenção da biodiversidade e apresentam condições ecológicas favoráveis para o desenvolvimento de samambaias que são indicadoras de qualidade ambiental. Entretanto, essas matas vêm sofrendo impactos negativos relacionados principalmente à alta densidade demográfica, ao modelo de ocupação urbana e à expansão agrícola em áreas rurais. Neste trabalho, a qualidade ambiental da mata ciliar do rio Cadeia no Sul do Brasil foi avaliada utilizando parâmetros fitossociológicos da comunidade de samambaias do sub-bosque e o Protocolo de Avaliação Rápida da Qualidade de Hábitat (PARH). Foram alocadas 120 parcelas de $25 \mathrm{~m}^{2}$ distribuídas equitativamente entre três fragmentos (FI, FII, FIII). Foram inventariadas todas as espécies de samambaias herbáceas em cada unidade amostral. Para a análise da estrutura comunitária foram calculados os parâmetros de densidade, frequência, dominância relativa e o valor de importância da espécie (VI). A menor riqueza de espécies foi registrada no FIII (sete espécies). A composição florística é mais heterogênea e a riqueza é maior no FI no qual as parcelas apresentaram cobertura de plantas e a nota do PARH mais elevadas. A pontuação do PARH diminuiu com o aumento do grau de urbanização da matriz do entorno e os FI e FII foram classificados como naturais enquanto que o FIII como impactado. Considerando as quatro espécies com maior VI nos três fragmentos (que somam 69,11\% do VI no FI, 78,36\% no FII e 91,06\% no FIII) podese afirmar que a estrutura comunitária de samambaias sofre uma simplificação com o aumento da antropização.

Palavras-chave: bioindicadores, fitossociologia, fragmentação, Sul do Brasil, urbanização.

\section{Community structure of ferns in riparian forest: evaluation in anthropization gradient}

\begin{abstract}
Riparian forests are essential to the maintenance of biodiversity and foster the development of ferns that are indicators of environmental quality. However, these forests have been degraded due mainly to high population density, pattern of urban settlement and agricultural expansion in rural areas. This study evaluated the environmental quality of
\end{abstract}


riparian vegetation of the Cadeia River in Southern Brazil, using phytosociological parameters of the understory's fern community and a Rapid Assessment Protocol of Habitat Quality (RAPQH). One hundred and twenty plots of $25 \mathrm{~m}^{2}$ were equally distributed among three fragments (FI, FII, FIII). All species of herbaceous ferns were inventoried in each sample unit. We calculated parameters of density, frequency, relative dominance and importance value (IV) of species for the analysis of community structure. The lowest species richness was recorded at FIII (seven species). The floristic composition is more heterogeneous and richness is higher in FI, where the parcels had greater plant cover and RAPQH scores. The RAPQH score decreased with increasing degree of urbanization in the surrounding matrix and FI and FII were classified as "natural," while FIII was classified as "impacted." Considering the four species with the highest IV in the three fragments (totaling $69.11 \%$ of IV in the FI, $78.36 \%$ and $91.06 \%$ in FII and FIII respectively), it can be affirmed that the fern community structure is degraded with increasing anthropization.

Keywords: bioindicators, fragmentation, phytosociology, Southern Brazil, urbanization.

\section{INTRODUÇÃO}

A vegetação natural às margens dos rios constitui as matas ciliares (Mueller, 1996), que devido à interação das condições físicas com o meio biológico caracterizam-se pela heterogeneidade florística em diferentes intensidades (Rodrigues e Nave, 2000). O ambiente ciliar protege os corpos d'água, a biodiversidade, o solo e contribui positivamente para a produção primária do sistema lótico (Gregory et al., 1992; Oliveira-Filho e Ratter, 1995; Mueller, 1996), sendo definidas como áreas de preservação permanente (APPs) pelo Código Florestal Brasileiro (Brasil, 2012).

No entanto, mesmo sendo essenciais para a manutenção da biodiversidade e da qualidade ambiental, em áreas urbanizadas, as matas ciliares sofrem impactos negativos relacionados principalmente à alta densidade demográfica e ao modelo de ocupação urbana (Dias et al., 2014), enquanto que em áreas rurais são degradadas pela expansão agrícola desordenada (Rodrigues e Gandolfi, 2000).

Estimativas recentes sobre a flora mundial de samambaias apontam para aproximadamente 11.500 espécies (Moran, 2008). Para o Brasil são descritas 1.111 espécies, distribuídas principalmente nas regiões Sul e Sudeste, e para o estado do Rio Grande do Sul são listadas 331 (Prado e Sylvestre, 2015). Pelas matas ciliares apresentarem condições ecológicas para o desenvolvimento de samambaias (Windisch, 1996), os estudos sobre a estrutura comunitária dessas plantas nesse tipo de ambiente vêm sendo realizados no Brasil em diferentes tipos vegetacionais, com diferentes estados de conservação, empregando metodologias distintas.

Dentre os que utilizaram área amostral fixa, destacam-se os de Forsthofer e Athayde-Filho (2012) que registraram 17 espécies em 1,5 ha, em Nova Xavantina; o de Kreutz et al. (2009) com oito espécies em 1 ha de dois córregos da Bacia do rio Pindaíba, o de Kreutz e Athayde-Filho (2009) que registraram nove espécies em 2 ha em quatro ordens do córrego Caveira em Barra das Graças e o de Athayde-Filho e Agostinho (2005) que registraram 10 espécies, em área total de 1 ha em duas veredas, em Campinápolis, todos no estado do Mato Grosso. No Rio Grande do Sul, Diesel e Siqueira (1991) registraram 24 espécies em 0,18 ha na Bacia Hidrográfica do Rio dos Sinos e Mallmann e Schmitt (2014) registraram 26 espécies em 0,3 ha em três fragmentos do rio Cadeia na Bacia Hidrográfica do Rio Caí. Estes inventários evidenciam alta heterogeneidade quanto ao número de espécies de samambaias em diferentes matas ciliares e áreas amostradas.

As samambaias são um importante grupo vegetal com grande potencial bioindicador, já 
que tem sua diversidade e capacidade de colonização diretamente vinculados aos fatores abióticos do meio (Silva et al., 2011). Além disso, respondem de forma mais intensa às variações das condições ambientais como topografia ou fatores edáficos, quando comparadas a outros grupos vegetais florestais (Costa et al., 2005).

A incidência de gradientes ambientais de antropização permite avaliar os impactos das atividades humanas sobre os ecossistemas naturais (McDonnell e Pickett, 1990). Contudo, a avaliação da qualidade ambiental exige métodos e ferramentas eficientes (Rodrigues e Castro, 2008), que permitam uma análise conjunta de um maior número de variáveis bióticas.

Baseado na constatação de Mallmann e Schmitt (2014), de que a riqueza média de samambaias muda em diferentes fragmentos num gradiente crescente de urbanização do habitat matriz, os objetivos desse trabalho foram: (i) avaliar a qualidade ambiental de três fragmentos de mata ciliar ao longo do rio Cadeia utilizando um Protocolo de Avaliação Rápida da Qualidade de Hábitat (PARH); (ii) comparar a florística entre comunidades de samambaias do sub-bosque destes três fragmentos, bem como, (iii) analisar a estrutura fitossociológica de samambaias nas mesmas áreas.

\section{MATERIAL E MÉTODOS}

\section{1. Área de estudo}

O rio Cadeia é um dos maiores afluentes do rio Cá́, principal curso d'água da bacia hidrográfica do Caí, localizada no nordeste do Rio Grande do Sul, com uma área de 4.945,70 $\mathrm{km}^{2}$ (Rio Grande do Sul, 2010). De acordo com a classificação de Köeppen-Geiger, o clima na bacia é do tipo Cfa, ou seja, temperado úmido (C) com ocorrência de chuvas durante todos os meses do ano (f), sendo a temperatura média do mês mais quente superior a $22^{\circ} \mathrm{C}$ (a) (Peel et al., 2007).

Em Santa Maria do Herval, 37,58\% da área de preservação permanente do rio Cadeia está sem a cobertura vegetal prevista pelo Código Florestal (Sarmento et al., 2001). Nesse município, foram selecionados três fragmentos de mata ciliar (Figura1) para a realização do estudo:

Fragmento I (FI): situa-se no extremo leste de Santa Maria do Herval, distante $11 \mathrm{~km}$ do Centro da cidade (29 $31^{\prime} 1.02^{\prime \prime} \mathrm{S}$ e $50^{\circ} 54^{\prime} 46.78^{\prime} \mathrm{O}, 509 \mathrm{~m}$ de altitude). A mata ciliar tem 160 $\mathrm{m}$ de largura considerando a margem do rio e a borda do fragmento, sendo que dados históricos indicam que não há ação antrópica intensa sobre a vegetação há cerca de 70 anos. $\mathrm{O}$ FI está inserido em uma matriz rural, com baixa densidade demográfica.

Fragmento II (FII): situa-se a 2,4 km de distância do Centro do município (29॰30'14.20"S e 5058'28.13'O, 399 m de atitude) e está inserido em uma matriz suburbana, que apresenta a segunda maior densidade demográfica do município. Neste ponto o fragmento tem $39 \mathrm{~m}$ de largura entre a margem do rio e a borda da mata ciliar.

Fragmento III (FIII): situa-se no Centro (29³0’07.30”S e 5059’52.27’O, $388 \mathrm{~m}$ de altitude), em uma matriz urbana que apresenta a maior parte da população do município. $\mathrm{O}$ fragmento apresenta $55 \mathrm{~m}$ de largura da margem do rio até o limite florestal.

\subsection{Inventário florístico}

Para o levantamento dos dados florísticos nos FI, FII e FIII (Figura 1) foram realizadas excursões mensais, durante um ano, para registrar todas as espécies de samambaias herbáceas ocorrentes em uma área de $100 \times 10 \mathrm{~m}$, subdividida em 40 parcelas contíguas de $5 \times 5 \mathrm{~m}$ distribuídas equitativamente em duas transecções, paralelas ao curso do rio Cadeia, de acordo com o desenho amostral de Mallmann e Schmitt (2014). O material foi coletado seguindo a metodologia proposta por Windisch (1992). As espécies de samambaias coletadas foram identificadas utilizando literatura especializada, comparação com material determinado em 
herbário e consultas a especialistas. A classificação adotada para as famílias seguiu a proposta de Smith et al. (2008). Exemplares férteis representativos foram depositados no Herbarium Anchieta (PACA) do Instituto Anchietano de Pesquisa - Universidade do Vale do Rio dos Sinos, no Rio Grande do Sul, Brasil.

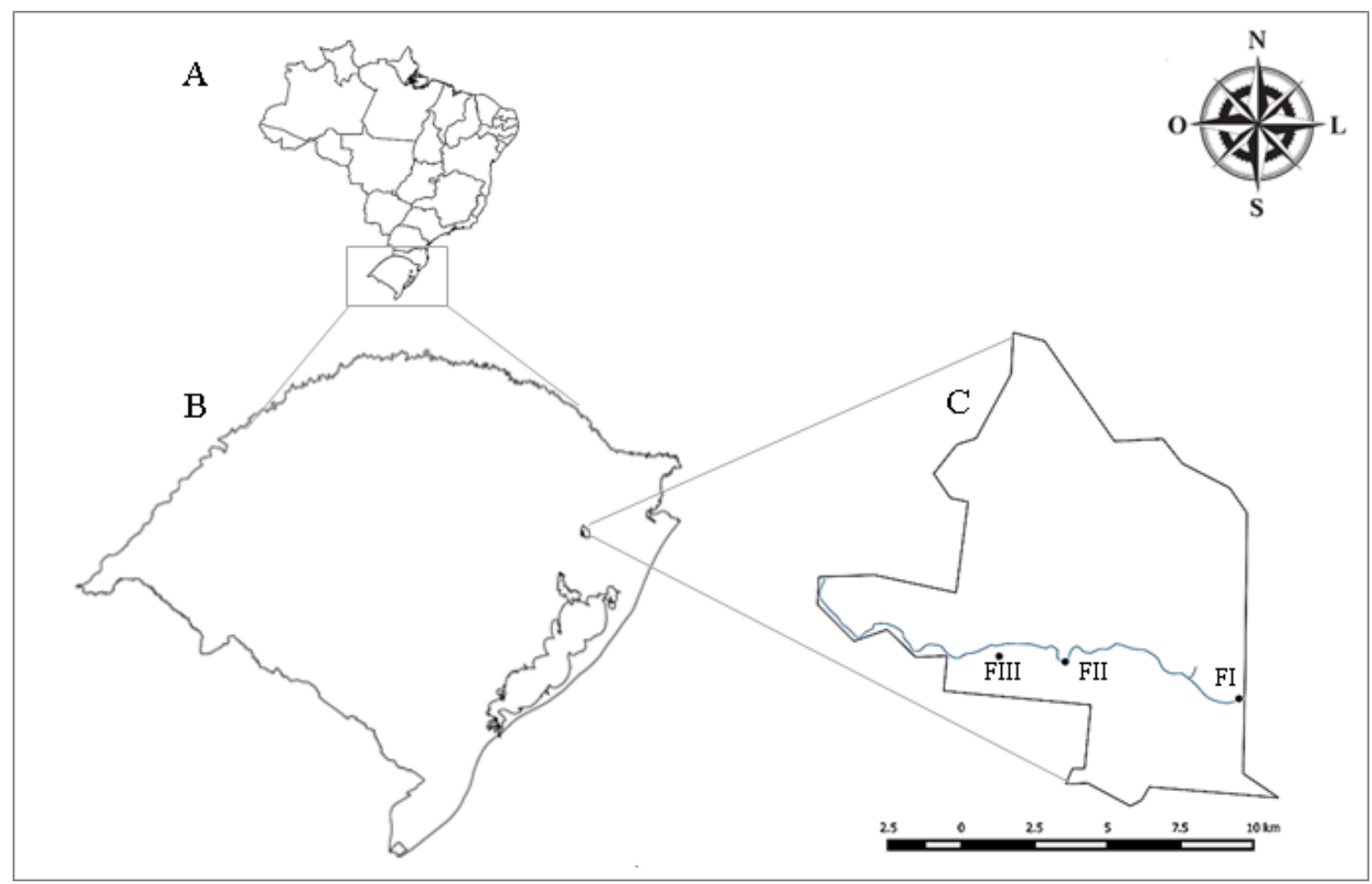

Figura 1. Mapa de localização dos fragmentos de mata ciliar (FI, FII e FIII) ao longo do rio Cadeia em Santa Maria do Herval (C), RS (B), Brasil (A).

\subsection{Estrutura comunitária}

Para a análise e comparação da estrutura da comunidade de samambaias herbáceas ocorrentes no sub-bosque dos fragmentos florestais, foram calculados os seguintes parâmetros fitossociológicos: densidade, frequência e dominância relativa, bem como, o valor de importância de acordo com Vuono (2002) adaptado por Condack e Sylvestre (2009) para esse grupo vegetal.

\subsection{Protocolo de Avaliação Rápida}

O Protocolo de Avaliação Rápida da Qualidade de Hábitat (PARH) é uma ferramenta criada para o monitoramento ambiental de sistemas hídricos e os ecossistemas que os abrangem (Bizzo et al., 2014). O PARH aplicado nos três fragmentos estudados foi modificado da proposta de Hannaford et al. (1997) e da Agência de Proteção Ambiental de Ohio (EUA) - (USEPA, 1987 adaptado por Callisto et al., 2002). Dos 20 parâmetros do PARH considerados neste estudo, os 10 primeiros analisam as características dos fragmentos e o nível dos impactos decorrentes da antropização e os demais, as condições de hábitat e o nível de conservação das condições naturais, sendo: 1: Ocupação das margens do rio (principal atividade); 2: Erosão das margens e assoreamento do rio; 3: Alterações antrópicas; 4: Cobertura vegetal no leito do rio; 5: Odor da água; 6: Oleosidade da água; 7: Transparência da água; 8: Odor do sedimento; 9: Oleosidade do sedimento; 10: Tipo de fundo; 11: Diversificação de hábitats submersos; 12: Tipos de substrato; 13: Percentual de deposição de lama; 14: Percentual de depósitos sedimentares; 15: Alterações no canal do rio; 16: Características do fluxo d'água; 17: Características da vegetação ripária; 18: Estabilidade das 
margens; 19: Extensão de mata ciliar; 20: Plantas aquáticas. As Pontuações aplicadas aos parâmetros do Protocolo de Avaliação Rápida da Diversidade de Hábitats utilizado para a avaliação dos fragmentos foram: parâmetros 1 - 10: pontuação máxima (5: situação natural), intermediária (3: alteração leve) e mínima: (0: alteração severa); parâmetros 11 - 20: pontuação máxima (5: situação natural), intermediária (3: alteração leve; 2: alteração mediana) e mínima (0: alteração severa). O somatório dos pontos de cada parâmetro do PARH classifica os trechos avaliados em: impactado ( 0 a 40 pontos); alterado (41 a 60 pontos) e natural (61 a 100 pontos).

\subsection{Análise estatística}

As médias de riqueza, abundância e área de cobertura de samambaias herbáceas por parcela no FI, FII e FIII foram comparadas entre si, utilizando o programa estatístico BioEstat versão 5.2. Para verificar a normalidade dos dados, utilizou-se o teste de Shapiro-Wilk. Como a hipótese de normalidade não foi satisfatória, os dados foram analisados pelo teste não paramétrico de Kruskal-Wallis, seguido pelo teste de Dunn, a 5\% de probabilidade.

Os dados de composição de espécies nos FI, FII e FIII foram submetidos à Análise de Coordenadas Principais (PCoA) para a visualização da similaridade florística em diagrama, adotando-se o índice de Sørensen-Dice. A Análise de Componentes Principais (PCA) foi utilizada na representação das relações entre as unidades amostrais com os parâmetros de riqueza, número de indivíduos e cobertura $\left(\mathrm{m}^{2}\right)$ de samambaias por parcela e a pontuação total do PARH. Ambas as análises foram conduzidas no software PAST (Paleontological Statistics Software Package for Education and Data Analysis), versão 2.17 (Hammer et al., 2001).

\section{RESULTADOS E DISCUSSÃO}

Os resultados da aplicação do PARH no rio Cadeia em Santa Maria do Herval classificaram os FI e FII como naturais e o FIII como impactado (Figura 2). Dessa forma, o nível de degradação de cada ponto avaliado acompanhou o grau de urbanização do entorno, evidenciando um gradiente decrescente de qualidade ambiental a partir do FI (pontuação - FI: 87; FII: 73 e FIII: 34).

Entretanto, a expressiva diferença do FIII em relação aos demais se deve também ao represamento desse trecho desde 1945, o que provocou modificações no canal do rio e no fluxo d'água. Estas alterações estão relacionadas à ocupação, erosão e estabilidade das margens; à deposição de lama e sedimentos; à redução dos tipos de substrato; além da homogeneidade de habitats submersos (Figura 2). De acordo com Rodrigues et al. (2010), obras de engenharia ou alterações no canal do rio causam a redução na densidade e diversidade de espécies aquáticas. Outro indicador do maior impacto no FIII é a presença de bancos de macrófitas, que são espécies flutuantes livres, cujo desenvolvimento está atrelado à menor velocidade da água (Thomaz, 2002), ao aumento da turbidez pela concentração de material em suspensão e outros atributos (Thomaz et al., 2004) que limitam sua ocorrência a este trecho.

Em contraponto, nos FI e FII onde o rio tem maior declividade, o fluxo d'água apresenta corredeiras que são indicadoras de alta qualidade do hábitat e da diversidade da fauna (Barbour et al., 1999). Estes fatores, dentre outros, influenciam inclusive na riqueza, estrutura e distribuição de espécies epifíticas em matas ciliares (Bonnet, 2006; Kersten e Kuniyoschi, 2009), como já constatado por Mallmann e Schmitt (2014) que registraram maior riqueza de samambaias epifíticas no FI e FII em relação ao FIII do presente estudo. Por fim, a melhor conservação da mata ciliar nos FI e FII confere proteção e estabilidade às margens do rio, o que contribui para a classificação desses pontos como sendo naturais, fato que também já foi descrito por Barrella et al. (2000). 


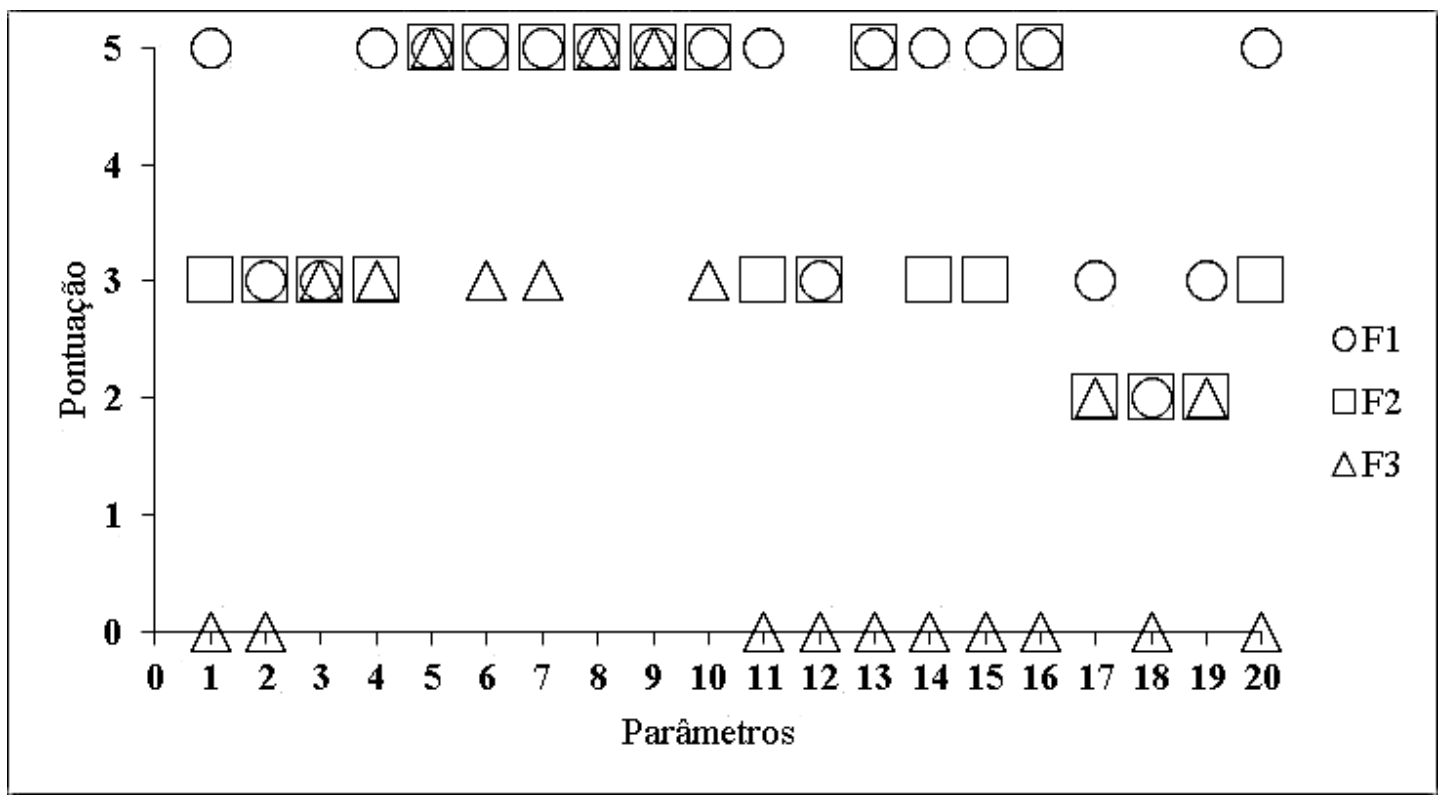

Figura 2. Pontuação aplicada aos parâmetros do Protocolo de Avaliação Rápida da Diversidade de Hábitats utilizado para a avaliação dos Fragmentos I (FI: ○), II (FII: $\square$ ) e III (FIII: $\Delta$ ) do rio Cadeia em Santa Maria do Herval. Parâmetros 1 - 10: pontuação máxima (5: situação natural), intermediária (3: alteração leve) e mínima: (0: alteração severa); parâmetros 11 - 20: pontuação máxima (5: situação natural), intermediária (3: alteração leve; 2: alteração mediana) e mínima (0: alteração severa). A relação entre o número e nome dos parâmetros pode ser encontrada no Material e Métodos.

$\mathrm{Na}$ análise da estrutura comunitária, verificou-se que no FI a riqueza específica total foi de 18 espécies variando de três a 10 por parcela, com média de 5,9 $( \pm 1,67)$ e as espécies com maior área de cobertura foram Megalastrum inaequale, Dicksonia sellowiana, Pteris deflexa e Ctenitis submarginalis, que somadas representam $90,68 \%$ do total da comunidade. Consequentemente estas mesmas espécies apresentaram os maiores valores de importância, somando $70,97 \%$ do total. Embora Dennstaedtia globulifera e P. deflexa apresentassem abundância menor que Thelypteris recumbens, o VI de importância mais elevado dessas duas espécies decorreu de valores expressivamente maiores de cobertura (Tabela 1). O mesmo ocorreu com $D$. sellowiana que apresentou baixa $D R$, devido ao número reduzido de indivíduos, mas foi a terceira espécie em valor de importância (VI) em virtude da sua elevada área de cobertura. Trata-se de uma espécie com folhas grandes, com até cerca de 2,40 $\mathrm{m}$ de comprimento (Fernandes, 2000).

Das espécies exclusivas encontradas no FI, Vandenboschia radicans é classificada como hemiepífita ou epipétrica, e ocorre geralmente em florestas fechadas e úmidas (Windisch, 2014). Da mesma forma, Eupodium kaulfussii é classificada como terrícola, sendo característica de ambientes montanhosos e úmidos (Tryon e Tryon, 1982) e integra a lista de espécies ameaçadas do Rio Grande do Sul na categoria vulnerável (Rio Grande do Sul, 2014). O registro dessas espécies evidencia o alto grau de conservação deste fragmento, corroborando com os resultados do PARH.

No FII foram registradas 21 espécies, com riqueza variando de 0 a 10 espécies por parcela e média de 4,7 $( \pm 2,26)$. Em relação à cobertura, Blecnhum brasiliense se destacou com 55,01\% da cobertura total, seguido de Ctenitis submarginalis e Deparia petersenii. O alto valor de cobertura de $B$. brasiliense se deve ao fato de ser uma espécie subarborescente, com cáudice ereto e robusto, com até $60 \mathrm{~cm}$ de altura e folhas de até 1,3 $\mathrm{m}$ de comprimento (Franz e Schmitt, 2005). Estas três espécies somam 81,84\% da cobertura total nesta área. As espécies B. brasiliense, C. submarginalis, D. petersenii e Anemia phyllitidis apresentaram os 
maiores VI representando 72,12\% do total (Tabela 2).

Tabela 1. Parâmetros fitossociológicos para as espécies de samambaias amostradas no sub-bosque do Fragmento I da mata ciliar do Rio Cadeia em Santa Maria do Herval, RS. Ni- número de indivíduos, Ui - número de parcelas de ocorrência da espécie, DR\%- densidade relativa, FR\%- frequência relativa, DoR\%- dominância relativa, VI- valor de importância.

\begin{tabular}{lccccccc}
\hline Espécie & $\mathrm{Ni}$ & $\mathrm{Ui}$ & $\mathrm{DR} \%$ & $\mathrm{FR} \%$ & DoR\% & VI \\
\hline Megalastrum inaequale (Kaulf. ex Link) A.R.Sm. \& & 436 & 39 & 38,58 & 16,32 & 45,82 & 33,57 \\
R.C.Moran & 240 & 35 & 21,24 & 14,64 & 10,74 & 15,54 \\
Ctenitis submarginalis (Langsd. \& Fisch.) Ching & 35 & 23 & 3,1 & 9,62 & 23,05 & 11,92 \\
Dicksonia sellowiana Hook & 70 & 30 & 6,19 & 12,55 & 11,07 & 9,94 \\
Pteris deflexa Link & 125 & 10 & 11,06 & 4,18 & 0,53 & 5,26 \\
Thelypteris recumbens (Rosenst.) C.F.Reed & 35 & 10 & 3,1 & 4,18 & 3,68 & 3,65 \\
Dennstaedtia globulifera (Poir.) Hieron. & 28 & 18 & 2,48 & 7,53 & 1,21 & 3,74 \\
Polystichum platylepis Fée & 47 & 11 & 4,16 & 4,6 & 1,54 & 3,43 \\
Diplazium herbaceum Fée & 33 & 10 & 2,92 & 4,18 & 0,09 & 2,4 \\
Vandenboschia radicans (Sw.) Copel. & 27 & 10 & 2,39 & 4,18 & 0,52 & 2,36 \\
Blechnum acutum (Desv.) Mett. & 16 & 11 & 1,42 & 4,6 & 0,17 & 2,06 \\
Thelypteris riograndensis (Lindm.) C.F.Reed & 12 & 9 & 1,06 & 3,77 & 0,1 & 1,64 \\
Anemia phyllitidis (L.) Sw. & 10 & 9 & 0,88 & 3,77 & 0,06 & 1,57 \\
Asplenium claussenii Hieron. & 7 & 6 & 0,62 & 2,51 & 1,36 & 1,5 \\
Dennstaedtia obtusifolia (Willd.) T.Moore & 5 & 5 & 0,44 & 2,09 & 0,04 & 0,86 \\
Campyloneurum nitidum (Kaulf.) C.Presl & 2 & 1 & 0,18 & 0,42 & 0,01 & 0,2 \\
Asplenium gastonis Fée & 1 & 1 & 0,09 & 0,42 & 0,01 & 0,17 \\
Thelypteris opposita (Vahl) Ching & 1 & 1 & 0,09 & 0,42 & 0 & 0,17 \\
Eupodium kaulfussii (J.Sm.) J.Sm. & $\mathbf{1 1 3 0}$ & & & & \\
\hline Total & & & & & \\
\hline
\end{tabular}

Ctenitis submarginalis foi ainda a espécie que esteve entre aquelas de maior VI independente do fragmento de ocorrência. De acordo com Mynssen e Windisch (2004), é uma espécie tanto de sub-bosque sombreado quanto mais exposta à luz solar, o que facilitaria sua distribuição mais generalizada, e nesse sentido aumentaria sua capacidade competitiva.

Há evidências botânicas da decrescente integridade ambiental a partir do fragmento I. A exemplo disso, B. brasiliense foi exclusivo do FII, que é menos preservado que o FI de acordo com a nota obtida no PARH. Essa espécie também foi encontrada em ambientes alterados nos estudos desenvolvidos por Paciencia e Prado (2005) e Silva (2013). O mesmo ocorreu com Macrothelypteris torresiana, que também foi exclusiva do FII sendo uma espécie genuinamente encontrada em ambientes alterados ou abertos, como beira de estradas e trilhas, terrenos baldios de áreas urbanas e florestas secundárias (Salino, 2000).

Cyathea atrovirens ocorreu apenas no FII. Essa espécie é preferencialmente encontrada em locais abertos e iluminados, tais como áreas degradadas ou clareiras florestais (Lorscheitter et al., 1999). Tryon e Tryon (1982) reportaram a ocorrência de C. atrovirens em áreas elevadas da Serra do Mar no sudeste brasileiro, destacando a sobrevivência de indivíduos em locais onde toda a cobertura do solo foi destruída pela ação de queimadas.

Asplenium gastonis e Campyloneurum nitidum ocorreram tanto em FI quanto FII, com 
VI baixos (Tabelas 1 e 2) e isso está relacionado diretamente ao fato de serem espécies preferencialmente epifíticas ou rupícolas e, neste caso, limitando seu desenvolvimento no estrato herbáceo.

Em contrapartida, Dicksonia sellowiana, que consta na lista de espécies ameaçadas do Rio Grande do Sul (Rio Grande do Sul, 2014), obteve o terceiro maior VI no fragmento I $(11,92)$ enquanto no fragmento II ficou na $18^{\mathrm{a}}$ posição $(\mathrm{VI}=0,32)$, com apenas um indivíduo. Essa espécie desempenha um papel fundamental como forófito de epífitos no sub-bosque das florestas, sendo que algumas epífitas são exclusivas ou ocorrem preferencialmente sobre seu cáudice (Fraga et al., 2008). Ademais, considerando o ritmo lento de crescimento de D. sellowiana, estimado por Schmitt et al. (2009) em 5,6 cm por ano, e a altura máxima de 6 $\mathrm{m}$ registrada no fragmento I, pode-se concluir que o fragmento possui mais de 100 anos.

Tabela 2. Parâmetros fitossociológicos para as espécies de samambaias amostradas no sub-bosque do Fragmento II da mata ciliar do Rio Cadeia em Santa Maria do Herval, RS: Ni- número de indivíduos, Ui - número de parcelas de ocorrência da espécie, DR\%- densidade relativa, FR\%- frequência relativa, DoR\%- dominância relativa, VI- valor de importância.

\begin{tabular}{|c|c|c|c|c|c|c|}
\hline Espécie & $\mathrm{Ni}$ & $\mathrm{Ui}$ & $\mathrm{DR} \%$ & $\mathrm{FR} \%$ & DoR\% & VI \\
\hline Blechnum brasiliense Desv. & 145 & 17 & 9,51 & 8,9 & 55,01 & 24,47 \\
\hline Ctenitis submarginalis (Langsd. \& Fisch.) Ching & 475 & 33 & 31,15 & 17,28 & 15,42 & 21,28 \\
\hline Deparia petersenii (Kunze) M.Kato & 377 & 23 & 24,72 & 12,04 & 11,41 & 16,06 \\
\hline Anemia phyllitidis (L.) Sw. & 198 & 31 & 12,98 & 16,23 & 1,73 & 10,31 \\
\hline Thelypteris riograndensis (Lindm.) C.F.Reed & 170 & 21 & 11,15 & 10,99 & 2,8 & 8,31 \\
\hline Thelypteris opposita (Vahl) Ching & 51 & 12 & 3,34 & 6,28 & 2,26 & 3,96 \\
\hline Megalastrum inaequale (Kaulf. ex Link) A.R.Sm. \& R.C.Moran & 16 & 9 & 1,05 & 4,71 & 2,33 & 2,7 \\
\hline Cyathea atrovirens (Langsd. \& Fisch.) Domin & 15 & 7 & 0,98 & 3,66 & 3,01 & 2,55 \\
\hline Macrothelypteris torresiana (Gaudich.) Ching & 14 & 9 & 0,92 & 4,71 & 1,67 & 2,43 \\
\hline Thelypteris dentata (Forssk.) E.P.St.John & 24 & 9 & 1,57 & 4,71 & 0,78 & 2,35 \\
\hline Alsophila setosa Kaulf. & 2 & 2 & 0,13 & 1,05 & 1,84 & 1,01 \\
\hline Campyloneurum nitidum (Kaulf.) C.Presl & 6 & 4 & 0,39 & 2,09 & 0,1 & 0,86 \\
\hline Asplenium gastonis Fée & 14 & 2 & 0,92 & 1,05 & 0,21 & 0,73 \\
\hline Pteris deflexa Link & 3 & 2 & 0,2 & 1,05 & 0,69 & 0,65 \\
\hline Pecluma truncorum (Lindm.) M.G.Price & 3 & 2 & 0,2 & 1,05 & 0,08 & 0,44 \\
\hline Blechnum acutum (Desv.) Mett. & 3 & 2 & 0,2 & 1,05 & 0,06 & 0,43 \\
\hline Doryopteris lorentzii (Hieron.) Diels & 3 & 2 & 0,2 & 1,05 & 0,04 & 0,43 \\
\hline Dicksonia sellowiana Hook & 1 & 1 & 0,07 & 0,52 & 0,38 & 0,32 \\
\hline Rumohra adiantiformis (G.Forst.) Ching & 3 & 1 & 0,2 & 0,52 & 0,13 & 0,28 \\
\hline Dennstaedtia globulifera (Poir.) Hieron. & 1 & 1 & 0,07 & 0,52 & 0,03 & 0,21 \\
\hline Adiantum raddianum C.Presl & 1 & 1 & 0,07 & 0,52 & 0,01 & 0,2 \\
\hline Total & 1525 & & & & & \\
\hline
\end{tabular}


No FIII a riqueza total foi de sete espécies variando de zero a cinco, com média de 1,95 $( \pm 1,06)$. Ctenitis submarginalis, Dennstaedtia globulifera e Thelypteris scabra apresentaram o maior valor de cobertura ( $83 \%$ da cobertura total da área) e somaram $78,78 \%$ do total do valor de importância (Tabela 3).

Tabela 3. Parâmetros fitossociológicos para as espécies de samambaias amostradas no sub-bosque do Fragmento III da mata ciliar do Rio Cadeia em Santa Maria do Herval, RS: Ni- número de indivíduos, Ui - número de parcelas de ocorrência da espécie, DR\%-densidade relativa, FR\%- frequência relativa, DoR\%- dominância relativa, VI - valor de importância.

\begin{tabular}{lcccccc}
\hline Espécie & $\mathrm{Ni}$ & $\mathrm{Ui}$ & $\mathrm{DR} \%$ & $\mathrm{FR} \%$ & DoR\% & VI \\
\hline Ctenitis submarginalis (Langsd. \& Fisch.) Ching & 164 & 31 & 44,57 & 39,74 & 42,56 & 42,29 \\
Dennstaedtia globulifera (Poir.) Hieron. & 96 & 12 & 26,09 & 15,38 & 20,33 & 20,6 \\
Thelypteris scabra (C.Presl) Lellinger & 59 & 9 & 16,03 & 11,54 & 20,1 & 15,89 \\
Pteris deflexa Link & 16 & 7 & 4,35 & 8,97 & 15,1 & 9,47 \\
Anemia phyllitidis (L.) Sw. & 29 & 15 & 7,88 & 19,23 & 1,28 & 9,46 \\
Asplenium claussenii Hieron. & 3 & 3 & 0,82 & 3,85 & 0,27 & 1,64 \\
Deparia petersenii (Kunze) M.Kato & 1 & 1 & 0,27 & 1,28 & 0,37 & 0,64 \\
\hline Total & $\mathbf{3 6 8}$ & & & & \\
\hline
\end{tabular}

Considerando as quatro espécies com maior VI nos três fragmentos amostrais (que somam $69,11 \%$ dos indivíduos no FI, 78,36\% no FII e 91,06\% no FIII) pode-se sustentar que a estrutura comunitária de samambaias difere de acordo com as condições ambientais observadas através dos parâmetros do PARH, uma vez que, quanto mais severas as intervenções antrópicas no fragmento, maior foi a soma do VI para essas espécies naquele fragmento. Apesar disso, foram registradas espécies mais tolerantes à redução da qualidade ambiental, como Anemia phyllitidis, Ctenitis submarginalis, Dennstaedtia globulifera e Pteris deflexa que ocorreram nos três fragmentos.

No interior desses três fragmentos foram amostradas 29 espécies de samambaias pertencentes a 13 famílias, sendo que as de maior riqueza foram Thelypteridaceae com seis espécies, Dryopteridaceae (quatro) e Pteridaceae (três) que juntas representam $44,82 \%$ do total de espécies registradas.

Os fragmentos I e II apresentaram riqueza específica total similar (18 e 21 espécies respectivamente). Considerando o maior grau de perturbação do FII em relação ao FI, registraram-se diferenças na composição de espécies dos fragmentos, como o percentual de cobertura médio de samambaias por parcela que foi expressivamente maior no FI quando comparado com os demais (Tabela 4). Já no FIII, a riqueza total foi reduzida para apenas sete espécies.

Tabela 4. Riqueza média, abundância média e área de cobertura média por parcela das samambaias do sub-bosque em três fragmentos de mata ciliar (FI, II e III) do rio Cadeia em Santa Maria do Herval, RS. Médias com mesma letra são iguais $(P<0,01)$. $(\mathrm{S}=$ riqueza média de samambaias herbáceas por parcela; $\mathrm{Ni}$ = número de indivíduos por parcela; Área cobertura = média da área de cobertura de samambaias por parcela $\mathrm{em}^{2}$; $\mathrm{DP}=$ desvio-padrão).

\begin{tabular}{lccc}
\hline & S (DP) & Ni (DP) & $\begin{array}{c}\text { Área cobertura }\left(\mathrm{m}^{2}\right) \\
(\mathrm{DP})\end{array}$ \\
\hline Fragmento I & $5,9 \pm 1,67(\mathrm{a})$ & $28,25 \pm 13,78(\mathrm{a})$ & $31,78 \pm 17,20(\mathrm{a})$ \\
Fragmento II & $4,7 \pm 2,26(\mathrm{~b})$ & $38,12 \pm 43,54(\mathrm{a})$ & $13,48 \pm 22,97(\mathrm{~b})$ \\
Fragmento III & $1,95 \pm 1,06(\mathrm{c})$ & $9,2 \pm 9,21(\mathrm{~b})$ & $4,45 \pm 4,58(\mathrm{~b})$ \\
\hline
\end{tabular}


A Análise de Coordenadas Principais (PCoA) aproximou os fragmentos I e II, demonstrando que eles são mais homogêneos sob o ponto de vista florístico, em decorrência de um maior compartilhamento de espécies. A análise de similaridade entre os fragmentos indicou que internamente o FI é o que apresenta composição florística mais homogênea. Entretanto, externamente ele é mais heterogêneo em relação ao FII e FIII (Figura 3).

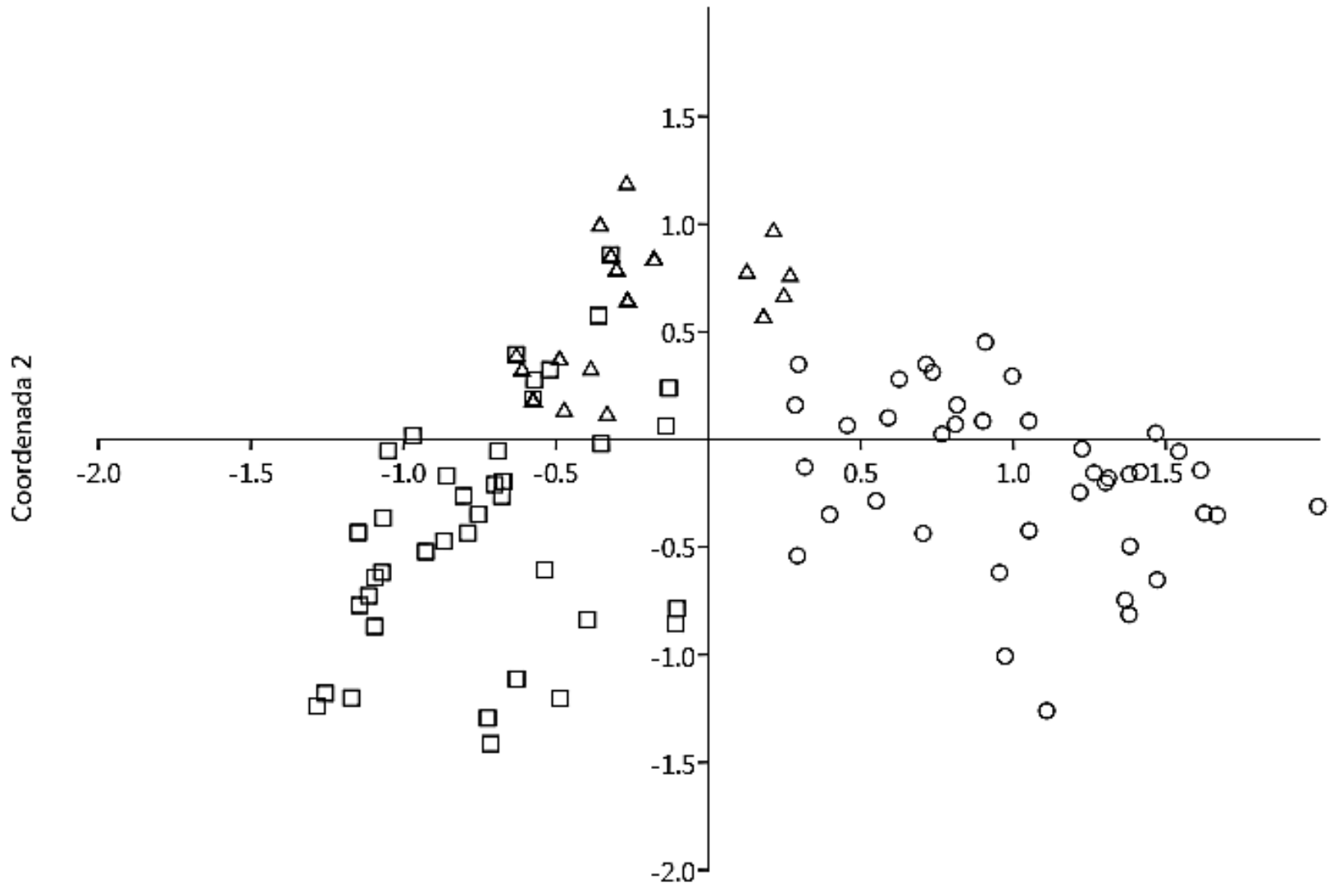

Coordenada 1

Figura 3. Análise de Coordenadas Principais (PCoA) entre as espécies de samambaias em suas respectivas parcelas: Fragmento I (०), Fragmento II ( $\square)$ e Fragmento III $(\Delta)$.

Os dois primeiros componentes da PCA explicaram $80,83 \%$ da variação e de maneira geral evidenciaram uma separação das parcelas de cada fragmento, em função das variáveis analisadas (Figura 4). Essa separação ocorreu principalmente pelo componente $1(64,05 \%)$, em que a riqueza de samambaias por parcela foi a variável mais relacionada $(0,55)$, seguida da pontuação do PARH $(0,50)$. Já pelo componente $2(16,78 \%)$, a variável com maior relação foi o número de indivíduos por parcela $(0,81)$, seguida da pontuação do PARH $(-0,55)$. A riqueza é maior nas parcelas nas quais a cobertura de plantas e a nota do PARH foram mais elevadas, demostrando que áreas mais antropizadas apresentam uma simplificação na estrutura da comunidade de samambaias herbáceas. O maior número de indivíduos associado ao FII pode ser devido ao fato de que espécies oportunistas e mais tolerantes podem ser favorecidas em função de alterações de condições ambientais locais. 


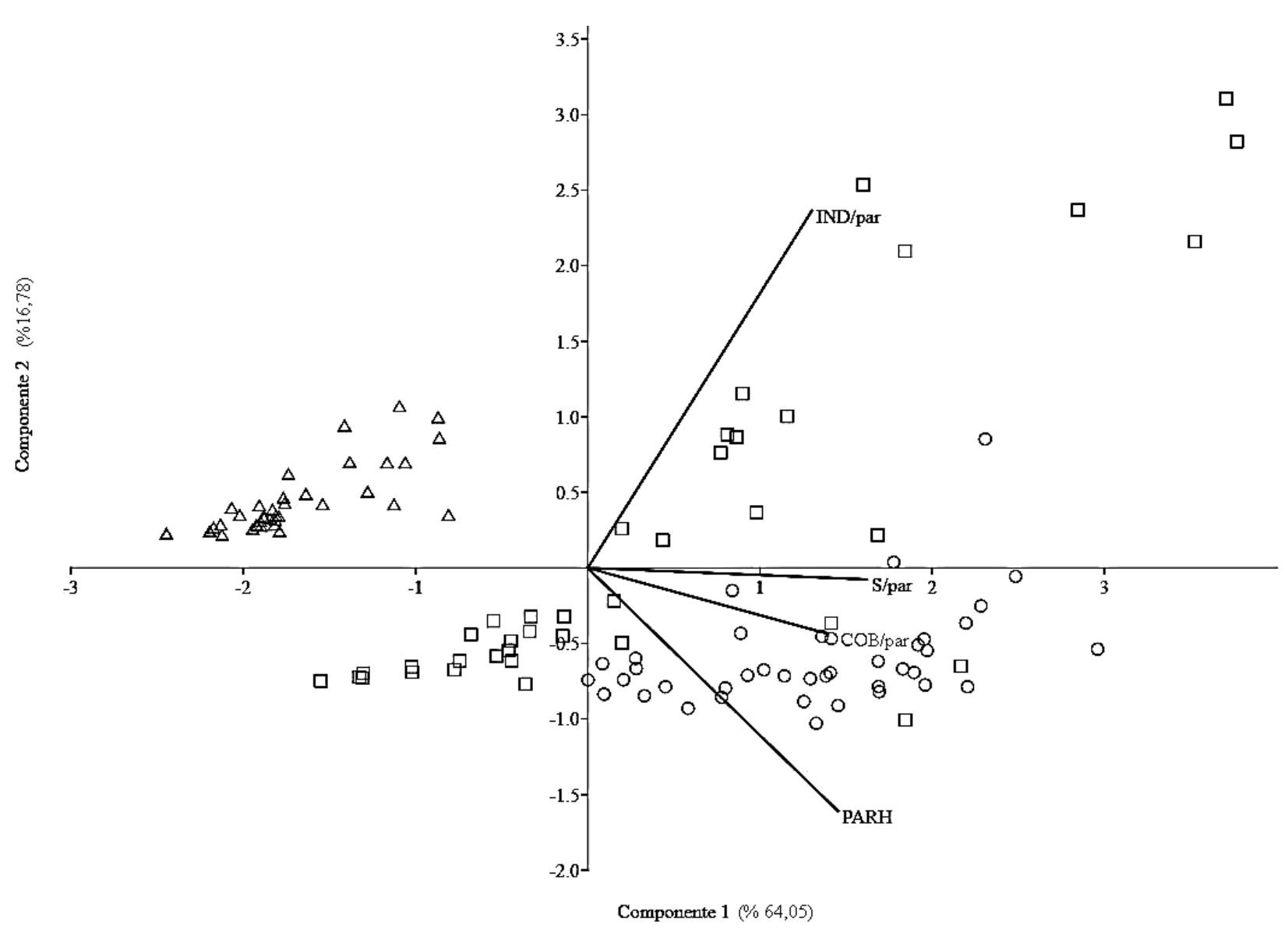

Figura 4. Análise de Componentes Principais (PCA), Fragmento I (०), Fragmento II ( $\square$ ) e Fragmento III $(\Delta)$. IND/par: Indivíduos por parcela; S/par: Riqueza por parcela; COB/par: Cobertura por parcela; PARH: Pontuação do Protocolo de Avaliação Rápida da Qualidade de Hábitat.

\section{CONCLUSÃO}

Embora se deva considerar a heterogeneidade característica das matas ciliares, mesmo em fragmentos muito próximos geograficamente como os do presente estudo, ficou evidente a simplificação da estrutura comunitária à medida que estes trechos perdem suas características naturais e têm sua qualidade ambiental diminuída de acordo com as categorias do PARH. Pode-se afirmar, inclusive, que quanto maior o grau de distúrbio de um fragmento, mais intensamente sua estrutura comunitária será afetada. Nesse sentido, o FIII, que é impactado, apresentou uma estrutura comunitária muito vulnerável, pois as quatro espécies com maior VI concentraram mais de $90 \%$ da comunidade de samambaias.

Por fim, dada a importância das funções deste tipo de formação vegetal e os impactos observados ao longo do rio Cadeia em Santa Maria do Herval, julga-se que a busca por estratégias eficientes para fins de recuperação destes fragmentos torna-se indispensável e são de caráter urgente.

\section{AGRADECIMENTOS}

Os autores agradecem a Coordenação de Aperfeiçoamento Pessoal de Nível Superior (CAPES) pelas bolsas de estudo concedidas para os dois primeiros autores e a Universidade Feevale pela infraestrutura disponibilizada para a realização deste estudo. 


\section{REFERÊNCIAS}

ATHAYDE-FILHO, F. P.; AGOSTINHO, A. A. Pteridoflora de duas veredas no município de Campinápolis, Mato Grosso, Brasil. Pesquisas, Botânica, v. 56, p. 145-160, 2005.

BARBOUR, M. T.; GERRITSEN, J.; SNYDER, B. D.; STRIBLING, J. B. Rapid bioassessment protocols for use in streams and wadeable rivers: periphyton, benthic macroinvertebrates and fish. 2. ed. Washington, D.C: USEPA, 1999.

BARRELLA, W.; PETRERE JR., M.; SMITH, W. S.; MONTAG, L. F. A. As relações entre as Matas ciliares, os rios e os peixes. In: RODRIGUE, R. R.; LEITÃO FILHO, H. F. Matas ciliares: conservação e recuperação. São Paulo: EDUSP/FAPESP, 2000. p. 187208.

BIZZO, M. R. O.; MENEZES, J.; ANDRADE, S. F. Protocolos de avaliação rápida de rios (PAR). Caderno de Estudos Geoambientais - CADEGEO, v. 4, n. 1, p. 5-13, 2014.

BONNET, A. Caracterização fitossociológica das bromeliáceas epifíticas e suas relações com os fatores geomorfológicos e pedológicos da planície do rio Iguaçu, Paraná, Brasil. 2006. 289f. Tese (Doutorado em Engenharia Florestal) - Universidade Federal do Paraná, Brasil, 2006.

BRASIL. Lei $\mathbf{n}^{0}$ 12.651, de 25 de maio de 2012. Dispõe sobre a proteção da vegetação nativa e dá outras providências. 2012. Disponível em: http://www.planalto.gov .br/ccivil_03/_Ato2011-2014/2012/Lei/L12651.htm. Acesso em: 12 jan. 2015.

CALLISTO, M.; FERREIRA, W.; MORENO, P.; GOULART, M. D. C.; PETRUCIO, M. Aplicação de um protocolo de avaliação rápida da diversidade de habitats em atividades de ensino e pesquisa (MG-RJ). Acta Limnologica Brasiliense, v. 14, n. 1, p. 91-98, 2002.

CONDACK, J. P. S.; SYLVESTRE, L. S. Structure of fern community in the high mountain forest of Itatiaia National Park, Brazil. In: VERMS, S. C.; KHULLAR, S. P.; CHEEMA, H. K. (Eds.). Perspective in Pteridophytes. Lucknow: Bishen Singh Mahendra Pal Singh, 2009, p. 113-126.

COSTA, F. R. C.; MAGNUSSON, W. E.; LUIZAO, R. C. Mesoscale distribution patterns of Amazonian understorey herbs in relation to topography, soil and watersheds. Journal of Ecology, v. 93, p. 863-878, 2005. http://dx.doi.org/10.1111/j.1365-2745.2005.01020.x

DIAS, R. M.; SALVADOR, N. N. B.; BRANCO, M. B. C. Identificação dos níveis de degradação de matas ripárias com o uso de SIG. Floresta e Ambiente, v. 21, n. 2, p. 150-161, 2014. http://dx.doi.org/10.4322/floram.2014.032

DIESEL, S.; SIQUEIRA, J. C. Estudo fitossociológico herbáceo/arbustivo da mata ripária da bacia hidrográfica do rio dos Sinos, Rio Grande do Sul. Pesquisas, Botânica, v. 42, n. 2 , p. 205-257, 1991.

FERNANDES, I. Taxonomia dos representantes de Dicksoniaceae no Brasil. Pesquisas, Botânica, v. 50, p. 5-26, 2000.

FORSTHOFER, M.; ATHAYDE-FILHO, F. P. A. Florística e Aspectos Ecológicos de Samambaias e Licófitas ao Longo do córrego Cachoeirinha, Nova Xavantina-MT. Pesquisas, Botânica, v. 63. p. 149-164, 2012. 
FRAGA, L. L.; SILVA, L. B.; SCHMITT, J. L. Composição e distribuição vertical de pteridófitas epifíticas sobre Dicksonia sellowiana Hook. (Dicksoniaceae), em Floresta Ombrófila Mista, no Sul do Brasil. Biota Neotrópica, v. 8, n. 4, p. 123-129, 2008.

FRANZ, I.; SCHMITT, J. L. Blechnum brasiliense Desv. (Pteridophyta, Blechnaceae): estrutura populacional e desenvolvimento da fase esporofítica. Pesquisas, Botânica, v. 56, p. 173-184, 2005.

GREGORY, S. V.; SWANSON, F. J.; McKEE, W. A.; CUMMINS, K. W. An ecosystem perspective of riparian zones. BioScience, v. 41, n. 8, p. 540-551, 1992. http://dx.doi.org/10.2307/1311607

HAMMER, O.; HARPER, D. A. T.; RYAN, P. D. Paleontological Statistics - PAST. Version 1.18. 2001. Disponível em: http://folk.uio.no/ohammer/past. Acesso em: jul. 2015.

HANNAFORD, M. J.; BARBOUR, M. T.; RESH, V. H. Training reduces observer variabillity in visual-based assessments of stream habitat. Journal of the North American Benthological Society, v. 4, p. 853-860, 1997. http://dx.doi.org/10.2307/1468176

KERSTEN, R. A.; KUNIYOSHI, Y. S. Conservação das florestas na bacia do alto Iguaçu, Paraná - Avaliação da comunidade de epífitas vasculares em diferentes estágios serais. Floresta, v. 39, n. 1, p. 51-66, 2009.

KREUTZ, C.; ATHAYDE-FILHO, F. P.; FERNANDES, L. R.; FORSTHOFER, M. Análise da distribuição espacial da flora pteridofítica em dois córregos da Bacia do Rio Pindaíba, MT. In: JORNADA CIENTÍFICA DA UNEMAT, 2., 2009, Barra do Bugres. Anais... Barra do Bugres: Unemat, 2009. 1 CD-ROM.

KREUTZ, C.; ATHAYDE-FILHO, F. P. Aspectos ecológicos da Flora Pteridofítica do Córrego Caveira, Barra do Garças - MT. In: CONGRESSO DE ECOLOGIA DO BRASIL, 9., São Lourenço. Anais... São Lourenço, 2009. p. 1-4.

LORSCHEITTER, M. L.; ASHRAF, A. R.; WINDSCH, P. G.; MOSBRUGGER, V. Pteridophyte spores of Rio Grande do Sul flora, Brazil. Part II. Palaeontographica, v. 251, p. 71-235. 1999.

MALLMANN, I. T.; SCHMITT, J. L. Riqueza e composição florística da comunidade de samambaias na mata ciliar do Rio Cadeira, Rio Grande do Sul, Brasil. Ciência Florestal, v. 24, n. 1, p. 97-109, 2014. http://dx.doi.org/10.5902/1980509813327

MCDONNELL, M. J.; PICKETT, S. T. A. Ecosystem structure and function along urbanrural gradient: an unexploited opportunity for ecology. Ecology, v. 71, n. 4, p. 12321237, 1990. http://dx.doi.org/10.2307/1938259

MORAN, R. C. Diversity, biogeography, and floristics. In: RANKER, T. A.; HAUFLER, C. H. (Eds.). Biology and evolution of ferns and lycophytes. New York: Cambridge University Press, 2008. p. 367-394.

MUELLER, C. C. Gestão de matas ciliares. In: LOPES, I. V.; BASTOS FILHO, G. S.; BILlER, D; BALE, M. (Orgs.). Gestão Ambiental no Brasil: experiência e sucesso. Rio de Janeiro: Fundação Getúlio Vargas, 1996. p. 185-214. 
MYNSSEN, C. M.; WINDISCH, P. G. Pteridófitas da Reserva Rio das Pedras, Mangaratiba, RJ, Brasil. Rodriguésia, v. 55, n. 85, p. 125-156, 2004.

http://www.jstor.org/stable/23497603

OLIVEIRA-FILHO, A. T.; RATTER, J. A. A. Study of the origin of central Brazilian forests by the analysis of plant species distribution patterns. Edinburg Journal of Botany, v. 52, n. 2, p. 141-194, 1995. http://dx.doi.org/10.1017/S0960428600000949

PACIENCIA, M. L. B.; PRADO, J. Distribuição espacial da assembléia de pteridófitas em uma paisagem fragmentada de Mata Atlântica no sul da Bahia, Brasil. Hoehnea, v. 32, n. 1, p. 103-117, 2005.

PEEL M. C; FINLAYSON B. L.; MCMAHON T. A. Updated world map of the KöppenGeiger climate classification. Hydrology and Earth System Science, v. 11, $n$. 5, p. 1633-1644, 2007.

PRADO, J.; SYLVESTRE, L. Samambaias e licófitas. In: INSTITUTO DE PESQUISAS JARDIM BOTÂNICO DO RIO DE JANEIRO. Lista de espécies da flora do Brasil. Rio de Janeiro, 2015. Disponível em: http://reflora.jbrj.gov.br/jabot/floradobrasil/ FB128483>. Acesso em: jul. 2015.

RIO GRANDE DO SUL. Decreto Estadual no 52.109, de 01 de dezembro de 2014. Declara as espécies da flora nativa ameaçadas de extinção do Estado do Rio Grande do Sul. Diário Oficial [do] Estado, Porto Alegre, n. 223, 02 dez. 2014, p. 2-11.

RIO GRANDE DO SUL. Secretaria do Ambiente e Desenvolvimento Sustentável - SEMA. Bacia hidrográfica do Rio Caí. 08 set. 2010. Disponível em: http://www.sema.rs.gov.br/conteudo.asp?cod_menu=56\&cod_conteudo=5864. Acesso em: jul. 2015.

RODRIGUES, A. S. L.; CASTRO, P. T. A. Protocolos de avaliação rápida: instrumentos complementares no monitoramento dos recursos hídricos. Revista Brasileira de Recursos Hídricos, v. 13, n. 1, p. 161-170, 2008.

RODRIGUES, R. R.; GANDOLFI, S. Conceitos, tendências e ações para a recuperação de florestas ciliares. In: RODRIGUES, R. R.; LEITÃO FILHO, H. F. Matas ciliares: conservação e recuperação. São Paulo: EDUSP/FAPESP, 2000. p. 235-248.

RODRIGUES, R. R.; NAVE, A. G. Heterogeneidade florística das matas ciliares. In: RODRIGUES, R. R.; H. F. LEITÃO-FILHO. Matas Ciliares: conservação e recuperação. São Paulo: EDUSP/FAPESP, 2000. p. 45-71.

RODRIGUES, A. S. L.; CASTRO, P. T. A.; MALAFAIA, G. Utilização dos protocolos de avaliação rápida de rios como Instrumentos complementares na gestão de bacias hidrográficas envolvendo aspectos da geomorfologia fluvial: uma breve discussão. Enciclopédia Biosfera, v. 6, n. 11, p. 1- 9, 2010.

http://www.repositorio.ufop.br/handle/123456789/4162

SALINO, A. Estudos Taxonômicos na família Thelyptetidaceae (Polypodiopsida) no estado de São Paulo, Brasil. 2000. 327f. Tese (Doutorado em Biologia Vegetal) Instituto de Biologia, Universidade Estadual de Campinas, Campinas, 2000. 
SARMENTO, E. C.; WEBER, E.; HASENACK, H. Avaliação da cobertura vegetal na microbacia Feitoria/Cadeia utilizando técnicas de geoprocessamento. 2001. Disponível em: http://www.ecologia.ufrgs.br/labgeo/artigos/cadeia.pdf. Acesso em Julho de 2015.

SCHMITT, J. L.; SCHNEIDER, P. H.; WINDISCH, P. G. Crescimento do cáudice e fenologia de Dicksonia sellowiana Hook. (Dicksoniaceae) no sul do Brasil. Acta Botanica Brasilica, v. 23, n. 1, p. 289-291, 2009. http://dx.doi.org/10.1590/S010233062009000100030

SILVA, I. A. A.; PEREIRA, A. F. N.; BARROS, I. C. L. Edge effects on fern community in an Atlantic Forest remnant of Rio Formoso, PE, Brazil. Brazilian Journal of Biology, v. 71, n. 2, p. 421-430, 2011. http://dx.doi.org/10.1590/S1519-69842011000300011

SILVA, V. L. Efeito de borda em Floresta com Araucária com diferentes graus de perturbação antrópica: uma análise da comunidade de samambaias e licófitas, de fatores microclimáticos e edáficos. 2013. 51f. Dissertações (Mestrado em Qualidade Ambiental) - Universidade Feevale, Novo Hamburgo, 2013.

SMITH, A. R.; PRYER, K. M.; SCHUETTPELZ, E.; KORALL, P.; SCHNEIDER, H.; WOLF, P. G. Fern classification. In: RANKER, T. A.; HAUFLER, C. H. (Eds.). Biology and Evolution of Ferns and Lycophytes. Cambridge: Cambridge University Press, 2008. p. 417-467.

THOMAZ, S. M.; PAGIORO, T. A.; BINI, L. M.; ROBERTO, M. C.; ROCHA, R. R. A. Limnology of the Upper Paraná Floodplain habitats: patterns of spatio-temporal variations and influence of the water levels. In: AGOSTINHO, A. A.; RODRIGUES, L.; GOMES, L.C.; THOMAZ, S. M.; MIRANDA, L. E. Structure and functioning of the Paraná River and its floodplain. Maringá: EDUEM, 2004. V. 1. p. 37-42.

THOMAZ, S. M. Fatores ecológicos associados à colonização e ao desenvolvimento de macrófitas aquáticas e desafios de manejo. Planta Daninha, v. 20, p. 21-33, 2002. http://dx.doi.org/10.1590/S0100-83582002000400003

TRYON, R. M.; TRYON, A. F. Ferns and allied plants with special reference to Tropical America. New York: Springer Verlag, 1982. 857p.

VUONO, Y. S. Inventário fitossociológico. In: SYLVESTRE, L. S.; ROSA, M. M. T. (Orgs.). Manual metodológico para estudos botânicos na Mata Atlântica. Rio de Janeiro: Seropédica, 2002. p. 51-65.

WINDISCH, P. G. Hymenophyllaceae (polypodiopsida) no estado do Rio Grande do Sul. Pesquisas, Botânica, v. 65, p. 15-48, 2014.

WINDISCH, P. G. Pteridófitas da região norte-ocidental do estado de São Paulo: guia para excursões. 2. ed. São José do Rio Preto: Universidade Estadual Paulista, 1992. $110 \mathrm{p}$.

WINDISCH, P. G. Towards assaying biodiversity in Brazilian pteridophytes. In: BICUDO, C. E. M.; MENEZES, N. A. (Eds.). Biodiversity in Brazil: a first approach. São Paulo: CNPq. 1996. p. 109-117. 


\begin{tabular}{|} 
Ambiente \& Água - An Interdisciplinary Journal of Applied Science \\
ISSN 1980-993X - doi:10.4136/1980-993X \\
www.ambi-agua.net \\
E-mail: ambi.agua@gmail.com
\end{tabular}

\title{
Mapa de aptidão do solo para a aplicação de lodo de esgoto: Bacia dos rios Piracicaba, Capivari e Jundiaí
}

\author{
doi:10.4136/ambi-agua.1714 \\ Received: 20 Jul. 2015; Accepted: 08 Dec. 2015 \\ Rodrigo Custódio Urban*; Ricardo de Lima Isaac \\ Universidade Estadual de Campinas (UNICAMP), Campinas, SP, Brasil \\ Faculdade de Engenharia Civil, Arquitetura e Urbanismo (FEC) \\ Departamento de Saneamento e Ambiente \\ *Autor correspondente: e-mail: rodrigo.urban@yahoo.com.br, \\ isaac@fec.unicamp.br
}

\section{RESUMO}

Considerando o problema técnico-econômico da disposição final de lodos gerados em estações de tratamento de esgoto, o presente trabalho teve como objetivo apresentar um mapa de aptidão do solo à recepção de lodo de esgoto da bacia dos Rios Piracicaba, Capivari e Jundiaí (PCJ). A bacia PCJ está localizada nos Estados de São Paulo (maior parte) e Minas Gerais. Caracteriza-se por apresentar grandes centros urbanos, importantes polos industriais e agrícolas, e constante crescimento populacional. Foram consideradas 6 classes de restrição da área ao uso de lodo de esgoto. Para definição das classes de restrição foram usados dados de relevo, susceptibilidade à erosão e tipo de solo, além de critérios relativos ao uso do solo, como presença de unidades de conservação, cursos d’água e áreas urbanas. Os mapas gerados demonstraram que menos de $30 \%$ do solo da bacia PCJ é apto à recepção de lodo de esgoto (conforme as classes de restrição de 0 a 3). Mesmo considerando apenas as classes de restrição de 0 a 2, foi possível observar que as áreas tem grande capacidade de recepção de

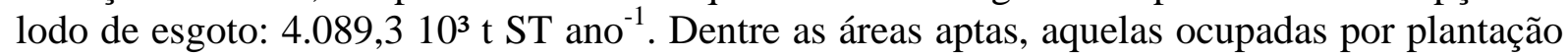
de cana de açúcar e reflorestamento foram identificadas como preferenciais à recepção. As áreas de reflorestamento aptas cobrem menos de 0,15 \% da área da bacia PCJ, mas podem ser úteis para as estações de tratamento de esgoto da parte oeste. Já as áreas cultivadas por cana de açúcar tem capacidade de recepção anual de lodo de esgoto de 2.582,7 $10^{3}$ t ST.

Palavras-chave: biossólidos, gerenciamento de lodo, resíduos de saneamento, SIG, uso do solo.

\section{Land feasibility map for sewage sludge application: watershed of the Piracicaba, Capivari and Jundiaí Rivers - Brazil}

\section{ABSTRACT}

Considering the technical and economic characteristics of the final disposal of sewage sludge, this paper sought to develop a land feasibility map for sewage sludge (biosolids) application in the watershed of the Piracicaba, Capivari and Jundiaí (PCJ) Rivers. The PCJ watershed is located in the States of São Paulo and Minas Gerais, and is characterized by large urban areas, important industrial and agricultural areas, and steady population growth. Six restricted classes of soils to receive biosolid applications were considered. The criteria 
used were susceptibility to erosion, soil type, and restrictive criteria as protected areas, water courses and urban areas. The maps generated have shown that less than $30 \%$ of the soil of the PCJ watershed is suitable to biosolids application. Considering only the classes of restriction 0,1 and 2, it was observed that the areas have great potential for biosolids application: 4,089.3 $10^{3} \mathrm{t}$ TS year-1. The sugar cane crop and reforestation areas were considered a priority to receive applications among suitable areas. Suitable reforestation areas cover less than $0.15 \%$ of the area of the PCJ watershed, but can be useful for the treatment stations of the west region. The areas covered by sugar cane have an annual sewage sludge reception capacity of $2,582.710^{3} \mathrm{t}$ TS.

Keywords: biosolids, GIS, land use, sanitation waste, sludge management, wastewater sludge.

\section{INTRODUÇÃO}

A disposição final dos lodos gerados em estações de tratamento de esgoto (ETE) urbanos é um problema crescente em regiões altamente urbanizadas. Os processos biológicos e físicoquímicos aplicados no tratamento de esgoto demandam destinação final dos resíduos sólidos gerados. O volume de lodo gerado em ETE gira em torno de $1 \%$ a $2 \%$ do volume total de esgoto tratado, entretanto, o tratamento e disposição final podem representar até $50 \%$ dos custos operacionais (Andreoli, 1999).

O gerenciamento de resíduos sólidos no Brasil teve um acréscimo de restrições legais após a publicação da Política Nacional dos Resíduos Sólidos - PNRS (Brasil, 2010). A destinação final ambientalmente correta de resíduos sólidos passa a ser um requisito para os geradores, incluindo as empresas de saneamento. Entretanto, os gestores têm dificuldades em encontrar áreas e alternativas que sejam economicamente viáveis e sustentáveis.

Andreoli (1999) considera a reciclagem agrícola do lodo de ETE como a solução mais adequada, pois reduz a utilização de recursos naturais e previne as vias inadequadas de destinação. Pesquisas ao redor do mundo indicam os possíveis benefícios do uso de lodo de ETE na agricultura: aumento da produtividade, melhora da qualidade da cultura e melhora das características físico-químicas e biológicas do solo (Andreoli, 1999; Murray et al., 2008; Singh e Agrawal, 2008; Kelessidis e Stasinakis, 2012; Lu et al., 2012). Entretanto, existem ressalvas em relação ao seu uso, para que não haja danos à saúde pública ou mesmo ao ambiente. Alguns trabalhos indicam os metais pesados, patógenos e micropoluentes orgânicos como os principais contaminantes presentes no lodo de ETE. (Pasda et al., 2006; Singh e Agrawal, 2008; Clarke e Smith, 2011; Kelessidis e Stasinakis, 2012).

Apesar da aplicação do lodo de ETE na agricultura acontecer a algumas décadas no mundo (Lu et al., 2012), a regulamentação da aplicação do lodo de esgoto no Brasil ocorre nos Estados do Paraná e de São Paulo durante a década de 1990 e no nível federal apenas em 2006 (Andreoli et al., 2008).

Os limites apresentados nas normas que regulamentam a reciclagem agrícola de lodo de ETE variam de país para país, mas, no geral, buscam a segurança sanitária desse material. Mais ou menos restritivas, as normas abrangem limites para os já citados metais pesados e microrganismos patogênicos (Beecher, 2008). Algumas normas também podem apresentar limites para a taxa de aplicação, critérios para monitoramento, controle de vetores e de emissão de odores (Lu et al., 2012).

Além da regulamentação dos limites de contaminantes permitidos, a aplicação do lodo de esgoto no solo agrícola deve considerar aspectos como a distância segura de corpos d'água e áreas residenciais, cultura recomendada para o uso e aptidão dos solos para o seu recebimento (Andreoli et al., 2001; CONAMA, 2006).

Considerando a reciclagem do lodo de ETE como uma alternativa viável, desde que as 
normas para segurança de saúde pública e ambiental sejam cumpridas, e visando facilitar a tomada de decisão dos gestores, o presente estudo tem como objetivo organizar um mapa de aptidão do solo à recepção de lodos de ETE em uma importante região do Estado de São Paulo: a bacia dos rios Piracicaba, Capivari e Jundiaí.

\section{MATERIAL E MÉTODOS}

\section{1. Área de estudo}

A bacia dos rios Piracicaba, Capivari e Jundiaí (PCJ) tem área total de 15303,67 km², sendo 92,6 \% localizados dentro do Estado de São Paulo e 7,4 \% no Estado de Minas Gerais. As bacias hidrográficas situam-se entre os meridianos $46^{\circ}$ e $49^{\circ} \mathrm{O}$ e latitudes $22^{\circ}$ e $23,5^{\circ} \mathrm{S}$ (COBRAPE, 2011).

Setenta e seis municípios estão, total ou parcialmente, dentro da bacia PCJ. Desses, 63 possuem sede administrativa dentro de áreas da bacia, totalizando uma população de 5.268.798 habitantes em 2010. A população urbana corresponde a 96,13\% dos habitantes da área. A cobertura do abastecimento de água alcança $96 \%$ na área dos municípios das bacias PCJ. A coleta de esgotos tem percentual médio de atendimento de 84,9\%. As maiores deficiências, em relação ao saneamento, são apresentadas no tratamento de esgotos, com índice médio de 41,8\% (COBRAPE, 2011).

Os usos do solo predominantes na região são o cultivo de cana-de-açúcar (33,61 \% da área) e as áreas de pastagem (39,06 \% da área). Por se tratar de uma região extensa e com relativa variação de relevo e geologia, existem vários tipos de solos na área de estudo (COBRAPE, 2011).

\subsection{Mapa de aptidão do solo à recepção de lodo de esgoto}

Para a organização do mapa de aptidão do solo à recepção de lodo de esgoto, foi adaptada a metodologia de Andreoli et al. (2000) e Souza et al. (2008), acrescentando critérios restritivos de uso do solo, considerados pertinentes ao estudo e respeitando as indicações da resolução 357 (CONAMA, 2006). A metodologia consiste na adoção de graus de restrição (0 a 5) para critérios relacionados às características do solo e de uso do solo. Mapas preliminares foram criados para cada critério, no formato de arquivo matricial. Foi realizada a sobreposição de todos os mapas elaborados, sendo atribuído a cada pixel o maior valor de grau dos critérios superpostos. O sistema de classificação é apresentado na Tabela 1. Todo o procedimento foi realizado em ambiente SIG. A base de dados foi obtida em Biociclo (2012).

A taxa de aplicação do lodo de esgoto no solo dependerá: do seu conteúdo em nutrientes, não devendo gerar aportes de nitrogênio superiores às quantidades necessárias ao desenvolvimento das culturas receptoras (Andreoli et al., 2001; CONAMA, 2006); da elevação do pH provocado pelo lodo de esgoto, que não deve ultrapassar o limite de 7,0 na mistura lodo-solo (CONAMA, 2006); e da concentração de substâncias inorgânicas, que não deverão exceder as indicações da resolução 375 (CONAMA, 2006).

Conforme a resolução 375 (CONAMA, 2006), a taxa de aplicação de lodo de esgoto depende do quociente entre a quantidade de nitrogênio recomendado para a cultura e o teor de nitrogênio disponível no lodo de esgoto. Entretanto, nem todas as ETE disponibilizam os dados de nitrogênio Kjeldahl, amoniacal, nitrato e nitrito. Por isso, para uma estimativa conservadora da capacidade de recepção, foi considerado metade do valor obtido para o solo mais restritivo estudado por Marin et al. (2010) no estado do Paraná. Foi utilizada metade do valor para considerar áreas com menor capacidade de recepção. O referido estudo utilizou o critério do poder corretivo de acidez do solo. Assim a capacidade anual de recepção considerada foi de 15 toneladas de matéria seca por hectare. 
Tabela 1. Critérios para classificação da aptidão do solo à recepção de lodo de esgoto. Critérios adaptados de Andreoli et al. (2000) e Souza et al. (2008).

\begin{tabular}{|c|c|c|}
\hline Critério & Restrição & Características \\
\hline \multirow{3}{*}{ Profundidade } & 0 -nula & Latossolos, cambissolos ou argissolos profundos. \\
\hline & 2-moderado & $\begin{array}{l}\text { Cambissolos ou argissolos com citação de pouca } \\
\text { profundidade }\end{array}$ \\
\hline & 3-forte & $\begin{array}{l}\text { Neossolos litólicos ou outras unidades com citação de solos } \\
\text { rasos }\end{array}$ \\
\hline \multirow{5}{*}{ Textura superficial } & 0 -nula & Textura argilosa (35-60 \% de argila) \\
\hline & 1 fraco & Textura muito argilosa (> $60 \%$ de argila) \\
\hline & 1-ігасо & Textura média (15-35 \% de argila) \\
\hline & 2-moderada & Textura siltosa ( $<35 \%$ de argila e $<15 \%$ de areia) \\
\hline & 3-forte & Textura arenosa $(<15 \%$ de argila) \\
\hline \multirow{5}{*}{ Susceptibilidade à erosão } & 0 -nula & Solos em relevo plano \\
\hline & 1-fraca & Solos argilosos ou muito argilosos em relevo suave ondulado \\
\hline & 2-moderada & $\begin{array}{l}\text { Textura média ou siltosa em relevo suave ondulado e textura } \\
\text { argilosa em relevo ondulado }\end{array}$ \\
\hline & 3-forte & $\begin{array}{l}\text { Relevo ondulado com textura arenosa e/ou caráter abrupto. } \\
\text { Relevo forte ondulado com textura muito argilosa }\end{array}$ \\
\hline & 4-muito forte & $\begin{array}{l}\text { Relevo forte ondulado, com textura média e arenosa. Relevo } \\
\text { montanhoso ou escarpado independente da classe textural. }\end{array}$ \\
\hline \multirow{5}{*}{ Relevo } & 0 -nula & Relevo plano (0-3 \%) \\
\hline & 1-fraco & Relevo suave ondulado (3-8 \%) \\
\hline & 2-moderada & Relevo ondulado (8-20 \%) \\
\hline & 3-forte & Relevo forte ondulado (20-45 \%) \\
\hline & 4-muito forte & Montanhoso ou escarpado (> 45 \%) \\
\hline \multirow[b]{2}{*}{ Hidromorfismo } & 0-nulo & Sem indicação \\
\hline & 3-forte & Hidromórfico \\
\hline \multirow{2}{*}{ Área de conservação } & 0-nulo & Área sem unidades de conservação \\
\hline & 5-total & Área de conservação \\
\hline \multirow{2}{*}{ Reservatórios } & 0-nulo & Área sem reservatório \\
\hline & 5- total & Reservatórios e área de 30 metros no entorno \\
\hline \multirow[t]{2}{*}{ Cursos d’água } & 0-nulo & Áreas sem rios \\
\hline & 5- total & Rios e respectiva área de preservação permanente \\
\hline \multirow{2}{*}{ Vulnerabilidade de aquíferos } & 0-nulo & Área sem recarga de aquíferos \\
\hline & 5- total & Área de recarga de aquíferos \\
\hline \multirow[t]{2}{*}{ Áreas urbanas } & 0 -nulo & Uso do solo diferente de urbano \\
\hline & 5- total & Uso do solo urbano \\
\hline
\end{tabular}

Foi adotado o maior valor dado ao pixel, independentemente do critério, como o valor do grau de restrição do solo. Pode-se classificar os solos, quanto aos critérios de restrição, conforme a lista a seguir, adaptada de Andreoli et al. (2001):

- Classe de restrição 0: Potencial muito alto para a aplicação de lodo de esgoto;

- Classe de restrição 1: Potencial alto para a aplicação de lodo de esgoto;

- Classe de restrição 2: Potencial moderado para a aplicação de lodo de esgoto. Devem ser recomendadas práticas rigorosas de conservação de solos, para a permissão do uso do 
material;

- Classe de restrição 3: Potencial baixo para a aplicação dos lodo de esgoto. Devem ser apresentados critérios atenuantes, como alternativas de manejo e práticas culturais. Se as medidas não forem efetivamente tomadas haverá risco;

- Classe de restrição 4: Não recomendada a aplicação do lodo de esgoto. Existe grave risco ao ambiente e à população no caso da aplicação do material;

- Classe de restrição 5: Restrição total à aplicação de lodo de esgoto.

\section{RESULTADOS E DISCUSSÃO}

Após a aplicação do método descrito anteriormente foi possível elaborar o mapa de aptidão para aplicação de lodo de esgoto na bacia PCJ (Figura 1).

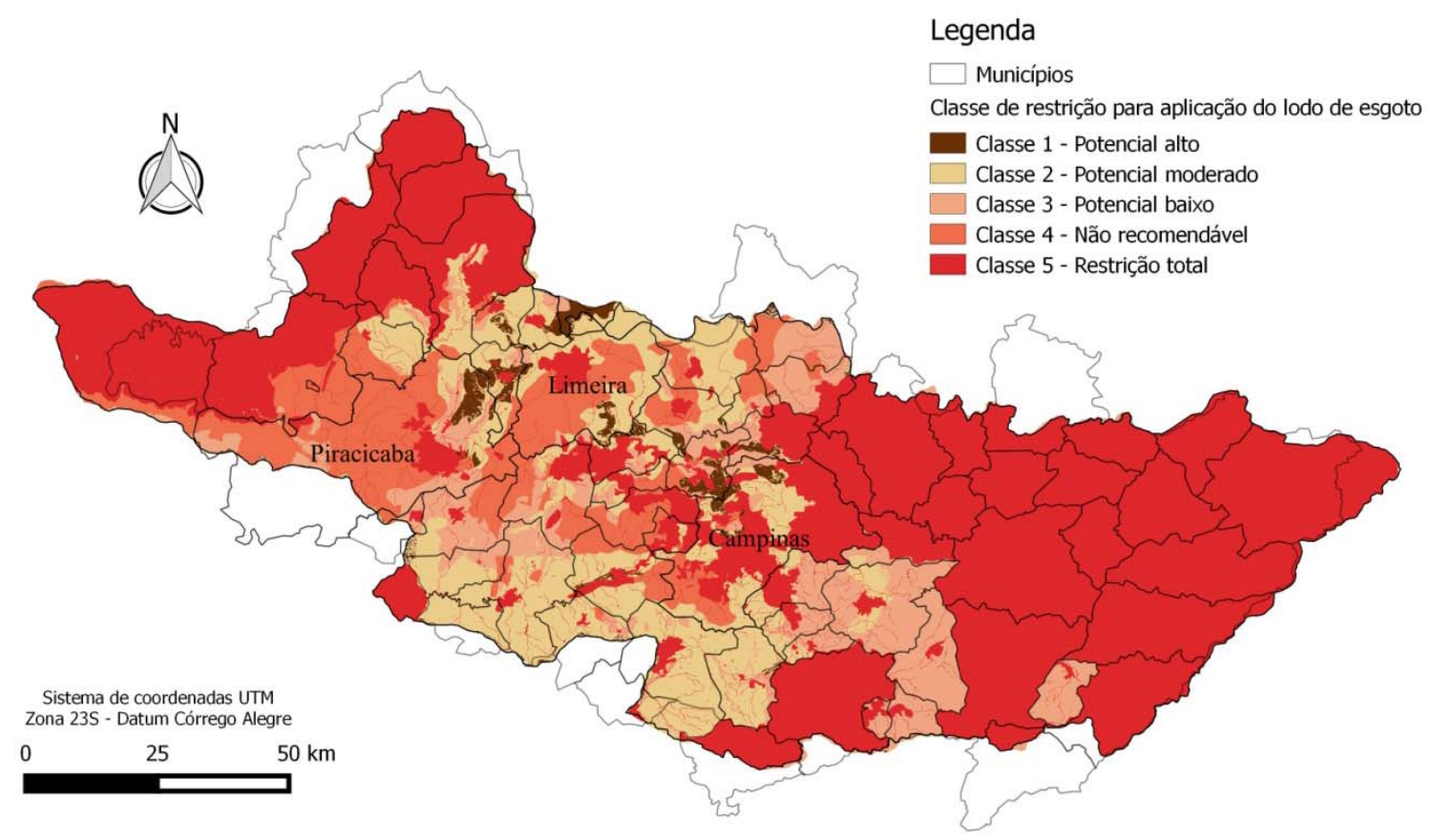

Figura 1. Mapa de aptidão do solo da bacia PCJ à aplicação de lodo de esgoto.

A partir do mapa pode-se perceber que a bacia PCJ tem grande parte de sua área proibida (classe 5) à aplicação de lodo de esgoto. Isso se deve principalmente a localização de quatro grandes áreas de preservação ambiental (APA): APA Corumbataí na parte leste da bacia, na parte oeste a APA Piracicaba - Juqueri Mirim Área 2, a APA Fernão Dias e a APA Sistema Cantareira. Enfatiza-se aqui que a resolução 375 (CONAMA, 2006) permite a aplicação de lodo de esgoto em APA, desde que estas não protejam mananciais de abastecimento. Entretanto, para evitar riscos ambientais, preferiu-se uma abordagem mais conservadora, excluindo-se todas as APA das áreas aptas à recepção de lodo de esgoto.

As áreas urbanas das grandes cidades da região (Campinas, Piracicaba e Limeira) também contribuíram para as áreas proibidas à aplicação de lodo de esgoto. É importante apontar essas cidades porque são elas também as maiores geradoras de lodo de ETE na bacia.

Não existem áreas na bacia PCJ com classe de restrição 0 , que seriam aqueles solos muito aptos a receberem o lodo de esgoto.

Conforme a lista baseada em Andreoli et al. (2001), as classes de restrição 4 e 5 não são adequadas para a recepção de lodo. Para tornar as áreas aptas mais visíveis foi feito um recorte do mapa apresentado na Figura 1. Esse novo mapa apresenta as classes de restrição 1, 
2 e 3 conjuntamente. Nessas áreas, consideradas aptas, é apresentado o uso e ocupação do solo, como última medida de restrição. A localização das ETE da bacia também é apresentada (Figura 2).

Visualmente, percebe-se no mapa, que as áreas cobertas por plantação de cana de açúcar e pastagem são predominantes nas classes de restrição 1, 2 e 3. Entretanto, a resolução 375 (CONAMA, 2006) proíbe a aplicação de lodo de esgoto em pastagem. A maior parte das ETE está concentrada nas proximidades das áreas consideradas aptas, com exceção de algumas da porção oeste da bacia PCJ.

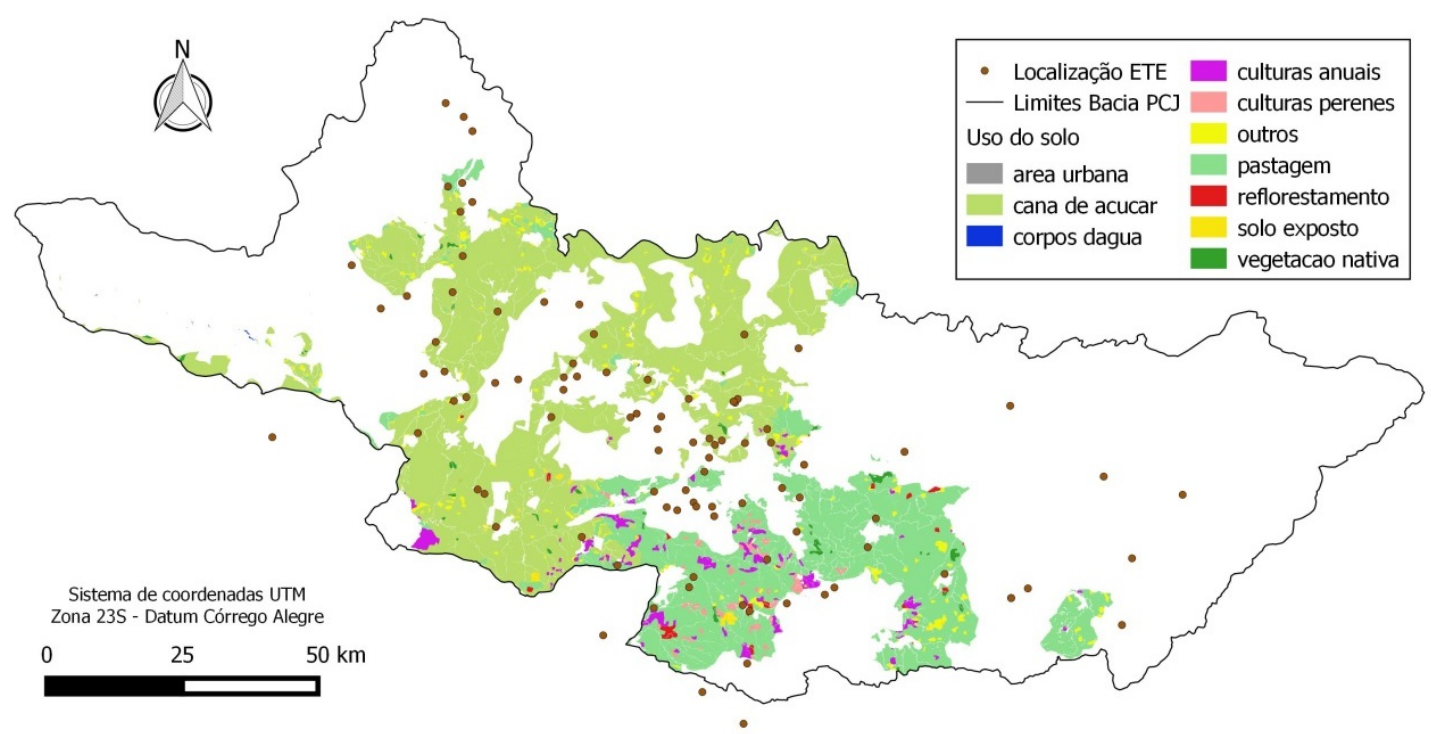

Figura 2. Uso e ocupação de áreas com classe de restrição 1, 2 e 3 à aplicação de lodo de esgoto bacia PCJ.

Entretanto, solos de classe 3 de restrição ainda tem risco de aplicação, conforme Andreoli et al. (2001). Por isso optou-se por fazer um segundo recorte, apenas com as áreas de classe 1 e 2 de restrição. Novamente as ETE foram representadas no mapa gerado (Figura 3).

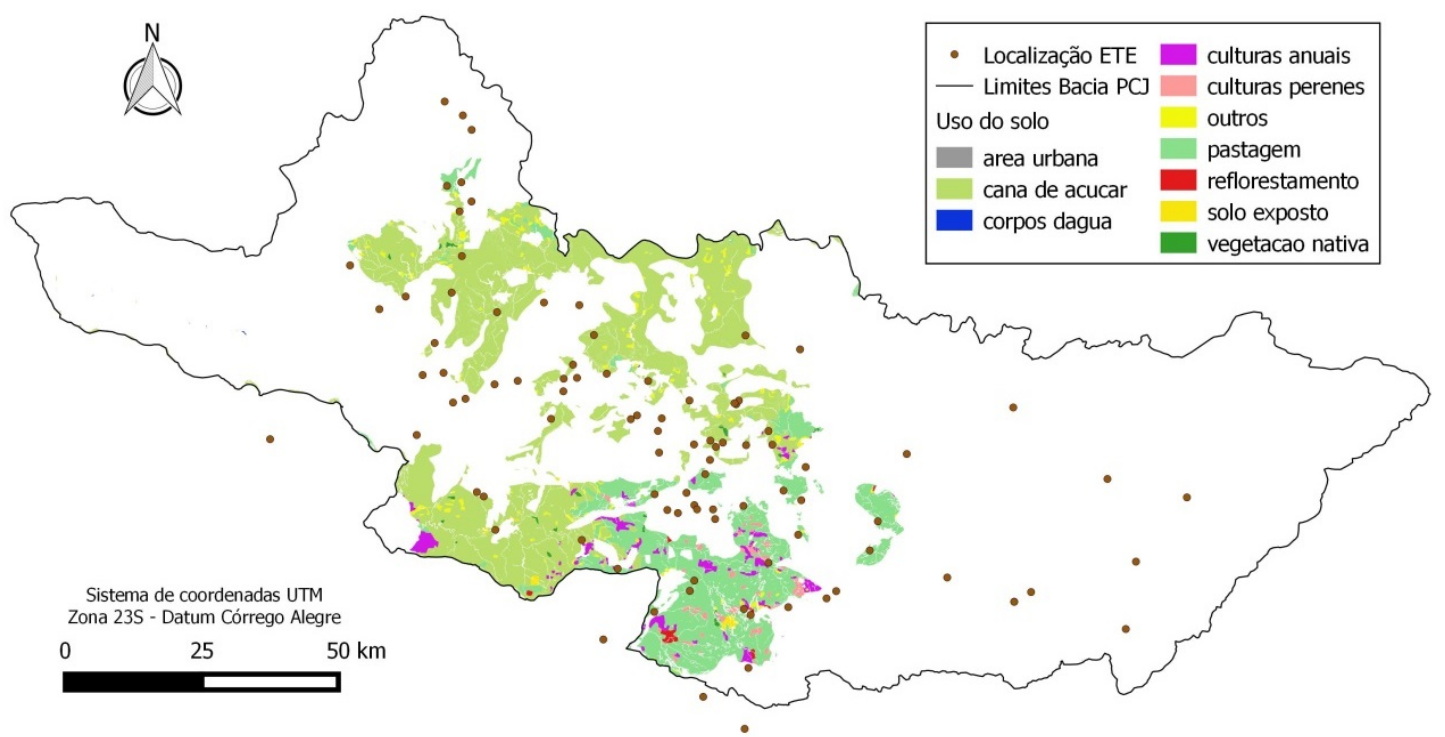

Figura 3. Uso e ocupação de áreas com classe de restrição 1 e 2 à aplicação de lodo de esgoto - Bacia PCJ. 
A análise do mapa permite observar que a área disponível para a aplicação do lodo de esgoto diminui consideravelmente com a retirada dos solos de classe 3 de restrição. As ETE da porção oeste da bacia ficam agora mais distantes dos possíveis pontos de aplicação, o que acarreta custos maiores com transporte para a disposição final.

Para confirmar as análises visuais realizadas, foram extraídas as áreas dos polígonos que caracterizam o uso e ocupação do solo das áreas com as classes de restrição 1, 2 e 3. Os valores percentuais em relação à área total da bacia PCJ são apresentados na Tabela 2.

A análise da Tabela 2 permite afirmar que a ocupação de cana de açúcar e pastagem é predominante em toda a área apta à recepção de lodo de esgoto. Novamente enfatizando a proibição da aplicação em pastagem pela resolução 375 (CONAMA, 2006). A classe de restrição 1 representa menos de $2 \%$ do total da bacia, apresentando pouca opção de área receptiva. A classe de restrição 2 cobre área maior que a classe de restrição 3, e deve ser usada prioritariamente, quando as áreas de classe 1 não estiverem disponíveis.

Menos de 30\% da área da bacia PCJ é apta à recepção de lodo. Esse valor diminui para menos de $17 \%$, quando considerados apenas as classes menos restritivas (1 e 2). Entretanto, a capacidade de recepção de lodo dessas áreas pode ser considerável.

Para a análise da capacidade de recepção, estimou-se que cada hectare poderia receber 15 toneladas de lodo de esgoto por ano, vide metodologia. Considerando as porcentagens da área total, tem-se uma capacidade de recepção anual, conforme a Tabela 2.

Tabela 2. Porcentagem da área ocupada e capacidade de recepção de cada uso do solo em relação ao grau de restrição à aplicação de lodo de esgoto.

\begin{tabular}{lccccccr}
\hline \multirow{2}{*}{ Classe } & \multicolumn{7}{c}{ Uso (\%) } \\
\cline { 2 - 8 } & Cana de açúcar & Culturas anuais & Culturas perenes & Pastagen & Reflores. & Outros & Total \\
\hline 1 & 1,42 & 0,01 & $<0,01$ & 0,11 & $<0,01$ & 0,06 & $\mathbf{1 , 5 9}$ \\
2 & 9,83 & 0,53 & 0,24 & 4,94 & 0,08 & 0,61 & $\mathbf{1 6 , 2 2}$ \\
3 & 5,96 & 0,17 & 0,03 & 5,24 & 0,06 & 0,54 & $\mathbf{1 2 , 0 0}$ \\
\hline Total & $\mathbf{1 7 , 2 1}$ & $\mathbf{0 , 7 0}$ & $\mathbf{0 , 2 7}$ & $\mathbf{1 0 , 2 8}$ & $\mathbf{0 , 1 4}$ & $\mathbf{1 , 2 0}$ & $\mathbf{2 9 , 8 1}$ \\
\hline \multicolumn{7}{c}{ Capacidade de recepção do uso $\left(10^{3}\right.$ t) } \\
\hline 1 & 325,9 & 1,2 & 0,7 & 24,6 & 0,1 & 13,0 & $\mathbf{3 6 5 , 5}$ \\
2 & $2.256,8$ & 121,1 & 54,6 & $1.133,2$ & 18,0 & 140,1 & $\mathbf{3 . 7 2 3 , 8}$ \\
3 & $1.367,1$ & 39,0 & 7,3 & $1.202,5$ & 14,5 & 123,1 & $\mathbf{2 . 7 5 3 , 6}$ \\
\hline Total & $\mathbf{3 . 9 4 9 , 8}$ & $\mathbf{1 6 1 , 3}$ & $\mathbf{6 2 , 6}$ & $\mathbf{2 . 3 6 0 , 3}$ & $\mathbf{3 2 , 6}$ & $\mathbf{2 7 6 , 3}$ & $\mathbf{6 . 8 4 2 , 9}$ \\
\hline
\end{tabular}

A classe de restrição 1, mesmo ocupando menos de 2 \% da área da bacia, ainda é capaz de receber 365,5 mil toneladas de lodo de esgoto anualmente. Com esta capacidade seria possível receber o equivalente ao lodo produzido por 7,5 cidades do porte de Curitiba, conforme dados apresentados por Pegorini et al. (2003).

Deve-se observar, assim como as pastagens, que nem todos os usos do solo representados podem receber o lodo de esgoto, apesar de serem considerados aptos. A resolução 375 (CONAMA, 2006) indica que, além da pastagem, a aplicação de lodo de esgoto é proibida em "cultivo de olerícolas, tubérculos e raízes e culturas inundadas, além de outras culturas cuja parte comestível entre em contato com o solo". Se houver a necessidade de cultivar as culturas citadas deve-se respeitar o prazo de 4 anos da última aplicação de lodo de esgoto. Isso acaba inviabilizando a aplicação nas culturas anuais ou mesmo nas perenes.

Andreoli et al. (2001) recomendam a aplicação de lodo de esgoto em "grandes culturas cujos produtos são industrializados ou não são consumidos in natura". Dessa forma, pode-se inserir nessa definição as áreas de cana de açúcar, que são aquelas que têm maior capacidade 
de recepção de lodo de esgoto (mais de $3.90010^{3}$ t ST ano ${ }^{-1}$ ). Conforme os mesmo autores, também é viável a aplicação em reflorestamento. Essa opção deve ser considerada, apesar de ocupar menos de 0,15 \% da área da bacia PCJ, pois pode ser um destino para as ETE que se encontram na parte oeste da bacia, e mais distantes das áreas de cana de açúcar. Os usos citados não desrespeitam as indicações da resolução 375 (CONAMA, 2006).

Considerando apenas as classes de restrição do solo que permitam a aplicação de lodo de esgoto, restringiram-se as áreas de plantação de cana de açúcar e reflorestamento para a recepção de lodo. Juntas essas áreas ocupam 17,36 \% da área total da bacia PCJ, e tem

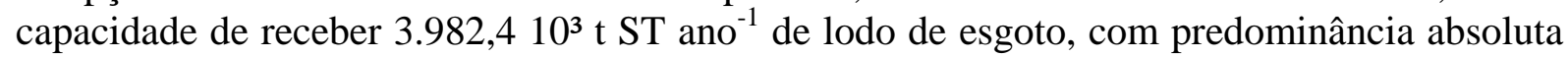
das áreas com cana de açúcar.

Excluindo a classe de restrição 3, pois demanda maior cuidado para a aplicação do lodo de esgoto, as áreas de cana de açúcar da bacia PCJ passam a ter capacidade de recepção de

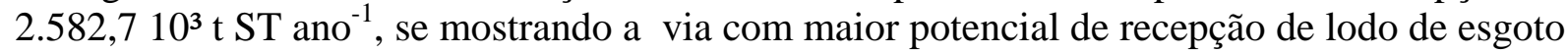
na área de estudo.

Uma importante ressalva sobre o uso das áreas de pastagem para a aplicação de lodo de esgoto deve ser feita, devido à possibilidade de receber pouco mais de $2.300 \mathrm{mil} \mathrm{t} \mathrm{ano}^{-1}$. Apesar de proibida a aplicação direta, existe a possibilidade de aplicação do lodo de esgoto e posterior implantação da pastagem. Entretanto, a entrada de animais deve ocorrer após 2 anos da última aplicação do lodo de esgoto (CONAMA, 2006), tornando essa opção pouco viável.

\section{CONCLUSÃO}

A metodologia adaptada para a elaboração do mapa de aptidão do solo à aplicação de lodo de esgoto da bacia PCJ se mostrou coerente e aplicável. O mapa gerado para a área de estudo pode ser usado como consulta auxiliar na tomada de decisão dos gestores das empresas de saneamento da bacia. A metodologia pode ser utilizada em outras áreas, desde que sejam consideradas, e incluídas nos critérios, as especificidades legais regionais.

Recomenda-se a aplicação do lodo de esgoto nos solos cultivados com cana de açúcar e reflorestamento. Considerando os usos citados e apenas as duas menores classes de restrição, 11,33\% da área da bacia PCJ é considerada apta à recepção de lodo, com uma capacidade estimada de recepção de 2600,8 mil t ST ano-1 ${ }^{-1}$ conforme o critério de limitação da quantidade de lodo pela alteração do $\mathrm{pH}$ do solo.

Os mapas apresentados são de abrangência regional, e devem ser usados em uma avaliação preliminar dos locais aptos à recepção. Estudos complementares devem ser realizados para a seleção dos locais específicos para a aplicação do lodo de esgoto de cada ETE. Para cada local escolhido deve-se fazer o cálculo da capacidade de recepção anual, considerando as características específicas do lodo da ETE e do solo onde este será lançado.

\section{REFERENCIAS}

ANDREOLI, C. V. (Coord.). Uso e manejo do lodo de esgoto na agricultura. Curitiba: SANEPAR $\backslash$ PROSAB, 1999.

ANDREOLI, C. V.; PEGORINI, E. S.; CASTRO, L. A. R. Diagnóstico do potencial dos solos da região de Maringá para a disposição final do lodo gerado pelos sistemas de tratamento de esgoto do município. Sanare - Revista Técnica da Sanepar, v. 13, n. 13, p. 40-50, 2000.

ANDREOLI, C. V.; PEGORINI, E. S.; FERNANDES, F. Disposição do lodo no solo. In: ANDREOLI, C. V.; VON SPERLING, M.; FERNANDES, F. (Eds.). Lodo de esgotos: tratamento e disposição final. Belo Horizonte: UFMG; SANEPAR, 2001. 
ANDREOLI, C. V.; GARBOSSA, L. H. P.; LUPATINI, G.; PEGORINI, E. S. Wastewater sludge management: a brazilian approach. In: LeBLANC, R. J.; MATTHEWS, P.; RICHARD, R. P. (Eds.). Global atlas of excreta, wastewater sludge, and biosolids management: moving forward the sustainable and welcome uses a global resource. Nairobi: UN-HABITAT, 2008.

BEECHER, N. Overview: moving forward the sustainable and welcome uses a global resource. In: LeBLANC, R. J.; MATTHEWS, P.; RICHARD, R. P. (Eds.). Global atlas of excreta, wastewater sludge, and biosolids management: moving forward the sustainable and welcome uses a global resource. Nairobi: UN-HABITAT, 2008.

BIOCICLO. Estudo de viabilidade para a instalação e operação de centrais de tratamento de lodo na bacia PCJ. Americana: Consórcio PCJ, 2012.

BRASIL. Lei $n^{0}$ 12305, de 02 de agosto de 2010. Institui a Política Nacional de Resíduos Sólidos; altera a Lei no 9.605, de 12 de fevereiro de 1998; e dá outras providências. Diário Oficial [da] União, Brasília, DF, 03 ago. 2010.

COMPANHIA BRASILEIRA DE PROJETOS E EMPREENDIMENTOS - COBRAPE. Plano das bacias hidrográficas dos rios Piracicaba, Capivari e Jundiaí 2010 a 2020 - relatório final. São Paulo, 2011. 815 p.

CONAMA. Resolução no 375, de 29 de agosto de 2006. Define critérios e procedimentos, para o uso agrícola de lodos de esgoto gerados em estações de tratamento de esgoto sanitário e seus produtos derivados, e dá outras providências. Diário Oficial [da] União, Brasília, DF, 30 ago. 2006.

CLARKE, B. O.; SMITH, S. R. Review of 'emerging' organic contaminants in biosolids and assessment of international research priorities for the agricultural use of biosolids. Environment International, v. 37, n. 1, p. 226-247, 2011. http://dx.doi.org/10.1016/j.envint.2010.06.004

KELESSIDIS, A.; STASINAKIS, A. S. Comparative study of the methods used for treatment and final disposal of sewage sludge in European countries. Waste Management, v. 32, p. 1186-1195, 2012. http://dx.doi.org/10.1016/j.wasman.2012.01.012

LU, Q.; HE, Z. L.; STOFFELLA, P. J. Land application of biosolids in the USA: a review. Applied and Environmental Soil Science, v. 2012, Article ID 201462, 11 p., 2012. http://dx.doi.org/10.1155/2012/201462

MARIN, L. M. K. S.; BITTENCOURT, S.; ANDREOLI, C. V.; CARAFINI, C.; LIMA, M. R.; SERRAT, B. M. et al. Determinação da taxa de aplicação máxima anual de lodo de esgoto higienizado por processo alcalino em solos da região metropolitana de Curitiba. Engenharia Sanitária e Ambiental, v. 15, n. 2, p. 113-118, 2010. http://dx.doi.org/10.1590/S1413-41522010000200003

MURRAY, A.; HORVATH, A.; NELSON, K. L. Hybrid life-cycle environmental and cost inventory of sewage sludge treatment and end-use scenarios: a case study from China. Environmental Science \& Technology, v. 42, p. 3163-3169, 2008. http://dx.doi.org/10.1021/es702256w 
PASDA, N.; PANICHSAKPATANA, S.; LIMTONG, P.; OLIVER, R.; MONTAGE, D. Evaluation of bangkok sewage sludge for possible agricultural use. Waste $\begin{array}{llllll}\text { Management \& } & \text { Research, v. 24, p. 167-174, }\end{array}$ http://dx.doi.org/10.1177/0734242X06063347

PEGORINI, E. S.; ANDREOLI, C. V., SOUZA, M. L. P.; FERNANDES, F. DOETZER, B; FERREIRA, A. C. Produção e disposição final de lodo de esgoto na reciclagem agrícola da região metropolitana de Curitiba - PR. In: SIMPÓSIO SOBRE BIOSSÓLIDOS NO ÂMBITO DO MERCOSUL, 3., São Paulo, 2003. Anais... Rio de Janeiro: ABES; AIDIS, 2003. 1 CD-ROM.

SINGH, R. P.; AGRAWAL, M. Potential benefits and risks of land application of sewage sludge. Waste Management, v. 28, p. 347-358, 2008.

http://dx.doi.org/10.1016/j.wasman.2006.12.010

SOUZA, M. L. P.; RIBEIRO, A. N.; ANDREOLI, C. V.; SOUZA, L. C. P.; BITTENCOURT, S. Aptidão das terras do Estado do Paraná para a disposição final de lodo de esgoto. Revista DAE, n. 177, 2008. http://dx.doi.org/10.4322/dae.2014.012 


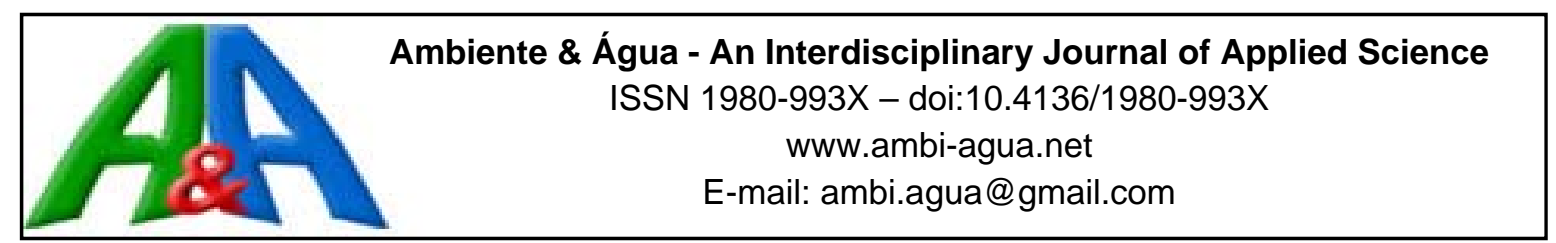

\title{
Teores de Arsênio em solos de três regiões do estado de Santa Catarina
}

\author{
doi:10.4136/ambi-agua.1746
}

Received: 03 Sep. 2015; Accepted: 17 Dec. 2015

\author{
Leticia Cristina de Souza $^{1 *}$; Mari Lucia Campos ${ }^{1}$; Gabriela Reichert ${ }^{2}$; \\ Carolina Natel de Moura ${ }^{3}$ \\ ${ }^{1}$ Universidade do Estado de Santa Catarina (UDESC), Lages, SC, Brasil \\ Departamento de Solos e Recursos Naturais \\ ${ }^{2}$ Universidade Federal do Paraná (UFPR), Curitiba, PR, Brasil \\ Departamento de Hidráulica e Saneamento \\ ${ }^{3}$ Universidade do Estado de Santa Catarina (UDESC), Lages, SC, Brasil \\ Departamento de Engenharia Ambiental \\ *Autor correspondente: e-mail: leti_quimica@yahoo.com.br, \\ mari.lucia03@gmail.com, gabrielareichertamb@gmail.com, \\ carolina.natel@hotmail.com
}

\section{RESUMO}

A determinação do teor natural de elementos-traço é essencial para monitorar a entrada de tais elementos no sistema solo e contribuir na remediação de áreas contaminadas. $\mathrm{O}$ objetivo desse trabalho foi determinar o teor natural de Arsênio em solos provenientes de três regiões do estado de Santa Catarina: Planalto Sul, Metropolitana e Litoral Sul. O teor de Arsênio foi obtido após digestão em forno de micro-ondas, seguindo o protocolo da USEPA 3051 A e quantificação em espectrometria de absorção atômica com atomização eletrotérmica. Os resultados foram analisados pelo teste Scott-Knott a 5\% de significância. Os atributos do solo que melhor se correlacionaram com os teores de Arsênio foram argila, carbono orgânico, capacidade de troca de cátions e óxidos de alumínio e de ferro. Os teores de Arsênio estão relacionados ao material de origem e ao relevo dos solos das regiões.

Palavras-chave: áreas contaminadas, elementos-traço, remediação.

\section{Arsenic content of soils from three regions of Santa Catarina State}

\section{ABSTRACT}

The determination of trace elements is necessary in order to monitor their entry into the soil system and to remediate contaminated areas. The purpose of this study was to determine the natural content of arsenic (As) in soils of three regions of Santa Catarina State (SC): the Southern Plateau, the Metropolitan area and the Southern Coast. Arsenic content was obtained after digestion in a microwave oven, following the USEPA 3051 A protocol and quantification was made by atomic absorption spectrometry with electrothermal atomization. The results were analyzed by the Scott-Knott test at a 5\% significance level. Soil attributes that best correlated with arsenic content were clay, organic carbon, cation exchange capacity and $\mathrm{Al}$ and $\mathrm{Fe}$ oxides. The arsenic levels are related to the source material and the slope of regional soils.

Keywords: contaminated areas, remediation, trace-elements. 


\section{INTRODUÇÃO}

O Arsênio (As) é o vigésimo elemento mais abundante na crosta terrestre e pertence ao grupo V da tabela periódica (Roy et al., 2015). Apresenta alta toxicidade para a saúde humana e animal e está extensamente distribuído na atmosfera, hidrosfera e biosfera, sendo associado tanto a fontes naturais, quanto antropogênicas. São fontes naturais, o intemperismo das rochas, atividades biológicas e emissões vulcânicas (Alonso et al., 2014). As fontes antropogênicas incluem herbicidas, fertilizantes fosfatados, atividades de mineração, resíduos industriais e atividades relacionadas à preservação da madeira (Chirenje et al., 2003; Alonso et al., 2014; Roy et al., 2015).

O As é um elemento calcófilo (Goldschmidt, 1958) e sua mobilidade no solo é regulada pelo seu estado de oxidação (Tarvainen et al., 2013). No solo, em condições óxicas (Eh > $200 \mathrm{mV}$; pH 5-8) o As é encontrado na forma $\mathrm{As}^{5+}$, enquanto que o $\mathrm{As}^{3+}$ é encontrado em condições anóxicas (Mcbride, 1994; Singh et al., 2015). O controle da solubilidade do As na solução do solo em condições aeróbias é feito pelas formas $\mathrm{Ca}_{3}\left(\mathrm{AsO}_{4}\right)_{2}, \mathrm{Mg}_{3}\left(\mathrm{AsO}_{4}\right)_{2}$ e $\mathrm{As}_{2} \mathrm{O}_{5}$ e em condições anaeróbias, $\mathrm{As}, \mathrm{As}_{2} \mathrm{~S}_{3}$ e $\mathrm{As}_{2} \mathrm{O}_{3}$ (Hayes e Traina, 1998).

O comportamento do arsenato no solo é semelhante ao do fosfato e vanadato (Mcbride, 1994; Rosas-Castor et al., 2014). O arsenato sofre adsorção em óxidos de $\mathrm{Fe}$ e $\mathrm{Al}$, aluminossilicatos não cristalinos e, em menor extensão, em argilossilicatos. $\mathrm{O}$ arsenato é o ânion do ácido forte $\mathrm{H}_{3} \mathrm{AsO}_{4}$ (ácido arsênico) que possui valores de pKa 2,24; 6,94 e 11,5; sendo adsorvido efetivamente em $\mathrm{pH}$ baixo (Mcbride, 1994). Os ânions $\mathrm{AsO}_{2}{ }^{-}, \mathrm{AsO}_{4}{ }^{3-}$, $\mathrm{HAsO}_{4}{ }^{2-} \mathrm{e}_{2} \mathrm{AsO}_{3}{ }^{-}$são formas móveis de As, sendo sorvidas em $\mathrm{pH}$ entre 7-9 (Mcbride, 1994). $\mathrm{O} \mathrm{As}^{3+}$ é 10 vezes mais tóxico que o $\mathrm{As}^{5+}$ (Rosas-Castor et al., 2014). O As está classificado pela Agency for Toxic Substances and Disease Registry - ATSDR (ATSDR, 2013) em primeiro lugar como a substância mais perigosa à saúde humana.

A maioria dos humanos está exposta a baixas concentrações de As, principalmente através de ingestão de alimentos e água (Singh et al., 2015). A ingestão de água contaminada com As talvez seja a via mais comum de intoxicação de seres humanos (Fitz e Wenzel, 2002). Entretanto, Zakharova et al. (2002) observaram que a exposição a As via ingestão de produtos agrícolas, contato com a pele e ingestão de solo resultam em alto risco à saúde. A ingestão de solo pode não ser uma via significante de contaminação para os adultos, porém pode ser considerável para crianças, particularmente em locais próximos a indústrias ou a locais que recebem descarte de resíduos de mineração e outros (WHO, 2001).

A importância de se conhecer os teores naturais de elementos-traço no solo, sem interferência humana, é necessária para o monitoramento e a remediação de áreas contaminadas e, além disso, contribui para o entendimento da magnitude dos riscos que a população está exposta diariamente (Tsuji et al., 2007). O acúmulo desses elementos, seja ele causado por atividades agrícolas ou industriais é preocupante, devido a uma possível transferência desses elementos para animais e seres humanos (Su e Yang, 2008).

A litologia e os processos de formação do solo estão interligados com os teores de elementos-traços no solo, sendo comum comparar a composição química do solo com o material de origem na avaliação de um solo contaminado (Parra et al., 2014). Para monitorar a contaminação do solo por um elemento, primeiramente, é necessário determinar a concentração do mesmo no solo em seu estado natural (Tarvainen et al., 2013) e, assim, obter os VRQ (Valor de Referência de Qualidade) dos solos (Biondi et al., 2011; Mendoza-Grimon et al., 2014). Os solos apresentam características específicas determinadas pela geologia, geomorfologia e clima, que determinam os processos pedogenéticos regionais, o que torna inadequado o uso dos valores genéricos para países e áreas diferentes do local de obtenção dos dados (Biondi et al., 2011). 
A determinação dos teores de As no estado de Santa Catarina, que possui uma área territorial de $95.913 \mathrm{~km}^{2}$, representando $16,61 \%$ da Região Sul e 1,11\% da área total do Brasil (Embrapa, 1998) é de suma importância no auxílio da identificação de áreas contaminadas e no gerenciamento das mesmas.

Considerando o exposto, o objetivo desse trabalho foi determinar os teores naturais de As em solos de três regiões do estado de SC sem ação antrópica, que possam ser aplicados em estudos de monitoramento e remediação no Estado.

\section{MATERIAL E MÉTODOS}

A pesquisa foi realizada em três regiões do estado de $\mathrm{SC}$, sendo Planalto Sul, Metropolitana e Litoral Sul (Figura 1).

A classificação das regiões utilizadas nesse estudo é a indicada pela Embrapa (1998) que realizou o levantamento de reconhecimento de solos do estado de SC. Os 14 solos avaliados (Tabela 1) são provenientes de um Banco de Solos da Universidade do Estado de Santa Catarina (UDESC) e foram coletados, descritos e classificados por Paes Sobrinho (2009); Costa (2013); Ferreira (2013); Lunardi Neto e Almeida (2013) e Teske et al. (2013).

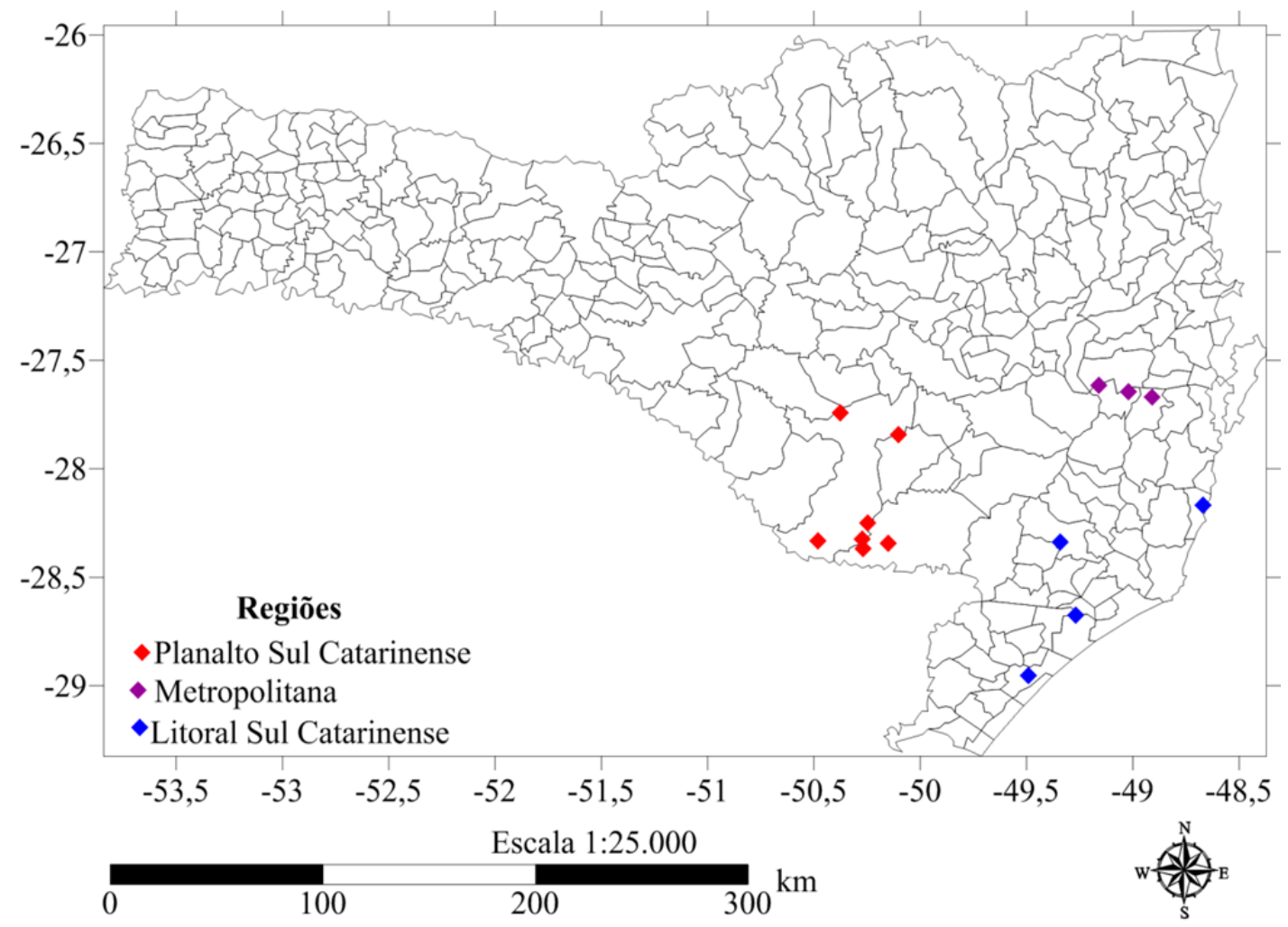

Figura 1. Mapa de Localização das Regiões Planalto Sul, Metropolitana e Litoral Sul no estado de SC.

Os perfis foram descritos e amostrados em corte de barranco de estrada, em locais sob vegetação natural de campo ou de mata, sendo que as amostras utilizadas neste estudo proveram do Horizonte A. Os mesmos autores que realizaram, descreveram e classificaram os solos deste estudo, também determinaram os atributos físicos e químicos dos solos, que se encontram na Tabela 2. 
Tabela 1. Regiões, classes pedológicas, material de origem e localização no Estado.

\begin{tabular}{|c|c|c|c|c|}
\hline Região & Classe & Material de Origem & Localização & $\begin{array}{l}\text { Profundidade } \\
\quad(\mathrm{cm})\end{array}$ \\
\hline \multirow{7}{*}{ Planalto Sul } & Nitossolo Bruno Distrófico típico - $\mathrm{NBd}^{*}$ & Basalto & Painel & $0-17$ \\
\hline & Cambissolo Húmico Distroférrico típico - CHd1 & Basalto & Lages & $0-22$ \\
\hline & Cambissolo Háplico Alumínico úmbrico - CXa & Riodacito & Lages & $0-13$ \\
\hline & Cambissolo Húmico Distrófico típico - CHd2 & Riodacito & Lages & $0-26$ \\
\hline & Nitossolo Vermelho Alítico típico - NVal & Basalto & Lages & $0-12$ \\
\hline & Cambissolo Háplico Alítico típico - Cxall & Riodacito & Lages & $0-14$ \\
\hline & Cambissolo Háplico Alítico típico - CXal2 & Riodacito & Lages & $0-12$ \\
\hline \multirow{3}{*}{ Metropolitana } & Argissolo Bruno-Acinzentado Alítico típico - PBACal & Argilitos e Siltitos & $\begin{array}{l}\text { Alfredo } \\
\text { Wagner }\end{array}$ & $0-13$ \\
\hline & Argissolo Amarelo Distrófico típico - PAd & Granito e Granulito & $\begin{array}{l}\text { Rancho } \\
\text { Queimado }\end{array}$ & $0-20$ \\
\hline & $\begin{array}{l}\text { Argissolo Vermelho-Amarelo Distrófico latossólico - } \\
\text { PVAd1 }\end{array}$ & Migmatito & $\begin{array}{l}\text { Águas } \\
\text { Mornas }\end{array}$ & $0-24$ \\
\hline \multirow{4}{*}{ Litoral Sul } & Neossolo Quartzârenico Órtico típico - Rqo1 & Sedimentos Arenosos & Imbituba & $0-28$ \\
\hline & Argissolo Vermelho Distrófico abrúptico - PVd & Siltito e Arenito & Içara & $0-10$ \\
\hline & Neossolo Quartzarênico Órtico típico - Rqo2 & Sedimentos Arenosos & Araranguá & $0-23$ \\
\hline & $\begin{array}{l}\text { Argissolo Vermelho-Amarelo Distrófico latossólico - } \\
\text { PVAd2 }\end{array}$ & Arenito e Siltito & $\begin{array}{l}\text { Lauro } \\
\text { Muller }\end{array}$ & $0-13$ \\
\hline
\end{tabular}

Nota: ${ }^{*}$ Simbologia das Classes de $1^{\circ}, 2^{\circ}$ e $3^{\circ}$ níveis de acordo com a Embrapa (2013).

Tabela 2. Atributos físicos e químicos ${ }^{1}$ e óxidos de $\mathrm{Al}$ e $\mathrm{Fe}^{2}$ dos solos.

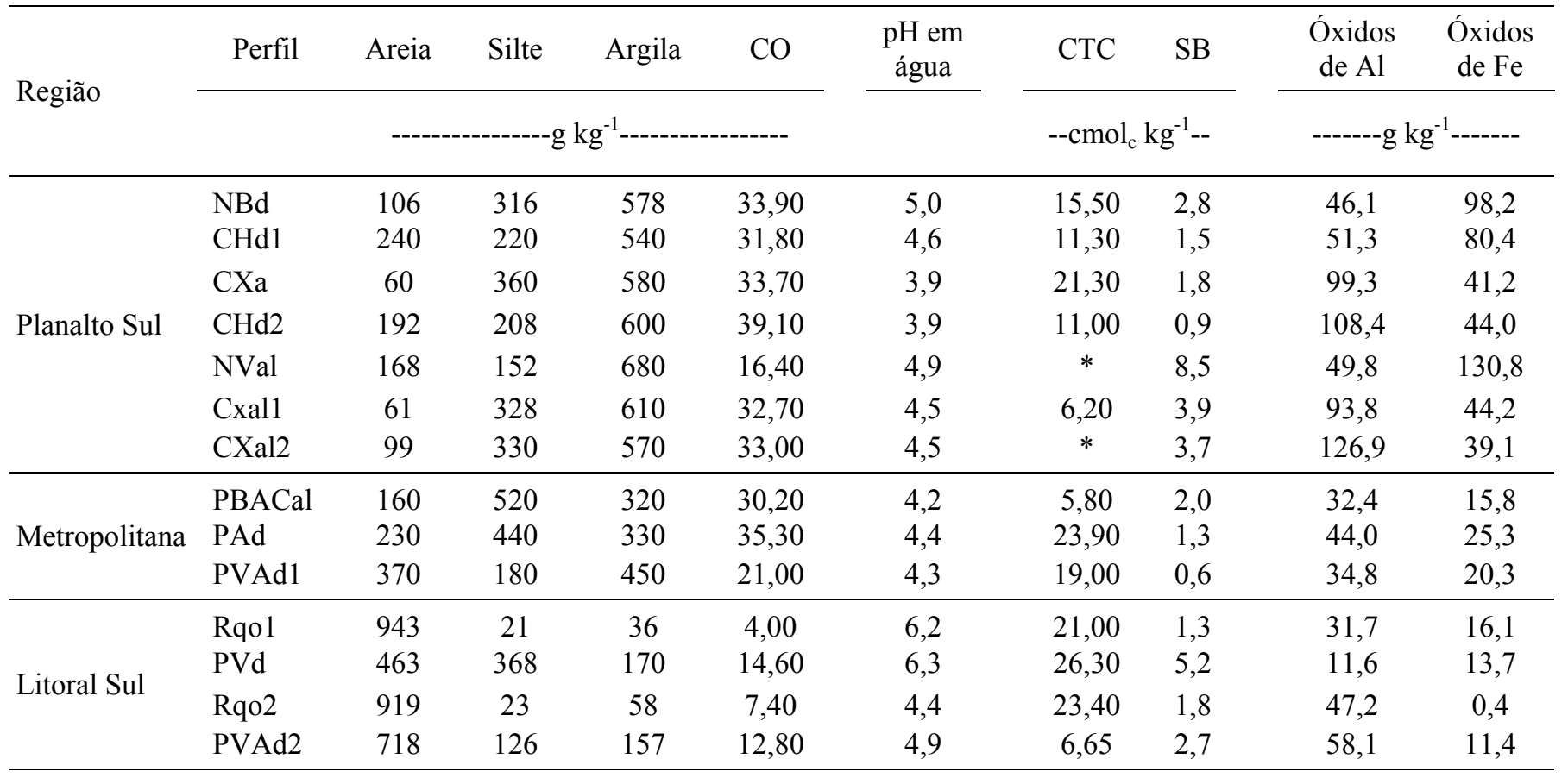

Fontes: ${ }^{1}$ Paes Sobrinho et al. (2009); Costa et al. (2013); Ferreira (2013); Lunardi Neto e Almeida (2013); Teske et al. (2013); conforme metodologia da Embrapa (1999); ${ }^{2}$ próprio autor, conforme Método USEPA 3051 A (USEPA, 1998); *Dados não determinados. CO: Carbono Orgânico; pH: potencial Hidrogeniônico; CTC: Capacidade de Troca de Cátions; SB: Soma de Bases; Al: Alumínio; Fe: Ferro.

As análises foram conduzidas ao Laboratório de Análise Ambiental do Departamento de Solos e Recursos Naturais do Centro de Ciências Agroveterinárias - UDESC/CAV. As amostras, após secas ao ar, foram moídas e passadas em peneira com abertura de $2 \mathrm{~mm}$. Após 
homogeneizadas, as amostras foram maceradas em almofariz de ágata até pó fino e tamisadas em peneira de 0,106 $\mathrm{mm}$ de abertura. Adicionaram-se $0,25 \mathrm{~g}$ de amostra de solo e $6 \mathrm{~mL}$ de solução de $\mathrm{HNO}_{3}$ 14,5 mol L${ }^{-1}$ em tubos de teflon, os quais foram levados para forno de Micro-ondas Multiwave 3000 para a digestão segundo método USEPA 3051 A descrito pela United States Environnmental Protection Agency - USEPA (1998).

Após a digestão e filtragem, o volume final foi completado até $10 \mathrm{~mL}$ com água ultrapura. As análises foram realizadas em duplicata. Em cada bateria foi inserido amostra referência SRM 2709 San Joaquin soil, certificada pelo National Institute of Standards and Technology (NIST) e provas em branco para cálculo do Limite de Detecção Qualitativo do Método Analítico (LDQM) (Tabela 3).

Tabela 3. Teores de As na amostra-controle SRM 2709 determinado e certificado, teores recuperados e LDQM.

\begin{tabular}{|c|c|c|c|}
\hline Teor & \multicolumn{2}{|c|}{ Recuperação } & \\
\hline Determinado Certificado & Determinado & Lixiviado $^{1}$ & LDQM \\
\hline $\mathrm{mg} \mathrm{kg}^{-1}$ & 0 & & \\
\hline $17,7 \pm 0,8$ & 47,7 & ** & 0,48 \\
\hline
\end{tabular}

Notas: ${ }^{1} \mathrm{O}$ certificado NIST SRM 2709 apresenta valores de \% de recuperação para lixiviação ácida para As método USEPA $3050 \mathrm{~B}$ em bloco digestor. **valores não determinados pelo NIST (2002).

O LDQM foi calculado segundo a equação LDQM $=\mathrm{Fd} \times(\mathrm{M} \pm \mathrm{t} \times \mathrm{s})$ (APHA et al., 1998), em que Fd é o fator de diluição das amostras, M é a média das provas em branco, t é o valor t-Student para um intervalo de confiança de $99 \%$, em função do número de graus de liberdade de medições repetidas; e s, o desvio padrão das provas em branco.

Os teores de As foram determinados por espectrofotometria de absorção atômica com atomização eletrotérmica (EAA-FG CONTRAA 700 ANALYTIK JENA). As curvas de calibração foram preparadas a partir de uma solução padrão de $1.000 \mathrm{mg} \mathrm{L}^{-1}$ certificada NIST, marca MERCK, utilizando-se água ultrapura para diluição. A condição de leitura utilizada para As foi a temperatura de pirólise de 700 a $1100^{\circ} \mathrm{C}$ e como modificador químico $\mathrm{Pd}\left(\mathrm{NO}_{3}\right)_{2}$ a $1 \%$ conforme recomendação do fabricante.

Não foi possível comparar a taxa de recuperação porque o protocolo não apresentou a porcentagem recuperada para o método lixiviado para As, método similar ao deste estudo.

As análises estatísticas foram conduzidas utilizando-se o programa Sisvar 5.3 (Ferreira, 2010) e o software $\mathrm{SAS}^{\circledR}$ (SAS Institute, 2003). Os resultados foram submetidos à análise de variância e teste de Scott-Knott. Para todos os testes, foi considerado o nível mínimo de 5\% de significância. Os solos da região Litoral Sul foram excluídos das análises estatísticas, pois todos os resultados analíticos ficaram abaixo do LDQM.

\section{RESULTADOS E DISCUSSÃO}

Os teores de As nesse estudo variaram de $<0,48$ a 9,36 $\mathrm{mg} \mathrm{kg}^{-1}$ (Tabela 4).

Tabela 4. Teores médios de As para as regiões Planalto Sul, Metropolitana e Litoral Sul.

\begin{tabular}{lc}
\hline Região & $\mathrm{As}\left(\mathrm{mg} \mathrm{kg}^{-1}\right)$ \\
\hline Planalto Sul & $6,79 \mathrm{~A}$ \\
Metropolitana & $5,55 \mathrm{~A}$ \\
Litoral Sul & $<0,48$ \\
\hline
\end{tabular}

Nota: As comparações entre as regiões foram testadas pelo teste de Scott-Knott a nível mínimo de 5\% de significância, sendo que médias seguidas da mesma letra não diferem entre si. 
Não houve diferença estatística entre as regiões Planalto Sul e Metropolitana (Tabela 4), enquanto que para a região Litoral Sul os teores de As dos solos ficaram abaixo do LDQM. Isso pode ser explicado pelas diferenças no material de origem e relevo das regiões. Os solos do Litoral Sul são formados por sedimentos recentes de rochas sedimentares, nos quais são encontrados altos teores de areia, que possivelmente não apresentam minerais que contenham As, além da baixíssima capacidade de adsorção química desse material. Outra questão é que os solos do Litoral Sul se encontram em relevo plano e são bem drenados (Costa et al., 2013), o que permite uma maior lixiviação dos elementos presentes nos mesmos.

Quando comparado às classes de solos dentro de cada região, verifica-se que não houve diferença significativa nos teores de As (Tabela 5).

Tabela 5. Teores médios de As dos solos das regiões Planalto Sul e Metropolitana.

\begin{tabular}{|c|c|}
\hline Classe de Solc & As $\left(\mathrm{mg} \mathrm{kg}^{-1}\right)$ \\
\hline \multicolumn{2}{|c|}{ Planalto Sul } \\
\hline NBd & $5,65 \mathrm{~A}$ \\
\hline CHd1 & $8,60 \mathrm{~A}$ \\
\hline $\mathrm{CXa}$ & $6,37 \mathrm{~A}$ \\
\hline $\mathrm{CHd} 2$ & $5,52 \mathrm{~A}$ \\
\hline NVal & $9,36 \mathrm{~A}$ \\
\hline CXal1 & $8,38 \mathrm{~A}$ \\
\hline CXal2 & $6,62 \mathrm{~A}$ \\
\hline \multicolumn{2}{|c|}{ Metropolitana } \\
\hline PBACal & $4,60 \mathrm{~A}$ \\
\hline PVA & $7,27 \mathrm{~A}$ \\
\hline PVAd1 & $4,78 \mathrm{~A}$ \\
\hline
\end{tabular}

Nota: As comparações entre classes de solos dentro de cada região foram testadas pelo teste de Scott-Knott a nível mínimo de 5\% de significância, sendo que médias seguidas da mesma letra não diferem entre si.

$\mathrm{Na}$ análise de correlação (Tabela 6) os teores de As tiveram correlação significativa e positiva com a argila $(0,89), \mathrm{CO}(0,72)$ e os óxidos de ferro $(0,74)$. Segundo Mcbride $(1994)$ e Kabata-Pendias (2011) a mobilidade desse elemento é reduzida devido à forte sorção por argilas, hidróxidos e matéria orgânica.

Tabela 6. Correlação entre os atributos físicos e químicos e os teores de As.

\begin{tabular}{cccccccccc}
\hline & Areia & Silte & Argila & $\mathrm{CO}$ & $\begin{array}{c}\mathrm{pH} \\
\text { em água }\end{array}$ & CTC & SB & $\begin{array}{c}\text { Óxidos } \\
\text { de Al }\end{array}$ & $\begin{array}{c}\text { Oxidos } \\
\text { de Fe }\end{array}$ \\
\hline As & $-0,86^{* *}$ & $\mathrm{~ns}$ & $0,89^{* *}$ & $0,72^{* *}$ & $\mathrm{~ns}$ & $0,59^{*}$ & $\mathrm{~ns}$ & $0,67^{* *}$ & $0,74^{* *}$ \\
\hline
\end{tabular}

Notas: **significativo ao nível de $1 \%$ de probabilidade $(\mathrm{p}<0,01)$; *significativo ao nível de $5 \%$ de probabilidade $(0,01 \leq \mathrm{p} \leq 0,05)$ e ns não significativo $(\mathrm{p} \geq 0,05)$; $\mathrm{CO}$ : Carbono Orgânico; $\mathrm{pH}$ : potencial Hidrogeniônico; CTC: Capacidade de Troca de Cátions; SB: Soma de Bases; Al: Alumínio; Fe: Ferro.

Segundo Wang e Mulligan (2006), a matéria orgânica pode aumentar a mobilidade de As, pois eles competem pelos sítios de adsorção disponíveis, no entanto, ácidos orgânicos podem reduzir a mobilidade, sendo agentes de ligação ou formando complexos insolúveis, especialmente quando saturado com cátions. $\mathrm{O}$ As tem alta afinidade por óxidos, motivo pelo qual houve significativa correlação entre As e óxidos de ferro. Segundo Chirenje et al. (2003), que obtiveram o mesmo comportamento para solos dos Estados Unidos, os óxidos geram superfícies reativas, em que o As é adsorvido, formando compostos estáveis e imóveis. Em 
geral, solos derivados de rochas ricas em óxidos de ferro, como o basalto, possuem maiores teores de As, devido à forte adsorção do elemento aos óxidos de Fe. Correlação significativa e positiva $(0,59)$ foi encontrada com a CTC. Isso ocorre devido à afinidade do oxiânion As de se ligar nos sítios disponíveis na CTC. Dudka e Market (1992) também encontraram correlação de $0,75^{* *}$ com a CTC.

Na região Planalto Sul ocorrem solos derivados de rochas ígneas, que originam solos mais argilosos, com maior capacidade de adsorver o As. São solos menos lixiviados, pois o relevo é suave ondulado a forte ondulado e moderadamente drenado (Costa et al., 2013; Ferreira, 2013; Teske et al., 2013).

Campos et al (2013) encontraram teores de 2,03 $\mathrm{mg} \mathrm{kg}^{-1}$ para solos do Bioma Cerrado. Biondi (2010) encontrou teor médio de As de $0,44 \mathrm{mg} \mathrm{kg}^{-1}$ para solos do Estado de Pernambuco. E para solos do Espírito Santo o teor médio determinado foi de $6,80 \mathrm{mg} \mathrm{kg}^{-1}$ (Paye et al., 2010). Para solos da Cuba, Alfaro et al. (2015) obtiveram 10,8 mg kg-1 de As. Dantu (2010) encontrou 10,65 $\mathrm{mg} \mathrm{kg}^{-1}$ de As para solos na Índia.

Essas diferenças dos teores de As encontrados para os solos de SC em relação a outros Estados brasileiros e a outros estudos encontrados na literatura internacional se deve às peculiaridades locais, como a composição do material de origem, a influência dos processos pedogenéticos e aos atributos físicos e químicos dos solos, que são intrínsecos para cada ambiente. Por isso, é essencial que os Estados brasileiros e os países estabeleçam seus VRQ.

\section{CONCLUSÃO}

Não houve diferença entre as classes de solos para as regiões Planalto Sul e Metropolitana, sendo que na Região Litoral Sul os teores ficaram abaixo do LDQM.

Os teores de As correlacionaram-se positivamente com os teores de argila, CO, CTC e óxidos de $\mathrm{Al}$ e de Fe.

\section{REFERÊNCIAS}

ALFARO, M. R.; MONTERO, A.; UGARTE, O. M.; NASCIMENTO, C.W. A. do; ACCIOLY, A. M. de A.; BIONDI, C. M. et al. Background concentrations and reference values for heavy metals in soils of Cuba. Environmental Monitoring Assessment,. v. 187, p. 1-10, 2015. http://dx.doi.org /10.1007/s10661-014-4198-3

ALONSO, D. L.; LATORRE, S.; CASTILlO, E.; BRANDÃO, P. F. B. Environmental occurrence of arsenic in Colombia: A review. Environmental Pollution, v. 186, p. $272-$ 281, 2014. http://dx.doi.org/10.1016/j.envpol.2013.12.009

AMERICAN PUBLIC HEALTH ASSOCIATION - APHA; AMERICAN WATER WORKS ASSOCIATION - AWWA; WATER ENVIRONMENT FEDERATON - WEF. Standard Methods for the examination of water and wastewater. $20^{\text {th }} \mathrm{ed}$. Washington, DC, 1998.

AGENCY FOR TOXIC SUBSTANCES AND DISEASE CONTROL - ATSDR. Priority list of hazardous substances. 2013. Disponível em: http://www.atsdr.cdc.gov/spl/. Acesso em: 13 jan. 2015.

BIONDI, C. M. Teores naturais de metais pesados nos solos de referência do Estado de Pernambuco. 2010. 70f. Tese (Doutorado em Ciência do Solo) - Universidade Federal Rural de Pernambuco, Recife, 2010. 
BIONDI, C. M.; NASCIMENTO, C.W.A.; NETA, A. B. F.; RIBEIRO, M. R. Teores de Fe, $\mathrm{Mn}, \mathrm{Zn}, \mathrm{Cu}$, Ni e $\mathrm{Co}$ em solos de referência de Pernambuco. Revista Brasileira de Ciência do Solo, v. 35, p. 1057-1066, 2011. http://dx.doi.org/10.1590/s010006832011000300039

CAMPOS, M. L.; GUILHERME, L. R. G.; MARQUES, J. J. G. de S. e M.; CURI, N.; ARAÚJO, A. S. A. A.; MIQUELLUTI, D. J. et al. Teores de arsênio e cádmio em solos do bioma cerrado. Revista Brasileira de Ciência do Solo, v. 37, n. 1, p. 281-286, 2013. http://dx.doi.org/10.1590/s0100-06832013000100029

CHIRENJE, T.; MA, L. Q.; CHEN, M.; ZILLIOUX, E. J. Comparison between background concentrations of arsenic In urban and non-urban areas of Florida. Advances in Environmental Research, v. 8, p. 137-146, 2003. http://dx.doi.org/10.1016/s1093$0191(02) 00138-7$

COSTA, A. da.; ALBUQUERQUE, J. A.; ALMEIDA, J. A.; COSTA, A. da.; LUCIANO, R. V. Pedotransfer functions to estimate retention and availability of water in soils of the state of Santa Catarina, Brazil. Revista Brasileira de Ciência do Solo, v. 37, p. 889910, 2013. http://dx.doi.org/10.1590/s0100-06832013000400007

DANTU, S. Geochemical patterns in soils in and around Siddipet, Medak District, Andhra Pradesh, India. Environmental Monitoring Assessment, v. 170, p. 681-701, 2010. http://dx.doi.org/10.1007/s10661-009-1267-0

DUDKA, S.; MARKERT, B. Baseline Concentrations of As, Ba, Be, $\mathrm{Li}, \mathrm{Nb}, \mathrm{Sr}$, and $\mathrm{V}$ in surface soils of Poland. The Science of the total Environment, v. 22, p. 279-290, 1992. http://dx.doi.org/10.1016/0048-9697(92)90046-u

EMPRESA BRASILEIRA DE PESQUISA AGROPECUÁRIA - EMBRAPA. Levantamento de Reconhecimento dos Solos do Estado de Santa Catarina. Rio de Janeiro, 1998. 735p (Boletim de Pesquisa, 6).

EMPRESA BRASILEIRA DE PESQUISA AGROPECUÁRIA - EMBRAPA. Manual de métodos de análise de solo. 2. ed. Rio de Janeiro, 1999. 212 p.

EMPRESA BRASILEIRA DE PESQUISA AGROPECUÁRIA - EMBRAPA. Sistema Brasileiro de Classificação de Solos. 3. ed. Brasília, 2013. 353p.

FERREIRA, D. F. SISVAR 5.3. Sistema de Análises Estatísticas. 2010. Disponível em: http://www.dex.ufla.br/. Acesso em: 10 jan. 2015.

FERREIRA, E. R. N. C. Química e Mineralogia de solos desenvolvidos de rochas alcalinas e ultrabásicas do Domo de Lages. 2013. 159f. Tese (Doutorado em Manejo do Solo) - Universidade do Estado de Santa Catarina, Lages, 2013.

FITZ, W. J.; WENZEL, W. W. Arsenic transformations in soil-rhizosphere-plant system: fundamentals and potential application to phytoremediation. Journal of Biotechnology, v. 99, p. 259-278, 2002. http://dx.doi.org/10.1016/s0168-1656(02)00218-3

GOLDSCHMIDT, V. M. Geochemistry. Londres: Oxford University Press, 1958. 425 p.

HAYES, K. F.; TRAINA, S. J. Metal speciation and its significance in ecosystem health. In: HUANG, P. M. Soil chemistry and ecosystem health. Madison: Soil Science Society of America Journal, 1998. p. 45-84. 
KABATA-PENDIAS, A. Trace elements in soils and plants. Boca Raton: CRC Press, 2011. $315 \mathrm{p}$.

LUNARDI NETO, A.; ALMEIDA, J. A. Mineralogia das frações silte e argila em Argissolos com horizontes subsuperficiais escurecidos em Santa Catarina. Revista de Ciências Agroveterinárias, v. 12, p. 282-293, 2013. http://dx.doi.org/10.1590/s010006832012000400001

MCBRIDE, M. B. Environmental chemistry of soils. New York: Oxford University Press, 1994. $406 \mathrm{p}$.

MENDOZA-GRIMÓN, V.; HERNÁNDEZ-MORENO, J. M.; MARTÍN, J. A. R.; FERNÁNDEZ-VERA, J. R.; PALACIOS-DÍAZ, M. P. Trace and major element associations in basaltic ash soils of El Hierro Island, Journal of Geochemical Exploration, v. 147, p. 277-282, 2014. http://dx.doi.org/10.1016/j.gexplo.2014.06.010

NATIONAL INSTITUTE OF STANDARDS AND TECHNOLOGY STANDARD REFERENCE MATERIALS - NIST. SRM 2709, 2710 and 2711. 2002. Disponível em: https://www-s.nist.gov/srmors/certificates/archive/2709.pdf. Acesso em: 10 jan. 2012.

PAYE, H. S.; MELLO, J. W. V.; ABRAHÃO, W. A. P.; FERNANDES FILHO, E. I.; DIAS, L. C. P.; CASTRO, M. L. O. et al. Valores de referência de qualidade para metais pesados em solos no Estado do Espírito Santo. Revista Brasileira de Ciência do Solo, v. 34, p. 2041-2051, 2010. http://dx.doi.org/10.1590/s0100-06832010000600028

PAES SOBRINHO, J. B.; ALMEIDA, J. A.; ERHART, J. Mineralogia da fração argila de Argissolos das Serras do Leste de Santa Catarina. Revista de Ciências Agroveterinárias, v. 8, p. 09-24, 2009.

PARRA, S.; BRAVO, M. A.; QUIROZ, W.; MORENO, T.; KARANASIOU, A.; FONT, O. et al. Distribution of trace elements in particle size fractions for contaminated soils by a copper smelting from different zones of the Puchuncaví Valley (Chile). Chemosphere, v. 111, p. 513-521, 2014. http://dx.doi.org/10.1016/j.chemosphere.2014.03.127

ROSAS-CASTOR, J. M.; GUZMÁN-MAR, J. L.; HERNÁNDEZ-RAMÍREZ, A.; GARZAGONZÁLEZ, M. T.; HINOJOSA-REYES, L. Arsenic accumulation in maize crop (Zea mays): A review. Science of the Total Environment, v. 488-489, p. 176-187, 2014. http://dx.doi.org/10.1016/j.scitotenv.2014.04.075

ROY, M.; GIRI, A. K.; DUTTA, S.; MUKHERJEE, P. Integrated phytobial remediation for sustainable management of arsenic in soil and water. Environment International, v. 75, p. 180-198, 2015. http://dx.doi.org/10.1016/j.envint.2014.11.010

SAS INSTITUTE INC ${ }^{\circledR}$ Statistical Analysis System. Versão 9.1.3. Cary, 2003. 1 CD ROM.

SINGH, R.; SINGH, S.; PARIHAR, P.; SINGH, V. P.; PRASAD, S. M. Arsenic contamination, consequences and remediation techniques: a review. Ecotoxicology and Environmental Safety, v. 112, p. 247-270, 2015.

http://dx.doi.org/10.1016/j.ecoenv.2014.10.009

SU, Y.; YANG, R. Background concentrations of elements in surface soils and their changes as affected by agriculture use in the desert-oasis ecotone in the middle of Heihe River Basin, North-west China. Journal of Geochemical Exploration, v. 98, p. 57-64, 2008. http://dx.doi.org/10.1016/j.gexplo.2007.12.001 
TARVAINEN, T.; ALBANESE, S.; BIRKE, M.; PONAVIC, M.; REIMANN, C. Arsenic in agricultural and grazing land soils of Europe. Applied Geochemistry, v. 28, p. 2-10, 2013. http://dx.doi.org/10.1016/j.apgeochem.2012.10.005

TESKE, R.; LUNARDI NETO, A.; HOFER, A.; ALMEIDA, J. A. Caracterização química, física e morfológica de solos derivados de rochas efusivas no planalto sul de Santa Catarina, Brasil. Revista de Ciências Agroveterinárias, v. 12, p. 175-186, 2013.

TSUJI, J. S.; YOST, L. J.; BARRAJ, L. M, SCRAFFORD, C. G.; MINK, P. J. Use of background inorganic arsenic exposures to provide perspective on risk assessment results. Regulatory Toxicology and Pharmacology, v. 48, p. 59-68, 2007. http://dx.doi.org/10.1016/j.yrtph.2007.01.004

UNITED STATES. Environmental Protection Agency - USEPA. Test Methods for Evaluation Solid Waste Physical and Chemical Methods: microwave assisted acid digest of sediments, sludges, soils and oils, SW 846; U. S. Washington: Government Printing Office, 1998.

WANG, S.; MULLIGAN, C. N. Effect of natural organic matter on arsenic release from soils and sediments into groundwater. Environmental and Health, v. 28, p. 197-214, 2006. http://dx.doi.org/10.1007/s10653-005-9032-y

WORLD HEALTH ORGANIZATION - WHO. Arsenic and arsenic compounds. 2001. Disponível em: http://www.who.int/ipcs/publications/ehc/ehc_224/en/ Acesso em: 12 jan. 2015.

ZAKHAROVA, T.; TATANO, F.; MENSHIKOV, V. Health cancer risk assessment for arsenic exposure in potentially contaminated areas by plants fertilizer: A possible regulatory approach applied to a case study in Moscow Region-Russia. Regulatory $\begin{array}{llllll}\text { Toxicology and Pharmacology, v. 36, p. 22-23, } 2002 . & \end{array}$ http://dx.doi.org/10.1006/rtph.2002.1561 


\begin{tabular}{|} 
Ambiente \& Água - An Interdisciplinary Journal of Applied Science \\
ISSN 1980-993X - doi:10.4136/1980-993X \\
www.ambi-agua.net \\
E-mail: ambi.agua@gmail.com
\end{tabular}

\title{
Potencial toxicológico de metais presentes em solos de cemitérios de Santa Maria - RS
}

\author{
doi:10.4136/ambi-agua.1723 \\ Received: 11 Aug. 2015; Accepted: 21 Nov. 2015 \\ Bruno Casanova Vilaverde Gomes ${ }^{1}$; Gislaine Kummer ${ }^{1}$; \\ Márcia Maria da Silva Monteiro Pereira²; Hécio José Izário Filho³; \\ Rodrigo Fernando dos Santos Salazar'; \\ Noeli Júlia Schüssler de Vasconcellos ${ }^{1 *}$ \\ ${ }^{1}$ Centro Universitário Franciscano (UNIFRA), Santa Maria, RS, Brasil \\ ${ }^{2}$ Laboratório Stollberg do Brasil, Guaratinguetá, SP, Brasil \\ ${ }^{3}$ Escola de Engenharia de Lorena (EEL/USP), Lorena, SP, Brasil \\ *Autor correspondente: e-mail: noejuabio@yahoo.com.br, \\ bruno.engenheiro.ambi@gmail.com, gislainekummer19@yahoo.com, \\ marcia.pereira@imerys.com, helcio@dequi.eel.usp.br, \\ r.f.s.salazar@gmail.com
}

\section{RESUMO}

A implantação de cemitérios sem projetos geoambientais e hidrogeográficos em solos sem propriedades estruturais e químicas necessárias para receber este tipo de atividade resulta que elementos tóxicos e lixiviados do cemitério percolem ao nível freático. Esta percolação leva a contaminação das águas subterrâneas, afetando drasticamente os recursos naturais e a saúde pública. Desta forma, a toxicidade de solos oriundos de cemitérios de Santa Maria foi avaliada. Paralelamente, as concentrações de Al, $\mathrm{Ca}, \mathrm{Cd}, \mathrm{Cr}, \mathrm{Cu}, \mathrm{Fe}, \mathrm{Mg}, \mathrm{Ni}, \mathrm{Pb}$ e $\mathrm{Zn}$ foram determinadas e comparadas com os limites de tolerância estabelecidos na Resolução 420 de 2013 do Conselho Nacional do Meio Ambiente. Oligochaetas da espécie Eisenia andrei foram empregadas como organismos bioindicadores em bioensaios, no formato ensaio-limite (controle versus tratamento), completamente casualizado, com três repetições, composto por sete tratamentos e dois níveis. Ao final do período de exposição, foram avaliados os efeitos de toxicidade aguda em oligochaetas, sendo feita a concentração letal média $\left(\mathrm{CL}_{50}\right)$. Os resultados da toxicidade aguda e crônica nos dois cemitérios analisados evidenciaram toxicidade $\left(\mathrm{CL}_{50}\right.$ 239,88 $\mathrm{mg} \mathrm{kg-}{ }^{1}$ e $\left.52,48 \mathrm{mg} \mathrm{kg}_{-}{ }^{1}\right)$ respectivamente e interferência no processo reprodutivo das oligochaetas. Pela análise química se detectou concentrações de metais tóxicos superiores aos valores de prevenção estabelecidos legalmente.

Palavras-chave: bioindicadores, oligochatas, metais tóxicos, toxicidade.

\section{Toxicological potential of metals found in soil of cemeteries in Santa Maria - RS}

\section{ABSTRACT}

Contamination with toxic elements and cemetery leachate may result when proper geoenvironmental and hydro geographic studies are not conducted and cemeteries are dug in soils 
without appropriate structure and chemical properties. This contamination may percolate through the water table, leading to groundwater contamination, which can dramatically affect natural resources and public health. The toxicity of soil from Santa Maria was evaluated in this context. In addition, concentrations of $\mathrm{Al}, \mathrm{Ca}, \mathrm{Cd}, \mathrm{Cr}, \mathrm{Cu}, \mathrm{Fe}, \mathrm{Mg}, \mathrm{Ni}, \mathrm{Pb}$ and $\mathrm{Zn}$ were determined and compared with the tolerance limits established by the National Environmental Council Resolution No. 420 of 2013. Oligochaetas of the Eisenia andrei species were used as bio indicators in the format bioassays-limit test (control versus treatment), in a completely randomized design with three replications, consisting of seven treatments and two levels. At the end of the exposure period, we evaluated the effects of acute toxicity in oligochaetas, considering the median lethal concentration $\left(\mathrm{LC}_{50}\right)$. The results of acute and chronic toxicity studies in the two analyzed cemeteries (urban and rural) showed toxicity $\left(\mathrm{CL}_{50} 239,88 \mathrm{mg} \mathrm{k}^{-1}\right.$ e 52,48 $\mathrm{mg} \mathrm{kg}^{1}$ ) and interference in the reproductive process of oligochaetas. We detected concentrations of toxic metals that exceeded legally established values.

Keywords: biomarkers, oligochaetas, toxic metals, toxicity.

\section{INTRODUÇÃO}

A Conferência de Estocolmo sobre o Meio Ambiente, realizada na capital da Suécia, Estocolmo, no ano de 1972, estreitou relações entre o homem e o meio ambiente, surgindo a expressão "Desenvolvimento Sustentável". No Brasil, como reflexo deste evento surgem as primeiras preocupações em proteger o meio ambiente (Matos, 2001; Barros et al., 2008) como por exemplo, a criação da lei de Política Nacional de Meio Ambiente (PNMA), n ${ }^{\circ}$ 6.938/81, alterada pela Lei $\mathrm{n}^{\mathrm{o}}$ 10.165, em 27 de dezembro 2000. Essa lei foi de extrema importância para a legislação ambiental, resultando na criação do Sistema Nacional de Meio Ambiente (SISNAMA) e do Conselho Nacional de Meio Ambiente (CONAMA), (Resoluções CONAMA, 2003; 2006; 2009; 2013). A Resolução CONAMA n 335/2003, alterada pela Resolução $\mathrm{n}^{\mathrm{o}} 368 / 2006$, normatiza os procedimentos de licenciamento ambiental de cemitérios no país, com o propósito de minimizar os riscos ambientais que possam impactar o ambiente (CONAMA, 2003; 2006), porém, apesar de vigente não vem sendo devidamente cumprida.

A importância dessa normatização se dá em razão do sepultamento de corpos diretamente no solo, prática utilizada no Brasil desde o século XVII, aliado a precariedade de infraestrutura e a má administração dos cemitérios, contribuírem significativamente para a contaminação das áreas ocupadas por esse tipo de atividade potencialmente geradora de passivos ambientais e de saúde pública (Matos, 2001). Não obstante, a maioria dos cemitérios existentes terem sido implantados sem levar em consideração os aspectos geológicos, hidrogeológicos e geotécnicos da área em questão, pois não eram consideradas fontes poluidoras, fornecendo riscos potenciais de contaminação à saúde pública (Barros et al., 2008; Pacheco et al., 1991; WHO, 1998). Embora a decomposição de corpos presentes nesses locais seja um processo inerente ao meio natural, os mesmos podem conter substâncias tóxicas, como metais tóxicos, que demandam um tratamento especial, por não serem degradados biológica ou quimicamente (Hong et al., 2002). Esta contaminação dependendo de suas características físico-químicas podem lixiviar e contaminar as águas subterrâneas (Leli et al. 2012).

Os testes toxicológicos são uma importante ferramenta para fornecer informações sobre a biodisponibilidade desses poluentes, bem como auxiliar na correlação da contaminação e dos efeitos adversos sobre a biota local e do seu entorno (Lowe e Butt, 2007).

As Oligochaetas, popularmente conhecidas como minhocas são um importante grupo de organismos constituintes da macrofauna do solo e representam uma alternativa para investigar 
toxicidade, pois se encontram distribuídas na maior parte da biomassa dos solos tropicais. Exímias bioindicadoras de ecotoxicidade, fornecem referências sobre a biodisponibilidade dos poluentes e o grau de contaminação (Andréa, 2010; Shuster et al., 2002). Por meio de seu deslocamento e de ingestão do solo, as oligochaetas tem contato direto com os poluentes que estão adsorvidos nos colóides, na matéria orgânica e na solução aquosa, mas, podem ainda absorver os poluentes por meio de contato direto e passagem pela cutícula apresentando sintomas adversos ou morte por intoxicação (Andréa, 2010). Somando-se a isto, no Brasil, poucos estudos abordam a contaminação do solo de cemitério por poluentes metálicos e biodisponibilidade destes contaminantes, como relatam Kemerich et al. (2012). Também, não são encontrados relatos sobre a influência desta contaminação na biota do solo.

Este estudo foi realizado com o objetivo foi avaliar a toxicidade e a concentração de metais tóxicos do solo de dois cemitérios de Santa Maria, Rio Grande do Sul, sobre oligochaetas como bioindicadoras de contaminação.

\section{MATERIAL E MÉTODOS}

As amostras de solo foram coletadas em dois cemitérios no município de Santa Maria - Rio Grande do Sul. O primeiro localizado em zona urbana do município, denominado Cemitério Municipal de Santa Maria, em solo classificado como Argissolo Vermelho distrófico arênico (Unidade de Mapeamento São Pedro) (EMBRAPA, 2006), latitude $29^{\circ} 41^{\prime} 43.20^{\prime \prime} \mathrm{S}$ e longitude 5349'28.63" W, e o segundo cemitério localizado em área rural do município, denominado Cemitério Municipal São Marcos, em solo classificado como Chernossolo Argilúvico férrico típico (Unidade de Mapeamento Ciríaco) (EMBRAPA, 2006), latitude $29^{\circ} 40^{\prime} 14.40^{\prime \prime} \mathrm{S}$ e longitude 53 40'54.73" W. Removeu-se $20 \mathrm{~cm}^{3}$ de solo na área central de ambos os cemitérios, respeitando-se uma distância entre 5 a $10 \mathrm{~m}$ uma da outra.

Uma pá foi introduzida no solo descartando-se os primeiros $10 \mathrm{~cm}$ do perfil, utilizando-se apenas a camada de 10 a $20 \mathrm{~cm}$ do perfil nas análises químicas e toxicológicas, coletando-se um total de $0,008 \mathrm{~m}^{3}$ de solo por amostra. A escolha de coletar a uma profundidade de $20 \mathrm{~cm}$ decorreu devido ás circunstâncias atípicas do local, com jazigos muito próximos, impossibilitando outra forma de coleta. As amostras de solo foram misturadas, embaladas em sacos plásticos resistentes, lacrados, etiquetados e encaminhados ao Laboratório de Engenharia Ambiental - LABEA do Centro Universitário Franciscano, homogeneizado e disposto em bandejas para secagem ao ar.

$\mathrm{O}$ pH foi determinado em solução saturada 1:2,5, com água deionizada, permanecendo em repouso por 60 min (Tedesco et al., 1995), e o bioensaio de toxicidade aguda adotado segue o formato ensaio-limite (controle versus tratamento), completamente casualizado, com a utilização de 10 oligochaetas adultas, da espécie Eisenia andrei (Vermelha da Califórnia), pesando entre 300 e $400 \mathrm{mg}$ e clitelo desenvolvido. O bioensaio foi realizado em triplicata, composto por quatro tratamentos e dois níveis (SCU - Solo de Cemitério Urbano e SCR - Solo de Cemitério Rural): T0 - 100\% solo de mata +10 oligochaetas adultas; T1 - 50\% solo de cemitério $+50 \%$ solo de mata +10 oligochaetas adultas; $\mathrm{T} 2-75 \%$ solo de cemitério $+25 \%$ solo de mata +10 oligochaetas adultas e T3 $-100 \%$ solo de cemitério +10 oligochaetas adultas.

Adotaram-se como unidades experimentais, vasos de PVC escuro furado no fundo, cobertos com plástico do tipo Sombrite, mantidos em ambiente com luz solar difusa. A exposição dos organismos teste se deu simultaneamente tanto às amostras de solo controle e contaminado como a umidade e alimentação, que foram mantidas pela adição de $500 \mathrm{~mL}$ de água destilada em intervalos de 3 dias e $15 \mathrm{~g}$ de esterco bovino seco e pulverizado, em intervalos de 7 dias durante um período de exposição de 90 dias, conforme as normas ISO 17512-1 - Soil quality - Avoidance test for determining the quality of soils and effects of 
chemicals on behaviour - Part 1: Test with earthworms (Eisenia fetida and Eisenia andrei) (ISO, 2008).

Após 96 horas de exposição realizou-se a avaliação do número de indivíduos mortos para o cálculo da $\mathrm{CL}_{50}$ do solo para as oligochaetas. Após a contagem dos indivíduos mortos remontou-se as unidades experimentais, colocando nas mesmas condições ambientais do teste agudo, por aproximadamente 90 dias, para avaliar o efeito tóxico crônico (reprodução). A $\mathrm{CL}_{50}$, concentração letal que mata $50 \%$ dos indivíduos expostos foi obtida pela transformação logarítmica das concentrações em probitos do percentual de mortos, que converte a curva dose-resposta, sigmoide, em uma reta que pode, assim, ser analisada por regressão linear simples, realizada, neste trabalho, no programa BioEstat 5.0.

Depois de realizado o ensaio da biodisponibilidade de metais tóxicos no solo, as amostras de solo de ambos os cemitérios determinou-se a concentração de metais tóxicos no Laboratório de Absorção Atômica do Departamento de Engenharia Química da Escola de Engenharia de Lorena - Universidade de São Paulo (USP), por Espectrometria de Emissão Ótica com Plasma Acoplado Indutivamente (ICP-OES).

Nas determinações realizadas, utilizou-se o ICP-OES modelo Optima DV 4300 da Perkin Elmer, facilitando a observação do plasma no modo de configuração axial e radial, assegurando uma observação mais sensível para cada elemento. As condições de análise do ICP-OES estão descritas na Tabela 1.

Para a digestão das amostras de solo foram empregados os procedimentos de abertura de amostras de origem ambiental por digestão ácida conforme proposto por Bianchi e colaboradores (2012) e Salazar e colaboradores (2011b).

Tabela 1. Parâmetros instrumentais do ICP-OES para a análise qualiquantitativa de metais tóxicos nos tratamentos com solo de cemitério com ou sem adição de oligochaetas.

\begin{tabular}{|c|c|}
\hline Parâmetro da análise & Valor \\
\hline Vazão de Argônio & $10 \mathrm{~L} / \mathrm{min}$ \\
\hline Vazão do Nebulizador & $0,54 \mathrm{~L} / \mathrm{min}$ \\
\hline Vazão de o Gás Auxiliar & $0,2 \mathrm{~L} / \mathrm{min}$ \\
\hline Referência de Leitura & $15 \mathrm{~mm}$ \\
\hline Potência da Rádio Frequência & $1450 \mathrm{~W}$ \\
\hline Vazão da Bomba Peristáltica & $1,50 \mathrm{~mL} / \mathrm{min}$ \\
\hline Equação da Curva & $\begin{array}{l}\text { Curva forçada ao zero/Cálculo } \\
\text { de integração de área }\end{array}$ \\
\hline Tipo de Nebulizador & $\begin{array}{l}\text { Nebulizador de fluxo cruzado } \\
\text { acoplado à câmara do Scott }\end{array}$ \\
\hline Posição da Tocha & -3 \\
\hline Comprimento de onda (nm) & $\begin{array}{l}\text { Al }(396,153), \mathbf{C a}(317,933), \\
\mathbf{C d}(228,802), \mathbf{C r}(267,716), \\
\mathbf{C u}(327,398), \mathbf{F e}(238,204), \\
\mathbf{M g}(285,213), \mathbf{N i}(231,604), \\
\mathbf{P b}(220,356), \mathbf{Z n}(206,203)\end{array}$ \\
\hline
\end{tabular}

Os dados das análises químicas dos metais tóxicos foram testados pela Análise de Variância Simples, utilizando o software ESTATISTICA. 


\section{RESULTADOS E DISCUSSÃO}

Nas Tabelas 2 e 3 encontram-se discriminadas as transformações dos dados resultantes do bioensaio de toxicidade aguda e o valor da concentração letal média $\left(\mathrm{CL}_{50}\right)$ das diferentes concentrações de solo dos cemitérios (urbano e rural) testados neste experimento. Nas figuras 1 e 2 são apresentadas as curvas dose-resposta para ambos os cemitérios avaliados (urbano e rural).

Considerando os valores da $\mathrm{CL}_{50}$ dos solos dos cemitérios urbano e rural $\left(239,88 \mathrm{mg} \mathrm{kg}^{-1}\right.$ e $52,48 \mathrm{mg} \mathrm{kg}^{-1}$ ) respectivamente, pode-se observar que o solo do cemitério rural pode ser classificado como muito tóxico $\left(\mathrm{CL}_{50} \leq 100\right)$, muito próximo de extremamente tóxico $\left(\mathrm{CL}_{50} \leq 50\right)$, para as oligochaetas. A razão desta afirmação é que a quantidade de solo do cemitério rural (contaminado), necessária para matar 50\% dos organismos expostos, é inferior a quatro vezes a quantidade de solo do cemitério urbano no qual a $\mathrm{CL}_{50}$ foi $>100$, o que representa uma toxicidade moderada. Ribeiro et al. (2012) verificaram que minhocas da espécie Eisenia andrei, de mesma espécie das utilizadas neste estudo, quando expostas a solos e húmus contaminado com cobre, na concentração de $500 \mathrm{mg} \mathrm{kg}^{-1}$ tiveram $90 \%$ de letalidade.

Tabela 2. Valores percentuais de mortos transformados em Probit e $\mathrm{CL}_{50}$, do bioensaio de toxicidade aguda em Oligochaetas (minhoca) expostas a diferentes concentrações de solo do cemitério urbano.

\begin{tabular}{cccccccc}
\hline $\begin{array}{c}\text { Grupo } \\
\text { Teste }\end{array}$ & $\begin{array}{c}\text { (a) } \\
\mathrm{N}^{\mathbf{0}} \text { org. } \\
\text { (oligochaetas) }\end{array}$ & $\begin{array}{c}\text { (b) } \\
\text { Conc. Solo } \\
\text { cemitério }\left(\mathrm{g} \mathrm{kg}^{-1}\right)\end{array}$ & $\begin{array}{c}\text { (c) } \\
\text { Log. Conc. }\end{array}$ & $\begin{array}{c}\text { (d) } \\
\mathrm{N}^{\mathrm{o}} \text { mortos }\end{array}$ & $\begin{array}{c}\text { (e) } \\
\% \text { mortos }\end{array}$ & $\begin{array}{c}\text { (f) } \\
\text { Pobits }\end{array}$ & $\begin{array}{c}\text { (f) } \\
\mathrm{CL}_{50} \mathrm{mg} \mathrm{kg}^{-1}\end{array}$ \\
\hline T0 & 30 & 0 & 0 & 0 & 0 & 0 & \\
$\mathrm{~T} 1$ & 30 & 500 & 2,70 & 4 & 13,3 & 3.87 & 239,88 \\
$\mathrm{~T} 2$ & 30 & 750 & 2,90 & 7 & 23,3 & 4.26 & \\
$\mathrm{~T} 3$ & 30 & 1000 & 3,00 & 28 & 93,3 & 6.48 & \\
\hline
\end{tabular}

Nota: Número de organismos testados (a), concentração de solo contaminado (b), log. da concentração (c), número de organismos mortos (d), percentual de organismos mortos (e), probito do percentual de organismos mortos (f) e $\mathrm{CL}_{50}(\mathrm{~g})$.

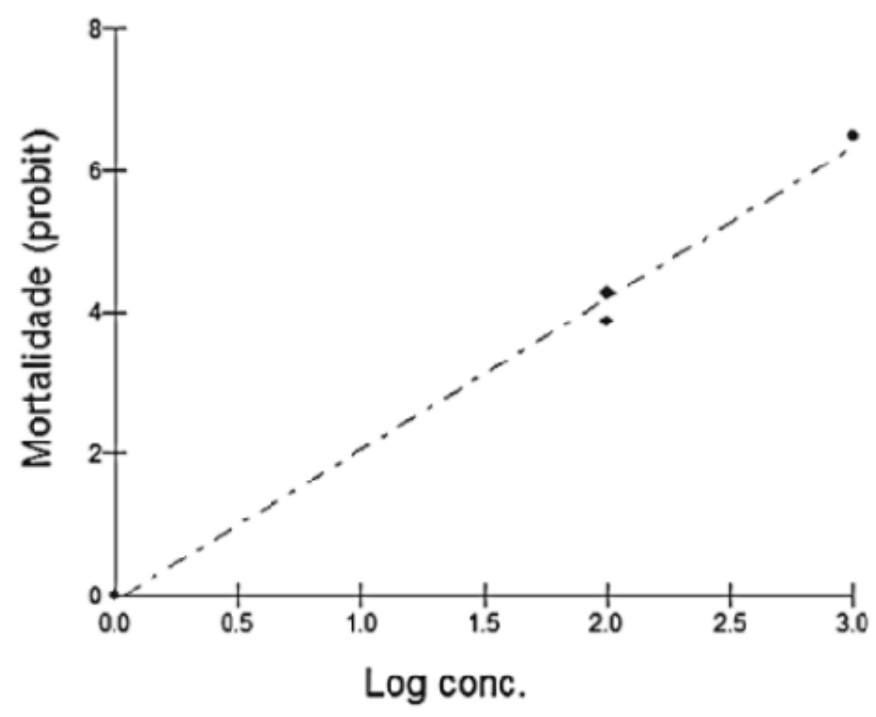

Figura 1. Relação dose-resposta do solo do cemitério urbano em oligochaetas com a transformação Probit 
Tabela 3. Valores percentuais de mortos transformados em Probit e CL50, do bioensaio de toxicidade aguda em Oligochaetas (minhoca) expostas a diferentes concentrações de solo do cemitério rural.

\begin{tabular}{|c|c|c|c|c|c|c|c|}
\hline $\begin{array}{l}\text { Grupo } \\
\text { Teste }\end{array}$ & $\begin{array}{c}\text { (a) } \\
\mathrm{N}^{\mathrm{o}} \text { org. } \\
\text { (oligochaetas) }\end{array}$ & $\begin{array}{c}\text { (b) } \\
\text { Conc. Solo } \\
\text { cemitério (g.kg- }{ }^{1} \text { ) }\end{array}$ & $\begin{array}{c}\text { (c) } \\
\text { Log. Conc. }\end{array}$ & $\begin{array}{c}\text { (d) } \\
\mathrm{N}^{\mathrm{o}} \text { mortos }\end{array}$ & $\begin{array}{c}\text { (e) } \\
\% \text { mortos }\end{array}$ & $\begin{array}{c}(f) \\
\text { Probits }\end{array}$ & $\begin{array}{c}\text { (g) } \\
\mathrm{CL}_{50} \cdot \mathrm{mg}^{-\mathrm{kg}^{-1}}\end{array}$ \\
\hline T0 & 30 & 0 & 0 & 0 & 0 & 0 & \multirow{4}{*}{52,48} \\
\hline $\mathrm{T} 1$ & 30 & 500 & 2,70 & 23 & 76,7 & 5.71 & \\
\hline $\mathrm{T} 2$ & 30 & 750 & 2,90 & 28 & 93,3 & 6.48 & \\
\hline T3 & 30 & 1000 & 3,00 & 30 & 100 & 8.09 & \\
\hline
\end{tabular}

Nota: Número de organismos testados (a), concentração de solo contaminado (b), log. da concentração (c), número de organismos mortos (d), percentual de organismos mortos (e), probito do percentual de organismos mortos (f) e $\mathrm{CL}_{50}(\mathrm{~g})$

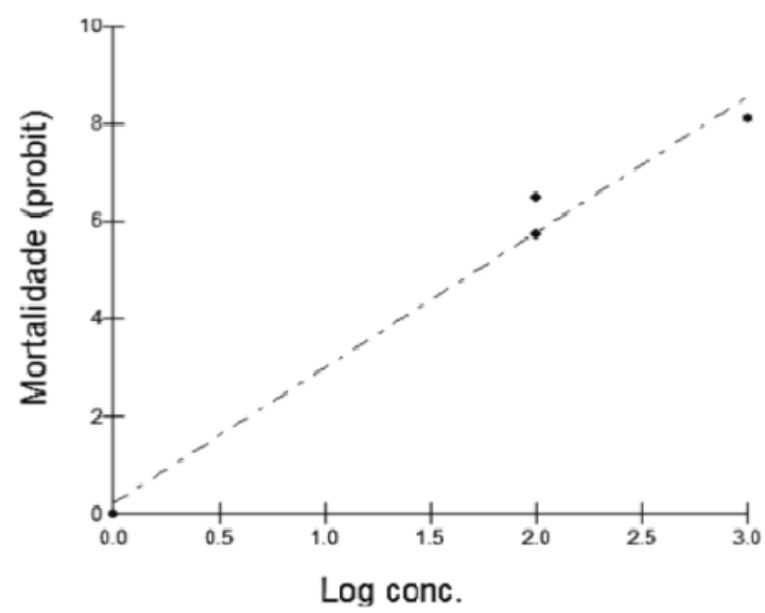

Figura 2. Relação dose-resposta do solo do cemitério urbano em oligochaeta com a transformação Probit.

A Figura 3 representa os percentuais de morte observados nos tratamentos T1, T2 e T3 nos quais se evidencia que quanto maior o percentual de solo contaminado adicionado maior é o número de indivíduos expostos mortos. Observa-se também que esses percentuais são superiores nos tratamentos que envolveram solo do cemitério rural. Considerando que foram adotados os mesmos tratamentos e procedimentos para ambos os cemitérios testados, pode-se inferir que o solo do cemitério rural, quer por suas características físico-químicas ou condições de construção, apresenta maior potencial tóxico que o solo do cemitério urbano.

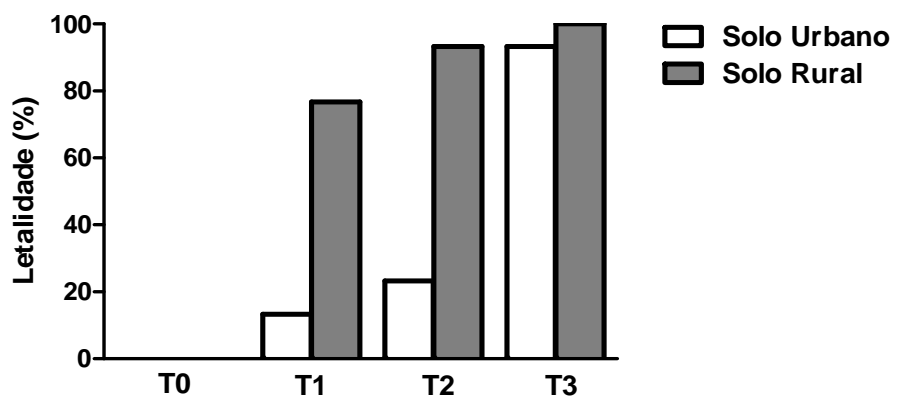

Figura 3. Letalidade nos diferentes tratamentos. T0 $100 \%$ solo de mata; $\mathrm{T} 1-50 \%$ solo contaminado $+50 \%$ solo de mata; $\mathrm{T} 2-75 \%$ solo contaminado $+25 \%$ solo de mata e T3 - 100\% solo contaminado. 
Os teores totais de Cádmio $(\mathrm{Cd})$, Cobre $(\mathrm{Cu})$, Cromo $(\mathrm{Cr})$, Chumbo $(\mathrm{Pb})$, Níquel $(\mathrm{Ni})$ e Zinco ( $\mathrm{Zn}$ ), detectados neste estudo, equiparados aos valores de referência propostos pela Resolução CONAMA 420, de dezembro de 2009 alterada pela Resolução CONAMA 460, de dezembro de 2013 (Tabela 4), evidenciam que apenas os metais $\mathrm{Cr}$ e $\mathrm{Ni}$ (em ambos os solos na concentração $100 \%$ ) estão acima dos valores de prevenção (VP).

Tabela 4. Concentração de metais tóxicos e valores de referência para o solo de mata e solo dos cemitérios urbano e rural.

\begin{tabular}{|c|c|c|c|c|}
\hline \multirow[b]{2}{*}{ Parâmetros } & \multicolumn{4}{|c|}{ Valores $\mathrm{mg} \mathrm{kg}^{-1}$} \\
\hline & $\begin{array}{c}\text { Prevenção } \\
\text { (CONAMA) }\end{array}$ & $\begin{array}{c}\text { Solo controle } \\
\text { (Mata) }\end{array}$ & $\begin{array}{l}\text { Solo urbano } \\
(100 \%)\end{array}$ & $\begin{array}{c}\text { Solo rural } \\
(100 \%)\end{array}$ \\
\hline $\mathrm{Cd}$ & 1,3 & 0,094 & $0,134^{\mathrm{ns}}$ & $0,106^{\mathrm{ns}}$ \\
\hline $\mathrm{Cr}$ & 75 & 9,34 & $163,68 * *$ & $172,56^{* *}$ \\
\hline $\mathrm{Cu}$ & 60 & 7,43 & $13,19^{\text {ns }}$ & $19,16^{\mathrm{ns}}$ \\
\hline $\mathrm{Ni}$ & 30 & 3,03 & $61,69^{* *}$ & $63,81 * *$ \\
\hline $\mathrm{Pb}$ & 72 & 7,43 & $16,20^{\text {ns }}$ & $22,47^{\mathrm{ns}}$ \\
\hline $\mathrm{Zn}$ & 300 & 17,79 & $95,08^{\text {ns }}$ & $53,02^{\text {ns }}$ \\
\hline
\end{tabular}

Ns $=$ não significativo; $*$ significativo a 0,$05 ; * *$ significativo a 0.10 . Análise de variância (ANOVA One-Way).

As concentrações totais dos demais metais analisados (Al, $\mathrm{Ca}, \mathrm{Fe}, \mathrm{Mg}$ ) ficaram abaixo dos Valores de Prevenção (VP- concentração que pode afetar negativamente a qualidade do solo), estabelecidos conforme CONAMA n $\mathrm{n}^{\mathrm{o}}$ 420/2009. Desta forma, $\mathrm{Cu}, \mathrm{Pb}$ e $\mathrm{Zn}$, estão enquadrados no Valor de Referência de Qualidade (VRQ), sendo atóxicos nas concentrações detectadas para ambos os solos. Cromo (Cr) e Níquel (Ni) não existem no organismo, porém são necessários para a manutenção do seu funcionamento e, portanto, requeridos em concentrações muito baixas, acima das quais se tornam extremamente tóxicos (Siqueira et al., 1994, Salazar et al, 2011a), provavelmente tenham sido os responsáveis pela mortalidade das oligochaetas expostas. Outra possível explicação para a toxicidade do $\mathrm{Cr}$ ter sido superior pode ser devido ao $\mathrm{Cr}^{+}$, apesar de ligar-se fortemente a sítios negativos de argila e matéria orgânica (Mengel e Kirkby, 1982), em solos mais arenosos como o solo do cemitério rural, tem maior mobilidade e, portanto, maior biodisponibilidade.

$\mathrm{Na}$ maioria dos solos a concentração não ultrapassa $60 \mathrm{mg} \mathrm{kg}^{-1} \mathrm{e}$, portanto, segundo WHO (1988) quase todo cromo hexavalente, forma mais tóxica tanto para plantas como para animais é resultante das atividades humanas. Por essa razão, no caso dos solos de cemitério, esses metais muito provavelmente são oriundos, principalmente, dos banhos de cromação dos adornos dos caixões e tintas e vernizes preservativos das madeiras usados na fabricação dos caixões e das urnas de metal. Relatos sobre a toxicidade do cromo em animais terrestes são escassos, porém, WHO (1988) cita também que a toxicidade para a maioria dos microorganismos ocorre na faixa de $0,05 \mathrm{mg} \mathrm{kg}^{-1}$.

A Figura 4 compara as concentrações de $\mathrm{Cd}, \mathrm{Cr}, \mathrm{Cu}, \mathrm{Ni}, \mathrm{Pb}$ e $\mathrm{Zn}$ detectadas neste experimento com os valores CONAMA 420\2009. Nesta figura, pode-se observar que tanto Cromo como Niquel foram encontrados acima dos valores de prevenção CONAMA $420 \backslash 2009$. 


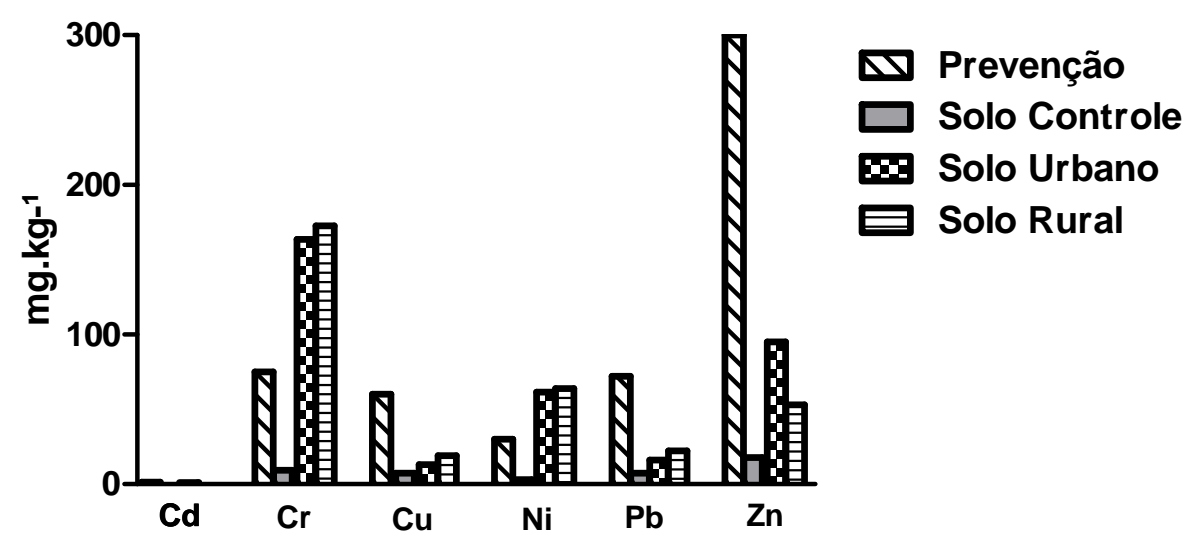

Figura 4. Concentração dos elementos em comparação com valores de referência (prevenção) CONAMA 420/2009.

Analisando os valores de cromo ( $\mathrm{Cr}$ ) detectados nos dois tipos de solos (urbano e rural), observam-se valores acentuados na concentração $100 \%$, com a ausência das oligochaetas, em ambos os casos (Figura 5 a e b).

Ao contrário, nos tratamentos que receberam oligochaetas, se observou comportamento diferenciado, no solo do cemitério urbano $(100 \%+$ oligochaetas $)$ houve decréscimo significativo na concentração do metal, porém, no solo do cemitério rural, na mesma condição o decréscimo foi superior.

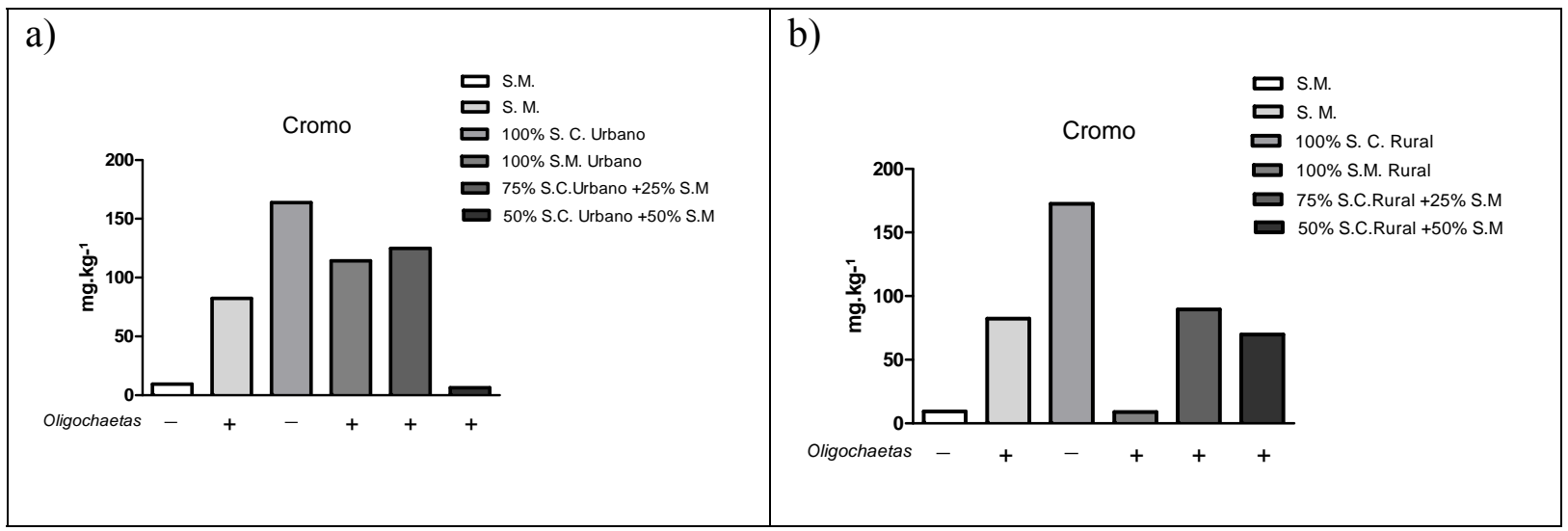

Figura 5. Comportamento do Cromo (Cr) em: a) solo de cemitério urbano com e sem oligochaetas, nas concentrações $0 \%, 100 \%, 75 \%$ e $50 \%$ e, b) solo de cemitério rural.

Segundo Macedo e Morril (2008), o Cromo, por participar dos processos metabólicos da glicose, tem sido considerado essencial para homens e animais e, por essa razão, acredita-se que a absorção do metal pelas oligochaetas foi superior em relação aos outros metais. Entretanto, quando presentes em amostras ambientais de água e solos acidificados esse elemento apresenta estado de oxidação hexavalente $\mathrm{Cr}(\mathrm{VI})$, podendo ser extremamente tóxico à biota local (Salazar et al., 2011b; Bianchi et al., 2012). Há que se considerar, ainda, a presença do metal no esterco bovino adicionado ao solo conforme propõem Gonçalves et al. (2007).

O comportamento do Níquel, ilustrado na Figura 6 (a e b), mostrou-se muito semelhante ao observado para o Cromo, em ambos os solos (urbano e rural). Contudo, ao compararmos o comportamento do metal no solo urbano com o comportamento apresentado no solo rural, o mesmo revela comportamento contrastante na concentração $100 \%$ solo de cemitério com presença de oligochaetas, no qual a concentração do metal reduziu. Além de apresentar, 
também, uma queda drástica no tratamento que continha solo de cemitério e solo de mata na mesma proporção. Nesta condição observa-se também que o Níquel teve um comportamento, principalmente no solo do cemitério rural, muito semelhante ao observado para o cromo.

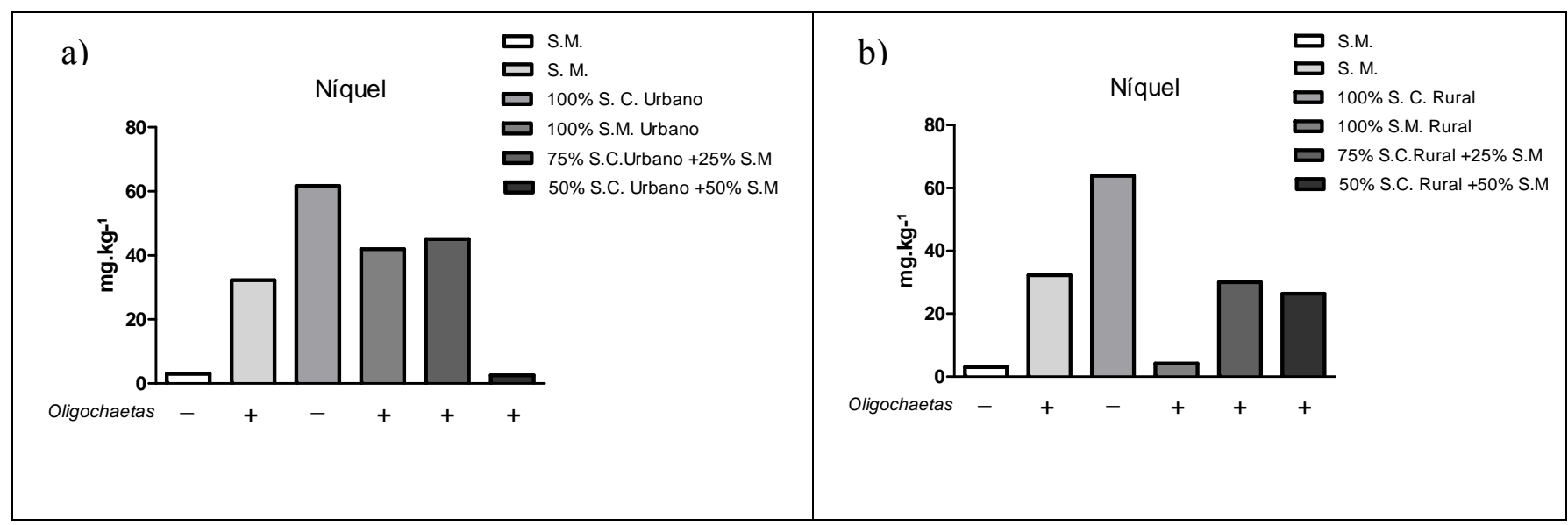

Figura 6. Comportamento dos metais tóxicos Níquel (Ni) em: a) solo de cemitério urbano; b) solo de cemitério rural e em solo de cemitério urbano.

Uma possível explicação para o comportamento do níquel seria de que como esse metal tem a propriedade de formar complexos na forma de quelatos com os compostos orgânicos do solo (Gonçalves et al., 2007; Salazar et al., 2011a), a matéria orgânica (esterco bovino) adicionada semanalmente às unidades experimentais tenha favorecido essa absorção do metal às partícula de solo e, consequentemente, a ingestão do metal junto com a matéria orgânica, reduzindo seu teor nos tratamentos contendo oligochaetas.

\section{CONCLUSÃO}

A presença de contaminantes como metais tóxicos nas concentrações detectadas neste trabalho comprometem a vida de um dos mais importantes grupos da macrofauna edáfica, as oligochaetas, indicadoras de toxicidade. Dentre os metais tóxicos que podem ser liberados no solo de cemitérios implementados em condições inadequadas, Cromo e Níquel, quando em concentrações elevadas são os que têm maior potencial para afetar a vida das oligochaetas. $\mathrm{O}$ cemitério é um empreendimento potencialmente poluidor, quando construído em local sem estudo prévio, principalmente em relação ao tipo de solo. Por fim, este aumento de substâncias tóxicas nos recursos naturais, deve ser analisado pelos órgãos competentes, pois o risco de tornar os recursos naturais desses locais, como o solo e os corpos hídricos subterrâneos inutilizáveis, é iminente.

\section{REFERÊNCIAS}

ANDRÉA, M. M. de. O uso de minhocas como bioindicadores de contaminação de solos. Acta Zoológica Mexicana, v. Especial 2, p. 95-107, 2010.

BARROS, Y. J.; MELO, V. F.; ZANELLO, S.; ROMANO, E. N. L.; LUCIANO, P. R.. Teores de metais pesados e caracterização mineralógica de solos do Cemitério Municipal de Santa Cândida, Curitiba (PR). Revista Brasileira de Ciência do Solo, v. 32, p. 1763-1773, 2008. http://dx.doi.org/10.1590/S0100-06832008000400041 
BIANCHI, S. R.; AMAIS, R. S.; PEREIRA, C. D.; SALAZAR, R. F. S.; NÓBREGA, J. A.; NOGUEIRA, A. R. A. Evaluation of a Collision-Reaction Interface (CRI) for Carbon Effect Correction on Chromium Determination in Environmental Samples by ICP-MS. Analytical Letters, v. 45, n. 18, p. 2845-2855, 2012. http://dx.doi.org/10.1080/00032719.2012.702176

CONSELHO NACIONAL DO MEIO AMBIENTE. Resolução 335, de 3 de abril de 2003. Dispõe sobre o licenciamento ambiental de cemitérios. Diário Oficial [da] União, Brasília, n. 101, 28 maio 2003, Seção 1, p. 98-99.

CONSELHO NACIONAL DO MEIO AMBIENTE. Resolução 368, de 28 de março de 2006. Altera dispositivos da Resolução 335, de 3 de abril de 2006, que dispõe sobre o licenciamento ambiental de cemitérios. Diário Oficial [da] União, Brasília, n. 61, 29 mar. 2006, Seção 1, p. 149-150.

CONSELHO NACIONAL DO MEIO AMBIENTE. Resolução 420, de 28 de dezembro de 2009. Dispõe sobre critérios e valores orientadores de qualidade do solo quanto à presença de substâncias químicas e estabelece diretrizes para o gerenciamento ambiental de áreas contaminadas por essas substâncias em decorrência de atividades antrópicas. Diário Oficial [da] União, Brasília, n. 249, 30 dez. 2009, Seção 1, p. 81-84.

CONSELHO NACIONAL DO MEIO AMBIENTE. Resolução 460, de 30 de dezembro de 2013. Altera a Resolução CONAMA 420, de 28 de dezembro de 2009, que dispõe sobre critérios e valores orientadores de qualidade do solo quanto à presença de substâncias químicas e dá outras providências. Diário Oficial [da] União, Brasília, n. 253, 31 dez. 2013, Seção 1, p. 153.

EMPRESA BRASILEIRA DE PESQUISA AGROPECUÁRIA - EMBRAPA. Sistema brasileiro de classificação de solo. 2. ed. Rio de Janeiro, 2006. 306 p.

GONÇALVES JR, A. C.; POZZA, P. C.; NACKE, H.; LAZZERI, D. B.; SELZLEIN, C.; CASTILHA, L. D. Homogeneização e níveis de metais em dejetos provenientes da bovinocultura de leite. Acta Scientiarum Technology, v. 29, p. 213-217, 2007. http://dx.doi.org/10.4025/actascitechnol.v29i2.712

HONG, K. J.; TOKUNAGA, S.; KAJIUCHI, T. Evaluation of remediation process with plant-derived biosurfactant for recovery of heavy metals from contaminated soils. Chemosphere, v. 49, p. 379-387, 2002. http://dx.doi.org/10.1016/S00456535(02)00321-1

INTERNATIONAL ORGANIZATION FOR STANDARDIZATION. ISO 17512-1: Soil quality - Avoidance test for testing the quality of soils and the toxicity of chemicals on behaviour - Part 1: Test with earthworms (Eisenia fetida and Eisenia andrei). Geneva, 2008.

KEMERICH, P. D. da C.; BORBA, F. de; SILVA, R. F. da; BARROS, G.; GERHARDT, A. E.; FLORES, C. E. B. Valores anômalos de metais pesados em solo de cemitério. Revista Ambiente \& Água, v. 7, n. 1, 2012. http://dx.doi.org/10.4136/ambi-agua.838

LELI, I. T.; ZAPAROLI, F. C. M.; SANTOS, V. C.; OLIVEIRA, M.; VIEIRA, F. A. G. Estudos ambientais para cemitérios: Indicadores, áreas de influência e impactos ambientais. Boletim de Geografia, v. 30, p. 45-54, 2012. http://dx.doi.org/10.4025/bolgeogr.v30i1.16348 
LOWE, C. N.; BUTT, K. R. Earthworm culture, maintenance and species selection in chronic ecotoxicological studies: a critical review. European Journal of Soil Biology, v. 43, p. 281-288, 2007. http://dx.doi.org/10.1016/j.ejsobi.2007.08.028

MACÊDO, L. S.; MORRIL, W. B. B. Origem e comportamento dos metais fitotóxicos: revisão da literatura. Tecnologia \& Ciência Agropecuária, v. 2, p. 2-38, 2008.

MATOS, B. A. Avaliação da ocorrência e do transporte de micronutrientes no aqüífero freático do cemitério de Vila Nova Cachoeirinha. 2001. 113 f. Tese (Doutorado em Recursos Minerais e Hidrogeologia) - Universidade de São Paulo, São Paulo, 2001.

MENGEL, K.; KIRKBY, E. A. Principles of plant nutrition. Worblanfe-Bern: International Potash Institute, 1982. $655 \mathrm{p}$.

PACHECO, A.; MENDES, J. M. B.; MARTINS, T.; HASSUDA, S.; KIMMELMANN, A. A. Cemeteries: a potential risk to groundwater. Water Science and Technology, v. 24, p. 97-104. 1991.

RIBEIRO, R.; HOEHNE, L.; MANICA, W.; CARLESSO, E. ETHUR, M.; STÜLP, S. A Absorção de cobre por minhocas da espécie Eisenia andrei em solos e húmus contaminados. Revista Destaques Acadêmicos, v. 4, n. 4, 2012.

SALAZAR, R. F. S.; ALCÂNTARA, M. A. K.; IZÁRIO FILHO, H. J. Evaluation of sample preparation methods and optimization of nickel determination in vegetable tissues. Revista Brasileira de Ciência do Solo, v. 35, n. 1, p. 241-248, 2011 la. http://dx.doi.org/10.1590/S0100-06832011000100022

SALAZAR, R. F. S.; GUERRA, M. B. B.; PEREIRA-FILHO, E. R.; NÓBREGA, J. A. Performance evaluation of collision-reaction internal stadardization in quadrupole ICPMS measurements. Talanta, v. 86, n. 1, p. 241-247, $2011 \mathrm{~b}$. http://dx.doi.org/10.1016/j.talanta.2011.09.009

SHUSTER, W. D.; SUBLER. S.; MCCOY. E. L. The influence of earthworm community structure on the distribution and movement of solutes in a chisel-tilled soil. Applied Soil Ecology, v. 21, p. 159-167, 2002. http://dx.doi.org/10.1016/S0929-1393(02)00063$\mathrm{X}$

SIQUEIRA, J. O.; MOREIRA, F. M. S.; GRISI, B. M.; HUNGRIA, M.; ARAÚJO, R. S. Microorganismo e processos biológicos do solo: perspectiva ambiental. Brasília, DF: EMBRAPA/CNPAF/CNPS, 1994. 142p. (Documentos, 45).

TEDESCO, M. J.; GIANELLO, C.; BISSANI, C. A. et al. Análise de solo, plantas e outros materiais. 2. ed. Porto Alegre: Departamento de Solos, UFRGS, 1995.174 p. (Boletim Técnico, 5).

WORLD HEALTH ORGANIZATION - WHO. Chromium. Geneva: Environmental Health Criteria, 1988. 61p.

WORLD HEALTH ORGANIZATION - WHO. The impact of cemeteries on the environment and public health: an Introductory briefing. Copenhagen: Regional Office for Europe, 1998. $11 \mathrm{p}$. 


\begin{tabular}{|} 
Ambiente \& Água - An Interdisciplinary Journal of Applied Science \\
ISSN 1980-993X - doi:10.4136/1980-993X \\
www.ambi-agua.net \\
E-mail: ambi.agua@gmail.com
\end{tabular}

\title{
Bioindicadores para avaliação do risco potencial de transmissão da esquistossomose no açude Apipucos, Pernambuco
}

\author{
doi:10.4136/ambi-agua.1756 \\ Received: 09 Sep. 2015; Accepted: 30 Nov. 2015 \\ Rodrigo Moraes Loyo*; Constança Simões Barbosa \\ Centro de Pesquisas Aggeu Magalhães (FIOCRUZ/CpqAM), Recife, PE, Brasil \\ Departamento de Parasitologia \\ *Autor correspondente: e-mail: rodrigoloyo@live.com, \\ constanca.barbosa@gmail.com
}

\section{RESUMO}

Em Pernambuco, a esquistossomose é endêmica na zona rural e inúmeros focos estão sendo detectados em localidades urbanas. Este artigo aferiu o risco para transmissão da esquistossomose em ambiente aquático urbano por meio de bioindicadores e biologia molecular. No Açude de Apipucos (bairro da região metropolitana do Recife) foram coletados caramujos vetores da esquistossomose em pontos com poluição fecal. Os Biomphalaria foram examinados por técnica molecular para detectar DNA de S. mansoni. Os coliformes fecais foram identificados por reação cromogênica. Os pontos de coleta foram georreferenciados e os resultados analisados com auxílio do aplicativo ArcGis. Foram coletados 77 B. straminea, 56 negativos e 21 positivos para $S$. mansoni. Coliformes fecais foram detectados em todas estações de coleta. O mapa de densidade Kernel mostrou o potencial do risco de transmissão da esquistossomose em ambiente aquático situado em bairro nobre da cidade.

Palavras chaves: indicadores ambientais, geoprocessamento para o ambiente, poluição, resíduos urbanos, saúde pública.

\section{Bio-indicators for the evaluation of potential risk of schistosomiasis transmission at the Apipucos reservoir, Pernambuco State}

\begin{abstract}
In Pernambuco, schistosomiasis is endemic in the countryside and large numbers of foci are being detected in urban areas. This paper evaluated the risk of schistosomiasis transmission in aquatic ecosystem based on biomarkers and molecular biology techniques. At the Apipucos reservoir (neighborhood of metropolitan Recife), schistosomiasis intermediate host snails were collected in contaminated sites with fecal coliforms. The Biomphalaria were examined by molecular methods to detect DNA of S. mansoni. The fecal coliforms were identified with a chromogenic reaction. The collection stations were georeferenced and the results analyzed with the ArcGIS software package. Out of 77 B. straminea collected, 56 were negative and 21 positive for $S$. mansoni. Fecal coliforms were detected in all collection
\end{abstract}


stations. The Kernel density map showed the potential risk of schistosomiasis transmission in upscale neighborhood of Recife city.

Keywords: environmental indicators, GIS of the environment, municipal waste, pollution, public health.

\section{INTRODUÇÃO}

O açude de Apipucos é uma parte integrante da sub-bacia do rio Capibaribe, localizado na região metropolitana do Recife em Pernambuco. Seu reservatório é composto por dois espelhos d'água, sendo o menor situado a leste com cerca de 62.500 metros quadrados de área e o maior a oeste perfazendo uma área de aproximadamente 96.250 metros quadrados. O ecossistema biótico desse reservatório de água doce é formado por seres diversos, dos mais simples como cianobactérias e rotíferos planctônicos até seres mais complexos como as traíras (Hoplias malabaricus), tambaquis (Colossoma macropomum) e piabas (Poecilia vivipara), já a parte abiótica é muito instável por estar localizado em uma área de urbanização crescente com relatos de contaminação fecal e de outros tipos de detritos como sacolas plásticas, garrafas e pneus que estão afetando o paisagismo e a qualidade hídrica do reservatório (Burgos e Arantes, 2009).

As enterobactérias são encontradas no trato gastrointestinal humano, no reino animal, na água, solo e vegetais. Sua presença em águas contaminadas por esgotos humanos é apontada como um bioindicador de contaminação fecal. Os indicadores biológicos são específicos a certos tipos de impacto, já que inúmeras espécies são comprovadamente sensíveis a um tipo de poluente, mas tolerantes a outros. Entretanto nenhum indicador é efetivamente perfeito, levando em consideração uma série de fatores tais como classe dos corpos de água, característica da água (água doce, salina), clima (temperado, tropical) além do fator econômico (Duarte, 2011).

A esquistossomose mansônica é uma doença de veiculação hídrica, transmitida por caramujos do gênero Biomphalaria que são contaminados por larvas do Schistosoma mansoni quando pessoas infectadas pelo parasito lançam seus dejetos contendo ovos do verme em ambientes aquáticos habitados por esses moluscos. Em Pernambuco a esquistossomose é endêmica na zona rural e inúmeros focos estão sendo detectados em localidades urbanas e litorâneas deste Estado mostrando a expansão desta endemia com índices preocupantes de casos agudos da doença em indivíduos que nunca tiveram exposição ao parasita $S$. mansoni (Souza et al., 2008; Paredes et al., 2010; Gomes et al., 2012).

Levantamento malacológico realizado no ano de 2012 em coleções hídricas da cidade do Recife identificou 30 criadouros do caramujo vetor Biomphalaria straminea e no açude de Apipucos foram encontrados dois pontos de coleta com moluscos apresentando DNA de $S$. mansoni. Na mesma ocasião, uma busca ativa realizada entre escolares residentes no entorno destes criadouros diagnosticou 14 crianças com esquistossomose, cinco delas comprovadamente casos autóctones, sendo uma delas usuária sistemática do açude (Barbosa et al., 2013).

Algumas técnicas moleculares que permitem a deteç̧ão do DNA de Schistosoma mansoni nos caramujos hospedeiros vêm sendo amplamente utilizadas, possibilitando diagnosticar rapidamente e com precisão caramujos infectados pelo parasito, permitindo assim identificar ambientes aquáticos onde existe o risco de transmissão da esquistossomose (Abath et al., 2000).

Levantamento malacológico realizado no ano de 2012 em coleções hídricas da cidade do Recife identificou 30 criadouros do caramujo vetor Biomphalaria straminea e no açude de Apipucos foram encontrados dois pontos de coleta com moluscos apresentando DNA de $S$. 
mansoni. Na mesma ocasião, uma busca ativa realizada entre escolares residentes no entorno destes criadouros diagnosticou 14 crianças com esquistossomose, cinco delas comprovadamente casos autóctones, sendo uma delas usuária sistemática do açude (Barbosa et al., 2013).

Existem poucos estudos locais que utilizam bioindicadores e técnicas moleculares para avaliar o risco de transmissão da esquistossomose em ambientes aquáticos, tendo em vista a importância histórica, ambiental e de lazer urbano do Açude de Apipucos, o presente trabalho tem como objetivo levantar indicadores biológicos (presença de moluscos vetores e de coliformes fecais) e usar técnicas de biologia molecular para detectar a presença de DNA do S. mansoni nos caramujos, a fim de estimar o risco ambiental de transmissão da esquistossomose para indivíduos que se expõem ao exercerem atividades de lazer aquático.

\section{MATERIAIS E MÉTODOS}

\section{1. Área de estudo}

O mapeamento da localidade de estudo foi realizado através da vetorização, com um equipamento de Global Positioning System (GPS) modelo Garmin montana 650. Abrangendo todo espaço geográfico dos limites do bairro de Apipucos, vias de acesso e a coleção hídrica formada pelo açude. Um croqui da área foi elaborado utilizando o software ArcGis 10.1 (http://www.esri.com/software/arcgis).

\subsection{Identificação dos criadouros e coleta das amostras}

A delimitação das estações de coleta no açude de Apipucos foi realizada considerando os seguintes critérios de importância epidemiológica: presença de caramujos vetores da esquistossomose, indícios de contaminação fecal, local de passagem de pedestres, de pesca e banho. No total foram demarcadas cinco estações de coleta dos moluscos vetores e dez estações de coleta de água. Três coletas de caramujo e água foram realizadas no estudo, uma em cada período climático (Seca, Chuva e Pós-chuva), considerando a influência pluviométrica sobre a densidade populacional dos caramujos Biomphalaria e concentração de dejetos. O tempo da coleta foi de 10 minutos em cada estação, pesquisando nas margens e nos diferentes estratos de vegetação.

\subsection{Análise dos moluscos coletados}

A infecção natural dos caramujos para o S. mansoni foi diagnosticada através da técnica clássica de exposição à luz que permite a visualização da forma infectante do parasita (cercárias) quando liberada pelo caramujo e pela identificação do DNA do parasita empregando o método molecular Nested PCR, modificado de Abath et al. (2000). No teste molecular as amostras foram divididas e analisadas por ponto de coleta, adicionado um controle de extração e reação. Foram utilizados como iniciadores externos os primers Unvfo2 e Schre19 e como iniciadores internos o Unvre6 e Schfo17.

\subsection{Análise das amostras de água}

As técnicas de coleta obedeceram aos procedimentos descritos no Standard Methods for the Examination of Water and Wastewate. Para a detecção e quantificação de coliformes totais (CT), coliformes fecais (CF) e Escherichia coli foi empregado o método cromogênico com o kit Colitag ${ }^{\mathrm{TM}}$.

As amostras hídricas foram obtidas nas mesmas estações de coleta dos moluscos vetores, em recipientes estéreis de $100 \mathrm{ml}$. O reagente cromogênico foi adicionado ao frasco da água coletada, homogeneizado para posterior incubação por 24 horas a uma temperatura de $35{ }^{\circ} \mathrm{C}$. A primeira leitura foi realizada por método direto, onde as amostras que migraram da 
coloração natural para um amarelo vibrante passaram para a fase dois. A segunda leitura foi feita com auxílio de um fotodocumentador da Loccus biotecnologia ${ }^{\mathrm{TM}}$ (L-Pix) que irradiou as amostras com um feixe de luz ultravioleta para determinar a presença de E. coli.

\subsection{Georreferenciamento dos dados}

As estações de coleta de caramujo e de amostras hídricas foram georreferenciadas. Os resultados dos dados biológicos foram tratados em planilha do Microsoft Excel 2007 para construção dos mapas temáticos usando o aplicativo ArcGis 10.1. A aplicação do estimador de densidade Kernel permitiu a agregação das informações gerando um aglomerado que destaca os locais quentes (hotspot) nas margens do açude Apipucos, salientando as áreas com maior potencial de risco para transmissão da esquistossomose, ao quais os frequentadores do açude estão expostos.

\section{RESULTADOS E DISCUSSÃO}

Ao longo da área marginal do açude, foram demarcadas cinco estações para coleta dos Biomphalaria e dez estações para análise da água, em locais frequentados pela população do bairro para pesca, banhos e lazer contemplativo. Nas coletas malacológicas realizadas ao longo do ano foram identificados dois criadouros e três focos de $B$. straminea no espelho d'água oeste do açude e em todas as dez estações de coleta foram detectados coliformes fecais evidenciando a existência poluição fecal disseminada nos dois espelhos d'água do açude de Apipucos - reservatório leste e oeste (Figura 1).

Nos criadouros identificados foram coletados 77 exemplares do caramujo B. straminea que se mostraram negativos para infecção ao $S$. mansoni pela técnica clássica de exposição à luz. Ocorreu a formação de um amplicon característico $(721 \mathrm{pb})$ do DNA do S. mansoni nas amostras de 21 caramujos o que indica que esses exemplares albergam algum estágio do parasito e portanto, estão passiveis de transmitir a parasitose.

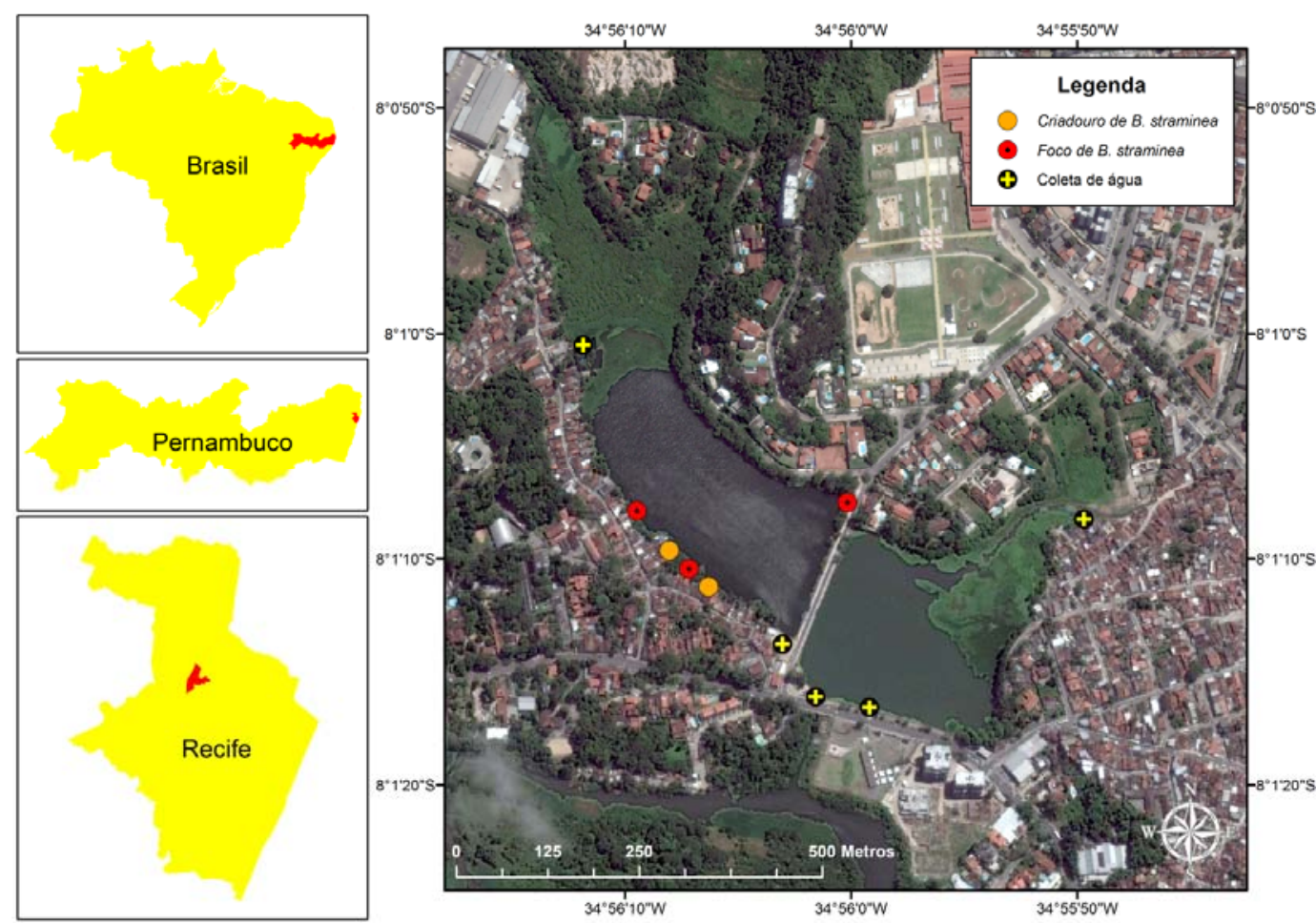

Figura 1. Açude Apipucos, região metropolitana do Recife, mostrando as dez estações de coleta georreferenciadas identificando os criadouros de $B$. straminea e potenciais focos de transmissão. 
O teste utilizado para dosagem de coliformes fecais (reagente cromogênico Colitag ${ }^{\mathrm{TM}}$ ) tem uma sensibilidade que permite a identificação de uma unidade formadora de colônia da enterobacteria de interesse, em $100 \mathrm{ml}$ da amostra hídrica. Os achados desse estudo mostram que houve reação positiva para $E$. coli com as amostras de nove locais de coleta sendo o mínimo encontrado de 240 UFC mL (Unidades Formadoras de Colônia) e pontos de coleta que passavam de 1600 UFC mL.

A análise espacial usando Kernel evidenciou as áreas com maior potencial de risco para transmissão da esquistossomose no Açude Apipucos, quando foram associados os dados: poluição fecal, presença e densidade populacional dos caramujos vetores nos pontos de coleta (Figura 2).

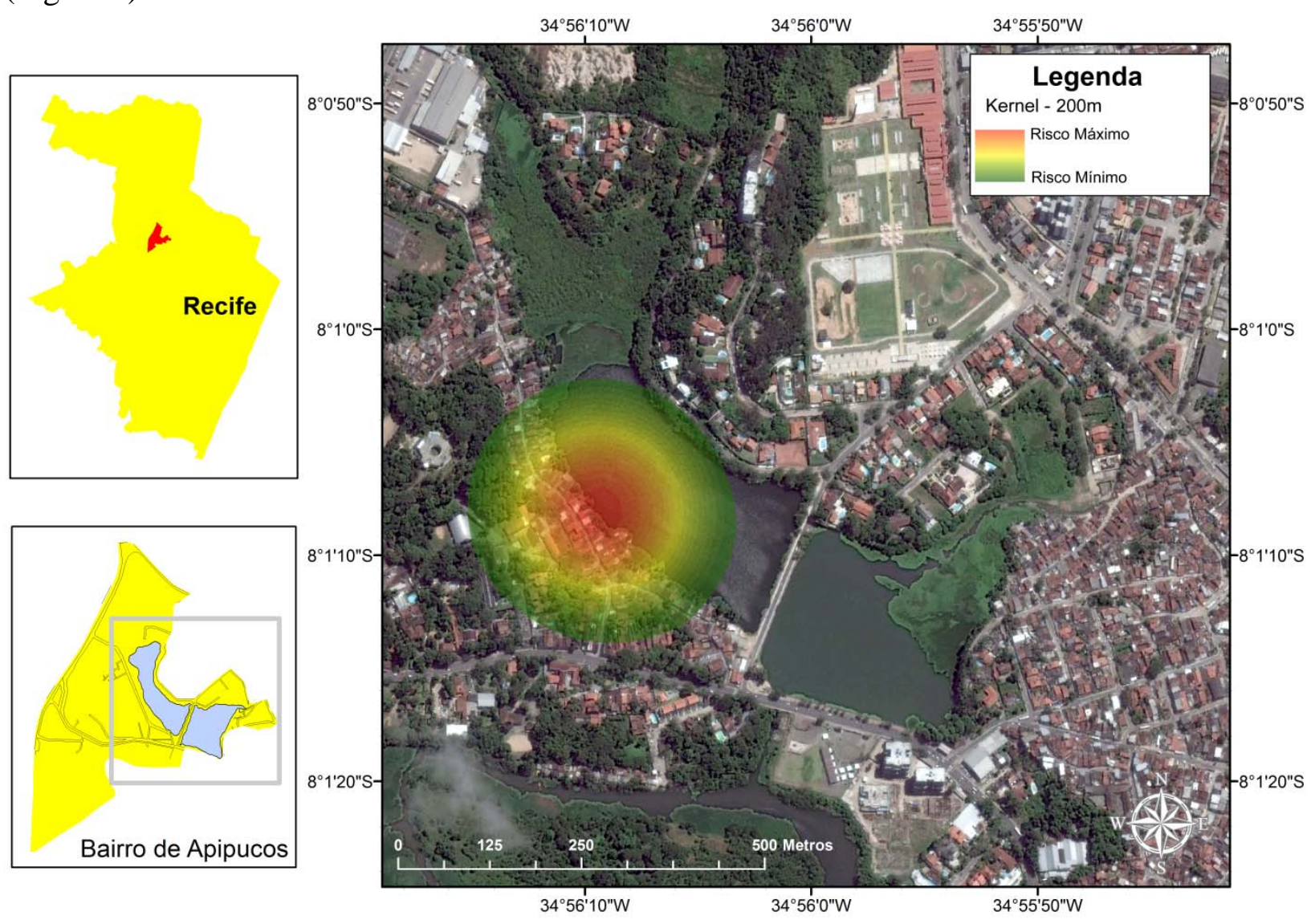

Figura 2. Mapa do Açude Apipucos mostrando a área de risco de transmissão para a esquistossomose em bairro nobre da cidade do Recife.

\section{CONCLUSÃO}

O presente estudo revelou pontos com indicadores biológicos de risco para a transmissão da esquistossomose no Açude de Apipucos que, apesar de localizado em bairro nobre do Recife, tem 81\% de residências sem sistema de esgotamento sanitário (Recife, 2015), condição que propicia a ocorrência de ambientes aquáticos insalubres. A cidade possui inúmeros criadouros do caramujo vetor da esquistossomose (Barbosa et al., 2013), mas ainda não se tem registro de vetores que eliminam as cercarias infectantes do parasito. Os achados desta pesquisa apontam potenciais riscos ambientais e biológicos para o estabelecimento do ciclo de transmissão do $S$. mansoni em Recife, o que demanda atuação urgente das autoridades de saúde no que se refere a medidas educativas e ao tratamento dos resíduos despejados pelas comunidades nos reservatórios dulciaquícolas desta cidade. 


\section{REFERÊNCIAS}

ABATH, F. G. et al. Characterization of Sm13, a tegumental antigen of Schistosoma mansoni. Parasitology research, v. 86, n. 9,p. 745-52, 2000. http://dx.doi.org/10.1007/PL00008562

BARBOSA, C. S. et al. Casos autoctones de esquistossomose mansonica em crianças de Recife, PE. Revista de Saúde Pública, v. 47, p. 684-690, 2013. http://dx.doi.org/10.1590/rsp.v47i4.76600

BURGOS, K.; ARANTES, E. Açude de Apipucos: história e ecologia. Recife: CEPE, 2009.

DUARTE, P. B. Microrganismos indicadores de poluição fecal em recursos hídricos. 2011. 52p. Monografia (Especialização em Microbiologia) - Instituto de Ciências Biológicas, Universidade Federal de Minas Gerais, Belo Horizonte, 2011.

GOMES, E. C. S. et al. Schistosomiasis transmission and environmental change: a spatiotemporal analysis in Porto de Galinhas, Pernambuco - Brazil. International Journal of Health Geographics, v. 11, p. 51, 2012.

PAREDES, H. et al. Spatial pattern, water use and risk levels associated with the transmission of schistosomiasis on the north coast of Pernambuco, Brazil. Cadernos de Saúde Pública, v. 26, n. 5, p. 1013-1023, 2010. http://dx.doi.org/10.1590/S0102311X2010000500023

RECIFE. Secretaria do Planejamento. Plano diretor do Recife: diagnóstico: a dinâmica físico-territorial. 2015. Disponível em: http://www.recife.pe.gov.br/pr/secplanejamento/ planodiretor/diagnostico_ii.html. Acesso em: 18 jan. 2015.

SOUZA, M. A. A. et al. Criadouros de Biomphalaria, temporários e permanentes, em Jaboatão dos Guararapes, PE. Revista da Sociedade Brasileira de Medicina Tropical, v. 41, n. 3, p. 252-256, 2008. http://dx.doi.org/10.1590/S0037-86822008000300006 


Ambiente \& Água - An Interdisciplinary Journal of Applied Science
ISSN 1980-993X - doi:10.4136/1980-993X
www.ambi-agua.net
E-mail: ambi.agua@gmail.com

\title{
Índices de qualidade da água e de estado trófico do rio Caiabi, MT
}

\author{
doi:10.4136/ambi-agua.1769 \\ Received: 02 Oct. 2015; Accepted: 30 Nov. 2015
Grasiane Andrietti $^{1}$; Rosane Freire ${ }^{2}$; Adriana Garcia do Amaral ${ }^{1}$; Frederico Terra de Almeida ${ }^{1}$; Milene Carvalho Bongiovani ${ }^{1}$; Roselene Maria Schneider ${ }^{1^{*}}$ \\ ${ }^{1}$ Universidade Federal de Mato Grosso (UFMT), Sinop, MT, Brasil \\ ${ }^{2}$ Universidade Estadual Paulista "Júlio de Mesquita Filho" (UNESP), Presidente Prudente, SP, Brasil \\ Departamento de Física, Química e Biologia \\ *Autor correspondente: e-mail: roselenems@yahoo.com.br, \\ grasiane_melo@yahoo.com.br, rofreire@gmail.com, adrianagamaral@gmail.com, fredterra@ufmt.br, \\ milene.bongiovani@gmail.com
}

\section{RESUMO}

Neste trabalho objetivou-se identificar a qualidade da água superficial do rio Caiabi, MT por meio do índice de qualidade de água (IQA) e índice de estado trófico (IET), bem como a avaliação das variações sazonais e espaciais, com a definição do delineamento do monitoramento mais adequado para o local de estudo. As coletas para o monitoramento da qualidade da água ocorreram em cinco pontos no rio Caiabi, entre julho de 2012 e junho de 2013. Foram quantificados os seguintes parâmetros de qualidade: $\mathrm{pH}$, temperatura, condutividade elétrica, oxigênio dissolvido, coliformes totais e termotolerantes, turbidez, nitrogênio Kjeldal, nitrito, nitrato, fósforo total, demanda bioquímica de oxigênio, série de sólidos e clorofila a. Os procedimentos de coleta e análises seguiram os definidos pelo Standard Methods for the Examination of Water and Wastewater. Os resultados dos índices IQA mostraram que as águas do rio Caiabi possuem boa qualidade. Os resultados de IET evidenciaram o baixo risco de eutrofização no rio Caiabi, com indicação de um ambiente lótico ultraoligotrófico. A análise de variância demonstrou que 10 dos 16 parâmetros de qualidade monitorados apresentaram diferenças de médias entre as estações de seca e chuva ou entre os pontos monitorados ou na interação entre estações e pontos. Esses resultados indicam que duas coletas anuais em dois pontos podem ser suficientes para descrever o comportamento da qualidade da água na bacia, desde que as condições de uso e ocupação do solo não sejam modificadas.

Palavras-chave: água superficial, bacia amazônica, recursos hídricos, uso e manejo do solo.

\section{Water quality index and eutrophication indices of Caiabi River, MT}

\section{ABSTRACT}

The objective of this study was to evaluate the water quality of the Caiabi River based upon the water quality index (WQI) and the trophic state index (TSI), considering seasonal and spatial variations, with the aim of determining the most appropriate monitoring design for this study site. Sampling for water quality monitoring was conducted at five points on the 
Caiabi River from July 2012 to June 2013. Quality parameters quantified were as follows: $\mathrm{pH}$, temperature, conductivity, dissolved oxygen, total and thermotolerant coliforms, turbidity, Kjeldahl nitrogen, nitrite, nitrate, total phosphorus, biochemical oxygen demand, series of solids, and chlorophyll a. Sampling procedures and analysis followed the methods recommended by the Standard Methods for the Examination of Water and Wastewater. The WQI results showed that the quality of the Caiabi River water is good. TSI results demonstrated the low risk of eutrophication in the Caiabi River, indicating an ultraoligotrophic lotic environment. Analysis of variance showed that 10 of the 16 monitored quality parameters presented differences of means between the dry and rainy seasons or among the monitored points or in the interaction between seasons and points. These results indicate that two annual sampling collections at two points may be sufficient to describe the water quality behavior in the basin, as long as the conditions of land use are stable.

Keywords: amazon basin, soil use and management, surface water, water resources.

\section{INTRODUÇÃO}

Pesquisas realizadas em bacias hidrográficas rurais têm demonstrado o potencial de degradação da qualidade das águas superficiais (Donadio et al., 2005; Chaves e Santos, 2009; Bateni et al., 2013; Rajankar et al., 2011). No Brasil, a região da Amazônia Legal sente as consequências do uso e ocupação do solo estabelecido aliado ao regime hídrico da região. Essas condições proporcionam um potencial teórico de degradação acentuado, considerando, principalmente, os eventos de intensa precipitação local (Souza et al., 2013). O transporte de sedimentos, o carreamento de nutrientes e matéria orgânica para o leito do rio traz agravos que podem se tornar irreversíveis, superando a capacidade de autodepuração do rio (Wittman et al., 2013).

Entre as bacias hidrográficas da região da Amazônia Legal, a do rio Caiabi chama a atenção por estar em uma região de grande importância social, econômica e ambiental. Localizado na região norte do estado de Mato Grosso, entre os biomas Amazônia e Cerrado (Marimon et al., 2006), é um curso d'água perene, de segunda ordem, possui $70 \mathrm{~km}$ de extensão e drena uma área de $499 \mathrm{~km}^{2}$, abrangendo os municípios de Sinop e Vera. O principal uso do solo dessa área é voltado para o plantio de soja e milho, culturas de extrema importância para a economia do estado de Mato Grosso, sendo a região norte um grande exportador de commodities agrícolas (Morales et al., 2013).

Quando a floresta é retirada dando lugar a sistemas de produção agrícola, ou pastagens, não apenas a paisagem é alterada, mas a qualidade da água da bacia hidrográfica também sofre modificações. Os usos múltiplos da água e as atividades antrópicas realizadas em uma bacia hidrográfica causa significativas alterações na qualidade dos recursos hídricos. Em prática, pouco se sabe sobre a qualidade das águas da bacia do rio Caiabi, o que representa que não se sabe qual é o real impacto do uso e ocupação do solo da bacia hidrográfica.

Em geral, decretos e resoluções sobre qualidade da água consistem em parâmetros de poluição das massas de água em monitoramento. Geralmente, são informações pouco expressivas para os cientistas, no entanto, significativas para os gestores e tomadores de decisão do setor de água e que querem saber sobre o estado de seus corpos hídricos (Nasirian, 2007). Nas últimas décadas, tem-se observado o crescente aumento de estudos quanto ao monitoramento da qualidade da água, como visto nos trabalhos realizados por Cunha et al. (2004), Prado e Moraes (2007), Pontes et al. (2012), Bucci e Oliveira (2014), em várias regiões do país. 
Desenvolvidos para integrar, interpretar e comunicar os dados obtidos no monitoramento ambiental, as análises estatísticas e os índices têm sido usados com sucesso para caracterizar o estado e as tendências da qualidade da água e transmitir essas informações para os grupos interessados. Não exigem grande número de parâmetros de qualidade da água para o desenvolvimento e a validação é necessária apenas para a concentração de um número limitado de parâmetros de qualidade da água.

A luz das problemáticas ambientais que envolvem a bacia hidrográfica do Rio Caiabi, com uso e ocupação predominantemente rural, o índice de qualidade de água (IQA) e o índice de estado trófico (IET) foram escolhidos nesse trabalho por representarem em um único número e a escala qualitativa correspondente, o estado da água de forma eficaz. Essa pesquisa também traz uma profunda avaliação das variações sazonais e espaciais da qualidade da água superficial do rio Caiabi, com a definição do delineamento experimental mais adequado para o local de estudo.

\section{MATERIAIS E MÉTODOS}

\section{1. Área de estudo}

A área de estudo foi a microbacia hidrográfica do rio Caiabi, localizada na região médio Norte do Estado de Mato Grosso, na área de abrangência da Amazônia Legal. O principal rio da microbacia é o Caiabi, com aproximadamente $51 \mathrm{~km}$ de extensão. Esta região apresenta clima tropical, de acordo com a classificação climática de Köppen-Geiger apresentando duas estações. Entre os meses de outubro a abril tem-se a estação de chuvas, sendo o restante do período caracterizado como seco, apresentando pouca ou nenhuma precipitação. A precipitação total anual é de aproximadamente $2.000 \mathrm{~mm}$ (Souza et al., 2013). O uso do solo da bacia é predominantemente de cultivo temporário, apresentando também áreas de pastagens.

Para as amostragens foram selecionados 5 locais de coleta ao longo canal principal do rio, considerando os aspectos de logística e acessibilidade ao local de coleta nos meses de seca e de chuva. Os locais foram denominados $\mathrm{P} 1, \mathrm{P} 2, \mathrm{P} 3, \mathrm{P} 4$ e $\mathrm{P} 5$, sendo o $\mathrm{P} 1$ próximo à nascente e o P5 próximo ao exutório da bacia (Figura 1).

Em relação aos locais escolhidos como pontos de monitoramento, o trecho definido como ponto 2 era o que apresentava maior área com influência de pastagens. Estradas rurais e carreadores foram identificados em todos os trechos que contribuíam para os pontos de coleta.

As campanhas de amostragem foram realizadas durante doze meses consecutivos, entre julho de 2012 e junho de 2013. A coleta da água era realizada no ponto central da seção transversal do rio à profundidade de $10 \mathrm{~cm}$, aproximadamente. Os parâmetros de qualidade investigados foram o oxigênio dissolvido (OD), turbidez (TB), pH, temperatura (T), coliformes totais (CT), coliformes termotolerantes (CTERM), sólidos totais (ST), sólidos dissolvidos (SD), sólidos suspensos (SS) demanda bioquímica de oxigênio (DBO), condutividade elétrica $(\mathrm{CE})$, nitrogênio total $(\mathrm{NT})$, nitrito $\left(\mathrm{NO}_{2}\right)$, nitrato $\left(\mathrm{NO}_{3}\right)$, fósforo total (PT) e clorofila (CL).

A concentração de oxigênio dissolvido (OD), a turbidez, a temperatura e o pH foram determinados in situ, por meio de sonda multiparâmetros Horiba. As amostras de água coletadas para a determinação dos outros parâmetros foram armazenadas em frascos de vidro ou polietileno e transportadas para a Universidade Federal de Mato Grosso campus Sinop em caixa de isopor contendo gelo. As coletas, preservações, transporte e procedimentos analíticos seguiram o descrito em APHA et al. (2005). 


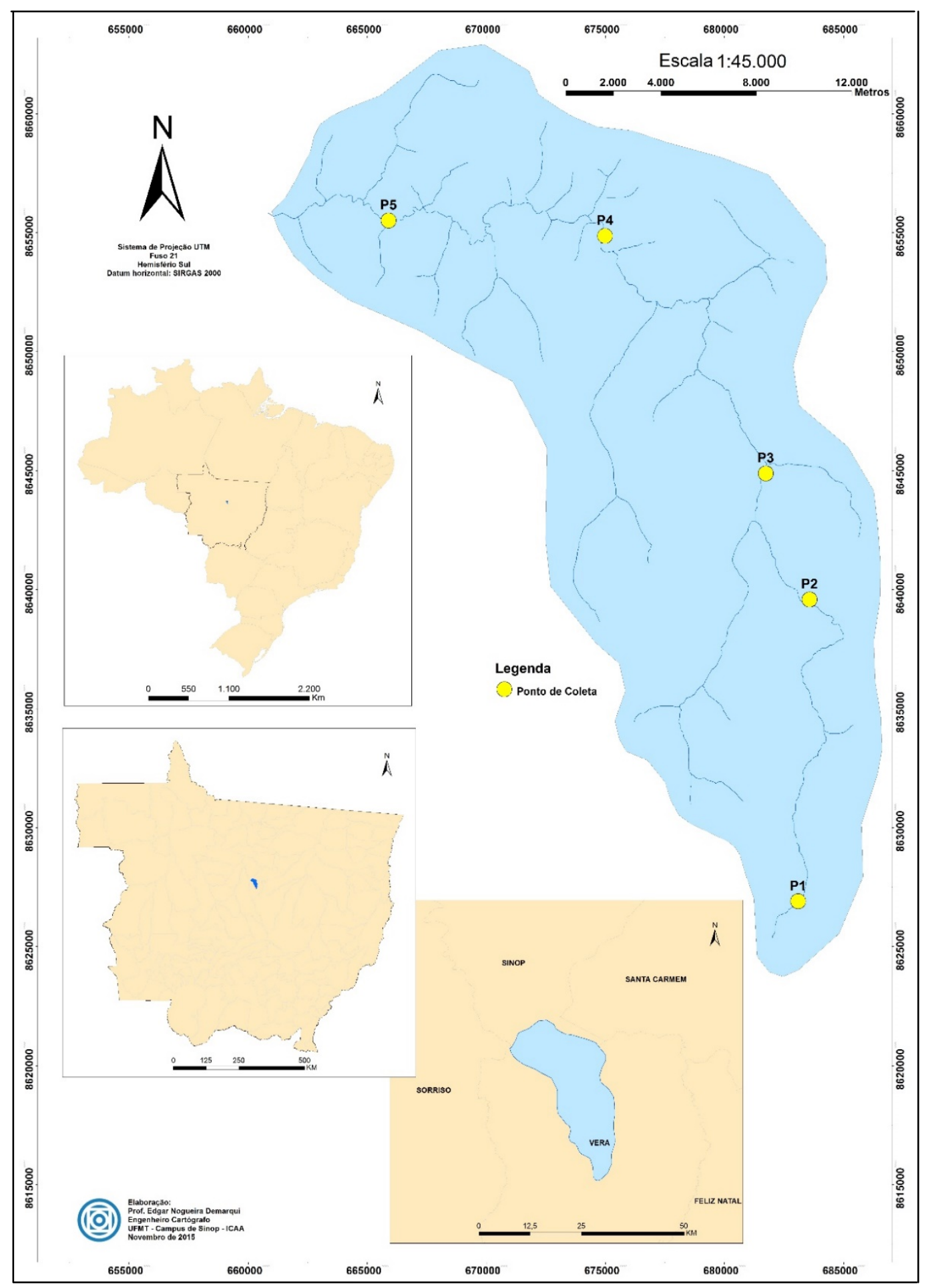

Figura 1. Localização regional da bacia hidrográfica do rio Caiabi no estado de Mato Grosso, com a ampliação da área da bacia e identificação dos pontos de amostragem.

Às amostras coletadas para análise de coliformes foram acondicionadas em frascos de Schott esterilizados. As amostras para análise de nutrientes (nitrogênio e fósforo) foram acidificadas logo após a coleta, com ácido sulfúrico. O método para as análises de coliformes totais e termotolerantes foi a de tubos múltiplos; o nitrogênio foi determinado pelo método 
micro-kjeldahl; o fósforo total pelo método do ácido ascórbico; o nitrito foi determinado pelo métdo colorimétrico; o nitrato pelo método de redução de cádmio; a demanda bioquímica de oxigênio pela incubação de amostras por cinco dias, a $20^{\circ} \mathrm{C}$; a condutividade elétrica foi medida em condutivímetro de bancada; para a determinação da clorofila a amostra foi filtrada em filtro de fibra de vidro, com posterior extração com acetona e determinação em espectrofotômetro; a determinação dos sólidos totais foi realizada por meio gravimétrico; os sólidos suspensos foram determinados pela filtração da amostra em filtro de fibra de vidro, secagem e pesagem; e os sólidos dissolvidos pela diferença entre sólidos totais e suspensos.

\subsection{Delineamento experimental e análise estatística}

Para verificar o efeito das fontes de variação (pontos de coleta e estações do ano) sobre os parâmetros de qualidade da água utilizou-se a análise de variância. Os testes foram realizados por meio do programa computacional $\mathrm{R}$ ( $R$ Development Core Team, 2011). O delineamento experimental adotado foi o Delineamento em Blocos ao Acaso, sendo que os tratamentos foram arranjados em esquema fatorial $5 \times 2$ : cinco pontos de coleta $\left(\mathrm{P}_{1}, \mathrm{P}_{2}, \mathrm{P}_{3}, \mathrm{P}_{4} \mathrm{e}\right.$ $\mathrm{P}_{5}$ ) e duas estações (seca: abril a setembro e chuvosa: outubro a março), com seis repetições. Nos meses de seca as precipitações foram muito pequenas $(<10 \mathrm{~mm}$ em abril e setembro) e nulas em maio, junho, julho e agosto. Após a análise de variância (ANOVA), os parâmetros que apresentaram diferença estatisticamente significativa $(\mathrm{p}<0,05)$ foram submetidos ao teste de Tukey para comparação de médias e o desdobramento da análise fatorial.

\section{3. Índice de qualidade da água e índice de estado trófico}

O Índice de Qualidade da Água é uma indicação aproximada do estado de qualidade do corpo hídrico. Para a determinação do IQA utilizou-se a Equação 1 (CETESB, 2008).

$$
I Q A=\prod_{i=1}^{9} Q_{i}^{w}
$$

em que:

$\mathrm{Q}_{\mathrm{i}}$ é o valor de qualidade do i-ésimo parâmetro, um número entre 0 e 100, obtido da respectiva curva média de variação de qualidade, em função de sua concentração ou medida (ANA, 2015) e;

wi é o peso correspondente ao i-ésimo parâmetro fixado em função da sua importância para a conformação global da qualidade, isto é, um número entre 0 e 1.

Cada um dos parâmetros que constitui o IQA possui um determinado peso, relativo à medida da sua contribuição para a qualidade da água. Os valores utilizados foram aqueles apresentados em ANA (2015).

A qualidade da água será em função do valor de IQA obtido, podendo ser de péssima (IQA $<25)$, ruim $(26<$ IQA $<50)$, regular $(51<$ IQA $<70)$, boa $(71<$ IQA $<90)$ ou ótima qualidade $(91<\mathrm{IQA} \leq 100)(\mathrm{ANA}, 2015)$.

O Índice de Estado Trófico (IET) auxilia na visualização geral grau de trofia (nutrição) e seu efeito na qualidade da água. O IET foi determinado com os resultados de clorofila $a$ (CL) e fósforo total (PT), seguindo o método descrito por Alves et al. (2012). As Equações 2, 3 e 4 foram utilizadas para a determinação do IET. 


$$
\begin{aligned}
& \operatorname{IET}(P T)=10 \cdot\left\{6-\left[\frac{0,42-0,36 \cdot \ln (P T)}{\ln (2)}\right]\right\}-20 \\
& \operatorname{IET}(C L)=10 \cdot\left\{6-\left[\frac{-0,7-0,6 \cdot \ln (C L)}{\ln (2)}\right]\right\}-20 \\
& \operatorname{IET}=\frac{\operatorname{IET}(P T)+\operatorname{IET}(C L)}{2}
\end{aligned}
$$

em que:

IET (PT) é o índice de estado trófico determinado para o fósforo;

IET (CL) é o índice de estado trófico determinado para a clorofila;

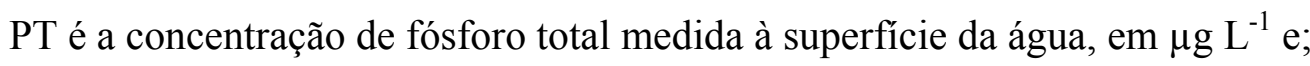

CL é a concentração de clorofila $a$ medida à superfície da água, em $\mu \mathrm{g} \mathrm{L^{-1 }}$.

A classificação do ambiente aquático quanto ao IET é dada em seis graus de trofia. Para rios tem-se: ultraoligotrófico (IET $\leq 47)$, oligotrófico $(47<$ IET $\leq 52)$, mesotrófico $(52<$ IET $\leq 59)$, eutrófico $(59<$ IET $\leq 63)$, supereutrófico $(63<$ IET $\leq 67)$ e hipereutrófico $($ IET $>67)$ (ANA, 2015).

\section{RESULTADOS E DISCUSSÃO}

\subsection{Variação sazonal e temporal dos parâmetros indicativos de qualidade hídrica}

A análise de variância mostrou que, dos parâmetros indicativos de qualidade analisados, sete apresentaram diferenças médias de concentração entre as estações seca e chuvosa $(p<0,05)$. As diferenças médias entre parâmetros foram identificadas nos parâmetros coliformes totais, DBO, nitrito, turbidez, temperatura, sólidos dissolvidos e sólidos totais (Tabela 1).

Tabela 1. Valores médios dos parâmetros indicativos de qualidade monitorados em função da estação.

\begin{tabular}{lccc}
\hline \multirow{2}{*}{ Parâmetro } & \multicolumn{2}{c}{$\begin{array}{c}\text { Valores médios nas } \\
\text { estações }\end{array}$} & $\begin{array}{c}\text { Valores de } \\
\text { referência* }\end{array}$ \\
\cline { 2 - 3 } & Chuva & Seca & \\
\cline { 1 - 3 } $\mathrm{CT}\left(\mathrm{NMP} 100 \mathrm{~mL}^{-1}\right)$ & 33,0 & 9,0 & -- \\
$\mathrm{DBO}\left(\mathrm{mg} \mathrm{L}^{-1}\right)$ & 1,2 & 0,5 & $<3$ \\
$\mathrm{NO}_{2}\left(\mathrm{mg} \mathrm{L}^{-1}\right)$ & 2,6 & 2,1 & $<1^{1}$ \\
$\mathrm{~TB}(\mathrm{NTU})$ & 11,36 & 6,26 & $<40$ \\
$\mathrm{~T}\left({ }^{\circ} \mathrm{C}\right)$ & 25,53 & 22,48 & $40 \pm 3{ }^{\circ} \mathrm{C}$ \\
$\mathrm{SD}\left(\mathrm{mg} \mathrm{L}^{-1}\right)$ & 68,0 & 29,0 & $<500$ \\
$\mathrm{ST}\left(\mathrm{mg} \mathrm{L}^{-1}\right)$ & 86,0 & 35,0 & -- \\
\hline
\end{tabular}

Nota: *Resolução CONAMA n $357 / 2005$.

Os poluentes $\mathrm{CT}, \mathrm{DBO}, \mathrm{NO}_{2}, \mathrm{~TB}, \mathrm{SD}$ e $\mathrm{ST}$ que apresentaram diferença significativa entre as estações revelaram a tendência de valores mais elevados durante o período de chuvas. 
Este comportamento é reflexo, principalmente, da ação do escoamento superficial das áreas agrícolas e de pastagens pela ocorrência de eventos de precipitação, em contraste com a estação seca.

Maiores concentrações de poluentes em função da sazonalidade estão descritas em literatura. Para os parâmetros coliformes totais (De Azevedo Lopes e Magalhães Jr., 2010; Chaves e Santos, 2009), DBO (Donadio et al., 2005), nitrito (Berka et al., 2001), turbidez, sólidos totais (Ríos-Villamizar et al., 2011; De Azevedo Lopes e Magalhães Jr., 2010), os autores reportam maiores concentrações nos períodos de chuva. De acordo com Grossi (2006), as estradas vicinais e carreadores servem de caminho para o escoamento superficial, ocasionando os processos erosivos, que se intensificam na estação chuvosa devido ao aumento na quantidade e na velocidade do fluxo.

Durante a estação seca os rios são abastecidos preponderantemente pelo escoamento subsuperficial, reduzindo a quantidade de sólidos e, consequentemente, as moléculas poluentes que adentram os recursos hídricos, aderidas às partículas coloidais. Apesar de ter sido identificada diferenças de concentrações entre estações, tem-se que os valores de referência para rios de classe 1 não foram ultrapassados.

A não observação de diferenças médias entre pontos, e somente entre estações, é um resultado bastante importante, pois indica que o acompanhamento do comportamento da qualidade da água não necessita de monitoramento constante (mensal). Duas coletas anuais em um único ponto são suficientes para demonstrar a qualidade da água em termos gerais, salvo situações de utilização pontual e específica da água.

Essa condição é de extrema importância, uma vez que a manutenção de um programa de monitoramento é dispendiosa. Se o monitoramento tem custos reduzidos, a possibilidade de manutenção da ação de monitoramento é maior.

Assim, para fins de acompanhamento do comportamento da qualidade da água e do estado trófico, a seção do rio próxima à confluência com o rio Teles Pires (P5) poderia ser uma estação constante de acompanhamento da qualidade da água, uma vez que este trecho apresenta uma seção de medição de vazão em operação desde o ano de 2010.

Em relação aos pontos de amostragem, os parâmetros indicativos de qualidade que apresentaram diferença significativa entre os trechos monitorados foram DBO e fósforo total (Tabela 2).

Tabela 2. Valores médios dos parâmetros indicativos de qualidade monitorados em função do local de amostragem.

\begin{tabular}{ccccc}
\hline \multirow{2}{*}{ Ponto } & \multicolumn{4}{c}{ Valores Médio } \\
\cline { 2 - 5 } & \multirow{2}{*}{$\mathrm{DBO}\left(\mathrm{mg} \mathrm{L}^{-1}\right)^{*}$} & $\mathrm{PT}\left(\mathrm{mg} \mathrm{L}^{-1}\right)^{*}$ & \multicolumn{2}{c}{ Sólidos suspensos $\left(\mathrm{mg} \mathrm{L}^{-1}\right)^{* *}$} \\
\hline $\mathrm{P}_{1}$ & $1,1 \mathrm{ab}$ & $0,09 \mathrm{ab}$ & $6,3 \mathrm{bA}$ & $35,0 \mathrm{aA}$ \\
$\mathrm{P}_{2}$ & $1,4 \mathrm{a}$ & $0,24 \mathrm{a}$ & $5,0 \mathrm{bA}$ & $21,7 \mathrm{aAB}$ \\
$\mathrm{P}_{3}$ & $0,8 \mathrm{ab}$ & $0,09 \mathrm{ab}$ & $3,8 \mathrm{aA}$ & $6,7 \mathrm{aB}$ \\
$\mathrm{P}_{4}$ & $0,8 \mathrm{ab}$ & $0,14 \mathrm{ab}$ & $7,5 \mathrm{aA}$ & $14,2 \mathrm{aB}$ \\
$\mathrm{P}_{5}$ & $0,6 \mathrm{~b}$ & $0,06 \mathrm{~b}$ & $6,3 \mathrm{aA}$ & $11,7 \mathrm{aB}$ \\
\hline
\end{tabular}

Nota: * Médias seguidas de mesma letra minúscula na coluna não apresentam diferença significativa. ${ }^{* *}$ Médias seguidas por letra minúscula na linha e maiúscula na coluna não diferem entre si a $5 \%$ de significância.

Os valores médios de concentração de $\mathrm{DBO}$ e fósforo total mostraram que os pontos $\mathrm{P}_{1}$, $\mathrm{P}_{3}$ e $\mathrm{P}_{4}$ apresentam comportamento semelhante entre si, enquanto os pontos $\mathrm{P}_{2}$ e $\mathrm{P}_{5}$ diferem completamente entre si e dos demais. $\mathrm{O}_{2}$ está situado logo abaixo de uma região com 
intensa criação animal, e à jusante do município de Vera (MT), fator que pode explicar o incremento de matéria orgânica e fósforo total no leito fluvial. Verificou-se que para o parâmetro fósforo, a qualidade da água estava em desacordo com o estipulado pela Resolução CONAMA n ${ }^{\circ} 357$, de 2005, que prevê concentração máxima de $0,1 \mathrm{mg} \mathrm{L}^{-1}$.

Toledo e Nicolella (2002) também constataram diferenças significativas entre pontos de água superficial monitorados. Zanini et al. (2010) observaram diferença significativa na análise de DBO entre os pontos amostrados e na interação entre pontos e estações.

A observação de diferenças médias desses parâmetros em relação aos pontos demonstra que, além de se monitorar o ponto 5, é preciso monitorar trecho a montante na bacia como, por exemplo, o_P2.

Observou-se que o efeito da interação do ponto de amostragem com a estação como fonte de variação para os parâmetros de qualidade somente ocorreu para o parâmetro sólidos suspensos. Comparando os valores médios de sólidos suspensos verificou-se maiores concentrações para os pontos 1 e 2 no período de chuva, enquanto que para os outros pontos esta diferença não ocorreu (Tabela 2).

A ocorrência das elevadas concentrações é dada em consequência das alterações antrópicas no local durante o período de monitoramento. $\mathrm{O}$ trecho representado pelo ponto 1 sofreu algumas intervenções ao longo do período de monitoramento. Porém, as alterações não se refletiram nos outros trechos provavelmente devido às características do canal, com possíveis pontos de menor velocidade que favoreceram a sedimentações dos sólidos. A produção de sedimentos está intimamente relacionada com a descarga líquida e com a combinação de fatores morfométricos da bacia (Antoneli e Thomaz, 2007). Segundo Chapman (1996), a concentração dos sólidos suspensos varia dramaticamente com as mudanças na descarga líquida da bacia.

No período de seca observou-se que a concentração de sólidos suspensos mantém-se constante ao longo de todo o canal. Ou seja, sem chuvas não se tem escoamento superficial e como a vazão mantém-se com pouca alteração, o arraste de sólidos nas águas do rio apresenta-se constante.

Os resultados do monitoramento para os outros elementos monitorados, clorofila a, coliformes termotolerantes, oxigênio dissolvido, condutividade elétrica e $\mathrm{pH}$, não apresentaram valores médios fora dos limites estabelecidos pela Resolução Conama ${ }^{\circ} 357$ de 2005 (Tabela 3), exceto para o $\mathrm{pH}$.

Tabela 3. Valores médios dos poluentes monitorados na bacia hidrográfica do rio Caiabi.

\begin{tabular}{lcccccc}
\hline Parâmetro & $\begin{array}{c}\text { Conc. } \\
\text { Média }\end{array}$ & DP & $\begin{array}{c}\text { Conc. } \\
\text { Mín. }\end{array}$ & $\begin{array}{c}\text { Conc. } \\
\text { Máx. }\end{array}$ & CV (\%) & $\begin{array}{c}\text { Valores de } \\
\text { Referência* }\end{array}$ \\
\hline $\mathrm{CL}\left(\mathrm{mg} \mathrm{L}^{-1}\right)$ & 0,43 & 0,49 & 0,00 & 2,80 & 0,68 & $<10,0$ \\
$\left.\mathrm{CTERM} \mathrm{(NMP} 100 \mathrm{~mL}^{-1}\right)$ & 4,02 & 2,24 & 3,00 & 23,00 & 1,56 & $<200,0$ \\
$\mathrm{NO}_{3}\left(\mathrm{mg} \mathrm{L}^{-1}\right)$ & 3,15 & 1,73 & 0,24 & 8,87 & 0,55 & $<10,0$ \\
$\mathrm{OD}\left(\mathrm{mg} \mathrm{L}^{-1}\right)$ & 6,61 & 1,33 & 4,30 & 10,50 & 0,21 & $>6,0$ \\
$\mathrm{CE}\left(\mu{\left.\mathrm{S} . \mathrm{cm}^{-1}\right)}_{\mathrm{pH}}^{3,31}\right.$ & 3,61 & 1,00 & 19,00 & 1,09 & -- \\
\hline
\end{tabular}

Nota. DP: Desvio-padrão; CV: Coeficiente de variação. * Resolução CONAMA 357 (2005) Segundo Frota Júnior et al. (2007), a condutividade elétrica é um parâmetro afetado por efeito acumulativo de sais totais. Portanto, poder-se-ia esperar resultados superiores no sentido nascente-foz, o que não foi observado nas águas do rio Caiabi. 
Apesar do valor médio do $\mathrm{pH}$ estar abaixo do que é indicado pela legislação, sabe-se que não é o uso e ocupação do solo que faz com que o pH seja baixo, e sim uma condição natural da bacia. Os solos da região são solos antigos e basicamente todas as bases do solo já foram lixiviadas (EMBRAPA, 2006).

Em relação aos parâmetros descritos na Tabela 3, a não observação de diferenças indica que o acompanhamento desses em um único ponto, com uma coleta anual é suficiente para demonstrar o comportamento deles.

\section{2. Índice de qualidade da água e índice estado trófico}

Apesar de não ter sido identificada diferença significativa entre pontos e estações, para fins de indicação da classe em termos de IQA e IET, valores médios dos pontos em cada uma das estações foram calculados. Estes valores indicaram que os trechos do rio Caiabi apresentaram boa qualidade da água, com baixo potencial de desenvolvimento de processo de eutrofização, conforme resultados apresentados na Tabela 4.

Tabela 4. Classificação da água do rio Caiabi em função do IET e IQA.

\begin{tabular}{cccccc}
\hline Ponto & Estação & IET & Classificação & IQA & Classificação \\
\hline \multirow{2}{*}{ P1 } & Seca & 26 & Ultraoligotrófico & 76 & Boa \\
& Chuva & 22 & Ultraoligotrófico & 74 & Boa \\
P2 & Seca & 20 & Ultraoligotrófico & 75 & Boa \\
& Chuva & 29 & Ultraoligotrófico & 74 & Boa \\
P3 & Seca & 29 & Ultraoligotrófico & 76 & Boa \\
& Chuva & 21 & Ultraoligotrófico & 74 & Boa \\
P4 & Seca & 17 & Ultraoligotrófico & 81 & Boa \\
& Chuva & 10 & Ultraoligotrófico & 76 & Boa \\
& Seca & 19 & Ultraoligotrófico & 87 & Boa \\
& Chuva & 25 & Ultraoligotrófico & 78 & Boa \\
\hline
\end{tabular}

Analisando a tendência temporal do IQA em 29 pontos de monitoramento na sub-bacia do rio das Velhas, Trindade (2013) constatou a influência da sazonalidade em apenas 3 pontos (10,34\%). Zanini et al. (2010) também não verificaram diferença significativa nos resultados médios de IQA entre as estações, entretanto, registrou diferenças entre os pontos analisados.

Inúmeros fatores possuem a propriedade de atenuar os efeitos negativos do uso do solo na qualidade da água. A presença de mata ciliar, segundo Donadio et al. (2005), auxilia na proteção dos recursos hídricos. O Caiabi possui mata ciliar ao longo de toda a sua extensão, o que pode ter contribuído com os altos resultados de IQA obervados. Outro fator que pode influenciar a qualidade da água é a cobertura vegetal. De acordo com Vanzela et al. (2010), as áreas de matas são áreas mais cobertas e estáveis, com maior capacidade de infiltração e armazenamento de água no solo, reduzindo o escoamento superficial.

É também importante ressaltar que com o início das chuvas há também o plantio da soja que ao nascer e se desenvolver protege o solo (cobertura) e que o manejo de solo (terraceamento) contribuem para a redução do carreamento de solo e outros elementos ligados a este.

O estudo de caso de Wu et al. (2013) confirmou a hipótese de que a reabilitação de águas poluídas com base no replantio de vegetação pode aliviar o aumento da poluição e possibilitar a reabilitação do ecossistema aquático degradado. 
Avaliando a ocupação no solo, fragmentação da paisagem e a qualidade da água da bacia do córrego Ponte de Terra (DF) entre os anos de 1992 e 2005, Chaves e Santos (2009), reportaram valores de IQA variando na faixa 71 - 81 no período de chuvas e 75 - 82 na estação seca. Os mesmos autores constataram um IQA ligeiramente superior no período de seca. Toledo e Nicolella (2002) observaram uma sutil deterioração na qualidade da água durante o período de chuvas, concluindo que os principais indicadores que contribuíram para os menores valores de IQA foram o OD, PT, amônia e CE. Magalhães Jr. et al. (2008) encontraram no ribeirão de Carrancas resultados de IQA inferiores aos encontrados no rio Caiabi, variando entre 37 e 64 . Os autores atribuíram a baixa qualidade da água à influência da atividade pecuária na região.

Em termos gerais, verificou-se, pelos valores de IET, que o nível trófico do rio se encontra pouco desenvolvido, pois os valores são baixos. De acordo com os resultados, as amostras coletadas apresentaram um índice ultraoligotrófico, sendo os valores de IET $<47$.

Affonso et al. (2011) encontraram valores de IET variando entre 32,5 e 75,5 na bacia hidrográfica do rio Taperoá, superiores aos valores encontrados no rio Caiabi. Cunha et al. (2008) avaliando a densidade fitoplanctônica e o estado trófico dos rios Canha e PariqueraAçu na bacia hidrográfica do rio Ribeira de Iguape (SP) concluíram que o IET aplicado a ambientes lóticos contribui para a superestimação dos resultados. Os autores afirmam que as formas fosfatadas que integram o cálculo podem contribuir para um incremento indevido do resultado, pois ocorre um aumento incompatível com a real resposta biológica associada, representada pelo crescimento fitoplanctônico.

O nível de estado trófico do rio Caiabi se encontra muito abaixo de uma faixa de risco e comprometimento em curto prazo. De acordo com Cunha et al. (2008), a eutrofização em ambientes lóticos seria incomum, devido as características próprias desse sistema, tais como o turbilhonamento, baixo tempo de residência e transporte contínuo da comunidade fitoplanctônica a jusante.

Os resultados da análise de variância para os índices de qualidade da água e de estado trófico demonstraram que não houve diferenças significativas dos valores entre os pontos, estações e nem na interação do conjunto. Apesar de ter-se identificado que concentrações dos elementos monitorados diferiram entre si em relação às estações e aos pontos, o efeito dessas diferenças nos índices obtidos foi inibido.

Quando índices são obtidos é importante considerar o efeito eclipse nos resultados. De acordo com Silva e Jardim (2006), o efeito eclipse advém do processo de agregar inúmeras variáveis ambientais em um único número, podendo atenuar o impacto negativo de uma das variáveis frente ao comportamento estável das demais.

A não observação de diferenças é um resultado bastante importante, pois indica que acompanhamento do comportamento da qualidade da água não necessita de monitoramento constante. Considerando apenas os resultados dos índices, uma única coleta anual em um único ponto é suficiente para demonstrar a qualidade da água em termos gerais, salvo situações de utilização pontual e específica da água.

Reitera-se que essa condição é importante, uma vez que a manutenção de um programa de monitoramento é dispendiosa. Se o monitoramento tem custos reduzidos, a possibilidade de manutenção da ação de monitoramento é maior.

Assim, para fins de acompanhamento do comportamento da qualidade da água e do estado trófico, a seção do rio próxima à confluência com o rio Teles Pires (P5) poderia ser uma estação constante de acompanhamento do índice de qualidade da água, uma vez que este trecho apresenta uma seção de medição de vazão em operação desde o ano de 2010.

Zhang et al. (2013) verificaram que a similaridade e a diferença temporal e espacial dos parâmetros podem permitir a otimização da frequência de monitoramento, o número de locais amostrados, de parâmetros monitorados, e, portanto, o custo associado. Assim, enquanto a 
bacia do rio Caiabi mantiver as mesmas atividades e mesmas práticas de uso e ocupação do solo, uma coleta anual no canal principal da bacia é suficiente para se identificar a qualidade da água em termos de IQA e IET.

\section{CONCLUSÕES}

Os resultados dos índices IQA apontam que as águas do rio Caiabi possuem qualidade boa. Os resultados podem ser atribuídos à presença de vegetação nativa, à conservação da mata ciliar e ao efeito de diluição motivado pelo acréscimo de tributários ao longo do trecho do rio.

Os resultados de IET evidenciam o baixo risco de eutrofização no rio Caiabi, uma vez o IET não excedeu o limite de 47. O ambiente pode ser classificado como ultraoligotrófico.

A análise de variância dos parâmetros monitorados demonstrou que o acompanhamento da qualidade da água pode ser realizado considerando-se apenas duas coletas anuais, uma no período de seca e outra no período de chuva, em dois pontos, sendo essas suficientes para descrever o comportamento da qualidade da água na bacia da maior parte dos parâmetros, desde que as condições de uso e ocupação do solo da bacia não sejam modificadas.

\section{AGRADECIMENTOS}

Ao Conselho Nacional de Desenvolvimento Científico e Tecnológico e à Coordenação de Aperfeiçoamento de Pessoal de Nível Superior pelo apoio financeiro.

\section{REFERÊNCIAS}

AFFONSO, A. G.; BARBOSA, C.; NOVO, E. M. L. M. Water quality changes in floodplain lakes due to the Amazon River flood pulse: Lago Grande de Curuaí (Pará). Brazilian Journal of Biology, v. 71, p. 601-610, 2011. http://dx.doi.org/10.1590/S151969842011000400004

AGÊNCIA NACIONAL DE ÁGUAS - ANA (Brazil). Portal da qualidade das águas. Indicadores de qualidade. Índice de qualidade das águas (IQA). 2015. Disponível em: http://portalpnqa.ana.gov.br/indicadores-indice-aguas.aspx. Acesso em: 15 abr. 2015.

ALVES, I. C. C.; El-ROBRINI, M.; SANTOS, M. L. S.; MONTEIRO; S. M.; BARBOSA, L. P. F.; GUIMARÃES, J. T. F. Qualidade das águas superficiais e avaliação do estado trófico do Rio Arari (Ilha de Marajó, norte do Brasil). Acta Amazônica, v. 42, n. 1, p. 115-124, 2012. http://dx.doi.org/10.1590/S0044-59672012000100014

AMERICAN PUBLIC HEALTH ASSOCIATION - APHA; AMERICAN WATER WORKS ASSOCIATION - AWWA; WATER ENVIRONMENT FEDERATION - WEF. Standard Methods for the Examination of Water and Wastewater. $14^{\text {th }} \mathrm{ed}$. Washington, D.C, 2005.

ANTONELLI. V.; THOMAZ, E. L. Caracterização do meio físico da bacia do arroio Boa Vista - Guamiranga, PR. Caminhos de Geografia, v. 8, p. 46-58, 2007.

BATENI, F.; FAKHERAN, S.; SOFFIANIAN, A. Assessment of land cover changes \& water quality changes in the Zayadhroud river basin between 1997-2008. Environmental Monitoring Assessment, v. 185, p. 10511-10519, 2013.

http://dx.doi.org/10.1007/s10661-013-3348-3 
BERKA, C.; SCHREIER, H.; HALL, K. Linking water quality with agricultural intensification in a rural watershed. Water, Air and Soil Pollution, v. 127, p. 389-401, 2001. http://dx.doi.org/10.1023/A:1005233005364

BUCCI, M. H. S.; OLIVEIRA, L. F. C. Índices de qualidade da água e de estado trófico na represa Dr. João Penido (Juiz de Fora, MG). Revista Ambiente \& Água, v. 9, n. 1, p. 130-148, 2014. http://dx.doi.org/10.4136/ambi-agua.1290

CHAPMAN, D. Water Quality Assessments: a guide to use of biota, sediments and water in environmental monitoring. $2^{\text {nd }}$ Edition. London: UNESCO/WHO/UNEP, 1996. p. 651.

CHAVES, H. M. L.; SANTOS, L. B. Ocupação do solo, fragmentação da paisagem e qualidade da água em uma pequena bacia hidrográfica. Revista Brasileira de Engenharia Agrícola e Ambiental, v. 13, p. 922-930, 2009.

http://dx.doi.org/10.1590/S1415-43662009000700015

COMPANHIA AMBIENTAL DO ESTADO DE SÃO PAULO - CETESB. Índices de qualidade das águas. 2008. Disponível em: http://www.cetesb.sp.gov.br/userfiles/ file/agua/aguas-superficiais/aguas-interiores/documentos/indices/01.pdf. Acesso em: 17 ago. 2014.

CONSELHO NACIONAL DE MEIO AMBIENTE - CONAMA. Resolução no 357, de 17 de março de 2005. Dispõe sobre a classificação dos corpos de água e diretrizes ambientais para o seu enquadramento, bem como estabelece as condições e padrões de lançamento de efluentes, e dá outras providências. Alterado pela Resolução CONAMA 397/2008. Disponível em: http://www.mma.gov.br/conama. Acesso em: 04 set. 2015.

CUNHA, D. G. F.; FALCO, P. B.; CALIJURI, M. C. Densidade fitoplanctônica e estado trófico dos rios Canha e Pariquera-Açu, bacia hidrográfica do rio Ribeira de Iguape, SP, Brasil. Revista Ambiente \& Água, v. 3, n. 2, 2008. http://dx.doi.org/10.4136/ambiagua. 55

CUNHA, A. C.; CUNHA, H. F. A.; BRASIL JUNIOR, A. C. P.; DANIEL, L. A. SCHULZ, H. E. Qualidade microbiológica da água em rios de áreas urbanas e periurbanas no baixo amazonas: o caso do Amapá. Engenharia Sanitária e Ambiental, v. 9, n. 4, p. 322-328, 2004. http://dx.doi.org/10.1590/S1413-41522004000400009

DE AZEVEDO LOPES, F. W.; MAGALHÃES JR., A. P. M. Influência das condições naturais de $\mathrm{pH}$ sobre o índice de qualidade das águas (IQA) na bacia do Ribeirão de Carrancas. Geografias, v. 6, n. 2, p. 134-147, 2010.

DONADIO, N. M. M.; GALBIATTI, J. A.; PAULA, R. C. Qualidade da água de nascentes com diferentes usos do solo na bacia hidrográfica do córrego Rico, São Paulo, Brasil. Engenharia Agrícola, v. 25, p. 115-125, 2005. http://dx.doi.org/10.1590/S010069162005000100013

EMPRESA BRASILEIRA DE PESQUISA AGROPECUÁRIA - EMBRAPA. Embrapa Arroz e Feijão. Cultivo do arroz de terras altas no estado de Mato Grosso. Sistemas de Produção, n. 7. 2006. Disponível em: https://sistemasdeproducao.cnptia.embrapa.br/ FontesHTML/Arroz/ArrozTerrasAltas/index.htm. Acesso em: 29 out. 2015.

FROTA JÚNIOR, J. I.; ANDRADE, E. M.; MEIRELES, A. C. M.; BEZERRA, A. M. E.; SOUZA, B. F. S. Influência antrópica na adição de sais no trecho perenizado da bacia hidrográfica do Curu, Ceará. Revista Ciência Agronômica, v. 38, p. 142-148, 2007. 
GROSSI, C. H. Diagnóstico e monitoramento ambiental da microbacia hidrográfica do rio Queima-Pé (MT). 2006. 122f. Tese (Doutorado em Ciências Agronômicas) Universidade Estadual Paulista Júlio De Mesquita Filho, Botucatu, 2006.

MAGAlHÃes JR., A. P.; DE AZEVEDO LOPES, F. W.; PEREIRA, J. A. A. P. Avaliação da qualidade das águas e condições de balneabilidade na bacia do ribeirão de CarrancasMG. Revista Brasileira de Recursos Hídricos, v. 13, p. 111-120, 2008.

MARIMON, B. S.; LIMA, E. S.; DUARTE, T. G.; CHIEGERATTO, L. C.; RATTER, J. A. Observations on the vegetation of Northeastern Mato Grosso, Brazil: an analysis of the Cerrado-Amazonian forest ecotone. Edinburgh Journal of Botany, v. 63, p. 323-341, 2006. http://dx.doi.org/10.1017/S0960428606000576

MORAlES, P. R. G. D.; D'AGOSTO, M. A.; SOUZA, C. D. R. Otimização de rede intermodal para o transporte de soja do norte do Mato Grosso ao porto de Santarém. Journal of Transport Literature, v. 7, n. 2, p. 29-51, 2013.

NASIRIAN, M. A new water quality index for environmental contamination contributed by mineral processing: a case study of Amang (Tin Tailing) processing activity. Journal of Applied Science, v. 7, n. 20, p. 2977-2987, 2007.

PONTES, P. P.; MARQUES, A. R.; MARQUES, G. F. Efeito do uso e ocupação do solo na qualidade da água na micro-bacia do Córrego Banguelo - Contagem. Revista Ambiente \& Água, v. 7, n. 3, p. 598-613, 2012. http://dx.doi.org/10.4136/ambiagua.962

PRADO, R. B.; MORAES NOVO, E. M. L. Avaliação espaço-temporal da relação entre o estado trófico do reservatório de Barra Bonita (SP) e o potencial poluidor de sua bacia hidrográfica. Sociedade \& Natureza, v. 19, n. 2, p. 5-18, 2007. http://dx.doi.org/10.1590/S1982-45132007000200001

RAJANKAR, P. N.; TAMBEKAR, D. H.; WATE, S. R. Groundwater quality and water quality index at Bhandara District. Environmental Monitoring Assessment, v. 179, p. 619-625, 2011. http://dx.doi.org/10.1007/s10661-010-1767-y

RÍOS-VILLAMIZAR, E. A.; MARTINS JUNIOR, A. F.; WAICHMAN, A. V. Caracterização físico-química das águas e desmatamento na bacia do rio Purus, Amazônia Brasileira Ocidental. Revista Geografia Acadêmica, v. 5, n. 2, 2011.

SILVA, G. S.; JARDIM, W. F. Um novo índice de qualidade das águas para proteção da vida aquática aplicado ao Rio Atibaia, região de Campinas/Paulínia - SP. Química Nova, v. 29, p. 689-694, 2006. http://dx.doi.org/10.1590/S0100-40422006000400012

SOUZA, A. P.; MOTA, L. L.; ZAMADEI, T.; MARTIN, C. C.; ALMEIDA, F. T.; PAULINO, J. Classificação climática e balanço hídrico climatológico no estado de Mato Grosso. Nativa - Pesquisas Agrárias e Ambientais, v. 1, n. 1, p. 34-43, 2013. http://dx.doi.org/10.14583/2318-7670.v01n01a07

TOLEDO, L. G.; NICOLELlA, G. Índice de qualidade de água em microbacia sob uso agrícola e urbano. Scientia Agrícola, v. 59, p. 181-186, 2002.

http://dx.doi.org/10.1590/S0103-90162002000100026 
TRINDADE, A. L. C. Aplicação de técnicas estatísticas para avaliação de dados de monitoramento de qualidade das águas superficiais da porção mineira da bacia do rio São Francisco. 2013. 165f. Dissertação (Mestrado em Saneamento, Meio Ambiente e Recursos Hídricos) - Universidade Federal de Minas Gerais, Belo Horizonte, 2013

VANZELA, L. S.; HERNANDEZ, F. B. T.; FRANCO, R. A. M. Influência do uso e ocupação do solo nos recursos hídricos do Córrego Três Barras, Marinópolis. Revista Brasileira de Engenharia Agrícola e Ambiental, v. 14, p. 55-64, 2010. http://dx.doi.org/10.1590/S1415-43662010000100008

WITTMAN, J.; WECKWERTH, A.; WEISS, C.; HEYER, S.; SEIBERT, J.; KUENNEN, B. et al. Evaluation of land use and water quality in an agricultural watershed in the USA indicates multiple sources of bacterial impairment. Environmental Monitoring Assessment, v. 185, p. 10395-10420, 2013. http://dx.doi.org/10.1007/s10661-013$3340-\mathrm{y}$

WU, J.; CHENG, S.; LI, Z.; GUO, W.; ZHONG, F.; YIN, D. Case study on rehabilitation of a polluted urban water body in Yangtze River Basin. Environmental Science and Pollution Research, v. 20, p.7038-7045, 2013. http://dx.doi.org/10.1007/s11356-012$1351-9$

ZANINI, H. L. H. T.; AMARAL, L. A.; ZANINI, J. R.; TAVARES, L. H. R. Caracterização da água da microbacia do córrego Rico avaliada pelo índice de qualidade de água e de estado trófico. Engenharia Agrícola, v. 30, p. 732-741, 2010. http://dx.doi.org/10.1590/S0100-69162010000400017

ZHANG, C.; APPEL, E.; QIAO, Q. Heavy metal pollution in farmland irrigated with river water near a steel plant - magnetic and geochemical signature. Geophysical Journal International, v. 192, p. 963-974, 2013. http://dx.doi.org/10.1093/gji/ggs079 


\begin{tabular}{|} 
Ambiente \& Água - An Interdisciplinary Journal of Applied Science \\
ISSN 1980-993X - doi:10.4136/1980-993X \\
www.ambi-agua.net \\
E-mail: ambi.agua@gmail.com
\end{tabular}

\title{
Análise multivariada para seleção de parâmetros de monitoramento em manancial de Juiz de Fora, Minas Gerais
}

\author{
doi:10.4136/ambi-agua.1590
}

Received: 29 Dec. 2014; Accepted: 04 Dec. 2015

\author{
Cézar Henrique Barra Rocha*; Amanda Maia Pereira \\ Universidade Federal de Juiz de Fora (UFJF), Juiz de Fora, MG, Brasil \\ Núcleo de Análise Geo Ambiental (NAGEA) \\ *Autor correspondente: email: barra.rocha@gmail.com, \\ amanda_maiap@hotmail.com
}

\section{RESUMO}

A necessidade de controlar o uso da terra e monitorar a qualidade da água para abastecimento público requer a avaliação de diversos parâmetros, os quais muitas vezes são difíceis de analisar e interpretar devido à grande quantidade de dados, além do custo financeiro. Nesse contexto, este trabalho teve como objetivo selecionar parâmetros mais relevantes para monitoramento da Represa Dr. João Penido, principal manancial de abastecimento de Juiz de Fora, MG. Essa avaliação foi realizada em uma série mensal de treze parâmetros fornecidos pela Companhia de Saneamento Municipal de Juiz de Fora entre os anos de 1998 a 2012, Estes dados foram avaliados estatisticamente usando Análise Fatorial e Análise de Componentes Principais, resultando na obtenção de parâmetros com maior variância. A componente 1 (FV1) refletiu a influência de sólidos dissolvidos associados aos processos de escoamento superficial na sub-bacia e erosão das margens. A FV2, assim como a FV3, associaram-se fortemente a possíveis lançamentos de esgoto no corpo hídrico. Com a análise multivariada, eliminaram-se cinco parâmetros que não apresentaram variância: dureza, oxigênio consumido, $\mathrm{pH}$, alcalinidade e manganês.

Palavras-chave: AF/ACP, qualidade da água, uso da terra.

\section{Multivariate Analysis for selection of monitoring parameters in water reservoir of Juiz de Fora, Minas Gerais}

\section{ABSTRACT}

The need to control land use and monitor water quality for public supply requires the evaluation of many parameters, which are often difficult to analyze and interpret due to cost and the large volume of data. In this context, this work selected the most relevant parameters for monitoring the Dr. João Penido Dam, the main supply source of Juiz de Fora, MG. The evaluation was conducted based upon a series of thirteen parameters of the catchment provided monthly by the Municipal Sanitation Company between 1998 and 2012. The data were statistically processed using Factor Analysis and Principal Component Analysis and parameters with highest variances were extracted. Component 1 (FV1) reflected the influence of dissolved solids associated with runoff processes in the watershed and with 
the erosion of margins. FV2, as well as FV3, are strongly associated to the potential discharge of sewage in the watershed area. Using multivariate analysis, we removed five parameters that showed no variance: hardness, consumed oxygen, $\mathrm{pH}$, alkalinity and manganese.

Keywords: FA/PCA, land use, water quality.

\section{INTRODUÇÃO}

No último censo oficial, a população de Juiz de Fora foi de 516.247 habitantes com um acréscimo de 13,37\% em relação ao ano de 2000 (IBGE, 2014). Isso tem ocasionado maiores demanda hídrica e pressão sobre os mananciais destinados ao abastecimento público. Paralelamente, a especulação imobiliária avançou sobre as sub-bacias de mananciais de Juiz de Fora, destacando a Represa Dr. João Penido. Ela tem área de aproximadamente $59,48 \mathrm{~km}^{2}$ e abastece cerca de $50 \%$ da cidade com vazão regularizada de 750 litros/segundo, chegando a até $65 \%$ no período da estiagem (CESAMA, 2014). A legislação e a fiscalização não foram capazes de evitar este processo, colocando em risco o futuro desse manancial.

Machado (2006) considera a Lei Municipal $n^{0}$ 6.087 / 1981 (Juiz de Fora, 1981) um avanço na proteção da represa, contudo, ressalva que ela é baseada em critérios pouco científicos, estando mais ligada ao processo de ocupação do que na manutenção das qualidades mínimas de suas águas. Esta ponderação ficou ainda mais evidente com a alteração do artigo $9^{\circ}$ pela Lei Municipal $n^{\circ} 11.817$ / 2009 (Juiz de Fora, 2009) que possibilita a "implantação de infraestrutura de alto interesse público" em áreas consideradas de preservação. Isso foi um artifício jurídico para a construção de uma Rodovia Estadual (Rodovia do Aeroporto) que induzirá ocupação antrópica a médio e longo prazo.

A Lei Municipal no 6.910 / 1986 (Juiz de Fora, 1986) instituiu a Bacia Hidrográfica da Represa Dr. João Penido como Zona Especial, sujeita a regime urbanístico específico que visa à preservação dos seus recursos naturais e a proteção ambiental e ecológica. $\mathrm{O}$ ideal seria a preservação de grandes fragmentos de floresta em toda a área da bacia, contudo, as matas ripárias nas margens já conseguiriam muitas vantagens para o manancial envolvido. São as funções previstas para as áreas de preservação permanente (APP), depreciadas na reformulação do Novo Código Florestal (Ab'Sáber, 2010; Casatti, 2010; Tundisi e Tundisi, 2010; Brasil, 2012a; 2012b, Rocha e Costa, 2015).

$\mathrm{O}$ adequado gerenciamento dos recursos hídricos tem sido uma constante preocupação dos gestores públicos. Para que haja fornecimento de água com boa qualidade e quantidade é necessário estabelecer um planejamento do uso e cobertura da terra nas bacias de mananciais de abastecimento (Bhattacharyya e Kapil, 2010; Tu, 2011; Sheela et al., 2012; Thurston et al., 2012; Abildtrup et al., 2013; Yu et al., 2013; Rocha et al., 2014).

Diante deste contexto, verifica-se a necessidade de um planejamento para a ocupação da região em que está inserida a Represa e também um monitoramento contínuo de suas águas. Tal monitoramento visa o controle da qualidade da água e busca avaliar diversos parâmetros, os quais muitas vezes são difíceis de analisar e interpretar devido à grande quantidade de dados, principalmente se as coletas e as análises de água ocorrerem por muitos anos. A fim de suplantar esse obstáculo é feita a utilização de abordagens multivariadas como a Análise Fatorial (AF) e a Análise de Componentes Principais (ACP) que são úteis para obter informações significativas, promovendo a redução do número de variáveis com o mínimo de perdas das informações (Liao et al., 2008; Bernardi et al., 2009; Autin e Edwards, 2010; Vanzela et al., 2010; Akbar et al., 2011; Mendonça e Souza, 2011; Spezia et al., 2011; Tu, 2011; Baborowski et al., 2012; Guedes et al., 2012; Sheela et al., 2012; Rocha et al., 2014).

Com base no que foi abordado, teve-se por objetivo selecionar os parâmetros mais 
relevantes para monitoramento da Represa Dr. João Penido, principal manancial de abastecimento de Juiz de Fora, MG, verificando pela Análise Fatorial / Análise de Componentes Principais os parâmetros que melhor explicam a variância total dos dados, ou seja, os mais relevantes para esta sub-bacia.

\section{MATERIAIS E MÉTODOS}

As informações limnológicas utilizadas foram fornecidas pela CESAMA - Companhia de Saneamento Municipal de Juiz de Fora e são referentes às análises da água na captação da Represa Dr. João Penido, com coordenadas geográficas: $2^{\circ} 41^{\prime} 03,3^{\prime \prime} \mathrm{S}$ e $43^{\circ} 24^{\prime} 12,68^{\prime \prime} \mathrm{W}$ (Datum WGS-84), localizada ao norte do município, $10 \mathrm{~km}$ da malha urbana. A construção da Represa Dr. João Penido se deu com o barramento do ribeirão dos Burros (córrego principal), contando também com os córregos Grama (ao centro), Vista Alegre (a leste) e outros tributários conforme a Figura 1.
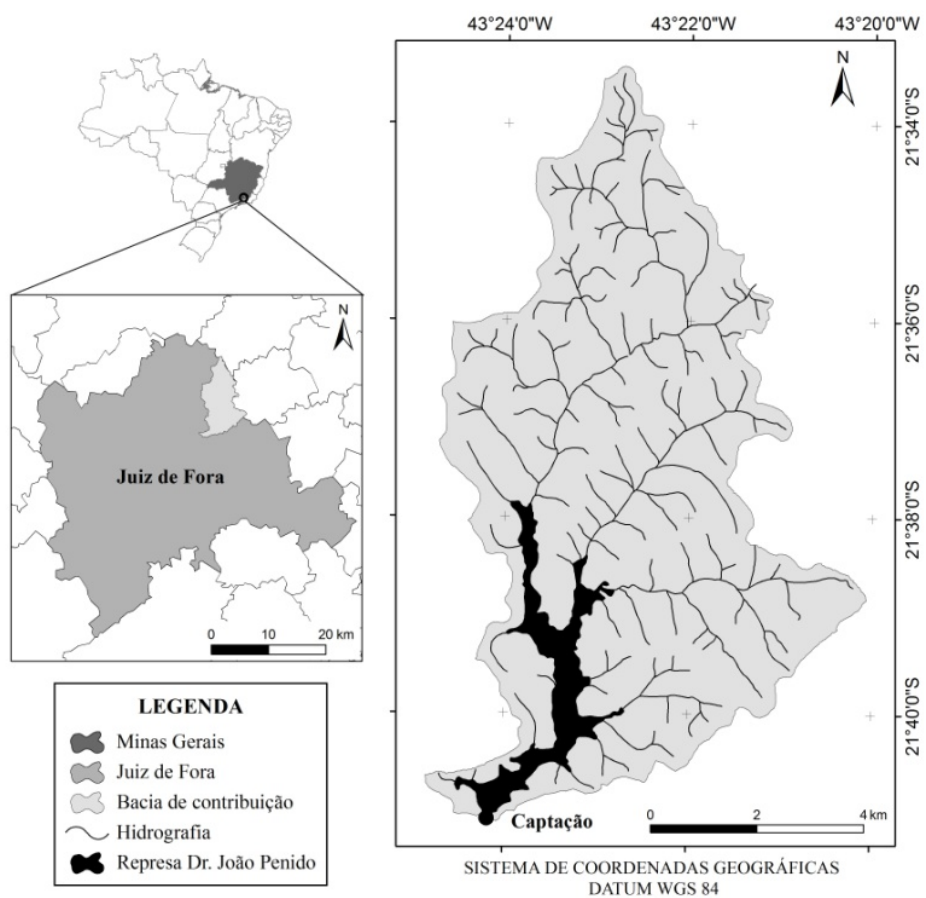

Figura 1. Mapa de Localização da área de estudo.

Fonte: Rocha et al. (2014).

Foram analisados os seguintes parâmetros: $\mathrm{pH}$, cor, turbidez, ferro (Fe), manganês (Mn), fósforo total (FT), alcalinidade $\mathrm{HCO}_{3}{ }^{-}$, cloreto, dureza, oxigênio consumido (OC), oxigênio dissolvido (OD), condutividade e demanda bioquímica de oxigênio $\left(\mathrm{DBO}_{5,20}\right)$ referentes às medições mensais realizadas entre o ano de 1998 a 2012, com exceção dos anos 2003 e 2004 não disponibilizados pela CESAMA, totalizando 13 anos de monitoramento.

Para a cidade de Juiz de Fora, o Plano Diretor de Desenvolvimento Urbano - PDDU (Juiz de Fora, 2000), estabelece que há uma sazonalidade bem definida no município, sendo a estação chuvosa de outubro a abril, com temperaturas mais elevadas e maiores precipitações (média de $20,9{ }^{\circ} \mathrm{C}$ e $199 \mathrm{~mm}$ por mês) e a estação seca de maio a setembro, período mais frio e com menor presença de chuvas (média de $17,1^{\circ} \mathrm{C}$ e $40 \mathrm{~mm}$ por mês).

Procedeu-se ao cálculo das médias de cada parâmetro para as duas estações anualmente e verificou-se a ocorrência de normalidade (Teste Kolmogorov-Smirnov). Foi aplicado o teste $\mathrm{t}$ de Student, com o auxílio do Microsoft Office Excel 2010, para verificar a ocorrência de variação estatisticamente significativa $(\mathrm{p}<0,05)$ entre as médias históricas das matrizes 
formadas com os dados de seca e chuva dos treze anos monitorados.

Alguns dados não foram mensurados durante vários meses de um mesmo ano, impossibilitando calcular uma média referente à estação daquele ano. Por esse motivo, o parâmetro ferro (Fe) foi excluído nos anos de 1998 e 1999; o manganês (Mn) e a condutividade foram excluídos de 1998 a 2002; os dados de fósforo total (FT) foram excluídos nos anos de 1998 a 2002 e 2005; os OD foram excluídos nos anos de 2000, 2002 e 2011; e a $\mathrm{DBO}_{5,20}$ nos anos de 1998 a 2002 e 2011.

Verificou-se a adequação dos dados à AF por meio dos testes de Kaiser-Meyer-Olkin (KMO) e esfericidade de Bartlett. As análises multivariadas (AF / ACP) foram realizadas no software SPSS ${ }^{\circledR}$ 15.0, e consistiram em três etapas, descritas por Toledo e Nicolella (2002): elaboração da matriz de correlação; extração dos fatores comuns com possível redução das variáveis explicativas e rotação dos eixos relativos aos fatores comuns, visando facilitar e simplificar a interpretação, somente quando necessário.

Os dados originais, em forma de matriz, foram expressos por $X=\left(X_{i, j}\right)$ em que, $i=1 \ldots n$ amostragens e $\mathrm{j}=1 \ldots \mathrm{p}$ variáveis limnológicas. As amostragens abrangeram os anos de $1998 \mathrm{a}$ 2012, com exceção dos anos 2003 e 2004, havendo, entretanto, ausência de dados em vários meses, os quais foram desconsiderados nas análises.

O coeficiente de correlação superior a 0,5 expressa uma forte correlação entre as variáveis limnológicas (Cohen, 1988 e Helena et al., 2000). Valores entre 0,3 e 0,49 expressam uma moderada correlação (Cohen, 1988). Para extração das componentes principais, utilizou-se a matriz de correlação com vista a eliminar o problema de escalas e unidades diferenciadas em que as variáveis foram medidas. A principal característica da $\mathrm{ACP}$, além da ortogonalidade, é que as componentes principais são obtidas em ordem decrescente de máxima variância. Assim, a primeira componente explica o máximo da variabilidade total dos dados; a segunda, explica o máximo da variabilidade dos dados restantes, não correlacionados com a primeira e assim sucessivamente (Guedes et al., 2012).

Os resultados da ACP, em alguns casos, podem apresentar dificuldades na sua interpretação. Objetivando superar tal obstáculo, adota-se o procedimento de rotação ortogonal da matriz das cargas fatoriais que possibilita melhor interpretação dos fatores ao redistribuir a variância explicada pelas componentes, não alterando na variância acumulada do conjunto de componentes. A utilização da rotação ortogonal pelo método Varimax permite um melhor ajuste ao modelo fatorial possível de explicação, sendo frequentemente utilizada em estudos de qualidade de água e processos hidrológicos (Aprile e Farias, 2001; Andrade et al., 2007; Girão et al., 2007; Guedes et al., 2012).

\section{RESULTADOS E DISCUSSÃO}

Os resultados das análises preliminares, que visaram testar a adequacidade dos dados à $\mathrm{AF} / \mathrm{ACP}$, possibilitaram o emprego de tal metodologia. Ao testar a hipótese de que a matriz de correlação é uma matriz identidade, teste de esfericidade de Bartlett $(\mathrm{p} \approx 0,000)$, foi descartada a hipótese nula, verificando a existência de correlações significativas entre as variáveis. Outro teste utilizado foi o índice de adequação da amostra (KMO), que segundo Hair et al. (2007), quantifica o grau de intercorrelação entre as variáveis. O resultado encontrado foi $\mathrm{KMO}=0,701$, o que permite a aplicação da análise.

Informações relevantes sobre a dinâmica das variáveis pode ser obtida através da matriz de correlação. Os parâmetros limnológicos que possuíram maior número de correlações (Tabela 1) tiveram maior importância na composição das componentes principais (CPs). A variável $\mathrm{pH}$ foi eliminada por apresentar correlações fracas com as outras variáveis e o teste foi refeito. No processamento da AF / ACP, a partir da matriz de correlação, foram extraídas as comunalidades, que representam o nível de associação entre a variável e os fatores 
extraídos, ou seja, a porcentagem da variância da variável explicada pelos fatores utilizados (Toledo e Nicolella, 2002). Valores abaixo de 0,5 indicam que a variável deve ser eliminada e a AF / ACP refeita (Figueiredo Filho e Silva Júnior, 2010). Norteados por tais considerações, eliminou-se a dureza, a alcalinidade e o manganês da análise.

Tabela 1. Matriz de correlação das variáveis limnológicas da Represa Dr. João Penido.

\begin{tabular}{lrrrrrrrr}
\hline & Cor & Turbidez & Fe & Ptotal & Cloreto & Condut. & DBO & OD \\
\hline Cor & 1,000 & & & & & & & \\
Turbidez & 0,470 & 1,000 & & & & & & \\
Fe & 0,736 & 0,324 & 1,000 & & & & & \\
Ptotal & $-0,208$ & $-0,124$ & $-0,049$ & 1,000 & & & & \\
Cloreto & 0,202 & 0,011 & 0,313 & 0,384 & 1,000 & & & \\
Condut. & 0,594 & 0,451 & 0,472 & $-0,102$ & 0,170 & 1,000 & & \\
DBO & 0,332 & $-0,177$ & 0,297 & $-0,225$ & 0,194 & 0,188 & 1,000 & \\
OD & 0,616 & 0,406 & 0,468 & $-0,133$ & 0,204 & 0,340 & 0,041 & 1,000 \\
\hline
\end{tabular}

As três primeiras componentes, explicaram cerca de $73 \%$ da variância total dos dados. Embora não exista um critério consensual sobre o número de fatores que devem ser extraídos na análise, o critério de Kaiser sugere a extração dos fatores com autovalor acima de um (Figueiredo Filho e Silva Júnior, 2010). Já Hair et al. (2007) sugerem que o número de componentes que representem $60 \%$ da variância acumulada, refletem uma ideia aceitável da variância original.

A Tabela 2 apresenta a matriz não rotacionada de pesos fatoriais que indica a contribuição que cada variável possui na componente principal. Esse valor deve ser maior que o valor absoluto de 0,5 e deve corresponder a uma única componente. O parâmetro OC apresentou valores maiores que 0,5 em mais de uma componente, mesmo após rotação. Optou-se por eliminá-lo e refazer o teste, resultando na Tabela 2. Caso semelhante aconteceu com a turbidez, porém, ao rotacionar a matriz pelo método Varimax, o problema foi superado conforme Tabela 3.

Tabela 2. Matriz de pesos fatoriais das variáveis limnológicas nas três componentes principais selecionadas.

\begin{tabular}{lccc}
\hline \multirow{2}{*}{ Variáveis } & \multicolumn{3}{c}{ Matriz(a) } \\
\cline { 2 - 4 } & \multicolumn{3}{c}{ Componente Principal (CP) } \\
\cline { 2 - 4 } & 0,915 & 2 & 3 \\
\hline Cor & 0,595 & $-0,058$ & $-0,080$ \\
Turbidez & 0,815 & $-0,291$ & 0,552 \\
Fe & $-0,196$ & 0,171 & $-0,108$ \\
Ptotal & 0,327 & 0,788 & 0,396 \\
Cloreto & 0,732 & 0,817 & $-0,016$ \\
Condutividade & 0,332 & $-0,044$ & 0,067 \\
DBO & 0,715 & 0,128 & $-0,858$ \\
OD & 3,16 & $-0,051$ & 0,211 \\
Autovalor & 39,45 & 1,43 & 1,27 \\
\% Variância Explicada & 17,82 & 15,81 \\
$\%$ Variância Acumulada & 39,45 & 57,27 & 73,09 \\
\hline
\end{tabular}

Nota: Os três tons de cinza destacam o peso de cada variável para aquela Componente Principal (CP). 
A primeira componente, após a aplicação do algoritmo Varimax (FV1), explicou cerca de $38,2 \%$ da variância total dos dados e teve como variável mais expressiva a cor, cujo valor foi de 0,879 (Tabela 3 ).

Tabela 3. Matriz de pesos fatoriais das três componentes principais após rotação Varimax.

\begin{tabular}{lccc}
\hline \multirow{2}{*}{ Variáveis } & \multicolumn{3}{c}{ Matriz(a) Rotacionada } \\
\cline { 2 - 4 } & \multicolumn{3}{c}{ Fator Varimax (FV) } \\
\cline { 2 - 4 } & 0,879 & $-0,029$ & 0,269 \\
\hline Cor & 0,722 & $-0,137$ & $-0,451$ \\
Turbidez & 0,754 & 0,184 & 0,320 \\
Fe & $-0,183$ & 0,844 & $-0,265$ \\
Ptotal & 0,238 & 0,809 & 0,251 \\
Cloreto & 0,731 & 0,007 & 0,091 \\
Condutividade & 0,132 & $-0,043$ & 0,918 \\
DBO & 0,745 & 0,030 & $-0,052$ \\
OD & 3,06 & 1,42 & 1,37 \\
Autovalor & 38,23 & 17,78 & 17,08 \\
\% Variância Explicada & 38,23 & 56,01 & 73,09 \\
\% Variância Acumulada & &
\end{tabular}

Nota: Os três tons de cinza destacam o peso de cada variável para o Fator Varimax - FV.

As outras variáveis desta componente também apresentaram resultados expressivos, cujos valores estão acima de 0,700 . Dentre as variáveis estudadas, a cor e o Fe foram as que apresentaram maior número de correlações, inclusive possuindo entre si, o maior coeficiente de Pearson da matriz (Tabela 1). Isso se justifica pelo fato do Fe, na ausência de oxigênio dissolvido, se apresentar em sua forma reduzida $\left(\mathrm{Fe}^{2+}\right)$, podendo se oxidar e precipitar causando coloração na água (Von Sperling, 2005). Os resultados obtidos pelo teste $t$ de Student destacam variação sazonal para a cor e o Fe com elevação de suas médias na estação chuvosa (Tabela 4), o que já foi observado por Rocha et al. (2014). Além disso, a turbidez está intrinsecamente relacionada com a cor presente na água, de forma que quando a cor aumenta, a turbidez também pode aumentar (Von Sperling, 2005). Para a CETESB (2009), a condutividade está relacionada com a presença de sais na água, indicando indiretamente uma medida da concentração de poluentes. A condutividade da água é proporcional ao aumento de sólidos dissolvidos, o que pode explicar a presença da condutividade nessa componente, visto que a cor também está relacionada com a presença dos sólidos dissolvidos. Uma fonte importante para o OD é a produção fotossintética (CETESB, 2009). Dessa forma, o OD se relaciona nessa componente, devido a presença de cor e turbidez que influenciam na passagem dos raios solares no corpo d'água e com isso podem diminuir a disponibilidade de luz para a fotossíntese e consequentemente diminuir a geração de OD na água.

A segunda componente (FV2) que explicou cerca de 17,8\% da variância dos dados foi composta pelos parâmetros fósforo total e cloreto. A presença de fósforo nas águas pode ter origem na dissolução de compostos do solo (escala muito pequena), despejos domésticos e/ou industriais, detergentes, excrementos de animais e fertilizantes. Concentrações elevadas de fósforo podem contribuir, da mesma forma que o nitrogênio, para a proliferação de algas e acelerar o processo de eutrofização. Assim como o fósforo total, a presença de cloreto também está relacionada ao lançamento de efluentes domésticos no corpo hídrico. Enquanto o fósforo é proveniente, principalmente de detergentes e excretas de animais, o cloreto está presente na urina humana e estima-se que cada pessoa expele através da urina cerca $6 \mathrm{~g}$ de 
cloreto por dia, o que faz com que os esgotos apresentem concentrações de cloreto que ultrapassam a $15 \mathrm{mg} . \mathrm{L}^{-1}$ (CETESB, 2009).

A componente FV3 foi representada por uma única variável, a DBO. Dentre todas as componentes, essa foi a mais expressiva, com valor de 0,918. Além disso, a variável explicou cerca de $17 \%$ da variância total dos dados. Esse resultado reforça a importância desse parâmetro, que é um dos mais utilizados para indicação da qualidade da água. A DBO (Demanda Bioquímica de Oxigênio) se refere a quantidade de oxigênio necessária para degradar a matéria orgânica presente no meio. Os maiores aumentos em termos de DBO, num corpo d'água, são provocados por despejos de origem predominantemente orgânica. A presença de um alto teor de matéria orgânica pode induzir ao completo esgotamento do oxigênio na água, provocando o desaparecimento de peixes e outras formas de vida aquática. Um elevado valor da DBO pode indicar um incremento da microflora presente e interferir no equilíbrio da vida aquática (CETESB, 2009).

Tabela 4. Resultados do Teste t-Student e médias e desvios padrões das variáveis monitoradas.

\begin{tabular}{|c|c|c|c|}
\hline \multirow{2}{*}{ Variável } & \multirow{2}{*}{ Teste T-Student } & \multicolumn{2}{|c|}{ Média e Desvio Padrão } \\
\hline & & Seca & Chuva \\
\hline $\mathrm{pH}$ & 0,082 & $7,037 \pm 0,216$ & $6,934 \pm 0,209$ \\
\hline Cor $(\mathrm{mg} \mathrm{PtCo} / \mathrm{L})$ & 0,000 & $38,654 \pm 13,009$ & $68,067 \pm 22,508$ \\
\hline Turbidez (UNT) & 0,001 & $4,825 \pm 0,990$ & $6,305 \pm 1,216$ \\
\hline $\mathrm{Fe}\left(\mathrm{mg} \cdot \mathrm{L}^{-1}\right)$ & 0,001 & $0,695 \pm 0,258$ & $1,718 \pm 0,802$ \\
\hline $\operatorname{Mn}\left(m g \cdot L^{-1}\right)$ & 0,932 & $0,207 \pm 0,200$ & $0,214 \pm 0,089$ \\
\hline P total $\left(\mu \mathrm{g} . \mathrm{L}^{-1}\right)$ & 0,941 & $0,346 \pm 0,181$ & $0,333 \pm 0,118$ \\
\hline Alcalinidade $\mathrm{CO} 3$ & 0,546 & $10,609 \pm 2,871$ & $11,164 \pm 2,730$ \\
\hline Cloreto (mg. $\left.\mathrm{L}^{-1}\right)$ & 0,372 & $9,100 \pm 5,098$ & $10,854 \pm 8,676$ \\
\hline Dureza $\left(m g . L^{-1}\right)$ & 0,633 & $11,782 \pm 5,877$ & $11,823 \pm 4,731$ \\
\hline OC $\left(m g \cdot L^{-1}\right)$ & 0,004 & $2,390 \pm 0,481$ & $3,038 \pm 0,747$ \\
\hline $\mathrm{OD}\left(\mathrm{mg} \cdot \mathrm{L}^{-1}\right)$ & 0,377 & $5,903 \pm 1,266$ & $6,327 \pm 0,835$ \\
\hline Condutividade (uS/cm) & 0,001 & $18,347 \pm 1,447$ & $21,134 \pm 0,874$ \\
\hline DBO (mg. $\left.\mathrm{L}^{-1}\right)$ & 0,408 & $2,728 \pm 3,015$ & $4,135 \pm 6,352$ \\
\hline
\end{tabular}

O comportamento das variáveis e sua distribuição pelas componentes permitem verificar as relações que estas apresentam entre si. Dessa forma, assim como feito por outros autores (Helena et al., 2000; Andrade et al., 2007; Guedes et al., 2012), é possível atribuir um nome que represente as características comuns entre tais variáveis de cada componente. Logo, a FV1 reflete a influência de sólidos dissolvidos associados ao escoamento superficial pelo fato das variáveis com os maiores coeficientes terem apresentado diferenças significativas entre as estações de seca e chuva. A FV2 se associa fortemente a possíveis lançamentos de esgoto na área da bacia. A concentração de fósforo total se mostrou muito elevada nas duas estações, pois a média para o período seco foi de $0,346 \mathrm{mg} . \mathrm{L}^{-1}$ e para o período chuvoso foi de 0,333 mg. $\mathrm{L}^{-1}$. Esses valores são cerca de 3 vezes maiores que o permitido pela Resolução CONAMA 357/2005 (Brasil, 2005) para águas doces de Classe $1\left(0,1 \mathrm{mg} . \mathrm{L}^{-1} \mathrm{P}\right)$. A FV3, assim como a FV2, caracteriza a influência do lançamento de esgotos na área da bacia, como já foi observado por Rocha et al. (2014).

Os resultados obtidos pelo teste t de Student apontaram as variáveis cor, turbidez, ferro, 
OC e condutividade com diferença sazonal significativa $(\mathrm{p}<0,05)$. Todas com aumento nas médias no período chuvoso. Contudo, não se descarta a hipótese de que outras variáveis também sofram interferência da precipitação, uma vez que é difícil mensurar temporalmente as influências das chuvas sobre as variáveis limnológicas (Fritzsons et al., 2003). Acrescenta-se o fato das amostras coletadas serem provenientes da captação, onde a qualidade da água pode ser resultado, dentre outros processos, do efeito de tamponamento ao longo do curso hídrico e da decantação na Represa, semelhante ao descrito por Carvalho et al. (2000) e Padial et al. (2009).

Observa-se também que o parâmetro $\mathrm{DBO}_{5,20}$ apresentou valores de desvio padrão, tanto para a estação seca quanto para a chuvosa, maiores que os valores das médias. Isso indica que os dados desse parâmetro estão variando muito. Esses altos valores de desvio padrão também poderiam ser explicados pela grande lacuna de dados desse parâmetro, entre 1998-2002 e 2011.

Ao se considerar que a componente FV1, a que mais explicou as variâncias totais dos dados, é resultante do transporte e lixiviação de materiais alóctones, deduz-se que o uso e a ocupação irregular do solo no entorno da sub-bacia influencia diretamente na qualidade da água deste manancial. Carneiro et al. (2010) afirmam que há uma desarticulação entre os instrumentos de gerenciamento dos recursos hídricos e os de planejamento do uso do solo, marcada pelo forte grau de informalidade e até mesmo ilegalidade na ocupação do solo. Este fato é nitidamente observado na sub-bacia de contribuição da Represa Dr. João Penido, sendo que resultados semelhantes foram encontrados por Rocha et al. (2014) num período de sete anos de monitoramento.

\section{CONCLUSÃO}

A utilização do teste $t$ de Student confirmou a necessidade de estudos que contemplem diferentes estações (seca e chuva), tendo em vista a variação observada para importantes parâmetros limnológicos em virtude da pluviosidade.

A Análise Fatorial / Análise de Componentes Principais promoveu a redução do número de variáveis de qualidade da água: dos treze parâmetros analisados, cinco foram eliminados, o que facilita a interpretação dos dados que mais influenciaram na dinâmica desta sub-bacia e diminuindo custos com monitoramento.

A análise permitiu a seleção de três componentes principais que explicaram $73,1 \%$ da variação total dos dados, indicando o escoamento superficial como principal determinante das variáveis que compõem a primeira componente e a contribuição orgânica, não associada à precipitação pluviométrica, como sugestiva das variáveis das segunda e terceira componentes.

A seleção de parâmetros de monitoramento de qualidade da água é relevante para o gerenciamento de recursos hídricos, consideradas as especificidades de cada sub-bacia e manancial correspondente. As informações obtidas podem apoiar decisões sobre planejamento e uso da terra, além de reduzir gastos com monitoramento.

\section{AGRADECIMENTOS}

Os autores agradecem a UFJF pelas bolsas concedidas e aos pesquisadores do NAGEA, LADINAA e PGECOL pelas discussões.

\section{REFERÊNCIAS}

AB'SÁBER, A. N. Do Código Florestal para o Código da Biodiversidade. Biota Neotrópica, v. 10, n. 4, p. 331-335, 2010. http://dx.doi.org/10.1590/S1676-06032010000400037 
ABILDTRUP, J.; GARCIA, S.; STENGER, A. The effect of forest land use on the cost of drinking water supply: A spatial econometric analysis. Ecological Economics, n. 92, p. 126-136, 2013. http://dx.doi.org/10.1016/j.ecolecon.2013.01.004

AKBAR, T. A.; HASSAN, Q. K.; ACHARI, G. A methodology for clustering lakes in Alberta on the basis of water quality parameters. Clean - Soil, Air, Water, v. 39, n. 10, p. 916-924, 2011. http://dx.doi.org/10.1002/clen.201100050

ANDRADE, E. M.; ARAÚJO, L. F. P.; ROSA, M. F.; DISNEY, W.; ALVES, A. B. Seleção dos indicadores da qualidade das águas superficiais pelo emprego da análise multivariada. Engenharia Agrícola, v. 27, n. 3, p. 683-690, 2007. http://dx.doi.org/10.1590/S0100-69162007000400011

APRILE, F. M.; FARIAS, V. P. Avaliação da qualidade da água da bacia do Rio Goiana, Pernambuco - Brasil. Bioikos, v. 15, n. 2, p. 109-114, 2001.

AUTIN, M. A.; EDWARDS, D. Nonparametric harmonic regression for estuarine water

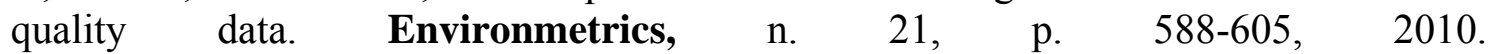
http://dx.doi.org/10.1002/env.1013

BABOROWSKI, M.; SIMEONOV, V.; EINAX, J. Assessment of water quality in the Elbe River at flood water conditions based on cluster analysis, principle components analysis, and source apportonment. Clean - Soil, Air, Water, v. 40, n. 4, p. 373-380, 2012. http://dx.doi.org/10.1002/clen.201100085

BERNARDI, J. V. E.; LACERDA, L. D.; DÓREA, J. G.; LANDIM, P. M. B.; GOMES, J. P. O.; ALMEIDA, R. et al. Aplicação da análise das componentes principais na ordenação dos parâmetros físico-químicos no alto Rio Madeira e afluentes, Amazônia Ocidental. Geochimica Brasiliensis, v. 23, n. 1, p. 79-90, 2009.

BHATTACHARYYA, K. G.; KAPIL, N. Impact of urbanization on the quality of water in a naturalreservoir: a case study with the DeeporBeel in Guwahaticity, India. Water and Environment Journal, n. 24, p. 83-96, 2010. http://dx.doi.org/10.1111/j.17476593.2008.00157.x

BRASIL. Lei n 12.651, de 25 de maio de 2012. Diário Oficial [da] União, Brasília, 28 mai. 2012a. Disponível em: http://www.planalto.gov.br/civil_03/_ Ato20112014/2012/Lei/L12651.htm. Acesso em: 08 out. 2013.

BRASIL. Lei $\mathrm{n}^{\circ}$ 12.727, de 17 de outubro de 2012. Diário Oficial [da] União, Brasília, 17 out. 2012b. Disponível em: http://www.planalto.gov.br/civil_03/_Ato20112014/2012/Lei/L12727.htm. Acesso em: 10 nov. 2013.

BRASIL. Resolução 357, de 17 de março de 2005. Diário Oficial [da] União, Brasília, 17 mar. 2005. Disponível em www.mma.gov.br/port/conama/res/res 05/res35705.pdf. Acesso em: 10 fev. 2014.

CARNEIRO, P. R. F.; CARDOSO, A. L.; ZAMPRONIO, G. B.; MARTINGIL, M. de C. A gestão integrada de recursos hídricos e do uso do solo em bacias urbano-metropolitanas: o controle de inundações na bacia dos rios Iguaçu/Sarapuí, na Baixada Fluminense. Ambiente e Sociedade, v. 13, n. 1, p. 29-49, 2010.

CARVAlHO, A. R.; SCHLITTLER, F. H. M.; TORNISIELO, V. L. Relações da atividade agropecuária com parâmetros físicos químicos da água. Química. Nova, v. 23, n. 5, p. 618-622, 2000. http://dx.doi.org/10.1590/S0100-40422000000500009 
CASATTI, L. Alterações no Código Florestal Brasileiro: impactos potenciais sobre a ictiofauna. Biota Neotrópica, v. 10, n. 4, p. 31-34, 2010. http://dx.doi.org/10.1590/S1676-06032010000400002

COMPANHIA DE SANEAMENTO MUNICIPAL DE JUIZ DE FORA - CESAMA. Represa Dr. João Penido. Disponível em: http://www.cesama.com.br/? pagina=joaopenido Acesso em: 29 dez. 2014.

COMPANHIA AMBIENTAL DO ESTADO DE SÃO PAULO - CETESB. Qualidade das águas interiores no estado de São Paulo. São Paulo, 2009. 43p.

COHEN, J. Statistical power analysis for the behavioral sciences. 2. ed. Hillsdale Lawrence Erlbaum Associates, 1988. 567p.

FIGUEIREDO FILHO, D. B.; SILVA JÚNIOR, J. A. da. Visão além do alcance: uma introdução à análise fatorial. Opinião Pública, v. 16, n. 1, p. 160-185, 2010. http://dx.doi.org/10.1590/S0104-62762010000100007

FRINTZONS, E.; HIND, E. C.; MANTOVANI, L. E.; RIZZI, N. E. As alterações da qualidade da água do Rio Capivari com o deflúvio: um instrumento de diagnóstico de qualidade ambiental. Engenharia Sanitária e Ambiental, v. 8, n. 4, p. 239-248, 2003.

GIRÃO, E. G.; ANDRADE, E. M.; ROSA, M. de F.; ARAÚJO, L. de F. P.; MEIRELES, A. C. M. Seleção dos indicadores da qualidade de água no Rio Jaibaras pelo emprego da análise de componente principal. Revista Ciência Agronômica, v. 38, n. 1, p. 17-24, 2007.

GUEDES, H. A. S.; SILVA, D. D.; EleSBON, A. A. A.; RIBEIRO, C. B. M.; MATOS, A. T.; SOARES, J. H. P. Aplicação da análise estatística multivariada no estudo da qualidade da água do Rio Pomba, MG. Revista Brasileira de Engenharia Agrícola e Ambiental, v. 16, n. 5, p. 558-563, 2012. http://dx.doi.org/10.1590/S141543662012000500012

HAIR, J. J. F.; ANDERSON, R. E.; TATHAM, R. L.; BLACK, W. C. Análise multivariada de dados. 5. ed. Porto Alegre: Bookman, 2007. 593p.

HELENA, B.; PARDO, R.; VEGA, M.; BARRADO, E.; FERNÁNDEZ, J. M.; FERNÁNDEZ, L. Temporal evolution of groundwater composition in an alluvial aquifer (Pisuerga River, Spain) by principal component analysis. Water Research, v. 34, n. 3, p. 807-816, 2000. http://dx.doi.org/10.1016/S0043-1354(99)00225-0

INSTITUTO BRASILEIRO DE GEOGRAFIA E ESTATÍSTICA - IBGE. Cidades@: MG Juiz de Fora. Disponível em: http://cod.ibge.gov.br/234B9. Acesso em: 07 maio 2014.

JUIZ DE FORA. Lei $\mathbf{n}^{\mathbf{0}} \mathbf{1 1 . 8 1 7}$ de 04 de agosto de 2009. Altera a Lei Municipal $\mathrm{n}^{\circ} 6.087$, de 04 de dezembro de 1981, e dá outras providências. Disponível em: http://www.leismunicipais.com.br/legislacao-de-juiz-de-fora/973791/lei-11817-2009juiz-de-fora-mg.html Acesso em: 07 jun. 2014

JUIZ DE FORA. Lei $\mathbf{n}^{\mathbf{0}} \mathbf{9 . 8 1 1}$ de 27 de junho de 2000. Institui o plano diretor de desenvolvimento urbano de Juiz de Fora. Disponível em: http://www.leismunicipais.com.br/legislacao-de-juiz-de-fora/1391368/lei-9811-2000juiz-de-fora-mg.html Acesso em 07 jun. 2014. 
JUIZ DE FORA. Lei $\mathbf{n}^{\mathbf{0}}$ 6.910 de 31 de maio de 1986. Dispõe sobre o ordenamento do uso e ocupação do solo no município de Juiz de Fora. Disponível em: http://www.leismunicipais.com.br/legislacao-de-juiz-de-fora/1060646/lei-6910-1986juiz-de-fora-mg.html. Acesso em: 02 jun. 2014.

JUIZ DE FORA. Lei $\mathbf{n}^{\circ} \mathbf{6 . 0 8 7}$ de 04 de dezembro de 1981. Dispõe sobre o parcelamento, uso e ocupação do solo, na área da bacia hidrográfica da Represa Dr. João Penido, em Juiz de Fora e dispõe outras providências. Disponível em: http://www.leismunicipais.com.br/legislacao-de-juiz-de-fora/1380937/lei-6087-1981juiz-de-fora-mg.html. Acesso em: 07 jun. 2014

LIAO, S. W.; GAU, H. S.; LAI, W. L.; CHEN, J. J.; LEE, C. G. Identification of pollution of Tapeng Lagoon from neighbouring rivers using multivariate statistical method. Journal of Environmental Management, v. 88, n. 2, p. 286-292, 2008. http://dx.doi.org/10.1016/j.jenvman.2007.02.010

MACHADO, P. J. O. Crescimento urbano e ocupação de mananciais: o caso de Juiz de Fora/MG. In: FÓRUM AMBIENTAL DA ALTA PAULISTA, 2., 2006, Estância Turística de Tupã. Resumos... Estância Turística de Tupã: ANAP/FACCAT/UNESP, 2006. 1 CD-ROM.

MENDONÇA, E. C. C. N.; SOUZA, P. S. de. Aplicação da técnica de análise de componentes principais para caracterização de águas de poços artesianos de áreas urbanas de Goiânia e Aparecida de Goiânia. Plurais, v. 1, n. 1, p. 19-36, 2011.

PADIAL, P. R.; POMPÉO, M.; MOSCHINI-CARLOS, V. Heterogeneidade espacial e temporal da qualidade da água no reservatório Rio das Pedras (Complexo Billings, São Paulo). Revista Ambiente \& Água, v. 4, n. 3, p. 35-53, 2009. http://dx.doi.org/10.4136/1980-993X.

ROCHA, C. H. B.; COSTA, H. F. Variação temporal de parâmetros limnológicos em manancial de abastecimento em Juiz de Fora, MG. Revista Brasileira de Recursos Hídricos, v. 20, n. 2, p. 543-550, 2015.

ROCHA, C. H. B.; FREITAS, F. A.; SILVA, T. M. Alterações em variáveis limnológicas de manancial de Juiz de Fora devido ao uso da terra. Revista Brasileira de Engenharia Agrícola e Ambiental, v. 18, n. 4, p. 431-436, 2014. http://dx.doi.org/10.1590/S141543662014000400011

SHEELA, A. M.; LETHA, J.; JOSEPH, S.; CHACKO, M.; SANAL KUMAR, S. P.; THOMAS, J. Water quality assessment of a tropical coastal lake system using multivariate cluster, principal component and factor analysis. Lakes e Reservoirs: Research and Management, n. 17, p. 143-159, 2012. http://dx.doi.org/10.1111/j.14401770.2012.00506.x

SPEZIA, L. FUTTER, M. N. BREWER, J. Periodic multivariate Normal hidden Markov models for the analysis of water quality time series. Environmetrics, n. 22, p. 304-317, 2011. http://dx.doi.org/10.1002/env.1051

THURSTON, L. P. SMITH, C. A. GENSKOW, K. PROKOPY, L. S. HARGROVE, W. L. The social context of water quality improvement evaluation. New Directions for Evaluation, n. 135, p. 41-58, 2012. http://dx.doi.org/10.1002/ev.20026 
TOLEDO, L. G. de; NICOLELLA, G. Índice de qualidade de água em microbacia sob uso agrícola e urbano. Scientia Agrícola, v. 59, n. 1, p. 181-186, 2002. http://dx.doi.org/10.1590/S0103-90162002000100026

TU, J. Spatially varying relationships between land use and water quality across an urbanization gradient explored by geographically weighted regression. Applied Geography, n. 31, p. 376-392, 2011. http://dx.doi.org/10.1016/j.apgeog.2010.08.001

TUNDISI, J. G.; TUNDISI, T. M. Impactos potenciais das alterações do Código Florestal nos recursos hídricos. Biota Neotrópica, v. 10, n. 4, p. 67-75, 2010. http://dx.doi.org/10.1590/S1676-06032010000400010

VANZELA, L. S.; HERNADEZ, F. B. T.; FRANCO, R. A. M. Influência do uso e ocupação do solo nos recursos hídricos do Córrego Três Barras, Marinópolis. Revista brasileira de Engenharia Agrícola e Ambiental, v. 14, n. 1, p. 55-64, 2010. http://dx.doi.org/10.1590/S1415-43662010000100008

VON SPERLING, M. Introdução à qualidade das águas e ao tratamento de esgotos. 3. ed. Belo Horizonte: Imprensa Universitária da UFMF, 2005. 452 p.

YU, D.; SHI, P.; LIU, Y.; XUN, B. Detecting land-use water quality relationships from the viewpoint of ecological restoration in an urban area. Ecological Engineering, n. 53, p. 205-216, 2013. http://dx.doi.org/10.1016/j.ecoleng.2012.12.045 


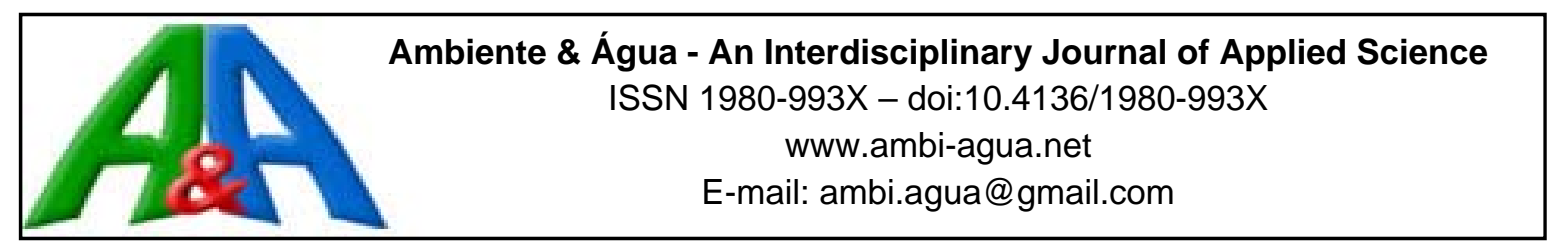

\title{
Remoção de microcistina-LR da Microcystis aeruginosa utilizando bagaço de cana-de-açúcar in natura e carvão ativado
}

\author{
doi:10.4136/ambi-agua.1785
}

Received: 15 Oct. 2015; Accepted: 04 Dec. 2015

\author{
Aline Rafaela de Almeida ${ }^{1 *}$; Fernando Hermes Passig'; Thomaz Aurélio Pagioro ${ }^{1}$, \\ Priscila Tiemi Higuti do Nascimento"; \\ Karina Querne de Carvalho ${ }^{2}$

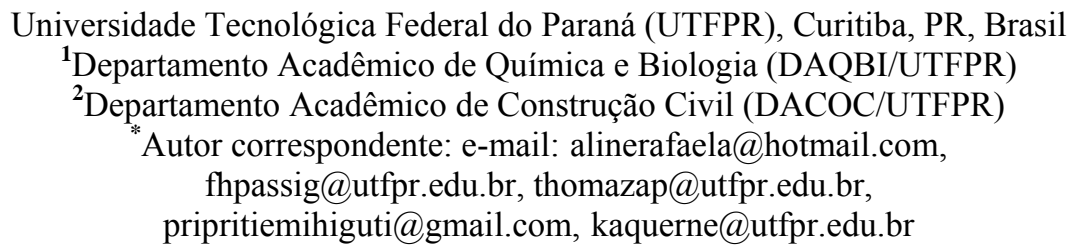

\section{RESUMO}

Microcistina-LR é um tipo de toxina liberada pela cianobactéria Microcystis aeruginosa encontrada em mananciais de água usados para abastecimento humano que pode causar doenças e até mesmo a mortandade do homem se não for totalmente removida no tratamento convencional da água. A retenção desta toxina é muitas vezes realizada através do processo de adsorção em carvão ativado nas estações de tratamento de água. Neste estudo foi avaliada a utilização do bagaço de cana-de-açúcar in natura como bioadsorvente em comparação ao carvão ativado na retenção da microcistina-LR. Primeiramente foi caracterizado o bioadsorvente e o carvão ativado em função de suas características físicas e químicas, e posteriormente foram realizados ensaios de adsorção. O desempenho da adsorção foi avaliado pela eficiência da retenção da toxina e pela capacidade máxima adsortiva. As eficiências médias de retenção da toxina no carvão ativado resultaram em 65,25; 41,74 e 11,75\%; e de 24,15; 18,92 e 12,27\% no bagaço de cana-de-açúcar para as concentrações de 2,36, 3,33 e $3,83 \mu \mathrm{g} \mathrm{L}{ }^{-1}$, respectivamente. $\mathrm{O}$ bioadsorvente apresentou eficiência de remoção da toxina similar àquela observada no carvão ativado para a concentração de $3,83 \mu \mathrm{g} \mathrm{L}^{-1}$. A capacidade máxima adsortiva obtida com o melhor ajuste linear para isoterma de Freundlich, foi de $6047,84 \mu \mathrm{g} \mathrm{g}^{-1}$ (concentração de toxina de 3,83 $\mu \mathrm{g} \mathrm{L}^{-1}$ ) para o bagaço de cana-de-açúcar e de $338,61 \mu \mathrm{g} \mathrm{g}^{-1}$ (concentração de toxina de $2,36 \mu \mathrm{g} \mathrm{L}^{-1}$ ) para o carvão ativado.

Palavras-chave: adsorção, isoterma de Freundlich, isoterma de Langmuir.

\section{Microcystin-LR removal from Microcystis aeruginosa using in natura sugarcane bagasse and activated carbon}

\section{ABSTRACT}

Microcystin-LR is a type of toxin released by the Microcystis aeruginosa cyanobacteria found in water sources used for human consumption. It can cause illness and even death if not completely removed in conventional water treatment. The retention of this toxin is often 
accomplished by the adsorption process in activated carbon in water treatment plants. In this study, a comparison was made between the retention of microcystin-LR by activated carbon and by sugarcane bagasse in natura applied as a bio-adsorbent. Adsorption experiments were performed after the physical and chemical properties of the bio adsorbent and the activated carbon were characterized. The adsorption performance was evaluated by the toxin removal efficiency and the maximum adsorption capacity. Average removal efficiencies of the toxin resulted in $65.25 ; 41.74$ and $11.75 \%$ for the activated carbon and $24.15 ; 18.92$ and $12.27 \%$ for the sugarcane bagasse for concentrations of $2.36,3.33$ and $3.83 \mu \mathrm{g} \mathrm{L}^{-1}$, respectively. The bio adsorbent presented removal efficiency for the toxin similar to that observed in the activated carbon for the concentration of $3.83 \mu \mathrm{g} \mathrm{L} \mathrm{L}^{-1}$. Maximum adsorption capacity obtained with better linear adjustment to the Freundlich isotherm was $6,047.84 \mu \mathrm{g} \mathrm{g}^{-1}$ (toxin concentration of $3.83 \mu \mathrm{g} \mathrm{L}^{-1}$ ) for sugarcane bagasse and $338.61 \mu \mathrm{g} \mathrm{g}^{-1}$ (toxin concentration of $2.36 \mu \mathrm{g} \mathrm{L}^{-1}$ ) for activated carbon.

Keywords: adsorption, Freundlich isotherm, Langmuir isotherm.

\section{INTRODUÇÃO}

A microcistina-LR é um tipo de toxina hepatotóxica produzida pela cianobactéria Microcystis aeruginosa, uma das mais frequentemente encontradas em mananciais de abastecimento de água para consumo humano. O valor máximo permitido desta toxina na água potável é de $1,0 \mu \mathrm{g} \mathrm{L}^{-1}$, conforme estabelecido na Portaria 2914/2011 do Ministério da Saúde (Brasil, 2011).

A presença de cianotoxinas em mananciais ocorre principalmente devido à aceleração do processo de eutrofização, comumente relacionada ao enriquecimento artificial dos ecossistemas aquáticos pelo excesso de compostos fosfatados e nitrogenados de origem sanitária e industrial de descargas sem tratamento prévio, e das regiões agricultáveis, devido ao uso intensivo de fertilizantes (Azevedo et al., 2002).

A quantidade excessiva destes nutrientes, atrelada às condições climáticas da região, favorece a floração de cianobactérias em águas de mananciais. O maior problema está no fato de que algumas espécies ocorrentes destas florações produzem e liberam toxinas para o ambiente no momento de senescência ou lise celular, o que consequentemente acarreta em danos à saúde da população (Belisário et al., 2011).

$\mathrm{O}$ caso mais grave registrado em relação aos efeitos das cianotoxinas ocorreu com a morte de 70 pessoas no município de Caruaru, estado de Pernambuco, durante tratamento de hemodiálise com a água contaminada com toxina de cianobactéria (Azevedo et al., 2002).

Como a prevenção de florações de cianobactérias envolve minimizar emissões de compostos nitrogenados e fosforados que muitas vezes são difusas, cabe aos processos convencionais de tratamento de água remover estes microrganismos e suas toxinas de modo a cumprir os padrões de potabilidade exigidos na legislação (Di Bernardo e Sabogal Paz, 2008).

As estações de tratamento de água para consumo humano em sua maioria são operadas com filtros de carvão ativado para eliminar gosto e odor da água, causados pelas cianobactérias, e também para reter suas toxinas (Di Bernardo e Sabogal Paz, 2008).

Devido ao maior custo do carvão ativado, e a disponibilidade do resíduo da produção de cana-de-açúcar no Brasil, um dos maiores produtores mundiais, a capacidade adsorvente do bagaço de cana-de-açúcar tem sido investigada como alternativa na remoção da microcistina da água (Jaguaribe et al., 2005; Albuquerque Jr. et al., 2008). Dentro deste contexto, neste trabalho é avaliada a eficiência do bagaço de cana-de-açúcar in natura em comparação ao carvão ativado na remoção de microcistina-LR de Microcystis aeruginosa.

\section{MATERIAIS E MÉTODOS}




\subsection{Preparação da amostra de toxina}

A amostra de toxina utilizada nos ensaios de adsorção foi proveniente de cultivo de Microcystis aeruginosa da cepa BB005, fornecida pelo Departamento de Botânica da Universidade Federal de São Carlos em março de 2011.

O cultivo foi realizado no Laboratório de Limnologia do Departamento Acadêmico de Química e Biologia da UTFPR - câmpus Curitiba, sede Ecoville, utilizando meio sintético de cultivo ASM-1, sendo que as principais condições para crescimento e manutenção do meio para a $M$. Aeruginosa foram: temperatura entre 22 e $28^{\circ} \mathrm{C}$; intensidade luminosa de $215 \mu \mathrm{mol} \mathrm{m} \mathrm{m}^{-2} \mathrm{~s}^{-1}$; fotoperíodo $12 / 12 \mathrm{~h}$ claro/h escuro e concentração inicial de $1,6.10^{3} \mathrm{cel} \mathrm{mL}^{-1}$ de acordo com procedimentos descrito por Jacinavicius et al. (2013).

A inoculação foi realizada na proporção de $10 \%$ de volume de inóculo para $100 \%$ de volume de meio de cultivo de acordo com procedimentos descritos por Jacinavicius et al. (2013).

A contagem de células foi realizada em hemacitômetro de Neubauer (profundidade 0,100 $\mathrm{mm}$ ) segundo metodologia proposta por Tortora et al. (2000), utilizando microscópio óptico Zeiss modelo Axiovert $40 \mathrm{C}$ até a verificação da concentração de $10^{6}$ cel $\mathrm{mL}^{-1}$.

Após ser quantificado, o cultivo foi colocado em um recipiente de 2,0 L de polietileno, homogeneizado, armazenado em freezer a aproximadamente $-8^{\circ} \mathrm{C}$, congelado e descongelado três vezes para rompimento da parede celular das cianobactérias e liberação da toxina para o meio de acordo com procedimento descrito por Jacinavicius et al. (2013).

Após este procedimento, uma amostra de $50 \mathrm{~mL}$ foi coletada para quantificação da toxina, e em seguida foram realizadas diluições do cultivo em água destilada para as concentrações de $2,36 \pm 0,34,3,33 \pm 0,25$ e $3,83 \pm 0,36 \mu \mathrm{g} \mathrm{L}^{-1}$ de toxina para posteriores ensaios de adsorção.

\subsection{Quantificação da toxina}

A quantificação da toxina do cultivo foi realizada no Laboratório do Centro de Análises e Ensaios Tecnológicos da TECPAR, Curitiba-PR, segundo metodologia descrita por Dahlmann e Luckas (2010), e analisada por Cromatografia Líquida de Alta Eficiência (Agilent 1100), acoplado a espectrômetro de massas triplo quadrupolo (Applied Biosystens API 4000) com fonte de ionização por electrospray.

A separação cromatográfica foi obtida em coluna analítica Eclipse XDB-C18 (4,6 x $150 \mathrm{~mm}, 5 \mu \mathrm{m}$, Agilent) e fase móvel constituída por: solvente A (10\% acetonitrila e $90 \%$ solução aquosa de acetato de amônia $5 \mathrm{mM}$ e ácido fórmico $1 \%)$ e solvente B $(90 \%$ acetonitrila e $10 \%$ solução aquosa de acetato de amônia $5 \mathrm{mM}$ e ácido fórmico $0,1 \%$ ), em eluição isocrática com $55 \%$ de A e $45 \%$ de B. O volume de injeção foi de $100 \mu \mathrm{L}$ e o limite de quantificação foi de $0,5 \mu \mathrm{g} \mathrm{L}^{-1}$.

\subsection{Preparo e caracterização dos materiais adsorventes}

O bagaço de cana-de-açúcar utilizado foi moído e seco em estufa a $100 \pm 10^{\circ} \mathrm{C}$ por $48 \mathrm{~h}$, triturado em liquidificador industrial por $5 \mathrm{~min}$ para posterior utilização nos ensaios de caracterização, conforme metodologia proposta por Belisário et al. (2011). Para o ensaio de adsorção foi utilizada a granulometria de 100 mesh de massa retida na peneira.

O carvão ativado utilizado (CAG) é de origem vegetal e composição granular, fabricado pela NORIT (modelo GAC 124W). Amostras de $10 \mathrm{~g}$ do carvão ativado foram colocadas em estufa a $60^{\circ} \mathrm{C}$ por $12 \mathrm{~h}$ e mantidas em dessecador. Para a caracterização, foram realizados os ensaios de granulometria segundo a NBR 11834:1991, e índice do número de iodo segundo NBR 12073:1991 (ABNT, 1991a; 1991b).

$\mathrm{O} \mathrm{pH}$ foi determinado em triplicata utilizando pHmetro pHTEK, modelo pHS-3E, conforme procedimentos descritos na norma ASTM D 3838-1980:1999 (ASTM, 1999). 
A microscopia eletrônica de varredura foi realizada em um microscópio Zeiss, modelo EVO/MAI 15 e em metalizador Quorum, modelo Q150RES.

A área superficial interna foi determinada por isotermas de adsorção/dessorção em $\mathrm{N}_{2}$ através do método Brunauer-Emmett-Teller (BET) no Instituto Lactec em um porosímetro Quantachrome, modelo NOVA1200.

\subsection{Isotermas de Adsorção}

Os ensaios de adsorção foram realizados no Laboratório de Saneamento do Departamento Acadêmico de Construção Civil da UTFPR, câmpus Curitiba, sede Ecoville, em uma incubadora Shaker da Solab, modelo SL 222, a temperatura controlada de $25 \pm 3^{\circ} \mathrm{C}$, agitação constante de $150 \mathrm{rpm}$, por $10 \mathrm{~h}$ com amostras em duplicata.

Foram utilizados erlenmeryes de $250 \mathrm{~mL}$, contendo $200 \mathrm{~mL}$ de solução de microcistina de 2,36 $\pm 0,34 \mu \mathrm{g} \mathrm{L}^{-1}, 3,33 \pm 0,25 \mu \mathrm{g} \mathrm{L}^{-1}$ e $3,83 \pm 0,36 \mu \mathrm{g} \mathrm{L}{ }^{-1}$ e $50 \mathrm{mg}$ de massa de material adsorvente para cada concentração.

Alíquotas de $10 \mathrm{~mL}$ das amostras do ensaio de adsorção foram coletadas em intervalos de duas horas, filtradas em membrana de acetato de celulose de $0,45 \mu \mathrm{m}$ e mantidas em recipientes de polietileno em refrigerador a $4^{\circ} \mathrm{C}$ para posterior quantificação da microcistinaLR.

A concentração da toxina adsorvida foi determinada utilizando a Equação (1).

$$
q_{e}=\frac{\left(C_{o}-C_{e}\right)}{M} \times V
$$

em que:

$q_{e}$ : é a concentração de equilíbrio da microcistina-LR em material adsorvente ( $\mu \mathrm{g}$ microcistina-LR $\mathrm{g}^{-1}$ de material adsorvente);

$C_{o}$ : é a concentração inicial de microcistina-LR ( $\mu$ g microcistina-LR $\mathrm{L}^{-1}$ de solução);

$C_{e}$ : é a concentração de equilíbrio de microcistina-LR ( $\mu \mathrm{g}$ microcistina-LR $\mathrm{L}^{-1} \mathrm{de}$ solução);

$V$ : é o volume da fase aquosa adicionada (L); e

$M$ : é a massa do material adsorvente $(\mathrm{g})$

A partir destes dados foram plotados gráficos da concentração de equilíbrio de microcistina-LR versus a quantidade adsorvida em $\mu \mathrm{g} \mathrm{g}^{-1}$ de material adsorvente e feito ajustes pelos modelos de Langmuir (Equação 2) e de Freundlich (Equação 3).

$$
q_{e}=\frac{q_{m a ́ x} \times b \times C_{e}}{1+b \times C_{e}}
$$

em que:

$q_{e}$ : é a concentração de equilíbrio de microcistina-LR em material adsorvente ( $\mu \mathrm{g}$ microcistina-LR $\mathrm{g}^{-1}$ de material adsorvente);

$q_{\text {máx }}$ : é a capacidade máxima de adsorção de microcistina-LR na monocamada do material adsorvente- limite de saturação ( $\mu \mathrm{g}$ microcistina-LR $\mathrm{g}^{-1}$ material adsorvente);

$C_{e}$ : é a concentração de equilíbrio de microcistina-LR ( $\mu \mathrm{g}$ microcistina-LR $\mathrm{L}^{-1} \mathrm{de}$ solução); e

$b$ : é a constante de equilíbrio da adsorção relacionada à energia de adsorção ( $\mathrm{L}$ de solução $\mu \mathrm{g}^{-1}$ de material adsorvente). 


$$
q_{e}=k C_{e^{\frac{1}{n}}}
$$

em que:

$q_{e}$ : é a concentração de equilíbrio de microcistina-LR em material adsorvente ( $\mu \mathrm{g}$ microcistina-LR $\mathrm{g}^{-1}$ de material adsorvente);

$k$ : é a constante da capacidade adsortiva ( $\mathrm{L}$ de solução $\mathrm{g}^{-1}$ de material adsorvente);

$C_{e}$ : é a concentração de equilíbrio de microcistina-LR ( $\mu \mathrm{g}$ microcistina-LR $\mathrm{L}^{-1} \mathrm{de}$ solução); e

$n$ : é a constante de equilíbrio da isoterma.

\section{RESULTADOS E DISCUSSÃO}

\subsection{Caracterização dos materiais adsorventes}

Os resultados da caracterização físico-química dos materiais adsorventes, bagaço de cana-de-açúcar e carvão ativado são apresentados na Tabela 1.

Tabela 1. Caracterização físico-química dos materiais adsorventes.

\begin{tabular}{lcc}
\hline \multicolumn{1}{c}{ Características } & Bagaço de cana-de-açúcar & Carvão Ativado \\
\hline Granulometria (\% massa) & 23,3 & 7,7 \\
$\mathrm{pH}$ & $4,1 \pm 0,05$ & $6,7 \pm 0,08$ \\
Área superficial interna $\left(\mathrm{m}^{2} \mathrm{~g}^{-1}\right)$ & 1,3 & 304,2 \\
Número de iodo $\left(\mathrm{mg} \mathrm{g}^{-1}\right)$ & nd & 665,86 \\
\hline
\end{tabular}

*nd: não determinado

A granulometria foi realizada para selecionar frações dos materiais adsorventes retidas na peneira com abertura nominal 100 mesh para o material adsorvente, que usualmente é o padrão utilizado no tratamento de água para consumo humano. Como o menor tamanho dos grãos do material adsorvente influencia na maior taxa de adsorção, é importante realizar este ensaio para conhecer o tempo requerido para o transporte do adsorvato nos poros do adsorvente (Di Bernardo e Sabogal, 2008).

$\mathrm{O} \mathrm{pH}$ do adsorvente é outro fator que influencia na adsorção, pois está diretamente relacionado à carga da superfície do adsorvente e ao grau de ionização dos solutos do adsorvato (Gonçalves et al., 2006). Huang et al. (2007) verificaram que o melhor adsorvente de microcistina-LR para três tipos de carvões ativados de origem vegetal, deve apresentar valor de $\mathrm{pH}$ menor no material adsorvente do que o da solução na qual a microcistina-LR está dissolvida. Sathishkumar et al. (2010) obtiveram melhor eficiência de retenção da microcistina-LR em pH de 3,0 no material adsorvente. De acordo com estes autores, o aumento do $\mathrm{pH}$ força as formas aniônicas da estrutura molecular da microcistina-LR, dificultando sua adsorção.

Além disso, a repulsão eletrostática entre os sítios vizinhos que causaria expulsão da microcistina-LR seria diminuída em menor $\mathrm{pH}$, aumentando as ligações de hidrogênio e a capacidade de adsorção. Os valores do $\mathrm{pH}$ do carvão ativado e do bagaço de cana-de-açúcar foram menores que 7,0, o que pode favorecer a adsorção da microcistina-LR de acordo com estudos previamente reportados.

A baixa área superficial do bagaço de cana-de-açúcar também foi verificada por Belisário et al. (2011) $\left(1,49 \mathrm{~m}^{2} \mathrm{~g}^{-1}\right)$ que atribuíram o potencial adsortivo à formação de monocamadas de paracetamol na superfície deste material devido à presença de grupos funcionais variados. Isto porque este adsorvente é um biopolímero natural constituído principalmente de celulose 
(50\%), hemicelulose (25\%) e lignina (25\%) de acordo com Meza et al. (2006). Assim como evidenciado por Albuquerque Jr. et al. (2008), não foi possível obter o número de iodo do bagaço de cana-de-açúcar por meio da norma de caracterização dos carvões ativados, provavelmente devido à reduzida área superficial.

De acordo com Müller et al. (2009), o número de iodo é um indicador de volume de microporos, e a remoção da microcistina-LR é influenciada pelo volume dos microporos secundários e mesoporos do carvão ativado, que não foram determinados neste trabalho.

Neste estudo foram verificados valores do número de iodo e da área superficial inferiores aos valores reportados por Donati et al. (1994) de 964 e $953 \mathrm{mg} \mathrm{g}^{-1}$ e de 1.196 e $1.366 \mathrm{~m}^{2} \mathrm{~g}^{-1}$, respectivamente, para carvões ativados de origem vegetal, o que pode ter influenciado na diminuição da remoção devido a maior concentração da microcistina-LR na solução.

\subsubsection{Análises do bagaço de cana-de-açúcar e do carvão ativado por MEV}

$\mathrm{Na}$ Figura 1 são apresentadas as fotomicrografias da superfície dos materiais adsorventes utilizados nos ensaios de adsorção.

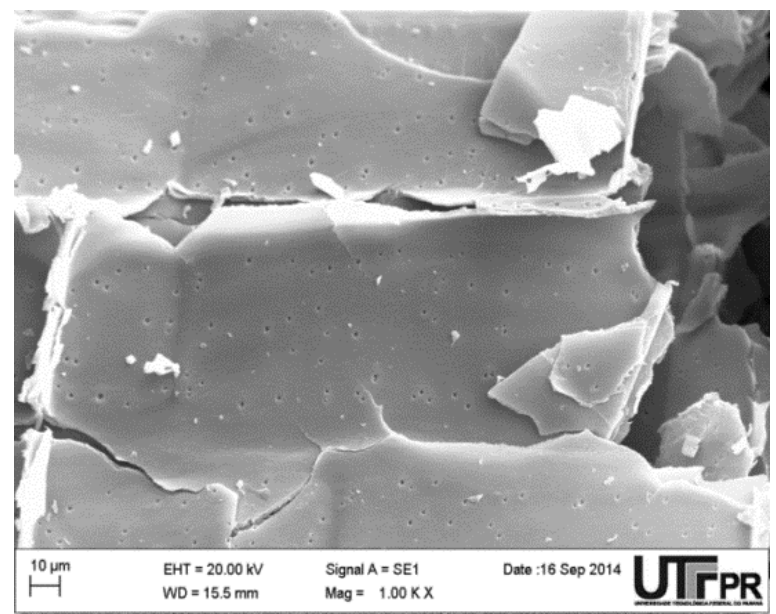

a)

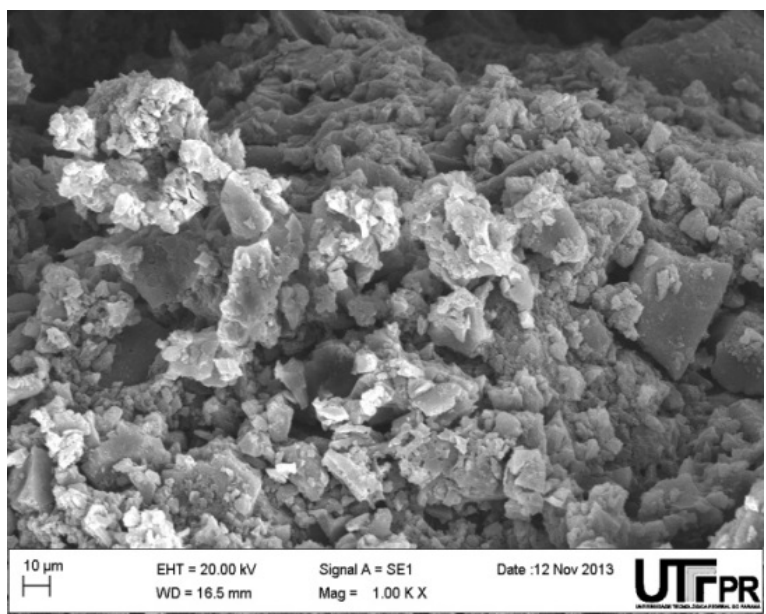

b)

Figura 1. Fotomicrografias eletrônicas de varredura da superfície do: a) bagaço de cana-de-açúcar com aproximação de 1,0 kX; b) carvão ativado com aproximação de 1,0 kX.

As imagens obtidas por MEV revelam que a superfície do bagaço de cana-de-açúcar apresenta-se lisa e com poros praticamente homogêneos e simétricos (macroporos) (Figura 1a); e a superfície do carvão ativado apresenta porosidade heterogênea com poros difusos (microporos), caracterizando sua maior área superficial (Figura 1b). Fotomicrografias similares foram verificadas por Belisário et al. (2011) para superfície do bagaço de cana-deaçúcar.

\subsubsection{Avaliação da remoção da microcistina-LR}

A variação da remoção da microcistina-LR em função do tempo de adsorção $(h)$ para os ensaios de adsorção com concentrações de $2,36 \pm 0,34 \mu \mathrm{g} \mathrm{L} \mathrm{L}^{-1}, 3,33 \pm 0,25 \mu \mathrm{g} \mathrm{L^{-1 }}$ e $3,83 \pm 0,36 \mu \mathrm{g} \mathrm{L}^{-1}$ é apresentada na Figura 2. 


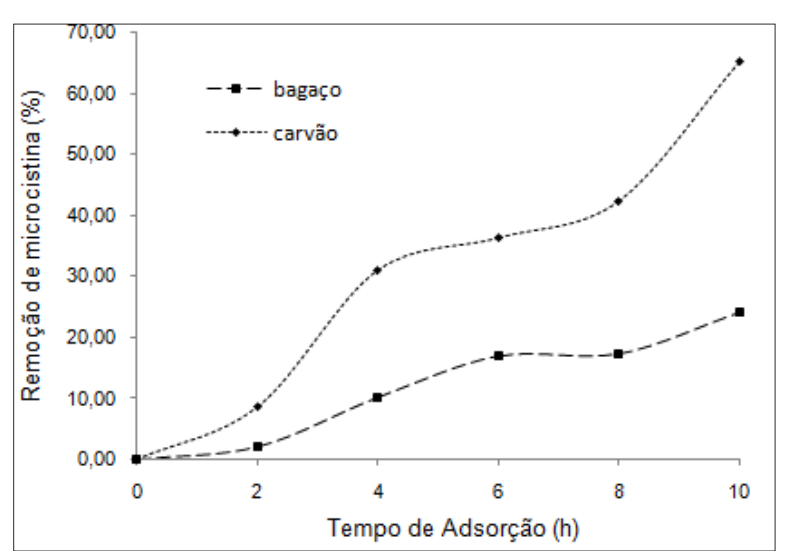

a) Amostra com concentração de $2,36 \mu \mathrm{g} \mathrm{L} \mathrm{L}^{-1}$

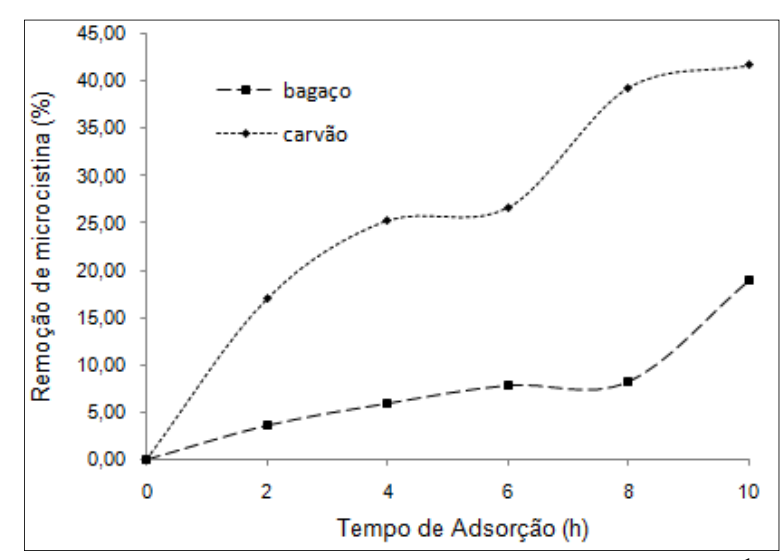

b) Amostra com concentração de $3,33 \mu \mathrm{g} \mathrm{L}{ }^{-1}$

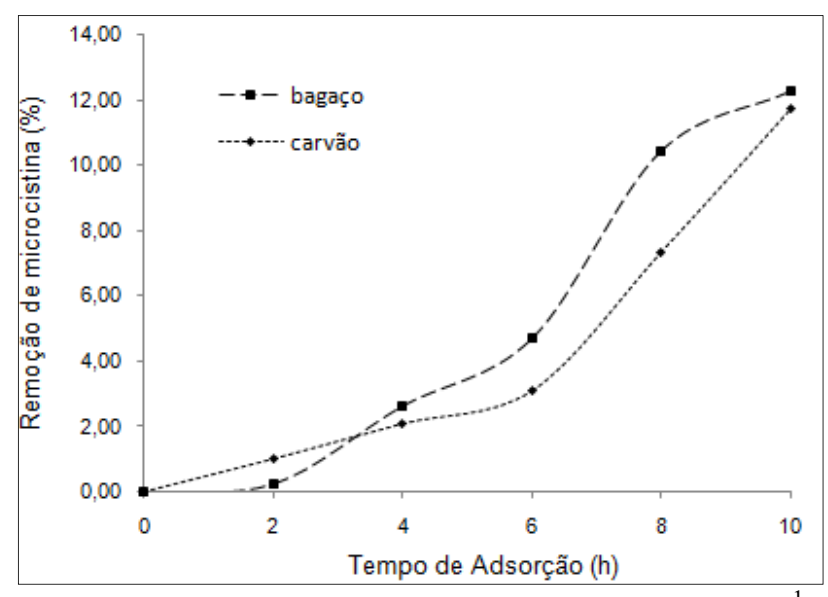

c) Amostra com concentração de $3,83 \mu \mathrm{g} \mathrm{L}{ }^{-1}$

Figura 2.Variação da retenção da microcistina-LR no intervalo de tempo de $10 \mathrm{~h}$ de adsorção.

As maiores eficiências de retenção foram observadas para o carvão ativado e podem ser justificadas em função da maior área superficial e do número de iodo em relação àquelas verificadas para o bagaço de cana-de-açúcar, independente da concentração de microcistinaLR na solução inicial.

As eficiências médias de retenção da toxina resultaram em 65,25; 41,74 e 11,75\% no carvão ativado e de 24,$15 ; 18,92$ e $12,27 \%$ no bagaço de cana-de-açúcar para as concentrações de 2,36, 3,33 e 3,83 $\mu \mathrm{g} \mathrm{L}^{-1}$, respectivamente. É possível notar tendência de similaridade da eficiência de retenção dos materiais em função do aumento da concentração da toxina para $3,83 \mu \mathrm{g} \mathrm{L}^{-1}$ nas seis primeiras horas dos ensaios, de $12,27 \%$ e $11,75 \%$ para o bagaço de cana-de-açúcar e carvão ativado, respectivamente.

Pyo e Moon (2005) obtiveram retenção da microcistina-LR de 39\% com concentração inicial de $1000 \mu \mathrm{g} \mathrm{L}^{-1}$ em carvão ativado com área superficial de $750 \mathrm{~m}^{2} \mathrm{~g}^{-1}$ e número de iodo de $1000 \mathrm{mg} \mathrm{g}^{-1}$. A menor retenção desta toxina neste trabalho indica que as características morfológicas deste carvão ativado não eram específicas para retenção desta toxina.

Belisário et al. (2011) obtiveram menor eficiência de retenção para paracetamol (23\%) sem qualquer ativação do bagaço de cana-de-açúcar. No entanto, Molica et al. (2013) justificaram a maior eficiência de retenção da microcistina-LR de até $70 \%$ devido ao aumento da área superficial do bagaço de cana-de-açúcar pela ativação física.

\subsubsection{Isotermas de adsorção}

As isotermas de adsorção de Langmuir e Freundlich, obtidas através da linearização dos dados experimentais utilizando as Equações (2) e (3) para obtenção das constantes e dos 
respectivos coeficientes de determinação $\left(\mathrm{r}^{2}\right)$, são apresentadas na Tabela 2.

Tabela 2. Resumo do ajuste dos parâmetros das isotermas de adsorção para o modelo de Langmuir e de Freundlich e do coeficiente de determinação $\left(\mathrm{r}^{2}\right)$

\begin{tabular}{|c|c|c|c|c|c|c|c|}
\hline \multicolumn{2}{|l|}{ Isoterma } & \multicolumn{3}{|c|}{ Langmuir } & \multicolumn{3}{|c|}{ Freundlich } \\
\hline Material adsorvente & $\begin{array}{c}C_{0} \\
\left(\mu g L^{-1}\right)\end{array}$ & $\underset{\left(\mu g g^{-1}\right)}{q_{\text {máx }}}$ & $\begin{array}{c}b \\
\left(L g^{-1}\right)\end{array}$ & $\mathbf{r}^{2}$ & $\begin{array}{c}k \\
\left(\mu g g^{-1}\right)\end{array}$ & $\mathbf{n}$ & $\mathbf{r}^{2}$ \\
\hline Bagaço de cana-de-açúcar & 236 & 49,26 & 5,49 & 0,94 & 962,72 & 1,66 & 0,94 \\
\hline Carvão ativado & 2,3 & 47,17 & 1,37 & 0,94 & 338,61 & 1,57 & 0,93 \\
\hline Bagaço de cana-de-açúcar & 333 & $-333,33$ & $-0,40$ & 0,95 & 214,44 & 1,06 & 0,84 \\
\hline Carvão ativado & & $-40,82$ & $-0,45$ & 0,93 & 0,83 & 0,65 & 0,93 \\
\hline Bagaço de cana-de-açúcar & 38 & 68,03 & 29,40 & 0,93 & 6047,84 & 2,37 & 0,98 \\
\hline Carvão ativado & J, J & 217,40 & 2,30 & 0,97 & 2154,27 & 1,59 & 0,91 \\
\hline
\end{tabular}

A capacidade de adsorção com melhor ajuste linear para isoterma de Langmuir foi obtida para o carvão ativado utilizando amostra com maior concentração de microcistina-LR com coeficiente de determinação $\left(\mathrm{r}^{2}\right)$ de 0,97 e $\mathrm{q}_{\text {máx }}$ de $217,40 \mu \mathrm{g} \mathrm{g}^{-1}$. Para o bagaço de cana-de-açúcar o melhor ajuste foi observado para a amostra com menor concentração de microcistina-LR com $\mathrm{r}^{2}$ de 0,94 e $\mathrm{q}_{\text {máx }}$ de 49,26 $\mu \mathrm{g} \mathrm{g}^{-1}$ (Tabela 2).

A capacidade máxima de adsorção e a constante de adsorção da isoterma de Langmuir para a amostra com concentração de $3,33 \mu \mathrm{g} \mathrm{L} \mathrm{L}^{-1}$ resultaram em valores negativos para os materiais, indicando a não representatividade deste modelo quanto à capacidade de adsorção para esta concentração.

A capacidade de adsorção com melhor ajuste linear para a isoterma de Freundlich foi obtida para o bagaço de cana-de-açúcar para a amostra com maior concentração de microcistina-LR com $\mathrm{r}^{2}$ de $0,98 \mathrm{e} \mathrm{k}$ de $6047,84 \mu \mathrm{g} \mathrm{g}^{-1}$. Para o carvão ativado o melhor ajuste foi observado para as amostras com concentração de 2,36 e $3,33 \mu \mathrm{g} \mathrm{L}^{-1} \mathrm{com} \mathrm{r}^{2}$ de $0,93, \mathrm{k}$ de $338,61 \mu \mathrm{g} \mathrm{g}^{-1}$ e k de $0,83 \mu \mathrm{g} \mathrm{g}^{-1}$, respectivamente. Porém para esta última amostra o valor de $\mathrm{n}$ resultou inferior a $1(0,65)$, indicando que a adsorção também não foi favorável segundo Bulut e Aydin (2006).

A constante $n$ está relacionada com a força de ligação entre o adsorvente e a microcistina-LR e a constante $\mathrm{k}$ à capacidade do adsorvente em reter a microcistina-LR. $\mathrm{O}$ bagaço de cana-de-açúcar apresentou a maior capacidade adsortiva à microcistina-LR $(\mathrm{k}=6047,84)$ e elevado valor de $\mathrm{n}(2,37)$, correspondendo a maior irreversibilidade do

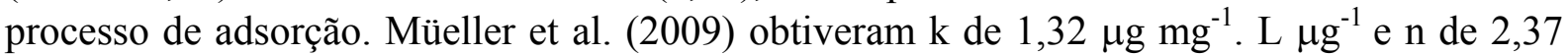
para carvão ativado de madeira na retenção de microcistina aeruginosa.

Para as demais amostras, os valores de $n$ variaram entre 1 e 10 , indicando que a adsorção foi favorável.

\section{CONCLUSÃO}

Com base na obtenção e na análise dos resultados, pode-se chegar as seguintes conclusões:

$\mathrm{O}$ bagaço de cana-de-açúcar apresentou área superficial interna de $1,3 \mathrm{~m}^{2} \mathrm{~g}^{-1}$, enquanto que o carvão ativado granular apresentou área superficial interna de $304,2 \mathrm{~m}^{2} \mathrm{~g}^{-1}$ e número de iodo de $665,86 \mathrm{mg} \mathrm{g}^{-1}$.

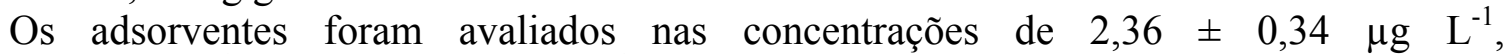
$3,33 \pm 0,25 \mu \mathrm{g} \mathrm{L}^{-1}$ e $3,83 \pm 0,36 \mu \mathrm{g} \mathrm{L}^{-1}$ e obtiveram melhor performance de retenção da Microcistina-LR de $65 \%$ para o carvão ativado granular e de $24 \%$ para o bagaço de cana-de- 
açúcar na concentração de $2,36 \pm 0,34 \mu \mathrm{g} \mathrm{L}{ }^{-1}$.

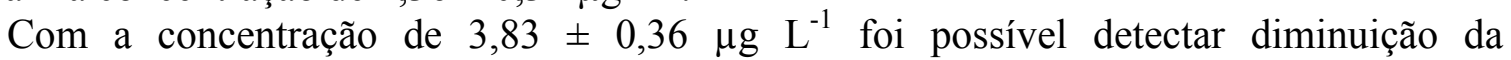
performance de retenção da Microcistina-LR para o carvão ativado granular (11,75\%) e para o bagaço de cana-de-açúcar (12,35\%). Nesta condição, foi possível constatar o potencial adsorvente do bagaço de cana-de-açúcar.

A capacidade de adsorção com melhor ajuste linear para isoterma de Langmuir foi obtida para o carvão ativado utilizando amostra com maior concentração de Microcistina-LR. Para o bagaço de cana-de-açúcar o melhor ajuste foi observado para a amostra com menor concentração de Microcistina-LR.

A capacidade de adsorção com melhor ajuste linear para a isoterma de Freundlich foi obtida para o bagaço de cana-de-açúcar para a amostra com maior concentração de microcistina-LR com r ${ }^{2}$ de 0,98 e $\mathrm{k}$ de $6047,84 \mu \mathrm{g} \mathrm{g}^{-1}$. Para o carvão ativado, o melhor ajuste foi observado para as amostras com menor concentração com $\mathrm{r}^{2}$ de $0,93 \mathrm{e} \mathrm{k} \mathrm{de} 338,61 \mu \mathrm{g} \mathrm{g}^{-1}$.

\section{AGRADECIMENTOS}

Os autores agradecem à equipe do Laboratório de Tecnologias Ambientais e Agronômicas do Instituto de Tecnologia do Paraná pelas análises quantitativas das toxinas da cianobactéria.

\section{REFERÊNCIAS}

ALBUQUERQUE JR., E. C.; MÉNDEZ, M. O. A.; COUTINHO, A. R.; FRANCO, T. T. Removal of cyanobacteria toxins from drinking water by adsorption on activated carbon fibers. Materials Research, v. 11, n. 3, p. 371- 380, 2008. http://dx.doi.org/10.1590/S1516-14392008000300023

ASSOCIAÇÃO BRASILEIRA DE NORMAS TÉCNICAS - ABNT. NBR 11834:1991. Carvão ativado pulverizado para tratamento de água - especificação. Rio de Janeiro, 1991a

ASSOCIAÇÃO BRASILEIRA DE NORMAS TÉCNICAS - ABNT. NBR 12073:1991. Carvão ativado pulverizado- Determinação do número de iodo. Rio de Janeiro, $1991 \mathrm{~b}$.

AMERICAN SOCIETY OF TESTING AND MATERIAL - ASTM. D 3838-1980:1999. Standard Test Method for $\mathrm{pH}$ of Activated Carbon. West Conshohocken, 1999.

AZEVEDO, S. M. F. O.; CARMICHAEL, W. W.; JOCHIMSEN, E. M.; RINEHART, K. L.; LAU, S.; SHAW, G. R. et al. Human in toxication by microcystins during renal dialysis treatment in Caruaru-Brazil. Toxicology, v. 181-182, p. 441-446, 2002. http://dx.doi.org/10.1016/S0300-483X(02)00491-2

BELISÁRIO, M.; GALAZZI, R. M.; BALTHAZAR, D. C.; PEREIRA, M. G.; RIBEIRO, A. V. F. N.; RIBEIRO, J. N. Emprego de resíduo de bagaço de cana de açúcar descartado por usinas de álcool como agente removedor de paracetamol em meio aquoso sob agitação. Revista Analytica, n. 50, p. 54- 62, 2011.

BRASIL. Ministério da Saúde. Secretaria de Vigilância em Saúde. Portaria $n^{0}$ 2914, de 12 de dezembro de 2011. Dispõe sobre os procedimentos de controle e de vigilância da qualidade da água para consumo humano e seu padrão de potabilidade. Diário Oficial [da] União, Brasília, DF, Seção 1, 14 dez. 2011. 
BULUT, Y.; AYDIN, H. A. Kinetics and thermodynamics study of methylene blue adsorption on wheat shells. Desalination, v. 194, p. 259-267, 2006. http://dx.doi.org/10.1016/j.desal.2005.10.032

DI BERNARDO, L.; SABOGAL PAZ, L. P. S. Seleção de tecnologias de tratamento de água. São Carlos: LDIBE, 2008. 878 p.

DAHLMANN, J.; LUCKAS, B. Analysis of selected microcystins in drinking and surface water using a highly sensitive direct injection technique. AB Sciex, n. 1830410-01, 2010 .

DONATI, C.; DRIKAS, M.; HAYES, R.; NEWCOBE, G. Microcystin-LR adsorption by powdered activate carbon. Water Research, v. 28, n.8, p. 1735-1742, 1994. http://dx.doi.org/10.1016/0043-1354(94)90245-3

GONÇALVES, G. C.; MENDES, E. S.; PEREIRA, N. C.; SOUSA, J. C. Produção de carvão ativado a partir de bagaço e melaço de cana-de-açúcar. Acta Scientiarum Technology, v. 28, n. 1, p. 21-27, 2006. http://dx.doi.org/10.4025/actascitechnol.v28i1.1203

HUANG, W. J.; CHENG, B. L.; CHENG, Y. L. Adsorption of microcystin-LR by three types of activated carbon. Journal of Hazardous Materials,v. 141, p. 115-122, 2007.

JACINAVICIUS, F. R.; GAMA JUNIOR, W. A.; AZEVEDO, M. T. P; SANT`ANNA, C. L. Manual para cultivo de cianobactérias. São Paulo: Secretaria do Meio Ambiente, 2013.

JAGUARIBE, E. F.; MEDEIROS, L. L.; BARRETO, M. C. S.; ARAUJO, L. P. The performance of activated carbons from sugarcane bagasse, babassu, and coconut shells in removing residual chlorine. Brazilian Journal of Chemical Engineering, v. 22, n. 01, p. 41-47, 2005. http://dx.doi.org/10.1590/S0104-66322005000100005

MEZA, J. C.; SIGOllot, J. C.; LOMASCOlO, A.; NAVARRO, D.; AURIA, R. New Process for Fungal Delignification of Sugar-Cane Bagasse and Simultaneous Production of Laccase in a Vapor Phase Bioreactor. Journal of Agricultural. Food Chemistry, v. 54, p. 3852-3858, 2006. http://dx.doi.org/10.1021/jf053057j

MOLICA, R. J. R.; DUARTE, M. M. B.; AVELAR, F. P.; LIMA FILHO, N. M.; NEVES, C. C. L.; BARAÚNA, O. S. et al. Adsorção de cianotoxinas em diferentes matrizes. In: FUNDAÇÃO NACIONAL DE SAÚDE. $5^{\circ}$ Caderno de Pesquisa em Engenharia de Saúde Pública. 2. ed. Brasília, 2013. p. 63-95.

MÜLLER, C. C.; RAYA-RODRIGUEZ, M. T.; CYBIS, L. F. Adsorção em carvão ativado em pó para remoção de microcistina de água de abastecimento público. Engenharia Sanitária e Ambiental, v. 14, n. 1, p. 29-38, 2009. http://dx.doi.org/10.1590/S141341522009000100004

PYO, D.; MOON, D. Adsorption of Microcystin LR by activated carbon fibers. Bulletin of the Korean Chemical Society, v. 26, n. 12, p. 2089-2092, 2005.

SATHISHKUMAR, M.; PAVAGADHI, S.; VIJAYARAGHAVAN, K.; BALASUBRAMANIAN, R.; ONG, S.L. Experimental studies on removal of microcystin-LR by peat. Journal of Hazardous Materials, v. 184, p. 417-424, 2010. http://dx.doi.org/10.1016/j.jhazmat.2010.08.051

TORTORA, G. J.; FUNKE, B. R.; CASE C. L. Microbiologia. 6. ed. Porto Alegre: Artmed, 2000 . 


Ambiente \& Água - An Interdisciplinary Journal of Applied Science
ISSN 1980-993X - doi:10.4136/1980-993X
www.ambi-agua.net
E-mail: ambi.agua@gmail.com

\title{
Estudo da qualidade da água por meio de bioindicadores bentônicos em córregos da área rural e urbana
}

\author{
doi:10.4136/ambi-agua.1672
}

Received: 26 May 2015; Accepted: 19 Nov. 2015

\author{
Sandro Morais Pimenta ${ }^{1 *}$; Geraldo Resende Boaventura ${ }^{2}$; \\ Alfredo Palau Peña ${ }^{3}$; Tiago Godoi Ribeiro ${ }^{1}$ \\ ${ }^{1}$ Instituto Federal de Goiás (IFG), Goiânia, GO, Brasil \\ ${ }^{2}$ Universidade de Brasília (UnB), Brasília, DF, Brasil \\ Departamento de Geociências \\ ${ }^{3}$ Pontifícia Universidade Católica de Goiás (PUC), Goiânia, GO, Brasil \\ Departamento de Arqueologia \\ *Autor correspondente: e-mail: moraisecologia@gmail.com, \\ grbunb@unb.br, alfredo.palau@gmail.com, godoiribeiro@gmail.com
}

\section{RESUMO}

Na avaliação da qualidade da água, além das análises físico-químicas e geoquímicas, outros métodos podem ser utilizados para realizar estudos ou monitoramentos. O objetivo deste estudo foi utilizar espécies de bioindicadores bentônicos intolerantes, tolerantes e resistentes às alterações na qualidade da água de dois córregos, considerando a granulometria dos sedimentos e sólidos voláteis. Foram amostrados oito pontos no córrego Bandeirinha e quatro pontos no córrego Josefa Gomes nos meses de janeiro e setembro de 2013. Os córregos estão localizados no município de Formosa-GO. A escolha dos dois córregos deu-se por estarem inseridos em ambientes distintos. Foram utilizados os índices de diversidade Shannon (H), Equitabilidade (E) e Similaridade de Bray-Curtis e o método de agrupamento UPGMA (Unweighted Pair Group Method with Arithmetic Mean). Foram identificadas no córrego Bandeirinha espécies bioindicadoras intolerantes às alterações na qualidade da água. Os reduzidos valores aferidos de sólidos voláteis, próprios de locais com pouca alteração antrópica, e as maiores dimensões granulométricas também corroboram uma paridade nos resultados. O córrego Josefa Gomes, completamente inserido em ambiente urbano apresentou alterações com espécies tolerantes e resistentes. Os resultados demostraram a distinção da qualidade da água em ambiente rural e urbano e a relação dos bioindicadores com os sedimentos. O estudo auxilia na interpretação das alterações na qualidade nas duas drenagens na região. A análise da qualidade da água por meio dos bioindicadores bentônicos pode ser associada às análises físico-químicas e geoquímicas da água e dos sedimentos para uma melhor interpretação dos resultados.

Palavras-chave: poluição, espécies resistentes, Formosa, Goiás, sedimento.

\section{Study of water quality using benthic biological indicators in rural and urban streams}

\section{ABSTRACT}

Methods other than physical-chemical and geochemical analyses can be used to assess 
water quality in surface drainage. This study used intolerant, tolerant and resistant benthic species as bioindicators of changes in water quality of two streams, taking in account particle size of sediments and volatile solids. Eight points were sampled in the stream Bandeirinha and four points in the stream Josefa Gomes in January and September 2013 in the municipality of Formosa-GO. The two streams were chosen in order to study two different environments. The study used the diversity indexes of Shannon $(\mathrm{H})$, Equitability $(\mathrm{E})$ and Bray-Curtis Similarity and the grouping analysis method UPGMA (Unweighted Pair Group Method with Arithmetic Mean). The results of particle size and volatile solids study of Bandeirinha stream located in rural areas showed bioindicator species intolerant of changes in water quality. The stream Josefa Gomes, completely inserted in an urban environment, showed alteration identified by tolerant and resistant species. The results demonstrate differences in water quality in the rural and urban environments and the relationship of bioindicators with sediments. The study aids the interpretation of changes in the water quality of the two drainages areas. Analysis of water quality based upon benthic biological indicators associated with physical-chemical and geochemical analyses of water and sediments provide a better interpretation of the results.

Keywords: pollution, Formosa, Goias, resistant species, sediment.

\section{INTRODUÇÃO}

A avaliação da qualidade da água, nas drenagens superficiais, pelos parâmetros físicoquímicos e geoquímicos pode ser associada à utilização de bioindicadores, que é a observação e acompanhamento da espécie ou grupo indicador durante um intervalo de tempo, visando obter informações sobre a condição do ambiente e mudanças nas comunidades biológicas (Mugnai et al., 2010).

A bioindicação usa alguns organismos que compõem um determinado ambiente para caracterizá-lo, a partir do conhecimento do comportamento das comunidades ecológicas quando submetidas a fatores estressantes, como a supressão da vegetação, na perda de habitats, reservatórios na mudança da paisagem, poluição e outras. As comunidades biológicas com a presença de poluentes na água sofrem alterações em densidade e riqueza, com isso são capazes de indicar o efeito, das ações antrópicas ou naturais no ambiente, o que permite uma avaliação biológica eficiente na identificação dos fatores atuantes (Chalar, 1994).

Para Callisto e Moreno (2006), espécie indicadora é aquela que possui pequena tolerância a variações ambientais e, quando presentes em determinada área, revela um conjunto de condições particulares daquele ambiente. A essência da bioindicação são as relações entre os seres vivos e os fatores ambientais. Assim, rápidas mudanças do ambiente provocadas pelo homem causam flutuações populacionais nos organismos. Cada espécie possui um padrão de variação característico: algumas são mais tolerantes, outras menos.

De acordo com Ribeiro e Uieda, (2005), ainda que as análises físico-químicas demonstrem o estado da água, o ideal é a associação desses métodos com métodos biológicos, permitindo uma caracterização mais completa, abrangendo os aspectos biológicos e a interação entre o meio físico, muitas vezes necessária para o manejo adequado dos recursos hídricos. Os indicadores biológicos têm sido considerados eficientes para medir a qualidade da água com baixos custos.

A Agência de Proteção Ambiental dos Estados Unidos (Environmental Protection Agency - EPA) recomenda a utilização de critérios biológicos que utilizam a condição de um organismo ou conjunto de organismos para descrever a integridade ecológica de uma área impactada, pouco impactada, ou áreas de referência, para complementar as informações sobre qualidade de água, tradicionalmente baseados em parâmetros químicos e físicos (USEPA, 2013) 
Callisto et al. (2004) apontam que a capacidade de sensibilidade às alterações do meio ambiente é o que determina a escolha dos bioindicadores, sendo os mais utilizados aqueles capazes de diferenciar entre oscilações naturais e alterações antrópicas, podendo indicar a presença e a dimensão de um determinado impacto ou anomalia no ambiente.

Milesi et al. (2008) afirmam que os macroinvertebrados bentônicos constituem um dos melhores bioindicadores de qualidade das águas nos ambientes lóticos, pois possuem características sésseis, ciclo de vida relativamente longo e são de fácil visualização.

Os macroinvertebrados referem-se à fauna de invertebrados que fica retida em uma malha de 0,2 mm, sendo ela constituída por diversos táxons, tais como Arthropoda, Mollusca, Annelida e Platyhelminthes, dentre outros. A maioria dessas espécies está associada aos habitats de fundo das drenagens superficiais e lagos, fixos ou não a um substrato, sendo denominada de comunidade bentônica (Mäenpää et al., 2003).

Callisto et al. (2004) destacam que as amostragens de bentos são relativamente fáceis e baratas. Os bentos são variavelmente sensíveis às condições ambientais, são sedentários, podem integrar e acumular condições em um ambiente, oferecendo um adequado nível de diagnóstico, considerando a natureza dos efeitos observados.

A utilização de bioindicadores para o monitoramento ambiental, com ênfase em insetos aquáticos é empregada em outros países, com métodos bem definidos e comunidades bioindicadoras conhecidas. Os macroinvertebrados bentônicos têm sido amplamente utilizados como bioindicadores de qualidade de água, por apresentarem as seguintes características: Ciclos de vida longo, podem viver entre semanas, meses e mesmo mais de 1 ano; em geral são organismos grandes (maiores que 125 ou $250 \mu \mathrm{m}$ ), sésseis ou de pouca mobilidade, portando mais fáceis de serem amostrados, com custos relativamente baixos; grande diversidade taxonômica e de identificação relativamente fácil (ao nível de família e alguns gêneros); e são organismos sensíveis a diferentes concentrações de poluentes no meio (Callisto e Moreno, 2006).

A utilização dos bioindicadores pode também ser mais direcionada a um tipo de poluição. Belmejo e Martos (2008) utilizaram bioindicadores sensíveis à poluição hídrica de derivados de petróleo em condições tropicais, impacto diretamente relacionado a drenagens localizadas em ambientes urbanos.

Neste entendimento, Milesi et al. (2008) realizaram o estudo dos efeitos dos metais Cobre e Zinco em comunidades de macroinvertebrados bentônicos, por estes íons metálicos estarem presente em fertilizantes e pesticidas além de resíduos orgânicos provenientes de áreas urbanas; portanto, sua correlação com os bioindicadores, pode ser utilizada na identificação de impactos e análise da qualidade da água em drenagens urbanas ou rurais.

Colpo et al. (2009) utilizando os macroinvertebrados bentônicos como indicadores do impacto ambiental promovido pelos efluentes de áreas orizícolas e os de origem urbana/industrial, concluíram que os efluentes produzidos pelas cidades e indústrias, são mais agressivos aos ambientes aquáticos que os efluentes drenados de uma lavoura manejada de acordo com as recomendações técnicas para a cultura.

Estudos mais recentes, como os de Yokoyama et al. (2012), destacam a importância dos bioindicadores na avaliação da qualidade da água e seu comportamento durante o ciclo hidrológico anual, com a flutuação da composição em resposta à variação da precipitação entre os períodos seco e chuvoso. Este efeito deve ser considerado ao realizar os estudos e monitoramento da qualidade da água em uma drenagem superficial.

O objetivo deste trabalho foi realizar o estudo da qualidade da água por meio de bioindicadores da comunidade bentônica, análise granulométrica dos sedimentos de corrente e sólidos voláteis, comparando os resultados do córrego Bandeirinha situado em ambiente predominantemente rural e o córrego Josefa Gomes, inserido no núcleo urbano do município de Formosa-GO. A aplicação da avaliação qualidade da água por bioindicadores, nas duas 
drenagens auxilia na determinação das possíveis contaminações provenientes das atividades antrópicas no ambiente rural e urbano do município de Formosa, bem como as consequências a médio e longo prazo, além de contribuir com a geração de dados e caracterização regional.

\section{MATERIAL E MÉTODOS}

\section{1. Área de Estudo}

Como área representativa para o desenvolvimento da pesquisa, optou-se pelos córregos Bandeirinha e Josefa Gomes localizados no município de Formosa-GO, e estes situam-se na microrregião do entorno de Brasília. O córrego Bandeirinha está inserido em ambiente rural e o córrego Josefa Gomes em ambiente urbano. A escolha das duas drenagens para o desenvolvimento do estudo, deu-se por possuírem características distintas; porém, localizadas na mesma região, o que permite a comparação da aplicação dos métodos de análise em ambos ambientes e a avaliação do comportamento destes, sujeitos a impactos ambientais diversos, do meio rural e do meio urbano. O município de Formosa-GO situa-se a $280 \mathrm{~km}$ da capital do estado, Goiânia e a 70 km de Brasília, possui uma população total de 100.085 habitantes, sendo distribuída em 92.023 habitantes em zona urbana e 8.062 em zona rural (IBGE, 2012). A agropecuária é atividade estruturante na economia de Formosa, principalmente a pecuária de corte e leite.

A região onde está inserido o município de Formosa possui clima Aw, segundo a classificação de Köppen. A distribuição pluviométrica é padrão típico do domínio morfoclimático dos cerrados, com precipitação média anual na ordem de $1.485 \mathrm{~mm}$, concentrando-se principalmente nos meses de outubro a abril. Nos meses de junho e julho as precipitações são praticamente ausentes. A temperatura média anual é em torno de $21,5^{\circ} \mathrm{C}$.

O córrego Bandeirinha tem suas nascentes localizadas na Fazenda Bandeirinha dentro do município de Formosa sob as coordenadas UTM (SAD 69) Zona 23L 235.931.00 m E; 8.285.262.00 m S, em altitudes médias de 1130 metros, com um percurso de 41,9 km em direção Norte, desaguando no Rio Paranã, em altitude aproximada de 600 metros, próximo à Serra Laranjeira. A drenagem principal da bacia dista do seu meandro mais próximo cerca de 2 km do núcleo urbano de Formosa, porém recebe contribuições antrópicas por alguns dos seus tributários, localizados na área urbana. Atualmente o córrego Bandeirinha é o principal manancial aproveitado pelo sistema de abastecimento de água no município.

A bacia hidrográfica que forma a cabeceira do Rio Preto está inserida dentro da área urbana de Formosa. Na área urbana este córrego tem o nome de Josefa Gomes, e tem percurso de 7,5 km em direção sudoeste. Sua nascente localiza-se no parque municipal Mata da Bica, sob as coordenadas UTM (SAD 69) Zona 23L 249.481 m E; 8.278.785 m S, com elevação de 945 metros desaguando na Lagoa Feia, em elevação de 873 metros. A área da nascente no Parque Municipal Ecológico Mata da Bica possui várias surgências de água, que se unem ao longo da área do parque formando a Lagoa da Mata da Bica e esta por consequência, alimenta a vazão do Córrego Josefa Gomes. Parte do percurso do córrego Josefa Gomes é retificado, principiando na lagoa da Mata da Bica, e se estendendo aproximadamente 1,5 km de percurso, e possuindo 2,5 metros de largura. Após a retificação o córrego Josefa Gomes percorre aproximadamente $6 \mathrm{~km}$ e deságua na Lagoa Feia. Em seguida a Lagoa Feia, o córrego é denominado Rio Preto e possui uma área de drenagem de aproximadamente $10.500 \mathrm{~km}^{2}$ abrangendo os Estados de Goiás, Distrito Federal e Minas Gerais. O Rio Preto é um dos principais tributários do rio Paracatu, no Estado de Minas Gerais, e é afluente do Rio São Francisco.

Conforme a carta geológica do Serviço Geológico Nacional, a região do córrego Bandeirinha no alto da bacia está situado em rochas do grupo Paranoá, e nas regiões média e baixa da bacia predomina as rochas do grupo Bambuí. O córrego Josefa Gomes está 
completamente inserido em rochas do grupo Paranoá da sua nascente ao seu deságue (Souza, 2004).

Faria (1995) descreveu que as aluviões da região são caracterizadas por sedimentos inconsolidados, dominantemente arenosos, representados por areias, com níveis de cascalhos, e lentes de material silto-argiloso.

\subsection{Amostragem e análises}

Para a realização do estudo foram determinados 8 pontos espacializados no córrego Bandeirinha, e 4 pontos no córrego Josefa Gomes (Figura 1). A seleção dos pontos de amostragem deu-se considerando as atividades antrópicas ao longo da drenagem e a incidência de tributários. As coletas ocorreram nos meses de janeiro e setembro de 2013 de acordo com o ciclo hidrológico da região. Os 8 pontos de amostragem no córrego Bandeirinha e os 4 pontos de amostragem no córrego Josefa Gomes seguem da nascente até o deságue das drenagens.

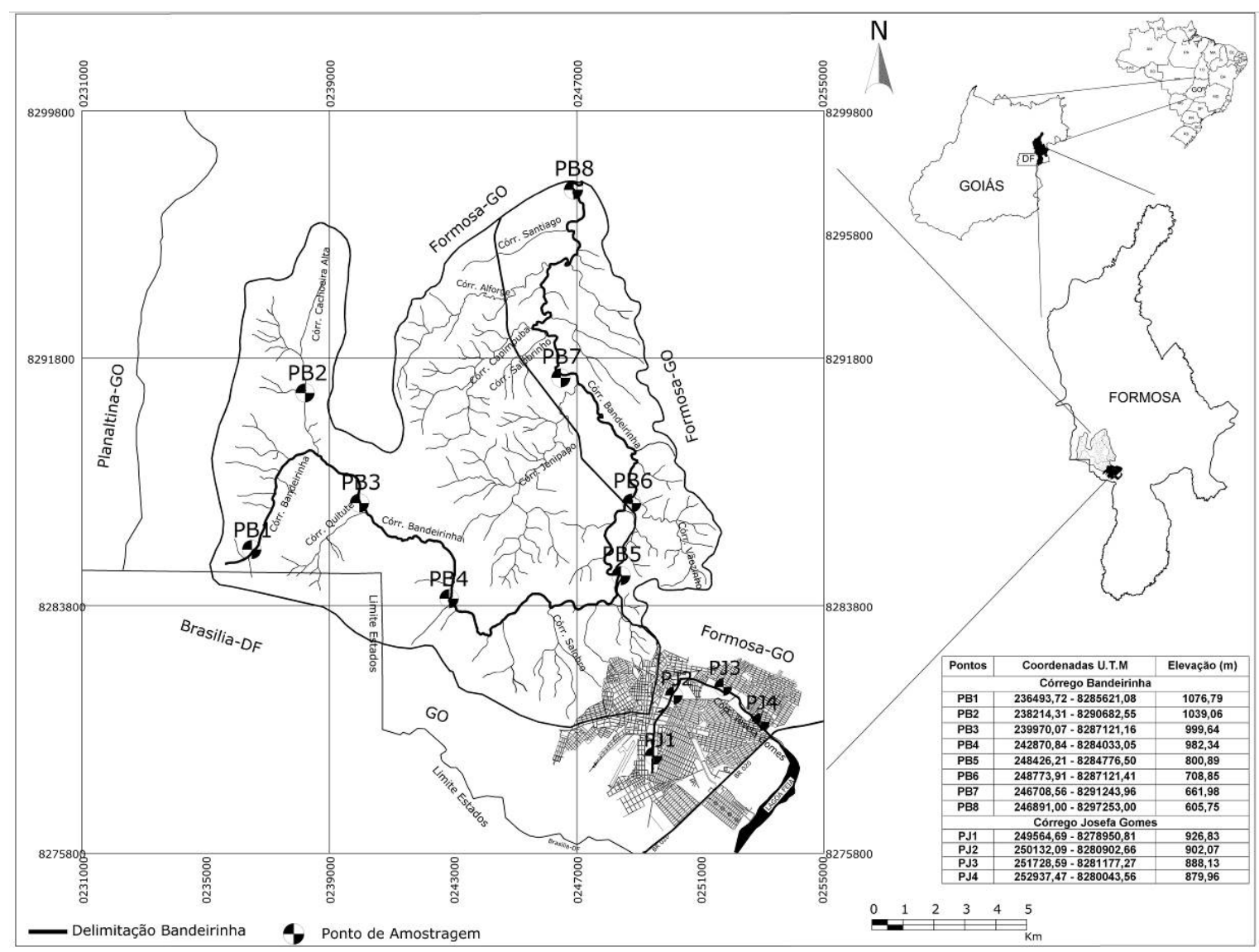

Figura 1. Localização da área, pontos de amostragem nos córregos Bandeirinha e Josefa Gomes, Formosa-GO.

Fonte: IBGE (2010).

As análises foram realizadas no Laboratório de Geoquímica da Universidade de Brasília - LabGeq e nos Laboratórios do Instituto Federal de Goiás, logo após a recepção das amostras. Nos pontos de amostragem foram coletadas amostras de sedimentos de corrente, uma vez que as dimensões das partículas que compõe as amostras de sedimentos são importantes para a caracterização dos habitats dos macroinvertebrados bentônicos. As amostras de sedimentos foram dispostas em bandejas previamente lavadas com $\mathrm{HNO}_{3} 20 \%$ $\mathrm{v} / \mathrm{v}$, cobertas com papel e colocadas para secar à temperatura ambiente. Após secagem, as 
amostras foram desagregadas e passaram por uma série de peneiras, a fim de identificar sua granulometria, retirando-se restos de folhas, plantas, pedregulhos e objetos maiores. Parte do material passante foi reservada para ser utilizado na determinação dos sólidos voláteis.

O procedimento para a avaliação da composição granulométrica seguiu a metodologia do Laboratório de Geotecnia do Instituto Federal de Goiás. Para a caracterização da granulometria das amostras, utilizou-se o jogo de peneiras com malhas nas seguintes ordens: $10 \mathrm{~mm}-5 \mathrm{~mm}-2,5 \mathrm{~mm}-0,8 \mathrm{~mm}-0,5 \mathrm{~mm}-0,125 \mathrm{~mm}-0,1 \mathrm{~mm}$. As peneiras foram previamente limpas e encaixadas de modo a formar um único conjunto com abertura de malha em ordem crescente da base para o topo, e fundo fechado. Após este procedimento promoveuse a agitação mecânica do conjunto, para permitir a separação e classificação prévia dos diferentes tamanhos de grão da amostra. A composição granulométrica no material retido é calculada em função do peso da amostra e apresentada em percentagem sobre o total da amostra.

A determinação dos sólidos voláteis nos sedimentos seguiu os procedimentos estabelecidos pela Standard Methods for the Examination of Water and Wastewater (APHA et al., 1999). Esta determinação foi realizada por gravimetria no laboratório de Geoquímica da UnB. Massas de $2 \mathrm{~g}$ das amostras foram pesadas em balança METLER modelo AG. 240 com variação de $\pm 0,00001 \mathrm{~g}$ e colocadas para secar em estufas a $105^{\circ} \mathrm{C}$ por 24 horas para determinar seu peso seco. Em seguida, as amostras foram levadas ao forno mufla, a temperatura de $450^{\circ} \mathrm{C}$, durante 24 horas, para que toda matéria orgânica e parte dos ânions volatilizassem. Posteriormente, foram novamente pesadas. O resultado é dado pela diferença entre o peso inicial subtraído do peso final.

Para análise dos bioindicadores foram utilizados os invertebrados bentônicos, coletados nos pontos onde se realizaram as amostragens de sedimentos de corrente. Foram utilizados os invertebrados bentônicos, coletados com um amostrador tipo Surber com armação dobrável em aço, tela de nylon 250 micras de abertura na malha, dimensões de abertura de 30 x $30 \mathrm{~cm}$ e comprimento de $60 \mathrm{~cm}$. Foram realizadas 5 réplicas de coleta por ponto em período sazonal. Após coletadas as amostras de bentos foram acondicionados individualmente em frascos plásticos e fixados com álcool $80 \%$, para identificação e contagem dos táxons encontrados, seguindo os trabalhos de Callisto et al. (2004), Borisko et al. (2007), Pimenta et al. (2009) e Mugnai et al. (2010).

A identificação dos táxons foi realizada por meio de Lupa binocular estereoscópica e os indivíduos foram identificados em ordem, família e gênero. Os bioindicadores foram divididos segundo Callisto et al. (2004) e Pimenta et al. (2009) em três grupos quanto a sua tolerância: organismos sensíveis ou intolerantes (representantes de Trichoptera, Plecoptera, Ephemeroptera), organismos tolerantes (representantes de Coleóptera, Heteroptera, Odonata) e organismos resistentes (Díptera, Oligochaeta).

Para o tratamento dos dados obtidos pelos bioindicadores, realizam-se cálculos dos índices ecológicos da diversidade Shannon $(\mathrm{H})$, Equitabilidade (E) conforme Magurran, (1988) e Similaridade de Bray-Curtis com o agrupamento UPGMA (Unweighted Pair Group Method with Arithmetic Mean), nestes cálculos foi utilizado o programa BioDiversity Professional Version 2.

\section{RESULTADOS E DISCUSSÃO}

Os resultados da composição granulométrica foram classificados de acordo com os tamanhos dos grãos, utilizando a escala de Wentworth, conforme os procedimentos adotados pelo Laboratório de Geotecnia do Instituto Federal de Goiás, assim classificados: Cascalho (8 a $2 \mathrm{~mm})$; Areia grossa (1 a $0,5 \mathrm{~mm})$; Areia média $(0,5$ a $0,25 \mathrm{~mm})$; Areia fina $(0,25 \mathrm{~mm}$ a $0,125 \mathrm{~mm}$ ) (Figura 2). 


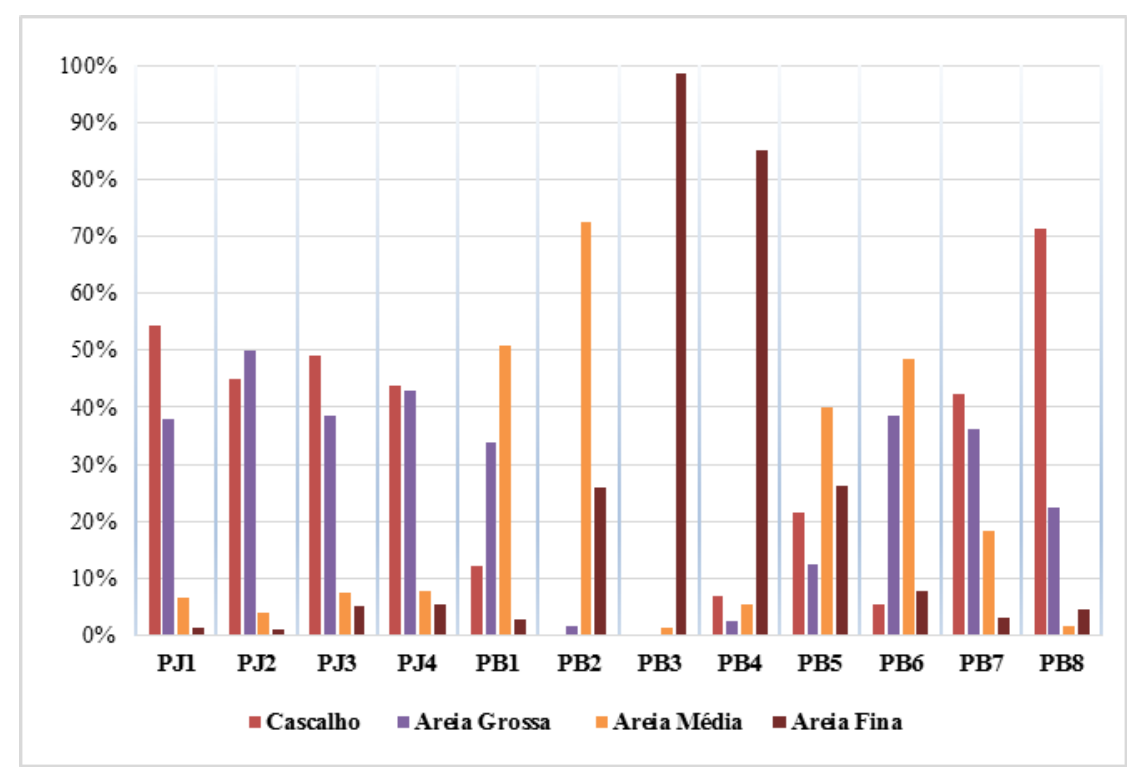

Figura 2. Percentagem de ocorrência das classes granulométricas dos sedimentos nos córregos Josefa Gomes e Bandeirinha nos pontos de amostragem.

Nos sedimentos amostrados, no córrego Bandeirinha destaca-se o ponto PB3, com o maior percentual composto por areia fina. A maioria dos pontos amostrados apresentou sedimento com distribuição entre Areia Fina - Areia Média - Areia Grossa, componentes naturais presentes no leito desta drenagem. Os sedimentos amostrados nos pontos do córrego Josefa Gomes demonstraram uma maior concentração de Areia Grossa a Cascalho. Partes deste material presente no leito desta drenagem provêm de contribuições antrópicas de construções, próximas ao córrego, lançadas pelas ligações do sistema de drenagem pluvial. A granulometria está em conformidade com as características geológicas da região, representadas pelo Grupo Paranoá e Grupo Bambuí, com aluviões recentes nas margens dos cursos de água, correspondendo a um depósito de sedimentos clásticos (areia e ou cascalho) formado pelo sistema fluvial no leito e nas margens dos córregos Bandeirinha e Josefa Gomes.

Os resultados da análise de sólidos voláteis representam parte da matéria orgânica e argilo minerais presentes nas amostras que se volatilizaram, pelo procedimento. Estes resultados são apresentados em percentagem pela diferença entre o peso inicial subtraído do peso final dado por: $\%=($ Pinicial - Pfinal $) * 100$. As análises de sólidos voláteis nos pontos do córrego Bandeirinha indicam tendência nos valores percentuais mantendo-se abaixo dos 10\% com média de 4,5\%, tendo seus maiores valores no PB2 (7\%), ponto localizado a cerca de 700 m de uma produção de hortaliça em área rural, e ponto PB5 (8\%), localizado a jusante do núcleo urbano do município, próximos a rodovia GO 116.

Para o córrego Josefa Gomes os percentuais encontrados são maiores que no córrego Bandeirinha. Da nascente no Parque Mata da Bica até próximo ao deságue na Lagoa Feia apresentou média de 16\%, tendo a maior aferição no PJ4 (21\%) ponto cerca de 850 metros de distância do deságue. Os maiores percentuais encontrados no córrego Josefa Gomes podem ser justificados por estar inserido em ambiente urbano e com diversas alterações antrópicas no seu canal e em suas margens.

$\mathrm{Na}$ amostragem dos bioindicadores realizada durante o ciclo hidrológico da região em ambas as drenagens foram identificados 64 táxons com um total de 1236 indivíduos, sendo 816 e 420 indivíduos respectivamente para o córrego Bandeirinha e córrego Josefa Gomes. Na estação seca foram amostrados 58 táxons e três vezes mais indivíduos $(\mathrm{N}=966)$ que a 
estação chuvosa $(\mathrm{N}=270)$ com 50 táxons.

Na Tabela 1 estão representados os índices de diversidade $(\mathrm{H})$ que variaram entre 1,372 (PJ2) a 3,15 (PB3) e equitabilidade (E) entre 0,5723 (PJ2) a 0,909 (PB3), com a evidência de maiores índices para os pontos mais a montante do córrego Bandeirinha, e mais a jusante no córrego Josefa Gomes.

Tabela 1. Índices de diversidade para os 12 pontos amostrados entre as drenagens dos córregos Bandeirinha (PB) e Josefa Gomes (PJ), Formosa-GO.

\begin{tabular}{ccccc}
\hline Pontos & Espécies & Espécimes & Shannon $(\mathrm{H})$ & Equitabilidade $(\mathrm{E})$ \\
\hline PJ1 & 15 & 125 & 1,904 & 0,7031 \\
PJ2 & 11 & 165 & 1,372 & 0,5723 \\
PJ3 & 17 & 90 & 2,202 & 0,7773 \\
PJ4 & 16 & 40 & 2,484 & 0,8958 \\
PB1 & 29 & 122 & 2,769 & 0,8223 \\
PB2 & 39 & 113 & 3,121 & 0,8520 \\
PB3 & 32 & 96 & 3,150 & 0,9090 \\
PB4 & 23 & 124 & 2,679 & 0,8545 \\
PB5 & 18 & 52 & 2,541 & 0,8792 \\
PB6 & 23 & 85 & 2,699 & 0,8608 \\
PB7 & 20 & 83 & 2,429 & 0,8110 \\
PB8 & 21 & 141 & 2,387 & 0,7841 \\
\hline
\end{tabular}

$\mathrm{Na}$ análise da similaridade entre os pontos amostrados no córrego Bandeirinha variou entre 24,32\% a 50,19\%, já para os pontos do córrego Josefa Gomes a variação foi maior entre $18,53 \%$ a $65,51 \%$ da amostragem (Figura 3) apresenta uma dissimilaridade entre as duas drenagens.

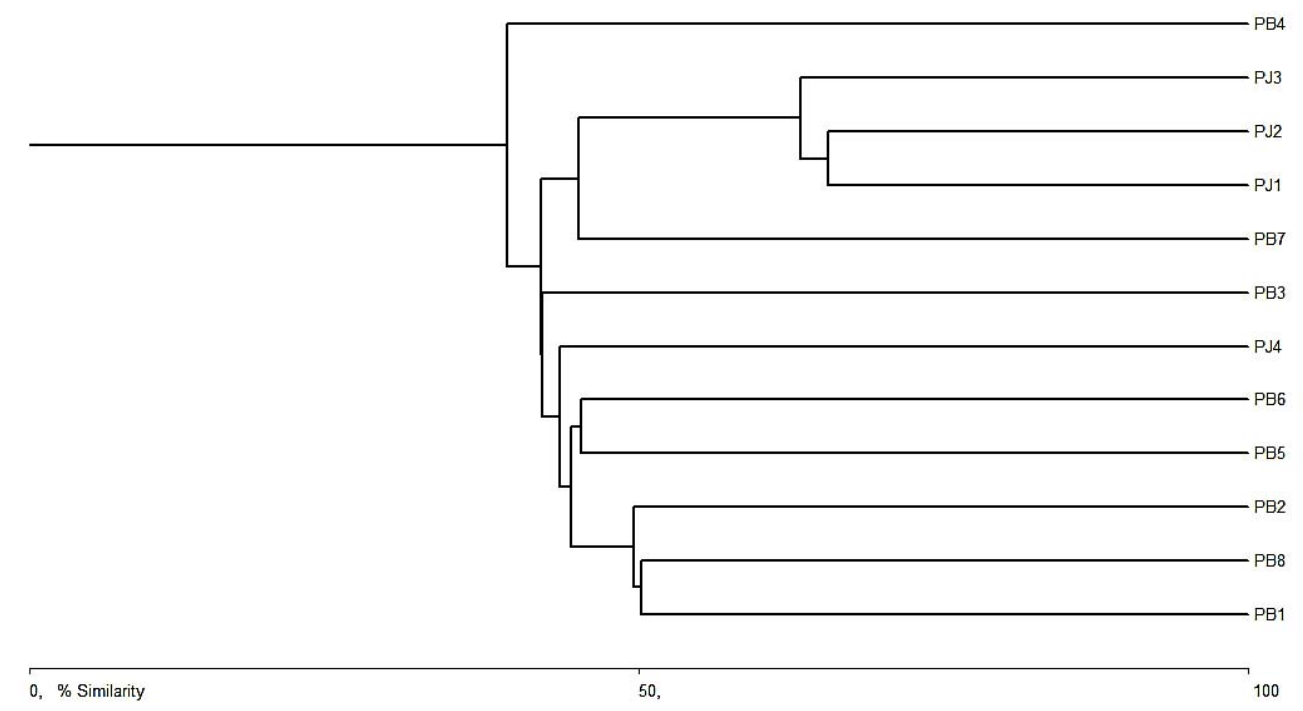

Figura 3. Análise de agrupamento (UPGMA) realizada a partir da matriz dos coeficientes de similaridade de Bray-Curtis, para os 12 pontos amostrados. Pontos córrego Bandeirinha (PB); Pontos córrego Josefa Gomes (PJ). 
O efeito sazonal marcado pelas amostragens dos períodos seco e chuvoso evidenciou um padrão espaço-temporal. Hauer e Resh (1996) afirmam que períodos secos e chuvosos também influenciam na distribuição desses organismos em seu habitat, pois alteram as características do substrato, disponibilidade de alimento e refúgio. O padrão espacial encontrado provavelmente pode estar relacionado ao tipo de substrato, que é fundamental para ocupação desses microhabitas tanto na fixação como na obtenção de alimento e refúgio pelos organismos (Hauer e Resh, 1996; Carvalho e Uieda, 2004).

Os macroinvertebrados bentônicos depois de identificados foram classificados, quanto a sua função de bioindicadores, sensíveis às mudanças que se sucedem na estrutura das comunidades bentônicas que variam de organismos próprios de águas limpas, intolerantes à poluição, a simples e de baixa diversidade, com organismos capazes de viver em águas poluídas. Os bioindicadores foram divididos em três grupos principais, existindo exceções dentro de cada grupo: organismos sensíveis ou intolerantes, organismos tolerantes e organismos resistentes (Tabela 2).

Tabela 2. Densidades dos táxons amostrados nos córregos Bandeirinha (PB) e Josefa Gomes (PJ), classificados por seu grau de tolerância, segundo Callisto et al. (2004). C = período chuvoso; $\mathrm{S}=$ período seco.

\begin{tabular}{|c|c|c|c|c|c|c|c|c|c|c|c|c|c|c|c|c|c|c|c|c|c|c|c|c|c|}
\hline \multirow{3}{*}{\multicolumn{2}{|c|}{$\begin{array}{l}\text { Bioindicadores } \\
\text { Ciclo Hidrológico }\end{array}$}} & \multicolumn{16}{|c|}{ Córrego Bandeirinha } & \multicolumn{8}{|c|}{ Córrego Josefa Gomes } \\
\hline & & \multicolumn{2}{|c|}{ PB1 } & \multicolumn{2}{|c|}{ PB2 } & \multicolumn{2}{|c|}{ PB3 } & \multicolumn{2}{|c|}{ PB4 } & \multicolumn{2}{|c|}{ PB5 } & \multicolumn{2}{|c|}{ PB6 } & \multicolumn{2}{|c|}{ PB7 } & \multicolumn{2}{|c|}{ PB8 } & \multicolumn{2}{|c|}{ PJ1 } & \multicolumn{2}{|c|}{ PJ2 } & \multicolumn{2}{|c|}{ PJ3 } & \multicolumn{2}{|c|}{ PJ4 } \\
\hline & & $\mathrm{C}$ & $\mathrm{S}$ & $\mathrm{C}$ & $\mathrm{S}$ & $\mathrm{C}$ & $\mathrm{S}$ & $\mathrm{C}$ & $\mathrm{S}$ & $\mathrm{C}$ & S & $\mathrm{C}$ & S & $\mathrm{C}$ & S & $\mathrm{C}$ & S & $\mathrm{C}$ & S & $\mathrm{C}$ & $\mathrm{S}$ & $\mathrm{C}$ & S & $\mathrm{C}$ & S \\
\hline \multirow{3}{*}{ 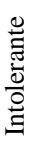 } & Trichoptera & 6 & 21 & 11 & 15 & 0 & 14 & 2 & 17 & 7 & 12 & 4 & 14 & 2 & 8 & 17 & 47 & 1 & 8 & 0 & 0 & 0 & 11 & 0 & 0 \\
\hline & Plecoptera & 2 & 5 & 0 & 0 & 2 & 1 & 0 & 0 & 0 & 0 & 1 & 4 & 0 & 0 & 0 & 0 & 0 & 0 & 0 & 0 & 0 & 0 & 0 & 0 \\
\hline & Ephemeroptera & 2 & 10 & 11 & 21 & 13 & 27 & 1 & 55 & 0 & 3 & 11 & 9 & 5 & 27 & 5 & 13 & 0 & 1 & 0 & 0 & 0 & 1 & 0 & 0 \\
\hline \multicolumn{2}{|c|}{ Somatória } & 10 & 36 & 22 & 36 & 15 & 42 & 3 & 72 & 7 & 15 & 16 & 27 & 7 & 35 & 22 & 60 & 1 & 9 & 0 & 0 & 0 & 12 & 0 & 0 \\
\hline \multirow{3}{*}{ 䒿 } & Coléoptera & 2 & 12 & 1 & 9 & 3 & 0 & 1 & 10 & 2 & 7 & 9 & 15 & 2 & 0 & 5 & 7 & 0 & 3 & 0 & 0 & 0 & 0 & 0 & 8 \\
\hline & Heteroptera & 0 & 0 & 1 & 3 & 1 & 0 & 0 & 2 & 2 & 3 & 0 & 0 & 0 & 0 & 0 & 0 & 0 & 0 & 1 & 0 & 0 & 1 & 0 & 3 \\
\hline & Odonata & 1 & 5 & 1 & 3 & 1 & 4 & 1 & 4 & 0 & 0 & 0 & 0 & 0 & 1 & 0 & 0 & 1 & 0 & 0 & 3 & 0 & 7 & 0 & 3 \\
\hline \multicolumn{2}{|c|}{ Somatória } & 3 & 17 & 3 & 15 & 5 & 4 & 2 & 16 & 4 & 10 & 9 & 15 & 2 & 1 & 5 & 7 & 1 & 3 & 1 & 3 & 0 & 8 & 0 & 14 \\
\hline \multirow{3}{*}{ 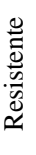 } & Díptera & 18 & 35 & 15 & 14 & 1 & 12 & 6 & 13 & 1 & 7 & 9 & 9 & 5 & 21 & 15 & 28 & 4 & 71 & 16 & 87 & 2 & 38 & 4 & 8 \\
\hline & Anellida & 0 & 1 & 0 & 1 & 0 & 4 & 0 & 0 & 0 & 3 & 0 & 0 & 0 & 5 & 0 & 0 & 0 & 23 & 8 & 35 & 2 & 17 & 4 & 1 \\
\hline & Mollusca & 0 & 1 & 4 & 1 & 0 & 0 & 7 & 3 & 0 & 0 & 0 & 0 & 0 & 1 & 0 & 1 & 0 & 5 & 1 & 8 & 0 & 3 & 2 & 3 \\
\hline \multicolumn{2}{|c|}{ Somatória } & 18 & 37 & 19 & 16 & 1 & 16 & 13 & 16 & 1 & 14 & 9 & 9 & 5 & 27 & 15 & 29 & 4 & 99 & 25 & 130 & 4 & 58 & 10 & 12 \\
\hline
\end{tabular}

Os resultados obtidos na avaliação de bioindicadores apontam a presença de táxons intolerantes em todos os pontos do córrego Bandeirinha. O maior número foi encontrado nos pontos PB2 e PB8, indicando condições ambientais favoráveis para espécies sensíveis às alterações ambientais. Os indivíduos tolerantes e resistentes foram também encontrados em todos os pontos de amostragem, com maior número de taxas nos pontos PB1 e PB2. A menor riqueza e diversidade foram encontradas no ponto PB8 próximo ao exutório do córrego Bandeirinha.

A presença da maioria dos grupos taxonômicos $(\mathrm{N}=33)$ das Ordens Ephemeroptera, Plecoptera e Trichoptera (EPT) nas amostragens de invertebrados bentônicos evidenciaram ambientes com melhor qualidade ambiental. As características de ambientes lóticos com corredeiras na maioria dos pontos amostrados auxiliaram na abundância e ou riqueza da maioria dos táxons. 
A qualidade ambiental dos pontos no córrego Bandeirinha pode ser verificada pela presença nos pontos PB1, PB3 e PB6 do gênero Anacroneuria (Plecoptera) que vive apenas em locais que apresentam boa qualidade da água. Nos pontos PB2 e PB3 foram registradas as maiores quantidades de táxons sensíveis Leptophlebiidae.

O tipo de substrato registrado em todos os pontos, conforme caracterização granulométrica foram areia e cascalho, favorecendo a presença de táxons mais sensíveis, como Ephemeroptera, Trichoptera e Plecoptera. Substratos formados por cascalhos e folhas apresentam as maiores riquezas, pois suportam uma maior quantidade de macroinvertebrados (Pierre e Kovalenko, 2014; Dias-Silva et al. 2013).

No córrego Josefa Gomes em todos os pontos predominaram táxons resistentes. Os pontos PJ3 e PJ4 apresentaram somente indivíduos resistentes, dos filos Annelida (classe: Oligochaeta) e Mollusca (classe: Gastropoda) indicando alterações antrópicas relevantes.

A não ocorrência espaço-temporal de alguns táxons registrados entre os 12 pontos amostrados (córregos Bandeirinha e Josefa Gomes) pode estar relacionada principalmente a capacidade de colonização dos grupos amostrados, com influência dos distúrbios gerados.

Na relação granulometria e biótica a heterogeneidade da composição física do sedimento pode ter influenciado na colonização em ambas as drenagens, mas tendo maior influência no córrego Josefa Gomes, com o menor número de táxons e indivíduos colonizadores.

Os teores de sólidos voláteis para ambas as drenagens indicam a distribuição e colonização no número de registros de táxons, evidenciando a maior influência na estação seca com maiores registros de riqueza. Os maiores resultados de sólidos voláteis presentes nos sedimentos amostrados são os do córrego Josefa Gomes, inserido no ambiente urbano, onde se percebe a relação com os bioindicadores pela ausência de táxons intolerantes e baixos resultados para táxons tolerantes, evidenciada no ponto PJ4. Os sólidos voláteis em sedimentos são indicativos indiretos de matéria orgânica de origem antrópica proveniente de lançamento de efluentes de forma contínua nesta drenagem.

\section{CONCLUSÕES}

Deve-se ressaltar que todas as áreas dos pontos amostrados possuem passivos ambientais gerados pelas atividades antrópicas, que contribuem de forma significativa para as alterações na qualidade da água, seja por áreas de preservação permanente desprovidas de cobertura vegetal, lançamentos de efluentes e resíduos sólidos, ou por atividades agropastoris. O córrego Bandeirinha, inserido em área rural do município, mesmo com os impactos gerados pelas atividades agropastoris, apresentou menores alterações na qualidade da água amostrada, em comparação com o córrego Josefa Gomes, inserido em ambiente urbano, onde os impactos gerados alteraram a qualidade da água nesta drenagem, e neste córrego predominaram táxons tolerantes e resistentes à poluição.

No córrego Josefa Gomes é visível a ausência da vegetação ciliar, pois grande parte das formações vegetais foi removida para propiciar, inicialmente, a prática da agropecuária e posteriormente, dar lugar à expansão urbana do município. É possível verificar que ao longo do córrego existem lançamentos de efluentes, ocupações no fundo de vale, lançamento de resíduos sólidos, desenvolvimento de processos erosivos, assoreamentos, dentre outros impactos, intensificados pelos processos de uso e ocupação do solo na área urbana.

Os resultados demostram a distinção entre as alterações na qualidade da água em ambiente rural e urbano e a relação dos bioindicadores com os sedimentos de corrente. $\mathrm{O}$ estudo auxilia na interpretação das alterações na qualidade da água além de contribuir com a geração de dados sobre as duas drenagens da região. A análise por meio dos bioindicadores bentônicos pode ser associada a análises físico-químicas e geoquímicas da água e dos 
sedimentos, proporcionando uma melhor interpretação dos resultados, para determinar a qualidade da água em drenagens superficiais.

A legislação brasileira, mais especificamente a Resolução CONAMA 357/2005 (CONAMA, 2005), classifica as drenagens superficiais, por meio dos padrões e valores máximos e mínimos permitidos para os parâmetros físico-químicos, não considerando as informações da dinâmica de interação dos organismos bioindicadores de qualidade de água. Contudo, os órgãos ambientais já solicitam dos empreendimentos relatório de qualidade de água em programas de monitoramento, que contenham além dos parâmetros físico-químicos de qualidade, indicação biológica para drenagens superficiais. É previsível que a legislação se atualize, direcionando uma metodologia que contemple a inter-relação da qualidade da água entre os parâmetros físico-químicos e os bioindicadores.

\section{AGRADECIMENTOS}

Os autores agradecem aos técnicos, professores e alunos do Laboratório de Geoquímica da UnB e ao Instituto Federal de Educação Ciência e Tecnologia Goiás - IFG, ao CNPq e a CAPES.

\section{REFERÊNCIAS}

AMERICAN PUBLIC HEALTH ASSOCIATION - APHA; AMERICAN WATER WORKS ASSOCIATION AWWA; WATER ENVIRONMENT FEDERATION - WEF. Standard Methods for the Examination of Water and Wastewater. 20th ed. Washington, 1999. $1496 \mathrm{p}$.

BELMEJO, L.; MARTOS. H. L. Utilização de Xiphophorus helleri como bioindicador de poluição hídrica de derivados de petróleo em condições tropicais. Revista Eletrônica de Biologia, v 1, n. 2, p. 1-17, 2008.

BORISKO. J. P; KILGOUR. B. W; STANFIELD. W. F; JONES. C. An evaluation of rapid bioassessment protocols for stream benthic invertebrates in Southern Ontario. Water Quality Research Journal, v. 42, n. 3, p. 184-193, 2007.

CALLISTO, M.; GOULART, M.; MEDEIROS, A. O.; MORENO, P.; ROSA, C. A. Diversity assessment of benthic macroinvertebrates, yeasts and microbiological indicators along a longitudinal gradient in Serra do Cipó, Brazil. Brazilian Journal of Biology, v. 61, n. 2, p, 259-266, 2004. http://dx.doi.org/10.1590/S1519-69842004000500003

CALLISTO, M.; MORENO, P. Bioindicadores como ferramenta para o manejo, gestão e conservação ambiental In: SIMPÓSIO SUL DE GESTÃO E CONSERVAÇÃO AMBIENTAL, 2., 2006, Erechim. Anais... Erechim: URI-Campus de Erechim, 2006.

CARVALHO, E. M.; UIEDA, V. S. Colonização por Macroinvertebrados Bentônicos em Substrato Artificial e Natural em um Riacho da Serra de Itatinga, São Paulo, Brasil. Revista Brasileira de Zoología, v. 21, n. 2, p. 287-293, 2004. http://dx.doi.org/10.1590/S0101-81752004000200021

CHALAR, G. Composicíon y abundancia Del zoobentos Del Arroyo Toledo (Uruguay) y su relación com La calidad de água. Revista Chilena de História Natural. v. 67, p. 129141, 1994. 
COLPO K. D.; BRASIL M. T.; CAMARGO, B.V. Macroinvertebrados bentônicos como indicadores do impacto ambiental promovido pelos efluentes de áreas orizícolas e pelos de origem urbana/industrial. Revista Ciência Rural, v. 39, n. 7, p. 2087-2092, 2009. http://dx.doi.org/10.1590/S0103-84782009005000161

CONSELHO NACIONAL DO MEIO AMBIENTE - CONAMA Resolução n. 357, de 17 de março de 2005. Dispõe sobre a classificação de corpos de água e diretrizes ambientais para o seu enquadramento, bem como estabelece as condições e padrões de lançamento de efluentes, e dá outras providencias. Diário Oficial [da] União, Brasília, DF, n. 53, 18 mar. 2005. Seção 1, p.58-63.

DIAS-SILVA. K.; CABETTE, H. S. R.; GIEHL, N. F. S.; JUEN, L. Distribuição de Heteroptera Aquáticos (Insecta) em Diferentes Tipos de Substratos de Córregos do Cerrado Matogrossense. EntomoBrasilis, v. 6 n. 2 p. 132-140. 2013.

FARIA, A. Estratigrafia e sistemas deposicionais do Grupo Paranoá nas áreas de Cristalina, Distrito Federal e São João d’Aliança - Alto Paraíso de Goiás. 1995. 199f. Dissertação (Mestrado em Geologia) - Instituto de Geociências, Universidade de Brasília, Brasília, 1995.

HAUER, F. R.; RESH. V. H. Benthic macroinvertebrates. In: HAUER, F. R.; LAMBERTI, G. A. (Eds.). Methods in Stream ecology. San Diego: Academic Press, 1996. p. 339369.

INSTITUTO BRASILEIRO DE GEOGRAFIA E ESTATÍSTICA - IBGE. Censo 2010. Disponível em: http://www.censo2010.ibge.gov.br/resultados_do_censo2010.php. Acesso em: 27 ago. 2012.

MÄENPÄÄ, K. A.; SORMUNEN, A. J.; KUKKONEN, J. V. Bioacumulation and toxicity of sediment associated herbicides (ioxynil, pendimethalin and bentazone) in Lumbriculus variegates (Oligochaeta) and chironomus riparius (Insecta). Ecotoxicology and Environmental Safety, v. 56 n. 3, p. 398-410, 2003. http://dx.doi.org/10.1016/S01476513(03)00010-1

MAGURRAN, A. E. Ecological diversity and its measurement. New Jersey: Princeton Universidad Press, 1988. 179 p.

MILESI, S. V.; BIASI, C.; RESTELLO, R. M; HEPP, L. U. Efeito de metais cobre (Cu) e Zinco (Zn) sobre a comunidade de macroinvertebrados bentônicos em riachos do sul do Brasil. Acta Scientiarum. Biological Sciences, v. 30, n. 3, p. 283-289, 2008. http://dx.doi.org/10.4025/actascibiolsci.v30i3.677

MUGNAI, R.; NESSIMIAN. J. L.; BAPTISTA, D. F. Manual de identificação de macroinvertebrados aquáticos do Estado do Rio de Janeiro. Rio de Janeiro: Technical Books, 2010.

PIMENTA, S. M.; PEÑA, A. P.; GOMES, P. S. Aplicação de métodos físicos, químicos e biológicos na avaliação da qualidade das águas em áreas de aproveitamento hidroelétrico da bacia do Rio São Tomás, Município de Rio Verde - Goiás. Sociedade \& Natureza, v. 21, n. 3, p. 393-412, 2009. http://dx.doi.org/10.1590/S198245132009000300013

PIERRE, J. I.; KOVALENKO. K. E. Effect of habitat complexity attributes on species richness. Ecosphere, v. 5, n. 2, 2014. http://dx.doi.org/10.1890/ES13-00323.1 
RIBEIRO, L. O.; UIEDA, V. S. Estrutura da comunidade de macroinvertebrados bentônicos de um riacho de serra em Itatinga, São Paulo, Brasil. Revista Brasileira de Zoologia, v. 22, n.3, p. 613-618, 2005. http://dx.doi.org/10.1590/S0101-81752005000300013

SOUZA, J. D. (Coord.). Carta geológica do Brasil ao milionésimo: Folha Brasília SD 23. Brasília: CPRM, 2004.

UNITED STATES. Environmental Protection Agency - USEPA. Rapid bioassessment protocols for use in streams and wadeable rivers: Periphyton, Benthic Macroinvertebrates and Fish. Disponível em: http://water.epa.gov/scitech/monitoring /rsl/bioassessment/. Acesso em: 22 maio 2013.

YOKOYAMA, E.; PACIENCIA, G. P.; BISPO, P. C.; OLIVEIRA, L. G.; BISPO, P. C. A sazonalidade ambiental afeta a composição faunística de Ephemeroptera e Trichoptera em um riacho de Cerrado do Sudeste do Brasil. Revista Ambiência Guarapuava, v. 8, n. 1, p. 73-84. 2012. 


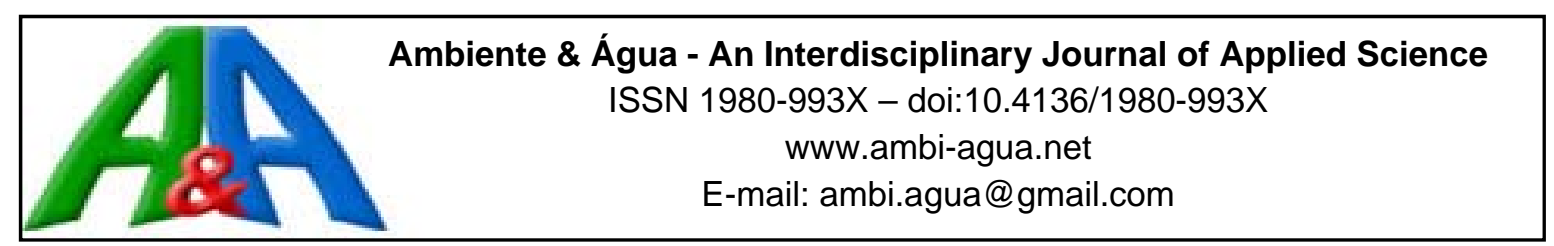

\title{
Pegada hídrica dos suínos abatidos na região do Corede Serra, RS, Brasil
}

doi:10.4136/ambi-agua.1688

Received: 22 Jun. 2015; Accepted: 02 Dec. 2015

\author{
Vania Elisabete Schneider; Sofia Helena Zanella Carra* \\ Universidade de Caxias do Sul (UCS), Caxias do Sul, RS, Brasil \\ Departamento de Ciência Exatas e Tecnologia (CCET) \\ *Autor correspondente: e-mail: sofi_carra@hotmail.com, \\ veschnei@ucs.br
}

\section{RESUMO}

Este trabalho teve por objetivo calcular a pegada hídrica dos suínos abatidos nos municípios que compõem o Conselho Regional de Desenvolvimento da Serra (Corede Serra), localizado na região nordeste do Estado do Rio Grande do Sul, no ano de 2014. A água consumida na produção dos grãos (milho e soja), destinados à alimentação dos animais e a água destinada à dessedentação animal e limpeza das áreas de criação, além da água incorporada aos suínos em crescimento e terminação, foram utilizadas no cálculo da pegada hídrica. A pegada hídrica total dos suínos abatidos foi de $0,19825 \mathrm{~km}^{3} \mathrm{ano}^{-1}$, na qual o consumo de água para o cultivo de milho e soja foi o mais representativo, 99,6\%. O município de Nova Prata apresentou a maior pegada hídrica total $\left(0,02343 \mathrm{~km}^{3}\right.$ ano $\left.{ }^{-1}\right)$, seguida pelos municípios de Paraí $\left(0,02187 \mathrm{~km}^{3}\right.$ ano $\left.{ }^{-1}\right)$ e por Serafina Corrêa $\left(0,01658 \mathrm{~km}^{3} \mathrm{ano}^{-1}\right)$. Os municípios de São Marcos $\left(0,000006 \mathrm{~km}^{3} \mathrm{ano}^{-1}\right)$, Bento Gonçalves $\left(0,00002 \mathrm{~km}^{3} \mathrm{ano}^{-1}\right)$ e Boa Vista do Sul $\left(0,00004 \mathrm{~km}^{3} \mathrm{ano}^{-1}\right)$ apresentaram as menores pegadas, resultado da baixa produtividade de milho associada ao rebanho suíno abatido pouco expressivo. A partir desta avaliação indica-se que a gestão dos recursos hídricos associada à cadeia suinícola deve contemplar a produção de grãos, destinados à atividade, além da demanda hídrica destinada ao confinamento.

Palavras-chave: gestão dos recursos hídricos, indicador de consumo de água, suinocultura.

\section{Water Footprint of pigs slaughtered in the northeast region of Rio Grande do Sul State, Brazil}

\begin{abstract}
This study estimated the water footprint of pigs slaughtered in the municipalities that participate in the Regional Council for the Development of Serra (Corede Serra, in Portuguese), located in the northeast region of Rio Grande do Sul State, in 2014. In order to determine the water footprint, we estimated the water consumed in the production of grains (corn and soybeans) used for animal feed, the water used for livestock watering, the water used for cleaning creation areas and the water consumed by animals during the growing and finishing phases. The total water footprint of slaughtered pigs was $0.19825 \mathrm{~km}^{3}$, the largest component of which was water used for the cultivation of grains (99.6\%). The municipality of
\end{abstract}


Nova Prata had the largest water footprint of Corede Serra $\left(0.02343 \mathrm{~km}^{3}\right.$ year $\left.^{1}\right)$, followed by the municipalities of Paraí $\left(0.02187 \mathrm{~km}^{3}\right.$ year $\left.{ }^{-1}\right)$ and Serafina Corrêa $\left(0.01658 \mathrm{~km}^{3}\right.$ year $\left.{ }^{-1}\right)$. The municipalities of São Marcos $\left(0.000006 \mathrm{~km}^{3}\right.$ year $\left.{ }^{-1}\right)$, Bento Gonçalves $\left(0,00002 \mathrm{~km}^{3}\right.$ year $\left.{ }^{-1}\right)$ and Boa Vista do Sul $\left(0.0004 \mathrm{~km}^{3}\right.$ year $\left.{ }^{-1}\right)$ had the lowest water footprints, due to low corn productivity associated with the low number of hogs slaughtered. From this assessment, it was found that the management of water resources associated with pig chain production should include water used in the production of feed grain as well as the water used directly in animal husbandry.

Keywords: swine, water consumption indicator, water resource management.

\section{INTRODUÇÃO}

De acordo com a Associação Brasileira de Proteína Animal (ABPA), o Brasil é o quarto maior produtor e o quarto maior exportador de carne suína, tendo uma produção de cerca de 3,344 milhões de toneladas no ano de 2014, com destaque para a região sul do Brasil, responsável por 69,03\% da produção nacional (ABPA, 2015).

A atividade suinícola, assim como as demais atividades pecuárias, é diretamente dependente de recursos naturais. Nesta evidencia-se o elevado consumo de água utilizada para o crescimento das commodities destinadas à alimentação dos suínos, dessedentação, higienização das áreas de criação, abate e processamento da carne. A falta de conhecimento acerca do impacto sobre os recursos hídricos na atividade pecuária pode comprometer o crescimento do setor, o que evidencia a necessidade de utilizar metodologias para avaliar o desempenho das atividades agropecuárias de forma a auxiliar os consumidores a optarem por produtos mais sustentáveis.

A avaliação da demanda hídrica é uma forma de geração deste tipo de informação que auxilia na tomada de decisão por produtos de menor impacto no uso da água e da terra (Jeswani e Azapagic, 2011; Pfister et al., 2011). De acordo com Hoekstra et al. (2011), a avaliação da pegada hídrica é uma ferramenta analítica que pode auxiliar na compreensão sobre como atividades e produtos interagem com a escassez e com a poluição da água e seus impactos relacionados, bem como o que pode ser feito para assegurar que as atividades e produtos contribuam para o uso sustentável dos recursos hídricos.

Hoekstra et al. (2011) classificam a água em três tipos:

Água Verde: água precipitada sobre a terra que não escoa ou recarrega os aquíferos, mas é armazenada no solo ou temporariamente sobre o solo ou a vegetação;

Água Azul: água superficial e subterrânea, isto é, a água em lagos, rios e aquíferos; e

Água Cinza: volume de água necessário para diluir os poluentes de modo que a qualidade da água em seu estado natural seja mantida nos padrões legais.

De acordo com Silva et al. (2013), o volume de água doce utilizada para produzir o produto é somado ao longo das várias fases da cadeia de produção, sendo essa a base para a compreensão do conceito de pegada hídrica. Segundo Palhares (2014), o cálculo da pegada hídrica e sua relação com o território geram informações com relevantes impactos sociais, ambientais e econômicos, necessários para implantação dos instrumentos de gestão contidos na Política Nacional de Recursos Hídricos e proporcionam impactos científicos de grande importância, fortalecendo o tema produção animal e recursos hídricos e possibilitando o desenvolvimento de pesquisas nas áreas de eficiência hídrica, tecnologias de tratamento de resíduos, mitigação dos impactos ambientais e zootecnia de precisão.

Neste contexto, o objetivo deste trabalho foi calcular as pegadas hídricas verde a azul dos suínos abatidos no ano de 2014 nos municípios que compõem o Conselho Regional de 
Desenvolvimento da Serra (Corede Serra).

\section{MATERIAIS E MÉTODOS}

Neste trabalho considerou-se o número de suínos abatidos no ano de 2014 na região do Corede Serra, conforme informações disponibilizadas pela Secretaria Estadual de Agricultura, Pecuária e Abastecimento do Estado do Rio Grande do Sul (SEAPA), para o cálculo da pegada hídrica.

A Figura 1 apresenta a distribuição dos municípios que compõem o Corede Serra e a localização da região no Estado do Rio Grande do Sul. A região do Corede Serra é composta por trinta e um municípios, localizados na região nordeste do Estado do Rio Grande do Sul (Rio Grande do Sul, 2015a). A presença de propriedades rurais de pequeno porte onde predominam a agricultura familiar, participação significativa da agropecuária na economia da região, além da localização da quase totalidade dos municípios sobre a mesma bacia hidrográfica, conforme apresentado na Figura 2, são características que tornam a região do Corede Serra uma unidade de referência ideal para a realização deste estudo.

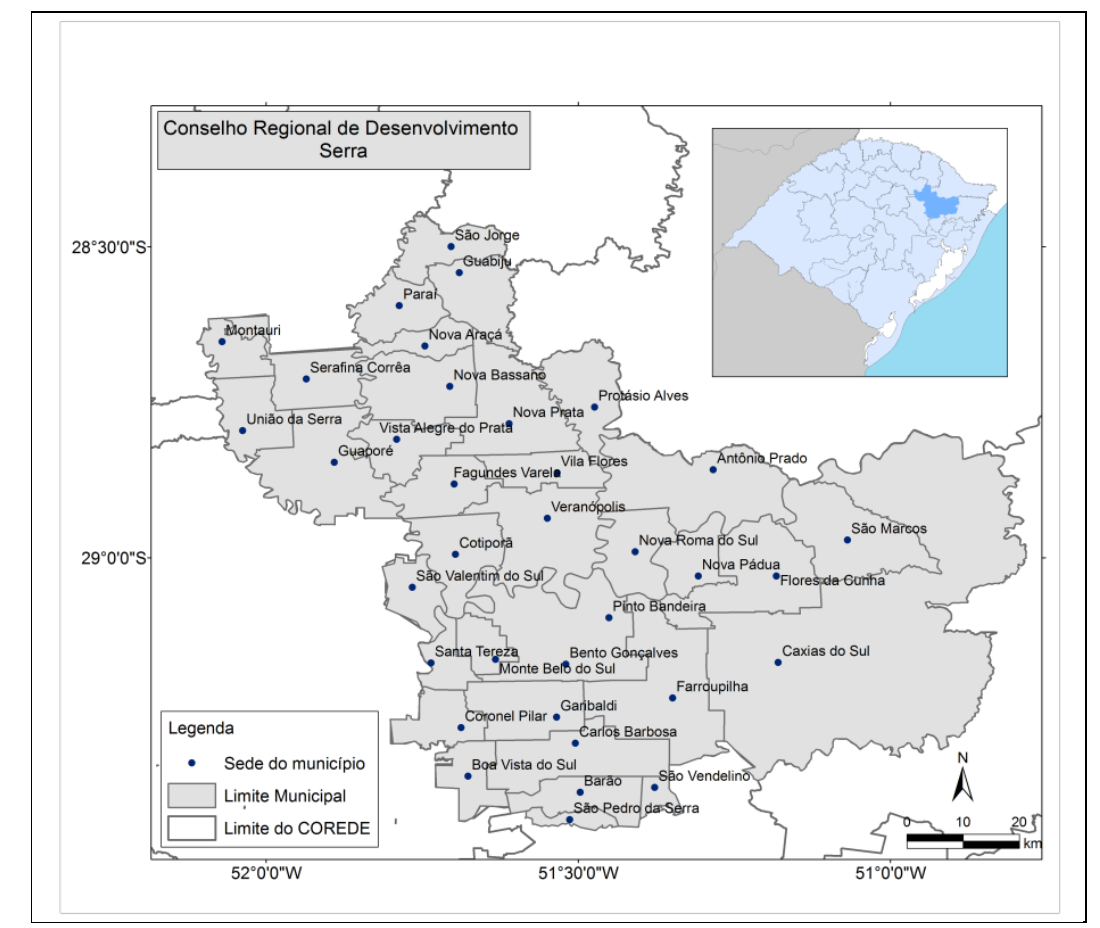

Figura 1. Mapa com a localização e identificação dos municípios que compõem o Conselho de Desenvolvimento da Serra (Corede Serra). Fonte: ISAM (2015).

A Figura 2 apresenta a localização do Corede Serra em relação à Bacia Hidrográfica Taquari-Antas e à Bacia Hidrográfica do Caí.

Neste estudo, utilizou-se a metodologia proposta por Chapaing e Hoekstra (2003) para o cálculo da pegada hídrica, considerando-se: a água consumida (evapotranspirada) na produção de grãos (milho e soja), a água destinada à dessedentação e a água utilizada na limpeza das instalações. Ressalta-se que, para a realização dos cálculos, foram consideradas as fases de crescimento e terminação. 


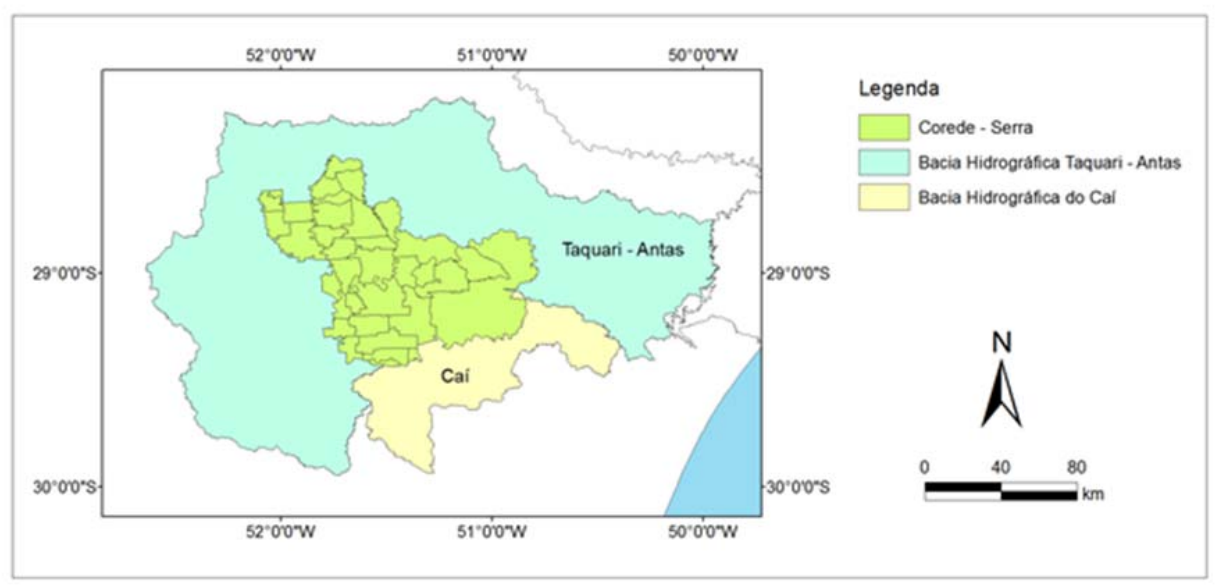

Figura 2. Mapa com a localização do Conselho de Desenvolvimento da Serra em relação às Bacias Hidrográficas Taquari-Antas e Caí.

Fonte: ISAM (2015).

Considerou-se 70 dias para a fase de crescimento e 93 dias para a fase de terminação, conforme proposto por Carra (2012). A Tabela 1 apresenta o número de suínos abatidos no ano de 2014 nos municípios do Corede Serra.

Tabela 1. Total de suínos abatidos no ano de 2014 nos municípios do Corede Serra.

\begin{tabular}{lc}
\hline Municípios & Suínos abatidos (2014) \\
\hline Antônio Prado & 27.253 \\
Bento Gonçalves & 83 \\
Boa Vista do Sul & 228 \\
Carlos Barbosa & 34.351 \\
Caxias do Sul & 23.587 \\
Coronel Pilar & 8.150 \\
Cotiporã & 19.745 \\
Fagundes Varela & 9.451 \\
Farroupilha & 4.212 \\
Flores da Cunha & 3.598 \\
Garibaldi & 304 \\
Guabiju & 13.249 \\
Guaporé & 26.242 \\
Montauri & 6.472 \\
Monte Belo do Sul & 0 \\
Nova Araçá & 37.073 \\
Nova Bassano & 55.064 \\
Nova Pádua & 787 \\
Nova Prata & 73.100 \\
Nova Roma do Sul & 25.858 \\
Paraí & 78.111 \\
Protásio Alves & 38.657 \\
Santa Tereza & 1.361 \\
São Jorge & 30.479 \\
São Marcos & 37 \\
São Valentim do Sul & 10.912 \\
Serafina Corrêa & 56.896 \\
União da Serra & 47.785 \\
Veranópolis & 25.272 \\
Vila Flores & 45.508 \\
Vista Alegre do Prata & 36.033 \\
\hline Total & 739.858 \\
Fonte: Rio Gran & \\
\hline
\end{tabular}

Fonte: Rio Grande do Sul (2015b). 
A Tabela 2 apresenta a constituição da dieta dos suínos em crescimento e terminação, utilizada como referência para o cálculo da quantidade de milho e soja consumidos pelos animais, conforme proposto por Palhares (2011).

Tabela 2. Consumo de ração, milho e farelo de soja durante as fases de crescimento e terminação.

\begin{tabular}{lccc}
\hline Tipo de ração & $\begin{array}{c}\text { Consumo de ração } \\
\left(\mathrm{kg} \mathrm{suíno}^{-1}\right)\end{array}$ & $\begin{array}{c}\text { Consumo de milho } \\
\left(\mathrm{kg} \mathrm{suíno}^{-1}\right)\end{array}$ & $\begin{array}{c}\text { Consumo de farelo } \\
\text { de soja }\left(\mathrm{kg} \mathrm{suíno}^{-1}\right)\end{array}$ \\
\hline Crescimento I & 47,20 & 31,62 & 13,21 \\
Crescimento II & 33,00 & 22,11 & 9,24 \\
Terminação I & 74,60 & 49,23 & 21,63 \\
Terminação II & 126,10 & 92,05 & 27,74 \\
\hline Total & $\mathbf{2 8 0 , 9 0}$ & $\mathbf{1 9 5 , 0 2}$ & $\mathbf{7 1 , 8 3}$ \\
\hline
\end{tabular}

Fonte: Adaptado de Palhares (2011).

Para o ciclo da cultura de milho considerou-se uma evapotranspiração de $450 \mathrm{~mm}$ $\left(0,45 \mathrm{~m}^{3} \mathrm{~m}^{-2}\right)$, o que resulta na necessidade de $4.500 \mathrm{~m}^{3}$ ha ${ }^{-1}$ (Palhares, 2011) e para o ciclo da cultura de soja considerou-se uma evapotranspiração de $600 \mathrm{~mm}\left(0,60 \mathrm{~m}^{3} \mathrm{~m}^{-2}\right)$, o que resulta na necessidade de $6.000 \mathrm{~m}^{3} \mathrm{ha}^{-1}$ (Couto e Sans, 2002).

A área total de milho e soja colhidos em cada município, bem como a quantidade produzida destes no ano de 2013 foram extraídas do IBGE (2013), visto que os suínos abatidos no ano de 2014 foram alimentados com as culturas produzidas no ano de 2013. A Tabela 3 apresenta as informações do IBGE (2013).

De acordo com Palhares (2011), os três principais produtos do denominado complexo soja são: grão, farelo e óleo. Desses, o farelo é a forma que os suínos consomem a soja. Portanto, nem toda a água consumida para a produção dos produtos do complexo soja pode ser contabilizada no cálculo da pegada hídrica, apenas a parcela de água consumida no farelo.

Utilizando-se os índices constantes nos Fatores de Conversão das Commodities Agropecuárias da FAO (FAO, 2006), considera-se que no caso brasileiro, de cada grão produzido, $77 \%$ são farelo e $23 \%$ são óleo. A partir das informações apresentadas na Tabela 3 foi calculado o consumo de água $\left(\mathrm{m}^{3} \mathrm{t}^{-1}\right)$ para as culturas de milho e soja a partir da Equação 1.

$$
\mathrm{PH}_{\text {verde }}=\frac{\mathrm{A}_{\text {colhida }} \cdot \mathrm{E}_{\mathrm{t}}}{\mathrm{Q}_{\text {prod }}} \cdot \mathrm{AB} \cdot \mathrm{AL}
$$

em que:

$$
\begin{aligned}
& \mathrm{PH}_{\mathrm{verde}}=\text { pegada hídrica verde }\left(\mathrm{m}^{3} \mathrm{ano}^{-1}\right) ; \\
& \mathrm{A}_{\text {colhida }}=\text { área colhida (ha); } \\
& \mathrm{E}_{\mathrm{t}}=\text { evapotranspiração da cultura }\left(\mathrm{mm} \mathrm{ha}^{-1}\right) ; \\
& \mathrm{Q}_{\text {prod }}=\text { quantidade produzida (t); } \\
& \mathrm{AB}=\text { animais abatidos no ano de } 2014 \text { nos municípios do Corede Serra e; } \\
& \mathrm{AL}=\text { quantidade de alimento consumido por animal (informações apresentadas na }
\end{aligned}
$$
Tabela 2).

A pegada hídrica verde total foi obtida através da Equação 2, na qual são somados os resultados das pegadas hídricas para o cultivo de milho e farelo de soja.

$$
\mathrm{PH}_{\mathrm{verde} \mathrm{total}}=\mathrm{PH}_{\mathrm{verde} / \text { milho }}+\mathrm{PH}_{\mathrm{verde} / \text { farelo de soja }}
$$
em que: 


$$
\begin{aligned}
& \mathrm{PH}_{\mathrm{verde}} \text { total = pegada hídrica verde total }\left(\mathrm{m}^{3} \mathrm{ano}^{-1}\right) \text {; } \\
& \mathrm{PH}_{\mathrm{verde} / \mathrm{milho}}=\text { pegada hídrica verde do milho }\left(\mathrm{m}^{3} \mathrm{ano}^{-1}\right) \text {; } \\
& \mathrm{PH}_{\mathrm{verde} / \text { arelo de soja }}=\text { pegada hídrica verde do farelo de soja }\left(\mathrm{m}^{3} \mathrm{ano}^{-1}\right) \text {. }
\end{aligned}
$$

Neste estudo considerou-se que todo o milho e o farelo de soja, destinados à alimentação dos suínos, sejam provenientes das áreas cultivadas nos municípios do Corede Serra, não havendo a necessidade de importação de alimento de outros municípios. Para o cálculo da pegada hídrica azul, referente ao processo de confinamento dos suínos, foram considerados apenas os usos diretos de água nas propriedades, sendo elas: dessedentação animal, lavagem e

\begin{tabular}{|c|c|c|c|c|}
\hline Município & $\begin{array}{l}\text { Área total de } \\
\text { colheita de } \\
\text { milho (ha) }\end{array}$ & $\begin{array}{l}\text { Quantidade } \\
\text { produzida de } \\
\text { milho (t) }\end{array}$ & $\begin{array}{l}\text { Área total de } \\
\text { colheita de } \\
\text { soja (ha) }\end{array}$ & $\begin{array}{l}\text { Quantidade produzida } \\
\text { de soja - somente farelo } \\
\text { - (t) }\end{array}$ \\
\hline Antônio Prado & 2.250 & 13.500 & - & - \\
\hline Bento Gonçalves & 900 & 3.150 & - & - \\
\hline Boa Vista do Sul & 600 & 2.880 & - & - \\
\hline Carlos Barbosa & 700 & 3.780 & - & - \\
\hline Caxias do Sul & 4.500 & 24.300 & - & - \\
\hline Coronel Pilar & 500 & 2.500 & - & \\
\hline Cotiporã & 1.150 & 6.900 & 75 & 207,9 \\
\hline Fagundes Varela & 2.000 & 13.000 & 400 & 924,0 \\
\hline Farroupilha & 600 & 3.240 & - & - \\
\hline Flores da Cunha & 700 & 3.150 & - & - \\
\hline Garibaldi & 340 & 1.768 & - & - \\
\hline Guabiju & 750 & 4.195 & - & - \\
\hline Guaporé & 3.300 & 24.090 & 2.000 & $11.242,0$ \\
\hline Montauri & 2.000 & 14.400 & 2.200 & $3.388,0$ \\
\hline Monte Belo do Sul & 290 & 1.450 & - & - \\
\hline Nova Araçá & 1.450 & 10.005 & 500 & $1.386,0$ \\
\hline Nova Bassano & 6.000 & 41.700 & 1.000 & $2.810,5$ \\
\hline Nova Pádua & 260 & 1.040 & - & - \\
\hline Nova Prata & 2.700 & 17.820 & 1.300 & $3.003,0$ \\
\hline Nova Roma do Sul & 1.300 & 7.365 & - & - \\
\hline Paraí & 4.000 & 28.400 & 800 & $2.217,6$ \\
\hline Protásio Alves & 525 & 4.054 & 900 & $2.079,0$ \\
\hline Santa Tereza & 680 & 2.856 & - & - \\
\hline São Jorge & 1.750 & 11.628 & 2.450 & $5.606,6$ \\
\hline São Marcos & 1.200 & 6.461 & - & - \\
\hline São Valentim do Sul & 250 & 1.800 & 600 & $1.663,2$ \\
\hline Serafina Corrêa & 2.000 & 13.000 & 2.200 & $6.098,4$ \\
\hline União da Serra & 3.100 & 22.320 & 1.200 & $2.772,0$ \\
\hline Veranópolis & 1.600 & 10.560 & 150 & 351,9 \\
\hline Vila Flores & 1.100 & 7.947 & 300 & 831,6 \\
\hline Vista Alegre do Prata & 2.000 & 12.000 & 300 & 762,3 \\
\hline Total & 50.495 & 321.259 & 16.375 & $45.344,1$ \\
\hline
\end{tabular}
higienização das instalações de criação e quantidade de água incorporada ao animal (produto).

Tabela 3. Quantidade de milho e soja produzidos nos municípios do Corede Serra.

Fonte: Elaborado pelos autores com base nas informações do IBGE (2013). 
Considerou-se um consumo de água, para fins de lavagem das instalações de criação de 0,004 $\mathrm{m}^{3} \mathrm{animal}^{-1}$, conforme proposto por Oliveira e Bellaver (2009), sendo que a mesma ocorre somente na desocupação da instalação, ou seja, ao final da terminação. Portanto, o volume de água para fins de lavagem das áreas de criação foi considerado no cálculo apenas para os suínos em terminação. Para a dessedentação animal considerou-se a metodologia citada por Perdomo et al. (2001), onde os suínos em crescimento consomem em torno de 0,003 $\mathrm{m}^{3} \mathrm{dia}^{-1}$ e os suínos em terminação consomem em torno de 0,007 $\mathrm{m}^{3} \mathrm{dia}^{-1}$.

No que tange à quantidade de água incorporada ao animal (produto), utilizada apenas no cálculo para os suínos em fase de terminação, considerou-se uma média de 66\% de água por quilo de carne, existente no animal vivo, conforme proposto por NEPA (2011), adição de 4\% de água (contida no sangue, glândulas, vísceras e conteúdo estomacal), rendimento de carcaça fria de 74\% (Palhares, 2011) e peso ao abate de 110,99 kg. O cálculo da pegada hídrica azul é apresentado na Equação 3.

$\mathrm{PH}_{\mathrm{azul}}=(\mathrm{CD} \cdot \mathrm{TP} \cdot \mathrm{AB})+\left(\mathrm{C}_{1} \cdot \mathrm{AB}\right)+\left(0,1109 \cdot 0,74 \cdot 0,66 \cdot \mathrm{A}_{\mathrm{p}} \cdot \mathrm{AB}\right)+\left(0,04 \cdot \mathrm{A}_{\mathrm{p}} \cdot \mathrm{AB}\right)(3)$

em que:

$\mathrm{PH}_{\mathrm{azul}}=$ pegada hídrica azul $\left(\mathrm{m}^{3} \mathrm{ano}^{-1}\right)$;

$\mathrm{CD}=$ consumo de água para dessedentação $\left(\mathrm{m}^{3} \mathrm{animal}^{-1}\right)$;

TP = tempo de permanência na fase de criação (70 dias para a fase de crescimento e 93 dias para a fase de terminação);

$\mathrm{AB}=$ quantidade de animais abatidos no ano de 2014 nos municípios do Corede Serra;

$\mathrm{C}_{\mathrm{l}}=$ consumo de água de limpeza, $0,04 \mathrm{~m}^{3}$ animal $^{-1}$ (Oliveira e Bellaver, 2009) e;

$\mathrm{A}_{\mathrm{p}}=$ quantidade de água no produto, considerando-se uma média de $66 \%$ de água por quilo de carne (NEPA, 2011), fator de adição de 4\% de água (água contida no sangue, glândulas, vísceras e conteúdo estomacal, entre outros), rendimento de carcaça fria de $74 \%$ e peso ao abate de 110,99 kg.

\section{RESULTADOS E DISCUSSÃO}

Conforme observado na Tabela 1, foram abatidos 739.858 suínos no Corede Serra no ano de 2014, no qual o município de Paraí apresentou a maior quantidade, com 78.111 animais, seguido por Nova Prata com 73.100 animais. Os municípios que apresentaram os menores números de suínos abatidos foram: São Marcos, com 37 animais e Bento Gonçalves, com 83 animais. O município de Monte Belo do Sul não apresentou nenhum suíno abatido no ano de 2014. A Tabela 4 apresenta a pegada hídrica verde para os suínos abatidos no ano de 2014, no Corede Serra.

De acordo com a Tabela 4, a pegada hídrica verde para o cultivo de milho corresponde a 50,34\% da pegada hídrica verde total, onde o mesmo resultado para o cultivo de soja corresponde a 49,66\%. O consumo de água para a produção de milho, considerando os suínos em crescimento resultou em $0,02740 \mathrm{~km}^{3} \mathrm{ano}^{-1}$ ao passo que na terminação resultou em de $0,07204 \mathrm{~km}^{3} \mathrm{ano}^{-1}$, corroborando em um volume de $0,09944 \mathrm{~km}^{3} \mathrm{ano}^{-1}$. Para a cultura de soja, o consumo de água, considerando os suínos em crescimento, resultou em $0,03066 \mathrm{~km}^{3} \mathrm{ano}^{-1} \mathrm{e}$ considerando a terminação resultou em $0,06743 \mathrm{~km}^{3} \mathrm{ano}^{-1}$, corroborando em um volume de pegada hídrica verde de $0,09810 \mathrm{~km}^{3} \mathrm{ano}^{-1}$. Desta forma, a pegada hídrica verde total resultou em 0,19754 $\mathrm{km}^{3} \mathrm{ano}^{-1}$, conforme apresentado na Tabela 4. 
Tabela 4. Pegada hídrica verde para os suínos abatidos no ano de 2014 no Corede Serra.

\begin{tabular}{|c|c|c|c|}
\hline Município & $\begin{array}{l}\text { Consumo de água - } \\
\text { cultura de milho } \\
\mathrm{km}^{3} \mathrm{ano}^{-1}\end{array}$ & $\begin{array}{l}\text { Consumo de água - } \\
\text { farelo de soja } \\
\mathrm{km}^{3} \mathrm{ano}^{-1}\end{array}$ & $\begin{array}{c}\text { Consumo de água } \\
\text { total } \\
\mathrm{km}^{3} \mathrm{ano}^{-1} \\
\end{array}$ \\
\hline Antônio Prado & 0,003986 & - & 0,00399 \\
\hline Bento Gonçalves & 0,000021 & - & 0,00002 \\
\hline Boa Vista do Sul & 0,000042 & - & 0,00004 \\
\hline Carlos Barbosa & 0,005582 & - & 0,00558 \\
\hline Caxias do Sul & 0,003833 & - & 0,00383 \\
\hline Coronel Pilar & 0,001430 & - & 0,00143 \\
\hline Cotiporã & 0,002888 & 0,003070 & 0,00596 \\
\hline Fagundes Varela & 0,001276 & 0,001760 & 0,00304 \\
\hline Farroupilha & 0,000684 & - & 0,00068 \\
\hline Flores da Cunha & 0,000702 & - & 0,00070 \\
\hline Garibaldi & 0,000051 & - & 0,00005 \\
\hline Guabiju & 0,002079 & - & 0,00208 \\
\hline Guaporé & 0,003155 & 0,002012 & 0,00517 \\
\hline Montauri & 0,000789 & 0,001035 & 0,00182 \\
\hline Monte Belo do Sul & 0,000000 & - & 0,00000 \\
\hline Nova Araçá & 0,004715 & 0,005760 & 0,01048 \\
\hline Nova Bassano & 0,006953 & 0,008440 & 0,01540 \\
\hline Nova Pádua & 0,000173 & - & 0,00017 \\
\hline Nova Prata & 0,009719 & 0,013640 & 0,02336 \\
\hline Nova Roma do Sul & 0,004005 & - & 0,00401 \\
\hline Paraí & 0,009654 & 0,012140 & 0,02180 \\
\hline Protásio Alves & 0,004393 & 0,007210 & 0,01160 \\
\hline Santa Tereza & 0,000284 & - & 0,00028 \\
\hline São Jorge & 0,004025 & 0,005740 & 0,00976 \\
\hline São Marcos & 0,000006 & - & 0,00001 \\
\hline São Valentim do Sul & 0,001330 & 0,001700 & 0,00303 \\
\hline Serafina Corrêa & 0,007681 & 0,008390 & 0,01653 \\
\hline União da Serra & 0,005824 & 0,008910 & 0,01474 \\
\hline Veranópolis & 0,003360 & 0,004640 & 0,00800 \\
\hline Vila Flores & 0,005528 & 0,007100 & 0,01260 \\
\hline Vista Alegre do Prata & 0,005270 & 0,006110 & 0,01138 \\
\hline Total & 0,099439 & 0,098100 & 0,19754 \\
\hline
\end{tabular}

Conforme observado a soja apresenta evapotranspiração 25\% superior se comparada a cultura de milho. Todavia, a cultura de soja foi produzida em 2013 em apenas 16 municípios, o que equivale a 51,61\% dos municípios que integram o Corede Serra. Ressalta-se que a cultura de soja não é predominante na Serra Gaúcha, em virtude da falta de tradição e de conhecimento sobre o manejo da cultura, o que pode refletir no resultado da pegada hídrica. 
A Tabela 5 apresenta a pegada hídrica azul para os suínos abatidos, ano de 2014, na região do Corede Serra.

Tabela 5. Pegada hídrica azul para o processo de confinamento dos suínos.

\begin{tabular}{|c|c|c|c|c|}
\hline Município & $\begin{array}{l}\text { Consumo de água } \\
\text { na dessedentação } \\
\left(\mathrm{km}^{3} \mathrm{ano}^{-1}\right)\end{array}$ & $\begin{array}{l}\text { Consumo de água n } \\
\text { lavagem }\left(\mathrm{km}^{3} \mathrm{ano}^{-1}\right)\end{array}$ & $\begin{array}{l}\text { a Água embutida no } \\
\text { produto }\left(\mathrm{km}^{3} \mathrm{ano}^{-1}\right)\end{array}$ & $\begin{array}{l}\text { Pegada hídrici } \\
\text { azul } \\
\left(\mathrm{km}^{3} \mathrm{ano}^{-1}\right)\end{array}$ \\
\hline Antônio Prado & 0,0000235 & 0,0000001090 & 0,00000257 & 0,0000261 \\
\hline Bento Gonçalves & 0,0000001 & 0,0000000003 & 0,00000001 & 0,0000001 \\
\hline Boa Vista do Sul & 0,0000002 & 0,0000000009 & 0,00000002 & 0,0000002 \\
\hline Carlos Barbosa & 0,0000296 & 0,0000001374 & 0,00000324 & 0,0000329 \\
\hline Caxias do Sul & 0,0000203 & 0,0000000943 & 0,00000222 & 0,0000226 \\
\hline Coronel Pilar & 0,0000070 & 0,0000000326 & 0,00000077 & 0,0000078 \\
\hline Cotiporã & 0,0000170 & 0,0000000790 & 0,00000186 & 0,0000189 \\
\hline Fagundes Varela & 0,0000081 & 0,0000000378 & 0,00000089 & 0,0000091 \\
\hline Farroupilha & 0,0000036 & 0,0000000168 & 0,00000040 & 0,0000040 \\
\hline Flores da Cunha & 0,0000031 & 0,0000000144 & 0,00000034 & 0,0000035 \\
\hline Garibaldi & 0,0000003 & 0,0000000012 & 0,00000003 & 0,0000003 \\
\hline Guabiju & 0,0000114 & 0,0000000530 & 0,00000125 & 0,0000127 \\
\hline Guaporé & 0,0000226 & 0,0000001050 & 0,00000247 & 0,0000252 \\
\hline Montauri & 0,0000056 & 0,0000000259 & 0,00000061 & 0,0000062 \\
\hline Monte Belo do Sul & 0,0000000 & 0,0000000000 & 0,00000000 & 0,0000000 \\
\hline Nova Araçá & 0,0000319 & 0,0000001483 & 0,00000349 & 0,0000356 \\
\hline Nova Bassano & 0,0000474 & 0,0000002203 & 0,00000519 & 0,0000528 \\
\hline Nova Pádua & 0,0000007 & 0,0000000031 & 0,00000007 & 0,0000008 \\
\hline Nova Prata & 0,0000629 & 0,0000002924 & 0,00000689 & 0,0000701 \\
\hline Nova Roma do Sul & 0,0000223 & 0,0000001034 & 0,00000244 & 0,0000248 \\
\hline Paraí & 0,0000673 & 0,0000003124 & 0,00000736 & 0,0000749 \\
\hline Protásio Alves & 0,0000333 & 0,0000001546 & 0,00000364 & 0,0000371 \\
\hline Santa Tereza & 0,0000012 & 0,0000000054 & 0,00000013 & 0,0000013 \\
\hline São Jorge & 0,0000262 & 0,0000001219 & 0,00000287 & 0,0000292 \\
\hline São Marcos & 0,0000000 & 0,0000000001 & 0,00000000 & 0,0000000 \\
\hline São Valentim do Sul & 0,0000094 & 0,0000000436 & 0,00000103 & 0,0000105 \\
\hline Serafina Corrêa & 0,0000490 & 0,0000002276 & 0,00000536 & 0,0000546 \\
\hline União da Serra & 0,0000411 & 0,0000001911 & 0,00000450 & 0,0000458 \\
\hline Veranópolis & 0,0000218 & 0,0000001011 & 0,00000238 & 0,0000242 \\
\hline Vila Flores & 0,0000392 & 0,0000001820 & 0,00000429 & 0,0000437 \\
\hline Vista Alegre do Prata & 0,0000310 & 0,0000001441 & 0,00000339 & 0,0000346 \\
\hline Total & 0,0006370 & 0,0000029594 & 0,00006970 & 0,0007097 \\
\hline
\end{tabular}


O consumo de água para a dessedentação dos suínos na fase de crescimento resultou em $0,0001554 \mathrm{~km}^{3} \mathrm{ano}^{-1}$ ao passo que na fase de terminação resultou em $0,000481 \mathrm{~km}^{3} \mathrm{ano}^{-1}$, corroborando em um volume de $0,000637 \mathrm{~km}^{3} \mathrm{ano}^{-1}$. Ressalta-se que o volume de água para lavagem/higienização e embutida no produto foram calculadas apenas para os suínos em terminação. Conforme observado na Tabela 5, a pegada hídrica azul total é de $0,0007097 \mathrm{~km}^{3} \mathrm{ano}^{-1}$.

A Tabela 6 apresenta o resultado da pegada hídrica para os suínos abatidos em 2014, no Corede Serra.

Tabela 6. Pegada hídrica dos suínos abatidos no ano de 2014 no Corede Serra.

\begin{tabular}{|c|c|c|c|}
\hline Municípios & $\begin{array}{l}\text { Pegada hídrica } \\
\text { verde total } \\
\left(\mathrm{km}^{3} \mathrm{ano}^{-1}\right)\end{array}$ & $\begin{array}{l}\text { Pegada hídrica azul } \\
\left(\mathrm{km}^{3} \mathrm{ano}^{-1}\right)\end{array}$ & $\begin{array}{l}\text { Pegada hídrica total } \\
\left(\mathrm{km}^{3} \mathrm{ano}^{-1}\right)\end{array}$ \\
\hline Antônio Prado & 0,00399 & 0,0000261 & 0,00401 \\
\hline Bento Gonçalves & 0,00002 & 0,0000001 & 0,00002 \\
\hline Boa Vista do Sul & 0,00004 & 0,0000002 & 0,00004 \\
\hline Carlos Barbosa & 0,00558 & 0,0000329 & 0,00562 \\
\hline Caxias do Sul & 0,00383 & 0,0000226 & 0,00386 \\
\hline Coronel Pilar & 0,00143 & 0,0000078 & 0,00144 \\
\hline Cotiporã & 0,00596 & 0,0000189 & 0,00598 \\
\hline Fagundes Varela & 0,00304 & 0,0000091 & 0,00305 \\
\hline Farroupilha & 0,00068 & 0,0000040 & 0,00069 \\
\hline Flores da Cunha & 0,00070 & 0,0000035 & 0,00071 \\
\hline Garibaldi & 0,00005 & 0,0000003 & 0,00005 \\
\hline Guabiju & 0,00208 & 0,0000127 & 0,00209 \\
\hline Guaporé & 0,00517 & 0,0000252 & 0,00519 \\
\hline Montauri & 0,00182 & 0,0000062 & 0,00183 \\
\hline Monte Belo do Sul & 0,00000 & 0,0000000 & 0,00000 \\
\hline Nova Araçá & 0,01048 & 0,0000356 & 0,01051 \\
\hline Nova Bassano & 0,01540 & 0,0000528 & 0,01545 \\
\hline Nova Pádua & 0,00017 & 0,0000008 & 0,00017 \\
\hline Nova Prata & 0,02336 & 0,0000701 & 0,02343 \\
\hline Nova Roma do Sul & 0,00401 & 0,0000248 & 0,00403 \\
\hline Paraí & 0,02180 & 0,0000749 & 0,02187 \\
\hline Protásio Alves & 0,01160 & 0,0000371 & 0,01164 \\
\hline Santa Tereza & 0,00028 & 0,0000013 & 0,00029 \\
\hline São Jorge & 0,00976 & 0,0000292 & 0,00979 \\
\hline São Marcos & 0,00001 & 0,0000000 & 0,00001 \\
\hline São Valentim do Sul & 0,00303 & 0,0000105 & 0,00304 \\
\hline Serafina Corrêa & 0,01653 & 0,0000546 & 0,01658 \\
\hline União da Serra & 0,01474 & 0,0000458 & 0,01478 \\
\hline Veranópolis & 0,00800 & 0,0000242 & 0,00803 \\
\hline Vila Flores & 0,01260 & 0,0000437 & 0,01265 \\
\hline Vista Alegre do Prata & 0,01138 & 0,0000346 & 0,01142 \\
\hline Total & 0,19754 & 0,0007097 & 0,19825 \\
\hline
\end{tabular}

Rev. Ambient. Água vol. 11 n. 1 Taubaté - Jan. / Mar. 2016 
Conforme observado na Tabela 6, a pegada hídrica total, considerando a soma das pegadas hídricas verde e azul resultou em $0,19825 \mathrm{~km}^{3} \mathrm{ano}^{-1}$. A Tabela 7 apresenta a representatividade percentual das pegadas hídricas verde e azul na pegada hídrica total dos processos analisados.

Tabela 7. Porcentagem que das pegadas hídricas verde e azul na pegada hídrica total.

\begin{tabular}{|c|c|c|}
\hline Municípios & $\begin{array}{l}\text { Pegada hídrica } \\
\text { verde total(\%) }\end{array}$ & $\begin{array}{c}\text { Pegada hídrica } \\
\text { azul(\%) }\end{array}$ \\
\hline Antônio Prado & 99,3 & 0,7 \\
\hline Bento Gonçalves & 99,6 & 0,4 \\
\hline Boa Vista do Sul & 99,5 & 0,5 \\
\hline Carlos Barbosa & 99,4 & 0,6 \\
\hline Caxias do Sul & 99,4 & 0,6 \\
\hline Coronel Pilar & 99,5 & 0,5 \\
\hline Cotiporã & 99,7 & 0,3 \\
\hline Fagundes Varela & 99,7 & 0,3 \\
\hline Farroupilha & 99,4 & 0,6 \\
\hline Flores da Cunha & 99,5 & 0,5 \\
\hline Garibaldi & 99,4 & 0,6 \\
\hline Guabiju & 99,4 & 0,6 \\
\hline Guaporé & 99,5 & 0,5 \\
\hline Montauri & 99,7 & 0,3 \\
\hline Monte Belo do Sul & - & - \\
\hline Nova Araçá & 99,7 & 0,3 \\
\hline Nova Bassano & 99,7 & 0,3 \\
\hline Nova Pádua & 99,6 & 0,4 \\
\hline Nova Prata & 99,7 & 0,3 \\
\hline Nova Roma do Sul & 99,4 & 0,6 \\
\hline Paraí & 99,7 & 0,3 \\
\hline Protásio Alves & 99,7 & 0,3 \\
\hline Santa Tereza & 99,5 & 0,5 \\
\hline São Jorge & 99,7 & 0,3 \\
\hline São Marcos & 99,4 & 0,6 \\
\hline São Valentim do Sul & 99,7 & 0,3 \\
\hline Serafina Corrêa & 99,7 & 0,3 \\
\hline União da Serra & 99,7 & 0,3 \\
\hline Veranópolis & 99,7 & 0,3 \\
\hline Vila Flores & 99,7 & 0,3 \\
\hline Vista Alegre do Prata & 99,7 & 0,3 \\
\hline Total & 99,6 & 0,4 \\
\hline
\end{tabular}

Fonte: Elaborado pelos autores.

Conforme observado na Tabela 7, a pegada hídrica verde é a mais significativa, correspondendo a $99,6 \%$ do total, enquanto a pegada hídrica azul correspondeu por apenas $0,4 \%$. Observa-se que o maior consumo de água está na produção das culturas de milho e soja, que são base para a alimentação dos animais. O resultado obtido corrobora com a afirmação dos autores Schlink et al. (2010) e Mekonnen e Hoekstra (2012), que a alimentação 
é o principal contribuinte para a pegada hídrica na atividade pecuária.

Quanto aos municípios observa-se que Nova Prata apresentou a maior pegada hídrica total $\left(0,02343 \mathrm{~km}^{3} \mathrm{ano}^{-1}\right)$, seguida por Paraí $\left(0,02187 \mathrm{~km}^{3} \mathrm{ano}^{-1}\right)$ e por Serafina Corrêa $\left(0,01658 \mathrm{~km}^{3} \mathrm{ano}^{-1}\right)$. Juntos, esses municípios foram responsáveis por um total de $28,12 \%$ dos suínos abatidos no ano de 2014 no Corede Serra. Os municípios de São Marcos (0,000006 $\left.\mathrm{km}^{3} \mathrm{ano}^{-1}\right)$, Bento Gonçalves $\left(0,00002 \mathrm{~km}^{3} \mathrm{ano}^{-1}\right)$ e Boa Vista do Sul $\left(0,00004 \mathrm{~km}^{3}\right)$ apresentaram as menores pegadas. Os três municípios representam 0,047\% do total de abates do Corede Serra no ano de 2014. Apesar de estes municípios apresentarem uma área colhida de milho (ha) próxima ou abaixo da média, se comparado aos demais municípios e uma produtividade $\left(\mathrm{t} \mathrm{ha}^{-1}\right)$ acima da média, se comparado aos demais municípios do Corede Serra, associado ao fato de não serem produtores de soja, a pegada hídrica total não resultou com um alto valor, em virtude do inexpressivo número de animais abatidos.

De acordo com um trabalho publicado por Palhares (2011), onde foi calculada a pegada hídrica dos suínos abatidos no ano de 2008 na região Centro-Sul do Brasil, o Estado do Rio Grande do Sul, com 6.863.059 suínos abatidos, apresentou uma pegada hídrica verde de $2,6967 \mathrm{~km}^{3} \mathrm{ano}^{-1}$. No mesmo trabalho obteve-se uma representatividade da cultura de milho de 58,30\% e da cultura de soja de $41,70 \%$ sobre o resultado da pegada hídrica verde total.

Na avaliação sobre o Corede Serra, realizada neste trabalho, a pegada hídrica verde da cultura de soja correspondeu a 49,66\% sobre o valor da pegada hídrica verde total, ao passo que a pegada hídrica verde da cultura de milho correspondeu a 50,34\%.

\section{CONCLUSÃO}

Sabendo que a atividade pecuária e, em especial, a atividade suinícola apresenta significativo valor econômico na região, com destaque para os municípios que apresentaram as maiores pegadas hídricas totais, este indicador expressa a necessidade de realizar a gestão dos recursos hídricos de forma prioritária evitando, assim, o risco de não haver oferta de água suficiente para manter a atividade suinícola.

Para reduzir a pegada hídrica verde é necessário aumentar a produtividade de milho e soja, visto que baixas produtividades elevam o seu valor, em virtude da menor eficiência hídrica. Com vistas a promover o aumento da produtividade das culturas e, respectivamente, a redução da pegada hídrica, é necessário investir em tecnologias associadas a políticas públicas que incentivem o aumento da produtividade. Todavia, cabe ressaltar a necessidade de se promover o aumento da produtividade sem causar impactos ao meio ambiente pelo uso excessivo de fertilizantes e outros insumos.

Apesar da pegada hídrica azul ter apresentado um valor bem inferior $(0,4 \%)$ comparado com a verde, esse resultado não diminui sua importância para a gestão dos recursos hídricos. Isso porque a criação de suínos é uma atividade realizada, na sua quase totalidade, de forma intensiva, estando concentrada em determinadas regiões, o que torna a água destinada à dessedentação e à limpeza das áreas de criação, uma ameaça à segurança hídrica das regiões de produção.

Em virtude da dificuldade de se obter informações fidedignas para a realização dos cálculos, tendo em vista diferenças das condições ambientais entre os municípios, variação entre os sistemas produtivos e a ausência de dados consistentes, ressalta-se que os resultados deste trabalho expressam tendências e não valores absolutos.

\section{AGRADECIMENTOS}

Secretaria de Desenvolvimento Econômico, Ciência e Tecnologia do Estado do Rio Grande do Sul; Secretaria de Agricultura, Pecuária e Abastecimento do Estado do Rio Grande 
do Sul; Escritório Regional da EMATER de Caxias do Sul; Dr. Julio César Palhares Pesquisador da EMBRAPA Pecuária Sudeste; Dr. Juliano Gimenez - Professor da Universidade de Caxias do Sul; Geise Macedo dos Santos - Monitora de pesquisa do Instituto de Saneamento Ambiental da Universidade de Caxias do Sul.

\section{REFERÊNCIAS}

ASSOCIAÇÃO BRASILEIRA DE PROTEÍNA ANIMAL - ABPA. Relatório anual de atividades 2014. São Paulo, 2015. Disponível em: http://abpabr.com.br/files/RelatorioAnual_UBABEF_2015_DIGITAL.pdf. Acesso em: 23 out. 2015.

CARRA, S. H. Z. Projeto de melhorias em uma propriedade suinícola de pequeno porte visando um modelo ideal. 2012. Trabalho de conclusão de curso (Engenharia Ambiental) - Universidade de Caxias do Sul, Caxias do Sul, 2012.

CHAPAGAIN, A. K.; HOEKSTRA, A. Y. Virtual water flows between nations in relation to trade in livestock and livestock products. Netherlands: Unesco-IHE, 2003. 45p.

COUTO, L.; SANS, L. M. A. Requerimento de água das culturas. Sete Lagoas: Embrapa Milho e Sorgo, 2002. (Circular Técnico n. 20).

FOOD AND AGRICULTURE ORGANIZATION OF THE UNITED NATIONS - FAO. Livestock Report 2006. Rome, 2006. Disponível em: ftp://ftp.fao.org/docrep/fao/009/a0255e/a0255e.pdf. Acesso em: 10 dez. 2014.

HOEKSTRA, A. Y.; CHAPAGAINS, A. K.; ALDAYA, M. M.; MEKONNEN, M. M. Manual de avaliação da pegada hídrica: estabelecendo o padrão global. São Paulo: Instituto de Conservação Ambiental, 2011.

INSTITUTO BRASILEIRO DE GEOGRAFIA E ESTATÍSTICA - IBGE. IBGE Cidades: produção agrícola municipal: lavoura temporária: 2013. Disponível em: http://www.cidades.ibge.gov.br/xtras/uf.php?lang=\&coduf=43\&search=rio-grande-dosul. Acesso em: 24 nov. 2014.

INSTITUTO DE SANEAMENTO AMBIENTAL DA UNIVERSIDADE DE CAXIAS DO SUL - ISAM. Mapas. Elaborados por Geise Macedo dos Santos. Caxias do Sul, 2015.

JESWANI, H. K.; AZAPAGIC, A. Water footprint: methodologies and a case study for assessing the impacts of water use. Journal of Cleaner Production, v. 19, p. 12881299, 2011. http://dx.doi.org/10.1016/j.jclepro.2011.04.003

MEKONNEN, M. M.; HOEKSTRA, A. Y. A global assessment of the water footprint of farm animal products. Ecosystems, v. 15, n. 3, p. 401-415, 2012. http://dx.doi.org/10.1007/s10021-011-9517-8

NÚCLEO DE ESTUDOS E PESQUISAS EM ALIMENTAÇÃO - NEPA. Tabela brasileira de composição de alimentos versão 2011. Disponível em: http://www.unicamp.br/nepa/taco/. Acesso em: 16 abr. 2015.

OLIVEIRA, P. A.; BELLAVER, C. Balanço da água nas cadeias de aves e suínos. Agricultura Industrial, v. 10, p. 39-44, 2009. 
PALHARES, J. C. P. Pegada hídrica de suínos abatidos nos estados da região centro-sul do Brasil. Revista Acta Scientiarum Animal Sciences, v. 33, n. 3, p. 309-314, 2011. http://dx.doi.org/10.4025/actascianimsci.v33i3.9924

PALHARES, J. C. P. Pegada hídrica de suínos e o impacto de estratégias nutricionais. Revista Brasileira de Engenharia Agrícola e Ambiental, v. 18, n. 5, p. 533-538, 2014. http://dx.doi.org/10.1590/S1415-43662014000500010

PERDOMO, C. C. et al. Produção de suínos e meio ambiente. In: SEMINÁRIO NACIONAL DE DESENVOLVIMENTO DA SUINOCULTURA, 9., 2001, Gramado. Anais... Concórdia: EMPBRAPA Suínos e Aves, 2001. p. 8-24.

PFISTER, S.; BAYER, P.; KOEHLER, A.; HELLWEG, S. Environmental impacts of water use in global crop production: Hotspots and trade-offs with land use. Environment. Science. Technology, v. 45, p. 5761-5768, 2011. http://dx.doi.org/10.1021/es1041755

RIO GRANDE DO SUL. Secretaria do Planejamento, Gestão e Participação Cidadã SEPLAG. Informações do Corede Serra. 2015a. Disponível em: http://www.sri.rs.gov .br/ coredes.asp?cod_corede=16. Acesso em: 14 maio 2015.

RIO GRANDE DO SUL. Secretaria Estadual de Agricultura, Pecuária e Abastecimento SEAPA. Informações sobre os suínos abatidos no ano de 2014 nos municípios que compõem o Corede Serra. Caxias do Sul, 2015b.

SILVA, V. P. R. et al. Uma medida de sustentabilidade ambiental: pegada hídrica. Revista Brasileira de Engenharia Agrícola e Ambiental, v. 17, n. 1, p. 100-105, 2013. http://dx.doi.org/10.1590/S1415-43662013000100014

SCHLINK, A. C.; NGUYEN, M. L.; VILJOEN, G. J. Water requirements for livestock production: a global perspective. Revue Scientifique et Technique (International Office of Epizootics), v. 29, n. 3, p. 603-619, 2010. 


Ambiente \& Água - An Interdisciplinary Journal of Applied Science
ISSN 1980-993X - doi:10.4136/1980-993X
www.ambi-agua.net
E-mail: ambi.agua@gmail.com

\title{
Análise de procedimentos para seleção de áreas prioritárias em programas de pagamento por serviços ambientais hídricos
}

\author{
doi:10.4136/ambi-agua.1782
}

Received: 19 Oct. 2015; Accepted: 15 Dec. 2015

\section{Ana Feital Gjorup ${ }^{1 *}$; Elaine Cristina Cardoso Fidalgo ${ }^{2}$; Rachel Bardy Prado ${ }^{2}$; Azeneth Eufrasino Schuler ${ }^{2}$}

\author{
${ }^{1}$ Universidade Federal Fluminense (UFF), Niterói, RJ, Brasil \\ Programa de Pós-Graduação em Engenharia de Biossistemas (PGEB) \\ ${ }^{2}$ Empresa Brasileira de Pesquisas Agropecuárias (Embrapa solos), Rio de Janeiro, RJ, Brasil \\ Departamento de Núcleo de Geomática (Ngeo) \\ *Autor correspondente: e-mail: ana_feital@hotmail.com, \\ elaine.fidalgo@embrapa.br, rachel.prado@embrapa.br, \\ marisolschuler@gmail.com
}

\section{RESUMO}

A abordagem de serviços ecossistêmicos tem se mostrado promissora para a avaliação das interações entre ecossistema e sociedade ao integrar conceitos ambientais e socioeconômicos o que requer conhecimento interdisciplinar. Porém, sua aplicação encontra limitações devido a lacunas de informação que orientem a tomada de decisão. Diante disso o presente trabalho foi desenvolvido buscando contribuir para a aplicação de princípios de serviços ecossistêmicos, no processo decisório em gestão de recursos hídricos. Seu objetivo é identificar procedimentos e metodologias empregados para a tomada de decisão visando a seleção de áreas prioritárias a serem contempladas em projetos ou programas de compensação por serviços ambientais. Para tal buscou-se na literatura técnica e científica publicações que descrevessem métodos e experiências para seleção de áreas prioritárias; identificaram-se as etapas-chave empregadas no processo de seleção de áreas prioritárias; em seguida fez-se um levantamento dos procedimentos adotados para cada etapa-chave, nos textos reunidos; e, por fim, as informações coletadas foram analisadas e classificadas. Considerando o universo amostral do presente trabalho, observou-se que a seleção de áreas prioritárias foi realizada com base no emprego direto dos critérios previamente determinados. A aplicação de indicadores, bem como a análise espacial, ainda são práticas pouco empregadas. Deve-se ressaltar, contudo, que a maioria dos documentos analisados não se propunha a descrever o processo de seleção de áreas prioritárias em detalhe, o que pode ter resultado em algumas omissões. Embora essas condições possam trazer limitações para as análises efetuadas neste trabalho, os resultados apresentados permitem identificar os principais objetivos, ações e critérios empregados para a seleção de áreas prioritárias em programas/projetos de compensação por serviços ambientais.

Palavras-chave: compensação por serviços ambientais, serviços ecossistêmicos, serviços ambientais hídricos, suporte à decisão. 


\title{
Analysis of selection procedures to determine priority areas for payment for water ecosystem services programs
}

\begin{abstract}
The approach of ecosystem services has shown promise for the evaluation of interactions between ecosystems and society, integrating environmental and socioeconomic concepts which require interdisciplinary knowledge. However, its usefulness in decision making is limited due to information gaps. This study was therefore developed in order to contribute to the application of principles of ecosystem services in the decision-making for water resources management. It aims to identify procedures and methodologies used for decision-making in order to select priority areas to be included in projects or compensation programs for environmental services. To do so, we searched technical and scientific literature describing methods and experiences used to select priority areas. Key steps in the process of selecting priority areas were identified; then a survey was conducted of the procedures adopted for each key step considering the literature selected; and, finally, the information collected was analyzed and classified. Considering the study's sample, we noted that the selection of priority areas was based on the direct use of predetermined criteria. The use of indicators and spatial analyses are practices still scarcely employed. We must highlight, however, that most of the analyzed documents did not aim to describe the process of selecting priority areas in detail, which may have resulted in some omissions. Although these conditions may limit the analysis in this study, the results presented here allow us to identify the main objectives, actions and criteria used to select priority areas for programs or compensation projects for environmental services.
\end{abstract}

Keywords: compensation for ecosystem services, ecosystem services, decision support, water ecosystem services.

\section{INTRODUÇÃO}

Os ecossistemas proporcionam uma variedade de produtos e serviços que contribuem, direta ou indiretamente, com o bem-estar humano (FAO, 2007). Apesar de sua importância para a vida, paradoxalmente, muitos estão seriamente ameaçados pelos impactos causados pelas diversas atividades humanas. Segundo Millennium Ecosystem Assesment (MEA, 2003) os ecossistemas e recursos naturais têm sido substancialmente explorados, degradados e destruídos no último século como consequência do crescimento econômico global.

Diante dos desafios colocados pelos êxitos e fracassos advindos do crescimento econômico, da necessidade de conciliar questões relativas ao desenvolvimento econômico e questões relativas ao meio ambiente, diversas iniciativas internacionais foram tomadas a partir da década de 1970 (Comissão Mundial Sobre o Meio Ambiente e Desenvolvimento, 1991; FAO, 2007; MEA, 2003). Em junho de 2001, o programa Millennium Ecosystem Assessment (MEA), lançado pelo então Secretário-Geral das Nações Unidas, Kofi Annan, analisou como as mudanças nos serviços dos ecossistemas têm afetado o bem-estar humano e podem afetar as pessoas nas próximas décadas e que tipos de respostas podem ser adotadas em escala local, nacional ou global para melhorar a gestão dos ecossistemas e contribuir, assim, para o bemestar humano e a redução da pobreza (MEA, 2003).

Peralta (2014) destaca que para construir uma nova mentalidade que vise à sustentabilidade ambiental, deverá ser reestruturado o processo de incentivos econômicos que conduzem os seres humanos a degradar o meio ambiente, de forma que as pessoas sejam orientadas a tomar decisões e desenvolver estilos de vida sustentáveis. Os instrumentos econômicos são um importante mecanismo para orientar, de maneira sustentável, as atividades dos agentes econômicos e distribuir de uma maneira mais adequada os custos 
socioambientais.

Neste contexto, visando reduzir a degradação dos ecossistemas, o conceito de serviços ecossistêmicos (SE) se tornou tema central no planejamento conservacionista (Koschke et al., 2012; Fisher e Turner, 2008). A abordagem de SE segundo Burkhard et al. (2009) tem sido bastante empregada, dentre vários motivos, por seu caráter integrador, que possibilita pesquisas interdisciplinares, reunindo conceitos ambientais e socioeconômicos.

Observa-se na literatura o emprego de dois diferentes termos: serviços ecossistêmicos e serviços ambientais. Os serviços ecossistêmicos, segundo definição de MEA (2003), refletem os benefícios diretos e indiretos providos pelo funcionamento dos ecossistemas, sem a interferência humana. Já nos serviços ambientais, os benefícios gerados estão associados também a ações de manejo do homem nos sistemas naturais ou agroecossistemas. Englobam tanto os serviços proporcionados ao ser humano por ecossistemas naturais (os serviços ecossistêmicos), quanto os providos por ecossistemas manejados ativamente pelo homem (agricultura agroflorestal, manejo florestal, reflorestamento) (Guedes e Seehusen, 2011). Muitas vezes os autores não diferenciam os termos, sendo comum o emprego de serviços ecossistêmicos na literatura internacional e serviços ambientais na nacional. Neste trabalho, empregamos o termo serviços ambientais a todos os serviços proporcionados pelos ecossistemas, manejados ou não pelo homem.

Considerando que o homem pode influenciar positivamente a oferta de serviços ambientais a partir da sua escolha em adotar práticas agrícolas diversificadas e sustentáveis (Muradian et al., 2009), tem-se intensificado a criação e aplicação de mecanismos de incentivo a essas práticas. Diversos autores salientam as vantagens de aplicação de mecanismos econômicos de gestão ambiental que utilizam sistemas de incentivos e desincentivos, financeiros ou fiscais visando orientar o mercado de forma que as diversas atividades econômicas sejam realizadas de maneira sustentável (Peralta, 2014; Rincón-Ruiz, 2014). Peralta (2014) ressalta o uso crescente do sistema de Pagamento por Serviços Ambientais (PSA) como um instrumento econômico de gestão ambiental com o intuito de internalizar externalidades ambientais positivas. No Brasil, segundo Pagiola et al. (2013), nos últimos anos tem sido observado um número crescente de esquemas de PSA. Um dos exemplos de emprego de mecanismo de PSA voltado à melhoria e proteção de recursos hídricos é o Programa Produtor de Água, da Agência Nacional de Águas, do Ministério do Meio Ambiente do Brasil. O Programa se efetiva por meio de articulações e parcerias entre instituições das esferas municipal, estadual, federal e privada, visando o desenvolvimento da política de PSA como um instrumento de incentivo aos produtores rurais que adotarem, de forma voluntária, práticas voltadas à conservação de água e solo (ANA, 2012).

Uma questão que se coloca a programas de PSA bem como a todos os programas e projetos que atuam através de mecanismos de incentivo é onde atuar prioritariamente. Essa questão é colocada em termos econômicos uma vez que os recursos financeiros para aplicação nesses programas são sempre limitados, mas também envolve a priorização para direcionar esforços de ação para áreas onde as intervenções produzirão os melhores benefícios (Orsi et al., 2011).

As vantagens da aplicação do conceito de serviços ecossistêmicos ou ambientais têm sido bastante exploradas. Do ponto de vista analítico, como salienta De Groot (2006), pode prover uma relação sistemática dos componentes e processos ecossistêmicos mais importantes e a dependência que as sociedades humanas têm deles. Entretanto, Burkhard et al. (2009) destacam que a aplicação do conceito de bens e serviços ecossistêmicos em nível de paisagem encontra limitações devido às lacunas de dados apropriados e de sua quantificação, ou seja, falta informação diretamente aplicável em escala regional ou local que oriente a tomada de decisão.

Nas últimas duas décadas, diferentes categorias de SE foram propostas buscando uma 
melhor compreensão dos mesmos (MEA, 2003; Haines-Young e Potschin, 2010), embora não haja consenso em torno de um único modelo de classificação. Haines-Young e Potcshin (2010) afirmam que dificilmente uma lista simples e genérica de classificação dos SE reuniria toda a diversidade de ecossistemas e regiões. Entretanto a classificação dos SE tem sido empregada como guia, orientando levantamentos e monitoramentos desses serviços. No caso dos serviços relacionados aos recursos hídricos, descrevem-se dois tipos: a) os serviços de provisão de água, que asseguram o abastecimento de água para a população; e os de regulação hídrica, que se relacionam às características regulatórias dos processos ecossistêmicos que refletem na quantidade e qualidade da água (MEA, 2003).

O presente trabalho foi desenvolvido buscando contribuir para a aplicação de princípios de serviços ecossistêmicos no processo decisório em gestão de recursos hídricos.

Seu objetivo geral foi identificar, compilar e analisar procedimentos e metodologias empregados para a tomada de decisão, visando à seleção de áreas prioritárias a serem contempladas em projetos ou programas de compensação por serviços ambientais.

\section{MATERIAL E MÉTODOS}

O trabalho foi desenvolvido como uma pesquisa exploratória descritiva, baseando-se no levantamento, análise e síntese de métodos propostos e de experiências relatadas destinadas à seleção de áreas prioritárias para pagamento ou outras formas de compensação pelo fornecimento de serviços ambientais, com maior enfoque nos serviços ambientais relacionados à provisão e regulação hídrica.

O trabalho iniciou com a identificação de palavras-chave para selecionar publicações ou relatórios que descrevessem métodos e experiências para a seleção de áreas prioritárias.

As palavras-chave foram empregadas em buscas em uma base de publicações sobre serviços ambientais hídricos organizada e descrita por Lyra et al. (2014). Essa base reunia à época 278 documentos sobre serviços ambientais. As principais fontes de informação são publicações catalogadas, documentos governamentais, relatórios de organizações internacionais e projetos locais em páginas da internet, incluindo artigos científicos, relatórios, manuais e comunicados que expressam experiências relevantes no tema. Dentre os documentos, há referências a programas de compensação por serviços ambientais no Brasil e em outras partes do mundo, sendo priorizadas as relacionadas aos serviços ambientais hídricos. Á época desta pesquisa, a base de publicações reunia documentos datados de 1996 a 2014, porém os documentos selecionados por sua relação com o tema abordado são do período entre 2006 e 2014.

A organização da informação relativa a procedimentos e métodos para a seleção de áreas prioritárias foi feita segundo as etapas deste processo, sendo dividida em:

- definição dos objetivos do processo de seleção de áreas prioritárias;

- definição das ações propostas para intervenção;

- estabelecimento de critérios para a seleção;

- realização de ponderação aplicada aos critérios;

- aplicação de método para integração de dados; e

- aplicação de método de validação dos resultados.

$\mathrm{Na}$ análise dos documentos foram compiladas as informações sobre cada um dos itens acima, quando existentes. No caso de experiências que se referiam a programas ou projetos de compensação por serviços ambientais, também foram registrados o nome do programa, o país, e quando informada, a localidade.

Para a organização e a análise das informações, foram adotados como referências o trabalho desenvolvido por Bennett et al. (2012) e o manual do Programa Produtor de Água da 
Agência Nacional de Águas (ANA, 2012). O trabalho de Bennett et al. (2012) foi adotado por trazer informações atualizadas oriundas da análise de vários programas de PSA em bacias hidrográficas que ocorrem em todo o mundo. O documento reúne informações de 205 programas ativos, sendo 76 em desenvolvimento. O manual do Programa Produtor de Água da Agência Nacional de Águas (ANA, 2012) também foi escolhido como referência porque estabelece orientações para o programa nacional que tem como objetivo geral apoiar projetos de PSA de proteção hídrica que visem promover a melhoria da qualidade e a ampliação da oferta das águas e a regularização da vazão dos corpos hídricos.

\section{RESULTADOS E DISCUSSÃO}

\subsection{Levantamento inicial}

A aplicação das palavras-chave para levantamento nos 278 documentos na base de dados permitiu selecionar previamente 57 deles que poderiam fazer referência à seleção de áreas prioritárias para programas de compensação por serviços ambientais. A análise dos 57 documentos resultou na identificação de 17 que apresentavam procedimentos para a seleção de áreas prioritárias. Um desses documentos, fornecido pela TNC no formato de relato de experiência, não foi publicado.

Os 17 documentos analisados (ANA, 2012; Bernardes, 2010; Bochner, 2010; Instituto Estadual de Florestas, 2011; Haaren et al., 2012; Micol et al., 2008; Guatemala, 2012; Mota et al., 2013; Muñoz-Piñaa et al., 2008; Naidoo e Ricketts, 2006; Padovezi et al., 2011; Zhang e Pagiola, 2011; Peralta, 2014; Pagiola et al., 2013; Pereira, 2013; Rio de Janeiro et al., 2012; Silva et al., 2013; Ulber et al., 2011), descreveram 29 métodos de seleção de áreas prioritárias, provenientes de seis países. No Brasil foram identificadas 20 experiências de programas que envolveram a seleção de áreas prioritárias.

Importante destacar que a base de dados consultada tem como principal foco experiências relacionadas aos serviços ambientais hídricos. Dos 29 métodos levantados, 21 tinham como foco recursos hídricos; sendo que seis incluíam biodiversidade e dois incluíam carbono. Esses oito relatos de experiências que não tratavam de forma explícita os recursos hídricos foram incluídos por contribuírem com a descrição de procedimentos para seleção de áreas prioritárias.

\subsection{Objetivos para seleção de áreas prioritárias}

Foram classificados três tipos de objetivos: 1) objetivos diretamente relacionados à melhoria dos serviços hídricos, 2) objetivos visando proporcionar benefícios socioeconômicos e 3) objetivos visando proporcionar melhoria de outros serviços ambientais (Figura 1). Cabe salientar que alguns documentos analisados não descreviam claramente seus objetivos. Eles foram recuperados ao longo da descrição do projeto ou programa, com base nas ações propostas, no processo para a seleção de áreas de intervenção ou mesmo nos resultados obtidos.

Os objetivos diretamente relacionados à melhoria dos serviços hídricos foram os mais relatados, o que era esperado, uma vez que este trabalho priorizou a análise de experiências da aplicação de serviços ambientais em gestão de recursos hídricos.

Considerando esse conjunto de objetivos, os mais citados são aqueles relacionados diretamente à provisão de água de qualidade: regular o fluxo hídrico e melhorar a qualidade da água. Os demais objetivos - controlar sedimentos, melhorar a infiltração da água no solo, conservar as bacias hidrográficas, proteger as nascentes e controlar a poluição da água embora expressem forte relação com os serviços e sejam capazes de alterá-los, são menos citados. 

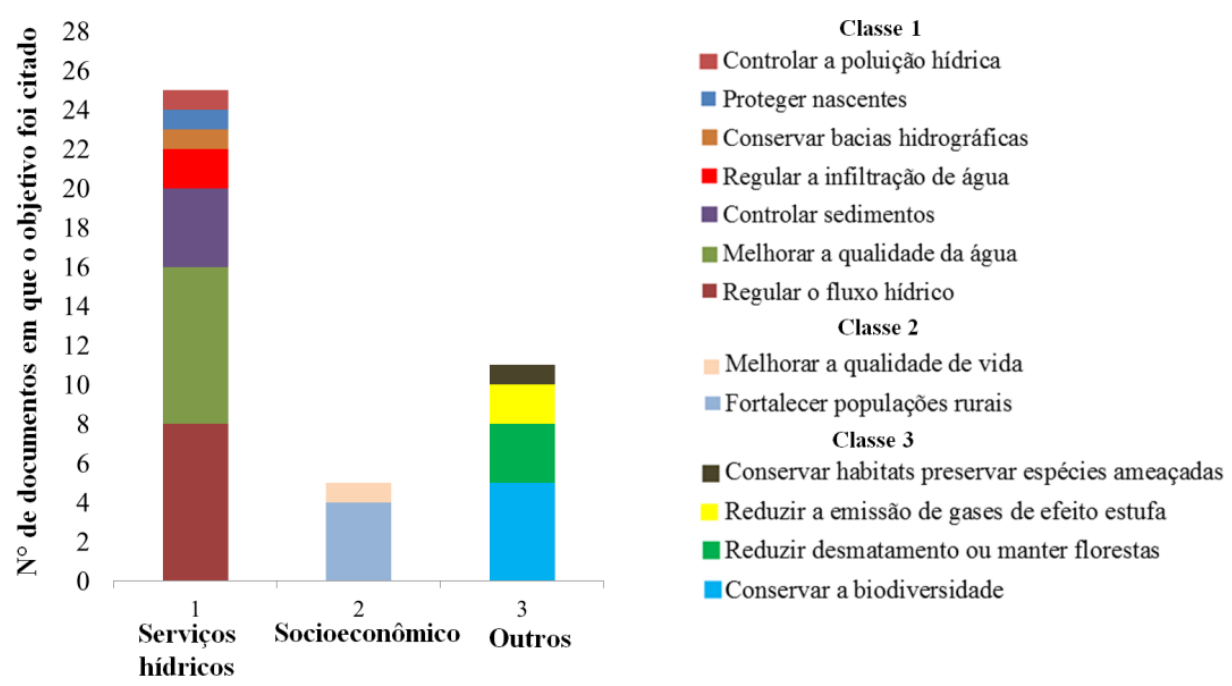

Figura 1. Objetivos do processo de seleção de áreas prioritárias observados nos documentos analisados.

Considerando os aspectos socioeconômicos, predominou o objetivo "fortalecer populações rurais", que reflete as características dos programas descritos na bibliografia considerada, os quais envolvem prioritariamente as populações rurais como fornecedoras de serviços ambientais. Como exemplo deste tipo de objetivo podemos citar "promover a permanência das populações rurais em suas comunidades”.

Dentre os objetivos que visam proporcionar melhoria de outros serviços ambientais, os mais frequentes foram conservar a biodiversidade (citado em cinco documentos), reduzir desmatamento ou manter florestas (3) e reduzir a emissão de gases de efeito estufa (GEE) (2).

\subsection{Ações propostas para intervenção}

As ações de intervenção foram analisadas segundo classificação proposta por Bennett (2012). A Figura 2 mostra o número de ações de intervenção propostas nos documentos analisados segundo essa classificação e a Tabela 1 apresenta um detalhamento dessas ações.

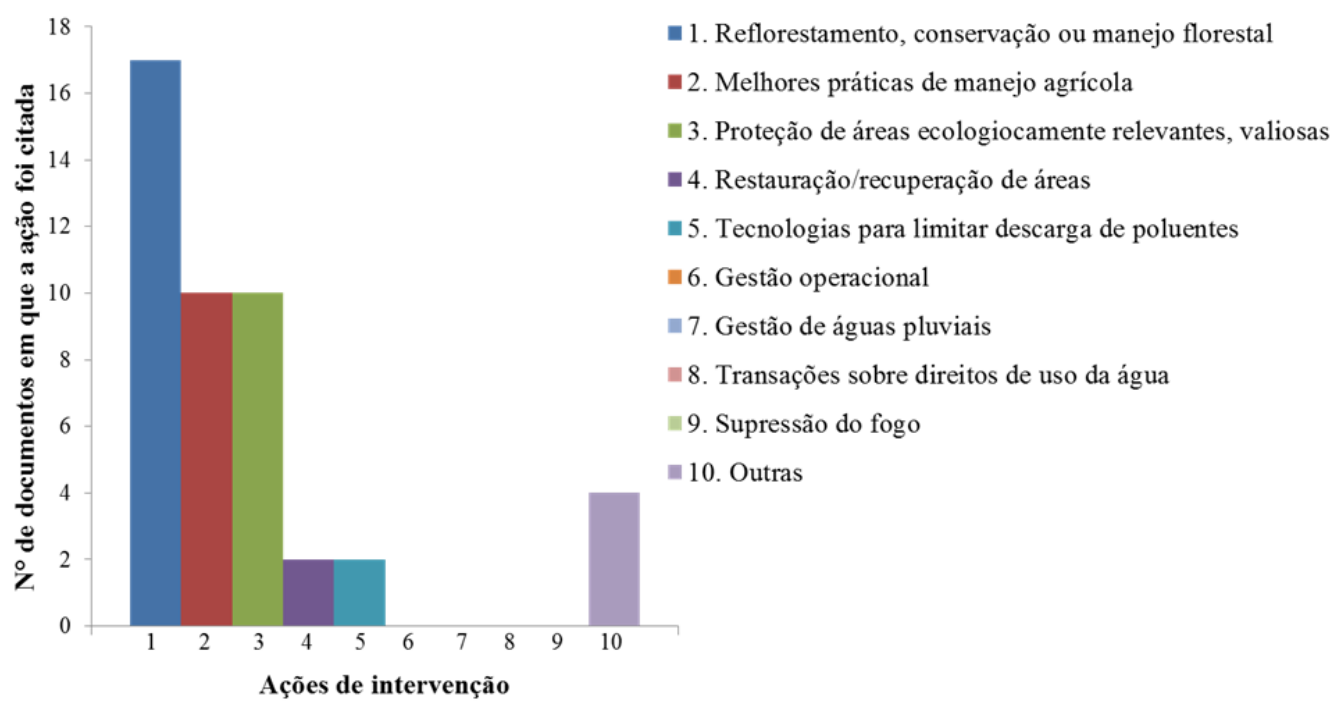

Figura 2. Ações propostas para intervenção observadas nos documentos analisados. 
Tabela 1. Ações propostas para intervenção.

\section{Ações}

Número de

casos

\section{Reflorestamento, conservação ou manejo florestal}

Manutenção ou proteção de áreas de floresta ou vegetação natural

Reflorestamento ou condução de regeneração com espécies nativas

Envolvem os dois tipos de ação (manutenção/proteção ou implantação de cobertura vegetal)

\section{Desenvolvimento de melhores práticas agrícolas}

Desenvolvimento de melhores práticas agrícolas

Apoiar/incentivar a produção em bases sustentáveis (aumento da diversidade

de plantas cultivadas, sistemas agroflorestais, manejo florestal)

\section{Proteção de áreas ecologicamente relevantes}

Manutenção/proteção ou implantação de cobertura vegetal em Área de

Preservação Permanente (APP)

Manutenção/proteção ou implantação de cobertura vegetal em APP e/ou

Reserva Legal (RL)

Manutenção/proteção ou implantação de cobertura vegetal em outras áreas (proximidade com unidade de conservação)

\section{Recuperação ou restauração de áreas}

Recuperação ou restauração de áreas degradadas

5. Tecnologias para limitar descarga de poluentes

Controle de disposição de resíduos sólidos e tratamento de efluentes

\section{Outras}

Medidas para conservação da biodiversidade

Medidas para redução das disparidades de desenvolvimento social

Não foram observadas nos documentos analisados as ações de intervenção classificadas como gestão operacional, gestão de águas pluviais, transações sobre direitos de uso da água e supressão do fogo.

\subsection{Critérios para priorização}

Os critérios para a seleção de áreas prioritárias ao desenvolvimento das ações propostas levantados nos documentos analisados foram divididos em dois grupos: os de elegibilidade e os de priorização de áreas à intervenção.

Os critérios de elegibilidade são aplicados para selecionar o público-alvo conforme as demandas do programa. A elegibilidade, em geral, é uma análise prévia, não estabelece prioridades, mas sim condições prévias para a participação, sendo empregada de forma independente ao processo de seleção de áreas prioritárias, por exemplo, a disposição do produtor em participar, ou a obrigatoriedade do produtor residir na bacia selecionada.

O levantamento dos critérios de priorização de áreas à intervenção revelou a diversidade destes. Alguns foram estabelecidos para identificar regiões prioritárias, que podem ser bacias hidrográficas, municípios ou outras delimitações definidas a priori pelo programa.

Outros critérios foram definidos para selecionar propriedades ou posses cujas áreas apresentam condições prioritárias para as ações propostas. Apresentamos a seguir os critérios 
levantados, destacando os casos em que são aplicados especificamente para a seleção de propriedades ou posses.

\subsubsection{Critérios relacionados aos recursos hídricos:}

- Importância da bacia para abastecimento;

- Ocorrência de alta escassez de água ou alto risco de inundação;

- Existência de conflito pelo uso da água;

- $\quad$ Nível de degradação da bacia e presença de usos conflitantes com conservação;

- $\quad$ Índice de urbanização;

- Potencial de redução de aporte de sedimentos mediante intervenção;

- $\quad$ Suscetibilidade a erosão;

- Características físicas, como limiar de declividade, limiar de gradiente altitudinal, limiar de densidade de drenagem;

- Limiar de média pluviomética; e

- Considerando a seleção de propriedades ou posses: localização na bacia hidrográfica (montante ou jusante), presença e/ou estado de conservação de APP, localização em áreas cársticas, localização em áreas de recarga, presença de cobertura vegetal, proximidade com Unidade de Conservação (UC), limiar de declividade.

\subsubsection{Critérios relacionados à biodiversidade:}

- Presença de alta biodiversidade;

- Localização em relação a áreas protegidas e corredores biológicos;

- Ocorrência de espécies ameaçadas ou em risco de extinção;

- Tamanho mínimo de fragmentos, tamanho e distância de fragmentos;

- Potencial de conectividade;

- Valor da área de vegetação natural - estabelecido segundo seu potencial de ocorrência de carne de caça, de madeira comercializável, potencial para bioprospecção, valor de existência, armazenamento de carbono, custo considerando conversão para agricultura; e

- Considerando a seleção de propriedades ou posses: proximidade com unidades de conservação; presença de áreas de vegetação natural ou proporção de área de vegetação natural; presença de vegetação em estágios mais avançados de sucessão; presença de reserva legal averbada; presença de vegetação em percentual superior ao mínimo destinado à reserva legal; e diversidade de plantas cultivadas.

\subsubsection{Critérios relacionados ao carbono:}

- Condição da área em 1990 e atualmente em relação à cobertura da vegetação nativa;

- Valor Potencial do PSA ${ }^{1}$.

\subsubsection{Critérios relacionados ao desenvolvimento social:}

- $\quad$ Potencial de redução de pobreza (índice de pobreza, concentração de pobreza na região, índice de desenvolvimento social); e

- Considerando a seleção de propriedades ou posses: produtores familiares; proprietários que recebam compensações por outro programa executado dentro da

${ }^{1}$ O Valor Potencial do PSA é dado pela expressão:

Valor Potencial do PSA = Adicionalidade Potencial - Custo Potencial em que, Adicionalidade Potencial $=$ Probabilidade de desmatamento $\mathrm{x}$ Massa de Carbono $\mathrm{x}$ Valor de mercado de carbono e Custo Potencial $=$ Custo de oportunidade de conservação. 
área; propriedades ou posses objeto de aplicação de financiamento na linha de conservação ambiental ou agroecológica; propriedades/posses vinculadas a projetos públicos de inclusão social no campo; propriedades/posses vinculadas a políticas públicas destinadas à juventude rural; propriedades/posses que participem de projetos associativos de produção; propriedades ou posses pertencentes a povos tradicionais; e propriedades ou posses localizadas em UCs.

\subsubsection{Outros critérios:}

- Áreas declaradas como prioritárias em regulamentação específica (legislação, planos diretores de bacia ou municipais) ou definidas em levantamentos previamente realizados;

- Existência de instituições parceiras com atuação local;

- Existência de projetos ambientais desenvolvidos por entidades locais;

- Município com equipe atuante na área de meio ambiente; e

- Considerando a seleção de propriedades ou posses: coerência do produtor com escopo do projeto ou cumprimento da legislação ambiental; e existência de práticas amigáveis de uso da terra - conservação do solo, produção sem uso de agrotóxico, prática de controle biológico, produção agroecológica.

O Programa Produtor de Água (ANA, 2012) descreve critérios para a seleção de subbacias para o desenvolvimento das ações do programa. Segundo o programa, a sub-bacia é elegível se atender a pelo menos um dos critérios descritos a seguir, sendo prioritária aquela que atender ao maior número deles:

- ser manancial de abastecimento de água para uso urbano ou industrial;

- $\quad$ ser manancial de fornecimento de água para a geração de energia elétrica;

- estar inserida em bacias hidrográficas que já tenham os instrumentos de gestão, previstos na Lei 9.443/97, implementados;

- estar inserida em uma bacia hidrográfica cujo Plano de Recursos Hídricos identifique problemas de poluição difusa de origem rural, erosão e déficit de cobertura vegetal em áreas legalmente protegidas;

- $\quad$ ter um número mínimo de produtores rurais interessados que possa viabilizar a aplicação do Programa;

- estar em situação de conflito de uso dos recursos hídricos; e

- estar sujeita a eventos hidrológicos críticos recorrentes.

Nas sub-bacias selecionadas, são realizados projetos individuais de propriedades, sendo priorizados os projetos que aportam maiores benefícios ambientais, ou seja, que alteram, de modo significativo, a qualidade da água da sub-bacia ou promovam a redução da erosão e a melhoria da infiltração de água. Em parte os critérios para a seleção de sub-bacias propostos pelo Programa Produtor de Água se assemelham aos critérios levantados nos demais documentos.

\subsection{Ponderação dos critérios}

Em relação à atribuição de peso aos critérios, observa-se que não é uma prática comum diferenciar os critérios segundo sua importância ou relevância na análise. Somente em um documento (Bochner, 2010) foi descrito o emprego de um método de ponderação. Nesse caso, foi feita uma atribuição de pesos aos critérios segundo sua importância. Os pesos, no intervalo entre 1 e 10, foram atribuídos individualmente por especialistas. Os resultados individuais foram reunidos e analisados utilizando o método Processo Analítico Hierárquico (AHP) (Saaty, 1980), para se obter o peso final para cada critério. 
A ausência de ponderação simplifica a análise, por outro lado, ela a restringe ao pressupor que todos os critérios têm o mesmo grau de importância. A vantagem de aplicação de ponderação é evidenciada no estudo de Bochner (2010) ao permitir considerar diferentes pesos aos critérios empregados, que podem refletir sua importância ou impacto para o alcance dos objetivos. Essa vantagem, aliada à escassez de métodos de ponderação empregados nos casos estudados, aponta para a necessidade de estudos visando a identificação e aplicação de técnicas de ponderação adequadas para a priorização de áreas de intervenção em PSA.

\subsection{Métodos de análise integrada}

O levantamento dos métodos aplicados para a análise integrada do conjunto dos critérios mostrou que há uma diversidade de abordagens. Dos 29 métodos ou procedimentos descritos, 28 descreveram algum tipo de método para integrar os critérios utilizados.

$\mathrm{Na}$ maioria dos casos, com 17 ocorrências, os critérios foram aplicados diretamente e selecionados aqueles que atendiam o maior número deles. Em quatro casos foram relatados o emprego de um indicador: a) o índice de suscetibilidade à erosão (The Nature Conservancy, 2013), b) o índice de percentual de abatimento de erosão (PAE) (Pagiola et al., 2013), c) o valor potencial da aplicação do PSA (Micol et al., 2008) e d) o aporte de sedimentos (The Nature Conservancy, 2013). Três documentos citam a sobreposição de mapas e aplicação de critérios: a) sobreposição de mapeamentos diversos (Zhang e Pagiola, 2011); sobreposição de mapeamento de suscetibilidade à erosão, principais mananciais e corredores ecológicos (relato de experiência fornecido por The Nature Conservancy em 2013); e 'área ativa de rio' que considera a manutenção da conectividade hidrológica e a variabilidade natural das áreas ripárias desde a nascente até a foz de um rio (Pagiola et al., 2013). Por último, com uma ocorrência cada, tem-se: análise custo-benefício espacial considerando estimativas dos custos de oportunidade de conservação (Naidoo e Ricketts, 2006); abordagem multicriterial em ambiente de Sistema de Informação Geográfica (SIG), empregando Combinação Linear Ponderada (CLP) (Bochner, 2010); avaliação da biodiversidade através de indicadores (Sistema MANUELA) (Haaren et al., 2012); e leilão baseado em propostas dos agricultores contendo listagem de serviços ambientais fornecidos e valor a receber (Ulber et al., 2011).

\subsection{Validação}

O emprego de métodos de validação foi observado em apenas dois casos: um que verificou a robustez aplicando outro método sobre os mesmos dados e comparando os resultados (Haaren et al., 2012); e o outro realizou testes aplicando o método em áreas nas quais havia PSA em andamento e comparou os resultados (Zhang e Pagiola, 2011).

A validação proporciona maior confiabilidade nos resultados. O número escasso de trabalhos que aplicam técnicas de validação justifica a necessidade de estudos visando a identificação, aplicação e avaliação dessas técnicas.

\section{CONCLUSÕES}

A análise dos documentos que relatam procedimentos para seleção de áreas prioritárias para programas de compensação por serviços ambientais revelou que os objetivos do processo de seleção de áreas prioritárias nem sempre são explicitados, constituindo-se em uma lacuna de informação que pode prejudicar a definição das intervenções necessárias e os critérios para seleção de locais prioritários à sua execução.

Dentre as intervenções mais frequentemente propostas para as áreas selecionadas tem-se o florestamento / reflorestamento ou manejo florestal, o desenvolvimento de melhores práticas agrícolas e a proteção de áreas ecologicamente relevantes.

A seleção de áreas prioritárias foi realizada na maior parte dos casos com base no 
emprego direto dos critérios previamente determinados. A aplicação de indicadores, bem como a análise espacial, ainda são práticas pouco empregadas.

Considerando a diversidade de critérios e a especificidade observada em alguns dos casos levantados, conclui-se que seu desenvolvimento e aplicação são bastante voltados às condições locais ou regionais ou a objetivos específicos do programa analisado.

É necessário que seja dada maior atenção à análise conjunta dos critérios, principalmente se considerarmos que pode haver alta correlação, seja positiva ou negativa, entre eles, o que pode levar a uma avaliação tendenciosa das áreas prioritárias, com sobrevalorização ou desvalorização de algumas de suas características.

Deve-se ressaltar, contudo, que a maioria dos documentos analisados não se propunha a descrever o processo de seleção de áreas prioritárias em detalhe, o que pode ter resultado em algumas omissões. Embora essas condições possam trazer limitações para as análises efetuadas neste trabalho, os resultados aqui apresentados permitem identificar os principais objetivos, ações e critérios empregados para a seleção de áreas prioritárias em programas/projetos de compensação por serviços ambientais. Além disso, indicam a necessidade urgente de que esses projetos ou programas sistematizem e relatem suas experiências na área.

\section{AGRADECIMENTOS}

Agradecemos à Embrapa pelo financiamento do projeto "Fortalecimento do conhecimento, organização da informação e elaboração de instrumentos de apoio aos Programas de Pagamentos por Serviços Ambientais Hídricos no meio rural”, no âmbito do qual este trabalho foi desenvolvido. Agradecemos a João Guimarães, da TNC, parceiro deste projeto, pelas informações fornecidas sobre o levantamento de áreas estratégicas para ações de conservação e produção de água no Espírito Santo.

\section{REFERÊNCIAS}

AGÊNCIA NACIONAL DE ÁGUAS - ANA (Brasil). Manual operativo do programa Produtor de Água. 2. ed. Brasília, 2012. 84 p.

BENNETT, G.; CARROLL, N.; HAMILTON, K. Charting new waters: state of watershed payments. Washington, DC: Forest Trends, 2012. Disponível em: <http://www.ecosystemmarketplace.com/reports/sowp2012>. Acesso em: 10 mar. 2015

BERNARDES, A. C. Pagamento por serviços ambientais: experiências brasileiras relacionadas à água. In: ENCONTRO NACIONAL DA ANPPAS, 5., 4-7 outubro 2010. Anais... Florianópolis: ANPPAS, 2010.

BOCHNER, J. K. Proposta metodológica para identificação de áreas prioritárias para recomposição florestal - estudo de caso: bacia hidrográfica do rio Macacu-RJ. 2010. 135f. Dissertação (Mestrado em Ciências) Pós-Graduação em Ciências Ambientais e Florestais, Universidade Federal Rural do Rio de Janeiro, Seropédica, 2010.

BURKHARD, B.; KROLL, F.; MÜLLER, F.; WINDHORST, W. Landscapes' capacities to provide ecosystem services: a concept for land-cover based assessments. Landscape Online, v. 15, p. 1-22, 2009.

COMISSÃO MUNDIAL SOBRE O MEIO AMBIENTE E DESENVOLVIMENTO. Nosso futuro comum. 2. ed. Rio de Janeiro: FGV, 1991. 428 p. 
DE GROOT, R. S. Function-analysis and valuation as a tool to assess land use conflicts in planning for sustainable, multi-functional landscapes. Landscape and Urban Planning, v. 75, p. 175-186, 2006. http://dx.doi.org/10.1016/j.landurbplan.2005.02.016

FOOD AND AGRICULTURE ORGANIZATION OF THE UNITED NATIONS - FAO. Land evaluation: towards a revised framework. Rome, 2007 (Land and Water Discussion Paper, n. 6).

FISHER, B.; TURNER, R. K. Ecosystem services: classification for valuation. Biological conservation, v. 141, p. 1167-1169, 2008.

http://dx.doi.org/10.1016/j.biocon.2008.02.019

GUATEMALA. Ministerio de Ambiente y Recursos Naturales. Lecciones aprendidas sobre los esquemas de compensación o pago por servicios ambientales implementados en el Corredor Seco con el apoyo del Efecto 4 del Programa Conjunto. Guatemala, 2012. 33 p.

GUEDES, F. B.; SEEHUSEN, S. E. (Orgs.). Pagamentos por serviços ambientais na Mata Atlântica: lições aprendidas e desafios. Brasília: MMA, 2011. 272 p. (Biodiversidade, n. 42)

HAAREN, C. VON; KEMPA, D.; VOGEL, K.; RÜTER S. Assessing biodiversity on the farm scale as basis for ecosystem service payments. Journal of Environmental Management, v. 113, p. 40-50, 2012. http://dx.doi.org/10.1016/j.jenvman.2012.07.033

HAINES-YOUNG, R.; POTSCHIN, M. The links between biodiversity, ecosystem services and human well-being. In: RAFAELLI, G. D.; FRID, C. L. J. (Eds.). Ecosystem ecology: a nex synthesis. Cambridge: Cambridge University Press, 2010. p. 110-139.

INSTITUTO ESTADUAL DE FLORESTAS. Diretoria de Desenvolvimento e Conservação Florestal. Bolsa verde: manual de princípios, critérios e procedimentos para a implantação da lei n.17.727 de agosto de 2008. Belo Horizonte, 2011.

KOSCHKE, L.; FÜRST, C.; FRANK, S.; MAKESCHIN, F. A multi-criteria approach for an integrated land-cover-based assessment of ecosystem services provision to support landscape planning. Ecological Indicators, v. 21, p. 54-66, 2012. http://dx.doi.org/10.1016/j.ecolind.2011.12.010

LYRA, W. D. S.; LIMA, A. P. M.; ALBUQUERQUE, R. H.; INÁCIO, M. C.; GJORUP, A. F.; MACARIO, C. G. N. M. et al. Methodological approach of a database as support to Programs of Payment for Ecosystem Water Services in Brazil. In: CONFERENCE OF THE ECOSYSTEM SERVICES PARTNERSHIP, 7., San José, CR. Anais... San José: [s.n.], 2014.

MICOL, L.; ANDRADE, J.; BÖRNER, J. Redução das emissões do desmatamento e da degradação (REDD): potencial de aplicação em Mato Grosso. Alta Floresta: Instituto Centro de Vida, 2008.

MILLENIUM ECOSYSTEM ASSESSMENT - MEA. Ecosystems and human well-being: a framework for assessment. Washington, D.C.: Island Press, 2003.

MOTA, A. M.; MENDES, A. S. R.; RIBEIRO, P. Projeto Ecocrédito - Montes Claros. In: PAGIOLA, S.; GLEHN, H. C. V.; TAFFARELLO, D. (Orgs.). Experiências de pagamento por serviços ambientais no Brasil. São Paulo: SMA/CBRN, 2013. 
MUÑOZ-PIÑAA, C.; GUEVARAB, A.; TORRESC, J. M.; BRAÑAA, J. Paying for the hydrological services of Mexico's forests: analysis, negotiations and results. Ecological Economics, v. 65, n. 4, p. 725-736, 2008. http://dx.doi.org/10.1016/j.ecolecon.2007.07.031

MURADIAN, R.; CORBERA, E.; PASCUAL, U.; KOSOY, N.; MAY, P. H. Reconciling theory and practice: an alternative conceptual framework for understanding payments for environmental services. Ecological Economics, v. 69, p. 1202-1208, 2009. http://dx.doi.org/10.1016/j.ecolecon.2009.11.006

NAIDOO, R. L.; RICKETTS, T. H. Mapping the economic costs and benefits of conservation. PLOS biology, v. 4, n. 11, p. 2153-2164, 2006. http://dx.doi.org/10.1371/journal.pbio.0040360

ORSI, F.; GENELETTI, D.; NEWTON, A. C. Towards a common set of criteria and indicators to identify forest restoration priorities: an expert panel-based approach.

Ecological Indicators, v. 11, n. 2, p. 337-347, 2011. http://dx.doi.org/10.1016/j.ecolind.2010.06.001

PADOVEZI, A.; DIEDERICHSEN, A.; VEIGA, F. (Orgs). Projeto conservador das águas passo a passo. Brasilia: The Nature Conservancy do Brasil, 2011. (Água, Clima e Floresta, v. 4).

PAGIOLA, S.; GLEHN, H. C. V.; TAFFARELLO, D. (Orgs.). Experiências de pagamento por serviços ambientais no Brasil. São Paulo: SMA/CBRN, 2013. 274 p.

PERALTA, C. E. O pagamento por serviços ambientais como instrumento para orientar a sustentabilidade ambiental: a experiência da Costa Rica. In: LAVRATTI, P.; TEJEIRO, G. (Orgs.). Direito e mudanças climáticas: pagamento por serviços ambientais: experiências locais e latino-americanas. São Paulo: Instituto O Direito por um Planeta Verde, 2014.143p. (Direito e Mudanças Climáticas, 7).

PEREIRA, P. H. Projeto conservador das águas - Extrema. In: PAGIOLA, S.; GLEHN, H. C. V.; TAFFARELLO, D. (Orgs.). Experiências de pagamento por serviços ambientais no Brasil. São Paulo: SMA/CBRN, 2013. p. 29-40.

RINCÓN-RUÍZ, A.; ECHEVERRY-DUQUE, M.; PIÑEROS, A. M.; TAPIA, C. H.; DAVID, A.; ARIAS-ARÉVALO, P. et al. Valoración integral de la biodiversidade y los servicios ecosistémicos: aspectos conceptuales y metodológicos. Bogotá, D. C.: IAvH, 2014. $151 \mathrm{p}$.

RIO DE JANEIRO. Secretaria Estadual do Ambiente; COMITÊ DE BACIA HIDROGRÁFICA DO RIO GUANDU; RIO CLARO. Prefeitura Municipal; THE NATURE CONSERVANCY; INSTITUTO TERRA DE PRESERVAÇÃO AMBIENTAL. Manual de orientação ao proponente de prestação de serviços ambientais, n' 01/12. Guandu, 2012.

SAATY, T. The analytic hierarchy process. New York: McGraw-Hill, 1980. 287p.

ULBER, L.; KLIMEK, S.; STEINMANN, H.; ISSELSTEIN, J.; GROTH, M. Implementing and evaluating the effectiveness of a payment scheme for environmental services from agricultural land. Environmental Conservation, v. 38, n. 04, p. 464-472, 2011. http://dx.doi.org/10.1017/S0376892911000385 
THE NATURE CONSERVANCY. Annual Report. 2013. Disponível em: http://www.nature.org/media/annualreport/2013-annual-report.pdf. Acesso em dez. 2015.

ZHANG, W.; PAGIOLA, S. Assessing the potential for synergies in the implementation of payments for environmental services programmes: an empirical analysis of Costa Rica. Enviromental Conservation, v. 38, n. 04, p. 406-416, 2011. http://dx.doi.org/10.1017/S0376892911000555 